\title{
THE INFLUENCE OF DESICCATION AND STRESS HISTORY ON MONOTONIC AND CYCLIC SHEAR RESPONSE OF THICKENED \\ GOLD TAILINGS
}

\author{
A thesis submitted to \\ the Faculty of Graduate and Postdoctoral Affairs \\ in Partial Fulfillment of the requirements for the degree \\ Doctor of Philosophy
}

by

Farzad Daliri

\begin{abstract}
Department of Civil and Environmental Engineering Carleton University
\end{abstract}

Ottawa-Carleton Institute of Civil and Environmental Engineering

September 2013

C2013 Farzad Daliri 
This Thesis is dedicated to:

\section{Mohammad Daliri}

Farzaneh Amiri

Farshad Daliri

and

Maryam Hosseini Fatemi 


\begin{abstract}
In thickened tailings technology, tailings are dewatered to the point where they exhibit a yield stress such that they form gently sloping stacks during deposition. Post-deposition, these tailings may densify due to settling, desiccation, and drainage. Fresh tailings subsequently deposited over the desiccated layer and this procedure is continued to facilitate construction of a stable stack. Tailings placed in the stack therefore undergo variable stress histories in terms of degree of desiccation and consolidation. A better understanding of the effects of this stress history on the geotechnical behaviour would lead to safe and economical designs. Most common design objectives in thickened tailings deposition are to improve stability, minimize deformation during earthquakes, facilitate reclamation, and maximize the deposition volume (stack slope).
\end{abstract}

This study investigated the influence of stress history on the monotonic and cyclic shear strength of thickened tailings. The field deposition process was simulated by desiccating layers to different degrees, and subsequently overlaying them with fresh tailings. This was done in a small column, as well as in a $0.7 \mathrm{~m}$ by $1 \mathrm{~m}$ plan "drying box", in which 5 layers were subsequently deposited, desiccated to different degrees, before placement of the next layer. Extracted samples were consolidated prior to shearing under simple shear and triaxial loading modes. Vane shear tests were also employed to complement the experiments. The effects of overconsolidation ratio (OCR) associated with desiccation and mechanical loading were also compared.

A profound effect of the degree of desiccation on the stress-strain behaviour of the tailings was measured. Tailings that have not experienced any desiccation exhibit strain-softening response under simple shear loading. A small degree of desiccation was enough to transform the response 
from contractive to dilative, and generally the degree of strain hardening increased with the degree of desiccation. Desiccated samples also exhibited more stiffness, and had a higher void ratio at a given consolidation pressure, than non-desiccated samples. These results have practical relevance, as the contribution of desiccation stress history has either been previously discounted or underestimated at best. 


\section{ACKNOWLEDGMENTS}

I would like to express the deepest appreciation to my supervisor Professor Paul H. Simms for his continuous support, guidance, motivation, patience, and encouragement throughout my doctoral study. Furthermore, I would like to express my sincere gratitude to my co-supervisor Professor Siva Sivathayalan for his guidance, support and constructive advices. Without their support and generous help this dissertation would have not been completed.

In addition to my advisors, I am also grateful to Professors D.G. Fredlund, Sai Vanapalli, Dharma Wijewickreme, Michael Bozozuk, Mamadou Fall, Mohammad Rayhani and Tim Newson for having enriched my knowledge with their excellent courses or guidance.

I would also like to appreciate the following staff of the Department of Civil and Environmental Engineering at Carleton University for their continuous support and help: Stanely Conley, Dr. Marie Tudoret, Payal Chadha, Pierre Trudel, Jason Arnott, Kay Casselman and Tim Stephens (from University of Western Ontario).

I would also like to thank my fellow graduate students, Dr. Adedeji Dunmola, Dr. Paramaguru Logeswaran, Dr. Farrokh Fazileh, Dr. Kasgin Khaheshi Banab, Dr. Pouria Ghods, Dr. Sahar Soleimani, Dr. S. Ali Etemad, Hyunseung Kim and Mohammad Shahsavari for their persistent friendship and enjoyable conversations regarding laboratory techniques and extracurricular activities. 
Financial support provided by the National Research Council of Canada (NSERC), Golder Associates and Carleton University is deeply appreciated. I would also like to acknowledge Barrick Gold Corporation for providing gold tailings.

I would like to thank all my family especially my father Mohammad Daliri, my mother Farzaneh Amiri and my brother Farshad Daliri. Finally, I would like to greatly appreciate my wife Maryam Hosseini Fatemi for her constant support, patience, understanding and help, which helped me to keep focused during my study period. 


\section{TABLE OF CONTENTS}

$\begin{array}{lll}\text { ABSTRACT } & \text { III }\end{array}$

ACKNOWLEDGMENT V V V V V

TABLE OF CONTENTS VII

$\begin{array}{ll}\text { LIST OF TABLES } & \text { XVI }\end{array}$

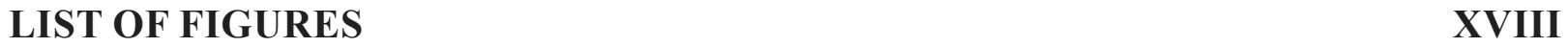

LIST OF NOTATIONS AND SYMBOLS $\quad$ XXXII

LIST OF APPENDICES KXXVI

CHAPTER 1: INTRODUCTION

$\begin{array}{ll}1.1 \text { Background } & 2\end{array}$

$\begin{array}{ll}1.2 \text { Objectives of the study } & 8\end{array}$

$\begin{array}{ll}1.3 \text { Outlines of the dissertation } & 9\end{array}$

CHAPTER 2: LITERATURE REVIEW 12

2.1 Introduction 12

2.2 Tailings deposition $\quad 13$

2.3 Tailings storage and disposal techniques 14

$\begin{array}{ll}\text { 2.3.1 Conventional methods of tailings storage } & 14\end{array}$

2.3.2 Upstream and downstream methods for tailing storage $\quad 14$

2.3.3 Rationale for dewatered tailings disposal $\quad 16$

2.4 Definition of tailings based on water content / solids content 17 
2.4.2 Thickened Tailings 19

$\begin{array}{lr}\text { 2.4.3 Paste Tailings } & 19\end{array}$

$\begin{array}{ll}\text { 2.4.4 Deposition of dewatered tailings } & 20\end{array}$

2.5. Geotechnical Properties of Tailings 22

2.5.1 Basic Characteristics 22

2.5.2 Grain Size Distribution and water retention curve 23

2.5.3 Consolidation Properties and Shrinkage Curve 24

$\begin{array}{ll}\text { 2.5.4. Saturated hydraulic conductivity } & 27\end{array}$

$\begin{array}{ll}\text { 2.5.5 Monotonic Shear Strength } & 28\end{array}$

$\begin{array}{ll}2.6 \text { Stress path and shear strength } & 30\end{array}$

2.6.1. Constant volume simple shear and triaxial tests 33

2.6.2 Comparison between simple shear and triaxial tests results 38

$\begin{array}{ll}\text { 2.7 Liquefaction phenomenon and steady state } & 40\end{array}$

2.7.1 Liquefaction Overview 40

2.7.2 Steady state and liquefaction mechanism 42

2.7.3 Earthquake characteristic in liquefaction phenomenon

2.8 Undrained behavior of sands 45

2.8.1 Critical Stress Ratio $\quad 46$

2.8.2 Phase Transformation 46

2.9 Cyclic Behavior of tailings $\quad 47$

2.10 Mechanics of Unsaturated Soils $\quad 50$ 
$\begin{array}{lr}2.10 .1 \text { Soil Suction } & 50\end{array}$

2.10.1.1 Matric Suction $\quad 52$

2.10.1.2 Osmotic Suction $\quad 53$

2.10.2 Measurement of Soil Suction 53

2.10.2.1 Tensiometer $\quad 54$

2.10.2.2 Dewpoint hygrometer (WP4-T) 56

2.10.3 Soil Water Characteristic Curve (SWCC) 57

2.10.3.1 Hysteresis effect of Soil Water Characteristic Curve 58

$\begin{array}{ll}\text { 2.10.3.2 Soil Water Characteristic Curve equations } & 60\end{array}$

2.10.3.3 Sensitivity of the Soil Water Characteristic Curve to net normal stress 62

2.10.4 Effective Stress of Unsaturated Soils 62

2.10.4.1 Stress state variables $\quad 64$

2.10.4.2 Suction Stress $\quad 64$

2.10.4.3 Validity and effectiveness of methods of predicting stress state of unsaturated 65 soils

2.10.4.4 Suction Stress Characteristics Curve (SSCC) 67

2.11 Microstructure investigation $\quad 73$

2.11.1 Mercury Intrusion Porosimetry (MIP) 74

2.11.1.2 Sources of possible errors in the MIP method 75

CHAPTER 3: MATERIAL AND METHODOLOGY 77

$\begin{array}{ll}3.1 \text { Introduction } & 77\end{array}$

3.2 Review of main objective and overall methodology 78 
3.3 Geotechnical Properties of Gold Mine Tailings used in the proposed study

3.4 Laboratory methods to simulate thickened tailings deposition

3.4.1 Small scale deposition simulation in a column

3.4.2 Multilayer deposition of thickened tailings in a drying box

3.4.2.1. Water content measurement

3.4.2.2. Soil Suction Measurement

3.4.2.2.1. Matric Suction measurement 93

3.4.2.2.2. Total Suction Measurement 95

3.4.2.3. Void ratio measurement

3.4.2.4. Sample Extraction Methods

3.4.2.4.1 Sample extraction by buried tubes 99

3.4.2.4.2 Sample extraction by pushing a thin wall tube using a hydraulic jack

3.5 Simple shear tests

101

3.5.1 Sample Preparation for SS tests

3.5.1.1 Reconstituted samples in the SS device

105

3.5.1.2 Undisturbed desiccated/rewetted samples in the SS device

3.5.1.3 Desiccated samples in the SS device

108

3.5.2 Consolidation Stage

3.5.2.1 Mechanically over-consolidated samples

3.5.3 Monotonic loading stage

3.5.3.1 Repeatability of monotonic simple shear responses 
3.5.5 Characteristics and limitations of the simple shear apparatus

3.6.1 Preparation methods

3.6.1.1 Reconstituted saturated samples (Slurry condition)

3.6.1.2 Desiccated/ rewetted undisturbed samples

3.6.2 Consolidation stage

3.6.3 Monotonic loading

3.6.4. Modified unsaturated triaxial test

3.6.4.1 Characteristics and limitations of modified triaxial tests

3.6.4.1.1 Void ratio measurement

3.6.4.1.2 Effective stress measurement

3.7.1 Sample preparation for vane shear test 
4.5 Fourth layer deposition 162

$\begin{array}{ll}\text { 4.6 First and second re-saturation } & 171\end{array}$

4.7 Fifth layer deposition $\quad 173$

$\begin{array}{ll}\text { 4.8 Cumulative Results } & 179\end{array}$

4.8.1 Water content results 179

4.8.2 Evaporation, electrical conductivity and temperature results 183

4.8.3 Matric and total suction results 186

$\begin{array}{ll}\text { 4.9 Tube extraction methods } & 192\end{array}$

$\begin{array}{ll}4.10 \text { Discussion } & 192\end{array}$

CHAPTER 5: APPLICABILITY OF CONSTANT VOLUME SIMPLE SHEAR 195 TESTS FOR SAND AND SILTY MATERIALS IN PARTIALLY-SATURATED CONDITION

$\begin{array}{ll}5.1 \text { Introduction } & 195\end{array}$

$\begin{array}{ll}5.2 \text { Test equipment and methodology } & 197\end{array}$

$\begin{array}{ll}5.3 \text { Materials } & 198\end{array}$

$\begin{array}{llr}\text { 5.3.1 Silica sand } & 199\end{array}$

$\begin{array}{lll}\text { 5.3.2 Glass beads } & 200\end{array}$

5.3.3 Synthetic Silt $\quad 200$

$\begin{array}{llr}5.4 & \text { Results } & 203\end{array}$

5.4.1 Silica Sand Results 203

5.4.1.1 Silica sand samples consolidated to $50 \mathrm{kPa} \quad 203$ 
5.4.1.2 Silica sand samples consolidated to $100 \mathrm{kPa}$

$\begin{array}{ll}\text { 5.4.2 Glass Beads results } & 205\end{array}$

$\begin{array}{ll}\text { 5.4.3 Synthetic Silt results } & 209\end{array}$

$\begin{array}{ll}\text { 5.4.3.1 Dry tests } & 210\end{array}$

5.4.3.2 Samples with $10 \%$ water content $(\mathrm{W}=10 \%)$

5.4.3.3 Samples with $14 \%$ water content $(\mathrm{W}=14 \%)$

5.4.3.4 Samples with $20 \%$ water content $(\mathrm{W}=20 \%) \quad 216$

$\begin{array}{ll}\text { 5.4.3.5 Comparisons of monotonic responses at same void ratios } & 218\end{array}$

$\begin{array}{ll}\text { 5.4.3.6 Phase transformation and failure angles } & 221\end{array}$

$\begin{array}{lll}5.5 & \text { Summary and Conclusion } & 221\end{array}$

CHAPTER 6: SIMPLE SHEAR, TRIAXIAL AND VANE SHEAR TEST 224 RESULTS

$\begin{array}{ll}\text { 6.1. Introduction } & 224\end{array}$

6.2 Results of simple shear tests $\quad 224$

6.2.1 Monotonic results of small scale laboratory tests 225

6.2.1.1 Monotonic results under $50 \mathrm{kPa}$ vertical effective consolidation pressure 225

6.2.1.2 Monotonic results under $100 \mathrm{kPa}$ vertical effective consolidation pressure $\quad 230$

6.2.1.3 Monotonic results under $400 \mathrm{kPa}$ vertical effective consolidation pressure 232

6.2.1.3.1 Desiccated-rewetted-consolidated samples 232

6.2.1.3.2 Desiccated-consolidated-rewetted samples 232

6.2.1.4 Monotonic results of desiccated-consolidated samples 233

6.2.1.5 Discussion on monotonic results of small scale method 238 
6.2.2 Monotonic Results of Mechanically over consolidated samples

\section{CHAPTER 7: DISCUSSION AND CONCLUSION}

7.1 Quantifying over-consolidation derived by desiccation 
7.4 Critical / steady state analysis

7.6 Applicability of the cyclic results of this study for different earthquakes

7.7 Influence of desiccation on strength and cost development in a TT stack

7.8 Important outcomes and conclusions

7.9 Recommendations for future research

REFERENCES

APPENDIX I 378

APPENDIX II

384

APPENDIX III

385

APPENDIX IV

387 


\section{LIST OF TABLES}

Table 2.1 Basic properties of mine tailings (Qiu and Sego, 2001)

Table 2.2 Consolidation behavior of tailings obtained by different researchers

Table 2.3 Saturated hydraulic conductivity of hard rock mine tailings obtained by different researchers

Table 2.4 Typical Effective shear strength parameters obtained by CU tests (Qui and Sego, 2001)

Table 2.5 Disadvantages and limitations of methods of predicting stress state of unsaturated soils

Table 5.1 Geotechnical properties of materials used in this study

Table 5.2 Constant volume simple shear tests on Silica Sand

Table 5.3 Characteristics of performed constant volume simple shear tests on synthetic 210 silt samples

Table 6.1 Monotonic simple shear tests performed on samples prepared with small scale deposition method

Table 6.2 Monotonic simple shear tests performed on desiccated samples under 400 $\mathrm{kPa}$ consolidation pressure

Table 6.3 Characteristics of cyclic simple shear tests performed on samples prepared by small scale deposition method

Table 6.4 Monotonic simple shear tests performed on samples obtained from Drying 273 box samples

Table 6.5 Monotonic simple shear tests performed on samples obtained from Drying 279 box samples 
Table 6.6 Modified triaxial tests performed on settled and desiccated-rewetted samples

Table 6.7 Characteristics of vane shear tests performed on settled and desiccated samples

Table 6.8 Characteristics of vane shear tests performed on settled and desiccatedrewetted samples

Table 7.1 Values of suction stress and $\mathrm{OCR}_{\mathrm{D}}$

Table 7.2 Drying time required to reach different values of water content obtained from the multi-layer deposition in the drying box 


\section{LIST OF FIGURES}

Figure 1.1 Volume change stress history of thickened tailings layers 6

Figure 2.1 Typical water retention type dam for tailings storage (after Vick, 1983) 15

Figure 2.2 Different tailings conventional deposition (after Vick, 1983) 15

Figure 2.3 Thickened tailings disposal during early deposition at the Bulyanhulu mine 17 (Photo courtesy of Crowder, 2004)

Figure 2.4 Slurry tailings discharged in tailings impoundment (after Bussiere, 2007)

Figure 2.5 Typical Tailing disposal facility at Bulyanhulu site (after Simms et al, 2007; 20 Shuttleworth et al., 2005)

Figure 2.6 Hydrologic process of thickened tailings stack (after Simms and Grabinsky, 2004)

Figure 2.7 Typical shrinkage curve with respect to residual strength and oxygen diffusion coefficient (after Simms et al., 2007)

Figure 2.8 General range of grain size distributions for hard rock tailings (Vick, 1990) 24

Figure 2.9 Shrinkage Curve of different mine tailings (after Qui and Sego, 2001) 26

Figure 2.10 Monotonic stress-strain responses of different tailings under $50 \mathrm{kPa} \mathrm{CU}$ triaxial test (after Qui and Sego, 2001)

Figure 2.11 Excess pore pressure generation of different tailings under $50 \mathrm{kPa} \mathrm{CU}$ triaxial tests (after Qui and Sego, 2001)

Figure 2.12 Monotonic triaxial response of fine grained mine tailings (after Sanin, 2005)

Figure 2.13 Stress conditions and shear deformations in a direct simple shear device

Figure 2.14 Comparison of undrained and constant volume test results (after Dyvik et al., 1987) 
Sanin, 2005)

Figure 2.16 Comparison of stress conditions in simple shear test and triaxial testing 37

Figure 2.17 Comparison of simple shear, triaxial compression and triaxial extension at $\quad 38$ same density and consolidation pressure (after Vaid and Sivathayalan (1996))

Figure 2.18 Comparison of simple shear, triaxial compression and triaxial extension 39 (after Vaid and Sivathayalan (1996))

Figure 2.19 Correction factors $\left(\mathrm{C}_{\mathrm{r}}\right)$ for converting cyclic triaxial CSR to the simple shear CSR at $\mathrm{N}_{\mathrm{L}}=10$ (after Vaid and Sivathayalan (1996))

Figure 2.20 reducing the contact forces during the liquefaction (after Al-Tarhouni et al.,2008)

Figure 2.21 Behavior of contractive and dilative soil under static and cyclic loading (Kramer, 1996)

Figure 2.22 Number of equivalent uniform cycles versus earthquake magnitude (Mw) for sands (Seed and Idriss, 1982)

Figure 2.23 Undrained monotonic responses of different relative densities sands a) Stress-Strain Response, b) stress path (after Vaid and Chern, 1985)

Figure 2.24 Cyclic stress ratio versus $\mathrm{N}_{\mathrm{L}}$ for gold mine tailings (after James M. et al., 2011)

Figure 2.25 Cyclic Stress Ratio of different tailings based on previous studies (after Sanin, 2005)

Figure 2.26 An early tensiometer (Ridley et al., 2008) 55

Figure 2.27 A high range tensiometer (suction probe) (after Ridley and Burland, 1995) 56

Figure 2.28 Schematic figure of tensiometer (after Ridley et al., 2008) 56

Figure 2.29 Typical Soil Water Characteristic Curve (after Vanapalli et al., 1997) 58

Figure 2.30 Hysteresis Effect in SWCC (after Fredlund and Rahardjo, 1993) 59

Figure 2.31 Typical desorption and adsorption curve (after Vanapalli et al., 1998) 59 
Figure 2.32 Van-Genuchten empirical fitting parameters for various soil types (Lu and Likos, 2010)

Figure 2.33 Typical SWCCs for various soil types based on average Van-Genuchten fitting parameters

Figure 2.34 Possible zones of SWCC for sand, silt and clay based on Van-Genuchten fitting parameters

Figure 2.35 Evolution of pore size distribution during a water retention curve test and after air and oven drying for a glacial till, PI $=10 \%$ (Simms and Yanful 2002)

Figure 3.1 Mixer used for remixing the settled tailings

Figure 3.2 Grain Size Distribution of tailings used in the study

Figure 3.3 Deposition of $10 \mathrm{~cm}$ tailings layer with $38 \%$ water content

Figure 3.4 Desiccated tailings at different water content

Figure 3.5 Typical extracted desiccated/rewetted samples from small scale simulation test

Figure 3.6 Proposed thin wall tubes for sampling (after Wijewickreme and Sanin 2004)

Figure 3.7 Buckets of tailings prepared at $38 \%$ water content

Figure 3.8 Schematic diagrams of steel reinforced Plexiglas drying box

Figure 3.9 Pictures of drying box in empty and filled conditions

Figure 3.10 A schematic plan for deposition of layers and location of sensors (All

Figure 3.12 A picture of the tipping bucket for drainage measurement

Figure 3.13 Layer of geotextile and thin wall tubes before first layer deposition

Figure 3.15 Comparison of calculated VWC and VWC obtained by sensors 92

Figure 3.16 A picture of UMS T5 tensiometer (Hoskin Scientific, 2009) 
Figure 3.17 Descriptions of different parts of a UMS T5 tensiometer (after Hoskin

Scientific, 2009)

Figure 3.18 a) schematic diagram and b) a picture of WP4-T device 96

Figure 3.19 A picture of TS-30S ultrasonic senix distance sensors to measure void ratio 98 changes

Figure 3.20 Four TS-30S ultrasonic senix distance sensors on top of the box before deposition

Figure 3.21 Triangle approximation of cross section of the cracks in the drying box

Figure 3.22 A picture of drying box with tubes prepared for fresh tailings and a buried 100 tube

Figure 3.23 Procedure of pushing thin wall tubes into the tailings using a hydraulic jack 102

Figure 3.24 Photograph of simple shear device at Carleton University

Figure 3.25 Schematic diagram of simple shear device at Carleton University (after Al- 104 Tarhouni, 2008)

Figure 3.26 Preparation of a reconstituted sample for simple shear test 107

Figure 3.27 Procedure for removing undisturbed samples from extracted tubes 109

Figure 3.28 a) A desiccated sample in the simple shear mould and b) incorrect stress- $\quad 110$ strain response of the desiccated sample

Figure 3.29 The procedure of preparing desiccated samples for the simple shear test

Figure 3.30 Repeatability of desiccated/rewetted samples in the simple shear device

Figure 3.31 Schematic diagram of triaxial apparatus at Carleton University

Figure 3.32 Preparing reconstituted slurry samples in the triaxial device

Figure 3.33 Sample preparation procedure for undisturbed desiccated rewetted samples for triaxial test 
Figure 3.34 Picture and schematic of T5 tensiometer inserted into modified base triaxial pedestal (Simms and Grabinsky, 2009)

Figure 3.35 Calibration by applying positive water pressure to the tensiometer

Figure 3.36 Calibration results of the tensiometer by applying positive water pressure

Figure 3.37 Schematic diagram of the modified unsaturated triaxial pedestal

Figure 3.38 Pictures of modified unsaturated triaxial pedestal

Figure 3.39 A picture of an inspector vane tester H-60

Figure 3.40 Schematic diagram of the Auto Pore IV Mercury Porosimeter (Manual of the Auto Pore IV)

Figure 3.41 Low pressure and high pressure ports of the Auto Pore IV Mercury Porosimeter

Figure 3.42 Freeze-drying procedure with liquid nitrogen

Figure 3.43 Samples placed in a vacuum desiccator after freeze drying

Figure 3.44 A picture of the penetrometer used in this study

Figure 4.1 A picture of the drying box with deposited layers

Figure 4.2 Drainage, evaporation and water content results of first layer deposition

Figure 4.3 Electrical conductivity, temperature and void ratio results of first layer deposition

Figure 4.4 Shrinkage curve of the tailings obtained in the first layer deposition

Figure 4.5 Matric suction results of first layer deposition

Figure 4.6 Total suction results of the first layer deposition

Figure 4.7 Desiccation process of the first layer deposition

Figure 4.8 Drainage, evaporation and water content results of second layer deposition

Figure 4.9 Electrical conductivity, temperature and void ratio results after second layer 150 deposition 
Figure 4.10 Matric and total suction results after deposition of second layer

Figure 4.11 Total suction results close and not close to cracks

Figure 4.12 Desiccation process in the second layer deposition

Figure 4.13 Evolution of cracks on the surface of the second layer during desiccation

Figure 4.14 Evaporation and water content results after deposition of third layer

Figure 4.15 Temperature, electrical conductivity and void ratio results after third layer deposition

Figure 4.16 Matric suction results after third layer deposition

Figure 4.17 Total suction results after third layer deposition

Figure 4.18 Desiccation process of the third layer deposition

Figure 4.19 Evaporation and water content results after deposition of fourth layer

Figure 4.20 Temperature, electrical conductivity and void ratio results after third layer 166 deposition

Figure 4.21 Matric suction results after fourth layer deposition

Figure 4.22 Total suction results after fourth layer deposition

Figure 4.23 Evolution of cracks on the surface of the fourth layer

Figure 4.24 Desiccation process of the fourth layer deposition

Figure 4.25 Water content results after first and second re-saturations

Figure 4.26 Actual evaporation rates of first and second re-saturations

Figure 4.27 The surface of the fourth layer after re-saturation prior deposition of the fifth layer

Figure 4.28 Water content results after the fifth layer deposition 
Figure 4.30 matric suction results after fifth layer deposition

Figure 4.31 Photos of drying box after deposition of the fifth layer

Figure 4.32 VWC of a) first layer and b) second layer during deposition of top fresh layers or re-saturations

Figure 4.33 VWC of all layers during depositions and re-saturations

Figure 4.34 Average GWC of all layers during depositions

Figure 4.35 Actual evaporation rates of all depositions and re-saturations

Figure 4.36 Temperature results of all layers during depositions and re-saturations

Figure 4.37 Bulk electrical conductivity results of all depositions and re-saturations

Figure 4.38 Matric suction of a) first layer and b) second layer after deposition of top layers

Figure 4.39 Matric suction of the deposited fresh layers

Figure 4.40 Surface total suction results of all depositions

Figure 4.41 Variation of surface total suction during dewatering process of all layers

Figure 4.42 SWCC of tailings based on matric and total suction results

Figure 4.43 Variations of a) matric suction, b) total suction with evaporation rate

Figure 5.1 Method of saturation used in this study

Figure 5.2 Particle size distributions of all tested materials

Figure 5.3 Figures of tested materials in the simple shear mould

Figure 5.4 SWCCs of fine grained synthetic silts and its comparison with gold tailings 202

Figure 5.5 Monotonic responses of Silica Sand samples consolidated to $50 \mathrm{kPa}$

Figure 5.6 Monotonic responses of Silica Sand samples consolidated to $100 \mathrm{kPa}$

Figure 5.7 Monotonic response of glass bead material under different saturation 208 condition 
Figure 5.8 Monotonic response of constant volume tests on dry synthetic silt under different void ratios

Figure 5.9 Monotonic response of constant volume tests on partial saturated synthetic 214 silt samples $(\mathrm{W}=10 \%)$ under different void ratios

Figure 5.10 Stress-strain monotonic response of partial saturated synthetic silts samples 215 $(\mathrm{W}=14 \%)$ under different void ratios

Figure 5.11 Stress path monotonic response of partial saturated synthetic silts samples 215 (W=14\%) under different void ratios

Figure 5.12 Excess pore pressure - shear strain monotonic response of partial saturated 216 synthetic silts samples $(\mathrm{W}=14 \%)$ under different void ratios

Figure 5.13 Monotonic response of constant volume tests on partial saturated synthetic 217 silt samples $(\mathrm{W}=20 \%)$ under different void ratios

Figure 5.14 Comparison of monotonic response of constant volume tests on synthetic 219 silts under same void ratio $\left(\mathrm{e}_{\mathrm{c}}=0.78 \pm 0.01\right)$ but different degrees of saturation

Figure 5.15 Comparison of monotonic response of constant volume tests on synthetic 220 silts under same void ratio $\left(\mathrm{e}_{\mathrm{c}}=0.66 \pm 0.01\right)$ but different degrees of saturation

Figure 5.16 Phase Transformation and failure angles of synthetic silt constant volume 222 simple shear tests

Figure 6.1 Volume change stress history of samples desiccated to a) $\mathrm{W}=23 \%$, b) $\mathrm{W}=12 \%$, rewetted and consolidated to $50 \mathrm{kPa}$

Figure 6.2 Monotonic results of desiccated and rewetted samples prepared by small 229 scale method under $50 \mathrm{kPa}$

Figure 6.3 Monotonic results of desiccated and rewetted samples prepared by small 231 scale method under $100 \mathrm{kPa}$

Figure 6.4 Monotonic shear response of desiccated and rewetted samples prepared by 234 small scale method under $400 \mathrm{kPa}$

Figure 6.5 Excess pore water pressure generation in desiccated and rewetted samples 235 prepared by small scale method under $400 \mathrm{kPa}$

Figure 6.6 Monotonic results of desiccated-consolidated-rewetted samples prepared 236 under $400 \mathrm{kPa}$ 
Figure 6.7 Monotonic shear responses of desiccated samples under $400 \mathrm{kPa}$

Figure 6.8 Normalized shear stress at PT and shear strength versus void ratio of samples consolidated under $400 \mathrm{kPa}$

Figure 6.9 Consolidation results of samples prepared based on small scale deposition method and consolidated under $400 \mathrm{kPa}$

Figure 6.10 Effect of rewetting on stress-strain response of tailings consolidated at 400 243 $\mathrm{kPa}$

Figure 6.11 Normalized shear stress at PT versus void ratio for all monotonic simple shear tests

Figure 6.12 Normalized shear stress at PT versus water content during desiccation

Figure 6.13 Monotonic simple shear response of mechanically over consolidated samples under $100 \mathrm{kPa}$

Figure 6.14 Comparison of Monotonic simple shear response of mechanically over consolidated samples and desiccated-rewetted samples under $100 \mathrm{kPa}$

Figure 6.15 Comparison of Monotonic simple shear response of mechanically over consolidated samples and desiccated-rewetted samples under $50 \mathrm{kPa}$

Figure 6.16 Cyclic simple shear response of settled sample $(\mathrm{Wd}=32 \%)$ under $\mathrm{CSR}=$ 251 0.05

Figure 6.17 Cyclic simple shear response of settled sample $(\mathrm{Wd}=32 \%)$ under $\mathrm{CSR}=$ 252 0.065

Figure 6.18 Cyclic simple shear response of settled sample $(\mathrm{Wd}=32 \%)$ under CSR $=$ 253 0.075

Figure 6.19 Cyclic simple shear response of settled sample $(\mathrm{Wd}=32 \%)$ under $\mathrm{CSR}=$ 254 0.125

Figure 6.20 Cyclic simple shear response of desiccated/rewetted sample $(\mathrm{Wd}=26 \%)$ 256 under $\mathrm{CSR}=0.05$

Figure 6.21 Cyclic simple shear response of desiccated/rewetted sample $(\mathrm{Wd}=26 \%)$ 257 under $\mathrm{CSR}=0.075$

Figure 6.22 Cyclic simple shear response of desiccated/rewetted sample $\left(\mathrm{W}_{\mathrm{d}}=26 \%\right)$ 258 under $\mathrm{CSR}=0.1$ 
Figure 6.23 Cyclic simple shear response of desiccated/rewetted sample $(\mathrm{Wd}=26 \%)$ under CSR CSR $=0.11$

Figure 6.24 Cyclic simple shear response of desiccated/rewetted sample $(\mathrm{Wd}=26 \%)$ 260 under $\mathrm{CSR}=0.125$

Figure 6.25 Cyclic simple shear response of desiccated/rewetted sample $(\mathrm{Wd}=20 \%)$ 261 under $\mathrm{CSR}=0.065$

Figure 6.26 Cyclic simple shear response of desiccated/rewetted sample $(\mathrm{Wd}=20 \%)$ 262 under $\mathrm{CSR}=0.075$

Figure 6.27 Cyclic simple shear response of desiccated/rewetted sample $(\mathrm{Wd}=12 \%)$ 264 under $\mathrm{CSR}=0.065$

Figure 6.28 Cyclic simple shear response of desiccated/rewetted sample $(\mathrm{Wd}=12 \%)$ 265 under $\mathrm{CSR}=0.075$

Figure 6.29 Comparison of cyclic simple shear response of samples with different desiccation history at $\mathrm{CSR}=0.125$

Figure 6.30 Comparison of cyclic simple shear response of samples with different desiccation history at CSR $=0.065$

Figure 6.31 Comparison of excess pore pressure generation of samples with different 269 desiccation histories at CSR $=0.065,0.075$ and 0.1

Figure 6.32 Cyclic stress ratio (CSR) versus numbers of cycles to reach liquefaction $\left(\mathrm{N}_{\mathrm{L}}\right)$ for all cyclic simple shear tests performed on samples prepared by small scale deposition method

Figure 6.33 The effect of desiccation history on cyclic resistance ratio of small scale prepared samples a) CRR10, b) CRR15

Figure 6.34 Monotonic simple shear response of tests performed on buried tubes under 275 $50 \mathrm{kPa}$ vertical effective stress

Figure 6.35 Results of simple shear tests on buried tubes under $100 \mathrm{kPa}$ vertical 276 effective stress

Figure 6.36 Monotonic simple shear response of tests performed on samples prepared 277 by sampling with thin wall tubes under $50 \mathrm{kPa}$ vertical effective stress

Figure 6.37 Difference between the responses of samples obtained from buried tubes 278 and thin wall tubes extracted by sampling methods 
Figure 6.38 Cyclic simple shear response of the sample obtained from Layer 1 at CSR $=0.075$

Figure 6.39 Cyclic simple shear response of the sample obtained from Layer 1 at CSR 281 $=0.1$

Figure 6.40 Cyclic simple shear response of the sample obtained from Layer 4 at CSR 282 $=0.2$

Figure 6.41 Cyclic simple shear response of the sample obtained from Layer 2 at CSR 283 $=0.075$

Figure 6.42 Cyclic simple shear response of the sample obtained from Layer 2 at CSR 284 $=0.125$

Figure 6.43 Cyclic simple shear response of the sample obtained from Layer 4 at CSR 285 $=0.2$

Figure 6.44 Cyclic simple shear response of the sample obtained from Layer 3 at CSR 287 $=0.075$

Figure 6.45 Cyclic simple shear response of the sample obtained from Layer 3 at CSR 288 $=0.1$

Figure 6.46 Cyclic simple shear response of the sample obtained from Layer 4 at CSR 289 $=0.15$

Figure 6.47 Cyclic simple shear response of the sample obtained from Layer 4 at CSR $=0.175$

Figure 6.48 Cyclic simple shear response of the sample obtained from Layer 4 at CSR $=0.2$

Figure 6.49 Comparison of excess pore pressure generation of samples from different layers at a) $\mathrm{CSR}=0.075, \mathrm{~b}) \mathrm{CSR}=0.1$

Figure 6.50 Comparison of excess pore pressure generation of samples from different layers at a) $\mathrm{CSR}=0.125, \mathrm{~b}) \mathrm{CSR}=0.2$

Figure 6.51 Cyclic stress ratio (CSR) versus numbers of cycles to reach liquefaction $\left(\mathrm{N}_{\mathrm{L}}\right)$ for all cyclic simple shear tests performed on samples obtained from different layers of the drying box

Figure 6.52 The effect of desiccation history on cyclic resistance (CRR10, CRR 15) of 296 samples obtained from different layers of the drying box 
Figure 6.53 Results of conventional triaxial test on saturated tailings 298

Figure 6.54 Comparison of result of conventional triaxial test and simple shear test on 299 saturated settled tailings

Figure 6.55 Variation of matric suction during desiccation-rewetting process of some 301 triaxial samples

Figure 6.56 Consolidation results of modified triaxial tests 300

Figure 6.57 Pictures of lateral strain in a modified triaxial test 302

Figure 6.58 Stress-strain responses of modified triaxial tests on settled and 304 desiccated/rewetted tailings

Figure 6.59 Excess pore pressure generation and stress path of modified triaxial tests on 305 settled and desiccated-rewetted tailings

Figure 6.60 Variation of q at PT and failure versus water content 306

Figure 6.61 Shape of failure surface obtained from a modified triaxial test performed on 307 a desiccated/rewetted sample

Figure 6.62 Comparison of conventional and modified triaxial tests on settled tailings

Figure 6.63 Comparison of the results of triaxial tests performed on desiccated tailings 311 with desiccated-rewetted samples

Figure 6.64 Variation of matric suction in modified triaxial tests on desiccated tailings

Figure 6.65 Cyclic modified triaxial response of settled tailings at CSR $=0.1$

Figure 6.66 Expess pore pressure and axial strain generation of settled tailings at $\mathrm{CSR}=$ 314 0.1

Figure 6.67 Cyclic modified triaxial response of settled tailings at CSR $=0.15$ 
Figure 6.68 Comparison of CSR vs $\mathrm{N}_{\mathrm{L}}$ for modified triaxial and simple shear tests

perfromed on settled tailings

Figure 6.69 Phase transformation and failure friction angles of simple shear tests under $50 \mathrm{kPa}$ and $100 \mathrm{kPa}$

Figure 6.70 Phase transformation and failure friction angles of modified triaxial tests

Figure 6.71Undrained shear strength versus water content for settled and desiccated tailings

Figure 6.72 Undrained shear strength versus water content for settled and desiccated/rewetted tailings

Figure 6.73 Undrained shear strength versus desiccated water content for settled and desiccated-rewetted tailings

Figure 6.74 Undrained shear strength versus desiccated water content for settled and desiccated-rewetted tailings

Figure 6.75 The influence of desiccation on monotonic simple shear response of reconstituted gold tailings prepared in the simple shear mould

Figure 7.2 The influence of consolidation under $100 \mathrm{kPa}$ on MIP results of thickened gold tailings

Figure 7.3 The comparison of dilative and contractive responses on MIP results of thickened gold tailings

Figure 7.4 Shear stress at PT and shear strength for different degrees of desiccation under $400 \mathrm{kPa}$ vertical effective consolidation pressure

Figure 7.5 Shear stress at PT and shear strength for different degrees of desiccation under $50 \mathrm{kPa}$ and $100 \mathrm{kPa}$ vertical effective consolidation pressure

Figure 7.6 Normalized shear stress at PT for different degrees of desiccation

Figure 7.7 Normalized shear stress at PT versus void ratio obtained from the simple shear tests

Figure 7.8 Compressibility of saturated, desiccated and desiccated/rewetted samples under $400 \mathrm{kPa}$ vertical effective consolidation pressures 
Figure 7.9 The influence of degree of desiccation on monotonic and cyclic shear strength of the tailings

Figure 7.10 shear strength and $q$ at PT for different degrees of desiccation under $50 \mathrm{kPa}$ consolidation cell pressure

Figure 7.11 Comparison of the monotonic results of simple shear tests and triaxial tests 341 under $50 \mathrm{kPa}$ consolidation pressure

Figure 7.12 Anisotropic behaviour of thickened tailings under extension and cyclic triaxial tests

Figure 7.13 Comparison of cyclic simple shear and triaxial results

Figure 7.14 Normalized shear strength of simple shear tests vs. void ratio

Figure 7.15 Comparisons of dilative and contractive responses

Figure 7.16 Impact of rewetting for simple shear samples consolidated under $400 \mathrm{kPa}$

Figure 7.17 Impact of rewetting for vane shear tests a) desiccated water content, b) current water content

Figure 7.18 Time required reaching different values of water contents 


\section{LIST OF NOTATIONS AND SYMBOLS}

\begin{tabular}{|c|c|}
\hline $\mathrm{AE}$ & Actual evaporation \\
\hline $\mathrm{AEV}$ & Air-entry value \\
\hline ARD & Acid rock drainage \\
\hline$a_{\max }$ & Peak ground acceleration \\
\hline CSR & Critical stress ratio/Cyclic stress ratio \\
\hline CRR & Cyclic resistance ratio \\
\hline $\mathrm{C}$ & Cohesion \\
\hline $\mathrm{C}_{\mathrm{c}}$ & Coefficient of curvature/Compression index \\
\hline $\mathrm{C}_{\mathrm{cr}}$ & Recompression index \\
\hline $\mathrm{C}_{\mathrm{v}}$ & Coefficient of consolidation \\
\hline $\mathrm{C}_{\mathrm{u}}$ & Coefficient of uniformity \\
\hline $\mathrm{D}_{\mathrm{r}}$ & Relative density \\
\hline DST & Direct Shear Test \\
\hline $\mathrm{EC}$ & Electrical conductivity \\
\hline EPT & Electronic Pneumatic Transducer \\
\hline ESEM & Environmental scanning electron microscopy \\
\hline e & Void ratio \\
\hline$e_{i}$ & Initial void ratio (before consolidation stage) \\
\hline $\mathrm{ec}_{\mathrm{c}}$ & Void ratio after consolidation stage \\
\hline $\mathrm{e}_{\max }$ & Maximum void ratio \\
\hline $\min$ & Minimum void ratio \\
\hline
\end{tabular}


$\mathrm{G}_{\mathrm{s}} \quad$ Specific gravity

GWC Gravimetric water content

Ks Saturated hydraulic conductivity

LL Liquid limit

LVDT Linear Variable Displacement Transducer

$\mathrm{M}_{\mathrm{w}} \quad$ Earthquake magnitude

MIP Mercury Intrusion Porosimetry

$\mathrm{N} \quad$ No. of cyclic

$\mathrm{N}_{\mathrm{L}} \quad$ No. of cycles to reach liquefaction

NGI Norwegian Geotechnical Institute

OCR Overconsolidation ratio

$\mathrm{OCR}_{\mathrm{D}}$ Overconsolidation ratio derived by desiccation

$\mathrm{OCR}_{\mathrm{M}}$ Mechanical overconsolidation

PE Potential evaporation

PGA Peak ground acceleration

PI Plasticity Index

PL Plastic limit

PSD Pore size distribution

PT Phase transformation

p' Mean effective stress

q Deviatoric stress

$\mathrm{R}$ The universal gas constant

RH Relative humidity 
$r_{d} \quad$ Stress reduction

$\mathrm{r}_{\mathrm{u}} \quad$ Excess pore pressure ratio

SL Shrinkage limit

SS Steady state/Simple shear

SSCC Suction stress characteristics curve

SWCC Soil-water characteristic curve

$\mathrm{S}_{\mathrm{r}} \quad$ Degree of saturation

$\mathrm{Su} \quad$ Steady-state shear strength

$\mathrm{T} \quad$ Absolute pressure/Temperature

TT Thickened tailings

TTT Thickened tailings technology

$\mathrm{T}_{\mathrm{s}} \quad$ Surface tension

$\mathrm{u}_{\mathrm{a}} \quad$ Pore air pressure

$\mathrm{u}_{\mathrm{w}} \quad$ Pore water pressure

USCS Unified soil classification system

VWC Volumetric water content

W Current Water content

Wd Water content after controlled desiccation

WRC Water retention curve

$\pi \quad$ Osmotic suction

$\emptyset_{\text {Failure }} \quad$ Friction angle at Failure

$\emptyset_{\mathrm{PT}} \quad$ Friction angle at phase transformation

$\gamma \quad$ Shear strain/Unit weight 


$\begin{array}{ll}\gamma_{\mathrm{d}} & \text { Dry unit weight } \\ \tau_{c y c} & \text { Cyclic shear stress } \\ \sigma^{\prime}{ }_{v c} & \text { Effective consolidation pressure/stress } \\ \sigma_{\mathrm{v}}^{\prime} & \text { Effective vertical stress } \\ \sigma^{\mathrm{s}} & \text { Suction stress } \\ \theta_{s} & \text { Saturated volumetric water content } \\ \psi & \text { Total suction } \\ v_{w 0} & \text { Specific volume of water } \\ \omega_{v} & \text { Molecular mass of water } \\ v_{V} & \text { Partial pressure of pore water vapour } \\ v_{V 0} & \text { Saturation pressure of vapour above pure water }\end{array}$




\section{LIST OF APPENDICES}

$\begin{array}{ll}\text { APPENDIX I: Drying box results } & 378\end{array}$

APPENDIX II: 3D volume change stress history of desiccated-rewetted samples

APPENDIX III: Generation of excess pore pressure during shearing for desiccated385 rewetted and desiccated-consolidated-rewetted samples

APPENDIX IV: Cyclic simple shear results 


\section{Chapter 1: Introduction}

\subsection{Background}

Mining is one of Canada's most important economic sectors. Just in 2011, the industry contributed \$36 billion to Canadian's gross domestic product (GDP) and employed 320,000 workers in the sectors of mineral extraction, processing and manufacturing (The Mining Association of Canada, 2012). Tailings, the by-product of milling of extracted rock or overburden, comprise the largest fraction of mine waste. A vast amount of mine tailings are produced all over the world every year. Due to increase in demand for metals, mine excavation and therefore mine waste productions have increased. The Canadian mining industry produces approximately 2 million tonnes of mine waste per day (Crowder, 2004).

The standard method for deposition is to transport the tailings from the mill at high water content (as a slurry) to an impoundment bounded by dams or dykes. For tailings that are deposited with 
such a high water contents, the consequences of dam failure can be devastating. Unfortunately, substantial failures have occurred on a regular basis over the last century (ICOLD 2001). One potential trigger is earthquake loading:

In 1978 the Izu-Ohshim-Kinkai earthquake in Japan caused two tailings dams owned by the Mochikochi gold mining company to fail due to liquefaction of the tailings materials behind the dams (Okusa and Anma, 1980). Tailings had been deposited in the pond since the dikes were constructed in 1964. The dam contained $476,000 \mathrm{~m}^{3}$ of deposits at the time of the earthquake (Okusa and Anma, 1980). The earthquake comprised of a main shock with magnitude 7 and a large after shock with magnitude 5.8. Dam number 1 failed during the main shaking. A flow of around $80,000 \mathrm{~m}^{3}$ of the dike materials and tailings containing toxic materials passed into the valley and travelled about 7-8 kilometers along the valley and river (Okusa and Anma, 1980). Dam number 2 failed about 24 hours after the main shock and about $3000 \mathrm{~m}^{3}$ of the dike materials and tailings escaped into the second valley. The delayed failure of the number 2 dam was postulated to be due to upward movement of the phreatic surface resulting from liquefaction of the tailings deposits behind the dam (Okusa and Anma, 1980). The geotechnical information obtained at the dam sites, in addition to the reported failure mechanism of both dams, makes this case one of the several unique case histories of dam failure caused by earthquake-induced liquefaction.

In October 1928, the Barahona tailings dam failure in Chile, which consisted of Teniente copper mine tailings, killed 54 people (Dobry and Alvarez, 1967). In March 1965, an intense earthquake (with a Richter magnitude of 7) in Chile triggered liquefaction in about 10 mining sites that 
consisted of slurry tailings and caused the death of about 400 people (Dobry and Alvarez, 1967). Earthquake loading, however, is responsible for only a fraction of dam failures. Other causes include poor control of the phreatic surface leading to instability or to dam washout, poor foundation control and /or improper characterization of dam foundation materials.

Some of the more recent tailings dam failures include:

- The Kolontár red mud dam failure (Hungary, October 2010)

The embankment of the Bauxite tailings dam failed and released a mixture of $600-700$ thousand cubic meters of red mud and water. The slurry flooded the lower sections of the settlements of Kolontár. Ten people were killed, and around 120 people were injured. The spilling red mud flooded 800 hectares of surrounding areas. The most catastrophic devastation was caused in the villages of Devecser and Kolontár, which are located near the reservoir. The cause of failure was mostly associated with a shear failure in the dike at the seepage point.

- Mianyang manganese tailings dam failure (China, July 2011)

Tailings dam damaged from landslides caused from heavy rainfalls. Tailings damaged residential roads and houses and around 272 people were forced to leave. Tailings were also washed into the Fujiang River and caused 200,000 people without drinking water supply. 
- Gullbridge copper tailings dam failure (Newfoundland, Canada, December 2012)

The tailings dam at the former Gullbridge copper mine in central Newfoundland failed while work was under way to stabilize it. The failure led to a breach of the $7 \mathrm{~m}$ high and approximately $25 \mathrm{~m}$ wide dam. The dam was impounding mine tailings that were partially covered by water forming a tailings pond. As a result of the failure, the pond fully drained over the next few hours, and a small amount of the tailings escaped.

An alternative to conventional deposition that minimizes or avoids the consequences of dam failures is thickened tailings technology (TTT). This technology improves the geotechnical performance of the tailings through dewatering, such that they exhibit minimal grain-size segregation and may be deposited with a gentle slope. This increases the density immediately upon deposition, provides a homogeneous deposit, and eliminates ponded water at the surface. Thickened tailings technology not only reduces the risk of dam failure, but can also potentially reduce the cost of the Tailings Storage Facility (TSF) for some projects by reducing dam construction costs and increasing water recycling.

One of the earliest sites was the Kidd Creek Mine in Northern Ontario, Canada, where the use of thickened tailings technology was pioneered by Eli Robinsky (Robinsky, 1975). Since then, thickened tailings technology has been employed quite commonly in the aluminum industry, with a smaller but growing uptake in metal mines. In the oil sands industry, various kinds of thickened tailings products are being considered to assist operators meet recent regulatory changes.

The tailings may be deposited from one or several spigots, after transport from the thickener either by pipeline or in a slurry launder. Cycling deposition between a number of spigots 
facilitates the development of thin lifts, and allows for a fresh layer to densify due to desiccation and/or drainage, before burial during subsequent deposition. Tailings deposited in this fashion will undergo a variable stress history, depending on the climate, deposition sequencing or "drying time", and layer thickness (Fisseha et al. 2010, Simms et al. 2007). In addition, the rate of dewatering of a fresh layer may depend on the stage of deposition: layers placed on top of multiple older layers may desiccate at a lower rate due to the moderating action of the underlying older tailings (Simms et al. 2010), and/or will have a variable degree of under-drainage.

Although generation of matric suction during desiccation may contribute to the shear strength, suction can be dissipated by rewetting due to the deposition of a fresh layer, or rainfall infiltration. The stress history induced by desiccation may not be eliminated by rewetting. Consequently, studying the influence of stress history on the strength of thickened tailings, which includes not only desiccation but subsequent rewetting and consolidation, is important to quantify the contribution of desiccation to the stability of a thickened tailings stack.

Figure 1.1 shows possible volume-stress histories of thickened tailings layers. After deposition, the thickened tailings layer initially has a reduction in volume due to settling under self-weight loading with no overburden pressure ${ }^{1}$. If the settled tailings are allowed to desiccate (case 1 and 2), the tailings layer generates matric suction during drying, which leads to shrinkage and decreasing void ratio due to drying. If the drying time is longer, desiccation continues to higher values of matric suction (case 2). With the deposition of a fresh layer on top of the desiccated layer, the bottom layer is subsequently rewetted by moisture migration due to gravity and

\footnotetext{
${ }^{1}$ In this thesis, the initial densification process due to self-weight loading in a thin lift is referred to as settling, to avoid confusion with consolidation due to burial of the lift by new material. This is not intended to suggest that this initial densification process could not be described by the mathematics of consolidation
} 
capillary action induced by placement of the top layer, which leads to a reduction in matric suction potentially up to zero (or a very low value). As other layers are deposited, rewetted tailings undergo mechanical consolidation induced by the weight of top layers.

If tailings do not experience desiccation, a fresh layer is added after initial settling of the bottom layer, and the bottom layer experiences settling and consolidation (case 3). The most important question could be the degree of desiccation before depositing a fresh layer as it relates to the strength and stability of the stack. Mining operators are interested in knowing an optimum water

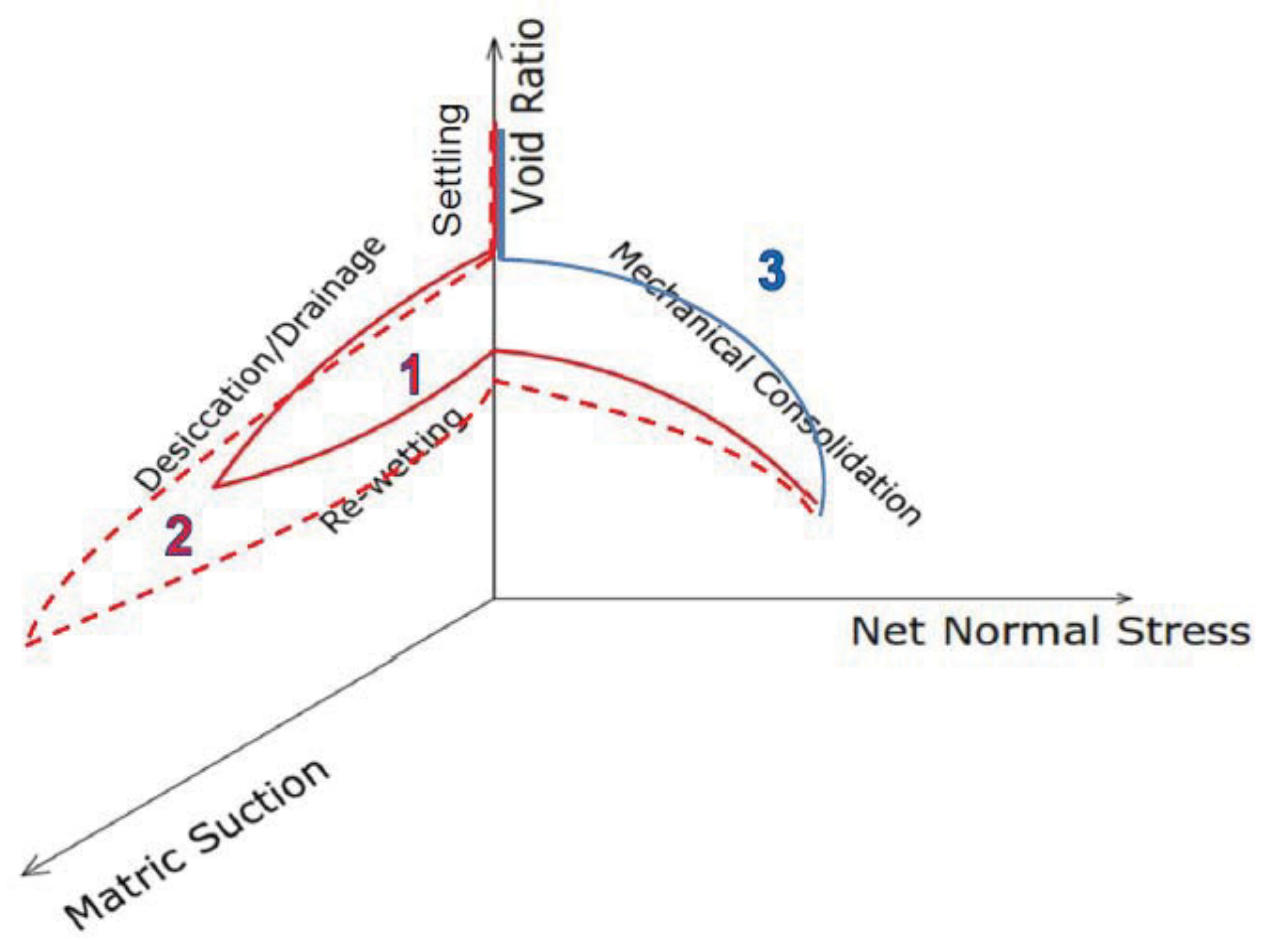

Figure 1.1 Volume change stress history of thickened tailings layers

content up to which they should continue desiccation. The time required for desiccation in this step is expected to significantly influence the final strength, but may also lead to acid rock 
drainage and higher costs (Simms and Grabinsky, 2004). The suction generated during desiccation is associated with decreasing water content during drying process. Longer time of desiccation results in lower values of water content and therefore higher values of suction. It has been suggested that if thickened tailings are dried to their shrinkage limit, that they will not exhibit flow liquefaction, and thus pose minimal risk of significant deformation during seismic events. ICOLD and UNEP (2001) contains the following text: "The thickened tailings do not segregate, so that all the particle sizes stay together forming a homogeneous material with a high capillary suction: when placed layer by layer, it dries to near its shrinkage limit and becomes dilative under earthquake strains, thus preventing liquefaction. It tends to remain saturated almost to the surface, thus preventing the development of acid drainage and so becomes suitable for eventual topsoil and vegetation." Though no additional source is provided, it suggests that drying to the shrinkage will result in dilative behaviour. However, there has only been limited experimental evidence to back up this supposition. Mining operators or regulators are naturally concerned about the remobilization of an unbounded tailings stack due to earthquake or heavy rainfall. This concern is of significant importance if the thickened tailings stack is constructed in an inclined area where long flows of failed tailings may occur.

This study aims to simulate thickened tailings deposition by small scale and multilayer laboratory tests and investigate the effect of drying time and stress history on final monotonic and cyclic shear strength of thickened gold tailings. The shear strength of the tailings will be measured using simple shear, triaxial, and vane shear tests. The effect of desiccation will also be compared to the effect of overconsolidation by mechanical loading. This last part is important as many practitioners believe that the behavior and response of desiccated and rewetted tailings can be 
simulated by mechanical overconsolidation. This hypothesis presumes that the equivalent effective stress generated during desiccation and alleviated during rewetting can be simulated by mechanical overconsolidation consisting of loading to higher effective stress and unloading to lower effective stress. This study aims to experimentally verify the validity of this assumption.

\subsection{Objectives of the study}

The primary goal of this study is to investigate the influence of stress history induced by desiccation and consolidation settlement on monotonic and cyclic shear strength of thickened gold tailings. Specific objectives in support of the primary goal include:

- Simulating thickened tailings field deposition in the laboratory using small scale and multilayer deposition methods and evaluating different methods of sampling to obtain samples for simple shear and triaxial tests.

- Investigating the response of mechanically overconsolidated tailings and comparing with the response of desiccated-rewetted tailings.

- Verifying the applicability of constant volume simple shear tests for desiccated-rewetted fine grained soils generating low values of suction.

- Comparing monotonic and cyclic simple shear test and triaxial tests and obtaining a correction factor for cyclic resistance $\left(\mathrm{C}_{\mathrm{r}}\right)$. 
- Obtaining Soil Water Characteristic Curve (SWCC) and Shrinkage Curve of Bulyanhulu gold tailings.

- Following variations of matric and total suctions, water content and void ratio during multilayer deposition of thickened gold tailings layers.

\subsection{Outlines of the dissertation}

This thesis consists of seven chapters. This first chapter presents a background and the objectives of the research. A literature review presented in chapter 2 includes:

a) An overview of deposition methods and geotechnical properties of tailings

b) Simple shear test and triaxial test methods, undrained response of sands, cyclic behavior and soil liquefaction

c) Mechanics of unsaturated soils, suction measurement devices, consolidation and shear strength in unsaturated soils, effective stress in unsaturated soils

d) Microstructure investigation, Mercury Intrusion Porosimetry (MIP)

Chapter 3, titled Materials and Methodology, presents details of sample preparation methods, the small scale field simulation in a bucket and multilayer deposition in a modular drying box. In addition, details of drying box measurement sensors and different methods of sampling are also addressed. Shear strength measurement devices such as vane shear, simple shear and triaxial 
devices at Carleton, and details of modified triaxial cell are explained. In the final sub-section of the chapter, the sample preparation method for MIP device is explained.

Chapter 4 presents the results of the simulation of multilayer deposition of thickened gold tailings in a drying box. Measurements of water content, evaporation, drainage, matric and total suction during multilayer deposition in the drying box are presented in the chapter. Chapter 5 addresses the applicability of constant volume simple shear tests for desiccated-rewetted gold tailings. Simple shear results of dry and partially saturated constant volume simple shear tests on Silica sand, glass bead and synthetic silts are presented and compared in order to verify the applicability of the findings of Dyvik et al. (1987). This chapter is a prerequisite for presenting simple shear results in chapter 6 . The final goal of this chapter is to verify the applicability of constant volume simple shear test for desiccated/rewetted tailings.

Chapter 6 presents all shear strength measurement test results, which includes the following:

a) Monotonic and cyclic simple shear tests on desiccated-rewetted samples prepared by small scale method

b) Monotonic and cyclic simple shear test on samples obtained from the drying box (sampling and buried tubes)

c) Monotonic simple shear test on desiccated tailings

d) Simple shear tests on mechanically overconsolidated samples

e) Conventional and modified triaxial tests on desiccated-rewetted tailings and desiccated tailings 
f) Vane shear tests on desiccated-rewetted tailings and desiccated tailings

Chapter 7 presents a summary and comparison of the results, discussion and conclusions. 


\section{Chapter 2: Literature Review}

\subsection{Introduction}

This literature review includes information on methods of tailings deposition (sections 2.2 and 2.3), thickened and paste tailings technology (section 2.4), geotechnical parameters of tailings (section 2.5), simple shear and triaxial test mechanics (section 2.6), liquefaction mechanisms (section 2.7), undrained behaviour of sands (section 2.8), cyclic behavior of tailings (section 2.9), mechanics of unsaturated soils, suction measurement devices, different approaches for interpreting effective stress of unsaturated soils (section 2.10), and finally Mercury Intrusion Method (MIP) (section 2.11). 


\subsection{Tailings deposition}

Mine wastes are among the largest amount of waste generated around the world. In Canada, the mining industry produces approximately 1 million tonnes of waste rock and 950000 tonnes of tailings per day, which in total it equals roughly 700 million tonnes per year (Crowder, 2004). The ore extraction rate and consequent tailings generation rate at the average mine is continually increasing, as ore bodies with lower concentrations of the desired mineral are increasing.

Mine waste can be categorized to two general types: waste rock and tailings. Waste rock is the residual material from excavation and blasting that is uneconomic to be sent to the mill due to the low concentration or absence of desired mineral. Waste rock particle size ranges from fine sands up to boulders. Waste rocks are typically deposited in piles or "dumps" on surface impoundment. On the other hand, rocks that contain an economic concentration of the desired mineral are sent to the mill for processing. Tailings are the waste product of the milling process, which includes grinding the rock to facilitate liberation of the different mineral grains. Most mills for hard rock mining grind the rock down to particles less than 100 microns in size, and with most of the particles greater than 10 microns. Tailings ground from rock are therefore predominantly silty material.

Most tailings are deposited in storage impoundments on the surface, though a significant fraction is returned underground as backfill in many operations. Historically, tailings were often deposited with no control in a nearby valley or water body close to the mine. In 1920s, some mining companies decided to confine tailings by dams to limit impoundment footprints (Robinsky, 1999). 


\subsection{Tailings storage and disposal techniques}

\subsubsection{Conventional methods of tailings storage}

Conventional tailings storage facilities normally employ surface impoundments confined by tailings dams. Vick (1990) categorized surface impoundment to two groups: "raised embankment" and "water-retention type dam". The majority of tailings impoundments consist of raised embankment (Vick 1990). Upstream and downstream methods have been widely used in slurry tailings as conventional methods for storing mine tailings.

\subsubsection{Upstream and downstream methods for tailing storage}

Upstream and downstream methods are conventional methods for tailing impoundments. In the upstream method, tailing materials are deposited from the top of the dyke (Vick, 1990). Figures 2.1 and 2.2 show water-retention, upstream and downstream methods for tailing storage. Though the water-retention type dam is relatively safe in terms of geotechnical stability, it is less frequently used due to higher cost of construction. In the upstream method, the dam or dyke is constructed in stages, with later stages being constructed on top of the tailings themselves. The underlying tailings typically undergo grain size segregation, coarser particles or sands settling closest to the dyke (Vick, 1983). While the sand fraction has better drainage characteristic than the fines, if the sands remain saturated they are susceptible to liquefaction. The upstream method has had the largest occurrence of tailings failures (Vick 1990). 


\title{
Water-retention type dam for tailings storage
}

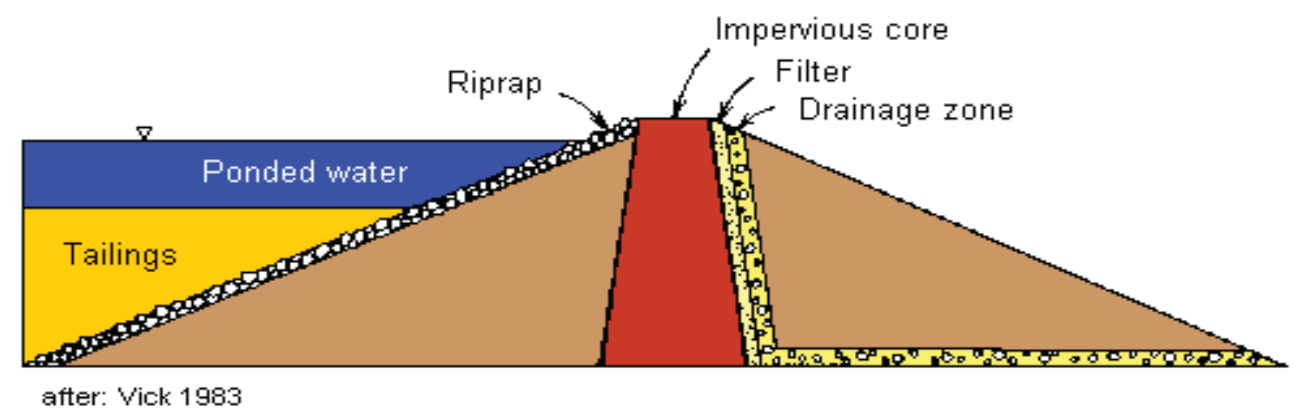

Figure 2.1 Typical water retention type dam for tailings storage (after Vick, 1983)

\section{Types of sequentially raised tailings dams}

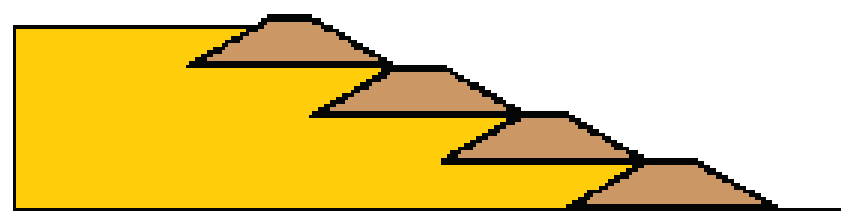

\author{
upstream
}

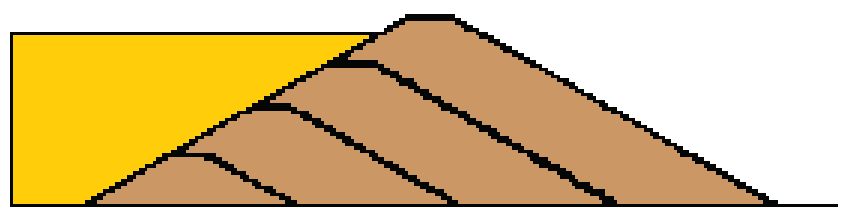

downstream

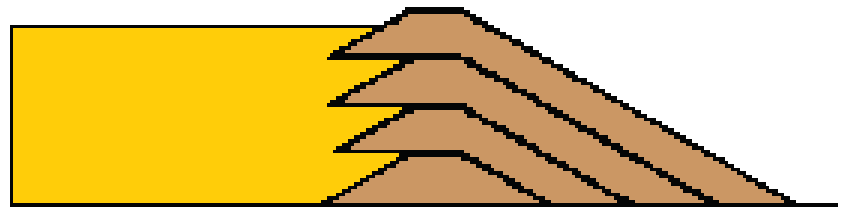

centerline

after: Vick 1983

Figure 2.2 Different tailings conventional deposition (after Vick, 1983) 
The downstream method has a similar starter dyke like the upstream method. However, the first dyke in the downstream method is not as permeable as the first dyke in the upstream method (Vick 1990). The most important difference between upstream and downstream method is that in downstream methods dykes are not allowed to be constructed on the unconsolidated tailing materials (Vick, 1990). Consequently, vulnerability to seismic loads is less than the upstream method and stability and resistance to failure are more than upstream method.

\subsubsection{Rationale for dewatered tailings disposal}

Dewatering tailings prior to deposition has some attractive potential advantages, including increased recycling of water, and improving stability of the deposited tailings. If the tailings are dewatered to the extent that they exhibit a yield stress upon deposition, they will form gently sloped stacks (Robinsky, 1999). This method of tailings deposition, which is called thickened or paste tailings, has been widely used since 1970's. Thickened tailings may gain shear strength through desiccation and self-weight consolidation subsequent to deposition. The increase in density due to desiccation not only decreases the total volume of the stack layers, but also increases the resistance of tailings stack to earthquake loading. In comparison to conventional methods, tailings stacks do not necessarily need dam containment. In addition, there is no overlying head of water on the top of the impoundment, which would result in reduction of seepage out of tailings impoundment in comparison to conventional methods (Crowder, 2004).

Due to limited water supply in many mine sites, dewatering the tailings would also help to have 
greater water recycling in a mining operation. Figure 2.3 shows a typical gold tailings disposal facility located at Bulyanhulu mine in Tanzania.

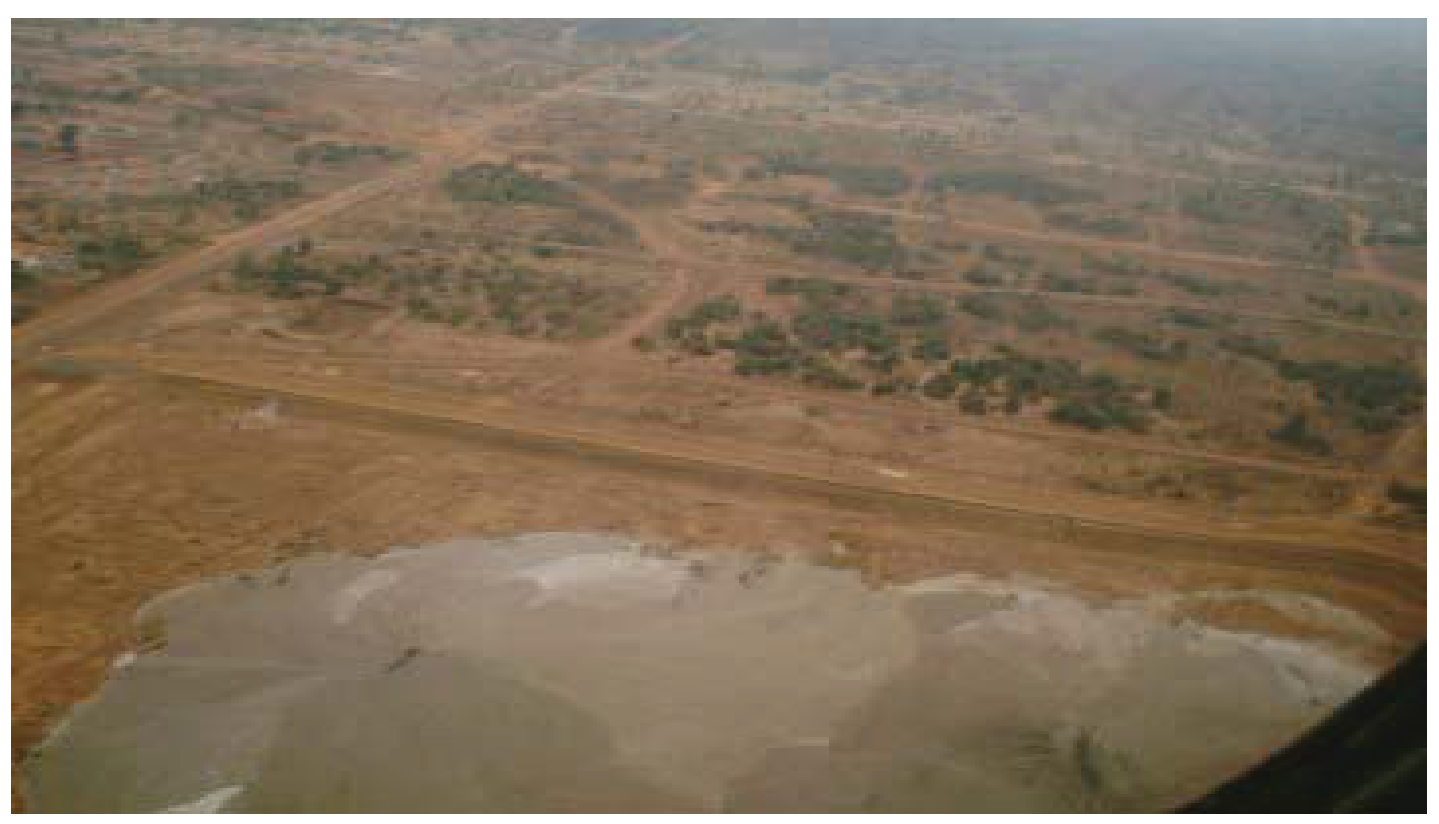

Figure 2.3 Thickened tailings disposal during early deposition at the Bulyanhulu mine (Photo courtesy of Crowder, 2004)

\subsection{Definition of tailings based on water content / solids content}

\subsubsection{Slurry Tailings}

Due to high water content of slurry deposition, the consequences of dam failure are significant. Slurry tailings may slowly gain strength and may remain in a state susceptible to flow 
liquefaction for a considerable time after deposition. Consequently, a breach in confinement may result in a considerable flow distance and associated catastrophic impacts.

Tailings coming out of the mill typically have high water contents due to water requirements for operations and transport during mineral processing. For example, solids concentrations $50 \%$ or lower are typical for hard rock mines. Slurry tailings are typically transported from the mill to the disposal area in the turbulent flow regime and above the critical flow velocity that defines onset of sedimentation in the pipeline. Figure 2.4 shows slurry tailings discharged in a tailings impoundment. Slurry tailings generally undergo grain size segregation during deposition, coarse particles settling closer to the deposition point. In most climates, slurried tailings form a pond that covers a fraction of the impoundment, with some beach of tailings forming near the deposition points or spigots. Slopes of slurry tailings deposits are usually less than $1 \%$ (Vick 1990).

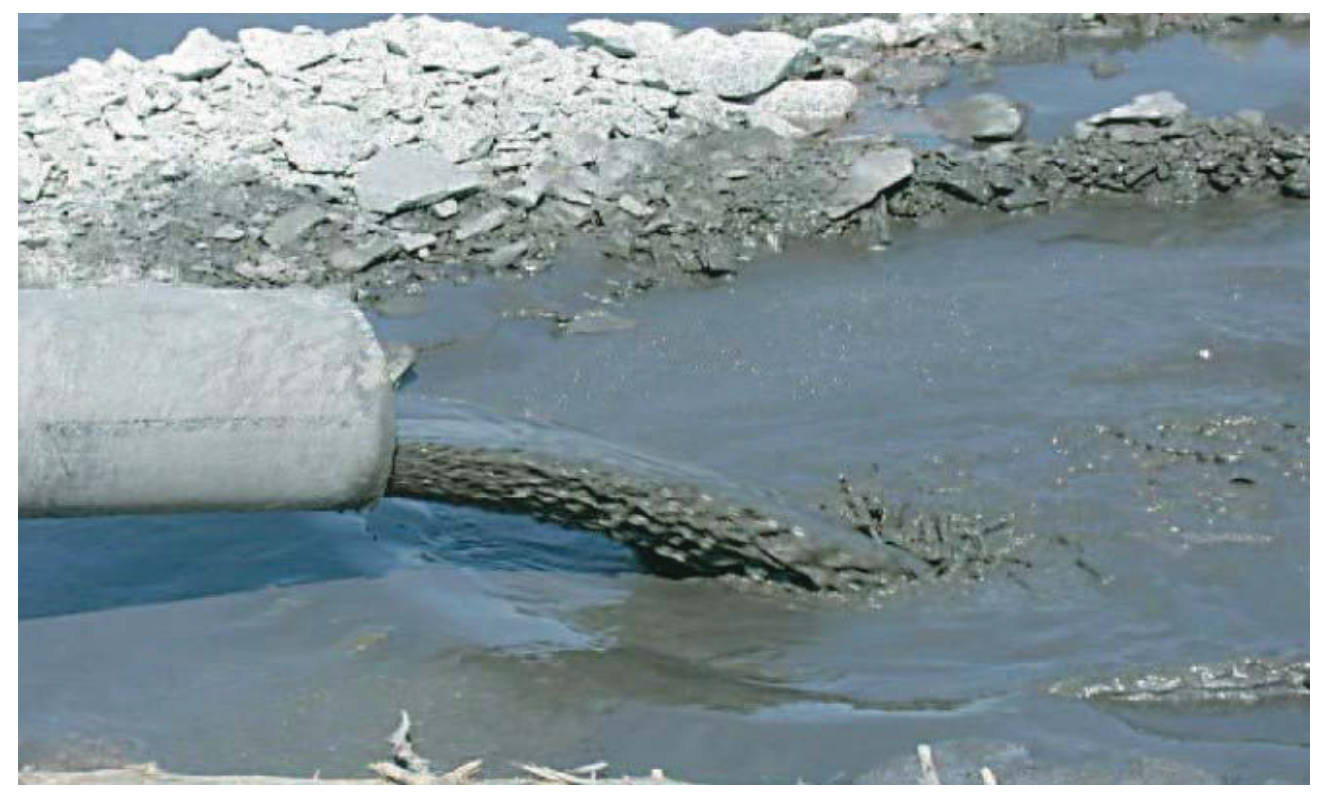

Figure 2.4 Slurry tailings discharged in tailings impoundment (after Bussiere, 2007) 


\subsubsection{Thickened Tailings}

One may define thickened tailings as tailings sufficiently dewatered such that they do not grainsize segregate during deposition, although they may still have a critical flow velocity during transport (Robinsky, 1975). Barbour et al. (1993) found no tangible difference between $\mathrm{D}_{10}$ and $\mathrm{D}_{60}$ at the Kidd Creek mine even at a distance of about $1 \mathrm{~km}$ from discharge, which shows the high relative homogeneity in thickened tailings in comparison to slurry tailings.

Thickened tailings generally also exhibit a yield stress by the time they come to rest in the disposal area, though they do not necessarily exhibit non-Newtonian behavior during pipeline transport. As a consequence of this manifestation of yield stress, thickened tailings naturally stack at a gentle slope. The slope gained varies between 2 to $5 \%$, and appears to depend on both operational and material parameters (Jewell, 2012; Simms et al., 2011). The prediction of beach slope is outside the scope of this thesis.

\subsubsection{Paste Tailings}

Certain tailings, given certain grains size requirements, may be dewatered to the point where they can be pumped as a laminar fluid with minimal sediment buildup in the pipeline. The solid content in paste tailings is approximately between $70 \%$ and $85 \%$. Figure 2.5 shows a typical method of paste tailings deposition from Bulyanhulu (Simms et al., 2007; Shuttleworth et al., 2005). Paste technology is more commonly used in cemented underground backfill, where 
transport is substantially aided by gravity, and a number of benefits, including homogeneity and quick strength gain, favour the relatively high degree of dewatering.

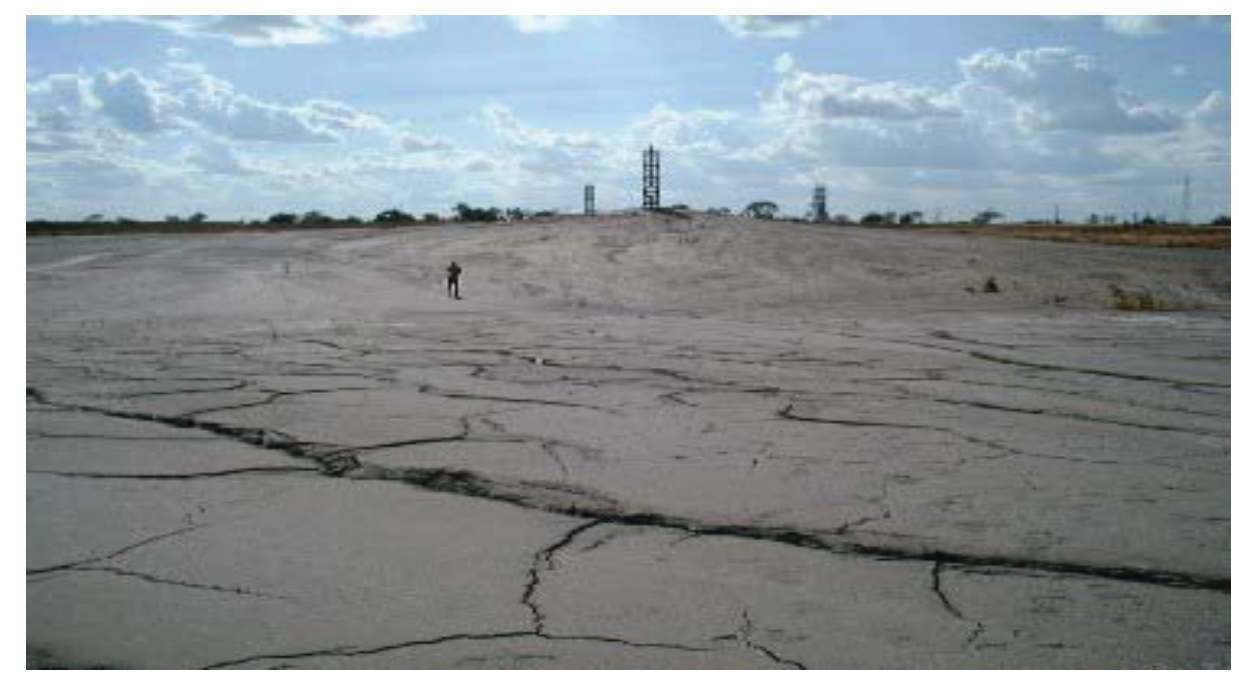

Figure 2.5 Typical tailings disposal facility at Bulyanhulu site (after Simms et al, 2007; Shuttleworth et al., 2005)

\subsubsection{Deposition of dewatered tailings}

It has been recognized that cycling deposition between different points in a tailings impoundment can accelerate overall strength gain. It allows for freshly deposited layers to dry and increase the shear strength and density. This may also prevent remobilization of the tailings when the next layer is deposited on top. However, the strength and stability of the stack as well as the environmental risks are highly affected by hydrological process in the tailings deposition area. Figure 2.6 presents the hydrological process and related stack design issues (Simms and Grabinsky, 2004). 


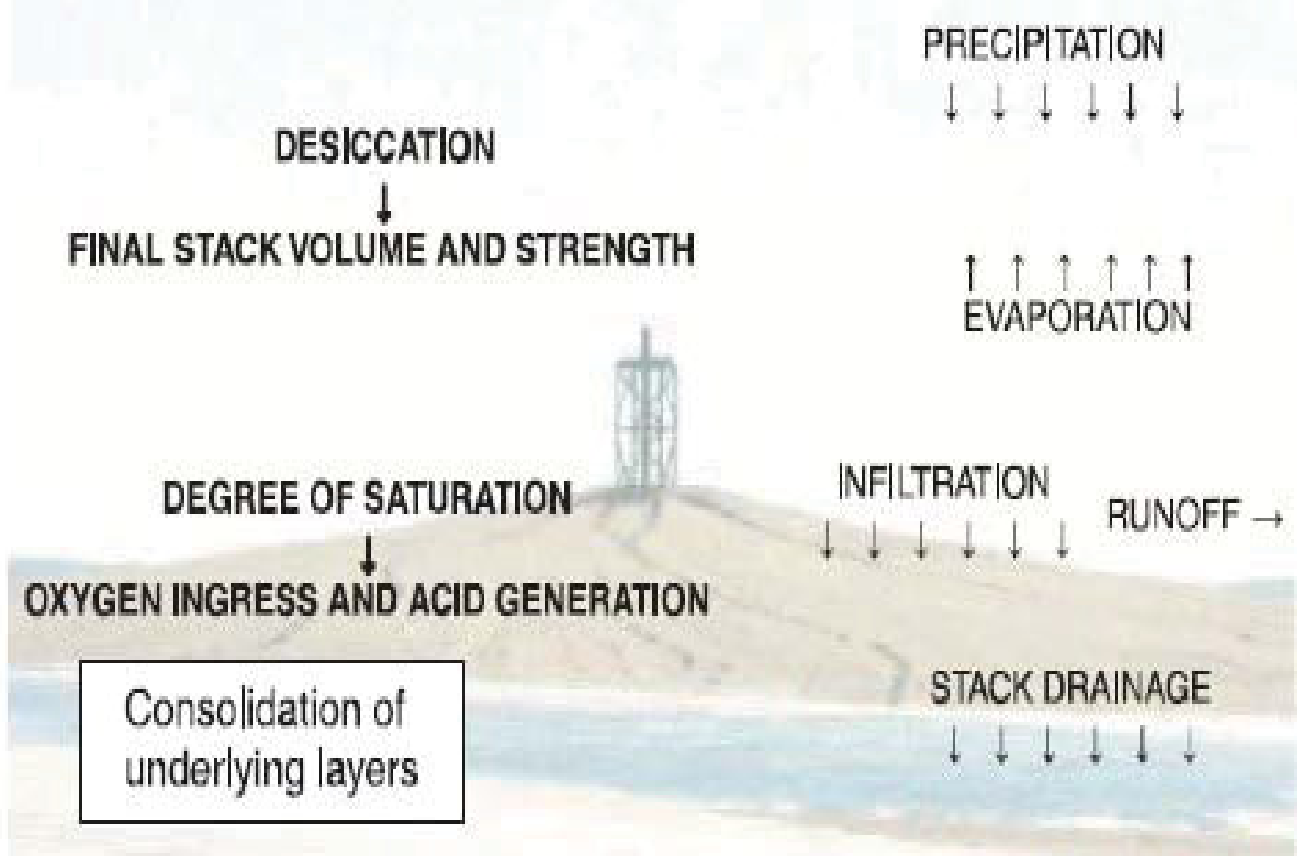

QUANTITY OF ACID DRAINAGE

Figure 2.6 Hydrological process of thickened tailings stack (after Simms and Grabinsky, 2004)

However, in the case of acid generation in tailings, a risk for generation and release of acid still remains in thickened and paste technology (Simms and Grabinsky, 2004). Although evaporation in a freshly deposited layer is helpful for densification and increase of shear strength, excess evaporation desaturates the tailings and increases the risk of acid generation. Figure 2.7 shows a typical shrinkage curve of thickened tailings with respect to relative residual strength and relative oxygen diffusion coefficient. It is clearly shown that as void ratio decreases, strength and density increases, but due to increasing the oxygen diffusion, the risk of acid generation increases once the tailings start to considerably desaturate (Yanful, 1993). Therefore, excessive drying may be problematic for acid generation. 


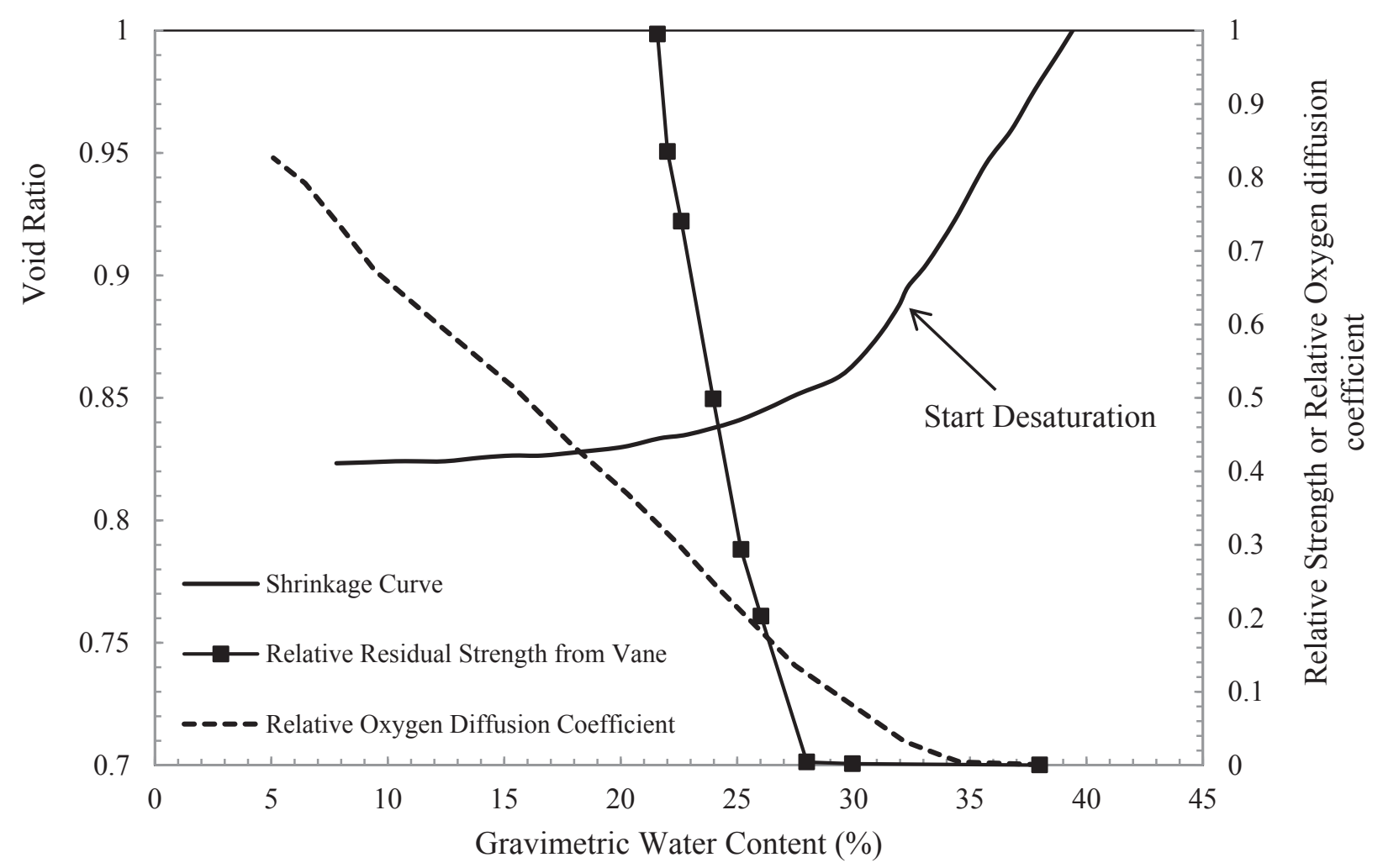

Figure 2.7 Typical shrinkage curve with respect to residual strength and oxygen diffusion coefficient (after Simms et al., 2007)

\subsection{Geotechnical Properties of Tailings}

\subsubsection{Basic Characteristics}

Generally, tailings that are produced from hard rock mining consist of fine sand or silt sized particles. Before deposition, $\mathrm{D}_{10}$ varies between 0.001 and $0.004 \mathrm{~mm}$, and $\mathrm{D}_{60}$ varies between 0.01 and $0.05 \mathrm{~mm}$. The coefficient of uniformity $\mathrm{C}_{\mathrm{u}}$ can vary between 8 and 18 (Bussiere, 2007). According to compaction test results for both standard and modified test, the optimal water content $\mathrm{w}_{\mathrm{opt}}$ varies between $10 \%$ and $20 \%$ and the corresponding unit weight $\gamma_{\mathrm{d}}$ varies between 
14.5 and $20 \mathrm{kN} / \mathrm{m}^{3}$. Generally, the metal mine tailings can be classified as ML (silt with low plasticity) or SM-ML (silty sands) for fine tailings and SP for coarse tailings (Bussiere, 2007).

Qiu and Sego (2001) also performed some experiments to study the basic characteristics of four different tailings. Table 2.1 shows the basic characteristic of three different tailings based on the investigations of Qiu and Sego (2001). Based on grain-size distributions and basic physical properties, tailings are usually non-plastic except for the coal wash tailings (Qiu and Sego, 2001).

Table 2.1 Basic properties of mine tailings (Qiu and Sego, 2001)

\begin{tabular}{cccc}
\hline Tailings type & Gold & Copper & Coal \\
\hline Specific Gravity, $\mathrm{G}_{\mathrm{s}}$ & 3.17 & 2.75 & 1.94 \\
Liquid Limit $(\%)$ & $24-30$ & - & 40 \\
Plasticity Index (\%) & $1-5$ & - & 16 \\
Shrinkage Limit (\%) & 21 & 24 & 21.1 \\
Clay size particles $(<2 \mu \mathrm{m}, \%)$ & 5 & 1.3 & 22.5 \\
Sand content $(>0.06 \mathrm{~mm}, \%)$ & 33 & 74.5 & 40 \\
Fines Content $(<74 \mu \mathrm{m}, \%)$ & 81 & 31.3 & 66.4 \\
$\mathrm{D}_{10}(\mu \mathrm{m})$ & 5 & 16 & 1.31 \\
$\mathrm{D}_{30}(\mu \mathrm{m})$ & 19 & 72 & 4.13 \\
$\mathrm{D}_{60}(\mu \mathrm{m})$ & 54 & 153 & 60 \\
USCS classification & $\mathrm{ML}$ & $\mathrm{SM}$ & $\mathrm{CL}$ \\
\hline
\end{tabular}

\subsubsection{Grain Size Distribution and water retention curve}

Comparisons of grain size distribution are reported in Vick et al. (1990), Qui and Sego (2001) and Bussiere (2007). In general, the grain size distribution of hard rock mines is generally in the 
range reported by Vick (1990), which has been shown in Figure 2.8. According to the soil mechanic reports from many projects, tailings are commonly classified in Unified Soil Classification System (USCS) as silt with low plasticity (ML) or Silty Sands (SM-ML).

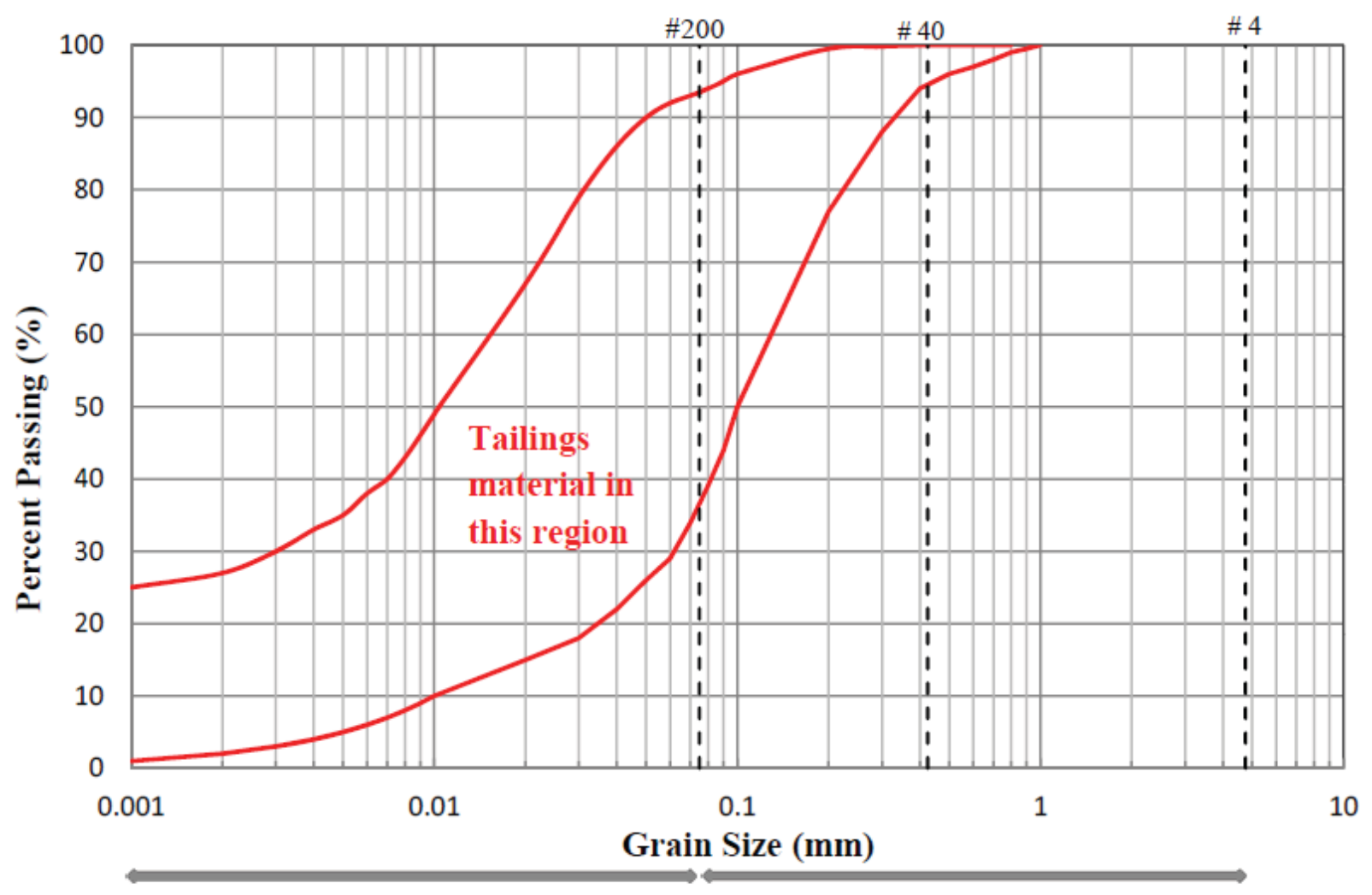

Clay and Silt

Sand

Figure 2.8 General range of grain size distributions for hard rock tailings (Vick, 1990)

\subsubsection{Consolidation Properties and Shrinkage Curve}

Bussiere (2007) reported consolidation characteristics of tailings. Compression index $C_{c}$, the recompression index $\mathrm{C}_{\mathrm{cr}}$ and the coefficient of consolidation $\mathrm{C}_{\mathrm{v}}$ were calculated in his tests. 
Typical value of compression index $C_{c}$ is estimated to be between 0.05 to 0.28 depending on the USCS classification and the type of the sample (Bussiere, 2007). The higher value of $C_{c}$ is generally related to the value of initial void ratio $e_{0}$. The recompression index usually varies between 0.003 and 0.03 . Basically, the consolidation process of coarse tailings is faster than fine tailings because of higher hydraulic conductivity. Table 2.2 demonstrates the consolidation test results for undisturbed hard rock mine tailings (Bussiere, 2007).

Table 2.2 Consolidation behavior of tailings obtained by different researchers

\begin{tabular}{cccccc}
\hline $\begin{array}{c}\text { Tailings } \\
\text { Classification }\end{array}$ & $\mathbf{e}_{\mathbf{o}}$ & $\mathbf{C}_{\mathbf{c}}$ & $\mathbf{C}_{\mathbf{c r}}$ & $\mathbf{C}_{\mathbf{v}}\left(\mathbf{c m}^{2} / \mathbf{s}\right)$ & Reference \\
\hline SM & 0.62 & 0.13 & 0.02 & - & Mabes et al. (1977) \\
ML & - & 0.28 & & $1.5 \times 10^{-1}$ & Volpo (1979) \\
ML & 0.75 & 0.05 & - & - & Matyas et al. (1984) \\
ML & 0.7 & 0.07 & - & $1.5 \times 10^{-3}$ & Blight and Steffen (1979) \\
ML & - & 0.054 & 0.056 & $6.2 \times 10^{-3}$ & Chen et al. (1988) \\
SP-SW & 1.06 & 0.08 & & - & Mittal and Morgenstern (1975) \\
SM & 0.95 & 0.13 & - & $1 \times 10^{-2}$ & Mittal and Morgenstern (1976) \\
\end{tabular}

In addition, Qiu and Sego (2001) also measured shrinkage limit and plotted shrinkage curves for different tailing materials. Shrinkage curve of different mine tailings are presented in Figure 2.9. Figure 2.9 clearly shows that when water content decreases, void ratio (volume) also decreases. The shrinkage curve shows that the void ratio changes with the moisture content and the 
shrinkage limit is the water content at which the volume of the soil does not decrease with decreasing moisture content (Qiu and Sego, 2001).

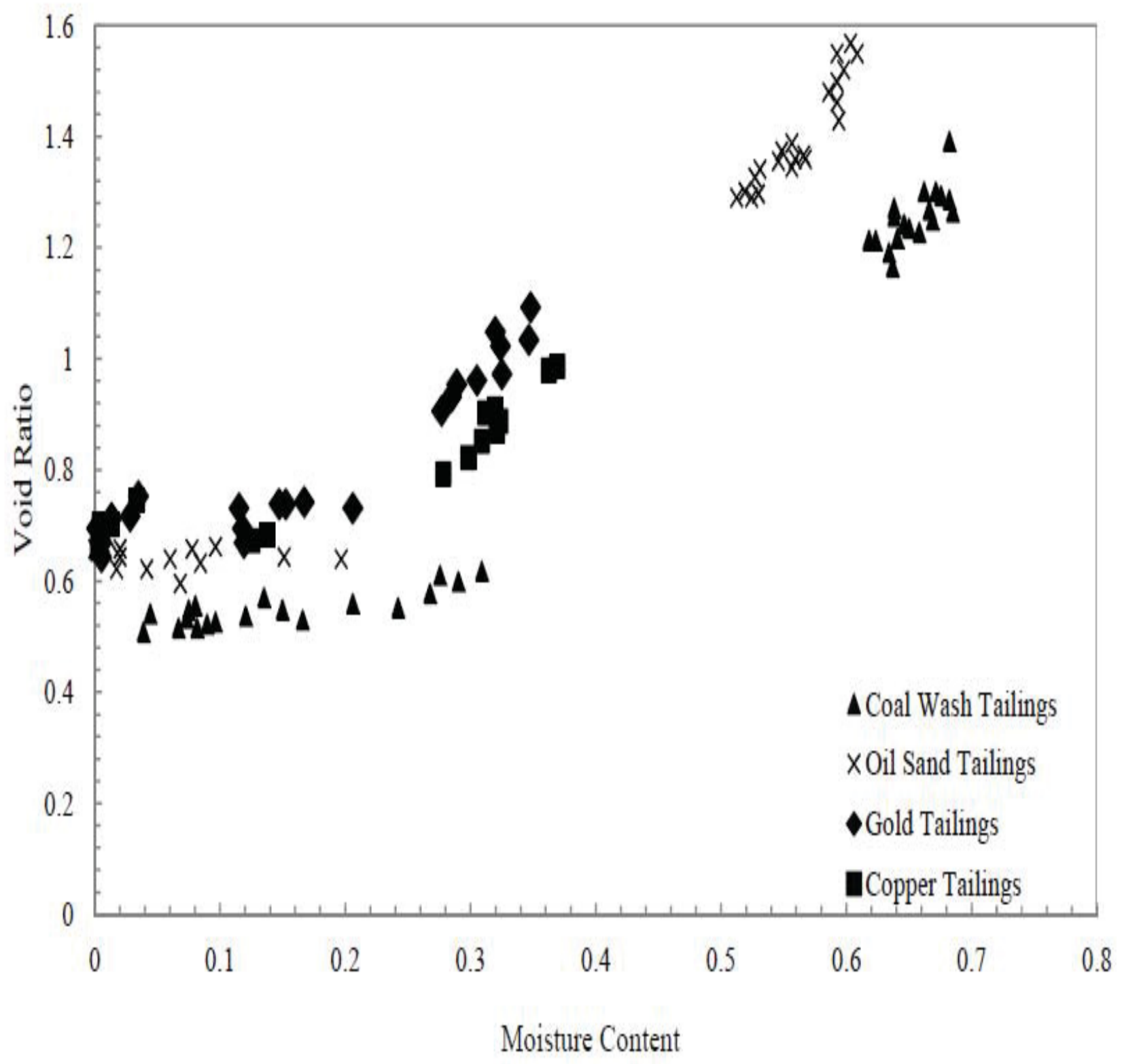

Figure 2.9 Shrinkage Curve of different mine tailings (after Qui and Sego, 2001) 


\subsubsection{Saturated hydraulic conductivity}

The saturated hydraulic conductivity is defined to evaluate the range of permeability in tailings materials. Due to the fact that the majority of tailings materials consist of fine grained materials, the coefficient of permeability of tailings is not so high. Because of heterogeneous nature of the tailings within the impoundment, it is not easy to determine the saturated hydraulic conductivity location by location in a tailings deposition. Bussiere et al. (2007) performed some tests to find the saturated hydraulic conductivity of tailings and they found it within the range of $3 \times 10^{-7} \mathrm{~m} / \mathrm{s}$

Table 2.3 Saturated hydraulic conductivity of hard rock mine tailings obtained by different researchers

\begin{tabular}{cccc}
\hline $\begin{array}{c}\text { Tailings } \\
\text { Classification } \\
\text { (USCS) }\end{array}$ & $\begin{array}{c}\text { void ratio } \\
(\mathbf{e})\end{array}$ & $\begin{array}{c}\text { Saturated hydraulic } \\
\text { conductivity }\left(\mathbf{K}_{\mathrm{s}, \mathbf{c m} / \mathbf{s})}\right.\end{array}$ & Reference \\
\hline ML & - & $6 \times 10^{-5}-6 \times 10^{-6}$ & Al and Blowes (2000) \\
ML & $0.68-1.14$ & $1.5 \times 10^{-5}-7 \times 10^{-6}$ & Barbour et al. (1993) \\
ML & $1.2-1.7$ & $1 \times 10^{-5}-7 \times 10^{-7}$ & Matyas et al. (1984) \\
ML & 0.7 & $1.6 \times 10^{-5}-9 \times 10^{-7}$ & Blight and Steffen (1979) \\
SM-ML & - & $6 \times 10^{-4}-4 \times 10^{-6}$ & Chen et al. (1988) \\
SM-ML & $0.65-1.1$ & $1 \times 10^{-2}-1 \times 10^{-3}$ & Mittal and Morgenstern (1975) \\
SM-ML & $0.6-1.25$ & $1 \times 10^{-4}-5 \times 10^{-7}$ & Mittal and Morgenstern (1976) \\
SP & $0.5-1.1$ & $6 \times 10^{-3}-2 \times 10^{-4}$ & Nelson et al. (1977) \\
ML & $0.6-0.9$ & $4 \times 10^{-5}-3 \times 10^{-6}$ & Brisson et al. (2002) \\
ML & 0.55 & $1.3 \times 10^{-4}$ & Guerra (1973) \\
ML & $0.45-1.2$ & $1 \times 10^{-5}-1 \times 10^{-6}$ & Mabes et al. (1977) \\
SM & 0.87 & $5 \times 10^{-4}$ & Bussiere et al. (1998) \\
\hline
\end{tabular}


and $1 \times 10^{-4} \mathrm{~m} / \mathrm{s}$. Table 2.3 presents typical saturated hydraulic conductivity results of remolded and undisturbed hard rock mine tailings from different researchers.

\subsubsection{Monotonic Shear Strength}

While the monotonic and cyclic response of saturated sands and clays have been the topic of extensive research during the past 50 years, the behavior of mine tailings materials especially in unsaturated condition have been considered only in recent years. The strength parameters of fine grained soils are typically used in design of mine tailings facilities, landfills, surface foundations, etc.

Qui and Sego (2001) performed around 20 triaxial undrained (CU) tests on different saturated tailings to determine the effective shear strength parameters of the tailings. After saturation and before applying deviator stress, the specimens were consolidated under different consolidation pressures such as 25, 50 and $100 \mathrm{kPa}$. Figures 2.10 and 2.11 present the stress-strain response and excess pore water pressure generation under $50 \mathrm{kPa}$ consolidation pressure respectively. Typical shear strength parameters of different tailings measured and tabulated in table 2.4 (Qui and Sego, 2001).

Table 2.4 Typical Effective shear strength parameters obtained by CU tests (Qui and Sego, 2001)

\begin{tabular}{ccc}
\hline Tailings type & $c^{\prime}$ & $\emptyset^{\prime}$ \\
\hline Copper & 0 & 34 \\
Gold & 0 & 33 \\
Coal & 10 & 32 \\
Oil Sand & 3 & 30 \\
\hline
\end{tabular}




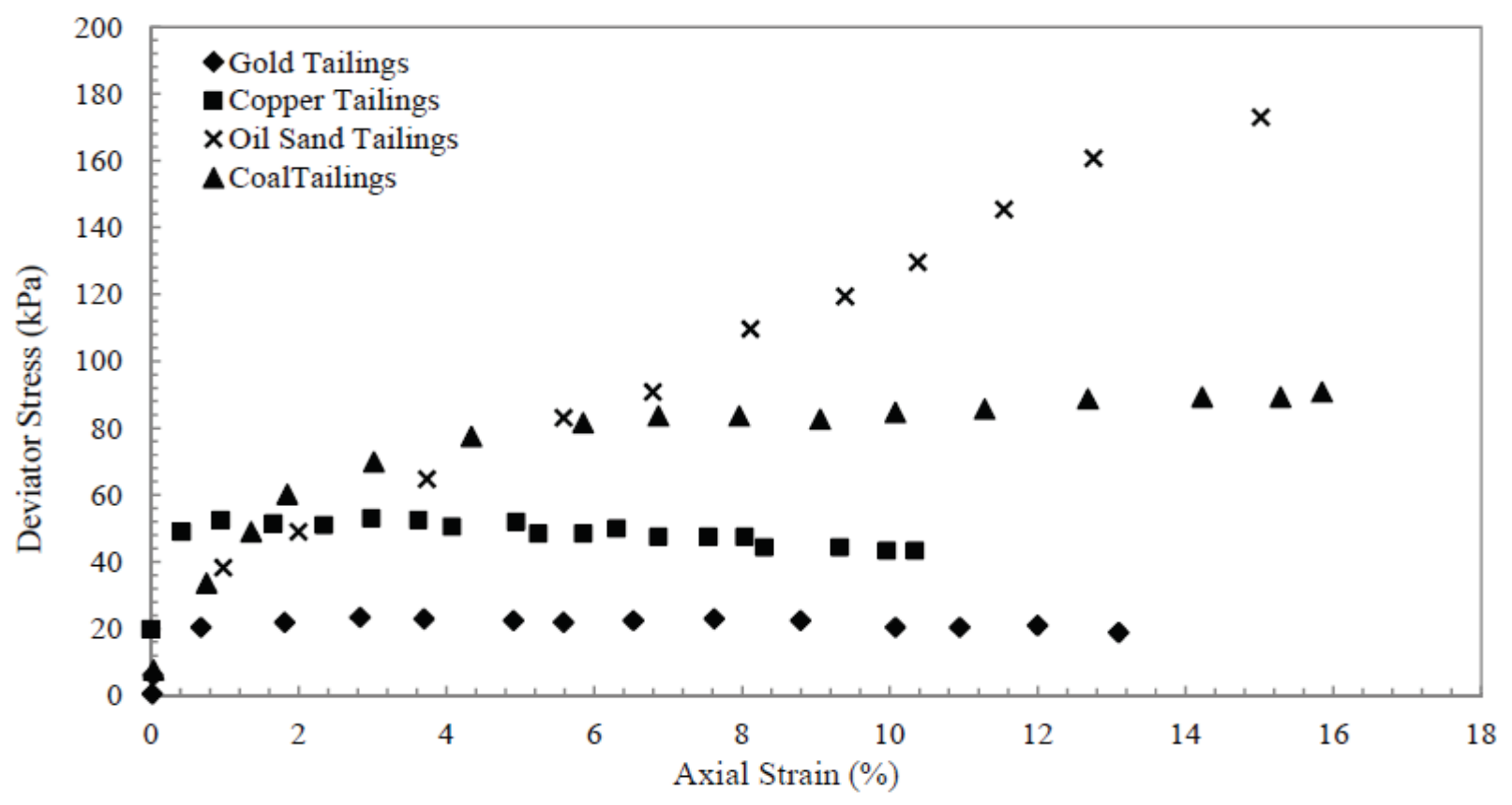

Figure 2.10 Monotonic stress-strain responses of different tailings under $50 \mathrm{kPa}$ CU triaxial test (after Qui and Sego, 2001)

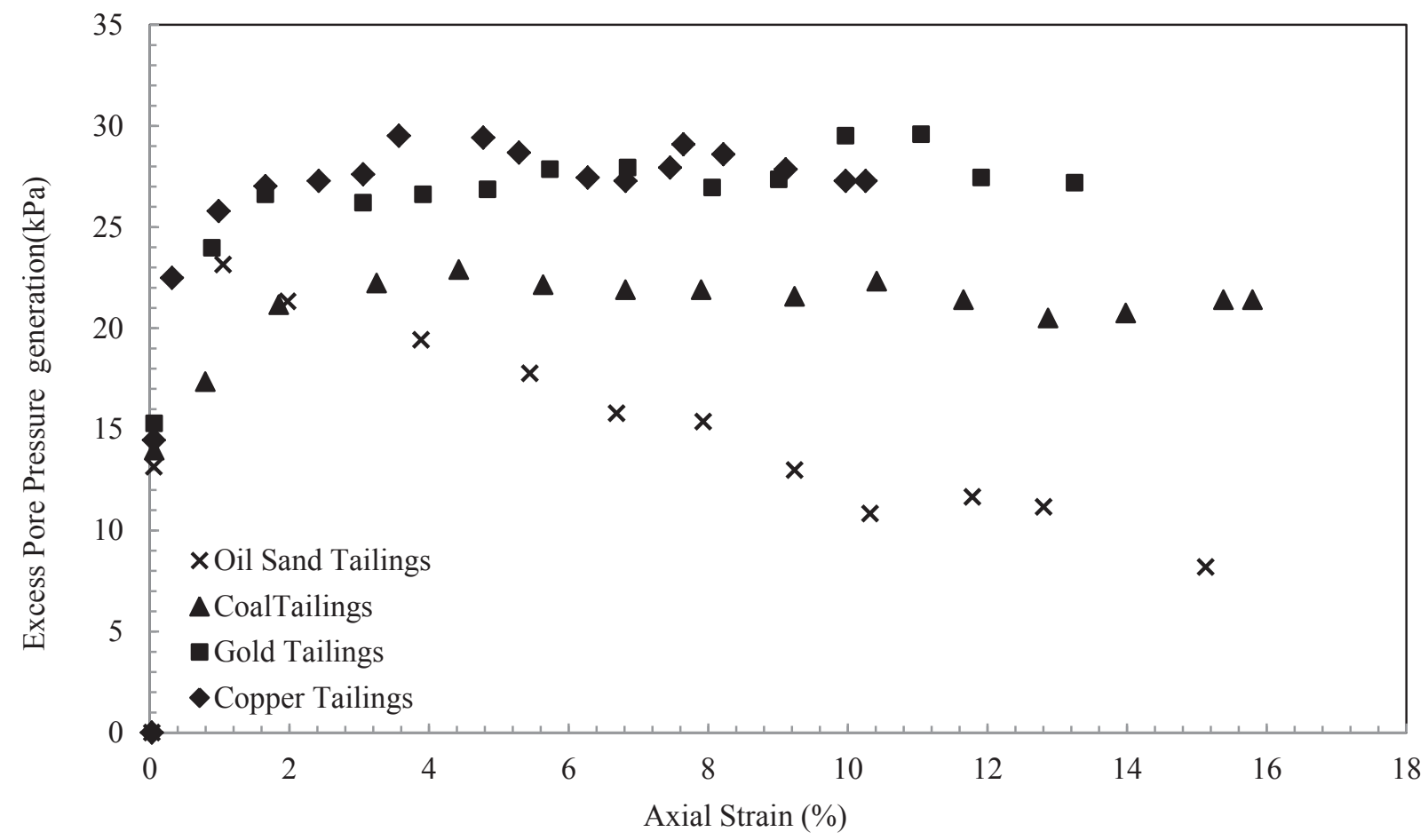

Figure 2.11 Excess pore pressure generation of different tailings under $50 \mathrm{kPa} C U$ triaxial tests (after Qui and Sego, 2001) 
Table 2.4 shows that the failure effective friction angle of different tailings were calculated within the range of $30^{\circ}-35^{\circ}$. Figure 2.10 clearly shows that Coal and CT tailings have strainhardening behavior. It should be noted that the shear behavior is a function of density.

Sanin (2005) also performed some monotonic CU triaxial tests on fine grained Fraser River delta silt at University of British Columbia. Figure 2.12 presents monotonic triaxial response of fine grained silt. It is shown that the samples at different consolidation pressures exhibited strainhardening response.

\subsection{Stress path and shear strength}

Different types of laboratory tests, such as simple shear test, triaxial test and unconfined compression test, are used to determine the shear strength parameters of saturated soils. The most preferred laboratory shear test should be the one that can simulate the in-situ field condition in an element test. Triaxial tests (compression and extension), direct shear test, simple shear test and hollow cylinder tests can be used in the laboratory shear tests to simulate different field conditions. Although triaxial and hollow cylinder tests represent the stress-strain relationships more distinctively, direct shear tests (DST) are widely employed because of its simplicity and quickness. Direct shear tests are typically performed to determine the soil strength parameters of a potential failure surface, which are more or less horizontal (Dyvik et al., 1987; Prevost and Høeg 1970). Bjerrum and Landva (1966) introduced an initial procedure for direct simple shear test which is fairly similar to a DST test but with improved uniformity and control. In the simple 

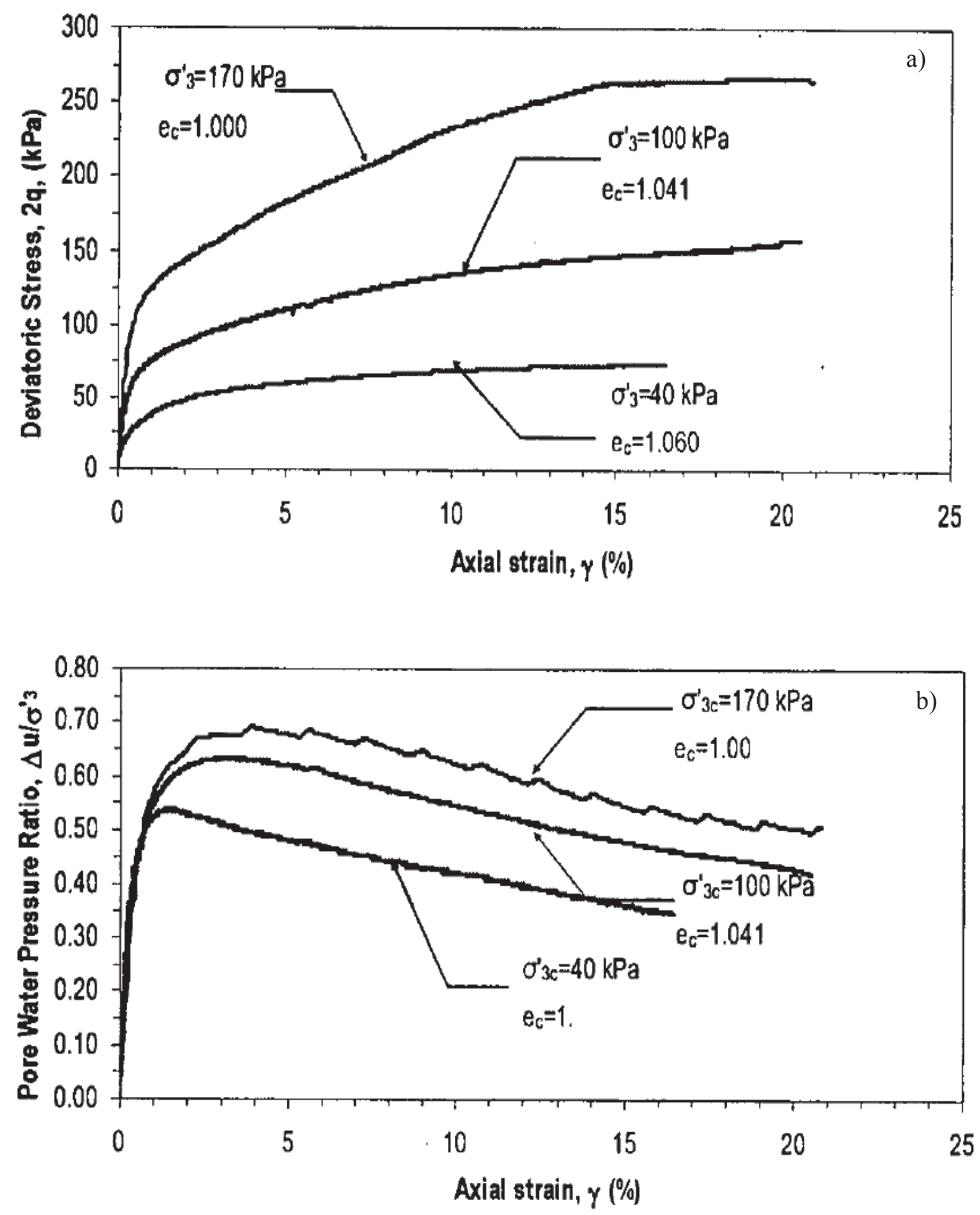

Figure 2.12 Monotonic triaxial response of fine grained Fraser River delta silt (after Sanin, 2005) 
shear device, the specimen is initially consolidated under one-dimensional vertical load and subsequently sheared by applying a shear stress in the horizontal plane.

Several researchers have used simple shear devices over the years (Peacock and Seed, 1968; Casagrande, 1976; Ansell and Brown, 1978; and Sidey et al., 1978), and these generally fall within two types: i) Norwegian Geotechnical Institute (NGI) and ii) Cambridge University simple shear devices (Bjerrum and Landva, 1966; Prevost and Høeg 1970; Dyvik et al., 1987). The sample cross-sectional area of the NGI type is cylindrical and the sample is constrained by a reinforced membrane or concentric rings; while the Cambridge University type has rectangular cross sectional area and the sample is constrained by hinged metallic walls (Prevost and Høeg 1970). However, in both types, samples experience a displacement boundary condition such that the lateral displacement is essentially $\operatorname{zero}\left(\varepsilon_{x}=\varepsilon_{y}=0\right)$. Figure 2.13 shows stress conditions and shear deformations in a simple shear specimen.

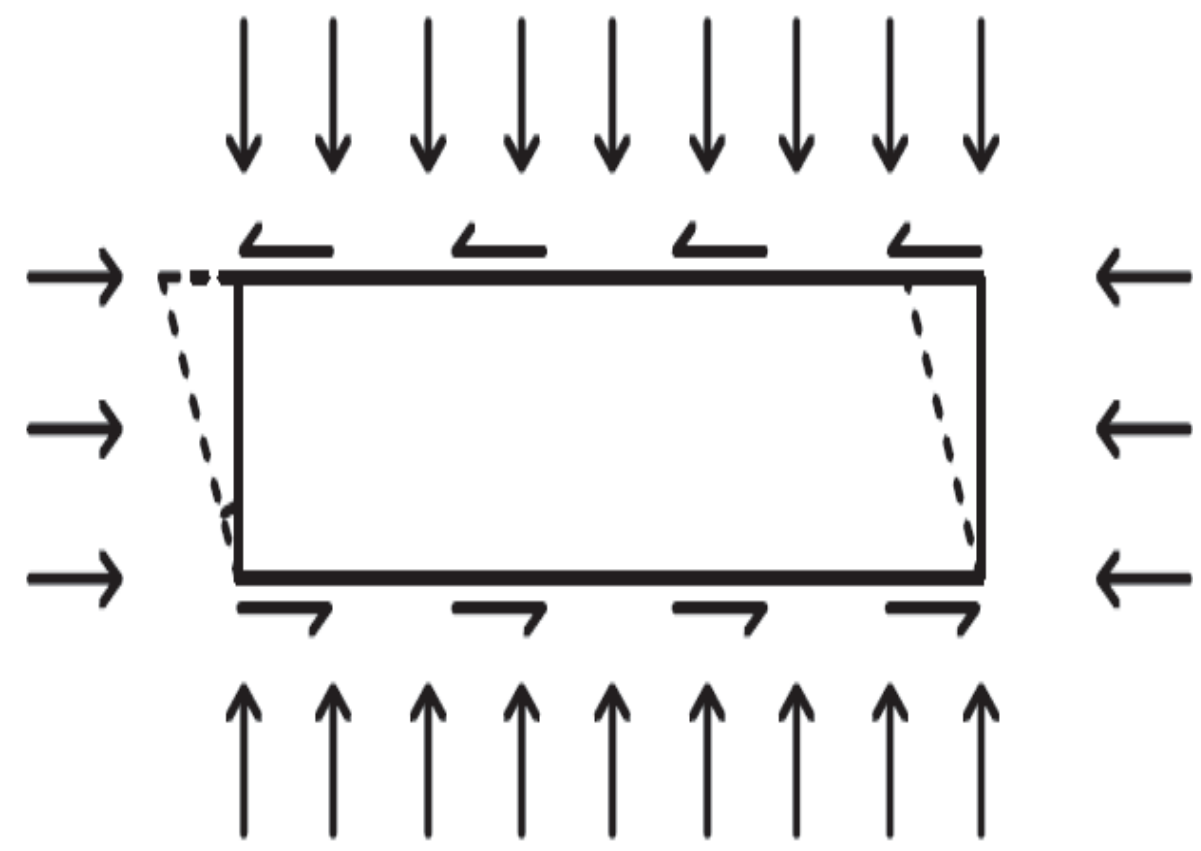

Figure 2.13 Stress conditions and shear deformations in a simple shear device 


\subsubsection{Constant volume simple shear and triaxial tests}

Realising truly undrained conditions is quite challenging in experimental soil mechanics due to systematic errors such as membrane penetration, and other factors that contribute to system compliance. Real-time feedback systems have been used to conduct truly undrained tests (Baldi and Nova, 1983; Vaid and Sivathayalan, 1996) but these add a significant level of complexity to the tests. Constant volume tests provide an alternate means to simulate the truly undrained condition (Bjerrum and Landva, 1966). Since the simple shear device does not allow lateral deformation $\left(\varepsilon_{x}=0, \varepsilon_{y}=0\right)$, only the height should be kept constant to achieve constant volume condition. The constant height condition can be enforced by clamping the top and bottom loading caps against vertical movement. In a constant volume test, it is assumed that the decrease (or increase) of vertical stress in a constant volume simple shear test is essentially identical to the increase (or decrease) of excess pore water pressure that would have measured in an undrained simple shear test conducted on the saturated specimen (Bjerrum and Landva, 1966; Finn et al. 1978; Dyvik et al. 1987). Dyvik et al. (1987) designed a chamber for NGI simple shear device that enabled an undrained test with pore water pressure measurement. They performed four parallel undrained and constant volume simple shear tests on consolidated Drammen clay to verify the validity of the assumption. Figure 2.14 shows the comparison of responses of truly undrained and constant volume tests. Comparing the responses of undrained and constant volume tests, Dyvik et al. (1987) reached the following conclusion: "The assumption that the change in applied vertical stress is equal to the pore pressure which would have developed in an undrained test is valid. This conclusion applies to saturated soils". Finn et al. (1978) have also demonstrated that the constant volume behavior of both saturated and dry 

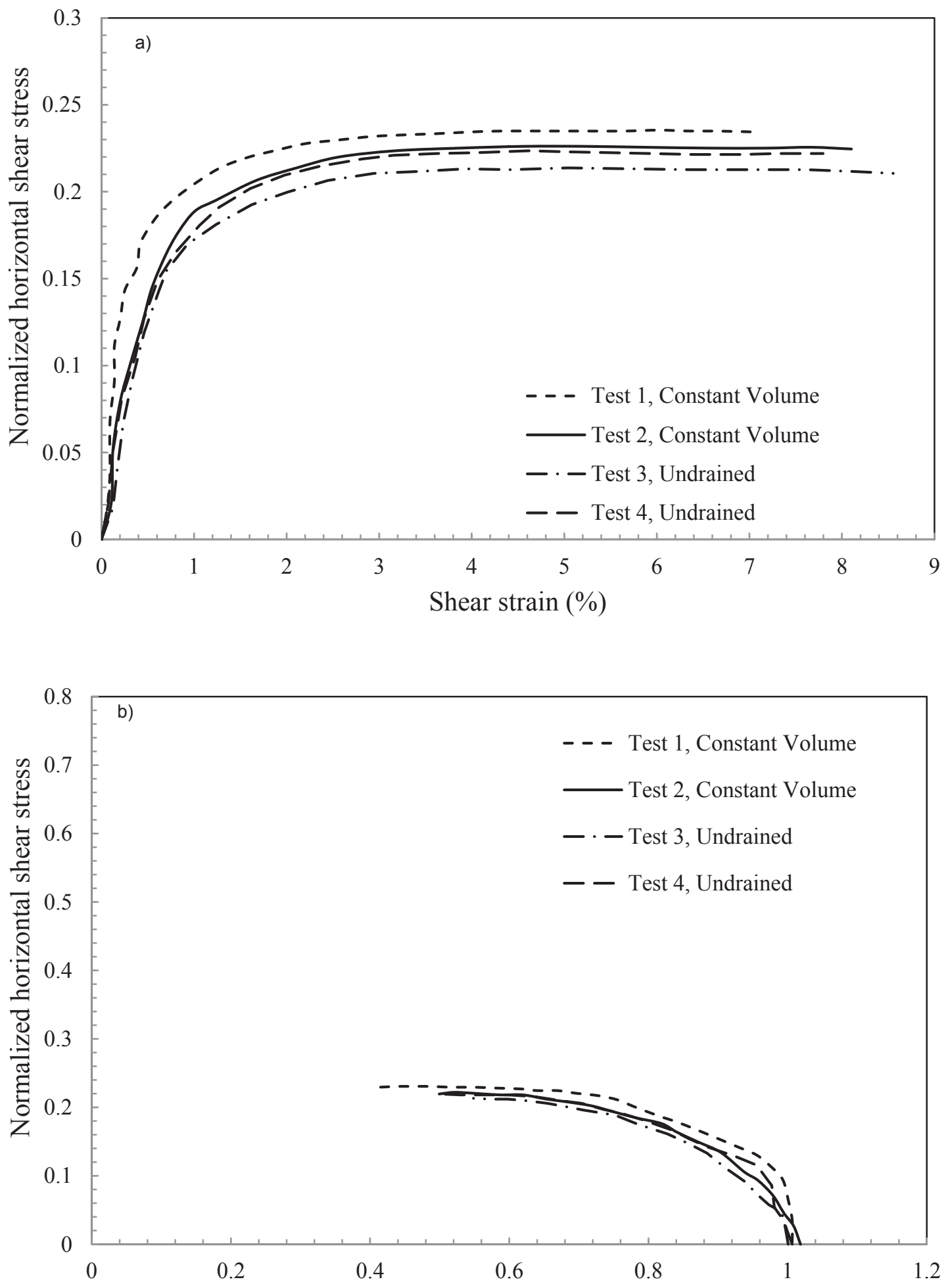

Normalized effective vertical stress

Figure 2.14 Comparison of undrained and constant volume test results (after Dyvik et al., 1987) 
sands is similar. Thus, if the equality of the constant volume response of saturated and dry sands under same initial and loading conditions is verified, it can be postulated that the constant volume behavior of sands is not dependent on the degree of saturation. Figure 2.15 presents the stress conditions under seismic loading and comparisons of stress conditions under simple shear test.

Although direct simple shear test is straightforward and easy to use, the triaxial test is also used in order to overcome some of the disadvantages of simple shear test. Sample preparation in triaxial test is more complicated than that in simple shear test, but a triaxial test is more versatile. The failure plane is predetermined during direct shear test. In triaxial test the specimen is allowed to have a failure plane based on material properties. However, it should be noted that specimens are generally hydrostatically consolidated in the triaxial test, which is not typical insitu. In addition, drainage in triaxial test can be controlled more accurately than direct shear test. Triaxial testing is conducted on a cylindrical soil sample and a non-reinforced latex membrane surrounds the sample. Depending on whether the vertical stress is higher or lower than lateral stress, a triaxial test may be compression or extension. It must be noted that in both compression and extension tests, vertical and horizontal stress directions coincide with the minor and major stress direction or vice versa. The stress path can be easily understood by using Mohr's circle analysis. Figure 2.16 presents the comparison of stress conditions in simple shear and triaxial tests. Simple shear test can simulate the plane strain field conditions fairly well, but in the triaxial test lateral deformation is inevitable. In addition, the cyclic simple shear test is capable of generating earthquake stress conditions more precisely than cyclic triaxial test. In the cyclic triaxial test, the deviator stress is applied cyclically. Generally, cyclic triaxial tests are conducted 
with the constant radial stress and the axial stress cycles with a frequency of $1 \mathrm{~Hz}(\mathrm{Kramer}$, 1996) or smaller.

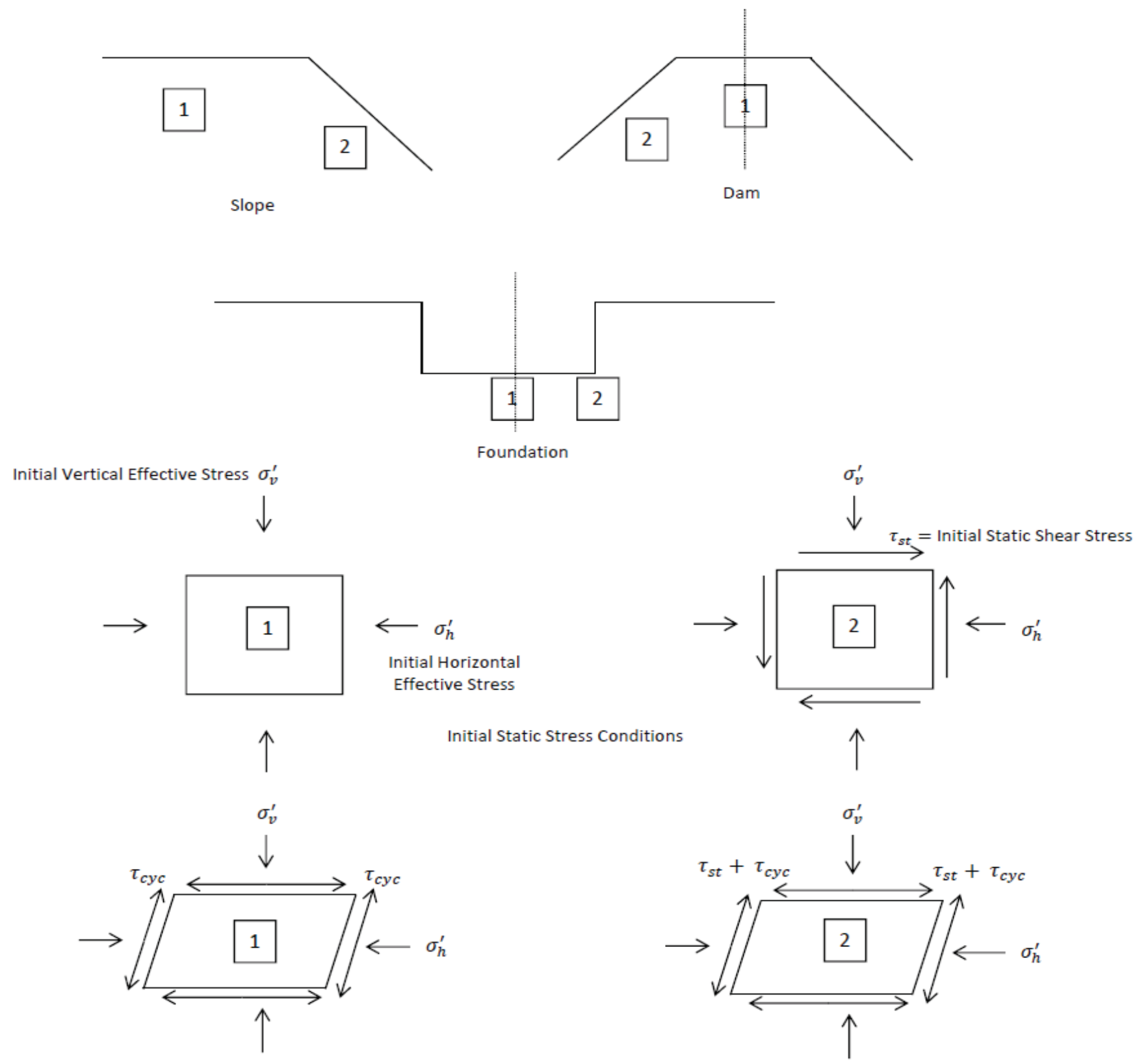

Stress Condition during Earthquake

Figure 2.15 Stress Conditions under monotonic and cyclic simple shear loading (Sanin, 2005) 
Simple Shear

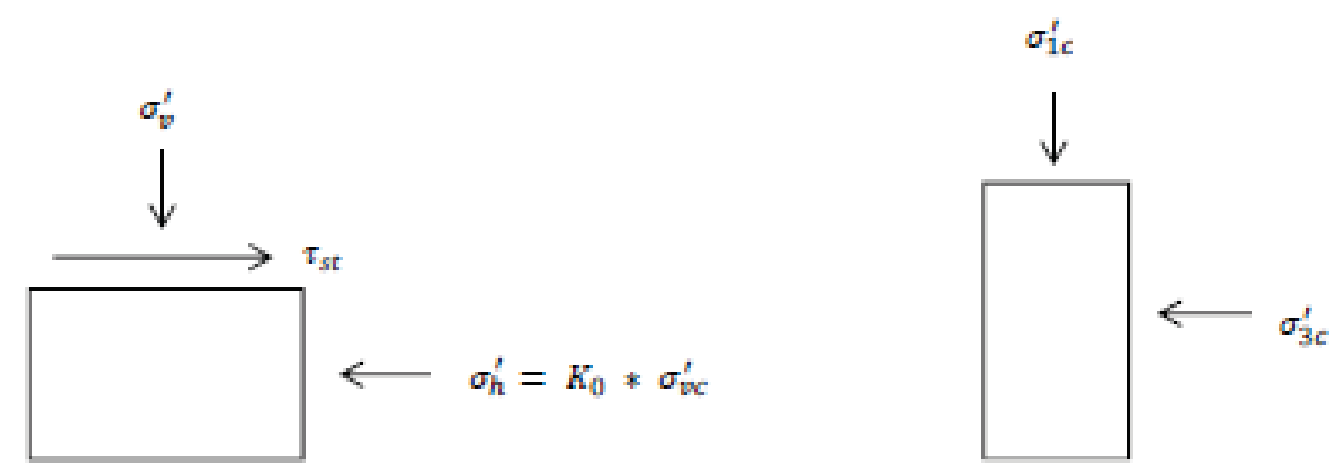

Monotonic simple shear

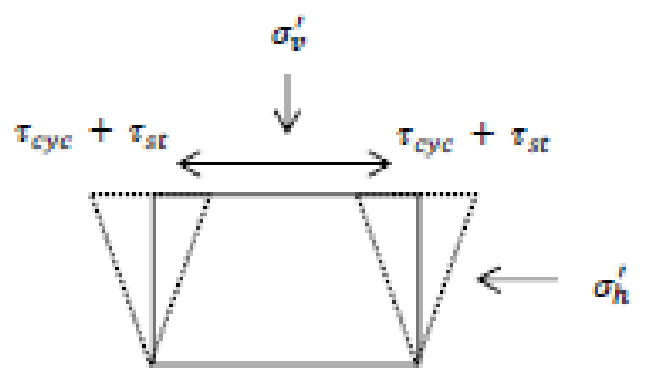

Cyclic simple shear Loading Condition

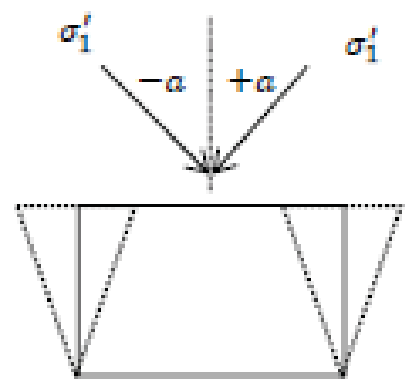

Principle Stress Direction during Cyclic Simple Shear Test
Triaxial

Monotonic Triaxial Compression

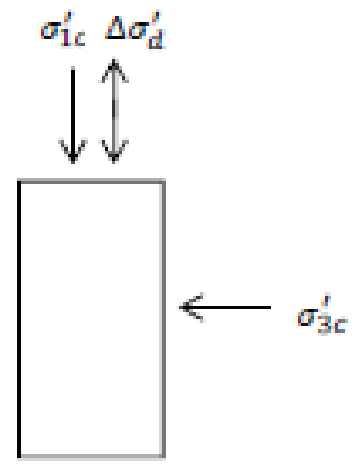

Cyclic Triaxial Loading Condition

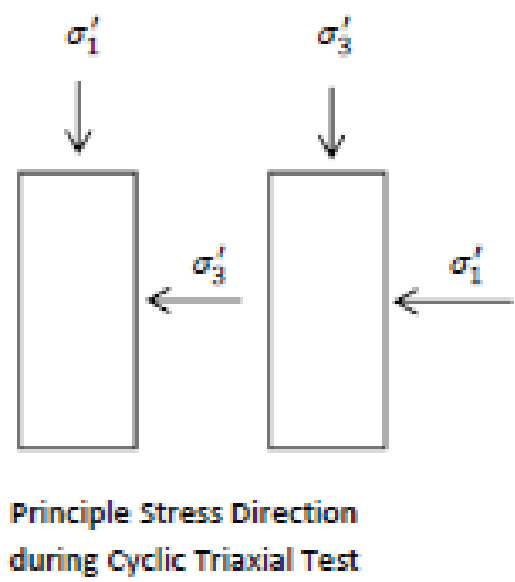

Figure 2.16 Comparison of stress conditions in simple shear and triaxial testing 


\subsubsection{Comparison between simple shear and triaxial tests results}

Vaid and Sivathayalan (1996) performed static and cyclic simple shear and triaxial tests on Fraser River delta sands, and compared the behavior under two different loading modes. The specific gravity of tested Fraser Delta sand was 2.72 and the minimal and maximal void ratio were 0.68 and 1.00 respectively. Fraser Delta sands are generally located in seismically active region in western Canada. Figure 2.17 and 2.18 compare the monotonic simple shear, triaxial compression and triaxial extension of Fraser Delta sand. Figure 2.17 shows that at the same void ratio and vertical effective consolidation pressure, the monotonic response of triaxial compression exhibited significantly higher shear stresses than the simple shear response. Response of triaxial extension was found to be a little bit weaker than the simple shear response.

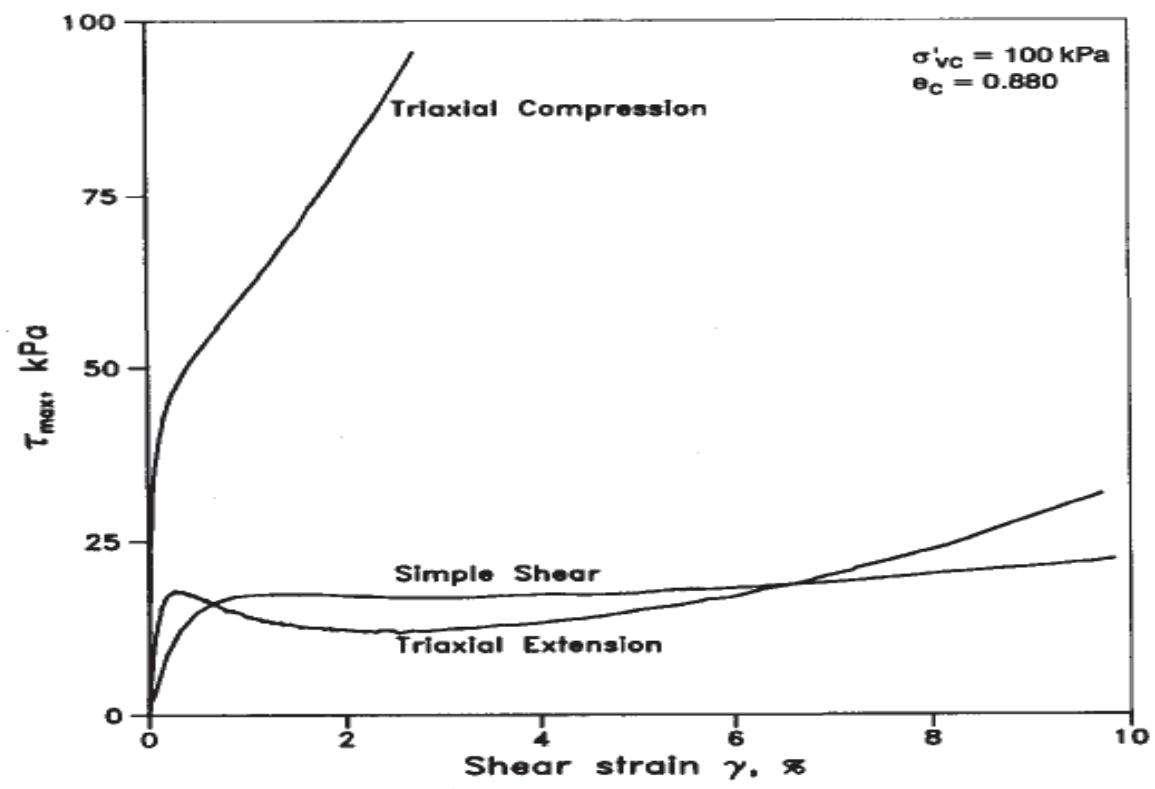

Figure 2.17 Comparison of simple shear, triaxial compression and triaxial extension at same density and consolidation pressure (after Vaid and Sivathayalan (1996)) 


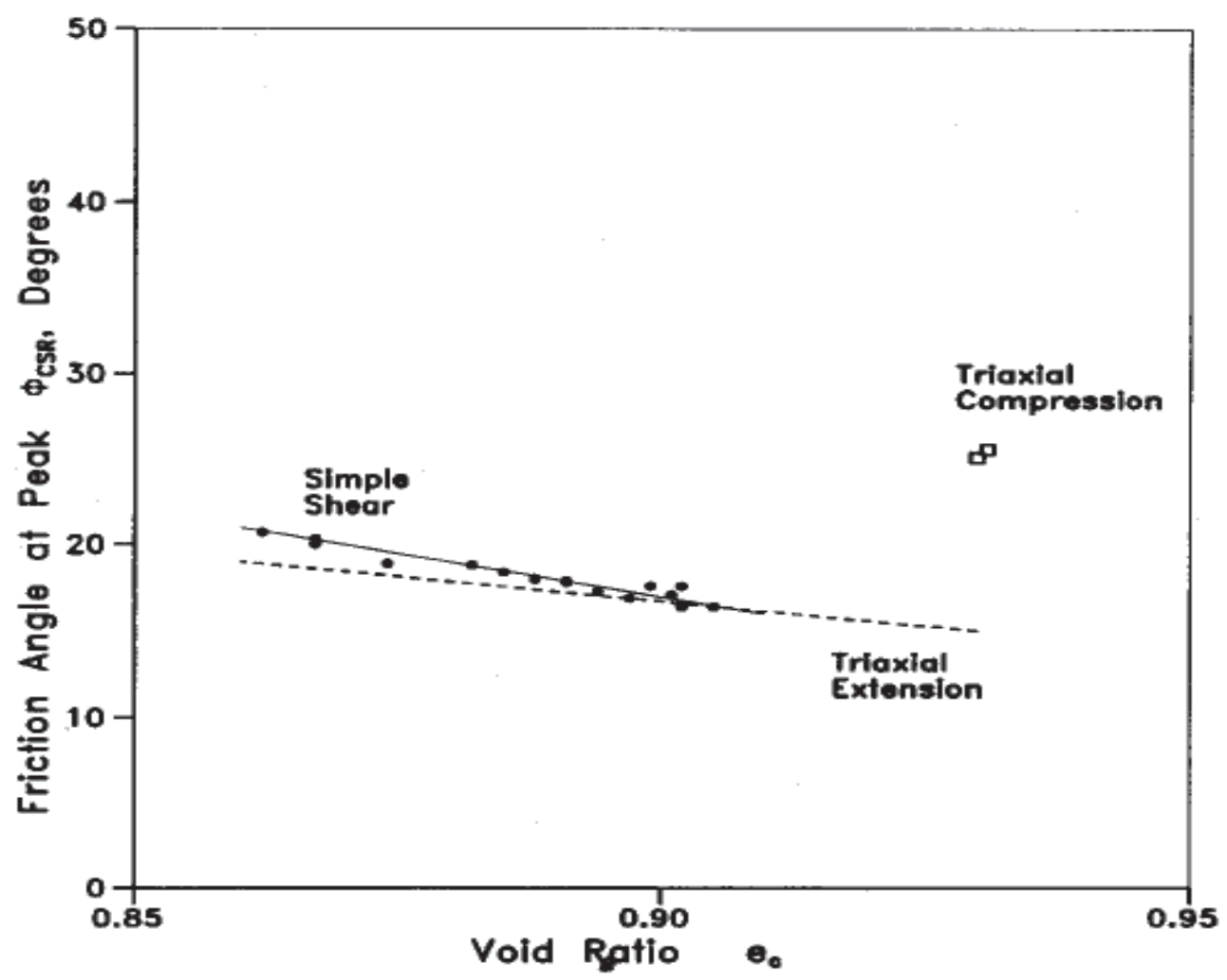

Figure 2.18 Comparison of simple shear, triaxial compression and triaxial extension (after Vaid and Sivathayalan (1996))

Cyclic simple shear tests were also performed at different confining stresses and different void ratios. Cyclic tests were performed on different confining stresses such as 50, 100, 200, $400 \mathrm{kPa}$. Liquefaction was deemed to have taken place when the single amplitude shear strain exceeds $3.75 \%$ in simple shear (equivalent axial strain in triaxial loading is $2.5 \%$ ). It was concluded that the cyclic resistance in triaxial test is usually higher than the cyclic resistance in simple shear test (Vaid and Sivathayalan, 1996). Figure 2.19 presents the values of correction factor $\left(\mathrm{C}_{\mathrm{r}}\right)$ for converting triaxial CSR $\left(\frac{\sigma_{d_{c y c}}}{2 \sigma_{\prime}^{\prime} 3 c}\right)$ to simple shear CSR $\left(\frac{\tau_{c y c}}{\sigma^{\prime} v c}\right)$ at $\mathrm{N}_{\mathrm{L}}=10$. It is clear that with increasing relative density, the correction factor becomes smaller $\left(\mathrm{C}_{\mathrm{r}}=0.6 \sim 0.7\right)$. 


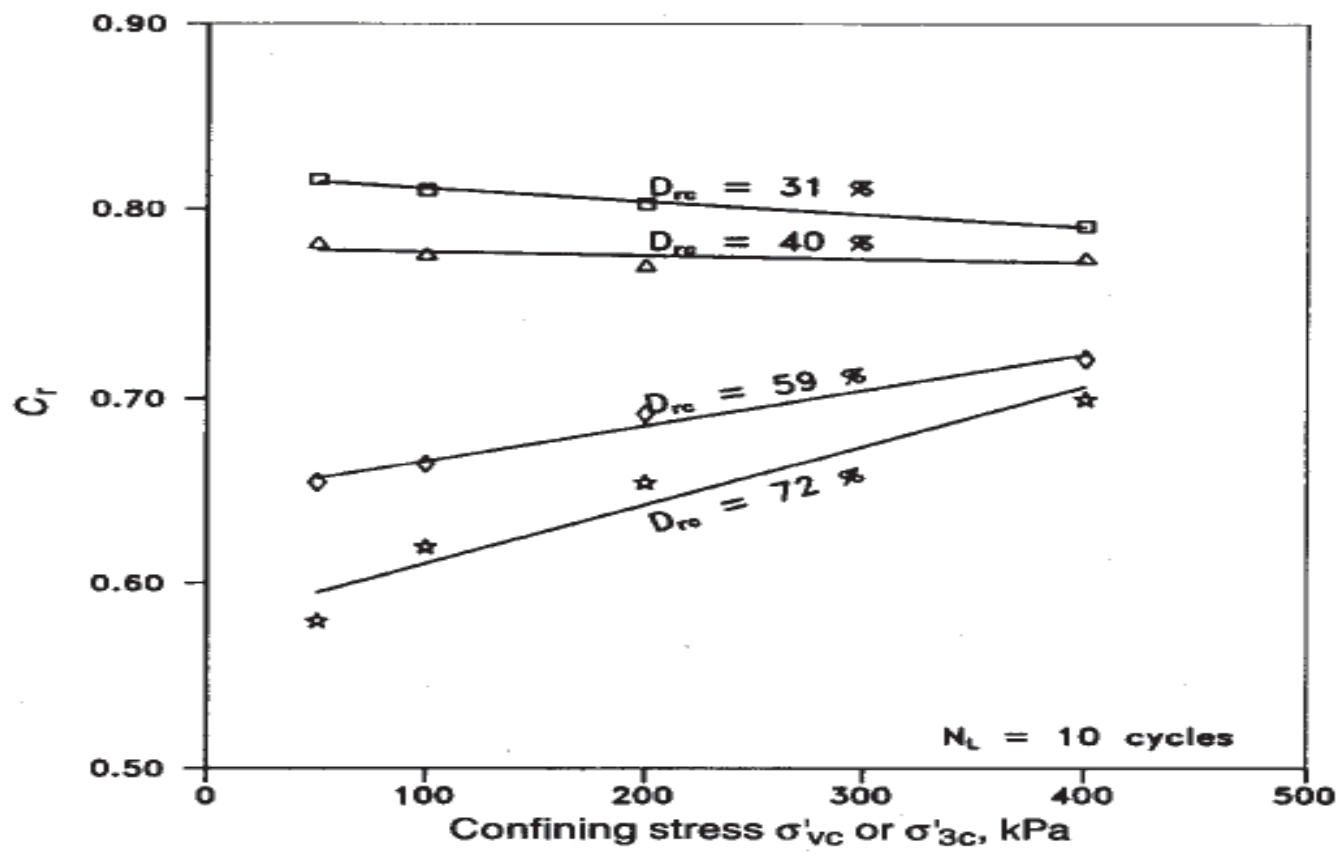

Figure 2.19 Correction factors $\left(\mathrm{C}_{\mathrm{r}}\right)$ for converting cyclic triaxial CSR to the simple shear CSR at $\mathrm{N}_{\mathrm{L}}=10$ (after Vaid and Sivathayalan (1996))

\subsection{Liquefaction phenomenon and steady state}

\subsubsection{Liquefaction Overview}

Ground displacement and associated geotechnical hazards due to earthquake loading have been one of the main concerns of geotechnical researchers. Liquefaction of saturated sands has been one of the major geotechnical research topics during the past 40 years. However, the behavior of fine-grained soils under earthquake loading has not been studied extensively and there has only been limited research reported in the literature for fine-grained soils. Significant strains or strength loss can contribute to ground deformations and slope instability during earthquake. One 
of the purposes of this study is to develop rational guidelines and analytical procedures for evaluating the potential for static or cyclic failure in low-plasticity gold mine tailings.

The undrained response of soils during the earthquake has been one of the major topics of geotechnical engineers. One of the earliest recorded observations was made by MacMurdo at an Indian earthquake in 1819, was the ejection of sand and water from the ground (Seed and Idriss, 1982). The term "Spontaneous liquefaction", introduced by Terzaghi in 1948, referred to the sudden change of a sand deposit into a deformable material which can flow like a fluid (Terzaghi and Peck, 1967; Ishihara, 1993). In 1964, several failures caused by two different earthquakes encouraged geotechnical engineers to develop their understanding of liquefaction (Seed and Idriss, 1982). After around 50 years, there has been a lot of research in order to define the liquefaction phenomenon and prevent and reduce its effect.

In general, liquefaction can be defined as a phenomenon in which effective stress decreases significantly and potentially due to the generation of excess pore water pressure. An increase in pore water pressure can occur because of either monotonic or cyclic loading. However, the liquefaction phenomenon is generally associated with dynamic forces especially earthquake. Under static loading, liquefaction is related to only strain softening; but under cyclic loading, strain softening or cyclic mobility can lead to liquefaction (Vaid and Sivathayalan, 1996). Figure 2.20 shows an illustration of the reduction of contact forces between soil particles during liquefaction. Liquefaction generally happens in cohesionless, saturated and contractive soils; Liquefaction of dilative soils generally requires higher intensity of cyclic loading. In addition to deformation, a general consequence of liquefaction in the ground surface is sand boils. Liquefaction phenomenon is usually associated with undrained loading condition in which the pore water pressure is not allowed to dissipate. 


\subsubsection{Steady state and liquefaction mechanism}

Steady state is defined as a state that the soil continuously deforms under constant effective confining pressure, and constant shear stress at constant volume and constant rate of shear strain (Castro, 1969). The steady state line is also defined as a boundary line between contractive and dilative behaviors in e-log $\sigma^{\prime}$ curve. Figure 2.21 demonstrates the generation of positive pore water pressure during cyclic loading within the critical state framework. The upper line shows contractive soil behavior and the lower line shows dilative soil behavior. Under static loading, for contractive soil, effective stress moves from A to B. Similarly, under static loading, for dilative soil, effective stress moves from D to E. Under cyclic loading, both dilative and contractive soils move to limited effective stress. In other words, under cyclic loads, both contractive (loose) and dilative (dense) soils are vulnerable and consequently in both of them liquefaction can take place (Kramer, 1996).

\subsubsection{Earthquake characteristic in liquefaction phenomenon}

The following earthquake parameters should be considered during the liquefaction phenomenon:

i) Intensity characteristics, ii) Frequency characteristics

Intensity characteristics are obtained from the magnitude and peak horizontal ground acceleration. PGA is the maximum horizontal acceleration measured on a ground motion record. 


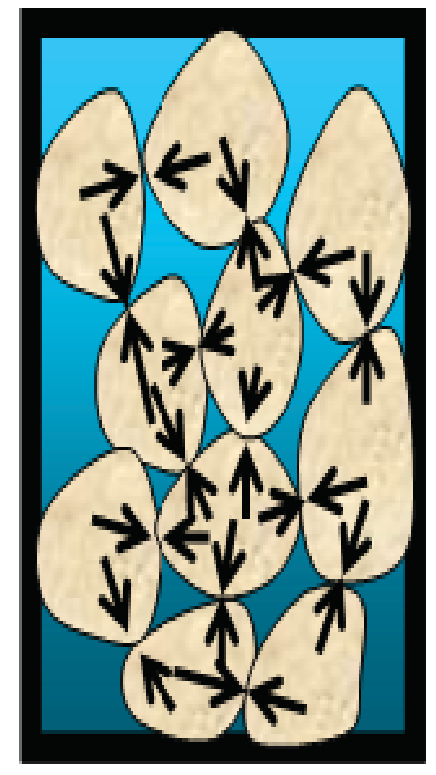

a) Initial State

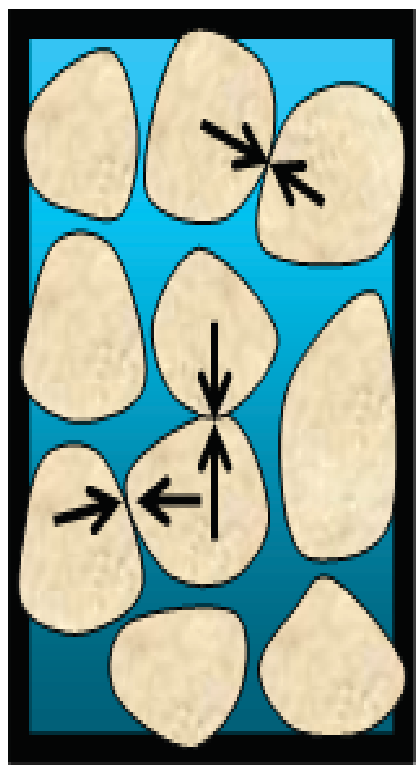

b) Liquefaction State

Figure 2.20 Reducing the contact forces during the liquefaction (after Al-Tarhouni et al., 2008)

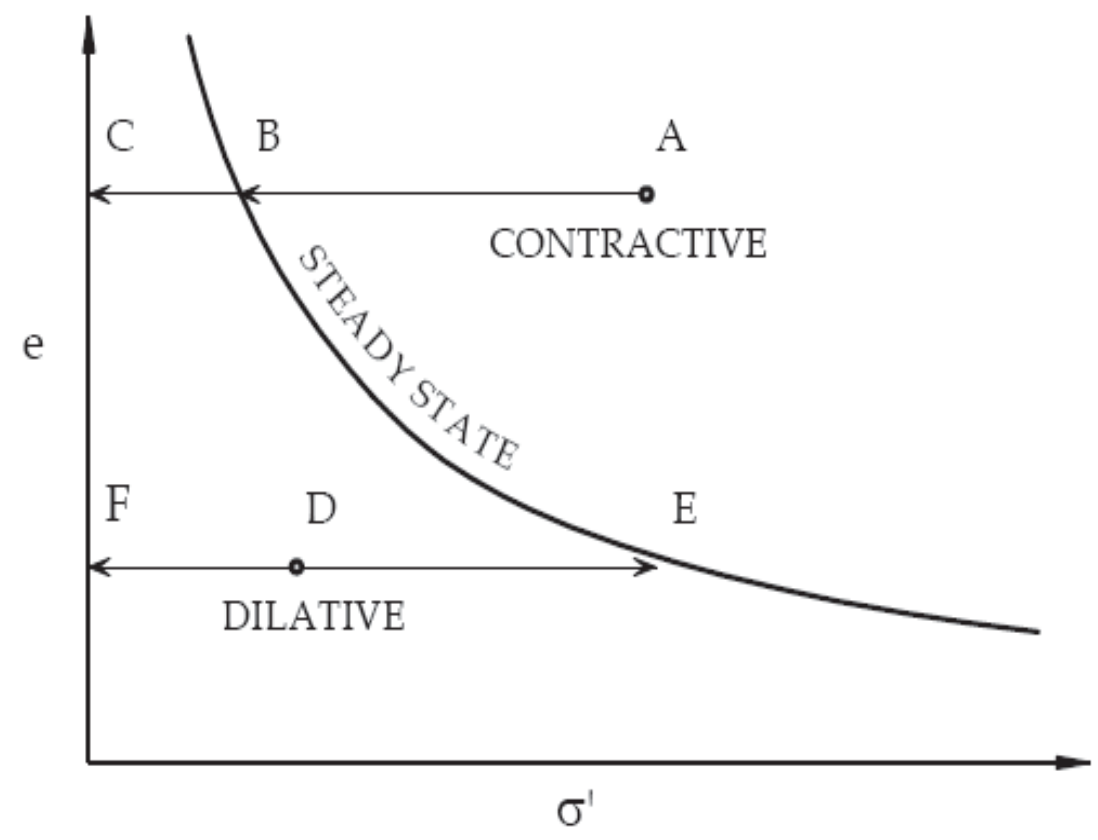

Figure 2.21 Behavior of contractive and dilative soil under static and cyclic loading (Kramer, 1996) 
Meanwhile, the magnitude of an earthquake is a function of the energy released from the ground movement (Kramer, 1996). Seeds and Idriss (1982) indicated the effects of the following earthquake characteristic on liquefaction phenomenon: i) the shear stress can increase with increasing peak ground acceleration, ii) Amplification of the ground motion decreases with increasing the contractiveness, iii) the number of cycles of shear stress increases with the magnitude.

Seed and Idriss (1971) and Youd and Idriss (2001) proposed the following equation for estimating the in situ cyclic stresses that are induced by the vertical propagation of shear waves:

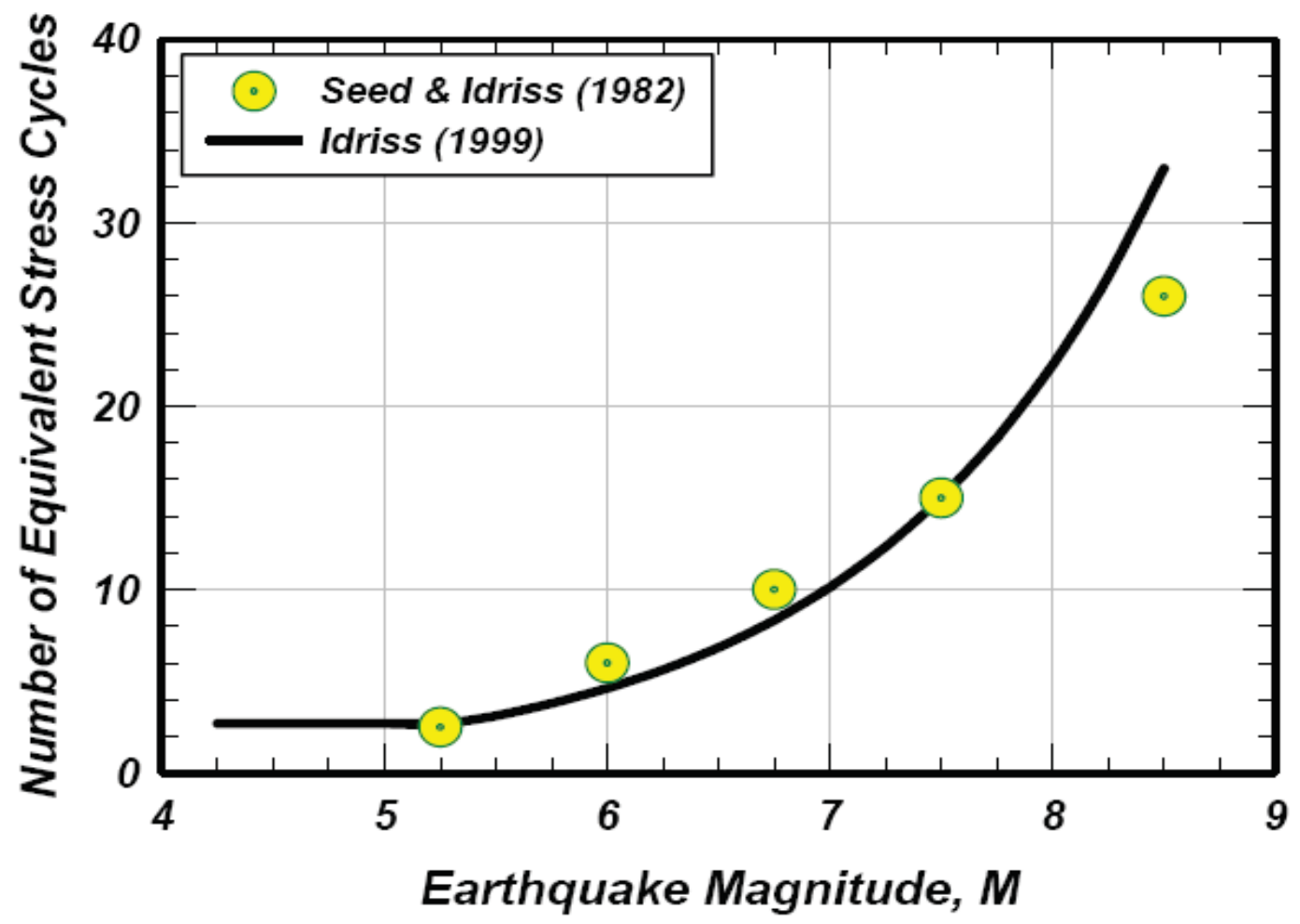

Figure 2.22 Number of equivalent uniform cycles versus earthquake magnitude (Mw) for sands (Seed and Idriss, 1982) 


$$
\mathrm{CSR} \approx 0.65 a_{\max } \frac{\sigma_{v c}}{\sigma^{\prime}{ }_{v c}} \cdot r_{d} \quad \text { Equation } 2.1
$$

where $\mathrm{a}_{\max }$ is the peak ground acceleration, $\sigma_{v c}$ is the total vertical stress, $\sigma^{\prime}{ }_{v c}$ is the effective consolidation, $r_{d}$ is the stress reduction coefficient. Figure 2.22 presents a typical number of equivalent uniform cycles versus earthquake magnitude (Seed and Idriss, 1982).

\subsection{Undrained behavior of sands}

Several researchers have conducted laboratory tests to study the undrained behavior of sands (e.g. Seed, 1979; Vaid and Chern, 1985; Vaid and Thomas, 1994). Vaid and Chern (1985) identified three types of undrained responses of sands under monotonic triaxial tests. Figure 2.23 presents monotonic stress-strain responses of sands with different densities. Curve 1 presents strain softening (or contractive behavior) in which sand experiences large deformation in low shear stress after reaching a peak strength. This behavior usually happens in loose sands and called "liquefaction" by Castro (1969) or "flow liquefaction" by Seed (1979) or "true liquefaction" by Chern (1985). Sand in curve 2 initially behaves through strain softening (contractive behavior) and after reaching minimum shear strength after the peak shear strength, the behavior changes to strain hardening. This behavior is called "limited liquefaction" by Castro (1969) and Chern (1985). For sand in dense condition (curve 3), the behavior is always strain hardening. It is clearly shown that the stress-strain response is highly dependent on initial 
stress state and relative density. As the relative density increases, the response changes from strain softening to strain hardening.

\subsubsection{Critical Stress Ratio}

Vaid and Chern, 1983 termed the effective stress ratio at the instant of the peak shear stress as the critical stress ratio. Thus, strain softening deformation will be triggered once the soil reaches this stress ratio. The response might be of the flow liquefaction type, or limited liquefaction type depending on the initial state. The locus of the critical stress ratio states on a stress path has been called the critical stress ratio line (Vaid and Chern 1983) or the collapse line (Sladen et al. 1991).

\subsubsection{Phase Transformation}

The phase transformation (PT) is the state when the behavior of sand changes from contractive behavior to dilative behavior (Ishihara et al. 1975). At this momentary state, the rate of excess pore water pressure generation is zero. Various studies have shown that the friction angle at the phase transformation is constant dependent on mineralogy only, and not affected by density, stress state and etc. (Ishihara et al., 1975; Vaid and Sivathayalan, 1996). 


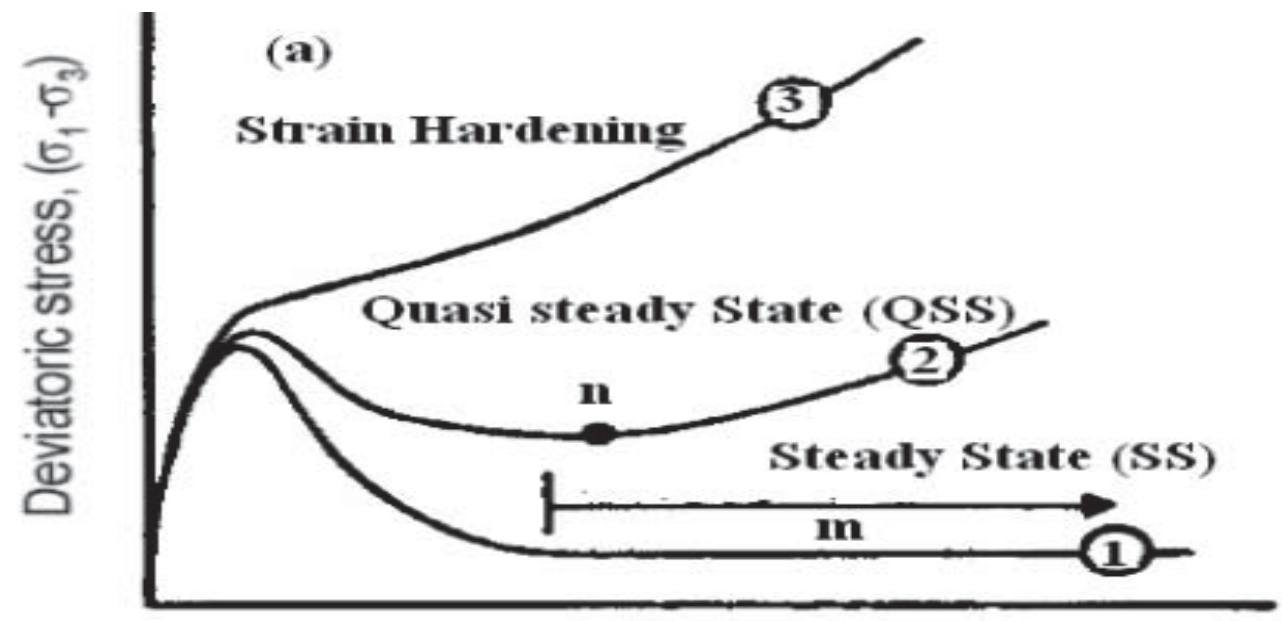

Axial strain, $\varepsilon_{\text {- }}$

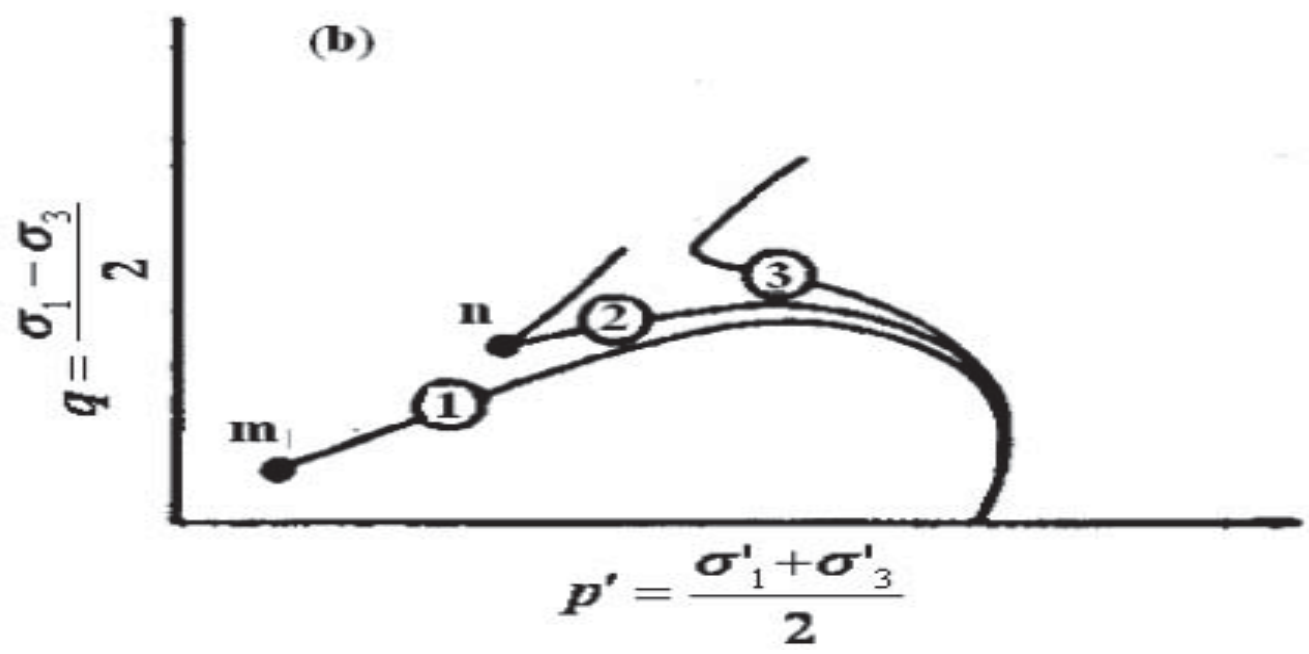

Figure 2.23 Undrained monotonic responses of different relative densities sands a) stress-strain response, b) stress path (after Vaid and Chern, 1985)

\subsection{Cyclic Behavior of tailings}

James et. al (2009) performed some cyclic simple shear tests on gold mine tailings obtained from the Abitibi region of the province of Quebec. Figure 2.24 presents cyclic simple shear results of the tailings. Moriwaki et al. (1982) also studied monotonic and cyclic undrained stress-strain response of copper tailings slimes. These tailings classified as CL or CL-ML and had Atterberg 
limits close to upper zone of A-line. Under monotonic and cyclic triaxial and DSS tests, tailings specimens exhibited clay-like behavior (Moriwaki et al., 1982). It was also concluded that the monotonic undrained shear strength ratio $\left(\frac{S_{u}}{\sigma_{v c}^{\prime}}\right)$ for triaxial tests was about $68 \%$ higher than the similar value for simple shear tests. Similarly, under cyclic loadings, the normalized cyclic shear strength $\left(\frac{\tau_{c y c}}{\sigma_{v c}^{\prime}}\right)$ for cyclic triaxial tests was about $35 \%$ greater than simple shear tests (Moriwaki et al., 1982). Consequently, the difference between triaxial and simple shear strength was greater in monotonic loading than cyclic loading.

Figure 2.25 presents cyclic resistance ratio (CRR) of different tailings materials based on the results of different researchers (Sanin, 2005). Generally, it was concluded from previous studies that tailings with low plasticity normally behaves like dilative sands in monotonic loading. Therefore, tailings with low plasticity have high strength to liquefaction during static loadings. However, they could be vulnerable to liquefaction during cyclic loading.

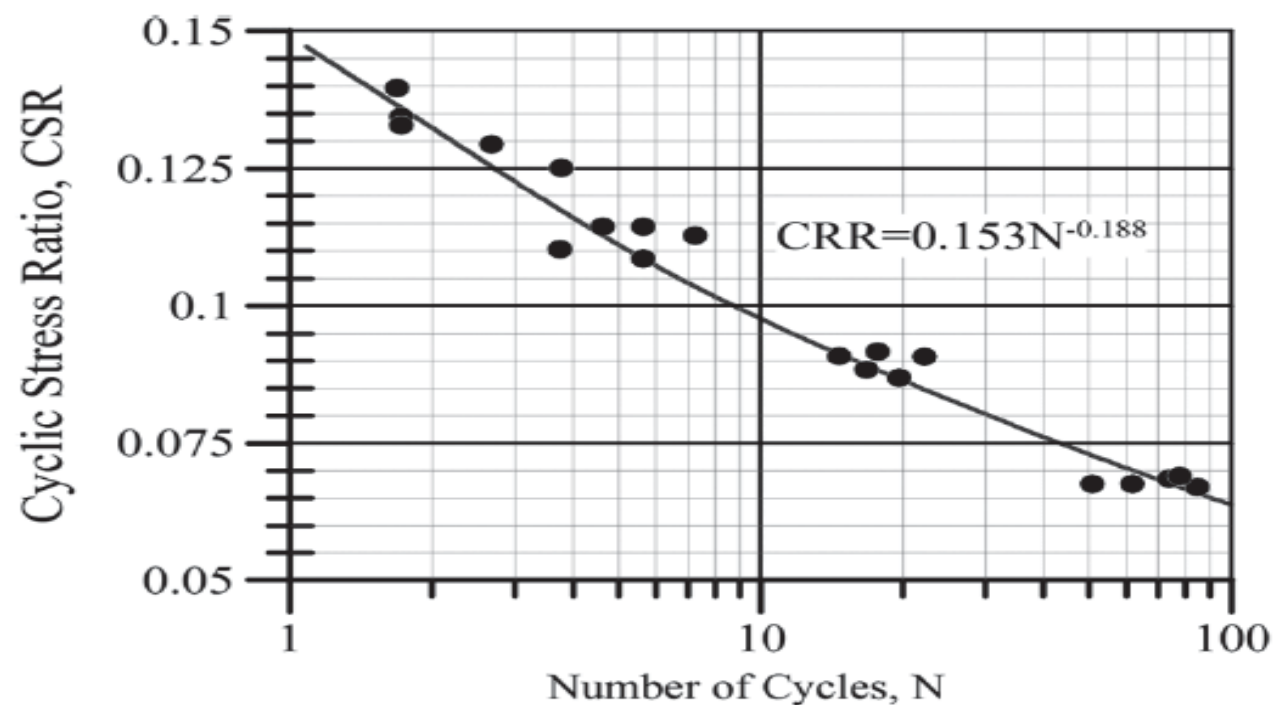

Figure 2.24 Cyclic stress ratio versus $\mathrm{N}_{\mathrm{L}}$ for gold mine tailings (after James M. et al., 2009) 


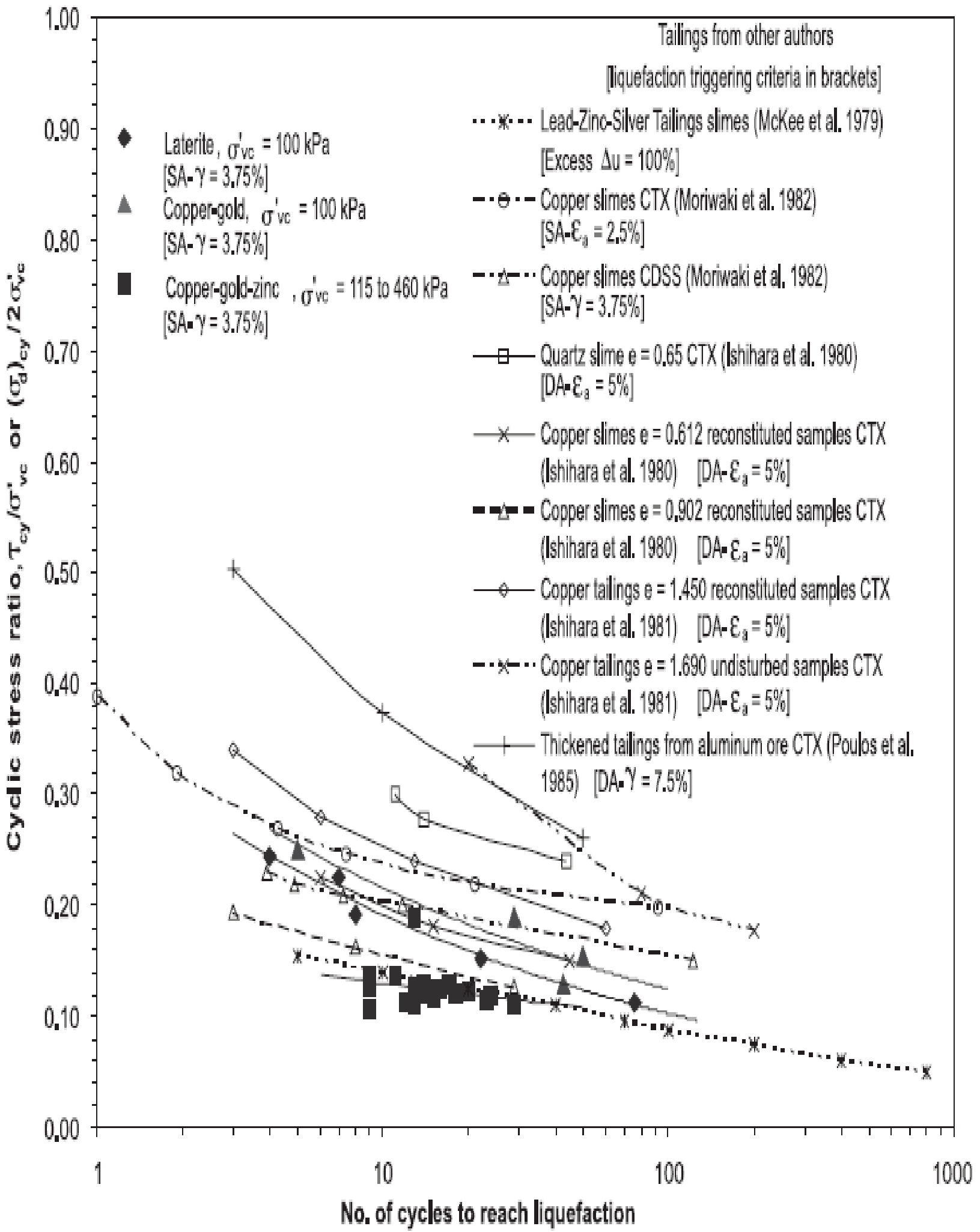

Figure 2.25 Cyclic Stress Ratio of different tailings based on previous studies (after Sanin, 2005) 


\subsection{Mechanics of Unsaturated Soils}

The soil is called saturated when the pore spaces between the solid particles are fully filled with water and unsaturated when the pore spaces are filled partially with water and partially with air. Unsaturated soil phases have been described as a three phase system consisting of:

a) water phase, b) air phase and c) soil particles.

Contractile skin is introduced by Fredlund et al. (1977) as another phase at the interface of air and water. When the air phase is continuous, the contractile skin contracts with the soil particles and influences the mechanical behavior of the soil. In the unsaturated region as the soil starts to desaturate, the pore pressure becomes negative. This negative pore-water pressure is associated with the soil suction.

\subsubsection{Soil Suction}

Soil suction is generally referred as the free energy state of soil water. The thermodynamic relationship between the free energy of soil water (or soil suction) and the partial pressure of the pore water vapour is given by Fredlund and Rahardjo (1993) as:

$$
\psi=-\frac{R T}{v_{w 0} \omega_{v}} \ln \left(\frac{v_{V}}{v_{V 0}}\right)
$$

where:

$\psi \quad$ : total suction 
$\mathrm{R}$ : the universal gas constant

$\mathrm{T}:$ absolute pressure

$v_{w 0}:$ specific volume of water

$\omega_{v}:$ molecular mass of water vapour

$v_{V}:$ partial pressure of pore water vapour

$v_{V 0}:$ saturation pressure of vapour above pure water

Equation 2.2 shows that the reference to quantify the components of suction is the vapor pressure above a flat surface of pure water (Fredlund and Rahardjo, 1993). The term $\frac{v_{V}}{v_{V 0}}$ is called relative humidity (RH):

$$
\text { Relative Humidity }=\mathrm{RH}=\frac{v_{V}}{v_{V 0}} \quad \text { Equation } 2.3
$$

Therefore, under given temperature there is a fixed relationship between total suction and relative humidity. The total suction is equal to zero when the relative humidity is equal to $100 \%$. When relative humidity becomes less than $100 \%$ in a soil, it is an indication of presence of suction in the soil.

The soil suction has two components, matric or capillary component of free energy and osmotic component of free energy. The total suction is the sum of two components:

$$
\psi=\left(u_{a}-u_{w}\right)+\pi \quad \text { Equation } 2.4
$$

where:

$$
\left(u_{a}-u_{w}\right)=\text { matric suction, } \mathrm{u}_{\mathrm{a}} \text { pore- air pressure, } \mathrm{u}_{\mathrm{w}} \text { pore water pressure }
$$


$\pi=$ Osmotic suction

\subsubsection{Matric Suction}

The matric suction component is associated with the capillary action resulting from the surface tension of water. The surface tension arises from intermolecular forces acting on molecules in the contractile skin (Fredlund and Rahardjo, 1993). The capillary action is commonly associated with the rise of a water surface in a capillary tube. The height of rise is higher if the diameter of the tube is smaller. The surface of the water in the capillary tube is curved compared to flat surface of water table. Therefore, the partial pressure of the water vapor above the curved surface of water is less than the partial pressure of the water vapor above a flat surface $\left(v_{V}<v_{V 0}\right)$, which causes relative humidity to be less than $100 \%$. The relative humidity reduces as the radius of curvature of the water surface decreases. In soils, the pores with small radii behave as capillary tubes. Therefore, the soil water rises above the water table. Since the air pressure is generally atmospheric $\left(\mathrm{u}_{\mathrm{a}}=0\right)$ in the field, the capillary water has a negative pressure. Matric suction is the difference between the pore air pressure, $\mathrm{u}_{\mathrm{a}}$, pore water pressure, $\mathrm{u}_{\mathrm{w}}$ in the air-water interface and is inversely proportional to the radius of curvature of the water surface in a capillary tube or pore (Fredlund and Rahardjo, 1993):

$$
\text { Matric suction }=\left(u_{a}-u_{w}\right)=-\rho g h_{c}=\frac{2 T_{s}}{R_{s}} \quad \text { Equation } 2.5
$$


where Ts is surface tension, Rs is radius of curvature and $h_{c}$ is height of rise (Considering $\left.h_{c}=\frac{2 T_{s}}{\rho g R_{s}}\right)$. This equation can be used to describe desaturation, which will occur when Rs is smaller than the radius of a given pore.

\subsubsection{Osmotic Suction}

Osmotic suction is associated with the presence of ionic concentration in pore water. The existence of ions in the pore water can decrease the equilibrium vapor pressure in the air phase due to molecular attraction between the ions and molecules of water. Osmotic suction is correlated with ionic conductivity. Several equations exist to relate osmotic suction to characteristics of the pore-water, such as the USDA equation given below:

$$
\pi=0.0191 E C^{1.074}(\text { USDA, 1950) } \quad \text { Equation } 2.6
$$

where $\pi$ is osmotic suction in $\mathrm{kPa}$ and $\mathrm{EC}$ is electrical conductivity in $\mu \mathrm{S} / \mathrm{cm}$

\subsubsection{Measurement of Soil Suction}


Two methods for measuring suction were used in this study: a tensiometer, and a chilled-mirror dewpoint hygrometer (WP4-T). Tensiometers were used for matric suction measurement up to around $90 \mathrm{kPa}$ and WP4-T dewpoint hygrometer was employed for measuring total suction.

\subsubsection{Tensiometer}

The instruments used for measuring pore water pressure are known as piezometers. When piezometers are used to measure the pore water pressure below the atmospheric pressure condition, the device is called tensiometer (Ridley et al., 2008). It consists of a high air-entry, porous ceramic cup and a measuring device, which are all placed in a small bore tube (Fredlund and Rahardjo, 1993). The tube and the cup are filled with de-aired water. After contacting the cup with the soil and achieving equilibrium between soils and measuring system, pore water in the soil and water in the tensiometer have identical pressures. Figure 2.26 shows an early tensiometer used by Richards in 1928 (Ridley et al., 2008). Tensiometers normally measure the matric suction from 0 to $90 \mathrm{kPa}$ and beyond this range measurement is not reliable due to cavitation of the water in tensiometer. Cavitation is the transformation from the liquid phase to the gas phase. Cavitaion takes place when the pressure of water phase drops below the saturation vapor pressure. Ridley and Burland (1993) introduced the concept of measuring suction greater than $100 \mathrm{kPa}$ with tensiometers. Ridley and Burland (1995) presented a new tensiometer using a combination of fine porous filters (capable of remaining saturated to 1,500 $\mathrm{kPa}$ ) and a technique

of preconditioning the instrument by pressurising the water in the instrument to very high pressures (Ridley et al. 2008). Figure 2.27 shows a high range tensiometer that is called "suction 
probe". The measured matric suction must not exceed the air-entry value of the ceramic cup. In fact, tensiometers work properly as long as the matric suction of the soil does not exceed the airentry value of the disk. Once the matric suction is higher than the air entry value of the disk, air will pass through the disk and enter the measuring system and leads to improper results (Fredlund and Rahardjo, 1993). Tensiometers are not able to measure the osmotic component of suction. Figure 2.28 demonstrates a schematic figure of a tensiometer.

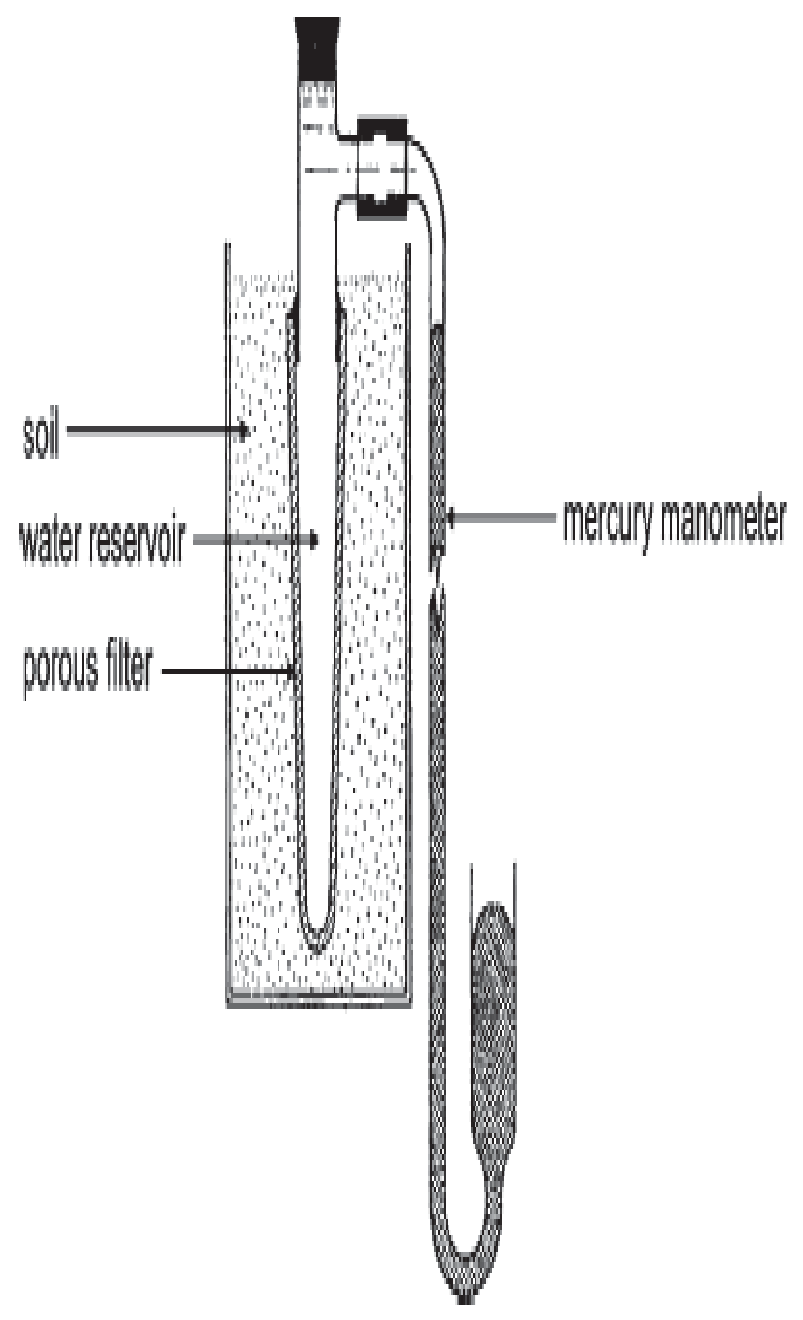

Figure 2.26 An early tensiometer (Ridley et al., 2008) 


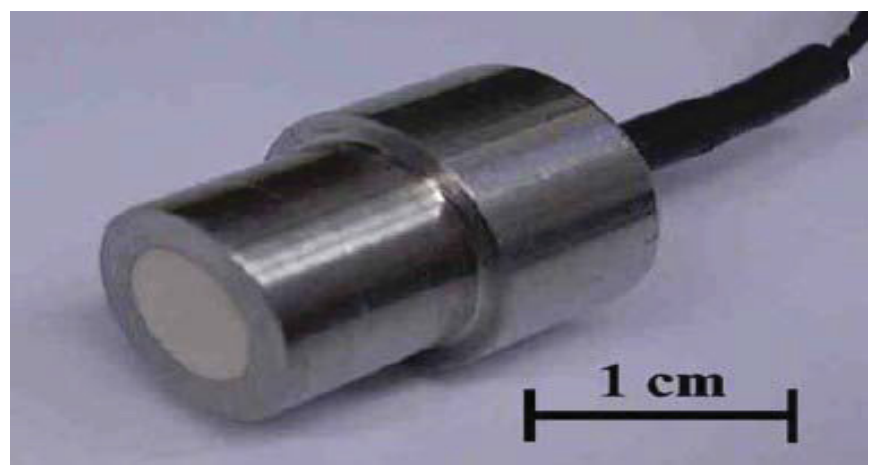

Figure 2.27 A high range tensiometer (suction probe) (after Ridley and Burland, 1995)

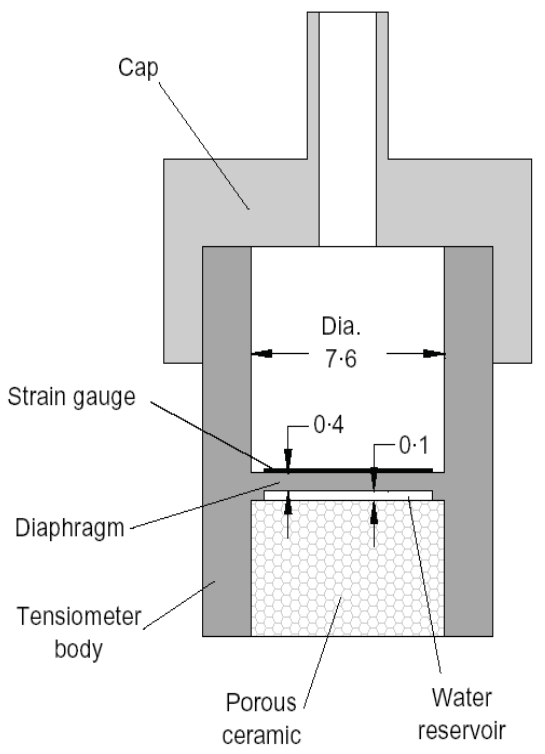

Figure 2.28 Schematic figure of tensiometer (after Ridley et al., 2008)

\subsubsection{Dewpoint hygrometer (WP4-T)}

WP4-T is one of the instruments that can be used for quickly measuring water potential. Water potential is a measurement of the energy status of water in a system. Water potential is a function 
of the vapor pressure of water in equilibrium with a sample in a sealed measurement chamber (Equation 2.2). WP4-T uses the chilled-mirror dewpoint technique to measure the water potential of a sample. At equilibrium, the water potential of the air in the chamber is the same as the water potential of the sample.

\subsubsection{Soil Water Characteristic Curve (SWCC)}

The Soil Water Characteristic Curve (SWCC) or Water Retention Curve (WRC) gives the water content of a soil at a given value of matric suction and stress history. The Soil Water Characteristic Curves have been correlated with the shear strength, coefficient of permeability, volume change of unsaturated soils (Vanapalli et al., 1996). Figure 2.29 shows a typical soil water characteristic curve, which shows the relationship between the degree of saturation and matric suction. The change in degree of saturation divided by the change in matric suction (i.e. slope of the curve) demonstrates the storage potential. The matric suction corresponding to the end of the first stage of dewatering the soil pores is referred as air-entry value (AEV). The degree of saturation at which the SWCC begins to flatten after the AEV is called residual degree of saturation and between the above points is called transition zone (Swanson, 1999). At zero degree of saturation, the soil suction is estimated to be $1,000,000 \mathrm{kPa}$ (Fredlund, 1993). Both the $\mathrm{AEV}$, the residual water content and the slope of the transition zone are moderately dependent on the grain size distribution of the soil through capillary effects, with sands having a much lower AEV than clays. In clays, the slope of the SWCC at high suctions $(>1 \mathrm{MPa})$ is related to their mineralogy, and has been correlated with the plasticity index (Marinho, 2005). 


\subsubsection{Hysteresis effect of Soil Water Characteristic Curve}

Soil Water Characteristic Curve of Unsaturated Soils has a different behavior during drying and wetting, and thus at a given matric suction the water content during drying and wetting processes are different (Fredlund and Rahardjo, 1993).

Figure 2.30 and Figure 2.31 present the hysteresis effect in Soil Water Characteristic Curve of an unsaturated soil. The non-uniformity in pore size distribution in a soil could result in hysteresis effect. The main curve shown in Figure 2.31 is desorption curve. Due to air entrapment in the soil, the starting point of desorption curve might be different from the end point of adsorption curve (Fredlund and Xing, 1994).

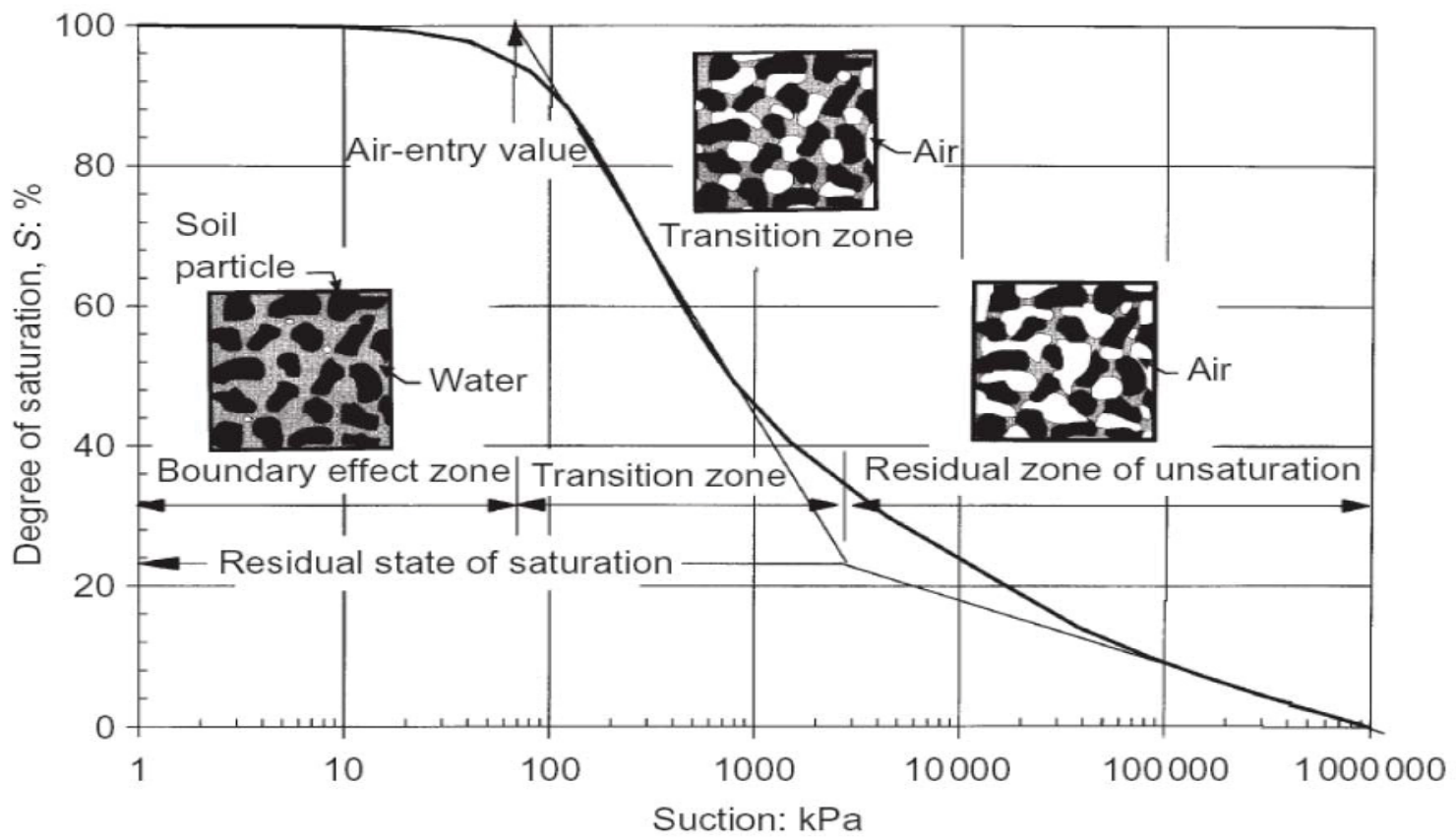

Figure 2.29 Typical Soil Water Characteristic Curve (after Vanapalli et al., 1997) 


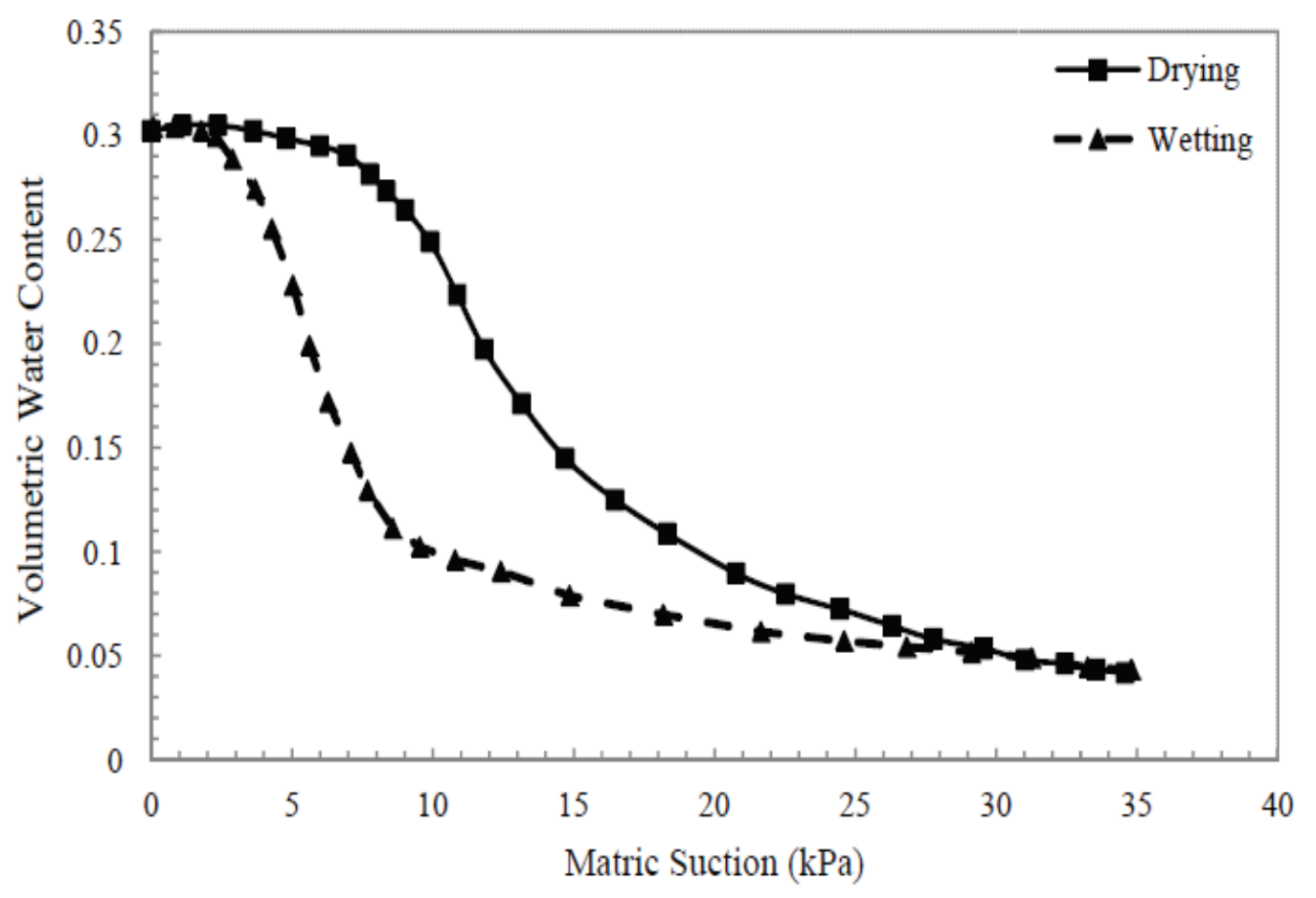

Figure 2.30 Hysteresis Effect in SWCC (after Fredlund and Rahardjo, 1993)

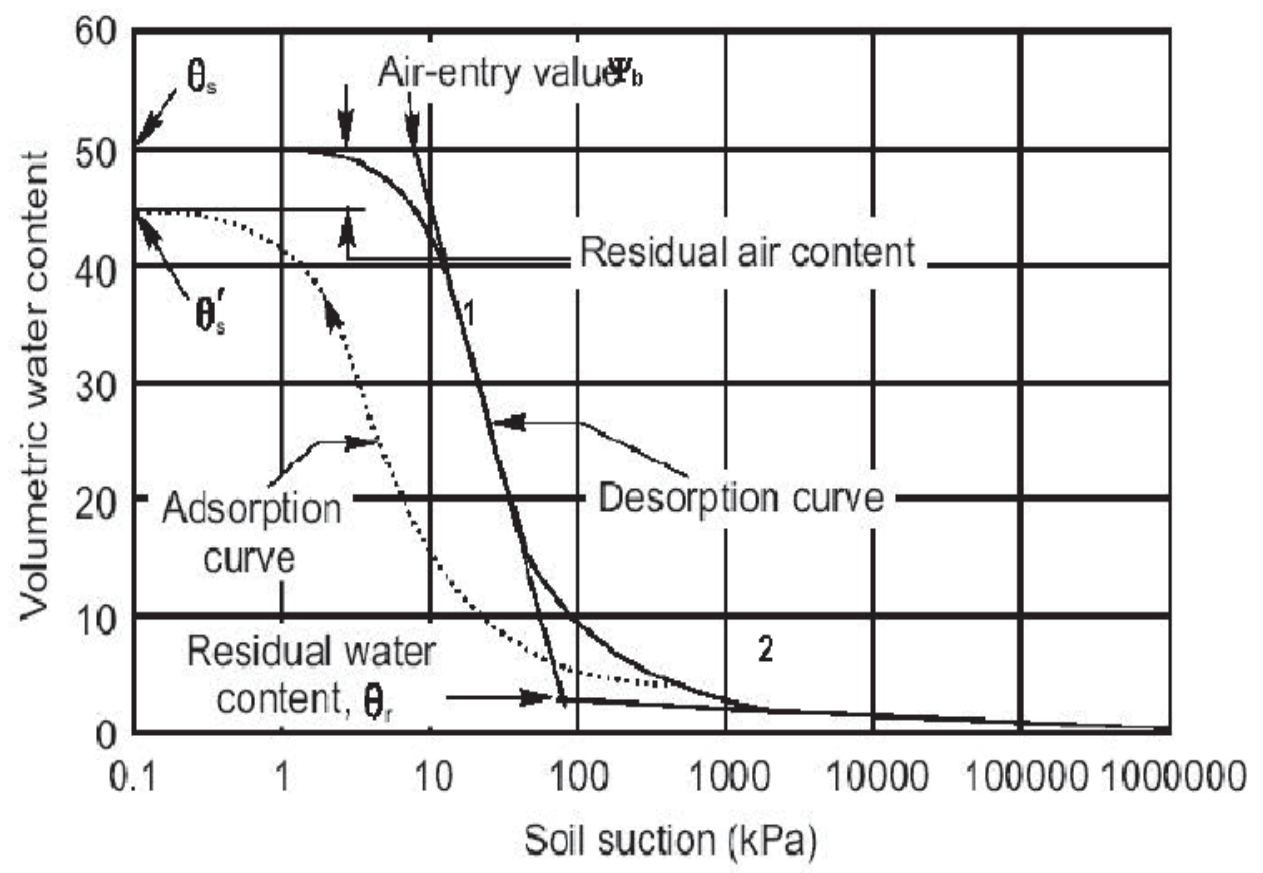

Figure 2.31 Typical desorption and adsorption curve (after Vanapalli et al., 1998) 
Vanapalli et al. (1998) proposed a method to empirically calculate the AEV and residual water content (degree of saturation) in SWCC. Figure 2.31 shows the procedure, which starts with drawing a tangent line through the inflection point (line 1) on the straight line portion of SWCC. A second line was drawn from either the starting point for $\mathrm{AEV}$ or point at $1,000,000 \mathrm{kPa}$ tangent to the basic curve for residual water content (line 2). The intersection would be the AEV or residual water content (degree of saturation) (Vanapalli et al., 1998).

\subsubsection{Soil Water Characteristic Curve equations}

A thorough review of different equations for the SWCC is given by Leong and Rahardjo (1997). Two of the more commonly used SWCC equations include those proposed by Fredlund and Xing (1994) and van-Genuchten (1980). van-Genuchten (1980) proposed a three parametric equation for soil water characteristic curve:

$$
w_{w}=w_{r v g}+\left(w_{s}-w_{r v g}\right)\left[\frac{1}{\left[1+[a \psi]^{n}\right]^{m}}\right] \quad \text { Equation } 2.7
$$

where:

$\mathrm{W}_{\mathrm{w}}$ : gravimetric water content at particular matric suction

$\mathrm{w}_{\text {rvg: }}$ residual gravimetric water content

$\mathrm{W}_{\mathrm{s}}$ : saturated gravimetric water content

$\psi$ : matric suction

a, n, m: fitting parameters 
Fredlund and Xing (1994) also proposed an analytical based equation for Soil Water Characteristic Curve. The equation is applicable for matric suction ranges between 0 to 1,000,000 kPa:

$$
\theta=C(\psi) \frac{\theta_{s}}{\left\{\ln \left[e+\left(\frac{\psi}{a}\right)^{n}\right]\right\}^{m}}
$$

Equation 2.8

where:

e is natural number and equals to 2.71828

a: approximate value of air-entry value

$\theta_{s}:$ saturated volumetric water content, which is equal to porosity

$\mathrm{m}, \mathrm{n}$ : fitting parameters

$\psi:$ matric Suction

$\mathrm{C}(\psi)$ : correction factor

Correction factor is equal to:

$$
C(\psi)=1-\frac{\ln \left\{1+\frac{\psi}{c_{r}}\right\}}{\ln \left[1+\frac{1000.000}{c_{r}}\right]}
$$

Equation 2.9

$\mathrm{C}_{\mathrm{r}}$ is the suction value corresponding to the residual water content. 


\subsubsection{Sensitivity of the Soil Water Characteristic Curve to net normal stress}

Thu et al. (2007) investigated the effect of net confining stress on the SWCC of the compacted silts. The comparison results from SWCC tests showed that the air entry value increases with increasing net confining stress. However, Heidarian (2012) examined the influence of $\mathrm{k}_{0}$ consolidation on the SWCC of gold tailings, and found that while there is a significant effect under high stresses $(>50 \mathrm{kPa})$, for the stress range likely encountered near the surface $(<20$ $\mathrm{kPa}$ ), the change in the AEV was insignificant.

\subsubsection{Effective Stress of Unsaturated Soils}

Karl Terzaghi in 1943 defined effective stress equation as a key parameter that produces measurable effects such as compaction or an increase of the shearing resistance (Terzaghi, 1943). The difference between total stress, $\sigma$ and pore-water pressure, $\mathrm{u}_{\mathrm{w}}$ is expressed as effective stress, $\sigma^{\prime}$ in a saturated soil:

$$
\sigma^{\prime}=\sigma-u_{w} \quad \text { Equation } 2.10
$$

The deformation and shear strength of soil in response to a change in stress conditions are associated with change in effective stress defined by equation 2.10. Terzaghi's effective stress 
has become the mainstay in current geotechnical engineering practice to characterize stress-strain relationships and strength in saturated soils.

In arid and semi-arid regions, many soils are commonly unsaturated. In these regions, the question would be the influence of forces in soil that are not explicitly included in the effective stress defined by Terzaghi but produce measurable effects on deformation and strength. Bolt (1956), Lambe (1960), Skempton (1960), Sridharan and Rao (1973) demonstrated that the missing forces are physicochemical in nature, namely van der Waals attraction and electrical double-layer repulsion. It was found that these forces play an important role in the structure of fine-grained soils such as clay ( $\mathrm{Lu}$ and Likos, 2006). More classical studies on unsaturated soils by Bishop (1959) and Mitchel (1976) have demonstrated that capillary forces should also be considered as an influential factor on effective stress of unsaturated soils.

Bishop (1959) proposed an effective stress equation for unsaturated soils:

$$
\sigma^{\prime}=\left(\sigma-u_{\mathrm{a}}\right)+\chi\left(\mathrm{u}_{\mathrm{a}}-\mathrm{u}_{\mathrm{w}}\right) \quad \text { Equation } 2.11
$$

where the "effective stress parameter" $\chi$ is generally considered to vary between zero and one as a function of the degree of saturation. The difference $\left(\sigma-u_{a}\right)$ is net normal stress and the difference $\left(u_{a}-u_{w}\right)$ is matric suction. For $\chi$ equal to unity, equation 2.11 reduces to Terzaghi's effective stress, which corresponds to saturated soil. For $\chi$ between zero and one, the second term in Equation 2.11, $\chi\left(u_{a}-u_{w}\right)$, describes the contribution of matric suction to effective stress and $\left(\sigma-\mathrm{u}_{\mathrm{a}}\right)$ expresses the contribution of net normal stress to effective stress. 


\subsubsection{Stress state variables}

Jennings and Burland (1962) proposed that Bishop`s effective stress equation might have some limitations to simultaneously interpret the mechanical behavior of unsaturated soils. Bishop and Blight (1963) re-evaluated the Bishop's effective stress equation and stated the following: (Vanapalli, 2010).

"a change in matric suction, $\left(\mathrm{u}_{\mathrm{a}}-\mathrm{u}_{\mathrm{w}}\right)$ did not result in the same change in effective stress as did a change in net normal stress, $\left(\sigma-\mathrm{u}_{\mathrm{a}}\right)$.”

Several investigators realized that the contribution of $\left(\sigma-u_{a}\right)$ and $\left(u_{a}-u_{w}\right)$ on effective stress of unsaturated soils should be considered as independent stress state variables. (Bishop and Blight (1963), Burland (1965), Matyas and Radhakrishna (1968), Fredlund and Morgenstern (1977), Fredlund and Rahardjo (1993) and Vanapalli (2010)). Fredlund and Rahardjo (1993) demonstrated that unlike saturated soils which need only one independent stress state variable $\left(\sigma-\mathrm{u}_{\mathrm{w}}\right)$ to investigate mechanical behavior of saturated soils, unsaturated soils require two independent stress state variables, net normal stress, $\left(\sigma-\mathrm{u}_{\mathrm{a}}\right)$ and matric suction $\left(\mathrm{u}_{\mathrm{a}}-\mathrm{u}_{\mathrm{w}}\right)$ to interpret the engineering behavior of unsaturated soils (Fredlund and Rahardjo 1993). In this case, although $\sigma_{3}, \mathrm{u}_{\mathrm{a}}$ and $\mathrm{u}_{\mathrm{w}}$ vary, the two stress-state variables remain constant and independent of soil properties (Fredlund and Rahardjo 1993).

\subsubsection{Suction Stress}


$\mathrm{Lu}$ and Likos (2006) proposed a series of particle-scale force analyses to distinguish and conceptualize three types of forces in saturated or unsaturated soil: 1) active "skeletal" forces distributed through the soil grains; 2) active "local" forces concentrated at or near the interparticle contacts; and 3) passive particle-particle contact forces which serve to counterbalance the skeletal and local forces. In saturated soils, only the first and third types of force is considered because the soil-water system might be treated as an equivalent continuum medium with macroscopic stresses defined at the boundary, as captured in Terzaghi's classic effective stress equation (Lu and Likos, 2006). Lu and Likos (2006) demonstrated that:

"Under unsaturated conditions, distinguishing between these three types of forces becomes necessary because pore pressure as a macroscopic stress disintegrates into several microscopic interparticle forces acting within the vicinity of the grain contacts". It is proposed here that the second type of force, which includes van der Waals forces, electrical double-layer forces, cementation forces, surface tension forces, and forces arising from negative pore water pressure, can be conceptually lumped into a macroscopic stress referred to as "suction stress" (Lu and Likos, 2006). In simple words, the contribution of matric suction to effective stress of unsaturated soils, i.e $\chi\left(\mathrm{u}_{\mathrm{a}}-\mathrm{u}_{\mathrm{w}}\right)$, is defined as suction stress.

\subsubsection{Validity and effectiveness of methods of predicting stress state of unsaturated soils}

In summary, there are currently three commonly used macroscale approaches for describing the state of stress in unsaturated soil: 
1) Modified effective stress approach, which is generally attributed to the work of Bishop (1959) and developed by Khalili and Khabaz (1998) and Lu and Likos (2006, 2010);

2) Independent stress state variable approach, which is generally attributed to the work of Fredlund and Morgenstern (1977); and

3) Modified stress variable and microstructure investigation approaches adopted by a number of researchers (Simms and Yanful 2004, 2005; Romero, 2008) for stress-strain analyses.

Each method has advantages and limitations. The validity and practicality of these approaches for describing the state of stress and corresponding behavior of unsaturated soil remain highly uncertain. There is disagreement among the researchers regarding the validity of first or second method. Difficulties associated with experimentally or theoretically determining the effective stress parameter $\chi$ have limited the general applicability of Bishop's approach in research and practice (Lu and Likos, 2006). Fredlund (2006) stated that approaches to integrate matric suction into effective stress following Bishop's approach violates continuum mechanics principles, as degree of saturation is a material property, not a stress state variable (Fredlund 2006). However, there is no doubt that the contribution of matric suction to strength and volume change behaviour decreases as degree of saturation also decreases. Lu and Likos (2010) demonstrated that "despite the popularity of the two independent stress state variable approach, it is quite controversial". Khalili and Khabbaz (1998) and Nuth and Laloui (2008) have also presented limitations of two independent stress state variables method. The method contains complexity by requiring additional material parameters that are usually variable or not easy to determine experimentally. 
Table 2.5 summarizes the disadvantages and limitations of methods of predicting effective stress of unsaturated soil.

\subsubsection{Suction Stress Characteristics Curve (SSCC)}

Considering the first approach to interpret the effective stress of unsaturated soils, plotting the value of suction stress versus saturation is defined as Suction Stress Characteristics Curve (SSCC). Lu and Likos (2006) proposed a modified form of Bishop's equation, effectively setting " $\chi "=S_{r}$, the degree of saturation:

$$
\sigma^{\prime}=\left(\sigma-u_{a}\right)-\sigma^{s}
$$

Equation 2.12

where $\sigma^{\mathrm{s}}$ is suction stress and defined as:

$$
\sigma^{\mathrm{s}}=-\left(\mathrm{u}_{\mathrm{a}}-\mathrm{u}_{\mathrm{w}}\right) \cdot S_{r} \quad(\text { Lu and Likos, 2006) } \quad \text { Equation } 2.13
$$

Therefore, if the SWCC is known in terms of degree of saturation, suction stress can be determined by multiplying the ordinates of degree of saturation and matric suction ( $\mathrm{Lu}$ and Likos, 2010). Lu and Likos (2010) utilized the van-Genuchten fitting parameters to quantify the SSCC for different coarse grained and fine grained soils. If van-Genuchten`s (1980) SWCC model is used, the normalized degree of saturation is expressed as: 
Table 2.5 Disadvantages and limitations of methods of predicting stress state of unsaturated soils

\begin{tabular}{|c|l|}
\hline Method & \multicolumn{1}{|c|}{ Disadvantages / Limitations } \\
\hline Suction Stress (Modified & 1) Difficulties associated with experimentally or \\
Bishops equation) & theoretically determining the effective stress parameter $\chi$ \\
Two independent stress & 2) Not consistent with continuum mechanics principles, \\
State variables & as degree of saturation is a material property, not a stress \\
(More popular method) & state variable \\
(Modified from Fredlund & parameters that are usually variable or not easy to \\
1977) & determine experimentally. \\
\hline
\end{tabular}

$$
S_{e}=\left\{\frac{1}{1+\left[\alpha\left(u_{a}-u_{w}\right]^{n}\right.}\right\}^{1-1 / n} \quad \text { (Lu and Likos, 2010) } \quad \text { equation } 2.14
$$

where " $\mathrm{n}$ " and " $\alpha$ " are empirical fitting parameters of unsaturated soil properties. " $\alpha$ " and " $n$ " are associated with the inverse of air entry pressure for water saturated soil and pore size 
distribution respectively. Figure 2.32 shows the range of values of " $\alpha$ " and " $n$ " parameters for various soil types ( $\mathrm{Lu}$ and Likos, 2010). Figure 2.33 shows typical soil-water characteristics curves based on van-Genuchten parameters. Figure 2.34 also presents three possible SWCC zones for sandy, silty and clayey soils based on van-Genuchten parameters. It is clear that clayey soils has higher air-entry value and generate high suction values in dewatering process.

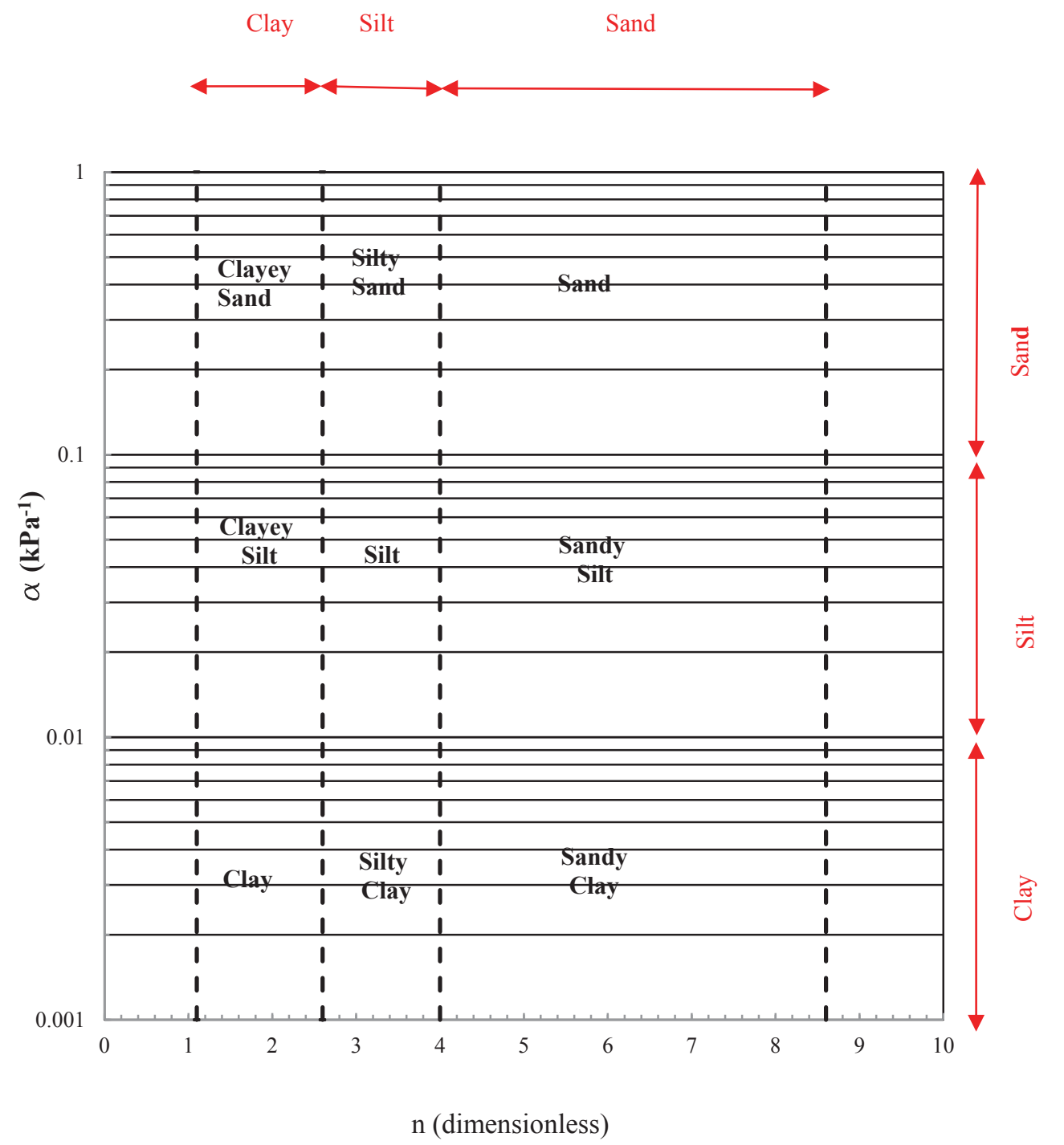

Figure 2.32 van-Genuchten empirical fitting parameters for various soil types (Lu and Likos, 2010) 


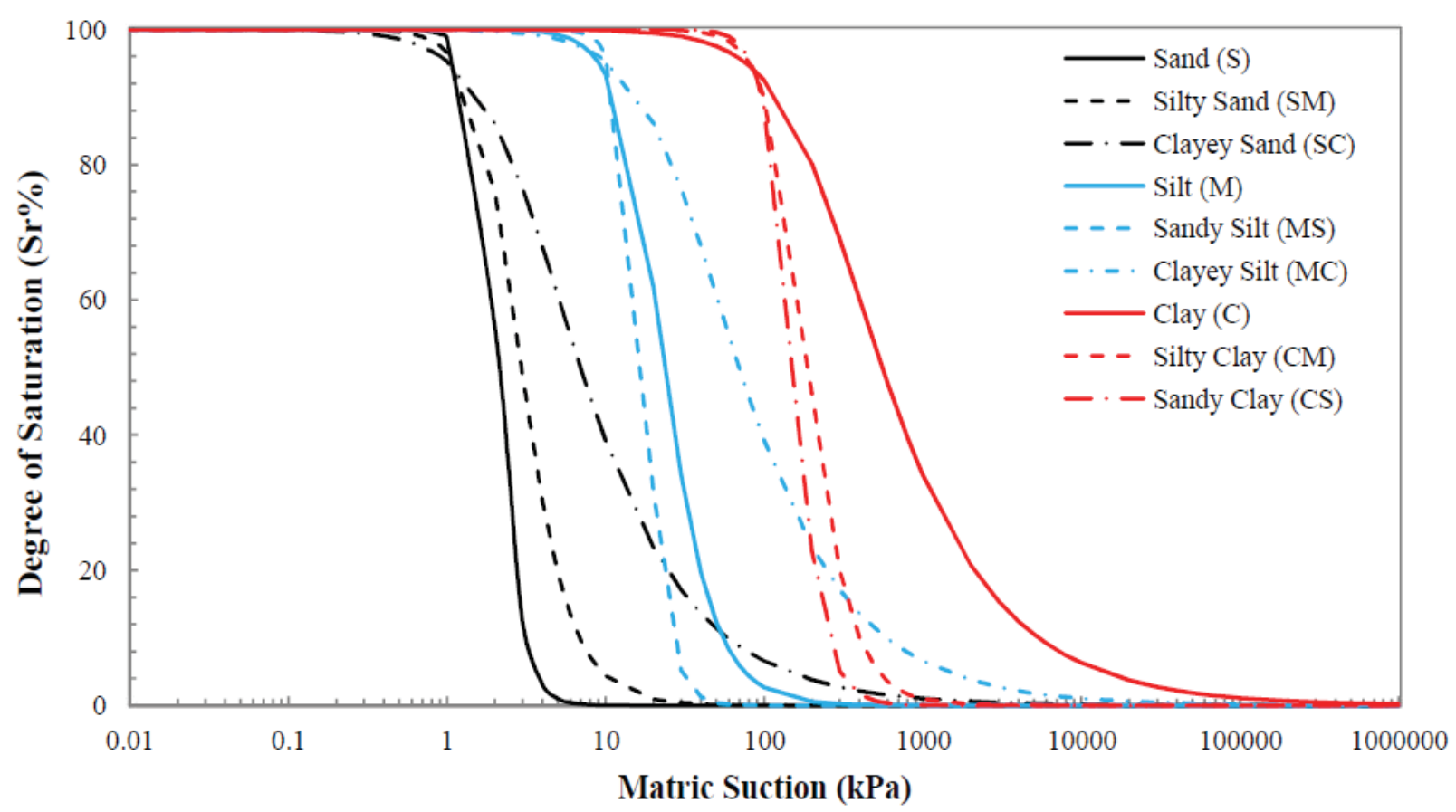

Figure 2.33 Typical SWCCs for various soil types based on average van-Genuchten fitting parameters

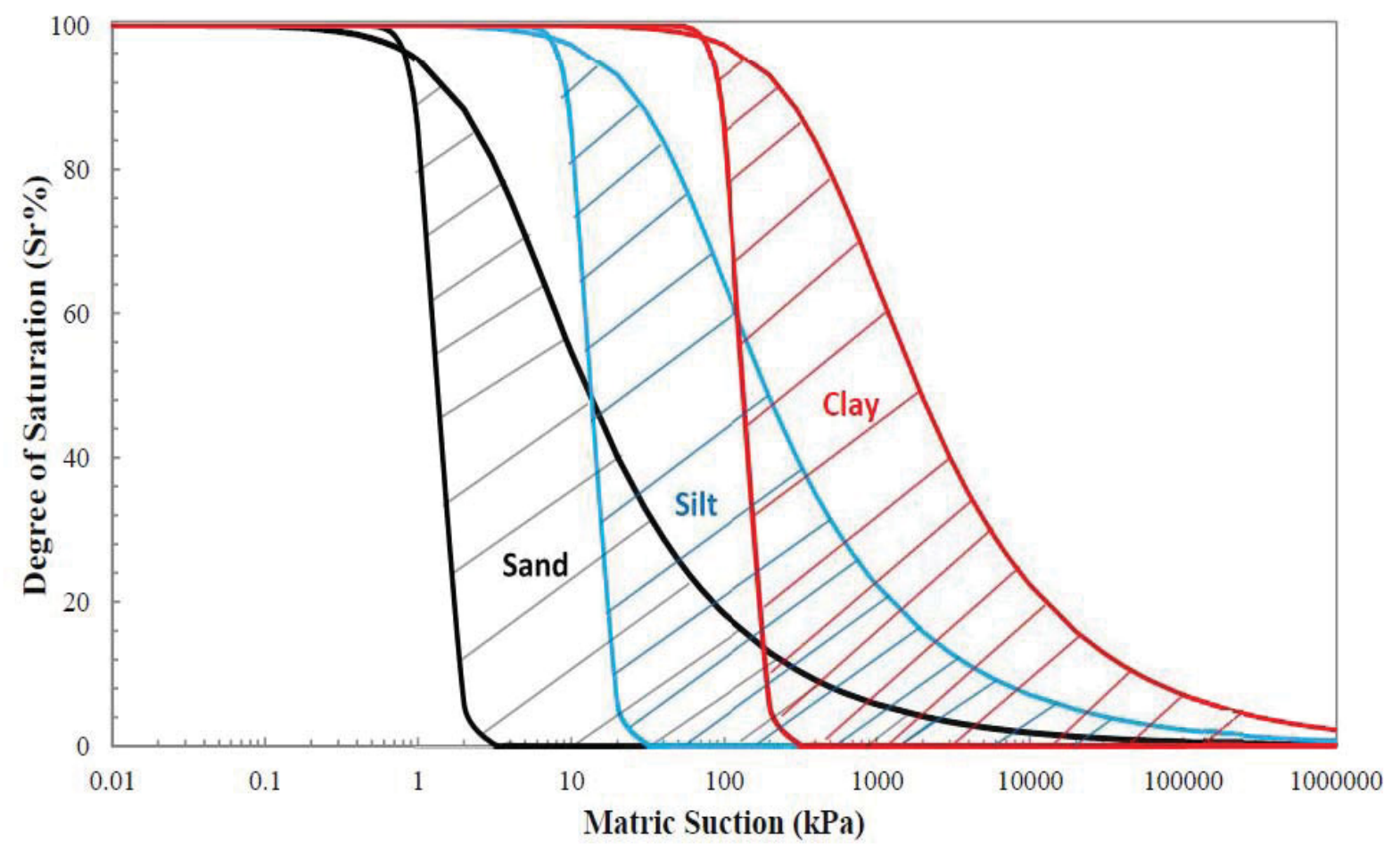

Figure 2.34 Possible zones of SWCC for sand, silt and clay based on van-Genuchten fitting parameters 
Lu and Likos (2010) obtained the value of suction stress substituting equation 2.14 in equation 2. 13:

$$
\sigma^{S}=-\frac{\left(u_{a}-u_{w}\right)}{\left(1+\left[\propto\left(u_{a}-u_{w}\right)\right]^{n}\right)^{(n-1) / n}} \quad \text { Equation } 2.15
$$

They used equation 2.15 to produce SSCC for various types of soils. Substituting equation 2.15 in equation 2.12, Lu and Likos (2010) obtained the following equation for effective stress of unsaturated soils:

$$
\sigma^{\prime}=\left(\sigma-u_{a}\right)+\frac{\left(u_{a}-u_{w}\right)}{\left(1+\left[\propto\left(u_{a}-u_{w}\right)\right]^{n}\right)^{(n-1) / n}} \quad(\text { Lu and Likos, 2010) } \quad \text { Equation } 2.16
$$

The effective stress of unsaturated soils can be also extrapolated from Fredlund et al. (1996) and Vanapalli et al. (1996a)'s shear strength equation as follows:

$$
\tau=c^{\prime}+\left(\sigma-u_{a}\right) \tan \emptyset^{\prime}+\left(u_{a}-u_{w}\right) S_{r}^{\mathrm{K}} \tan \emptyset^{\prime} \quad(\text { Vanapalli, 2010) } \quad \text { Equation } 2.17
$$

$$
\begin{array}{ll}
\sigma^{\prime}=\left(\sigma-u_{\mathrm{a}}\right)+\left(u_{a}-u_{w}\right) S_{r}^{\mathrm{K}} & \text { Equation } 2.18
\end{array}
$$


where $\mathrm{S}_{\mathrm{r}}$ is degree of saturation and $\mathrm{K}$ is a fitting parameter associated with plasticity index. Vanapalli and Fredlund (2000) Graven and Vanapalli (2006) and Oliviera and Marinho (2003) provided the following relationships between the fitting parameter, $\mathrm{K}$ and plasticity index, PI, using shear strength data sets of shear strength as below:

$\mathrm{K}=-0.0008(P I)^{2}+0.0801(P I)+1 \quad$ Vanapalli and Fredlund (2000) $\quad$ Equation 2.19

$\mathrm{K}=-0.0016(P I)^{2}+0.0975(P I)+1 \quad$ Graven and Vanapalli (2006) Equation 2.20

$\mathrm{K}=-0.0044(P I)^{2}+0.22455(P I)+0.9715 \quad$ Oliviera and Marinho (2003) Equation 2.21

Equations 2.19 and 2.20 show that a fitting parameter $\mathrm{K}=1$ is required for predicting the contribution of suction on effective stress of non-plastic unsaturated soils (i.e. PI $=0$; coarse grained soils).

In summary, the contribution of net normal stress to the effective stress of unsaturated soils is equal to $\left(\sigma-\mathrm{u}_{\mathrm{a}}\right)$; the contribution of matric suction to effective stress of unsaturated soils, which covers active local forces close to inter-particle contact (i.e. van der Waals forces, electrical double-layer forces, cementation forces, surface tension forces, and forces arising from negative pore water pressure) is called suction stress $\left(\sigma^{S}\right)$ and calculated from equation 2.16. SSCC is a representation of the value of suction stress as soil dewaters. In fact, the value of effective stress obtained by contribution of matric suction is plotted for different degrees of saturation. Obviously, SSCC does not include the net normal stress and does not represent the 
final effective stress of unsaturated soils. Readers may be aware that adding or increasing net normal stress on unsaturated soils might also affect the value of matric suction. Since the SWCC of the soil might be changed by increasing the net normal stress, the SSCC should be plotted considering SWCC of the soil with a very low value of net normal stress. Therefore, SSCC shows the value of suction stress including the contribution of matric suction on effective stress

of unsaturated soils, assuming that there is no net normal stress in the soil. Obviously, omitting the net normal stress or overburden pressure is not possible in the field or a soil sample. However, this assumption helps to employ the SWCC of the soil and quantify the contribution of matric suction and other active local forces as a value of suction stress in different degrees of saturations as a SSCC. The SSCC is helpful to find the variations of suction stress of different soils during dewatering.

\subsection{Microstructure investigation}

Microstructural studies consist of using techniques at particle scale $(<100 \mu \mathrm{m})$ to analyse the arrangement and distribution of particles, particle assemblies and pores in different soils. Microstructure investigations studies are increasingly used to facilitate understanding of the macroscopic behavior and physical properties of different soils (Romero and Simms 2008; Collins and McGowan, 1974; Mitchell and Soga, 2005). There are two well-known techniques to study unsaturated soil microstructure: mercury intrusion porosimetry (MIP) and environmental scanning electron microscopy (ESEM). The MIP technique has been frequently used while the ESEM is a fairly new technique with great potential because of minimal sample preparation 
requirements. Some of the macroscopic soil properties can be explained in terms of microstructural characteristics such as distribution and connectivity of pores, particle size distribution, void ratio, etc. In addition, understanding microstructural behavior of unsaturated soils has been used in geotechnical applications in which soils undergo water content and volume changes (Romero and Simms, 2008).

\subsubsection{Mercury Intrusion Porosimetry (MIP)}

A typical method to obtain a quantitative investigation of the microstructure of materials associated with porosity is mercury intrusion porosimetry (MIP). Pore size distribution (PSD) can be obtained directly from the MIP method. The PSD is an essential fabric element that is used in geotechnical engineering applications and can lead to obtaining macroscopic properties such as type of compaction for clays (Ahmed et al., 1974; Kong et al,. 2005) or water retention properties (Simms and Yanful 2002, 2005).

In the MIP technique an absolute pressure $\mathrm{p}$ is applied to a non-wetting liquid (mercury) in order to enter the empty pores. The pressure required to push the liquid into pores with a specific entrance diameter, d, is given by the Washburn equation (Washburn, 1921):

$$
P=\frac{4 T_{s} \cos \theta}{d}
$$

Equation 2.22

where $\mathrm{P}$ is the absolute pressure, $\theta$ is the contact angle between the mercury and the soil, and $\mathrm{T}_{\mathrm{s}}$ is the surface tension. This equation supposes a cylindrical-shaped pore and for slit-shaped pores, $\mathrm{d}$ is equal to twice the inter-particle distance (Simms and Yanful, 2004). Figure 2.36 presents an 
example of employing MIP to investigate the effect of water retention curve on PSD of glacial till performed by Simms and Yanful (2002).

\subsubsection{Sources of possible errors in the MIP method}

Simms and Yanful (2004) have proposed some possible sources of errors in the MIP method:

a) The effects of sample preparation to remove water,

b) The effect of pressure generated during MIP on soil structure,

c) The influence of pore accessibility on the generated PSD.

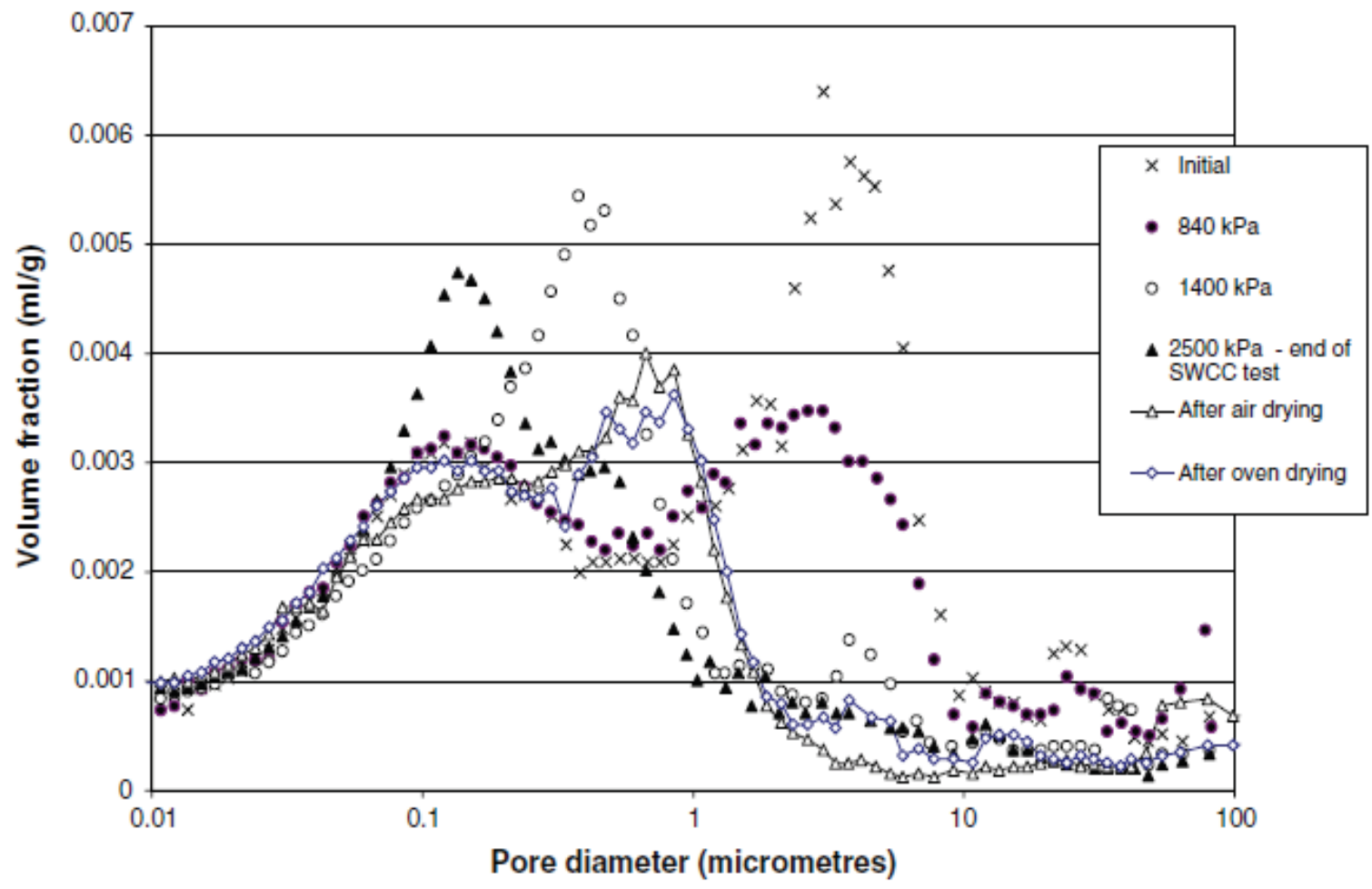

Figure 2.35 Evolution of pore size distribution during a water retention curve test and after air and oven drying for a glacial till, PI $=10 \%$ (Simms and Yanful 2002) 
A soil sample must be fully dried before undergoing MIP. Two most common methods to remove water are oven-drying and freeze-drying (Simms and Yanful, 2004). Freeze-drying with sublimation has the least effect on soil structure, due to fast rate of freezing (Simms and Yanful, 2004). The accessibility of pores and the differentiation of apparent from actual pore distributions can be addressed using percolation theory (Simms and Yanful, 2002). Simms and Yanful (2005) proposes that " if a larger pore is cut off from intruding mercury by surrounding smaller pores, it will not be intruded until the pressure is high enough to intrude into the smaller pores, and hence the measured PSD will tend to exaggerate the frequency of actual smaller pores and underpredict the frequency of actual larger pores."

The literature review explained in this chapter will be used in next chapters to investigate the influence of stress history induced by desiccation and consolidation settlement on monotonic and cyclic shear strength of thickened gold tailings. 


\section{Chapter 3: Material and Methodology}

\subsection{Introduction}

Laboratory experiments of this study consist of i) experiments conducted to simulate the stress history of thickened tailings layers and ii) shear strength measurement tests performed on samples prepared in the simulations. Several experimental aspects such as sample preparation procedures, selection of test devices and test procedures are discussed in this chapter. Details of small scale laboratory tests as well as multilayer drying box deposition are described. Suction measurement devices (both matric and total suctions), void ratio measurement methods and methods of measuring the rate of evaporation and water content in the drying box experiment are explained. In addition, details of laboratory strength measurement devices i.e. simple shear test, triaxial test and vane shear device are also described. 


\subsection{Review of main objective and overall methodology}

As discussed earlier, in thickened tailings technology, tailings are pumped from the underflow of thickeners and deposited in a flat area. Initially, the deposited layer experiences settling due to self-weight with no overburden pressure. The layer is allowed to densify by desiccation and/or drainage. When the next fresh layer is deposited on the desiccated bottom layer, the bottom layer is rewetted due to capillary action and flow of water under gravity. This process is sequentially continued to reach a stable stack, which is more resistant to static and cyclic loading than conventional slurry deposition. The geotechnical behavior of a thickened tailings layer is a function of stress history that the tailings layer experiences during desiccation, rewetting and consolidation induced by the weight of top layers. Potentially, three factors are expected to influence the final strength of unsatuared deposited tailings: i) density ii) stress history (loading and suction history), and iii) current stress and suction state. Any aging effects, such as cementation due to mineral precipitation, are not considered in this research. As described in Chapter 1, different stress histories are possible, depending on the degree of desiccation of the fresh layer (Figure 1.1). The objective of this thesis is to consider the influence of the extent of desiccation on the geotechnical behavior of subsequently rewetted and buried tailings. Most of our tests impose complete rewetting (zero suction after rewetting), and subject the samples to target consolidation stresses.

In order to appropriately simulate the thickened tailings field process, two small scale tests in a column (Diameter $\sim 250 \mathrm{~mm}$ ) with two-layer deposition, and simulation of multilayer deposition in a drying box ( $1 \mathrm{~m}$ by $0.7 \mathrm{~m}$ in plan) were employed in this study to simulate 
desiccation and subsequent rewetting. Samples are then extracted and placed in simple shear and triaxial tests to undergo consolidation and subsequent monotonic or cyclic loading. Details of the experimental methods are explained in later sections.

\subsection{Geotechnical properties of gold mine tailings used in this study}

The material tested consists of gold mine tailings from Bulyanhulu mine in Tanzania. The gravimetric water content (GWC) of the tailing at deposition at the mine, when the mine employed thickened tailings, was $38 \%$. This water content corresponds to a yield stress of about 30-50 Pa, as determined using a cylinder and using the equation of Pashias et al. (1996). The tailings were collected at a deposition point and then shipped to Ottawa. The tailings were maintained in saturated condition in plastic bags. Though the tailings were shipped at the pumping water content $(38 \%)$, it was found that due to settling during transport, the water content reduced to around $22-25 \%$ and it was required to remix the tailings with the bleed water produced by settling in order to re-produce the tailings with $\mathrm{W}=38 \%$. Subsequent to increasing the water content, if the tailings are left in columns with no evaporation or drainage, the tailings inevitably settle to a water content of $30 \%$ within 48 hours. This occurs for heights of tailings ranging from 0.1 to $1 \mathrm{~m}$ (Simms et al., 2012). Figure 3.1 shows the mixer used for remixing the tailings. The specific gravity of gold mine tailings was determined to be 2.89 (ASTM D854, 2000). The liquid limit of the tested materials was measured by the Casagrande test (ASTM D4318) and the results showed that $L L=24 \%$. Plastic limit was also obtained as $P L=21.5 \%$ by the rolling test (ASTM D4318). Thus, the plasticity index of materials is $2.5 \%$. Shrinkage Limit 
of Bulyanhulu gold mine tailings was measured as $18 \%$ (Simms et al., 2007). Based on the Unified Soil Classification System (USCS), the tailings are classified as ML, which means silt with low plasticity. Figure 3.2 also presents the grain size distribution of the tailings obtained by hydrometer test (ASTM D421, ASTM D422). The chemical analysis of tailings liquid phase showed the concentration of important dissolved ionic species: Sodium (394 mg/ L), Arsenic (95.3 mg/L), Copper (126 mg/L) Magnesium (2010 mg/L) Calcium (7030 mg/L), and iron (31100 mg/L) (Al- Tarhouni, 2008).

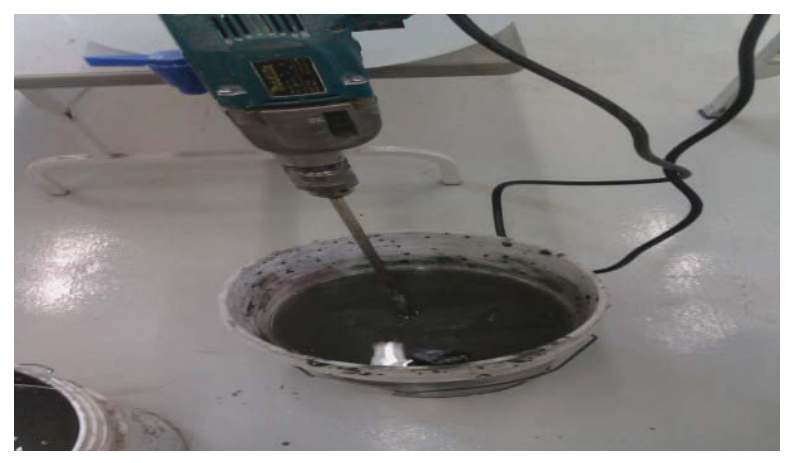

Figure 3.1 Mixer used for remixing the settled tailings

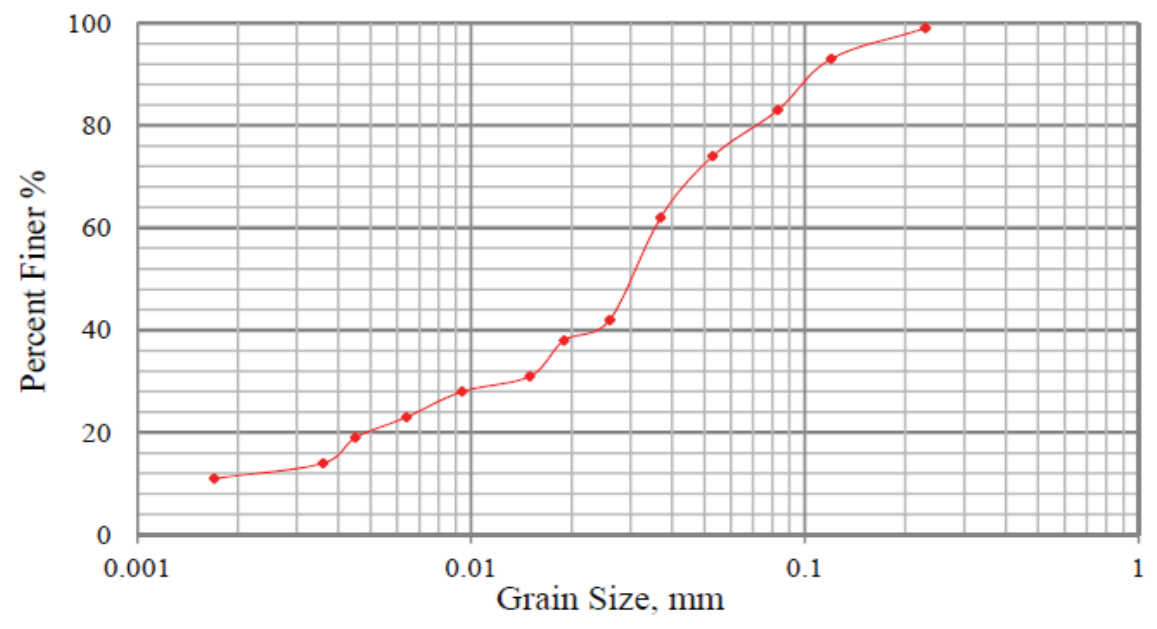

Figure 3.2 Grain Size Distribution of tailings used in the study 


\subsection{Laboratory methods to simulate thickened tailings deposition}

Two laboratory methods were used in this study to simulate the deposition of thickened tailings layers: 1) small scale laboratory deposition in a bucket 2) multilayer drying box deposition. Both methods impose regulated evaporation followed by rewetting and finally extraction by sampling methods. The drying box simulated multilayer deposition (up to 5 layers) and included sensors for monitoring void ratio, water content, evaporation, drainage and suction. Two sampling methods were also used to extract the undisturbed samples from the drying box. The details of both methods are described in the following sections.

\subsubsection{Small scale deposition simulation in a column}

The tailings were re-mixed using a paint mixer and combined with bleed water to the pumping water content before deposition in a column of approximately $250 \mathrm{~mm}$ diameter. The column was placed on a scale and the weight was recorded, as is the relative humidity and temperature in the laboratory. To simulate the stress history that occurs in the field, two layers of tailings of thickness of about $100 \mathrm{~mm}$ were sequentially deposited in the cylindrical column. Two commercial fans were located horizontally $250 \mathrm{~mm}$ above the top of the column in order to accelerate desiccation. The rate of potential evaporation was measured $9.1 \mathrm{~mm} /$ day. The tailings were deposited at the pumping water content (38\%) and allowed to settle and desiccate to water content values of $30,28,25,23,19,17,12$ and $4 \%$, which represent states above and below the 
shrinkage limit. A thin wall tube (approx. $70 \mathrm{~mm}$ ID and $1.4 \mathrm{~mm}$ wall thickness) was buried inside the deposited tailings. Under these conditions, drying invariably produced a uniform profile of water content with depth, as determined using grab samples for oven-drying. Similar observations were made by Fisseha et al. (2010) and Bryan et al. (2010) on drying tests on these tailings. Volume change during the drying and rewetting phase of the experiments was measured approximately using a 1-D ultrasonic displacement sensor mounted over the centre of the tailings. After desiccation was complete, the first layer of tailings was then overlaid with fresh tailings $(\mathrm{W}=38 \%)$. The tailings were left for 12 hours to allow for re-saturation of the underlying sample, but before substantial evaporation could occur. In this step, the thickened tailings deposition process is simulated and a sample can be extracted from the bottom layer to be placed in the shear strength measurement device. The extraction of the thin wall tube consisted of extracting the buried tube from the bottom layer. The water content of the desiccated and rewetted second layer at sampling was always between $21 \%$ and $22 \%$, though the degree of

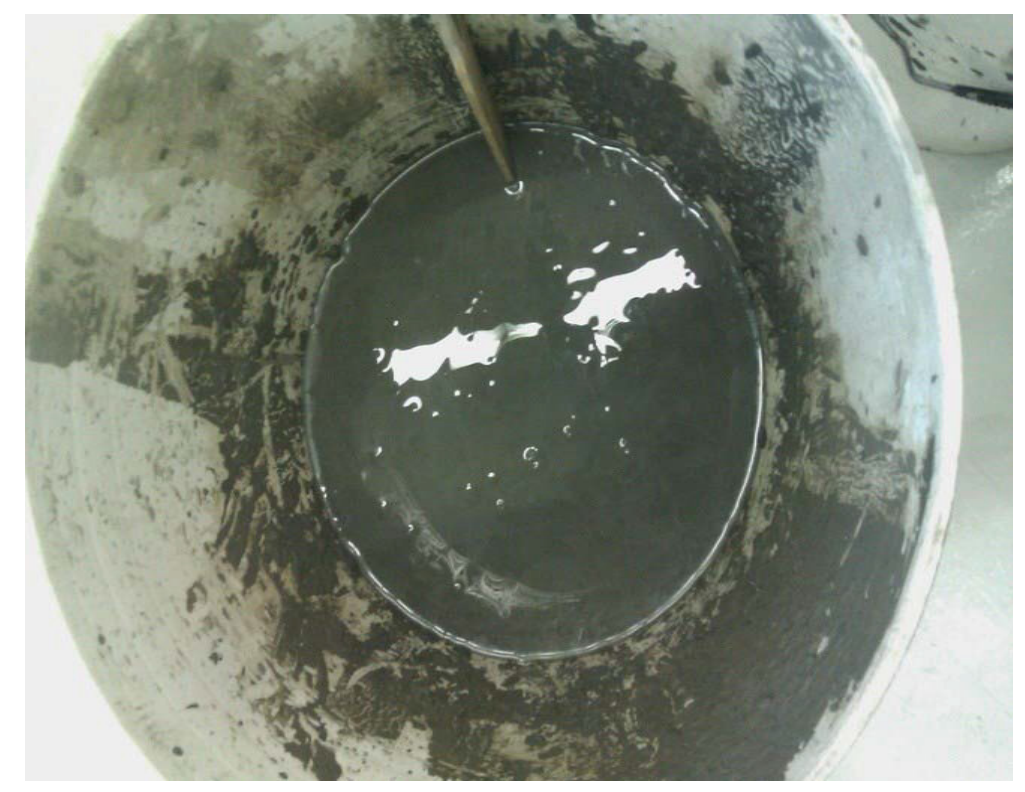

Figure 3.3 Deposition of $10 \mathrm{~cm}$ tailings layer with $38 \%$ water content 

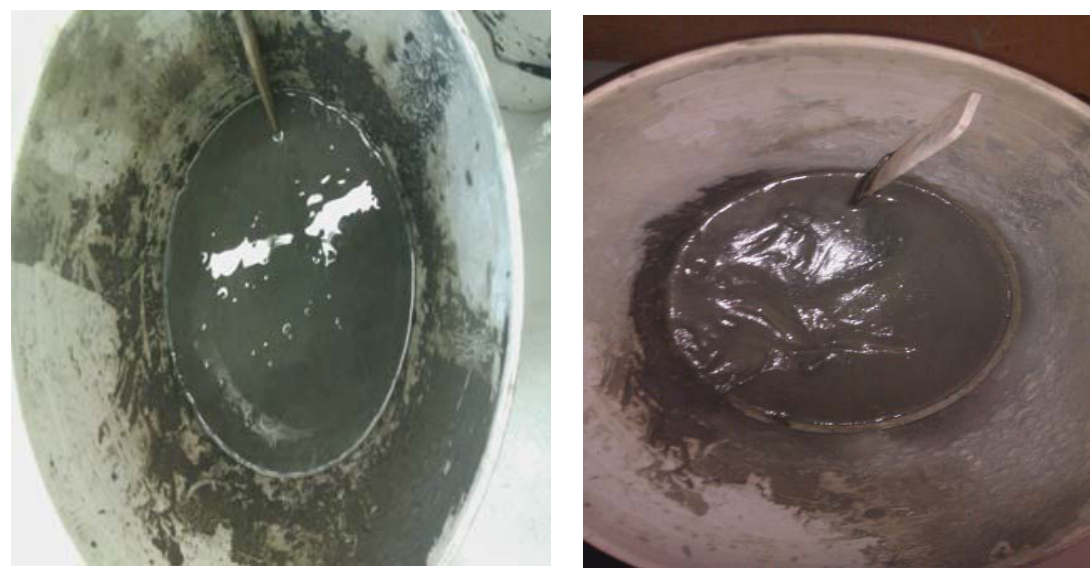

a) $\mathrm{W}=38 \%$

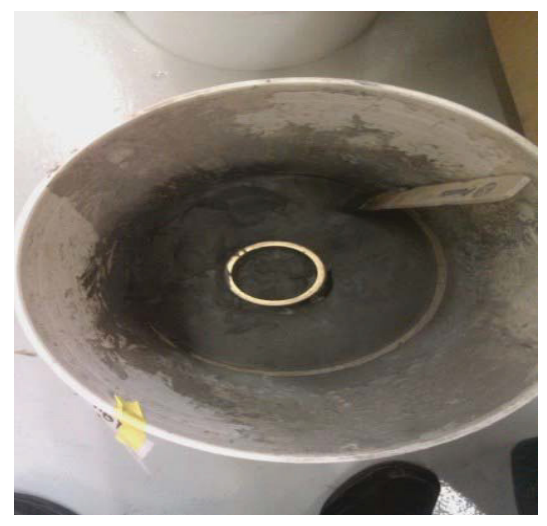

d) $\mathrm{W}=22 \%$ b) $\mathrm{W}=27 \%$

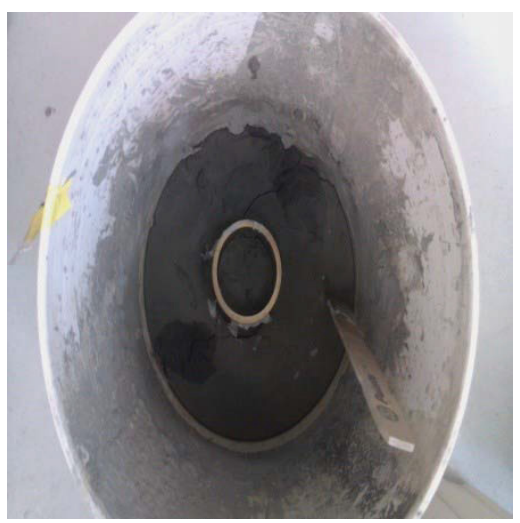

e) $\mathrm{W}=18 \%$

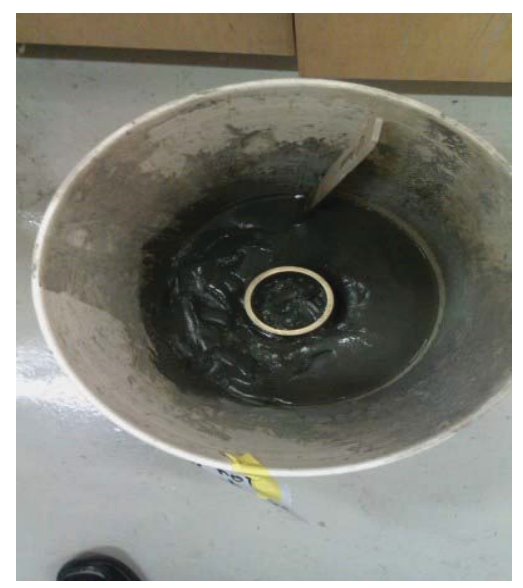

c) $\mathrm{W}=25 \%$

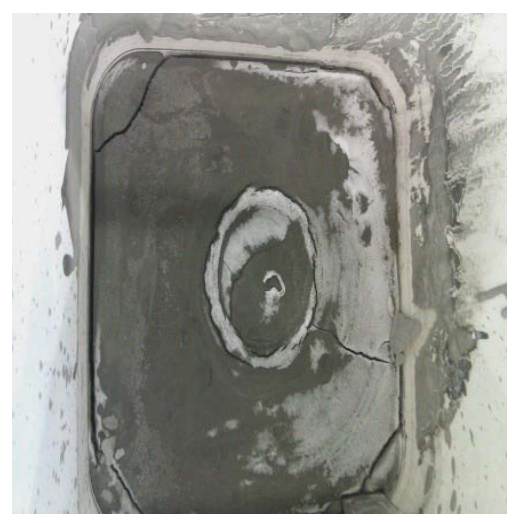

f) $\mathrm{W}=13 \%$

Figure 3.4 Desiccated tailings at different water content

saturation varied between 0.95 and 0.8 . Extracted samples were then placed in the simple shear device and subsequently consolidated under $50 \mathrm{kPa}, 100 \mathrm{kPa}, 200 \mathrm{kPa}$ and $400 \mathrm{kPa}$ consolidation pressures. Figure 3.3 shows deposition of first layer with $100 \mathrm{~mm}$ thickness in the bucket. Figure 3.4 and 3.5 also show desiccated tailings at different water contents and typical extracted samples respectively. 


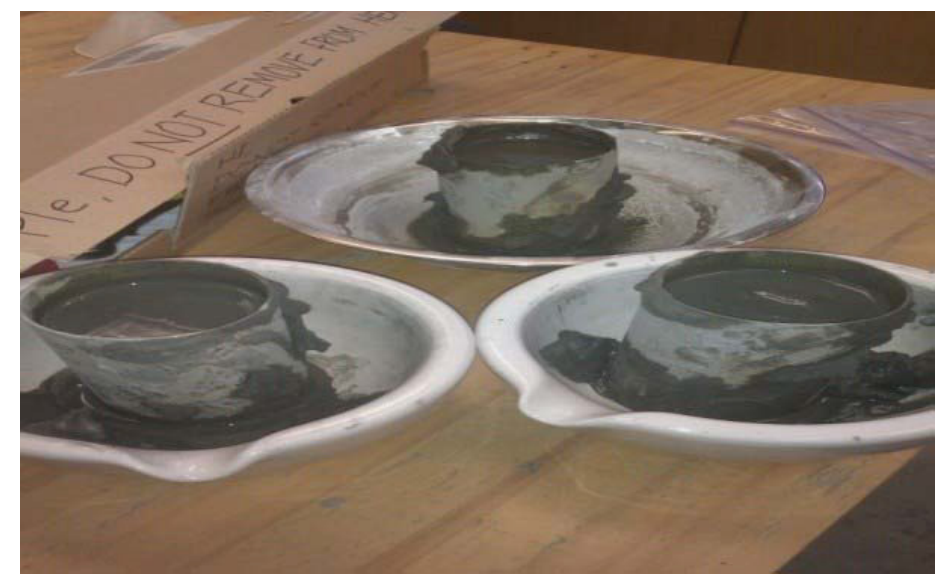

Figure 3.5 Typical extracted desiccated-rewetted samples from the small scale simulation test

For sampling, thin wall tubes with inner diameter of $69.95 \mathrm{~mm}$ were employed. In order to minimize the disturbance to undisturbed samples, thin-wall tubes with the length to diameter ratio of 1.42 were employed (Wijewickreme and Sanin 2004). Figure 3.6 shows characteristics of the thin wall tubes used in the sampling.
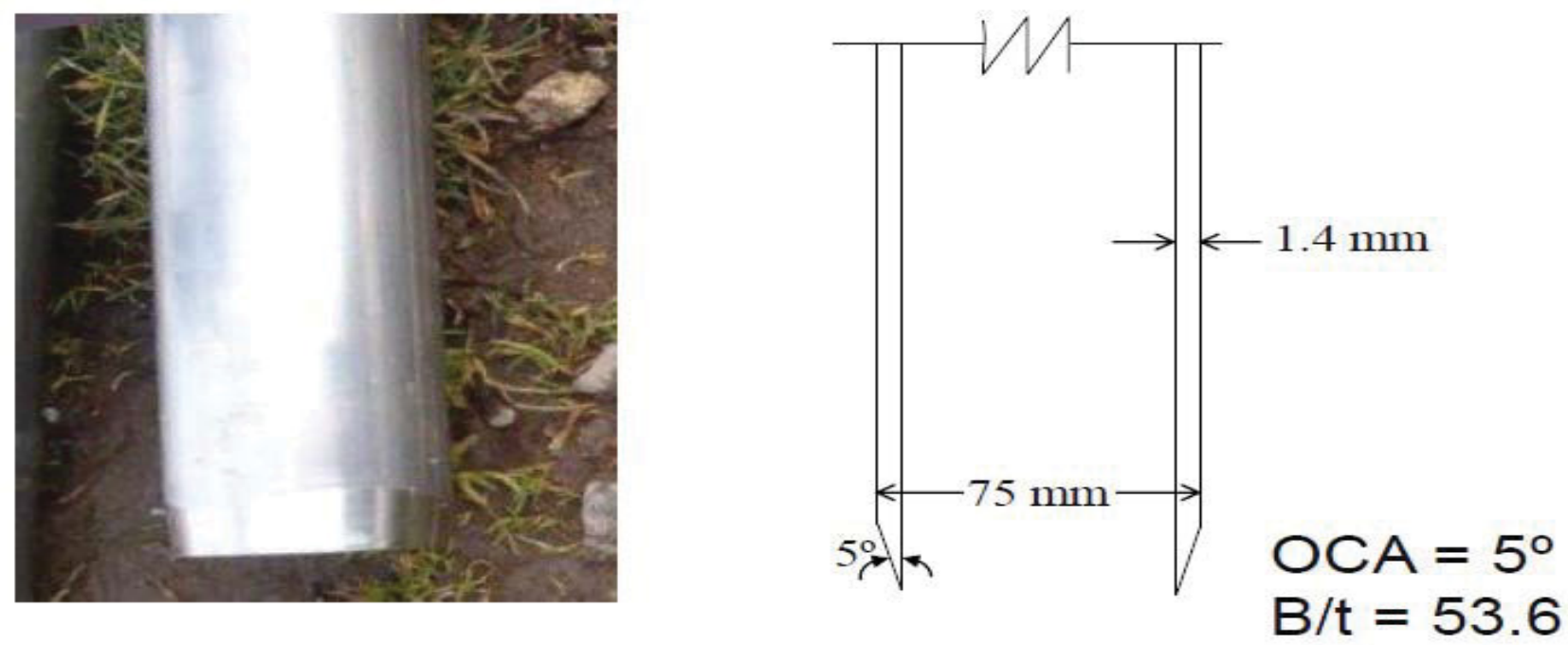

Figure 3.6 Proposed thin wall tubes for sampling (after Wijewickreme and Sanin 2004) 


\subsubsection{Multilayer deposition of thickened tailings in a drying box}

A modular drying box was designed and built in the civil engineering laboratory at Carleton University to enable sequential deposition of thickened tailings layers. The plexiglas box was reinforced by steel members that can be detached to facilitate extraction of the buried tubes from the bottom layers. The plan area of the box was $0.7 \mathrm{~m} \times 1 \mathrm{~m}$, and the height was $1.20 \mathrm{~m}$. The box was modular (two vertical sections) so as to minimize variation in the distance from the top of the tailings to the top of the box, in order to maintain a consistent imposed potential evaporation rate. Tailings were prepared at $38 \%$ water content in the buckets before deposition, and the water content was verified by oven-drying samples obtained from the mixed tailings (Figure 3.7). Figure 3.8 presents schematic diagrams of the drying box. Figure 3.9 also shows the drying box in empty and filled states.

Figure 3.10 presents a schematic plan for deposition and the location of water content sensors and tensiometers within the drying box. The first and second layers were planned to have desiccation beyond the shrinkage limit. The three top layers were planned to have desiccation to the shrinkage limit.

The drying box was mounted on load cells to measure water loss. Four load cells (WM-II model from Artech Industries Inc.) were placed on the floor to carry each corner of the box. Each load cell had a capacity of $2272 \mathrm{~kg}$. The total weight was recorded using a data logger every $1 \mathrm{~min}$. Drainage was monitored out of the bottom using a tipping bucket. Each tip was designed to collect $10 \mathrm{~mL}$ of water. A small opening at the bottom of the box connected to a valve was designed to collect the drainage through the tipping bucket. Figure 3.11 and 3.12 show pictures 
of load cells (hidden between the plates) and the drainage tipping bucket respectively. A geotextile was placed at the bottom of the box before placement of tailings to prevent passing solid particles through the drainage valve. Figure 3.13 shows the layer of geotextile at the bottom of the drying box prepared for deposition of the first layer deposition. The picture also shows some thin wall tubes prepared to be buried after deposition of the first layer.

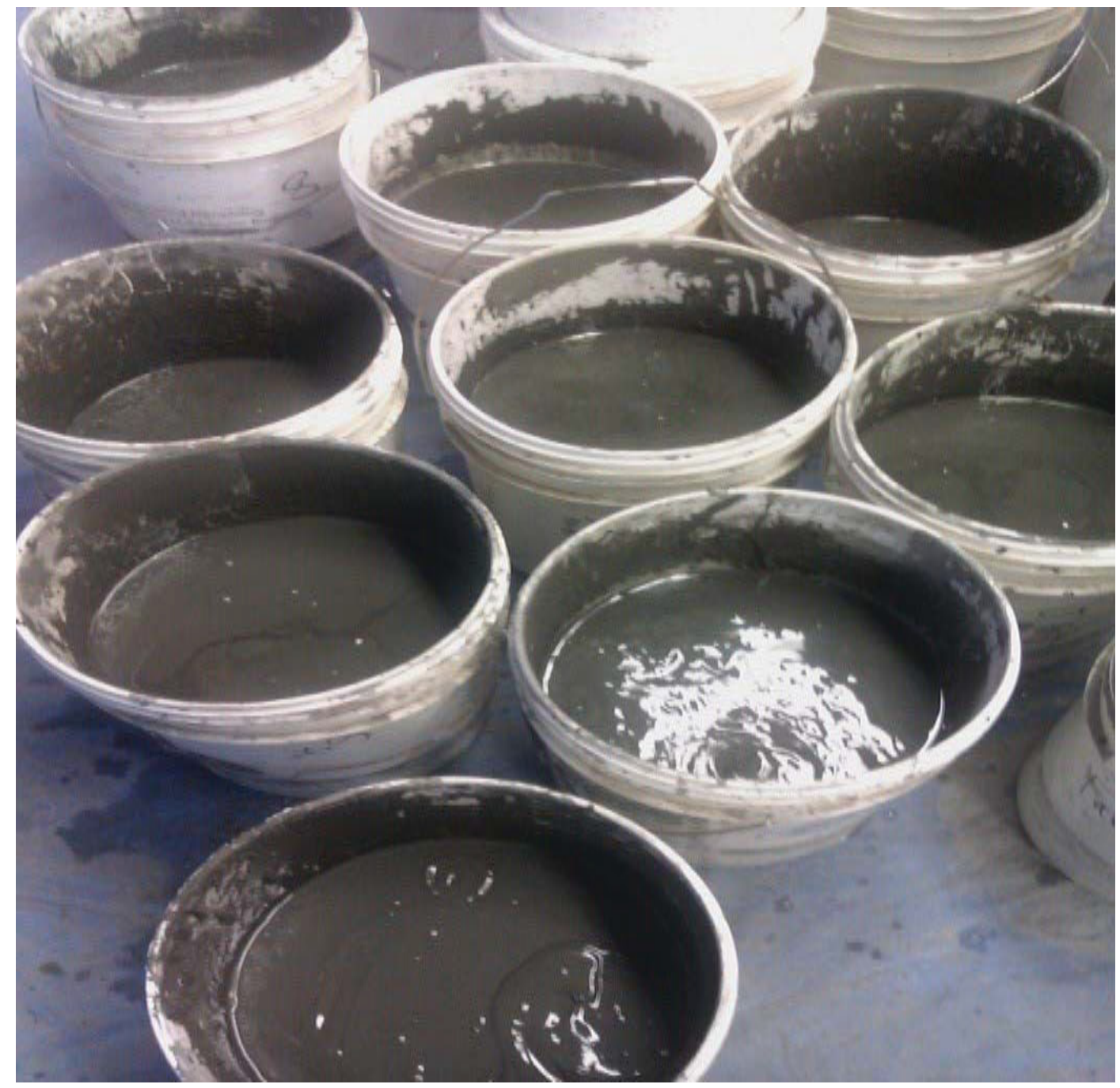

Figure 3.7 Buckets of tailings prepared at $38 \%$ water content 
Side View

(ADEF)

Samples are extracted from $\mathrm{BCGH}$ side

Dimensions : cm

Tensiometer Sensor

(T)

Water Content Sensor

(W)
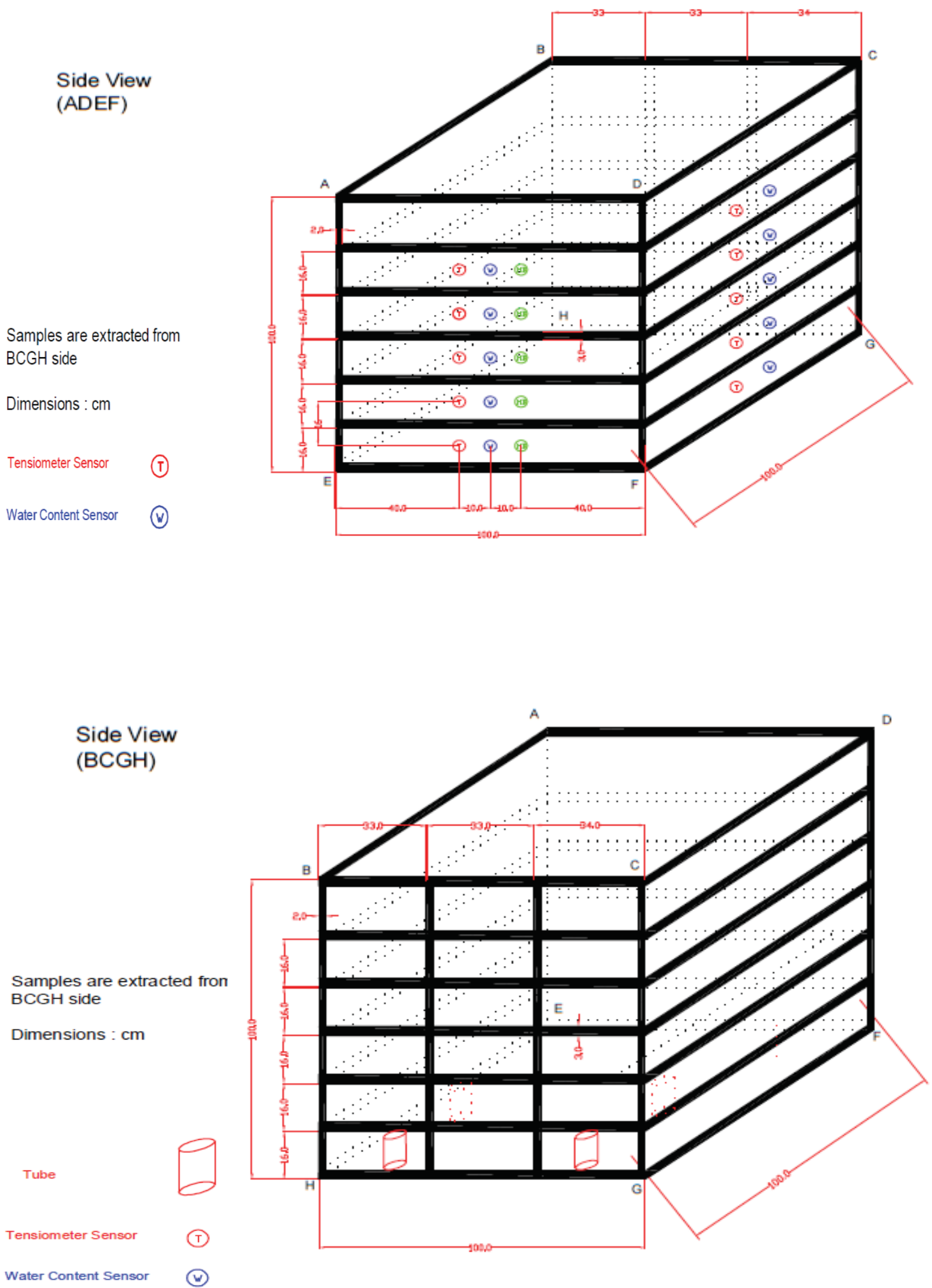

Figure 3.8 Schematic diagrams of steel reinforced Plexiglas drying box 


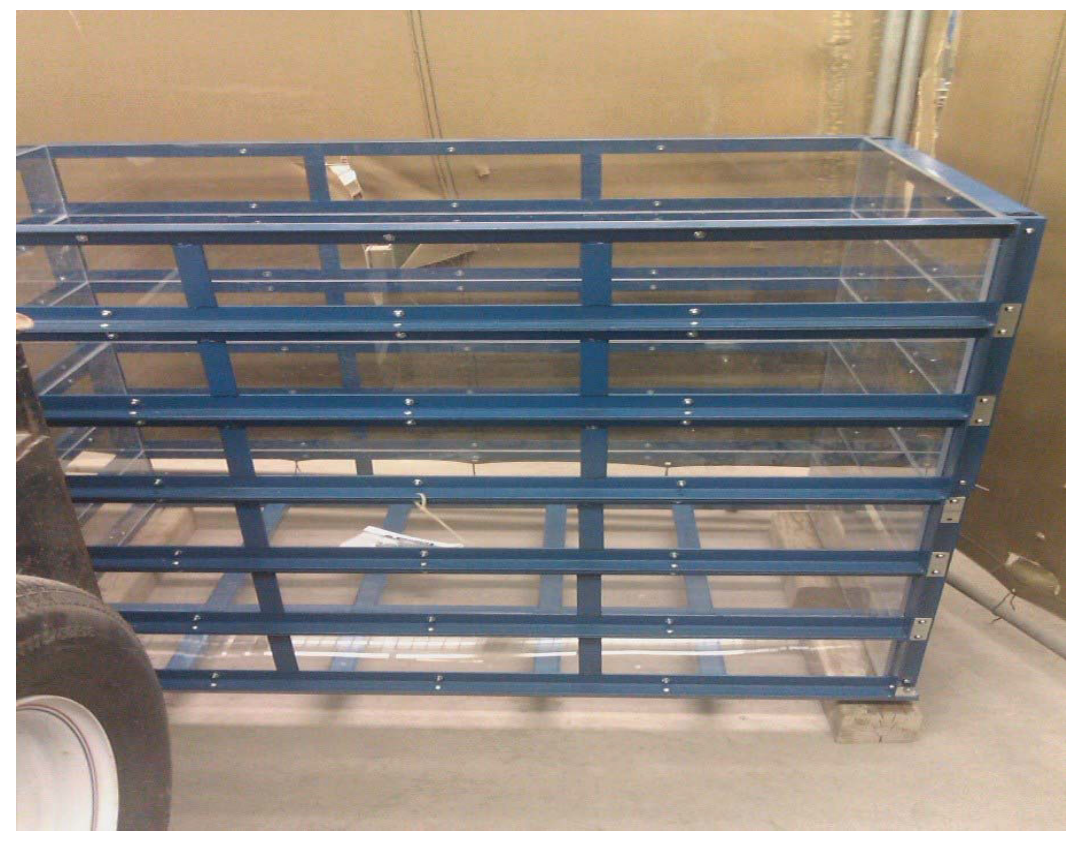

(a)

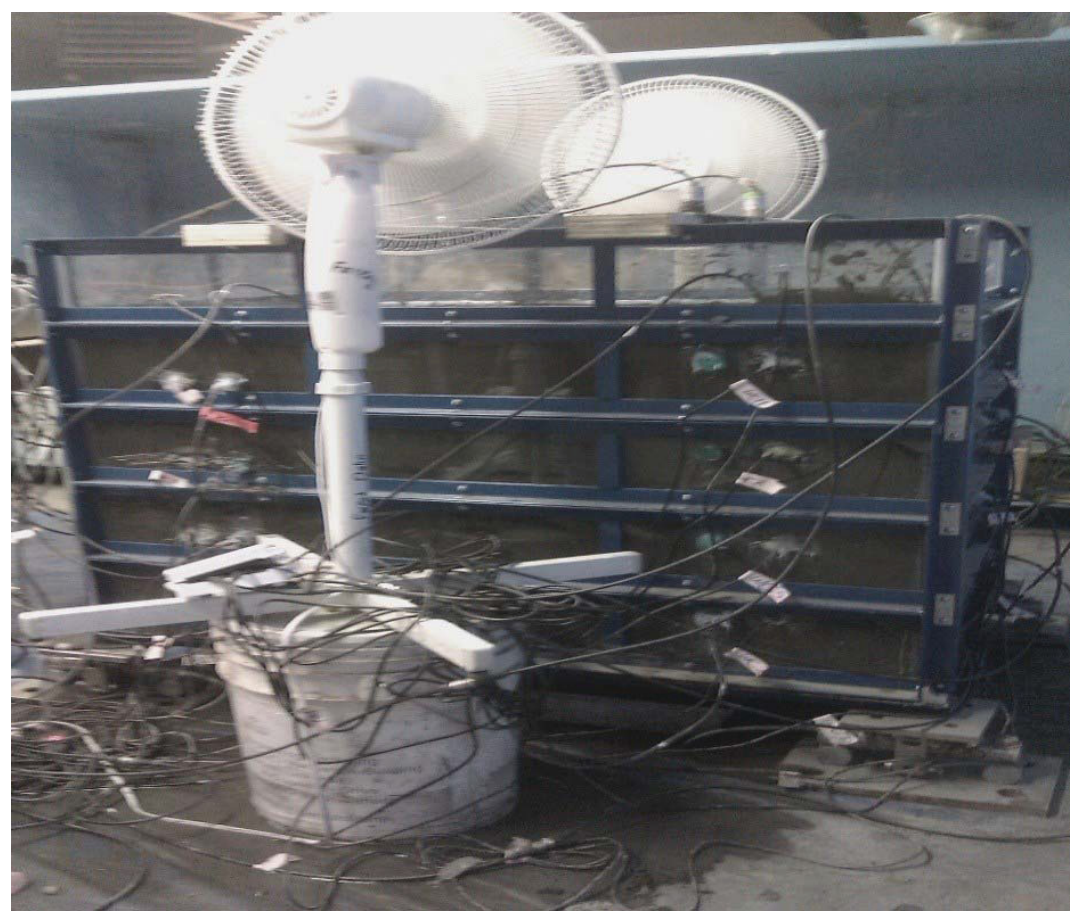

(b)

Figure 3.9 Pictures of the drying box in empty and filled conditions 


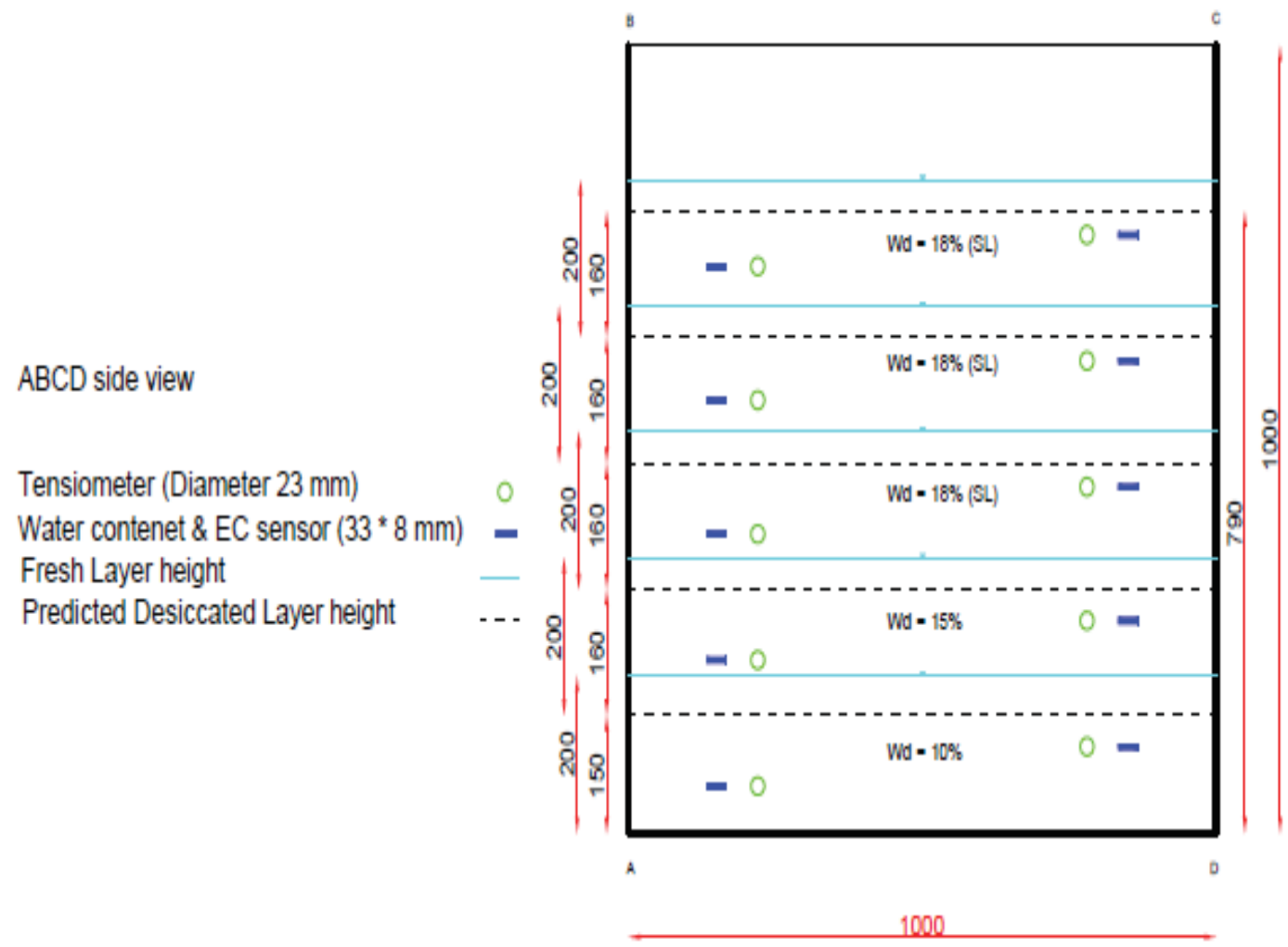

Figure 3.10 A schematic plan for deposition of layers and location of sensors (All dimensions are in $\mathrm{mm}$ )

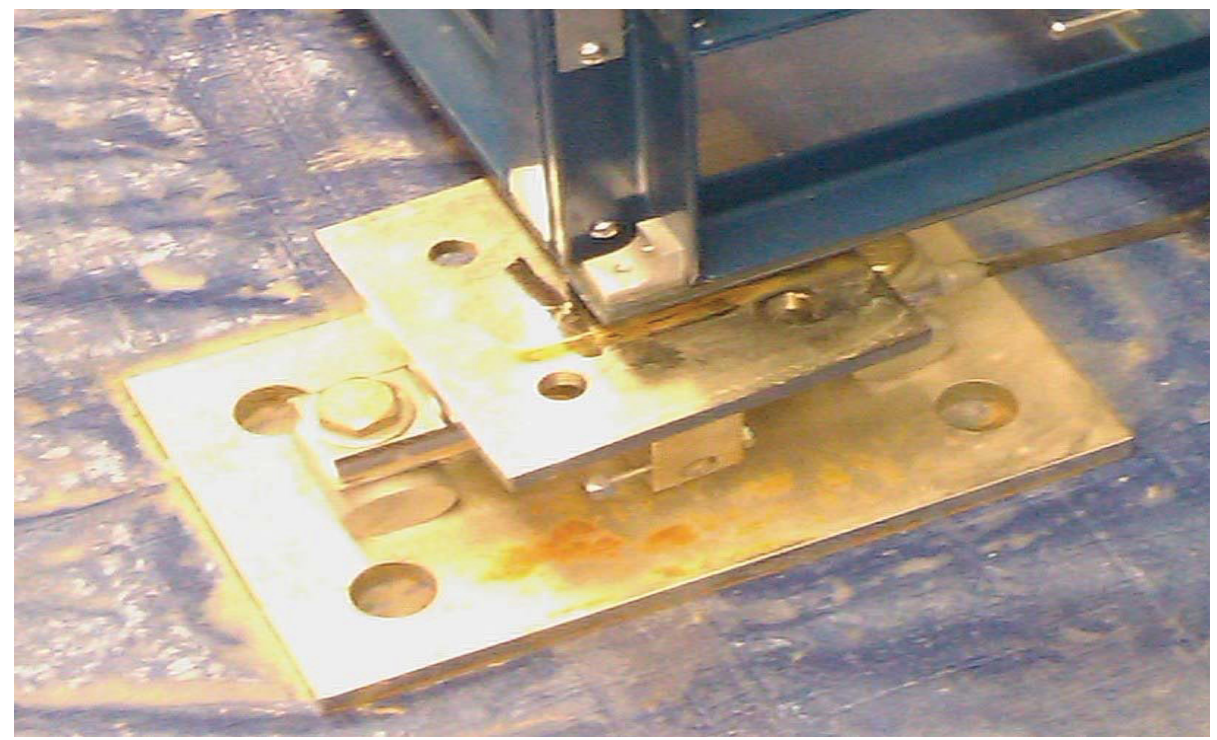

Figure 3.11 A picture of a WM-II load cell (hidden between the plates) 


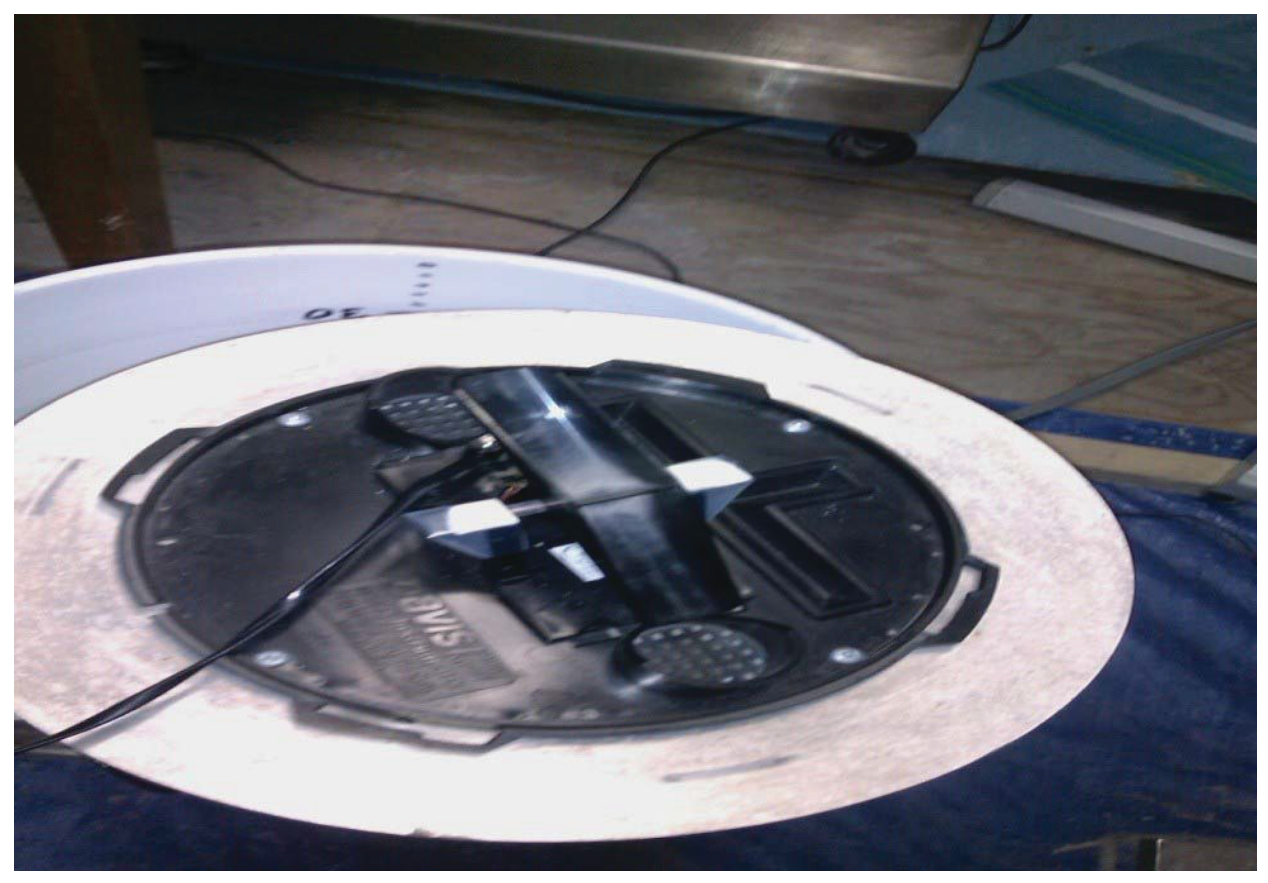

Figure 3.12 A picture of the tipping bucket for drainage measurement

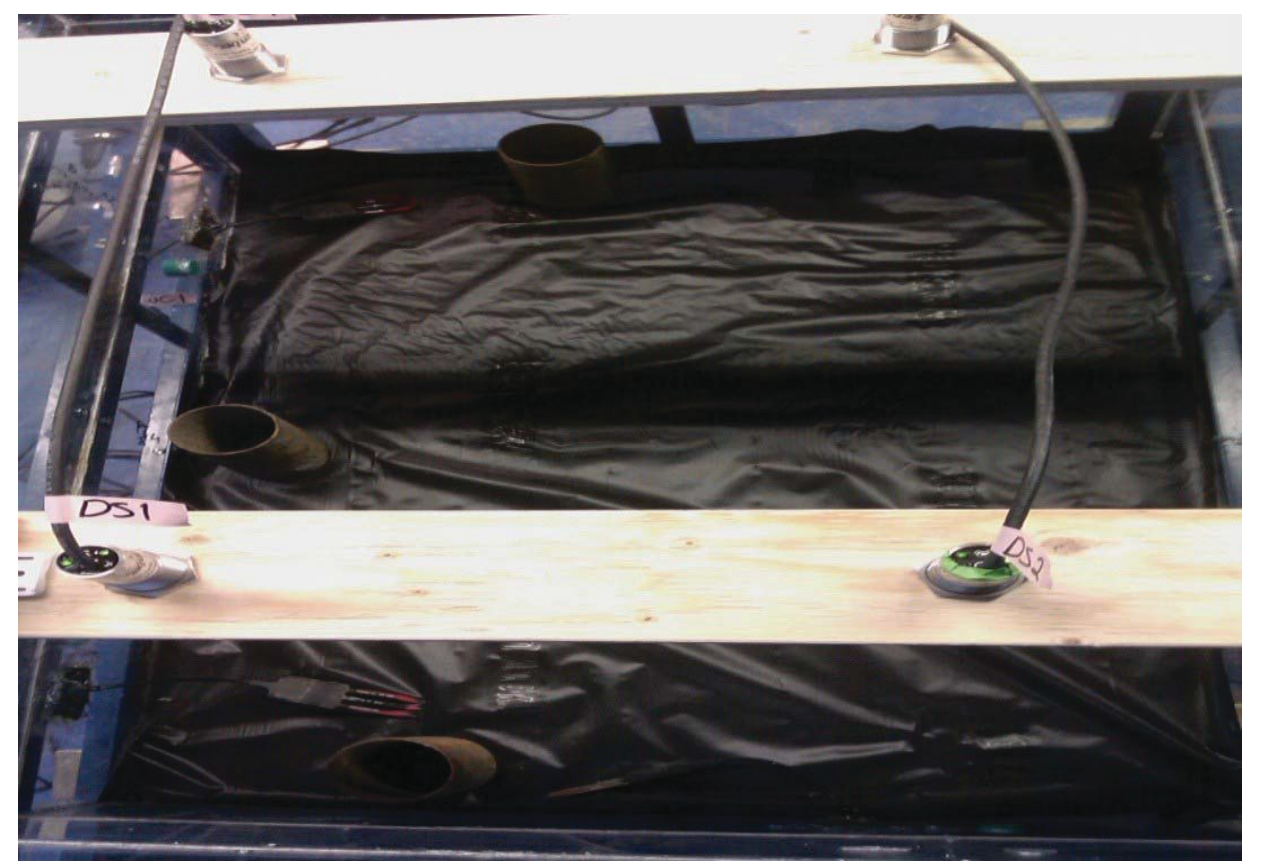

Figure 3.13 Layer of geotextile and thin wall tubes before first layer deposition 


\subsubsection{Water content measurement}

5TE volumetric water content (VWC) sensors were used in the drying box to measure the water content of layers. In addition to the volumetric water content, 5TE sensors are designed to measure electrical conductivity and temperature of soil. Water content, electrical conductivity and temperature of each layer were recorded at five minutes intervals by three 5TE sensors. The sensors were located at the top, middle and bottom of each layer. The sensor uses an oscillator running at $70 \mathrm{MHz}$ to measure the dielectric permittivity of soil to determine volumetric water content. A thermistor in thermal contact with the sensor prongs measure the soil temperature, while the screws on the surface of the sensor are responsible for measuring electrical conductivity. Figure 3.14 shows a picture of 5TE sensor. In order to evaluate accuracy of the volumetric water content sensors and calibrate them, the water content obtained from mass balance equations were compared with volumetric water content of sensors. In this method, the amount of removed water by both evaporation (from load cells) and drainage (from tipping buckets) were calculated. Calculating the total mass, amount of removed water and volume of the tailings by distance sensors, the volumetric water content was calculated. Figure 3.15 shows comparison of the values of calculated VWC by mass balance and VWC of three sensors during desiccation of the first layer. It is clear that both calculated VWC and VWC obtained by sensors are fairly similar in higher water contents after deposition. For lower water contents (lower than shrinkage limit), two sensors exhibited somehow higher VWC. Calibration of 5TE sensors was also performed by Innocent (2013) in a smaller chamber. 


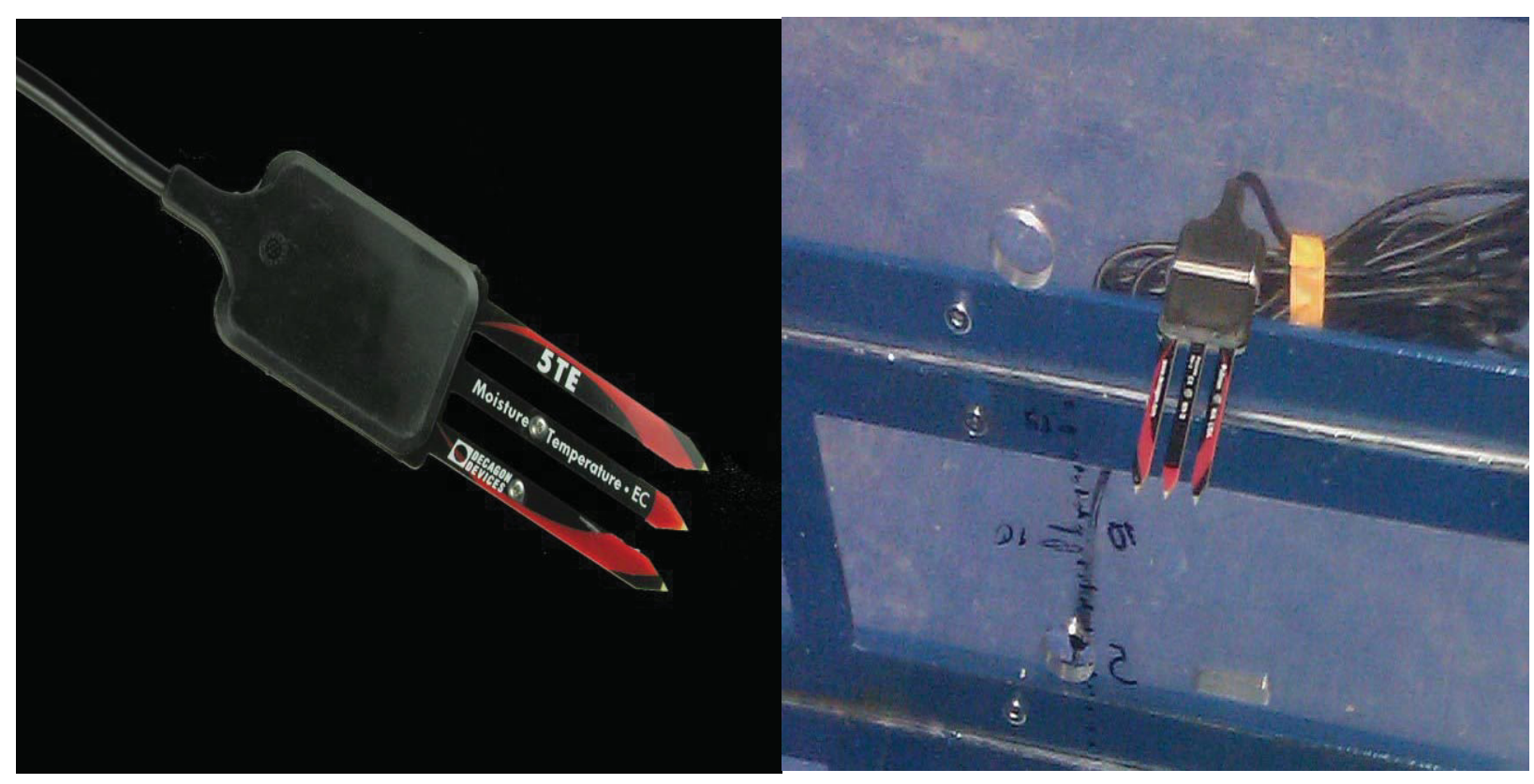

Figure 3.14 Pictures of a 5TE sensor

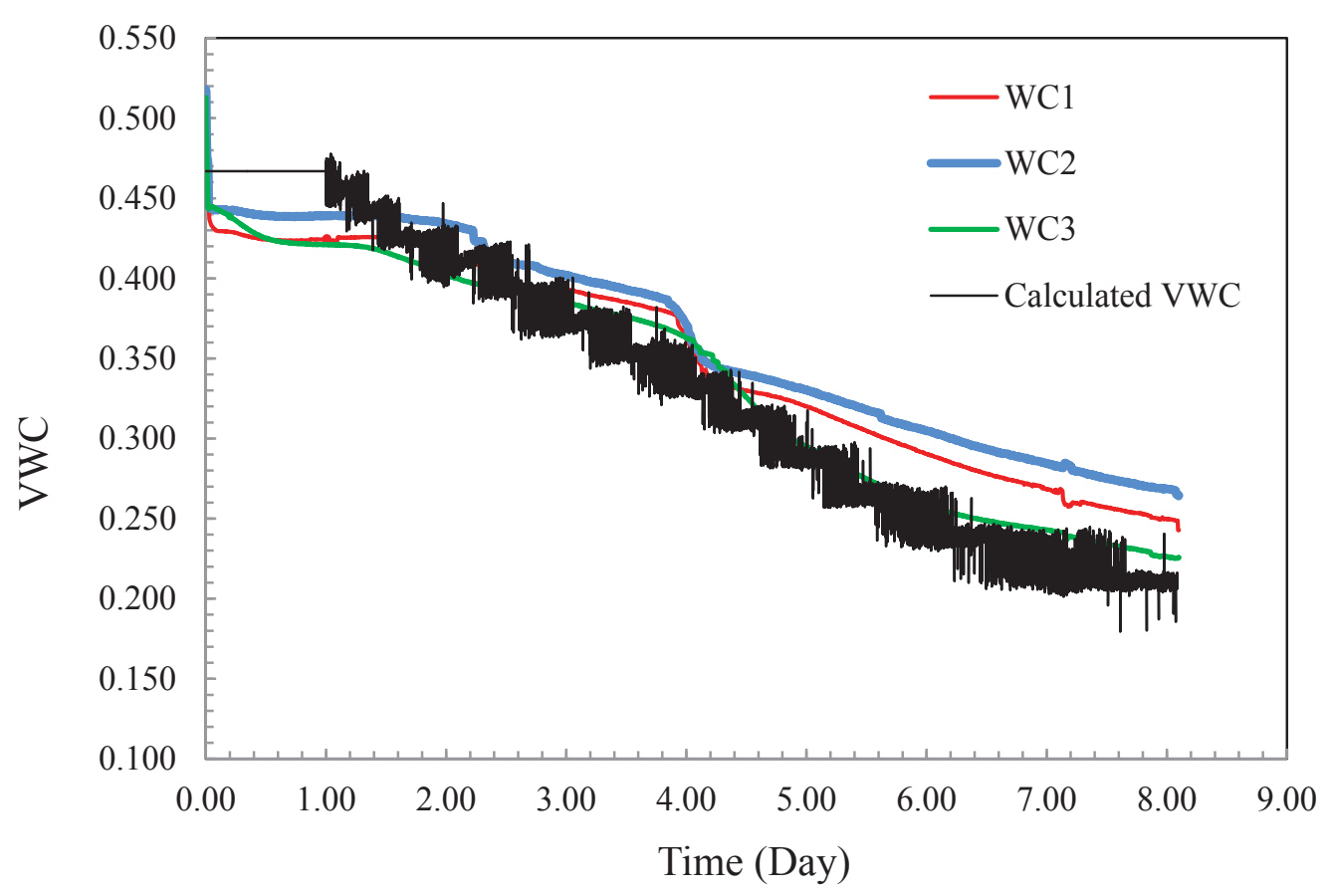

Figure 3.15 Comparison of calculated VWC and VWC obtained by sensors 


\subsubsection{Soil Suction Measurement}

\subsection{Matric Suction measurement}

UMS T5 tensiometers were used in this study to measure the value of matric suction during desiccation of layers. These tensiometers are designed to measure pore water pressure from +100 $\mathrm{kPa}$ (water pressure) to $-95 \mathrm{kPa}$ (suction). Soil water and tensiometer water have contact through the porous ceramic. If the soil dries out to higher suction values, the tensiometer runs empty and cavitation occurs. Each layer of tailings in the drying box had two or three tensiometers and the value of matric suction was recorded by a data logger at 5 minutes intervals. For layers that have a layer of water on top after initial settlement, tensiometers measured positive pore pressure. The sensor body of T5 tensiometers is made of acrylic glass. To transfer the soil water tension as a negative pressure (suction) into the tensiometer, a semi-permeable diaphragm is needed. This must have good mechanical stability and water permeability. The tensiometer cup consists of porous ceramic $\mathrm{Al}_{2} \mathrm{O}_{3}$ material. The pressure transducer offers the soil water tension as a linear output signal, with $1 \mathrm{mV}$ corresponding to $1 \mathrm{kPa}$.

The tailings layers during desiccation usually go beyond the cavitation range. For example, desiccation to the shrinkage limit for Bulyanhulu tailings generate around $200 \mathrm{kPa}$ matric suction, which is higher than cavitation range. Therefore, most of the tensiometers cavitated during desiccation and they required refilling after re-wetting process. In this case, tensiometers were replaced with prepared tensiometers after rewetting to measure the value of suction after rewetting. Figure 3.16 and 3.17 show a picture and description of different parts of a T5 tensiometer respectively. 


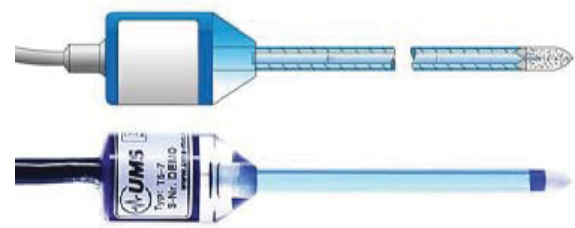

Figure 3.16 A picture of UMS T5 tensiometer (Hoskin Scientific, 2009)

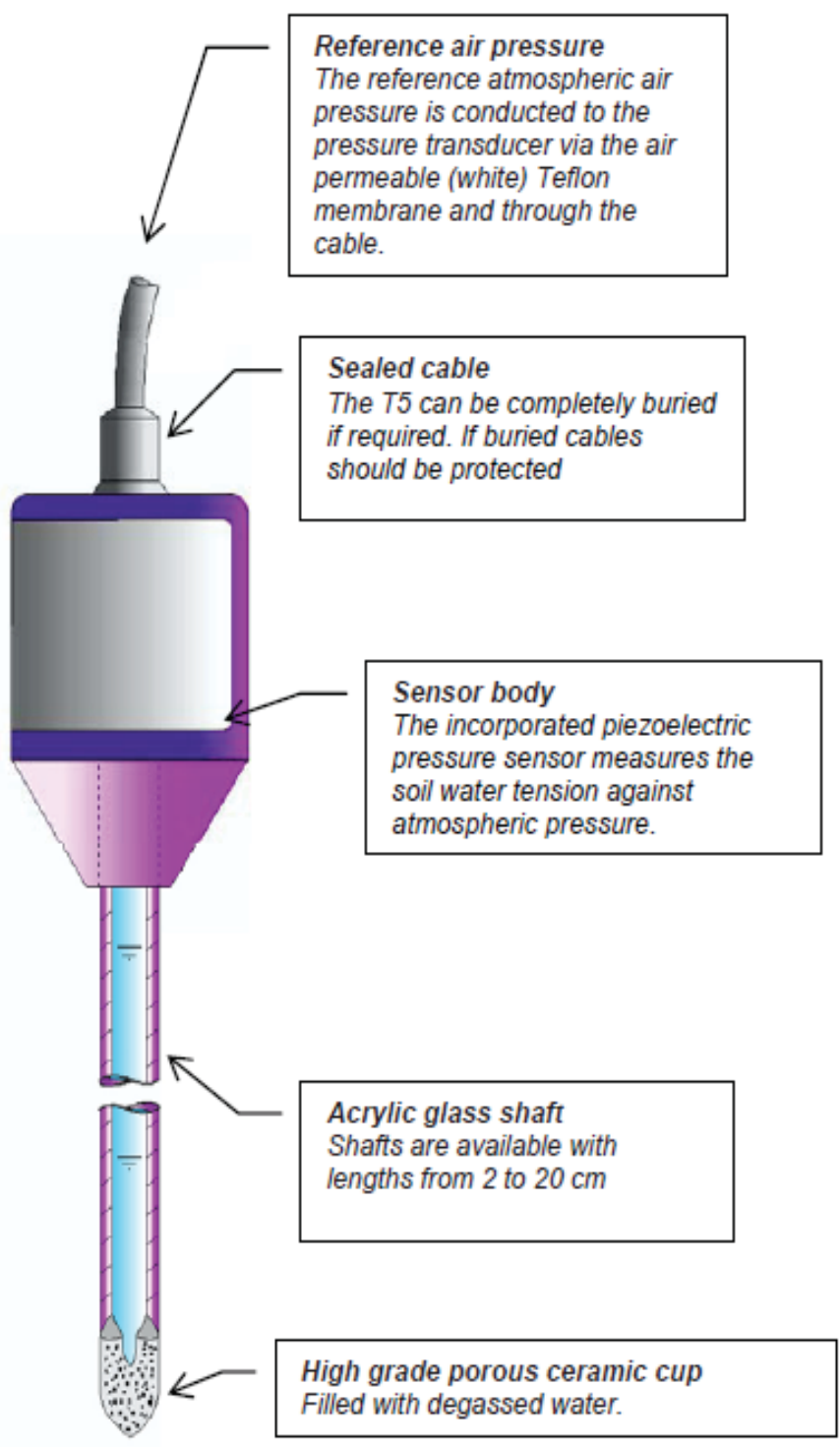

Figure 3.17 Descriptions of different parts of a UMS T5 tensiometer (after Hoskin Scientific, 


\subsection{Total Suction Measurement}

As discussed in the previous section, tensiometers are not reliable for high values of suction due to problems associated with cavitation. Therefore, a WP4-T Dewpoint Potensiometer was employed to measure the total suction of the tailings layers during desiccation. In this case, samples were collected from the top of layers and covered by plastic wraps. The wrapped sample was then moved to the WP4-T device to measure the total suction. WP4 is one of the fastest instruments for measuring water potential, giving readings directly in MPa. It measures water potential from 0 to - $60 \mathrm{MPa}$, with an accuracy of $\pm 0.1 \mathrm{MPa}$ from 0 to $-10 \mathrm{MPa}$ and $\pm 1 \%$ from 10 to $-60 \mathrm{MPa}$ (WP4 manual, Decagon devices, 2003). The instrument is easy to use and maintain, with simple checking of calibration. Water potential is a measurement of the energy status of the water in a system. Water potential is a function of the vapor pressure of air in equilibrium with a sample in a sealed measurement chamber. WP4-T uses the chilled-mirror dewpoint technique to measure the water potential of a sample. In fact, the sample is equilibrated with the headspace of a sealed chamber that contains a mirror and a means of detecting condensation on the mirror. At equilibrium, the water potential of the air in the chamber is the same as the water potential of the sample. In the WP4, the mirror temperature is accurately controlled by a thermoelectric cooler. The exact point at which condensation first appears on the mirror is observed with a photoelectric cell. A beam of light is directed onto the mirror and reflected into a photodetector cell. The photodetector senses the change in reflectance when condensation occurs on the mirror. A thermocouple attached to the mirror then records the temperature at which condensation occurs. WP4 then signals this by flashing a green LED and/or beeping. The final water potential and temperature of the sample is then displayed. WP4 also 
uses an internal fan that circulates the air within the sample chamber to reduce time to equilibrium. Since the device is very sensitive to contamination, calibration check is necessary before each test. Verification standards are specially prepared salt solutions that have a specific molality and water potential. The potassium chloride $(\mathrm{KCl})$ verification standards are accurate, easy to use, and readily available from Decagon Devices (WP4 manual, Decagon devices, 2003). For sample preparation, the sample was placed in a disposable sample cup, completely covering the bottom of the cup. WP4 was able to accurately measure a sample that does not (or cannot) cover the bottom of the cup. A larger sample surface area speeds up the reading by shortening the time needed to reach vapor equilibrium. Figure 3.18 presents a schematic diagram and a picture of a WP4-T device. Figure $3.18 \mathrm{~b}$ also shows a tailings sample located in the device in a cup.

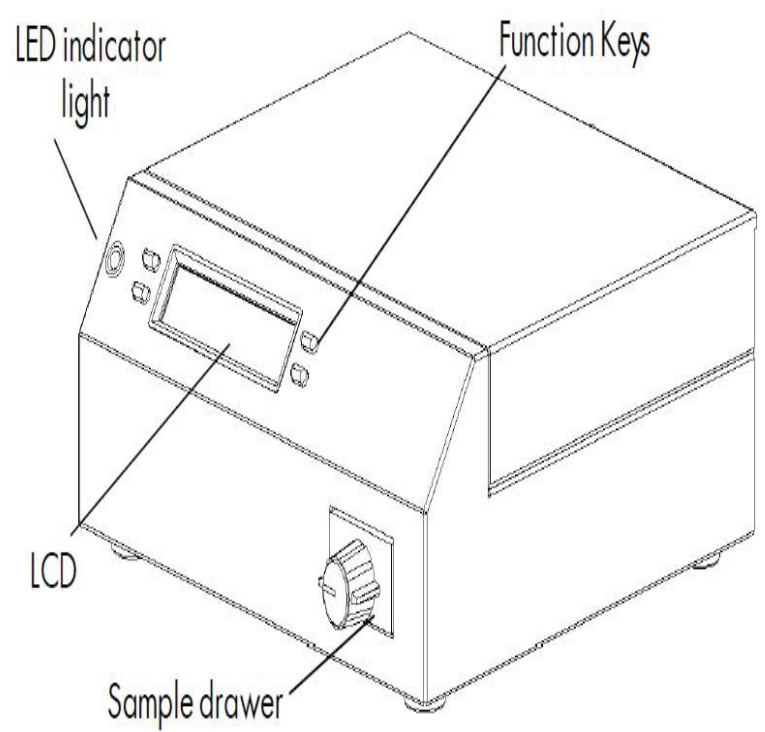

(a)

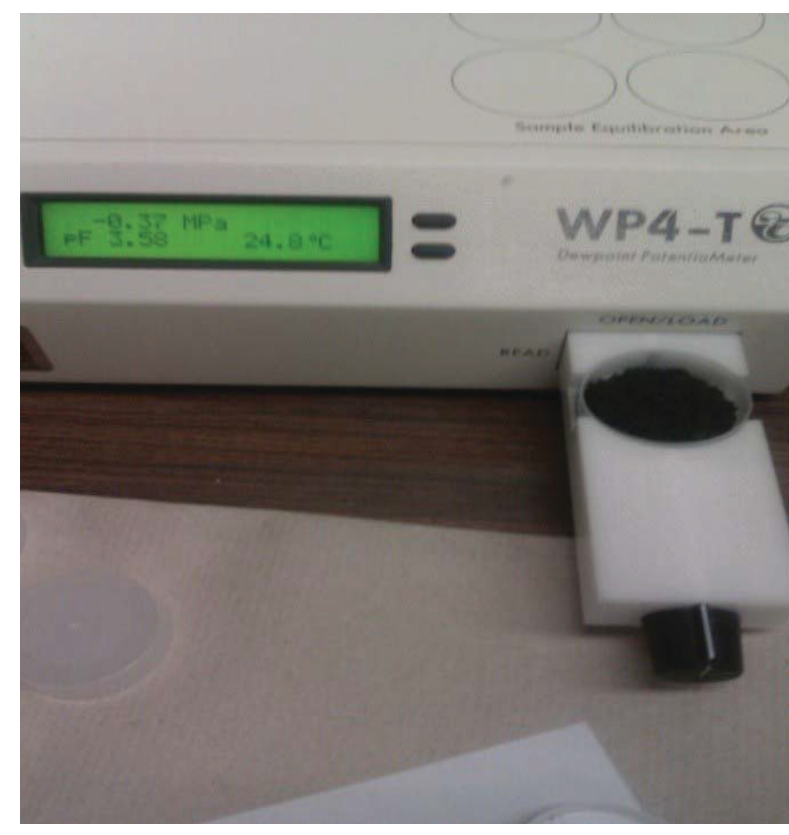

(b)

Figure 3.18 a) schematic diagram and b) a picture of WP4-T device 


\subsubsection{Void ratio measurement}

TS-30S ultrasonic senix distance sensors were employed in the drying box tests to monitor and record the height of deposited tailings during drainage and desiccation. TS-30S ultrasonic distance sensors consist of a rugged transducer in a stainless steel case for long service life in tough environments. The height of deposited tailings decreased rapidly one day after deposition due to high rate of drainage and evaporation. The height was reduced more gradually in the next days. By calculating the height reduction using TS-30S sensors and volumetric water content by 5TE sensors, the void ratio reduction was calculated. Figure 3.19 shows a picture of TS-30S ultrasonic senix distance sensors. Four TS-30S sensors were located on top of the box to measure the height deposited tailings. An average value was calculated considering all four sensors. Two wood beams were also designed to stabilize the sensors on top of the box. Figure 3.20 shows a picture of the TS-30S ultrasonic distance sensors located on top of the box.

After appearance of cracks on the surface of deposited tailings, volume of voids should also cover the air pores of cracks. Accurate measurement of cracks was not possible due to variation in the crack thickness. However, it was attempted to approximately measure the volume of cracks in the deposited tailings by assuming a triangle cross section in the crack. In this case, two void ratios were calculated for each deposition: i) void ratio based on vertical height, ii) void ratio based on vertical height and horizontal shrinkage. Figure 3.21 shows method of triangle approximation of the cross section of the cracks. 


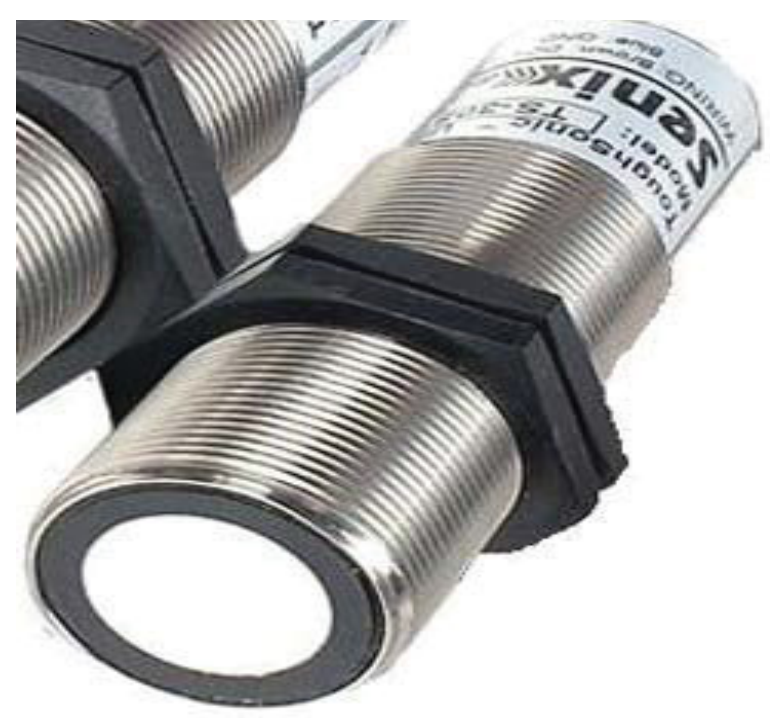

Figure 3.19 A picture of a TS-30S ultrasonic senix distance sensor to measure void ratio changes

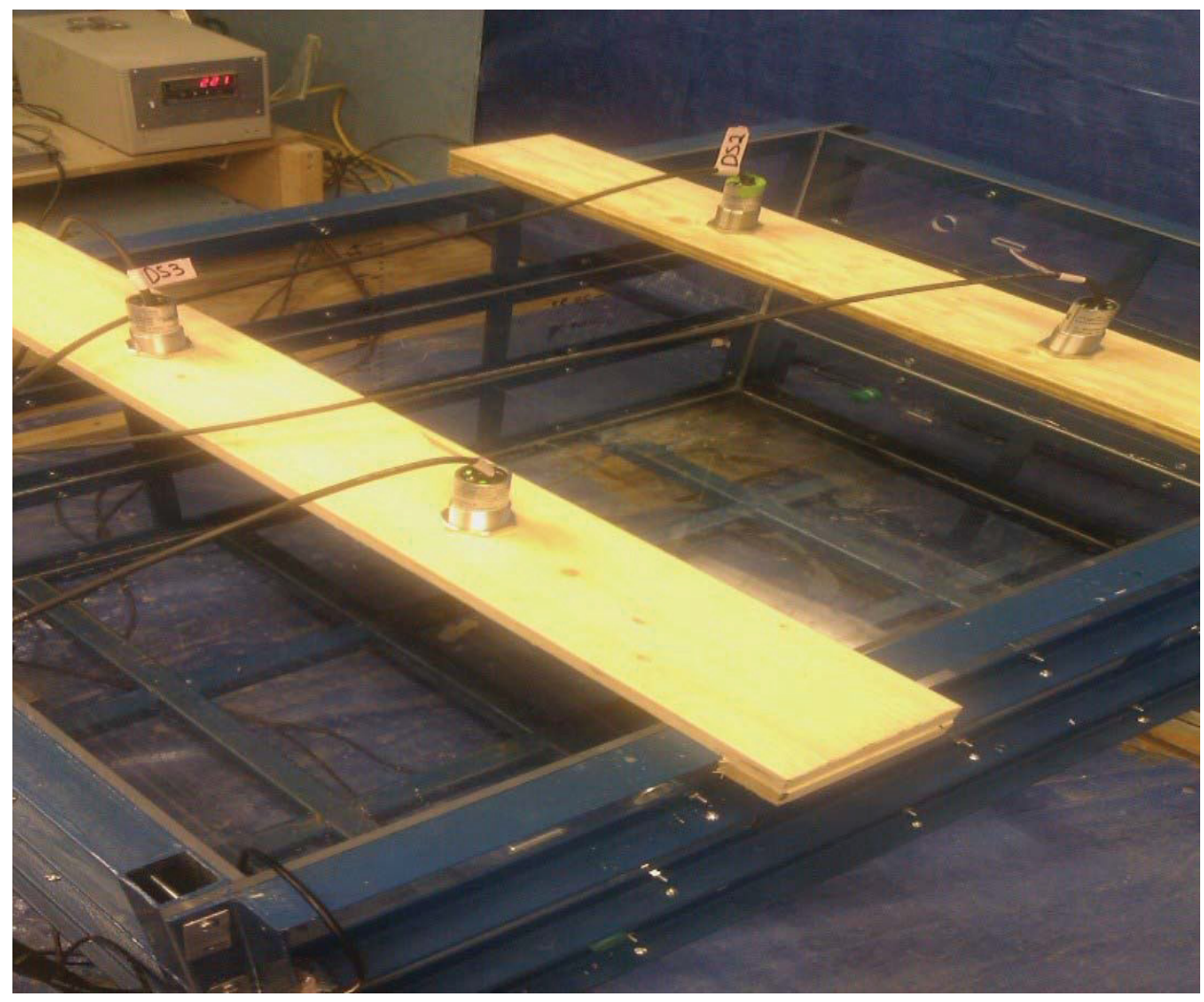

Figure 3.20 Four TS-30S ultrasonic senix distance sensors on top of the box before deposition 


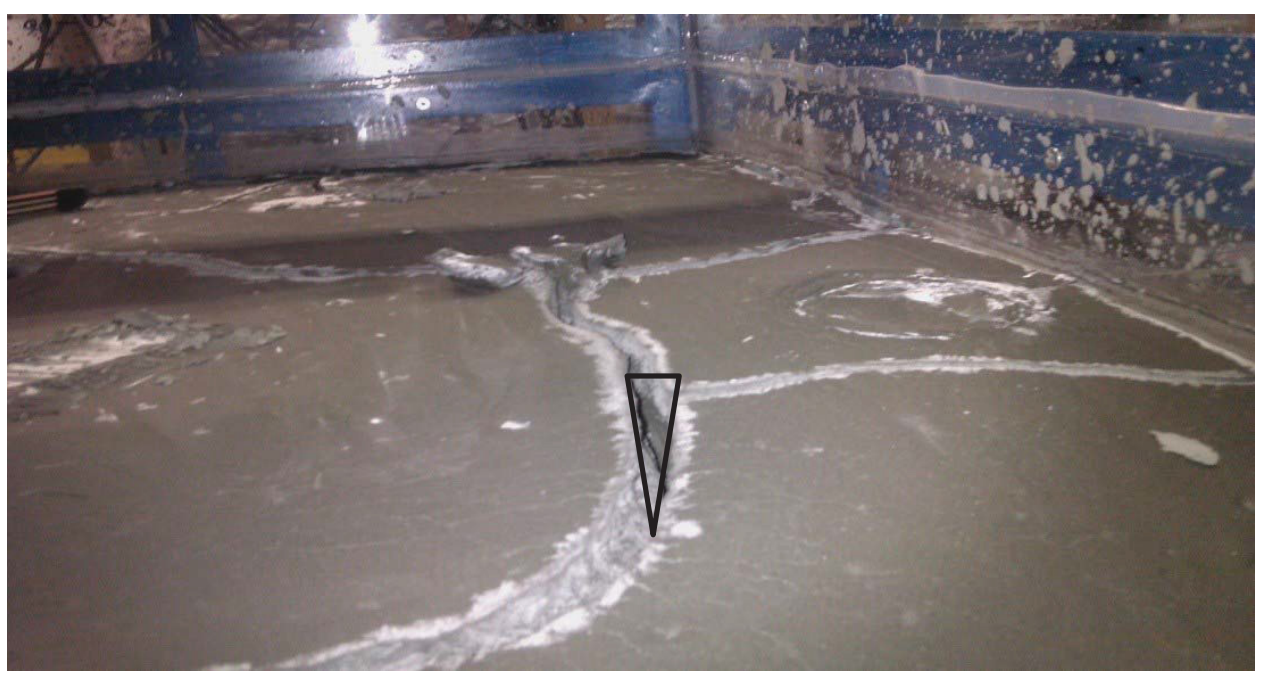

Figure 3.21 Triangle approximation of cross section of the cracks in the drying box

\subsubsection{Sample Extraction Methods}

Two sample extraction methods were used in this study to extract samples from different layers of the drying box. Extracted samples were placed in the simple shear device for strength measurement. The results of these two methods will be compared in Chapter 6. The following sections explain the details of the methods.

\subsection{Sample extraction by buried tubes}

In this method, thin wall tubes (Figure 3.6) were placed vertically in the box before deposition of tailings. When tailings at 38\% water content are deposited, the tubes are buried in the fresh 
tailings. Each layer in the drying box contained three tubes. Since tailings inside the tubes are contacted with other tailings from the top and bottom of the tubes, tailings inside the tubes were expected to experience desiccation, rewetting and consolidation. In fact, the tailings inside the tubes were expected to experience the same stress history as the layer. Therefore, the tailings in the tubes can provide appropriate samples for strength measurement by simple shear tests. Figure 3.22 shows three tubes prepared for depositing fresh top tailings layer and a buried tube that have been buried in the bottom layer. Buried tubes were extracted after all layers were deposited. To extract the tubes, the steel parts of the box were detached and the tubes were extracted manually. Each tube provides two or three samples for simple shear test. The method for providing a simple shear sample from the extracted tubes will be discussed in later sections.

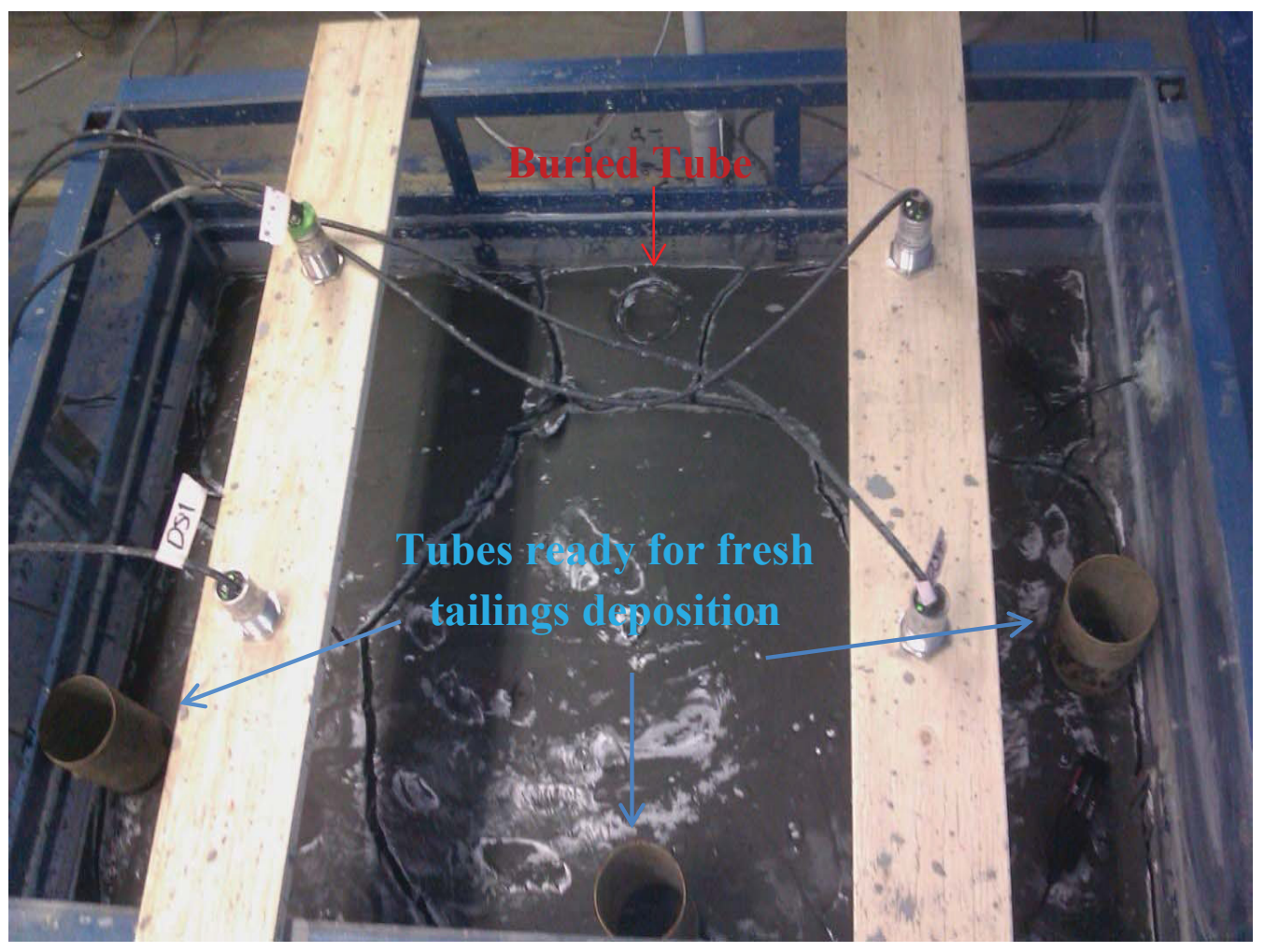

Figure 3.22 A picture of drying box with tubes prepared for fresh tailings and a buried tube 


\subsection{Sample extraction by pushing a thin wall tube using a hydraulic jack}

In this method, two thin wall tubes (Figure 3.6) with the length of $700 \mathrm{~mm}$ were adopted to be pushed into the tailings using a hydraulic jack. The tubes were pushed in the middle of the box where there was no sensor. Figure 3.23 shows the procedure of sample extraction by pushing thin wall tubes into the tailings.

\subsection{Simple shear tests}

The Carleton University simple shear test is a NGI (Norwegian Geotechnical Institute) type apparatus. The cylindrical soil specimen, $70 \mathrm{~mm}$ in nominal diameter and approximately $20 \mathrm{~mm}$ height is placed in a reinforced rubber membrane. The reinforced rubber membrane constrains the specimen from lateral displacement. Therefore, the soil specimen during consolidation and shear loading would be in a state of zero lateral strain ( $\varepsilon_{x}$ and $\varepsilon_{y}$ equals to zero). A photograph and schematic diagram of the apparatus at Carleton University are shown in Figures 3.24 and 3.25 respectively.

The apparatus basically consists of a vertical acting air piston, horizontal double acting lowfriction air piston, simple shear load frame, a constant speed motor drive, load cells, Electronic Pneumatic Transducer (EPT) and Linear Variable Displacement Transducers (LVDT). The vertical load system is located at the bottom of the apparatus and consists of a simple single 


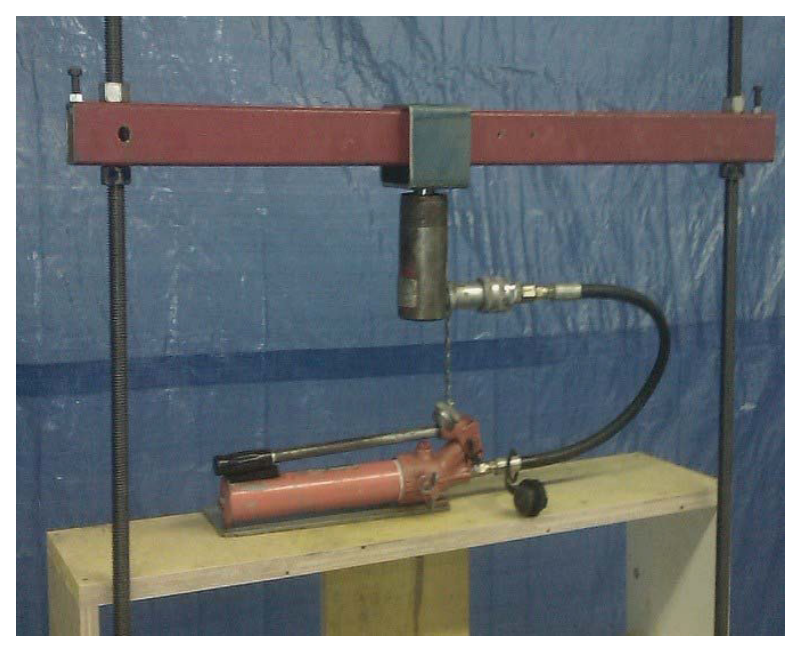

a)

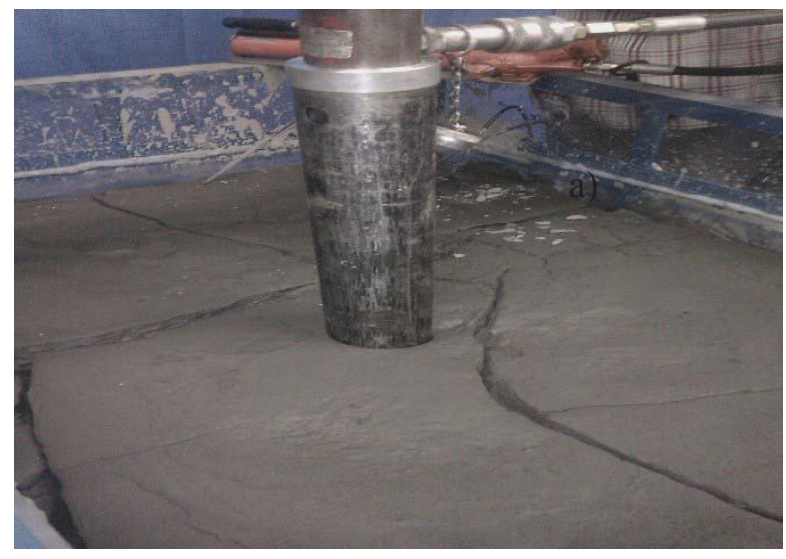

c)

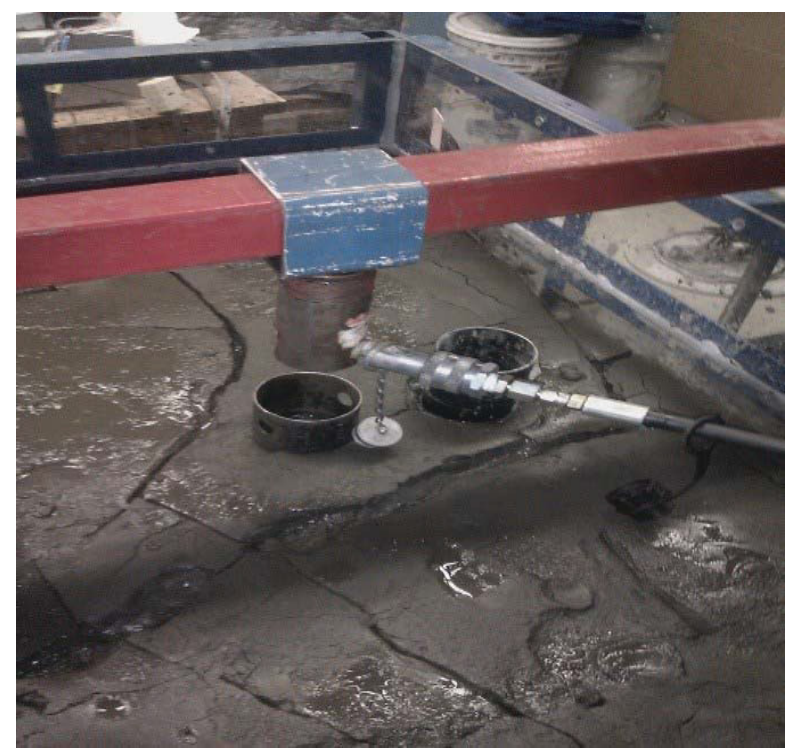

e)

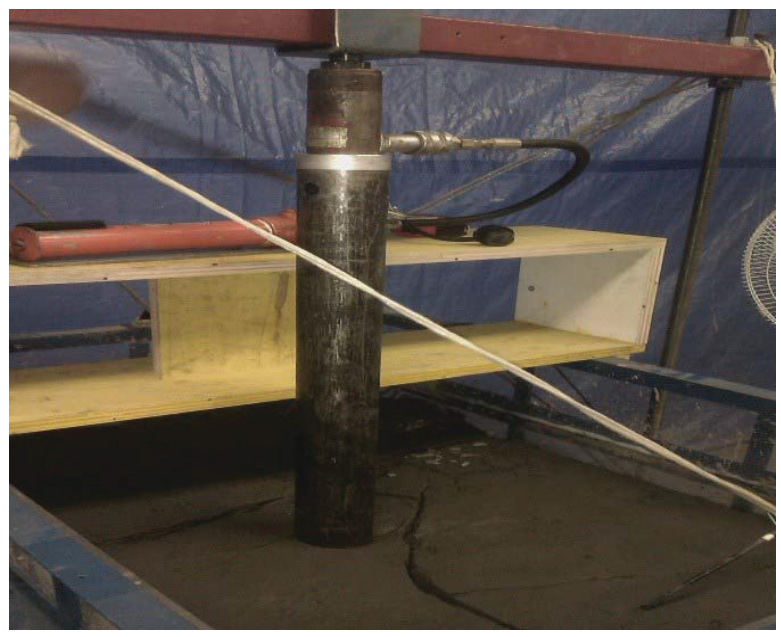

b)

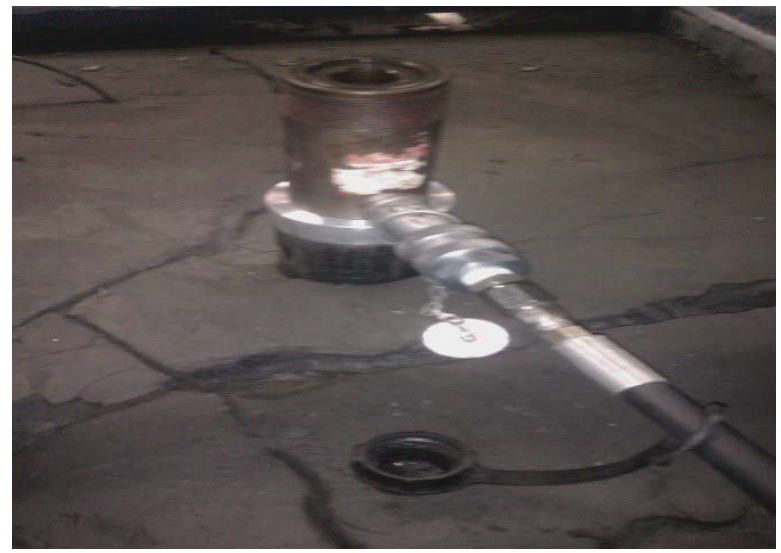

d)

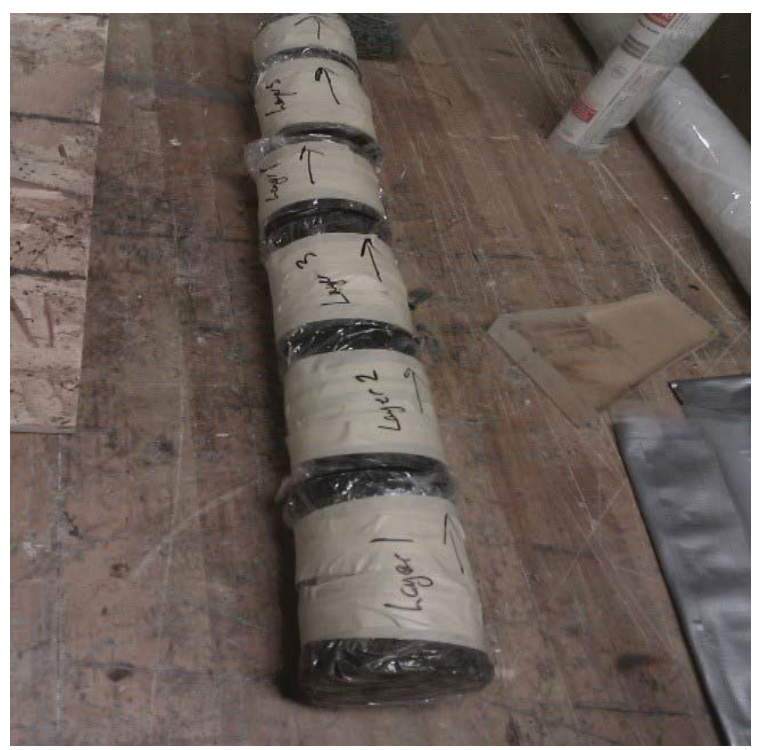

f)

Figure 3.23 Procedure of pushing thin wall tubes into the tailings using a hydraulic jack 
acting air piston and it is controlled manually by an external pressure regulator. Horizontal load can be applied by either double acting low-friction air pistons or a motor driving system connected to a constant speed motor drive. The vertical and shear load are measured using vertical and horizontal load cells, and the vertical and horizontal displacements are measured using two Linear Variable Displacement Transducers (LVDTs). In addition, an additional LVDT in the horizontal direction is used to increase the scope of LVDT measurement range. The cyclic loading is applied by double acting frictionless pistons which are controlled by Electronic Pneumatic Transducer (EPT) connected to a computer. The horizontal loading system allows a smooth transition from stress-controlled to strain-controlled loading.

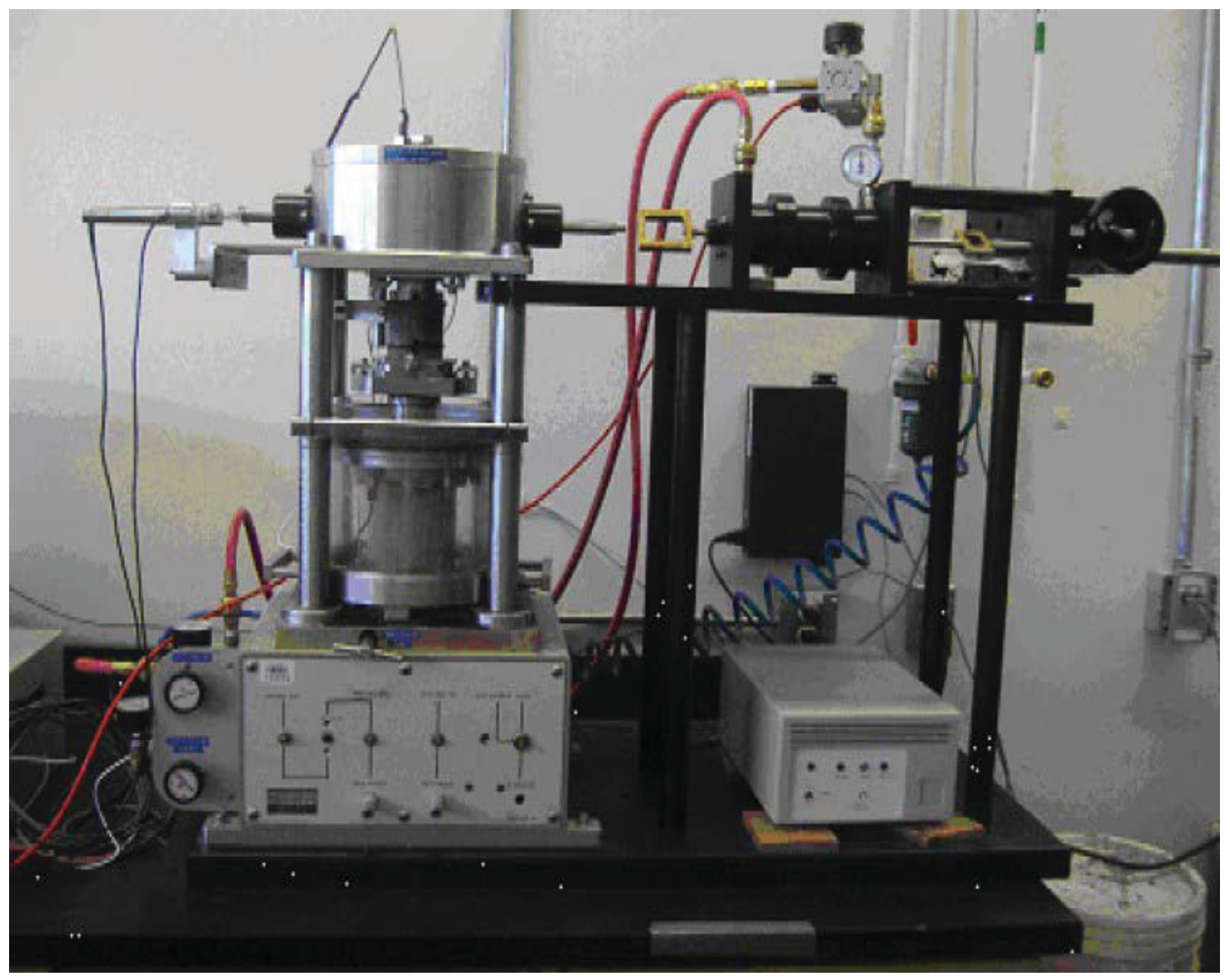

Figure 3.24 Photograph of simple shear device at Carleton University 
Constant volume condition is achieved during shear loading by keeping the height of the sample constant. This has been the case in all shear tests undertaken, and as a result no volume change of the sample occurred during the shear loading phase.

In a constant volume test, the specimen diameter is constrained by the reinforced membrane and any vertical displacement is restricted by clamping the top and bottom loading cap against vertical movement. It has been demonstrated that the decrease or increase of vertical stress in a constant volume SS test is essentially equivalent to the increase (or decrease) of excess pore water pressures in an undrained test (Dyvik et al., 1987; Finn et al., 1978). In other words, the

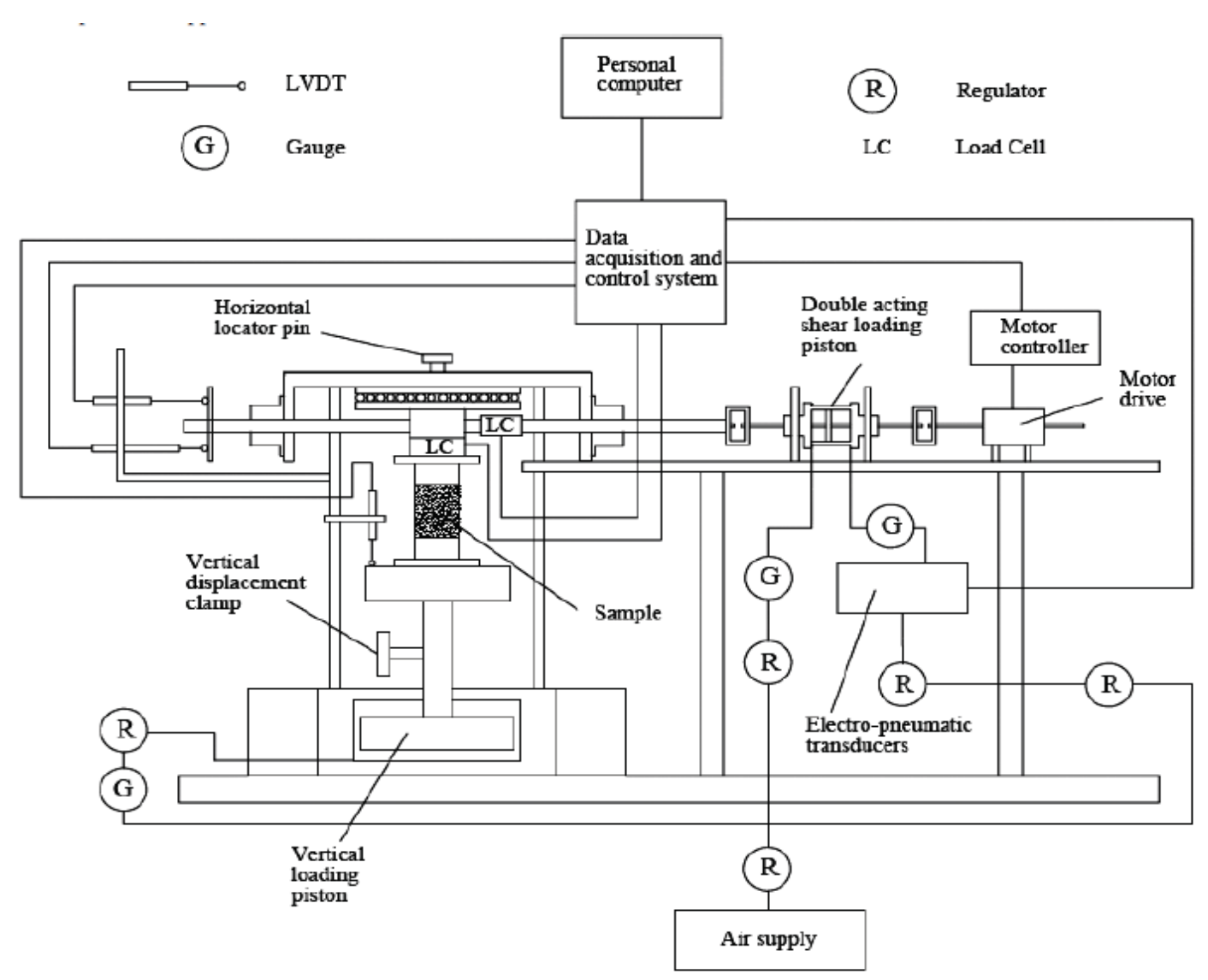

Figure 3.25 Schematic diagram of simple shear device at Carleton University (after Al-Tarhouni, 2008) 
change in applied vertical stress has been shown to be equivalent to the excess pore pressure $(\Delta u$ $=\sigma_{\mathrm{vc}}^{\prime}-\sigma_{\mathrm{v}}^{\prime}$ ), which would have been measured in a truly undrained test. The applicability of this assumption of partial saturated coarse and fine grained soils will be discussed in Chapter 5 .

The Carleton University SS test is equipped with a high speed data acquisition and control system. All transducers are supplied with $5 \mathrm{~V}$ dc voltage. The high speed data acquisition has the potential to gather about 500 sets of data per second.

\subsubsection{Sample preparation for SS tests}

\subsubsection{Reconstituted samples in the SS device}

Reconstituted tailings are prepared at $38 \%$ water content and deposited in the simple shear mould. To setup the specimen, a saturated porous stone is initially placed in the lower pedestal of the SS device. Two porous stones are attached to two steel grooved disks. The grooves are used to prevent samples from slipping during shear loading. A reinforced rubber membrane is placed in position and the bottom of the membrane is constrained with the bottom pedestal using an Oring. Afterwards, a two piece split mould surrounds the reinforced membrane and $20 \mathrm{kPa}$ suction is subsequently applied to the membrane to stretch it. Tailings in their initial water content are spooned and distributed inside the cavity of the reinforced membrane. The specimen is tamped

gently in order to remove the air bubbles and create homogenous tailings mass. Tailings should be leveled by spatula and the top cap is placed, and the initial height is recorded. Samples settle under the weight of the top cap at this stage. After that, the reinforced membrane is carefully 
flipped on the top cap and sealed using another O-ring. In the next step, the brass ring was carefully removed and the sample height is measured. The current height of the sample is recorded and then the mould is moved and placed under the simple shear apparatus. Around 5 $\mathrm{kPa}$ vertical pressure is applied to the sample before the two-piece split mould is removed. The height of the sample is measured from the computer using vertical LVDT from this stage onwards. The sample height before placing the sample in the apparatus is considered as initial sample height. Figure 3.26 shows the procedure for preparing reconstituted samples for the simple shear device.

\subsubsection{Undisturbed desiccated-rewetted samples in the SS device}

As noted earlier, the undisturbed desiccated-rewetted samples have been already prepared in thin wall tubes using either small scale laboratory test or multilayer drying box test. Upon retrieval from the bucket or the drying box, the extracted sample is covered by plastic wrap and waxed to prevent excessive evaporation during storage. Extracted tubes and the simple shear specimen ring were placed on a mechanical jack and by applying pressure from the bottom of the tube, the sample was pushed into the ring. Using a wire-cutter, a sample with approximately $22 \mathrm{~mm}$ height was prepared in the ring. The ring was placed on the top of the mould and the sample was transferred into the cavity by using a plastic cylinder of the same inner diameter. The procedure after placing the undisturbed sample to the simple shear mould is similar to that of reconstituted samples. Figure 3.27 shows the procedure of removing the sample from tubes using a jack and push them into the simple shear mould. 


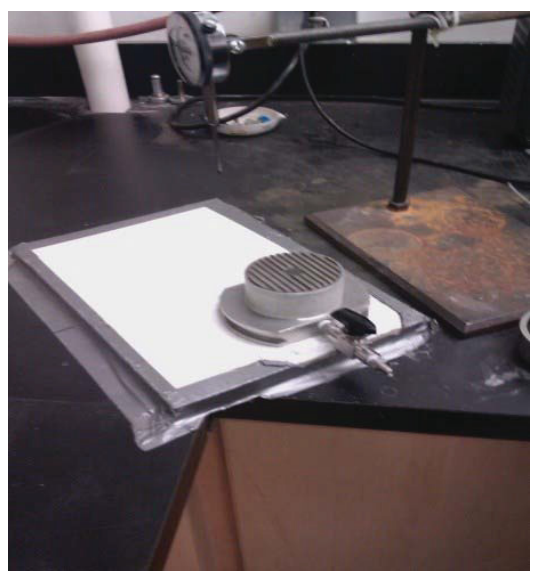

(a)

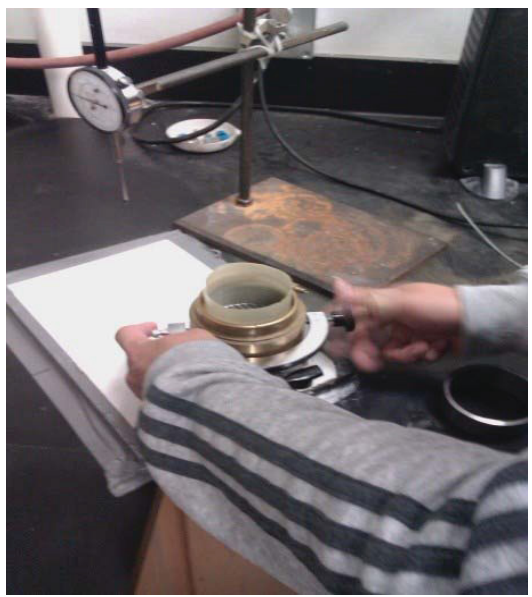

(d)

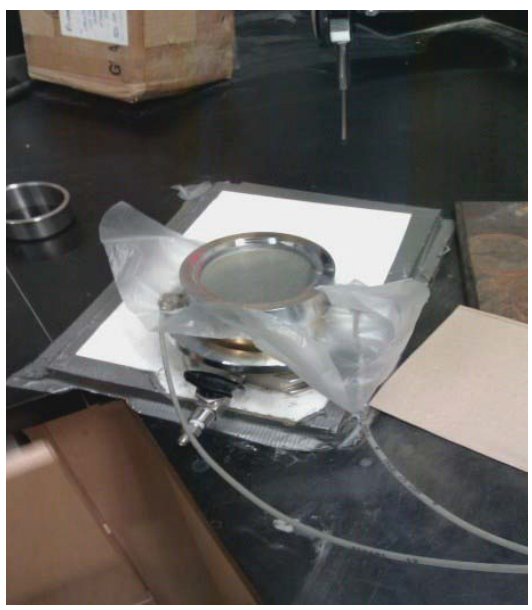

$(\mathrm{g})$

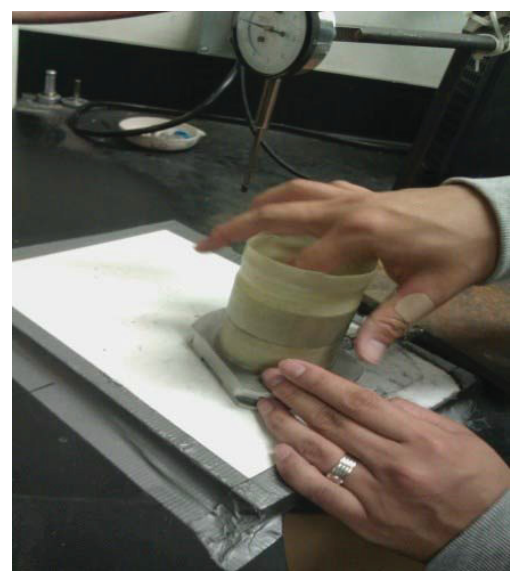

(b)

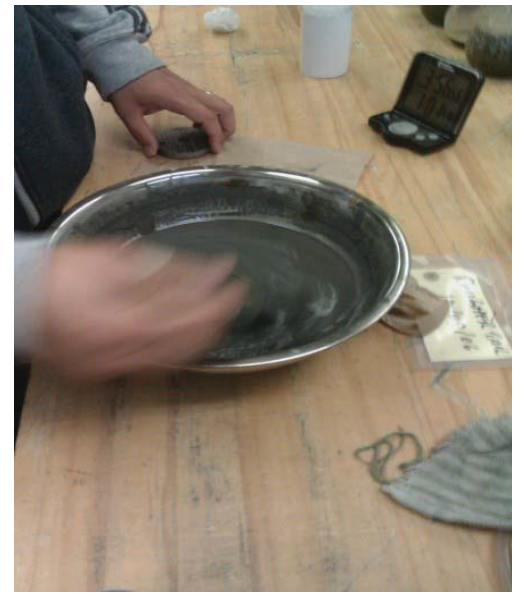

(e)

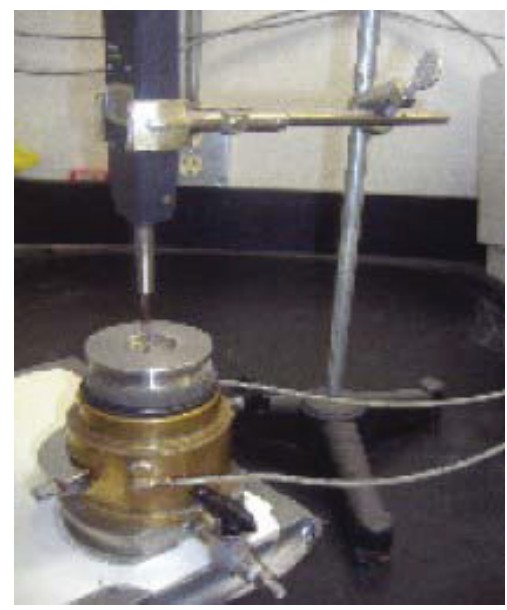

(h)

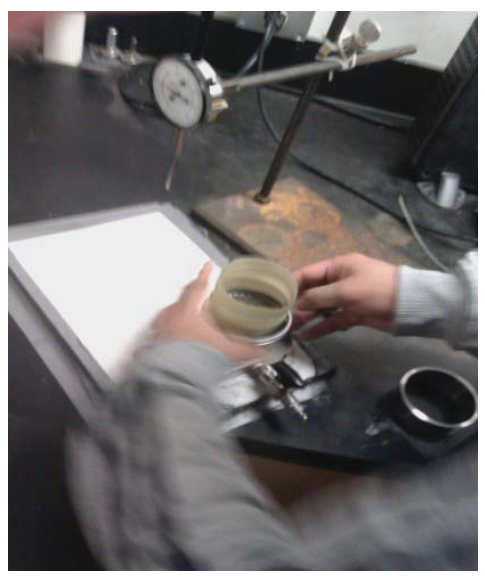

(c)

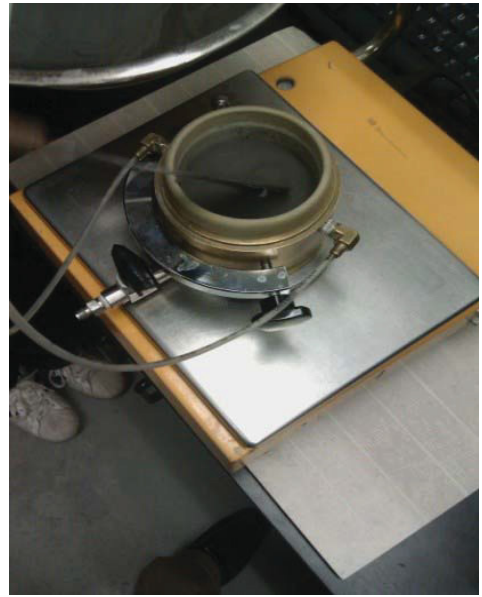

(f)

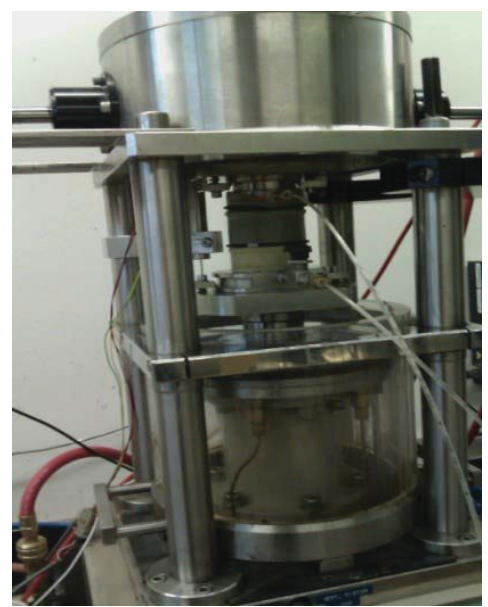

(i)

Figure 3.26 Preparation of a reconstituted sample for simple shear test 


\subsubsection{Desiccated samples in the SS device}

In addition to desiccated-rewetted tailings, some desiccated samples were also tested under simple shear. It was attempted to prepare desiccated tailings at different degrees of desiccation. Initially, the first trial consisted of depositing tailings in a simple shear mould and allowing them to desiccate. However, this method did not work properly due to the formation of a gap between the sample and the reinforced membrane during desiccation. Tailings experienced lateral shrinkage during desiccation, which results in decreasing volume and therefore diameter of the sample. Therefore, a groove appears between the sample and the membrane, and this affects the measured stress-strain response quite significantly. These specimens yielded a strain softening response following the peak (unlike the response of extracted samples that closely fit the SS mould, as will be shown in Chapter 6) possibly on account of the lack of constant volume condition during shearing. Figure 3.28 shows a desiccated sample in the simple shear mould as well as the measured (albeit incorrect) stress-strain response of the sample. Highly desiccated samples are expected to exhibit strain hardening response under undrained loading but the noted behavior is similar to the drained behaviour of dense or overconsolidated soils. A second method was used to avoid the response associated with the gap. This method consisted of preparing desiccated samples in a larger tube, pushing the simple shear ring into the desiccated sample, and then transferring the sample into the mould after trimming the sample. Preparing the sample using this method makes an appropriate desiccated sample with no gap. However, in this method, it is not possible to continue desiccation to high degrees of desiccation since the sample is too tough to be able to get extracted by the simple shear ring. The lowest reachable water content in this method was $\mathrm{W}=16.7 \%$. As mentioned, beyond this water content, because of 
high values of suction, the specimen ring could not be pushed into the tailings properly. Figure 3.29 shows the procedure of preparing desiccated samples for a simple shear test.

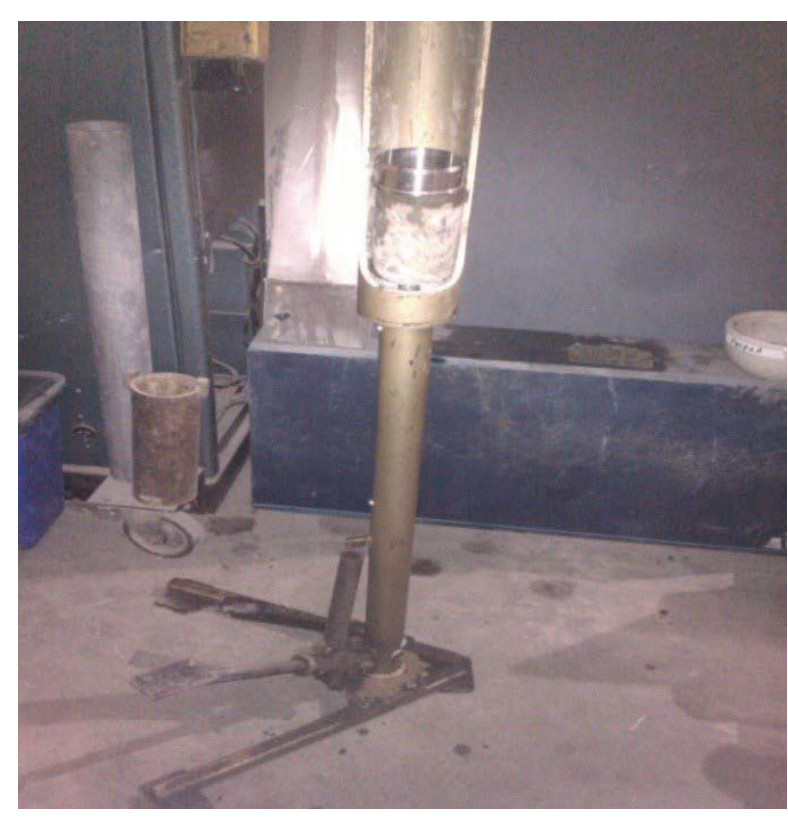

(a)

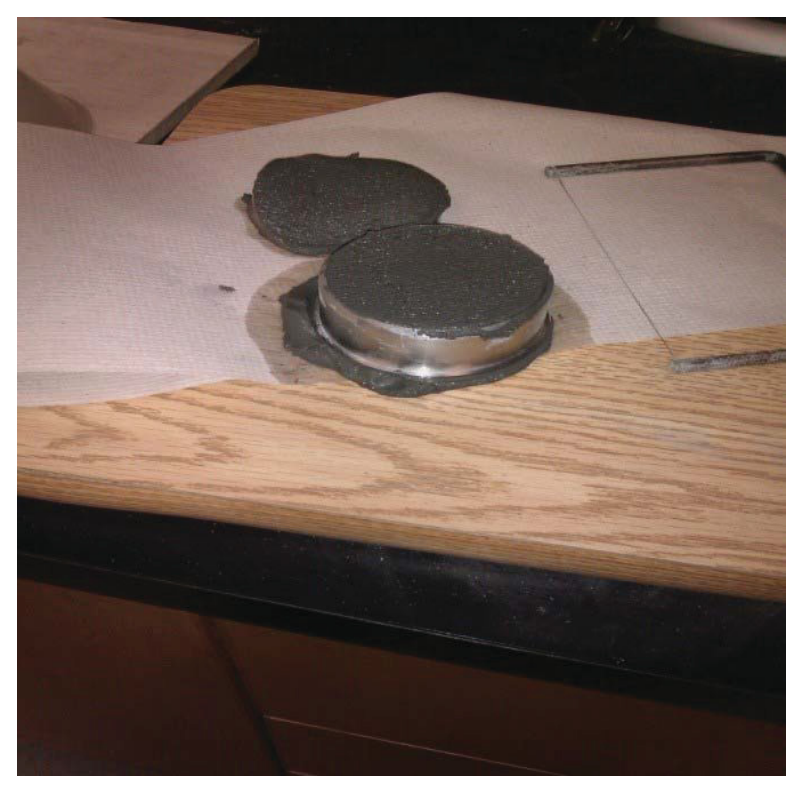

(c)

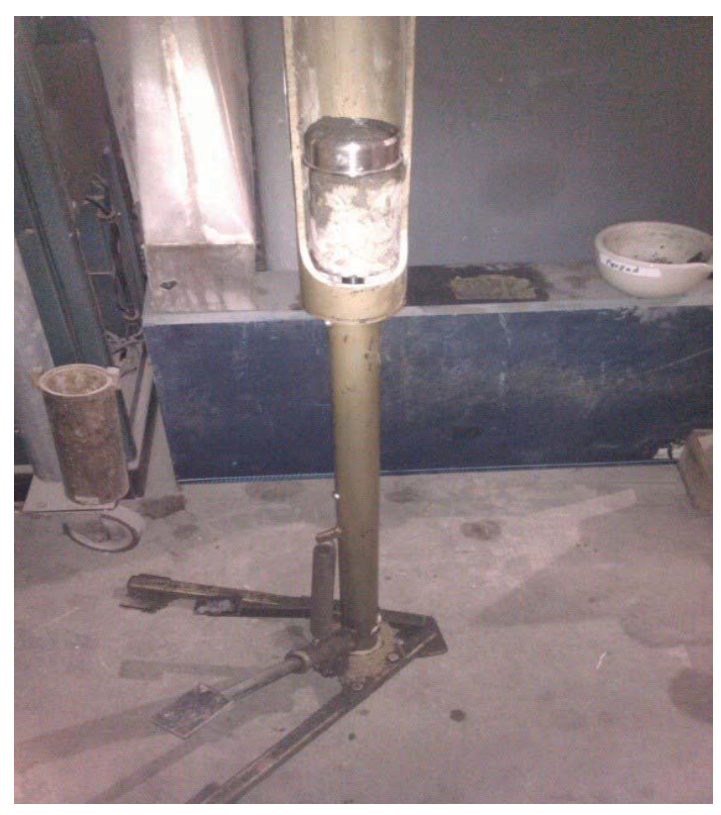

(b)

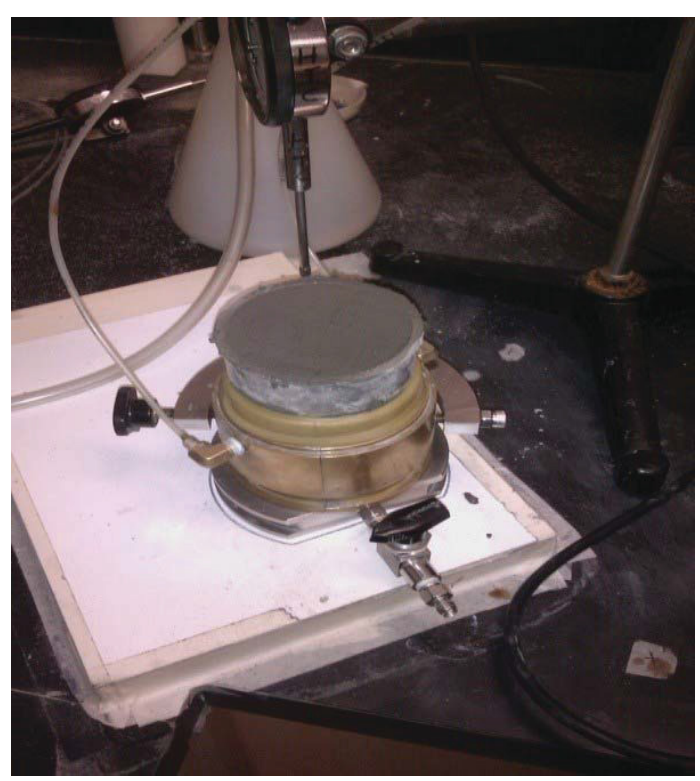

(d)

Figure 3.27 Procedure for removing undisturbed samples from extracted tubes 
Desiccated to $\mathrm{W}=10 \%$

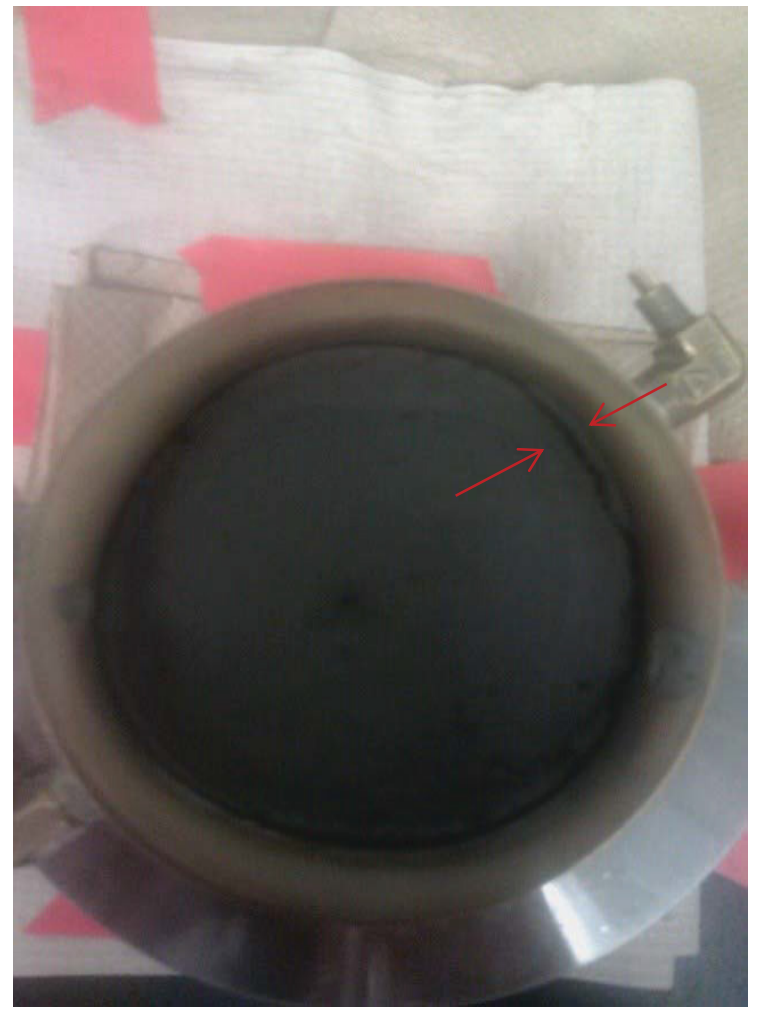

a)

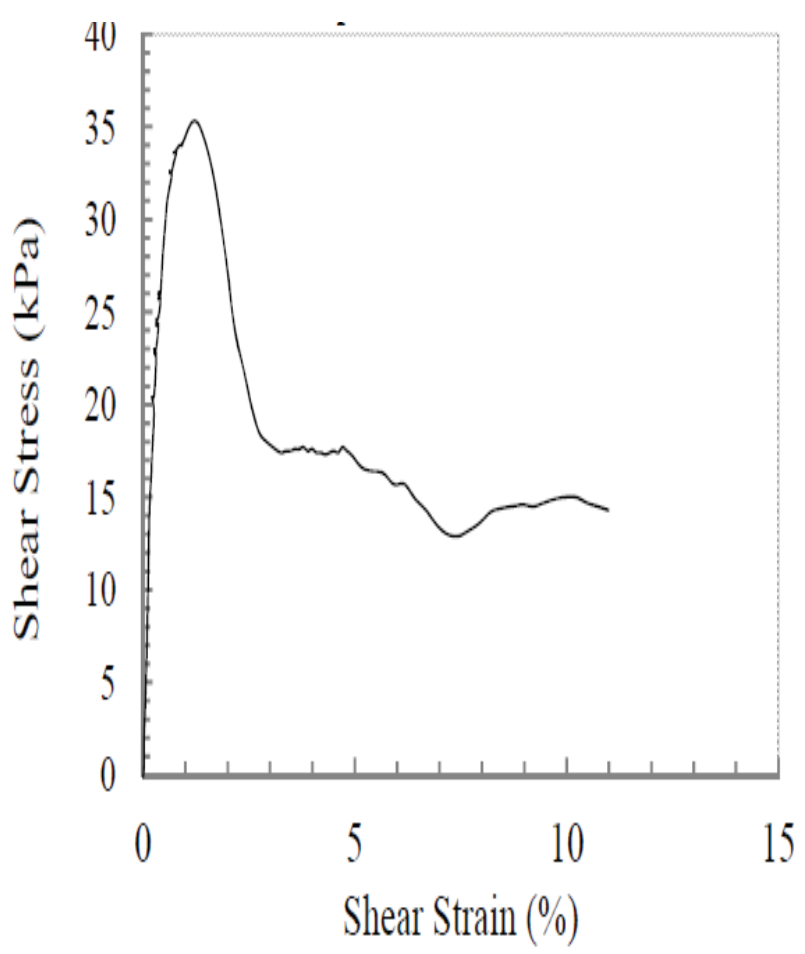

(b)

Figure 3.28 a) A desiccated sample in the simple shear mould and b) incorrect stress-strain response of the desiccated sample

\subsubsection{Consolidation Stage}

Simple shear specimens were consolidated to vertical stress $\sigma_{\mathrm{vc}}^{\prime}$ ranging from 50 to $400 \mathrm{kPa}$.

During the one dimensional consolidation stage in the simple shear test, the change in height of the specimen (representative of the volume change) is recorded using the data acquisition system. The vertical stresses during consolidation stage is applied until reaching a desired 
consolidation pressure. Saturated (low value of suction) and desiccated-rewetted samples were consolidated under $50 \mathrm{kPa}, 100 \mathrm{kPa}, 200 \mathrm{kPa}$ and $400 \mathrm{kPa}$. Desiccated samples were consolidated under just $400 \mathrm{kPa}$, to see if the influence of desiccation could be removed by imposition of a high consolidation pressure.

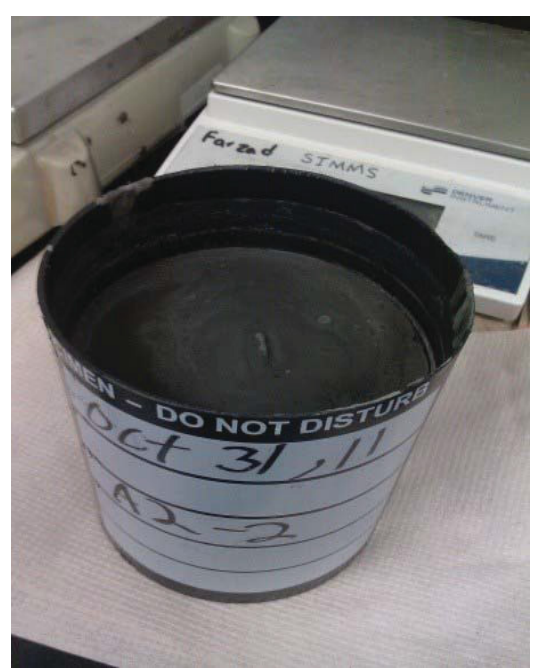

a)

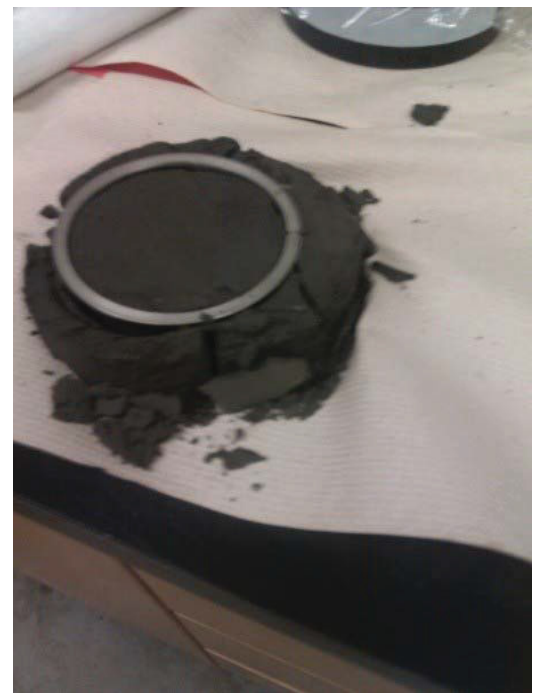

d)

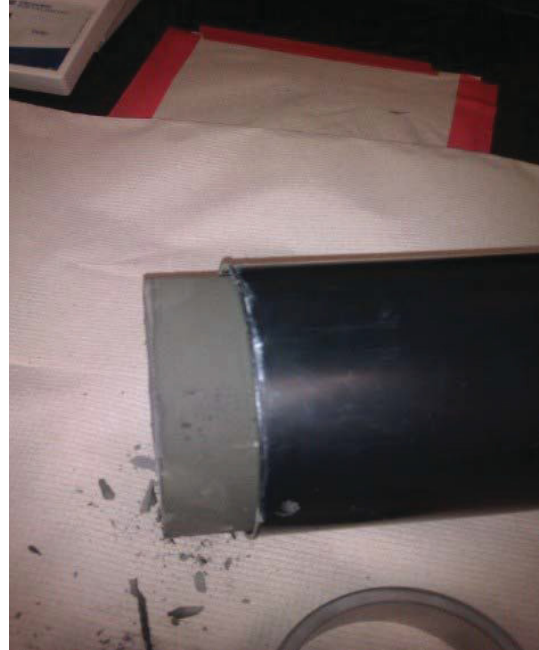

b)

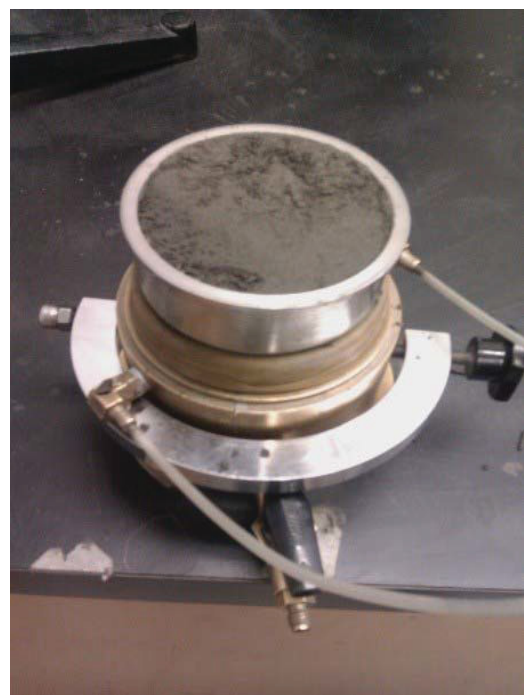

e)

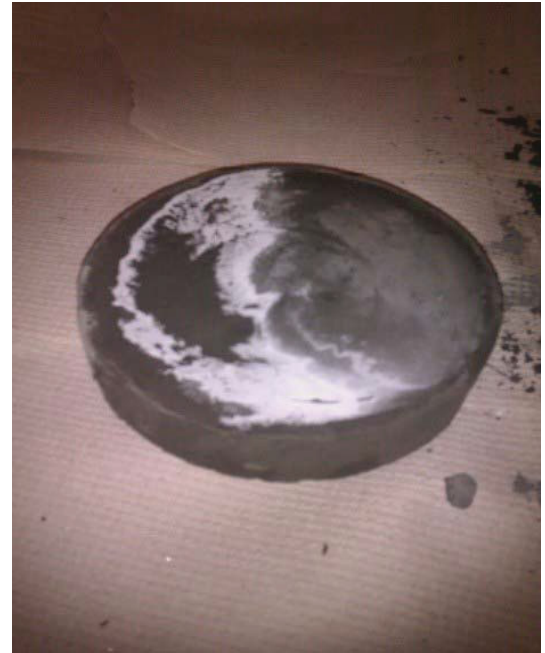

c)

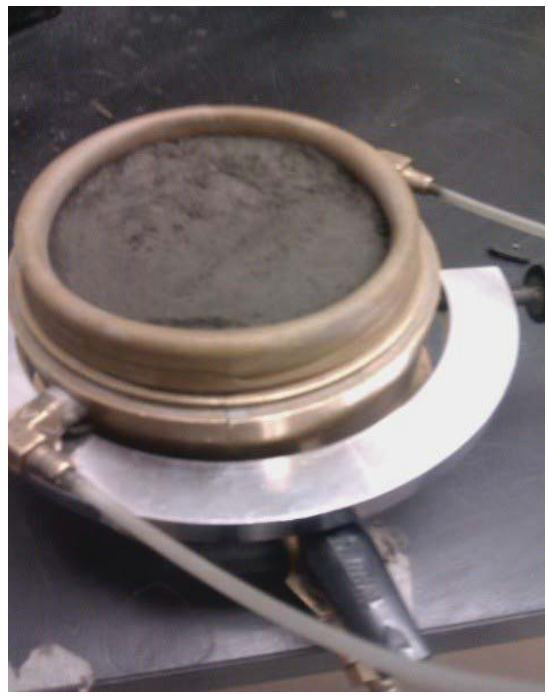

f)

Figure 3.29 The procedure of preparing desiccated samples for the simple shear test 


\subsubsection{Mechanically over-consolidated samples}

In order to achieve mechanical overconsolidation, samples should be initially consolidated to a higher consolidation pressure and then unloaded to a lower current confining pressure. Samples were prepared to reach mechanical overconsolidation ratio $\left(\mathrm{OCR}_{\mathrm{M}}\right)$ equal to 1,2 and 4 . For instance, for reaching $\mathrm{OCR}_{\mathrm{M}}=2$, samples were initially consolidated to $400 \mathrm{kPa}$ and unloaded to $100 \mathrm{kPa}$.

\subsubsection{Monotonic Loading stage}

After finishing the consolidation stage, the samples were sheared monotonically under constant volume. Since the reinforced membrane does not allow lateral deformation $\left(\varepsilon_{x}=0, \varepsilon_{y}=0\right)$, only the height should be kept constant to achieve constant volume condition. The constant height condition can be applied by clamping the top and bottom loading caps against vertical movement. The loading rate of the motor drive is controlled by a control system which is connected between the data acquisition and motor drive. The shear strain rate during monotonic loading was about 20\%/hour similar to previous studies. Al-Tarhouni et al (2008) demonstrated that there is no appreciable difference between the responses of simple shear test on Bulyanhulu tailings at shear strain loading rates of $20 \% / \mathrm{hr}$ to $80 \% / \mathrm{hr}$. The monotonic shear loading was applied until reaching $11 \%$ shear strain and this strain was assumed to represent the failure state. 


\subsubsection{Repeatability of monotonic simple shear responses}

In order to ensure that the SS testing yields reliable results, two identical desiccated-rewetted samples of tailings were tested under the same conditions. Samples were prepared in a bucket at $38 \%$ water content and allowed to desiccate close to the shrinkage limit (SL $=18 \%)$. After rewetting and extraction, both samples had fairly same water content ( $\mathrm{W}=22 \%$ ). Both samples were placed in the device and consolidated under $400 \mathrm{kPa}$. The void ratio after consolidation was similar in both tests $\left(\mathrm{e}_{\mathrm{c}}=0.61\right)$. Figure 3.30 shows the results of tested samples that were consolidated to the $400 \mathrm{kPa}$ effective consolidation pressure and a void ratio of 0.61 . It can be seen from the figure that the response of both samples are fairly similar. This infers that the sample preparation technique is repeatable, and the performance of simple shear apparatus is excellent.

\subsubsection{Cyclic Loading Stage}

For cyclic loading stage, the shear load was applied in the form of a sinusoidal wave with a frequency of $0.1 \mathrm{~Hz}$ (i.e., period of the cyclic loading is $10 \mathrm{sec}$ ). The double acting piston is used to apply cyclic shear loading. Similar to monotonic condition, the undrained behavior is considered for cyclic tests by using the constant volume condition. US National Research Council (NRC 1985) suggested that the development of $3.75 \%$ single amplitude shear strain of the sand samples should be considered as the triggering of liquefaction under cyclic loading. 

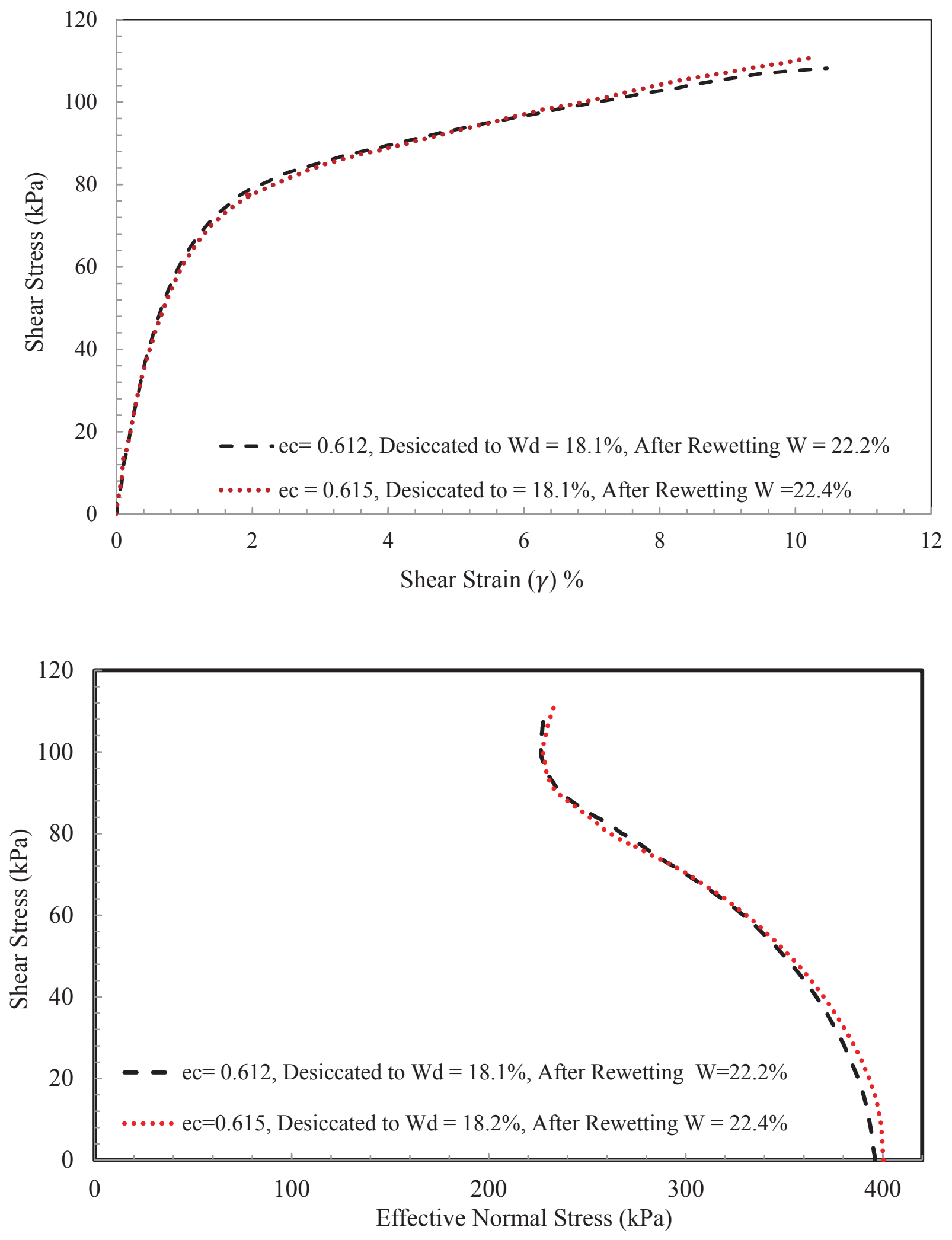

Figure 3.30 Repeatability of desiccated-rewetted samples in the simple shear device 
This shear strain might correspond to the development of $100 \%$ pore pressure ratio for contractive sands where no static shear stress exists on the sample. This criterion has been also used to investigate different types of fine-grained mine tailings under simple shear loading. (Wijewickreme et al., 2005). It should be mentioned that for fine grained soils including tailings, the development of $100 \%$ pore pressure ratio might not occur even at high shear strain levels. Therefore, the criteria based on the $100 \%$ pore pressure ratio might not be valid (Singh, 1996). However, using the shear strain level as a criterion provides a consistent means of evaluating the triggering of liquefaction in the samples and allows a rational comparison of cyclic shear resistance data. Cyclic shear stress ratio (CSR) was defined as the ratio of maximum cyclic shear stress to the effective vertical confining pressure during consolidation $\left(\operatorname{CSR}=\frac{\tau_{c y c}}{\sigma_{v c}^{\prime}}\right)$. Tests were conducted at CSR values ranging from 0.05 to 0.2 for desiccated-rewetted tailings.

\subsubsection{Characteristics and limitations of the simple shear apparatus}

Simple shear apparatus imposes stress conditions on the sample that are very similar to those experienced in the in situ condition, especially during earthquake loading. Although this is a great advantage, there are some drawbacks in the conventional simple shear device. Since most of desiccated-rewetted tailings were not fully saturated and had degree of saturation around $90 \%$, the most important issue in accuracy of the results of simple shear tests is the effect of degree of saturation and applicability of the Dyvik et al. (1987)'s assumption for fine grained soils. This concern was experimentally investigated in this study by performing simple shear tests on various materials e.g. synthetic silt, glass bead, silica sand and gold tailings. The results of the investigation will be discussed in chapter 5. In addition, simple shear apparatus is not able to 
produce complimentary shear stress on the lateral boundaries of the soil sample. In fact, simple shear apparatus does not produce a pure shear condition. Due to the lack of complimentary shear stress on the lateral boundaries and shear failure zones introduced to the sample after a certain level of shear strain, simple shear apparatus creates non-uniformities of the stresses and strains across the top and bottom of the samples.

Incapability of measuring suction during consolidation and shearing is also another limitation of the Carleton's simple shear device. To analyze the results of desiccated samples, the value of matric suction during shearing was assumed to be equal to unloaded condition (SWCC) or was measured after the test using the WP4 dewpoint Hygrometer. Neither approach might be accurate.

\subsection{Triaxial Tests}

In this study, three possible test methods were considered for conducting triaxial tests:

1) Conventional triaxial test using back pressure to saturate re-constituted and extracted desiccated-rewetted undisturbed samples and reaching acceptable B values (normal triaxial test).

2) Unsaturated triaxial test using tensiometer at the bottom of the sample and monitor the matric suction during triaxial test without saturation stage (Simms and Grabinsky, 2009). 
3) Unsaturated triaxial test using axis-translation technique and perform Fredlund and Rahardjo (1993) unsaturated triaxial test by controlling matric suction under different confining pressures.

Since the axis-translation technique assumes that the water pressure is fixed at a specific value and the pore-air pressure is regulated to control matric suction, the self-desiccation behavior of thickened tailings in the field would not be appropriately simulated. As a result, this study concentrates on first and second methods.

Triaxial apparatus at Carleton University is capable of performing both stress and strain controlled triaxial tests under compression and extension modes. Figure 3.31 shows a schematic diagram of triaxial apparatus at Carleton University. The triaxial specimen has a nominal diameter of $63.5 \mathrm{~mm}$ and height of $127 \mathrm{~mm}$. Two $60 \mathrm{~mm}$ diameter pore stones are placed on either end of the sample to facilitate drainage during consolidation. The apparatus is capable of applying $450 \mathrm{~kg}$ vertical load (with the precision of $\pm 0.01 \mathrm{~kg}$ ). Pressures are measured using high performance millivolt output pressure transducer with the precision of better than $0.1 \mathrm{kPa}$ and over the range of $700 \mathrm{kPa}$ gauge pressure. The axial strain is measured using precision gauge head with a precision of better than $0.001 \mathrm{~mm}$ and a range of $\pm 25 \mathrm{~mm}$. Volumetric change during the test in saturated soils is measured by a Differential Pressure Transducer (DPT). All transducers were calibrated before starting the tests. The double acting frictionless air piston is used to apply stress controlled test. 


\subsubsection{Preparation methods}

Sample preparation for reconstituted and desiccated-rewetted samples in triaxial tests was similar to sample preparation in the simple shear device. Extraction method for laboratory undisturbed desiccated samples is similar to undisturbed samples for the simple shear device.

\subsubsection{Reconstituted saturated samples (Slurry condition)}

In reconstituted samples, tailings were prepared at $38 \%$ water content and deposited in the triaxial mould, allowed to dewater to reach initial settling. Figure 3.32 shows the procedure of sample preparation for reconstituted samples in slurry condition.

The top cap was carefully placed on top of the deposited tailings with minimum sample disturbance. The membrane is flipped on the top cap and an O-ring is placed around the top cap to seal the sample appropriately. Around $30 \mathrm{kPa}$ suction is applied before removing the sample mould i.e. all the specimens experienced some desiccation. Application of suction provides the confinement required to hold the sample after removing the sample mould. Initial void ratio of the sample was calculated using the cavity area of the mould together with the measured initial height. Subsequent measurement of height and water expelled from the specimen can help to accurately measure the void ratio in saturated samples. In order to achieve fully saturated condition, back pressure should be applied from the bottom of the sample. Skempton's B-value test is used as an indicator of the degree of saturation of the specimen in a triaxial test. If the 
specimen is fully saturated the increment of $\Delta \sigma_{3}$ in undrained condition would yield an equal $\Delta u$.

Testing under back pressure involves raising the pore water pressure artificially by connecting a source of constant pressure to the specimen. The purpose of applying a back pressure is to ensure

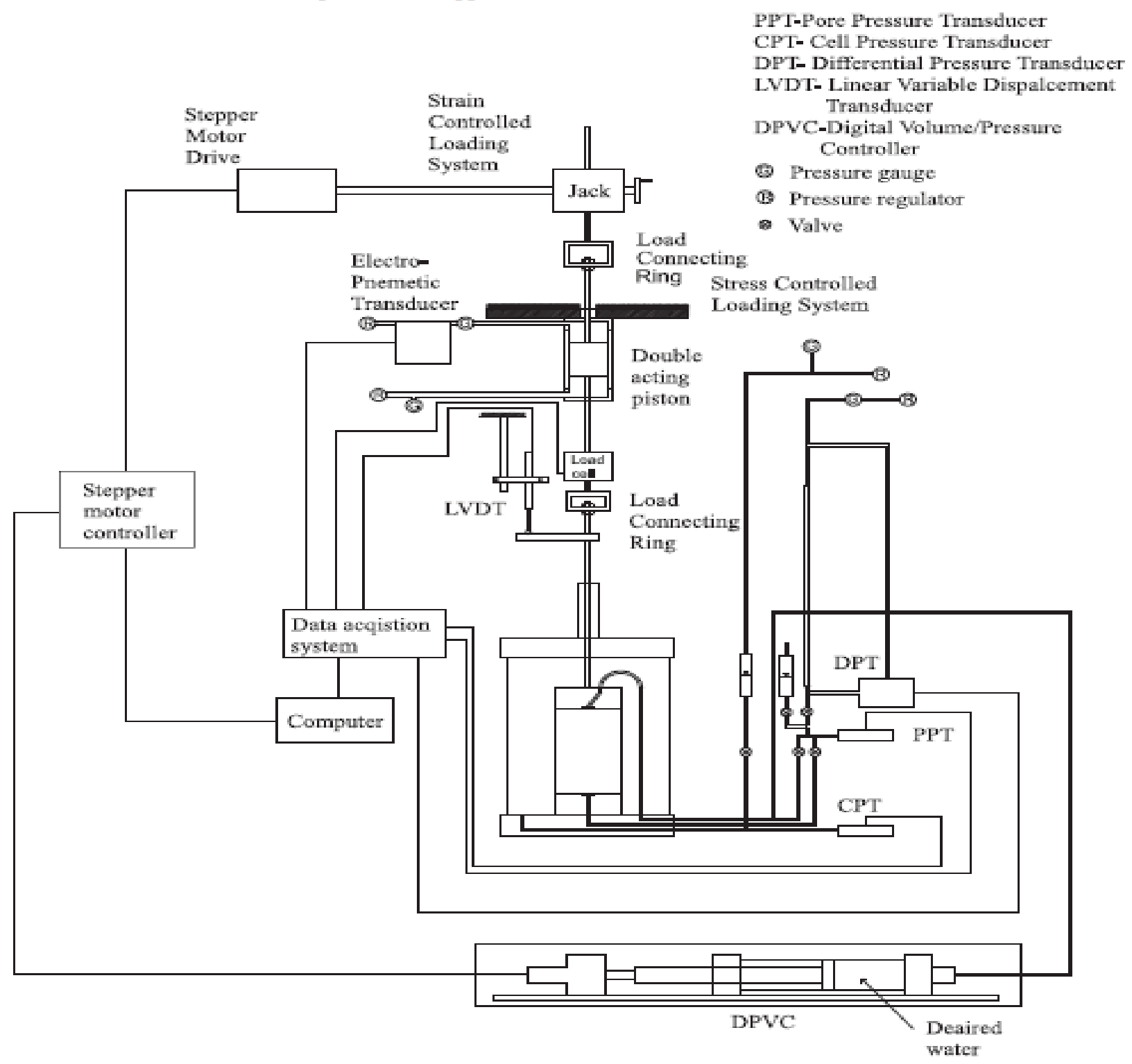

Figure 3.31 Schematic diagram of triaxial apparatus at Carleton University 


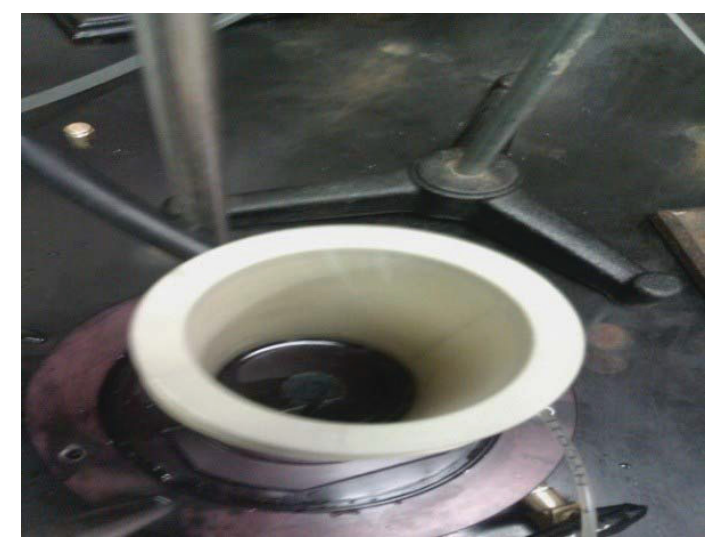

a)

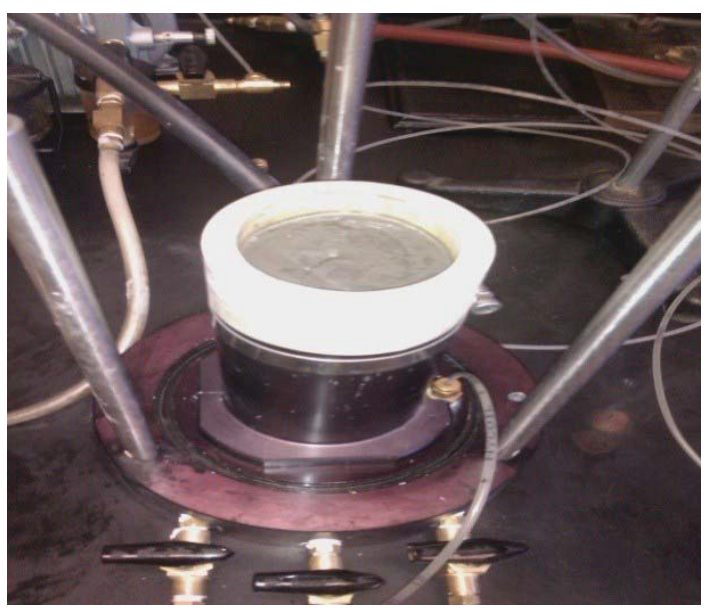

c)

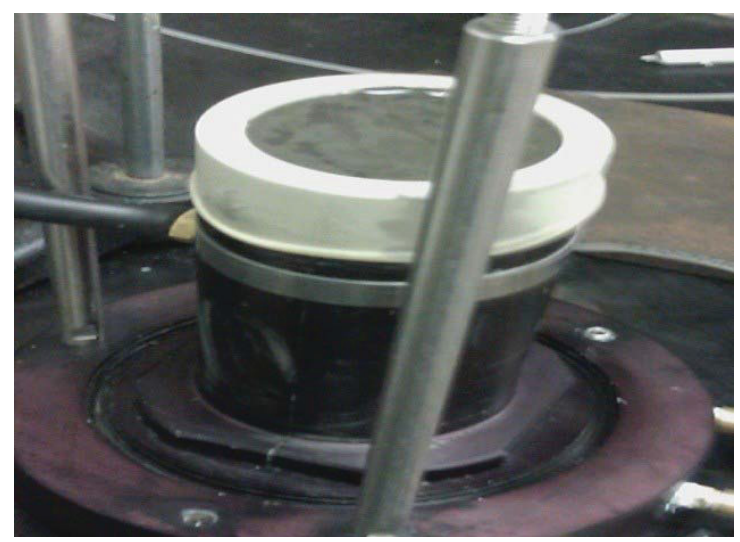

b)

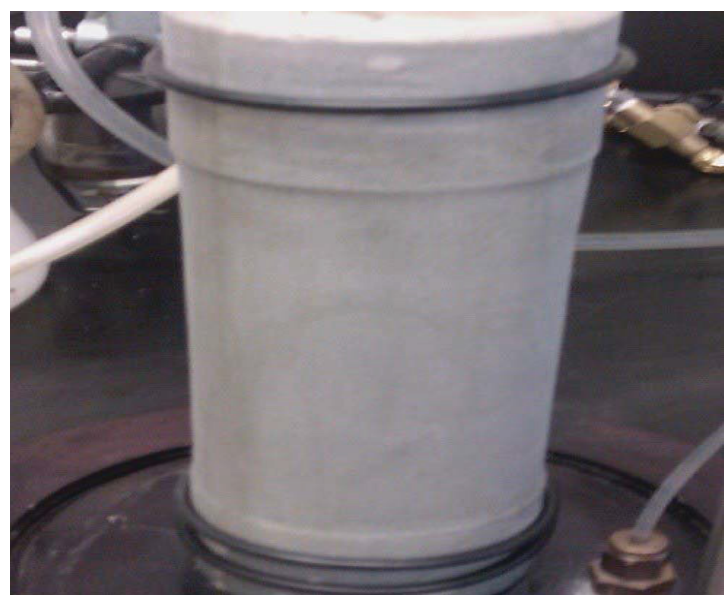

d)

Figure 3.32 Preparing reconstituted slurry samples in the triaxial device

full saturation of the specimen. In other words, saturation by back pressure is associated with increasing the specimen pore pressure to obtain saturation. During application of back pressure the difference between cell pressure and pore pressure is kept constant in order to maintain a constant effective stress. 


\subsubsection{Desiccated - rewetted undisturbed samples}

Desiccated-rewetted undisturbed triaxial sample preparation is similar to the small scale method that was described for the simple shear device. In this method, tailings are prepared at $38 \%$ water content in a bucket and allowed to desiccate to different values of water content. Samples are extracted after rewetting using thin wall tubes (63.5 $\mathrm{mm}$ diameter). Extracted samples were carefully placed into the triaxial mould to avoid sample disturbance. Figure 3.33 presents the procedure for preparing undisturbed desiccated-rewetted tailings for the triaxial device. Thin wall tubes for triaxial tests had $130 \mathrm{~mm}$ height and $63.5 \mathrm{~mm}$ diameter. In order to reach an acceptable B value, water should be circulated in the sample by applying back pressure. Since the device did not have capability of applying two lines of back pressure from the top and bottom of the sample; Unfortunately, the B value of desiccated-rewetted samples even under high back

pressures did not go beyond $80 \%$. Consequently, the normal triaxial test on desiccated-rewetted tailings, which has degree of saturation close to $90 \%$, was not possible. As a result, modified unsaturated triaxial test was employed for desiccated-rewetted tailings.

\subsubsection{Consolidation stage}

Following the saturation phase, specimens were consolidated to a hydrostatic effective stress state of $100 \mathrm{kPa}$. Volume and height change during consolidation were monitored and used to determine the void ratio at the end of consolidation. 


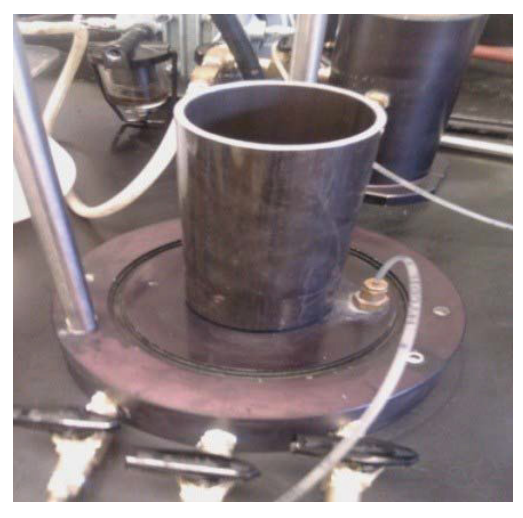

a)

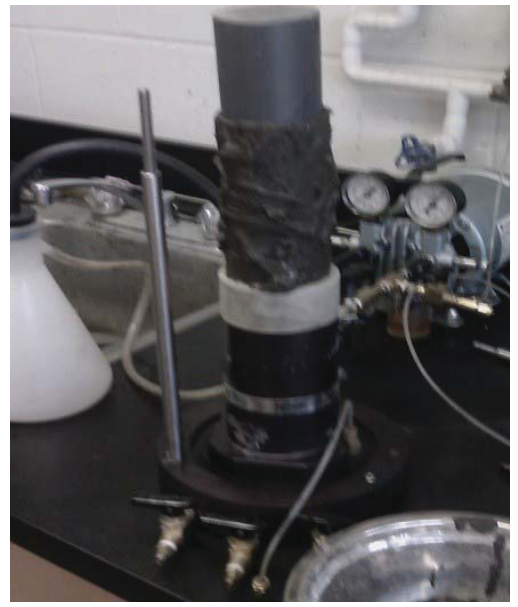

d)

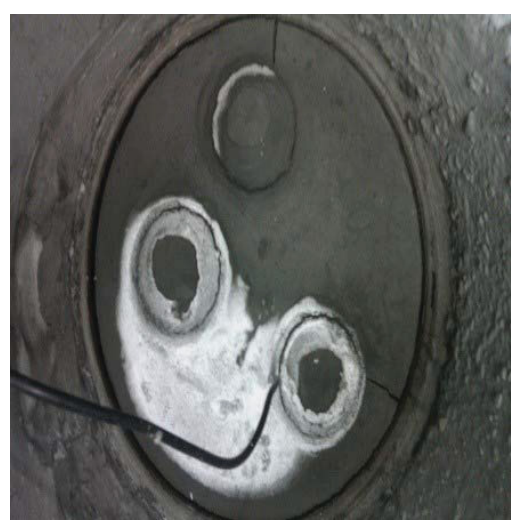

b)

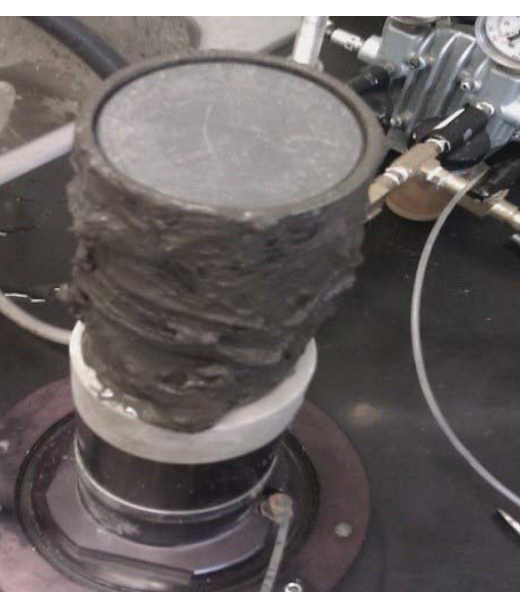

e)

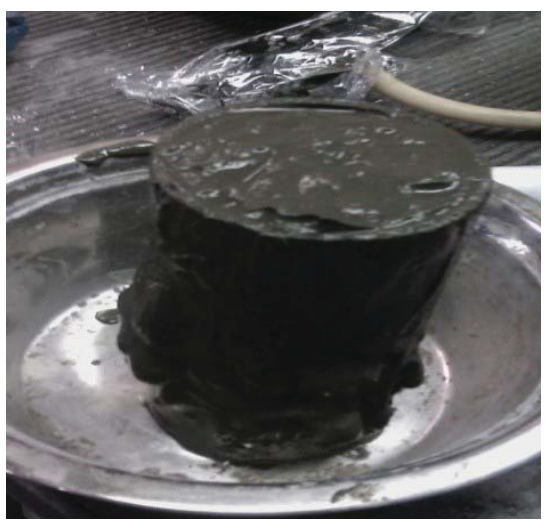

c)

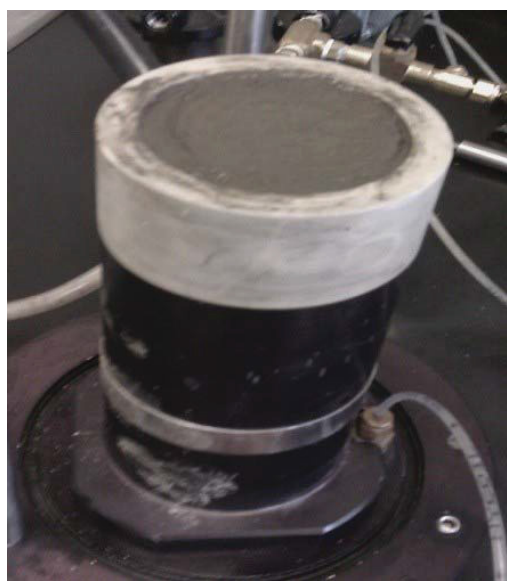

f)

Figure 3.33 Sample preparation procedure for undisturbed desiccated-rewetted samples for triaxial test

\subsubsection{Monotonic loading}

Following consolidation, the samples were monotonically sheared at a rate of $12 \%$ axial strain per hour with no drainage permitted and pore water pressure was measured. Consolidation 
pressures and monotonic and cyclic loading characteristics for normal triaxial tests are fairly similar to those noted in simple shear.

\subsubsection{Modified unsaturated triaxial test}

Modified unsaturated triaxial test was designed to measure the value of suction (or pressure) during the triaxial test. Direct measurement of suction (or pore pressure) is conducted by using a T5 tensiometer. Since B value is not measured and matric suction is monitored from the initial phase of tests by the tensiometer, removing the air-bubbles is not essential and the test is performed under unsaturated condition. Therefore, the saturation part is omitted and the pore water pressure (either positive or negative) is measured by the tensiometer during the test. Simms and Grabinsky (2009) also employed this method to perform unsaturated triaxial test on cemented paste tailings. Figure 3.34 shows a picture of the device that they used. In this method, a tensiometer is inserted into the sample from the bottom of the sample and the tensiometer is responsible for measuring pore water pressure in saturated condition and matric suction in unsaturated condition. Obviously, the positive pore water pressure should not pass the tensiometer's positive pressure limitation. In addition, since tensiometers are not capable of measuring high values of suction due to cavitation problems, samples should not have high values of suction. This issue does not matter for desiccated-rewetted samples, which has high degree of saturation $(\mathrm{S}>0.9)$ i.e. low values of suction (or positive pore pressure). However, desiccated samples with high values of suction (more than $200 \mathrm{kPa}$ ) should not be prepared. In some tests, tensiometers were inserted after consolidation stage, when matric suction is expected 

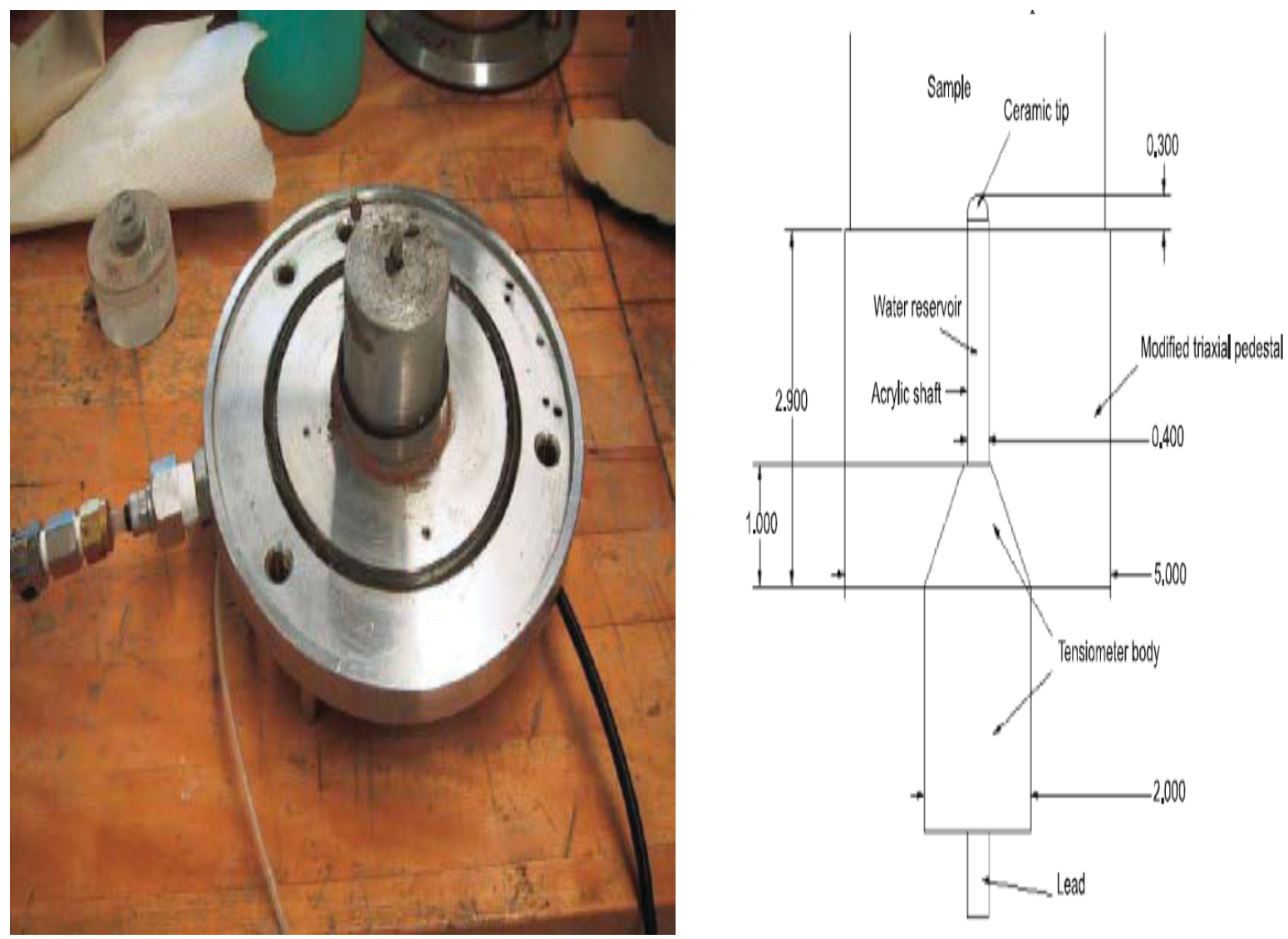

Figure 3.34 Picture and schematic of T5 tensiometer inserted into modified base triaxial pedestal (Simms and Grabinsky, 2009)

to get alleviated by loading. Another method of increasing matric suction measurement capability is applying a positive pressure during preparation to the ceramic cup. In order to apply positive water pressure and also calibrate the tensiometers, the back pressure line was employed. In this method, a positive water pressure was applied to the ceramic cup. The pressure was also monitored by the pore pressure transducer. Figure 3.35 presents the method of tensiometer's calibration. Figure 3.36 presents results of the calibration. The results showed that the response of the tensiometer to positive pore pressure is excellent. 


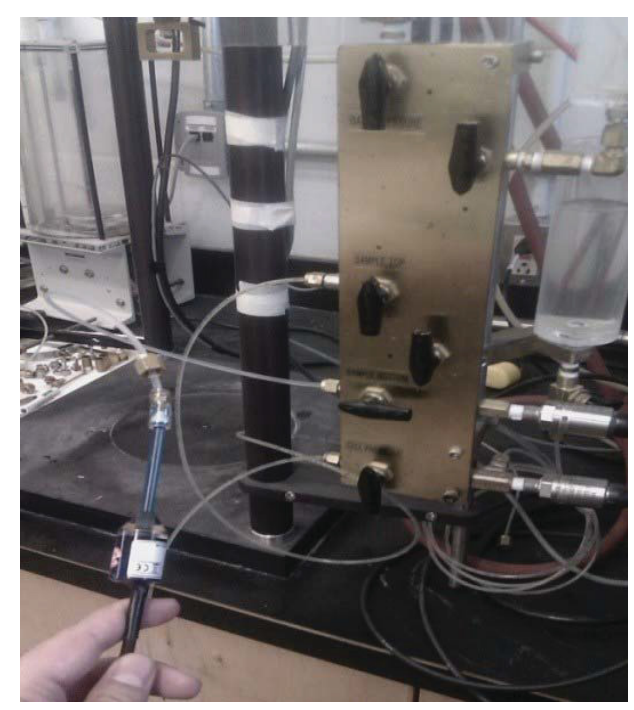

Figure 3.35 Calibration by applying positive water pressure to the tensiometer

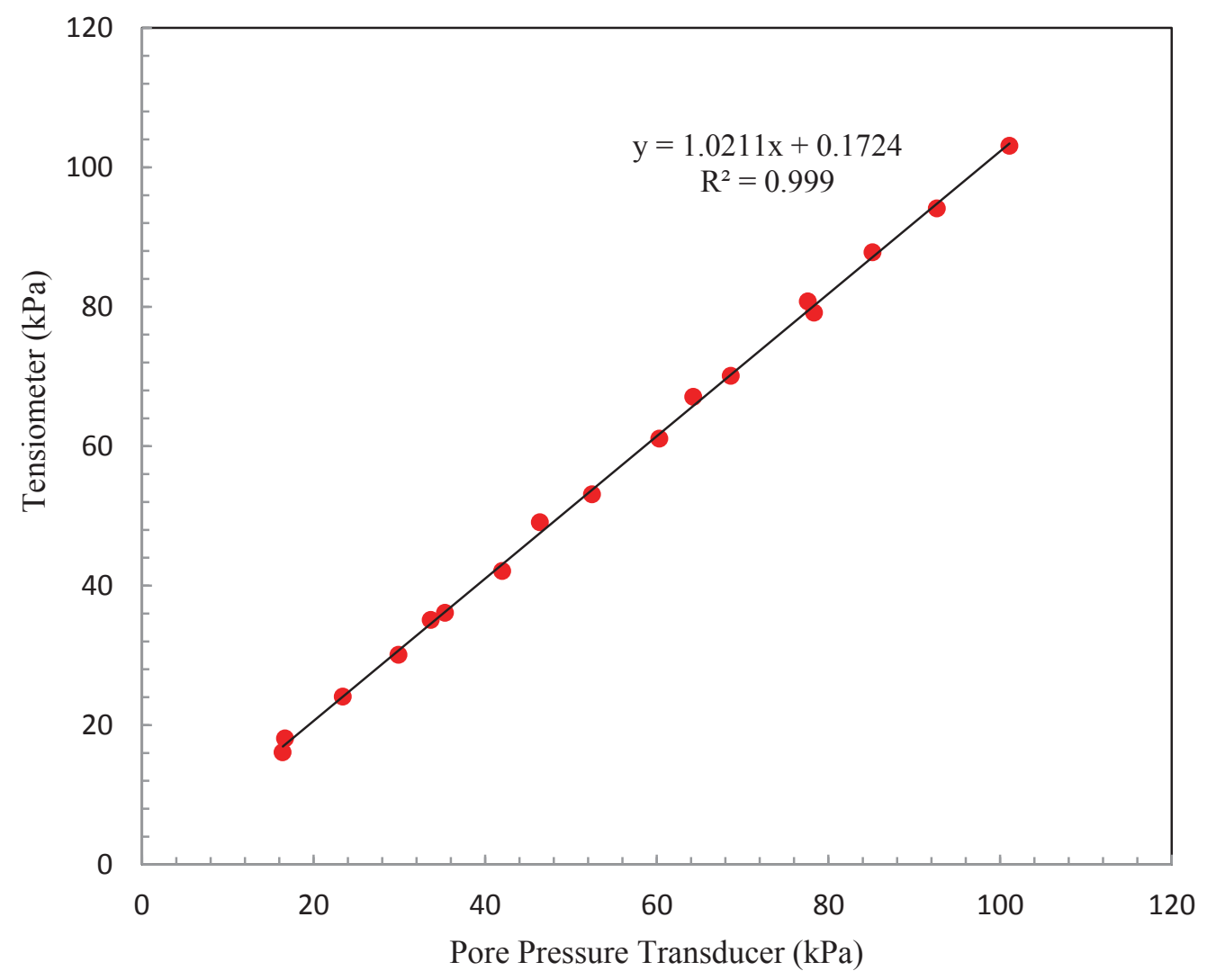

Figure 3.36 Calibration results of the tensiometer by applying positive water pressure 
Modified unsaturated triaxial pedestal was designed and built at Carleton University. Figure 3.37 shows a schematic diagram of the modified triaxial pedestal. The pedestal was located on top of three legs, which provide a space for inserting a tensiometer. The tensiometer was sealed and fixed by an O-ring. Figure 3.38 also shows pictures of modified triaxial device. In order to evaluate the efficiency of the tensiometer, one triaxial test in slurry condition (positive pore water pressure) was conducted. In this test, the excess pore pressure generation measured by pore pressure transducer during undrained triaxial test was compared to the pore pressure measurement of the tensiometer.

\subsubsection{Characteristics and limitations of modified triaxial tests}

Conventional triaxial tests were not applicable for desiccated-rewetted samples due to the fact that reaching appropriate B value even under one line of back pressure was not successful. The recommendation could be adding another line of back pressure to the device to have back pressure in both sample top and sample bottom, which is not in the scope of this thesis. In order to deal with this problem, the modified triaxial cell was designed to monitor the pore water pressure (or matric suction) during the test and eliminate the saturation part. However, since the specimen was not fully saturated, there were some approximations in void ratio measurement

and stress calculation, which make some limitations to the modified triaxial test accuracy. It should be also noted that specimens were hydrostatically consolidated in the triaxial test, which is not typical in-situ. Most natural soils will be subjected to non-hydrostatic initial state. 


\subsection{Void ratio measurement}

Two methods were used in this study to approximately estimate void ratios after consolidation: i) for higher degrees of saturation $(0.95<\mathrm{S}<1)$ the volume of removed water during consolidation was assumed to be the same as volume change of the sample. Therefore, recording the height change during consolidation, the area and therefore the diameter were calculated to be used for void ratio measurement; ii) for lower degrees of saturation $(0.89<\mathrm{S}<0.95)$ the diameter was approximately estimated during the test by taking pictures or using a caliper. Obviously, due to variation of the diameter in different heights of the sample, the estimation is not expected to be as accurate as the first method.

\subsection{Effective stress measurement}

The diameter was also influential in calculating effective stresses during shearing. The diameter was calculated assuming no volumetric strain during shearing. In other words, considering compressibility of air, the volume was assumed to be constant during shearing. Consequently, the area and the diameter were calculated by recording the height change based on constant volume. However, the constant volume change was checked during shearing by tracking the level of water of the cell. It was obtained that in all modified triaxial tests, there was no tangible change in the level of water of the cell, which can confirm no tangible volume change during shearing. In order to calculate the effective stresses during shearing and calculating friction 


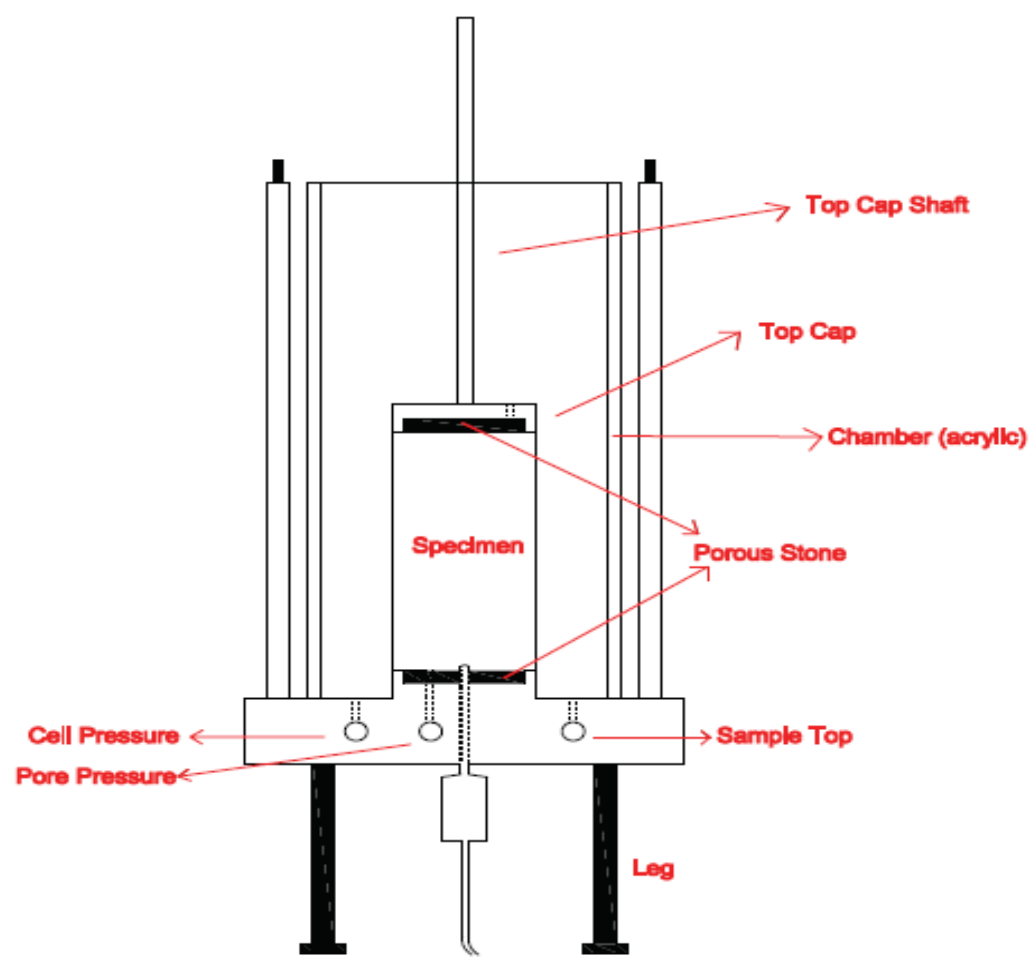

(a)

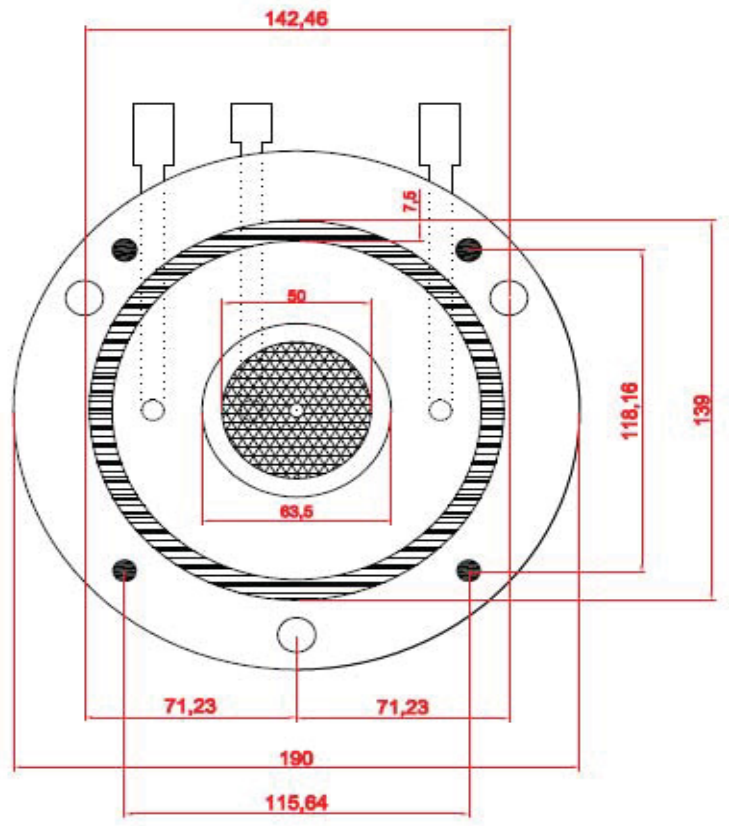

(b)

Figure 3.37 Schematic diagram of the modified unsaturated triaxial pedestal 


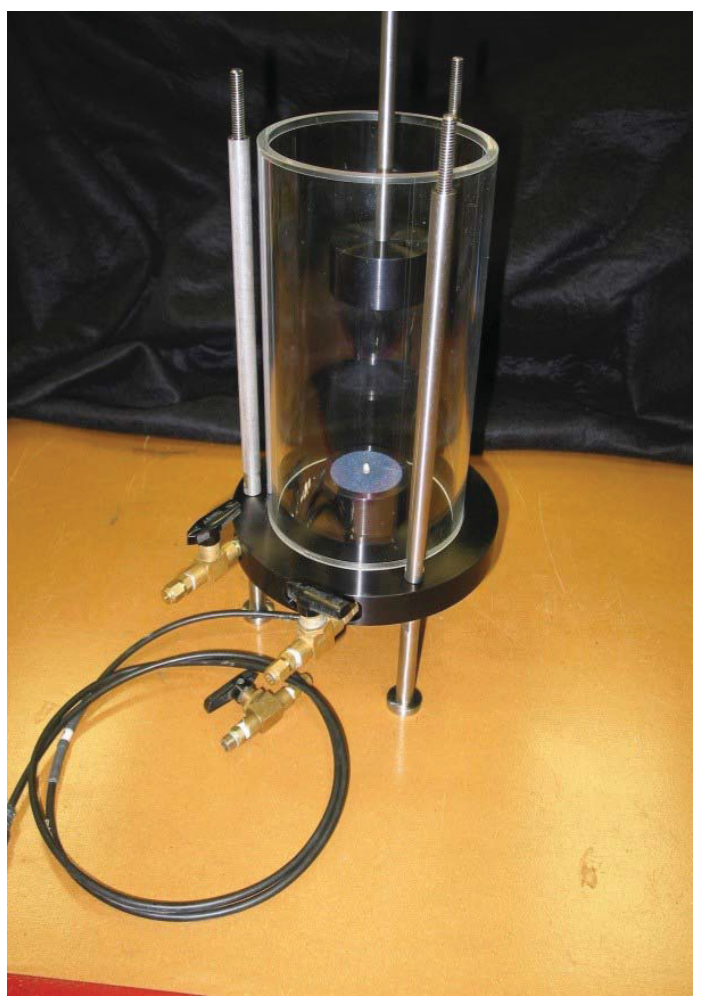

(a)

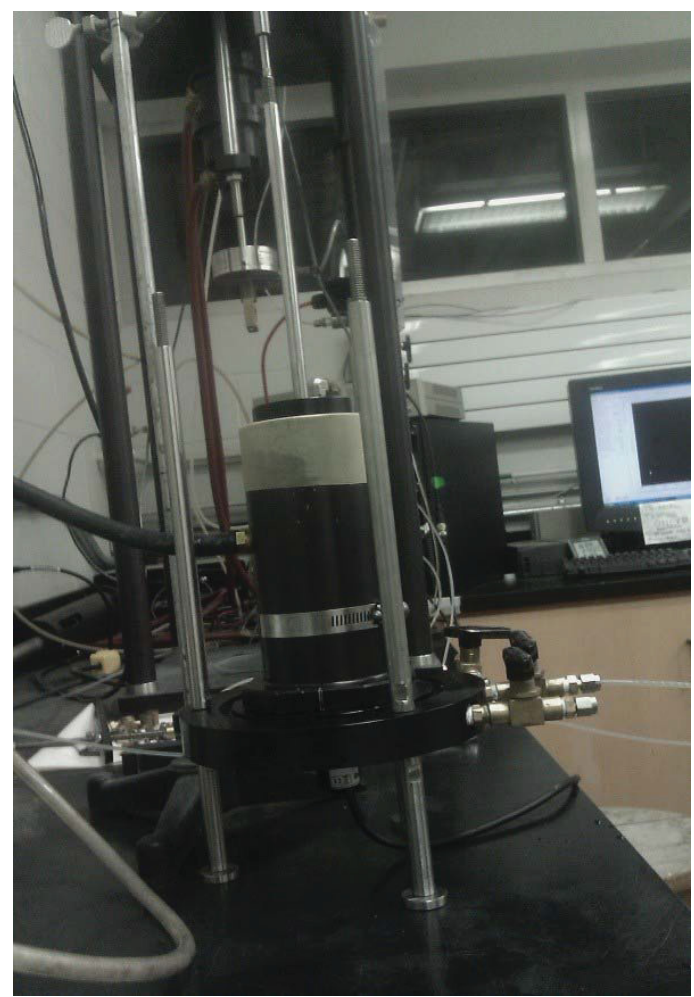

(b)

Figure 3.38 Pictures of modified unsaturated triaxial pedestal

angles, the effective stresses were also modified by Bishop's equation assuming $\mathrm{S}=\chi$ and atmospheric air pressure.

\subsection{Vane shear tests}

Inspector vane tester H-60 was used in this study to investigate the in situ undrained shear strength of the tailings at different conditions. The range of the instrument ranges from $0-260$ 
$\mathrm{kPa}$ when three different sized vanes are used. The accuracy of the instrument is expected to be within $10 \%$ of the reading. The measuring part of the instrument is a spiral-spring. When the handle is turned, the spring deforms and the upper part and the lower part of the instrument show a mutual angular displacement. When torque is applied, the scale-ring follows the upper part of the instrument and when failure is obtained, the scale-ring remains in its position and shows the value of undrained shear strength. There were three sizes of four blade vanes:

- $16 \times 32 \mathrm{~mm}$ (small, $0-260 \mathrm{kPa})$

- $20 \times 40 \mathrm{~mm}$ (standard, $0-130 \mathrm{kPa})$

- $25.4 \times 50.8 \mathrm{~mm}($ large, $0-65 \mathrm{kPa})$

The ratio of cross sectional area of vane to the area to be sheared is 14,16 and $24 \%$. Figure 3.39 shows a picture of an Inspector vane tester H-60 with three vane sizes.

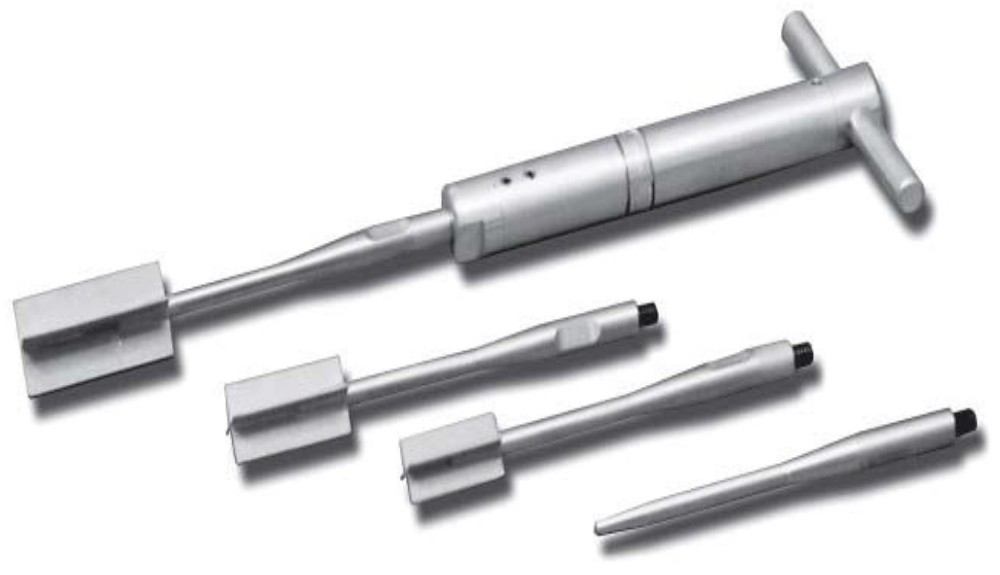

Figure 3.39 A picture of an inspector vane tester H-60 


\subsubsection{Sample preparation for vane shear test}

The sample preparation of small scale vane shear tests is fairly similar to small scale simple shear tests. Tailings at 38\% water content were deposited in a bucket and allowed to desiccate to different values of water content. For each water content, four or five vane tests were performed and the average value was considered as the undrained shear strength of the saturated or desiccated tailings. The same procedure was conducted after rewetting for desiccated-rewetted tailings in a bucket to measure the undrained shear strength of desiccated-rewetted tailings with different desiccation history.

\subsection{MIP device and sample preparation}

In order to investigate the effect of re-wetting, consolidation, OCR and shearing on the microstructure, an investigation was conducted using Mercury Intrusion Porosimetry (MIP). As discussed in Chapter 2, in this technique an absolute pressure is applied to a non-wetting liquid (mercury) in order to enter the empty pores of a flash freeze-dried sample.

\subsubsection{Device Description}

Auto Pore IV Mercury Porosimeter at University of Western Ontario was used in this study to measure the pore diameters. Figure 3.40 presents schematic diagram of the Auto Pore IV Mercury Porosimeter. The device consists of low pressure and high pressure analyses. All steps 
of low pressure and high pressure analyses, data collection, recording and display are controlled by computer. The device consists of four ports for low pressure analysis and two ports of high pressure analysis. The range of measurement of pore diameter in the low pressure stage is from $360 \mu \mathrm{m}$ to $3.6 \mu \mathrm{m}$. The high pressure analysis has measurement capacity of $6 \mu \mathrm{m}$ to $0.003 \mu \mathrm{m}$. Figure 3.41 presents low and high pressure ports of the Auto Pore IV Mercury Porosimeter.

\subsubsection{Sample preparation and testing procedure}

Sample preparation consisted of preparing a cubical sample in the simple shear device, freezedrying and placing in the MIP device. It was attempted to cut and collect the samples from the middle of the simple shear samples. The size of the sample was chosen to be from $5 \mathrm{~mm} \times 5 \mathrm{~mm}$ $\times 5 \mathrm{~mm}$ to $10 \mathrm{~mm} \times 10 \mathrm{~mm} \times 10 \mathrm{~mm}$. The samples were then wrapped with plastics to avoid evaporation. In the freeze drying step, the samples were contacted with liquid nitrogen in order to become freeze-dried. Pentane was also used to facilitate the process. Figure 3.42 presents the process of freeze-drying with a container filled with liquid nitrogen. Samples were dried in a vacuum desiccator after the freeze-drying method, which is shown in Figure 3.43. All samples were then collected and prepared at Carleton University and transferred to University of Western Ontario to be placed in the Auto Pore IV Mercury Porosimeter device. Before starting the MIP device, the weight of the sample and penetrometer were measured. To begin the pressure analysis, the sample was placed in a penetrometer and the penetrometer was loaded in a low pressure port. Figure 3.44 shows a picture of the penetrometer used in this study. The first step of low pressure analysis was the evacuation of gases from the penetrometer. In the next step, the Penetrometer is automatically filled from the back with mercury. The second phase of low 
pressure analysis is applying $0-345 \mathrm{kPa}$ and recording the intrusion. After finishing the low pressure analysis, the penetrometer is removed from the low pressure port and installed in the high pressure port. The high pressure is applied by oil and collects data up to $228 \mathrm{MPa}$ (Manual of the Auto Pore IV, 2004). Pore volume data are calculated by measuring the volume of mercury remaining in the penetrometer. With increasing pressure, mercury moves into the sample's pores, which is called Intrusion. Different size pores intrusion requires different pressures.

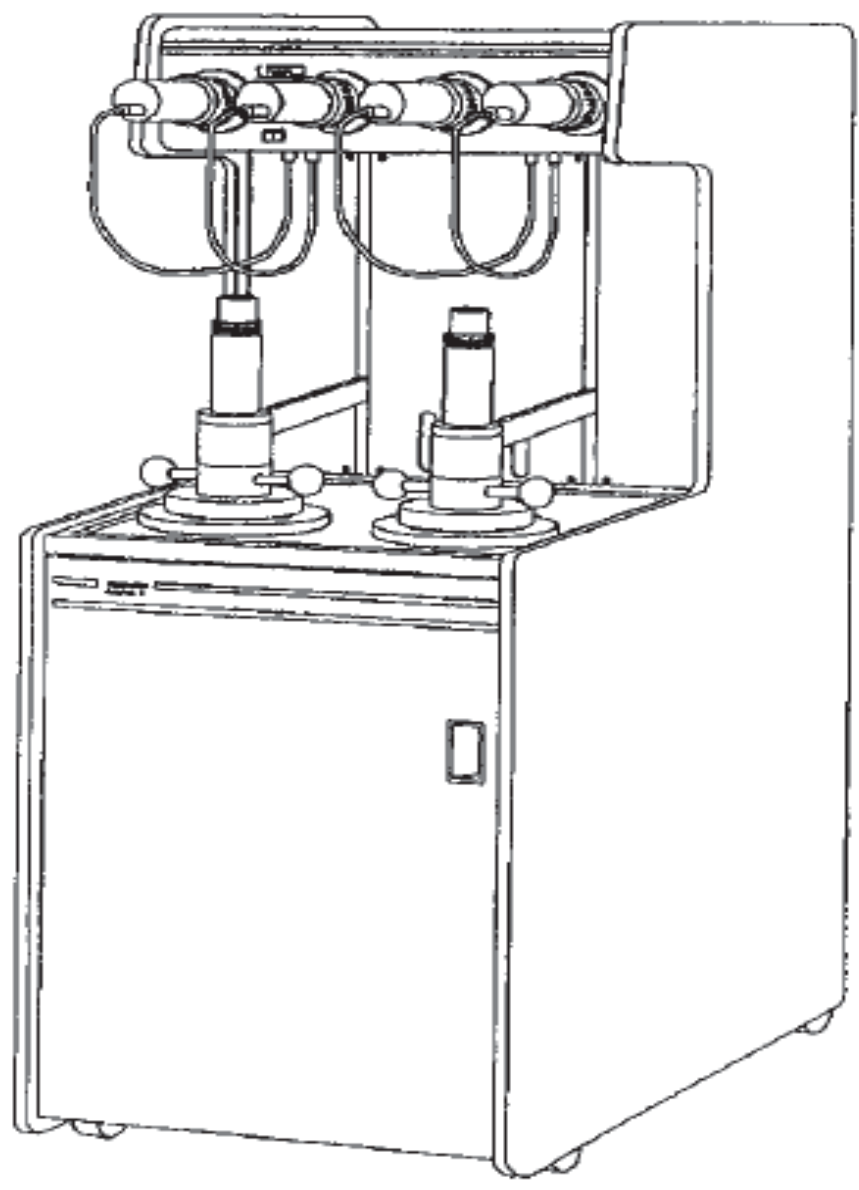

Figure 3.40 Schematic diagram of the Auto Pore IV Mercury Porosimeter (Manual of the Auto Pore IV) 


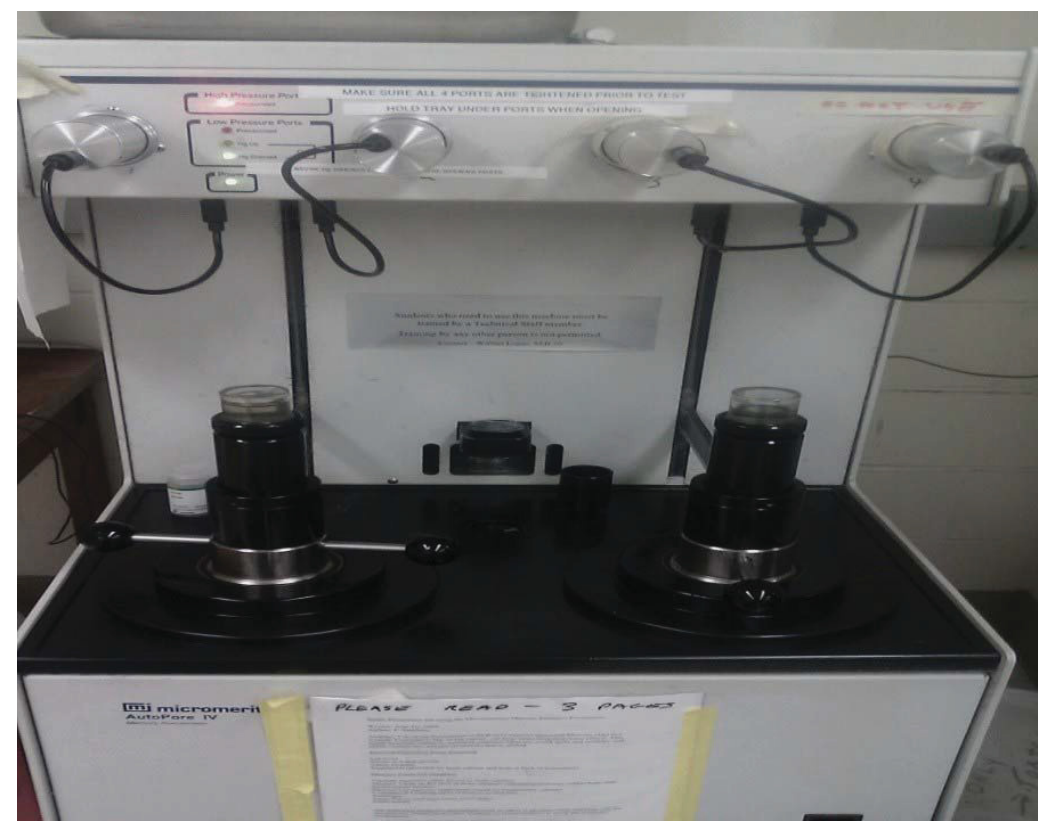

Figure 3.41 Low pressure and high pressure ports of the Auto Pore IV Mercury Porosimeter

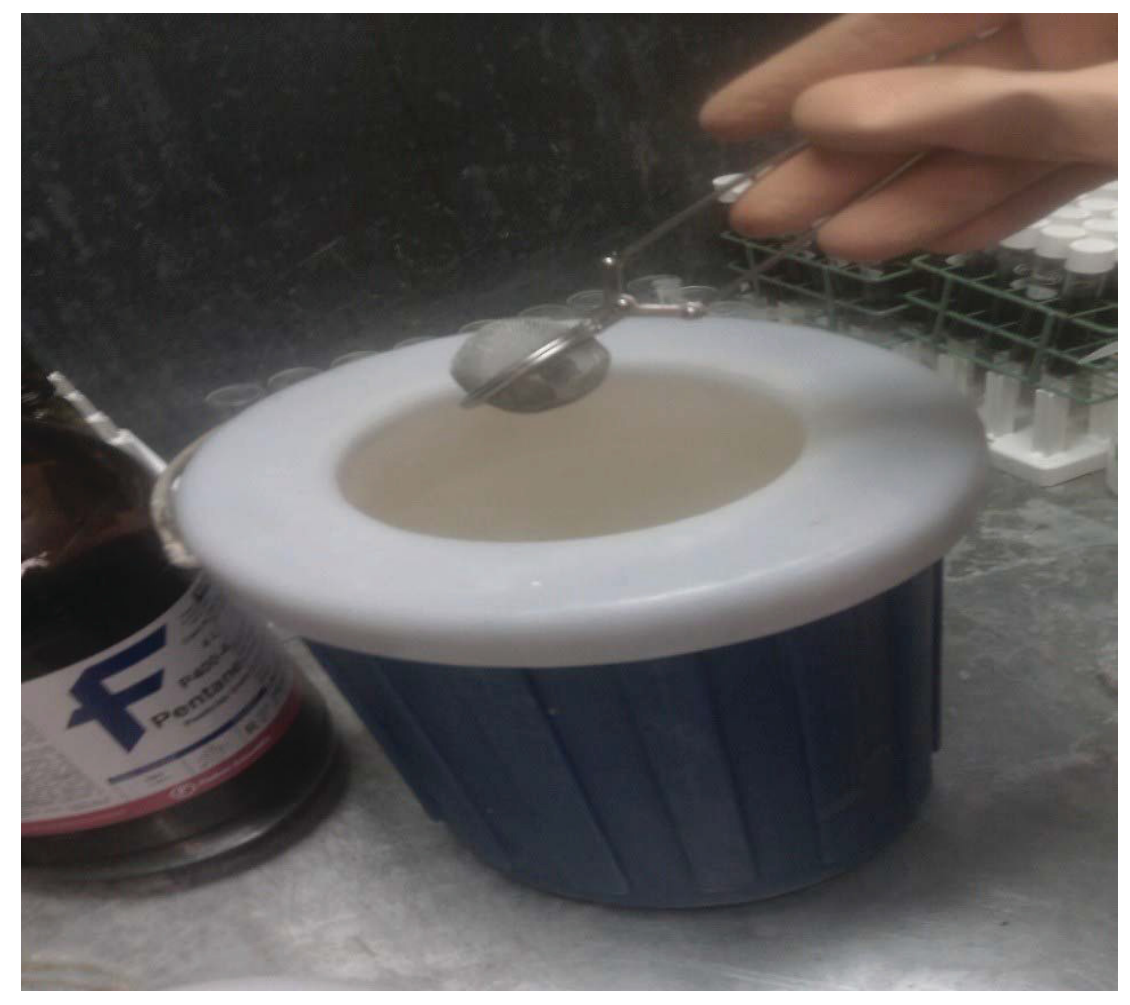

Figure 3.42 Freeze-drying with liquid nitrogen 


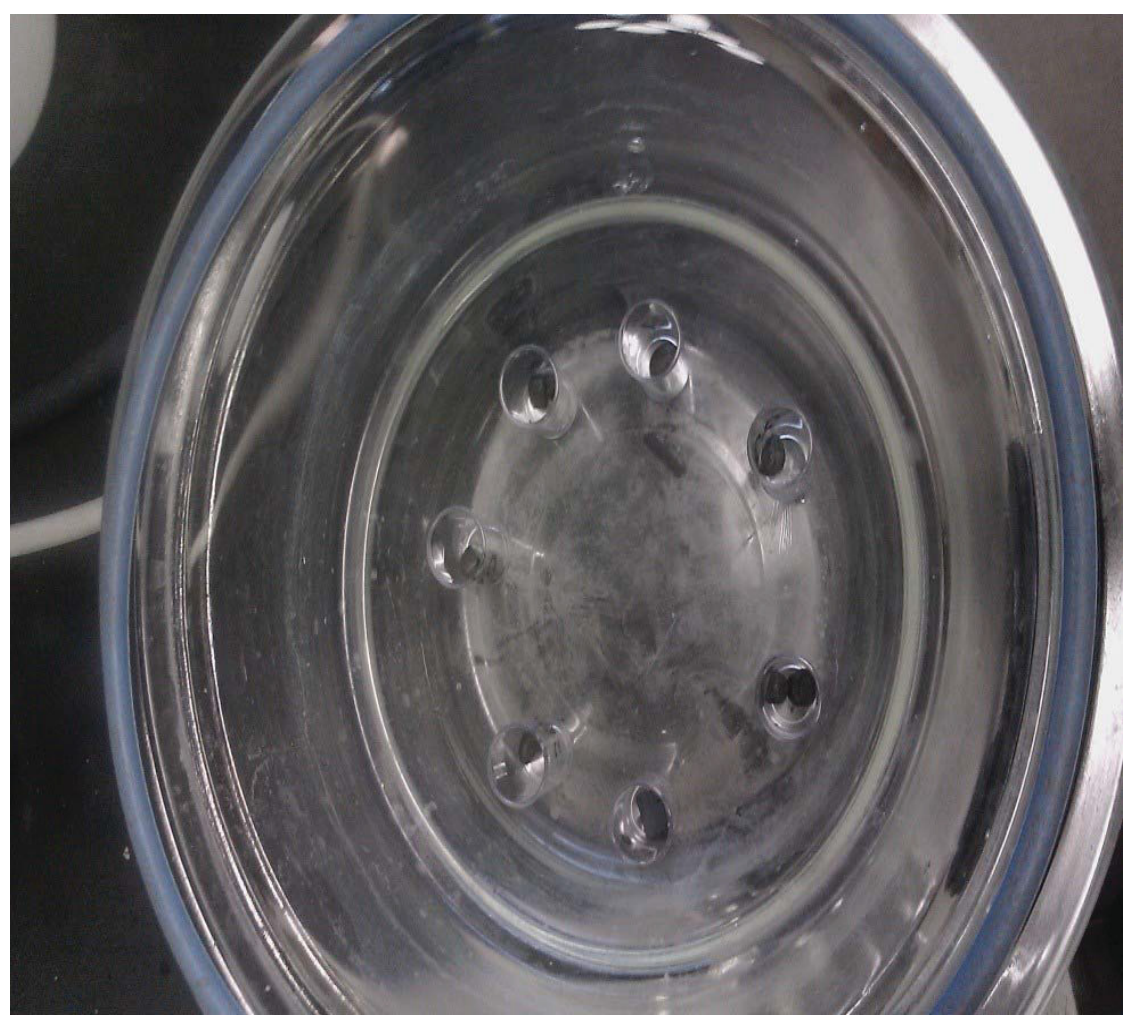

Figure 3.43 Samples placed in a vacuum desiccator after freeze drying

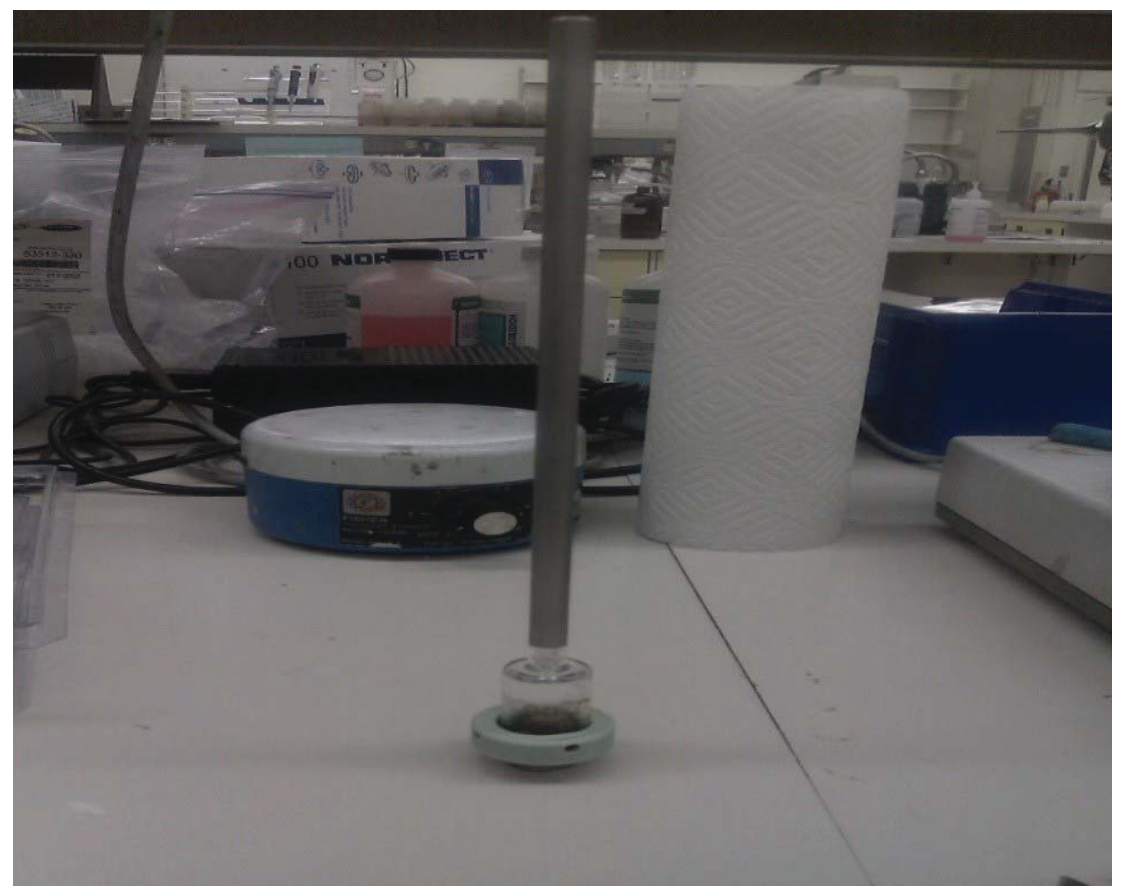

Figure 3.44 A picture of the penetrometer used in this study 


\section{Chapter 4: Simulation of multilayer deposition of thickened gold tailings in a drying box}

\subsection{Introduction}

As discussed in chapters one and two, thickened tailings are dewatered prior to deposition to gain some yield stress to enable stable stacking in tailings storage facilities. In order to evaluate the influence of stress history of thickened tailings layers on final static and cyclic stability of the stack, appropriate methods of deposition simulation are required. The small scale deposition simulation in a bucket was explained in chapter 3. Additionally, a larger scale of simulation was performed by designing a modular drying box to deposit thickened tailings layers. The box was designed to be suitable for depositing five thickened tailings layers. Monitoring water content, matric suction, evaporation, drainage and void ratio facilitate applying various stress histories to the deposited layers. The box was modular so as to minimize variation in the distance from the top of the tailings to the top of the box. Details of the dimensions of the drying box, methods of direct measurements of the mentioned parameters have been discussed in section 3.5. Figure 4.1 shows a picture of the drying box with deposited layers. 
As discussed in previous chapters, this study aims to simulate the stress history of thickened tailings layers based on the following steps: initial settling, drainage and desiccation, rewetting, consolidation and shearing. Except consolidation and shearing, other steps were simulated in the multilayer deposition in the drying box. Two last steps were simulated using simple shear tests on extracted tubes from different layers, which will be explained in chapter 6 . The deposited layers were supposed to experience different desiccation histories, which consists of low values of water content for the first and second layers and shrinkage limit for other top layers. The target water contents for the first and second layers were $12 \%$ and $16 \%$, and $18 \%$ for the third, fourth, and fifth layer. Two wet-dry cycles were also added to simulate rainfall events between deposition of the fourth and fifth layers. This chapter presents the results of multilayer deposition of thickened tailings layers in the drying box.

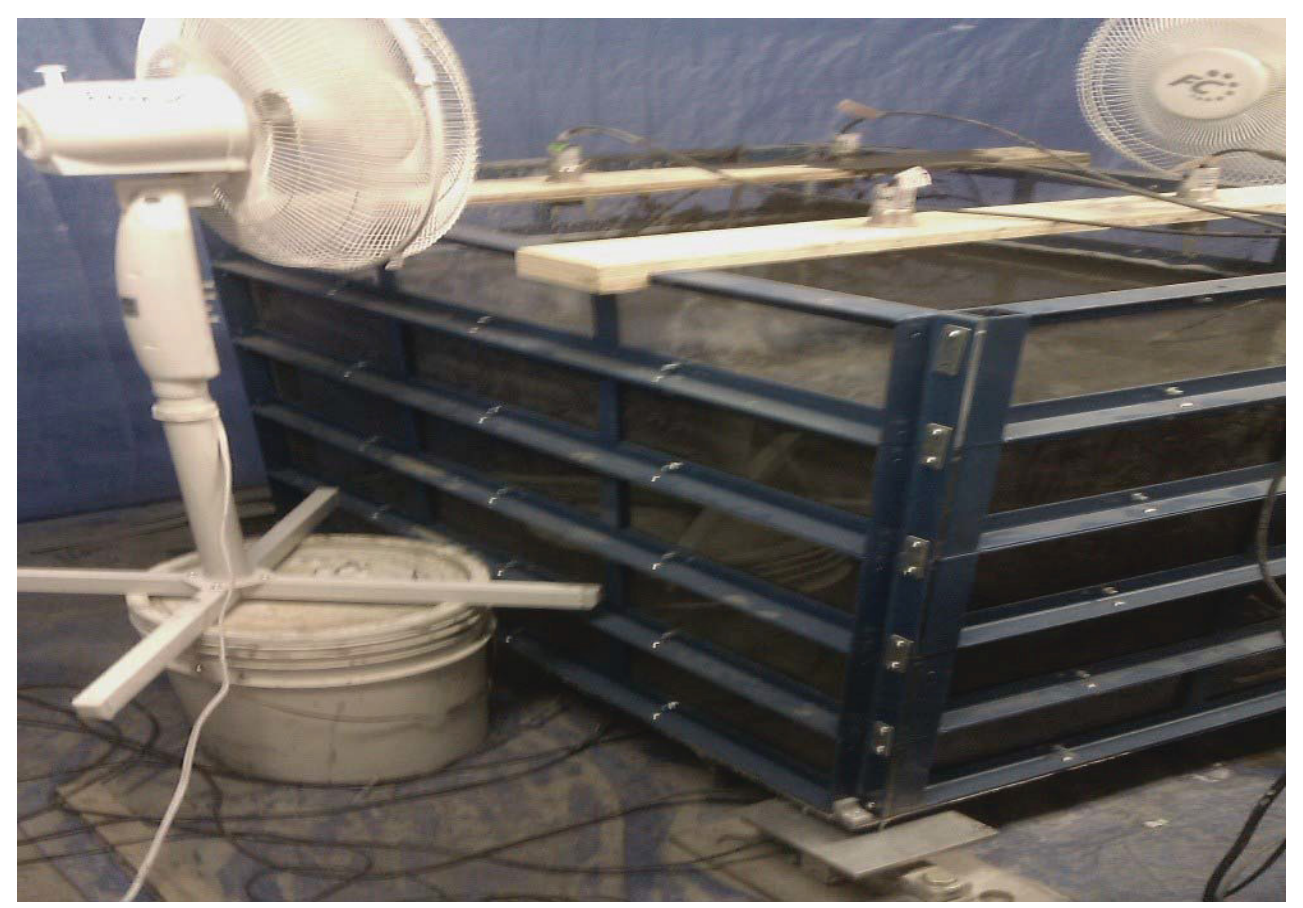

Figure 4.1 A picture of the drying box with deposited layers 


\subsection{First Layer Deposition}

Tailings were prepared at 38\% water content in different buckets and deposited as the first layer. The thickness of the first layer after deposition was around $180 \mathrm{~mm}$. Drainage, evaporation and water content were monitored following deposition and Figure 4.2 presents these measurements. It can be seen that it took eight days for the first layer to reach the target water content $(\mathrm{W}=$ $12.4 \%$ ). Figure $4.2 \mathrm{a}$ clearly shows that the drainage rate immediately after deposition was significantly high $(5 \mathrm{~mm} /$ day). However, just one hour after deposition, the rate of drainage dramatically decreased to $0.42 \mathrm{~mm} /$ day. The reason could be attributed to initial settling. Tailings at $38 \%$ water content behaved as a fluid and have hydrostatic pressure. After initial settling, tailings become denser and the drainage flow rate decreases. The rate of drainage gradually decreases to $0.038 \mathrm{~mm} /$ day at the end of the first day and finally stopped at day 3 . Figure $4.2 \mathrm{~b}$ presents the rate of actual evaporation (AE) for the first layer deposition. Since there was a layer of water on top of the settled tailings at the first day, the amount of AE in the first day was 9.9 $\mathrm{mm} /$ day and close to potential evaporation $(\mathrm{PE})$ rate. The rate of potential evaporation was measured using a column of water. The rate of evaporation slightly decreased to $9.37 \mathrm{~mm} /$ day at day 3 and more rapidly decreased to $3.2 \mathrm{~mm} /$ day at day 8 . The reduction in evaporation rate shows that dewatering process requires more time at lower water contents. Figure 4.2c presents the calculated gravimetric water content for the first layer. The calculation was associated with the mass balance equations. Figure $4.2 \mathrm{~d}$ compares the calculated average GWC with surface GWC. As expected, the GWC of the surface is one or two percent lower than the average GWC of the layer. However the trend of reduction is similar. Calculated volumetric water content 

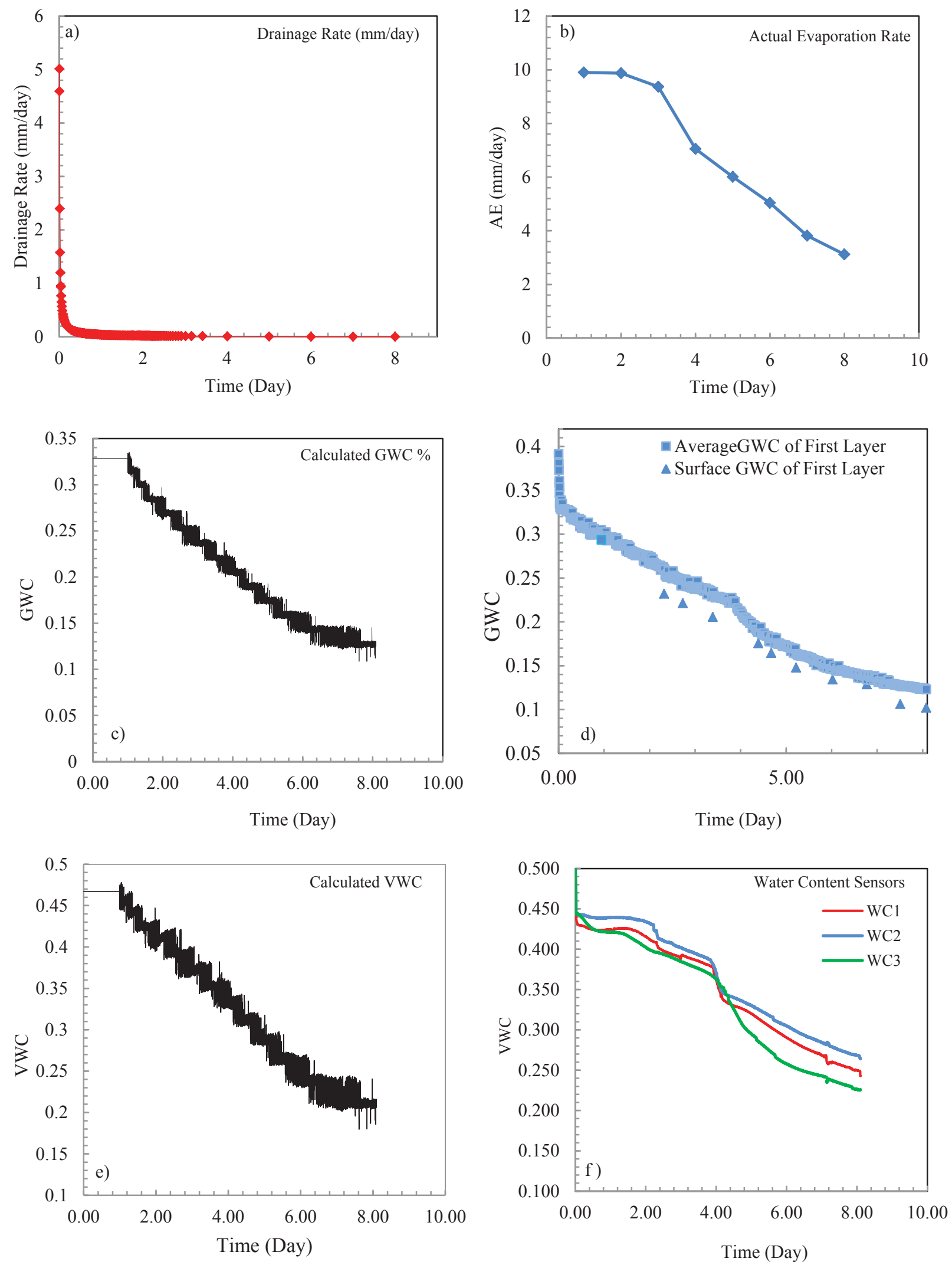

Figure 4.2 Drainage, evaporation and water content results of first layer deposition 
(VWC) and VWC obtained by three 5TE sensors are presented in Figures 4.2e and 4.2f respectively. Comparison shows that the calculated VWC works well until the tailings starts to desaturate. After desaturation and at low water contents, the sensors exhibited slightly higher water contents than calculated VWC $(1 \% \sim 3 \%)$

Figure 4.3 presents electrical conductivity, temperature and void ratio results of the first layer deposition. Electrical conductivity is associated with osmotic suction. Figure 4.3a shows that the bulk electrical conductivity decreases with continuing desiccation, which is attributed to increasing osmotic suction during desiccation. Figure $4.3 \mathrm{~b}$ shows the value of temperature inside the tailings measured by 5TE sensors. The approximation of the volume of cracks is shown in Figure 4.3c. The method used to determine the volume of cracks was explained in chapter three in Figure 3.22. No cracks were observed in the first two days. The cracks appeared at day 3 and the volume of the cracks increased significantly until reaching the shrinkage limit. After passing the shrinkage limit, the rate of increase is gentler and becomes constant for higher degrees of desiccation. Figure $4.3 \mathrm{~d}$ shows the variation of void ratio during desiccation. The void ratio of settled tailings was calculated to be around 0.96 . The void ratio decreases during the dewatering process and the rate of reduction becomes gentler as the water content gets closer to the shrinkage limit. After passing the shrinkage limit, there is no tangible change in the void ratio. The void ratio of tailings prepared by small scale method varied within the range of $0.52-0.7$. The black line considers the variation of void ratio based on just change in vertical height obtained by ultrasonic distance sensors. The red line considers the variation of void ratio based on change in vertical height and also shrinkage obtained by cracks, which is a more accurate method. Obviously, both lines are the same until cracks appeared. Cracks appeared at $\mathrm{e} \sim 0.78$, and the void ratio decreased to $\mathrm{e} \sim 0.66$ at the end of the desiccation process. 

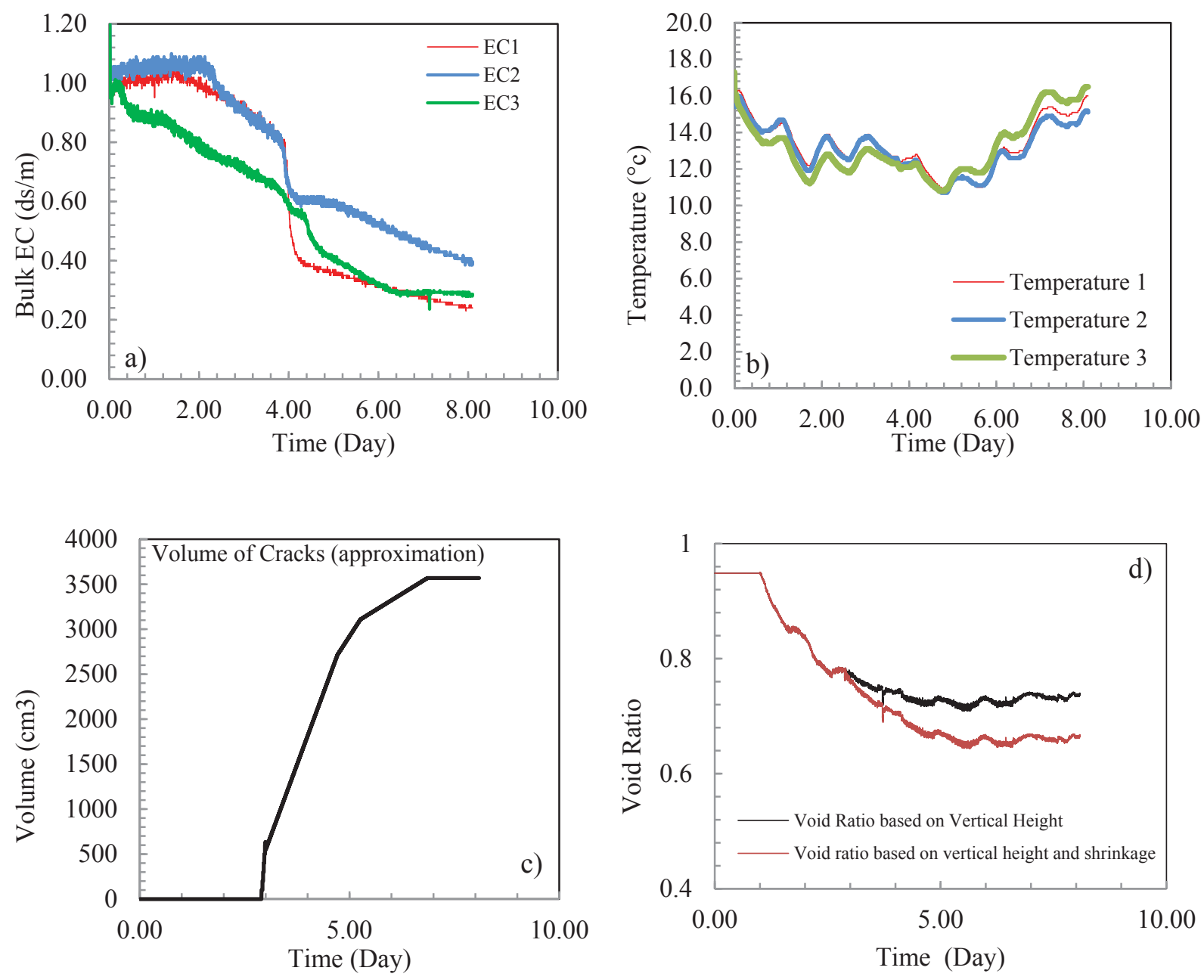

Figure 4.3 Electrical conductivity, temperature and void ratio results of first layer deposition

Figure 4.4 presents the shrinkage curve of the tailings obtained in the first layer deposition. It is clear that after deposition, the void ratio of the tailings decreased linearly with increasing degree of desiccation (decreasing water content). This linear reduction continues until the soil remains saturated. Once the soil becomes unsaturated (at GWC $\sim 21.7 \%$ ), the reduction in GWC does not lead to linear reduction in void ratio. The point where the linear correlation between GWC and void ratio becomes nonlinear is attributed to air-entry value (AEV) of the tailings. The reduction in void ratio of the tailings during desiccation continues up to shrinkage limit (SL 17.3\%). 
After this point, reduction in GWC does not lead to tangible decrease in void ratio. In other words, as expected, the volume of the tailings does not decrease during desiccation after the shrinkage limit.

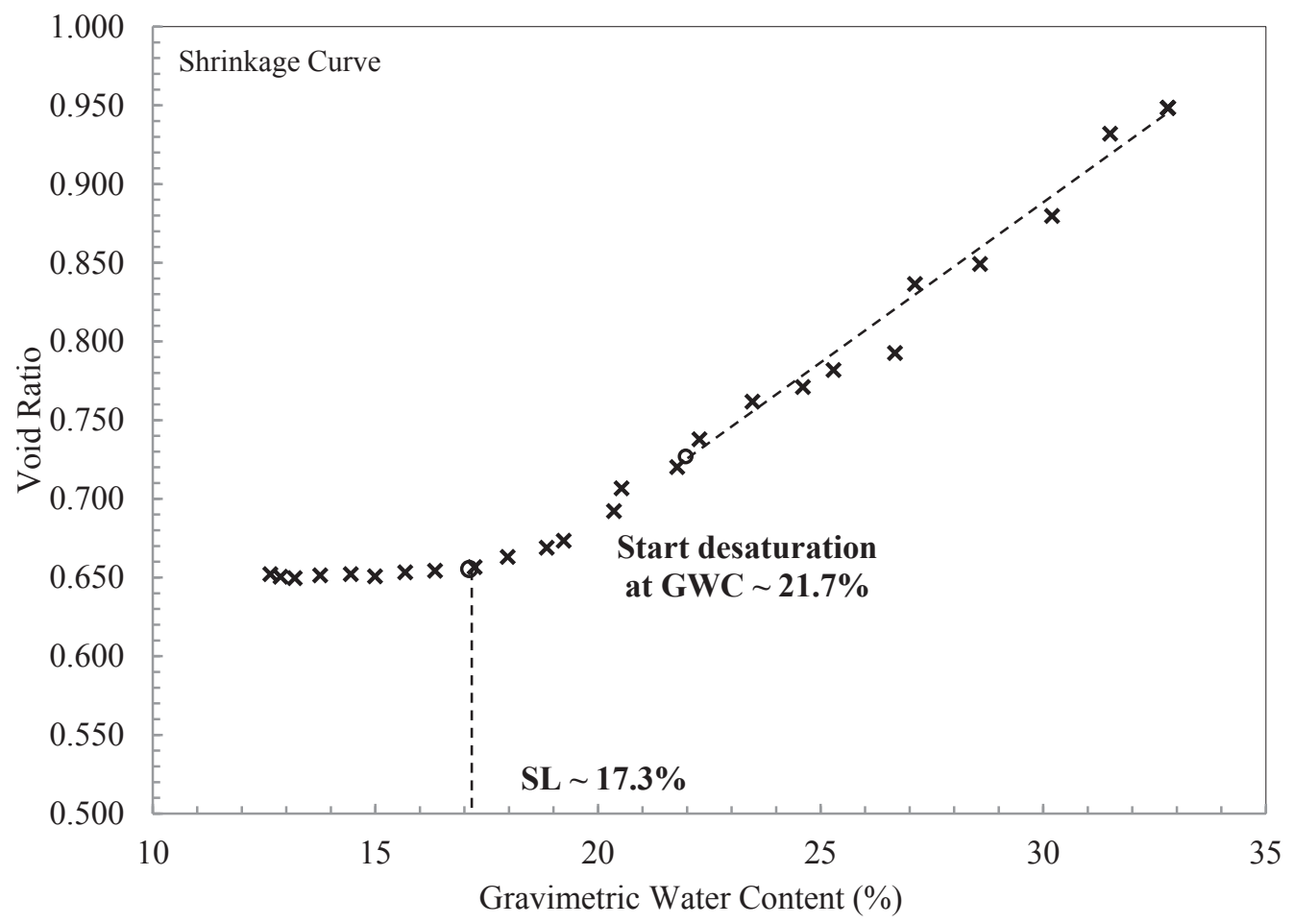

Figure 4.4 Shrinkage curve of the tailings obtained in the first layer deposition

Figure 4.5 presents the matric suction results of the first layer deposition obtained by tensiometers inserted in the deposited layers. As mentioned before, three tensiometers were inserted in each layer of the drying box. Figure $4.5 \mathrm{~b}$ shows that all tensiometers measured a low value of negative suction (positive pore pressure) after deposition. This positive pore pressure is 

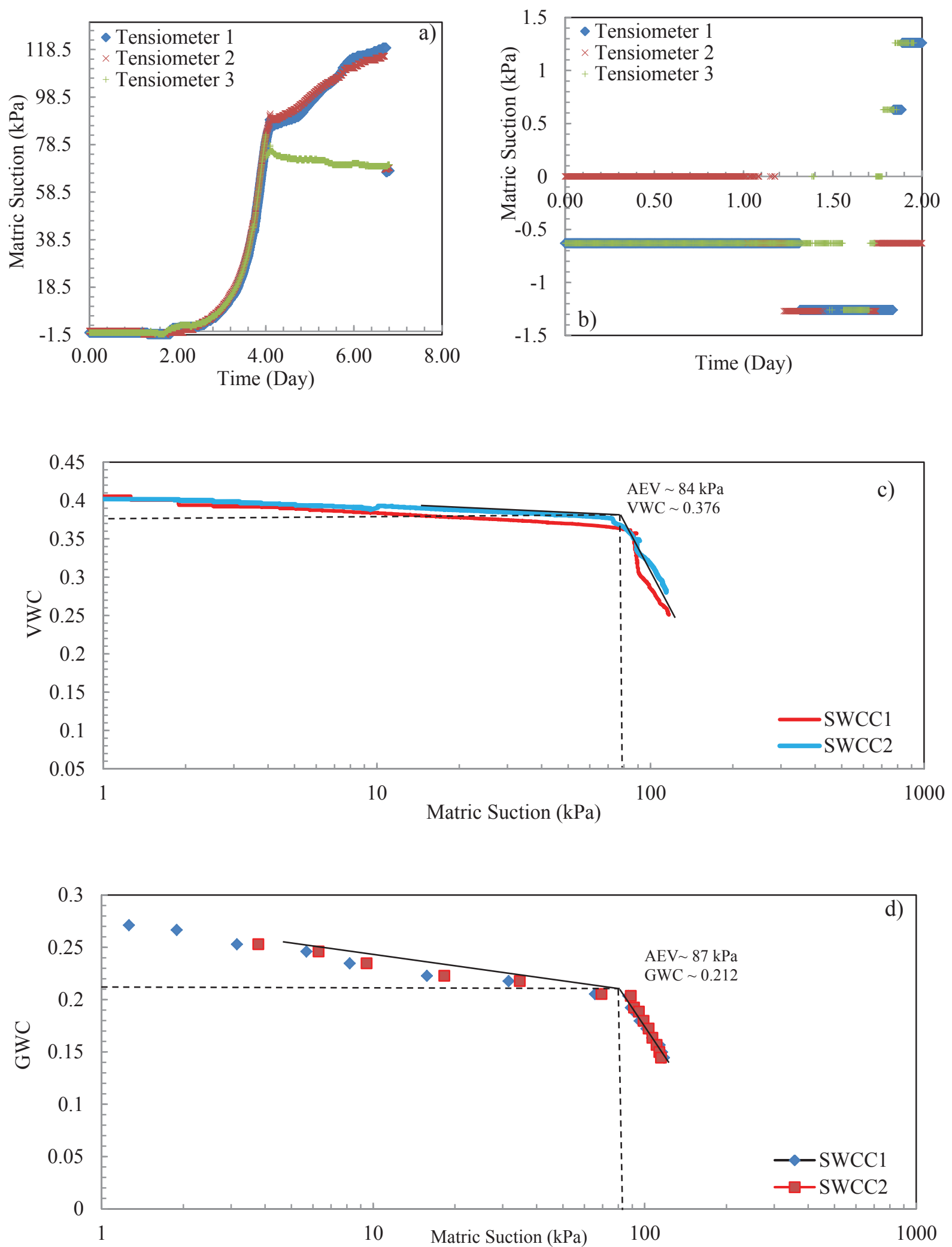

Figure 4.5 Matric suction results of first layer deposition 
attributed to hydrostatic pressure of the tailings at high water content after deposition. Improper tensiometer preparation and limitation of the loggers have led to no variation in matric suction in the first day. It is clear that after initial settling, tailings do not behave as a liquid and the positive pressure disappears. Figure 4.5a shows that matric suction values obtained by tensiometers 1 and 2 start to increase at day 2 . The increase in matric suction continues significantly to $85 \mathrm{kPa}$ up to day 4. After day 4, increase in matric suction is not as high as before day 4. The increase continued until cavitation occurs in tensiometers. The point where the rate of increase in matric suction becomes gentler can be attributed to air-entry value (AEV). Tensiometer 3 cavitated earlier than the other two. Figures $4.5 \mathrm{c}$ and $4.5 \mathrm{~d}$ present the plot of matric suction obtained by tensiometers 1 and 2 versus VWC and GWC respectively. It is clear that the value of AEV is around $85 \mathrm{kPa}$ and it corresponds to GWC $\sim 21 \%$. In other words, until reaching $21 \%$ gravimetric water content, the dewatering process generates matric suction without significant decrease in the degree of saturation. However, after passing GWC $=21 \%$, further increase in matric suction leads to significant reduction in degree of saturation and water content.

In order to investigate the potential behaviour of the layer of geotextile as a capillary barrier layer, drainage and suction results of the first layer were considered. It was noted that the rate of drainage significantly decreased when matric suction appears in the layer (around day 2.5). The rate of drainage stops at day 4 when around $55 \mathrm{kPa}$ matric suction is generated in the layer. Therefore, the layer of geotextile is not expected to behave as a capillary barrier layer.

Figure 4.6 presents the total suction results of first layer deposition obtained by WP-4 total suction measuring device. The values correspond to total suction at the surface of the desiccated layer. Figure 4.6a shows that the values of total suction increase from $400 \mathrm{kPa}$ to $1250 \mathrm{kPa}$ with continuing desiccation from $\mathrm{GWC}=16.5 \%$ to $\mathrm{GWC}=12.5 \%$. This significant increase could be 

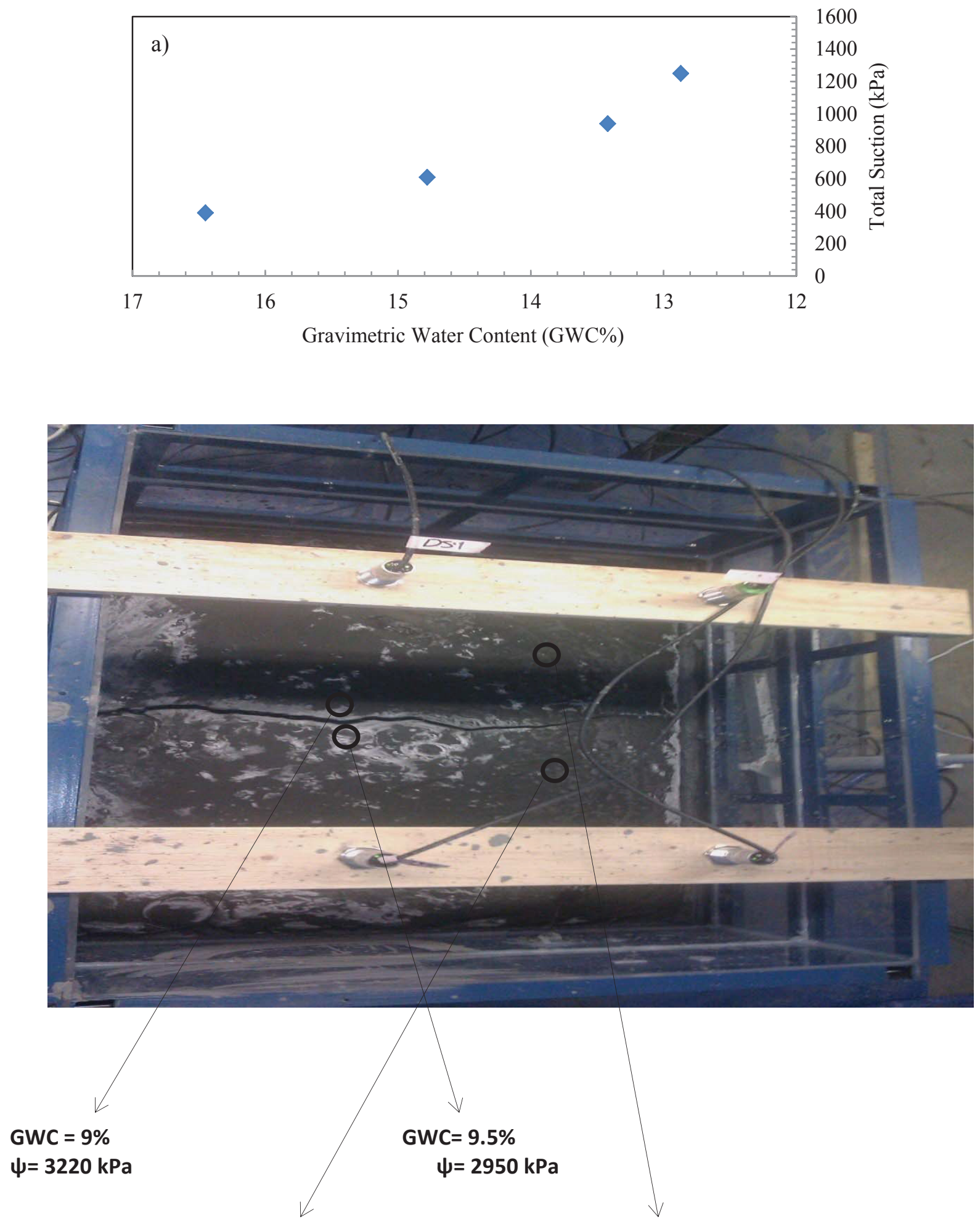
a) $1 \mathrm{~mm}$ Depth: GWC $=10.6 \%, \Psi=1580 \mathrm{kPa}$
a) $1 \mathrm{~mm}$ Depth: GWC $=10 \%, \psi=1690 \mathrm{kPa}$

b) $3 \mathrm{~mm}$ Depth: GWC $=11.3 \%, \psi=1410 \mathrm{kPa}$

b) $3 \mathrm{~mm}$ Depth: GWC $=10.9 \%, \psi=1570 \mathrm{kPa}$

Figure 4.6 Total suction results of the first layer deposition 


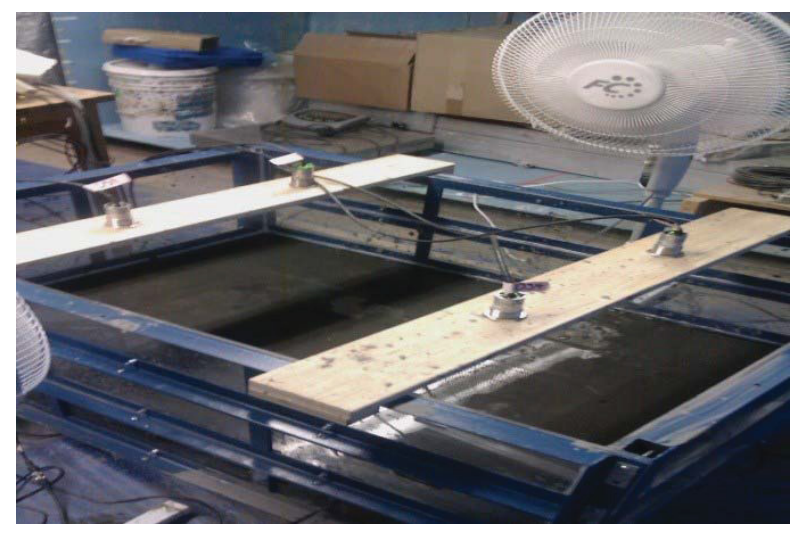

Day 1

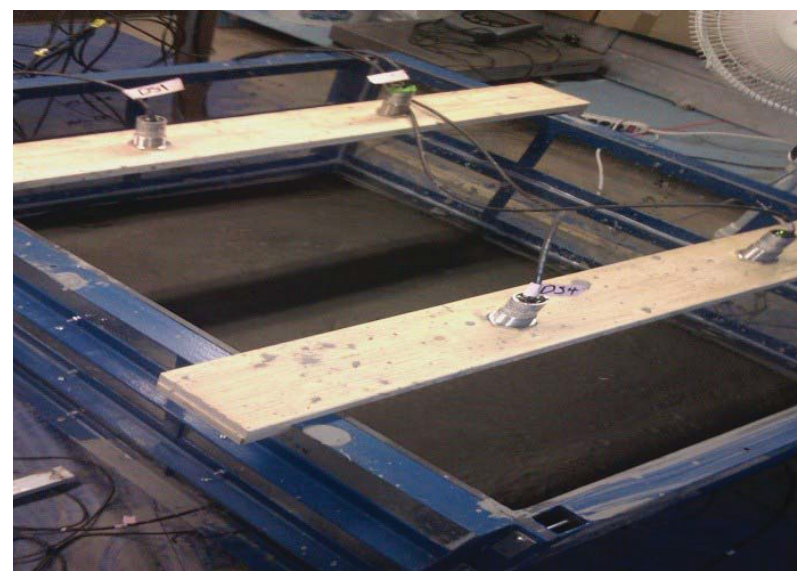

Day 4

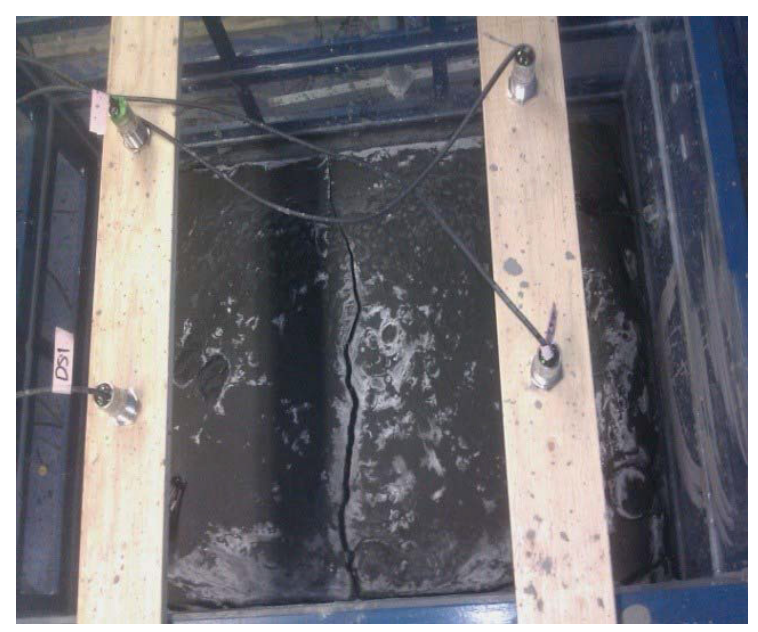

Day 7

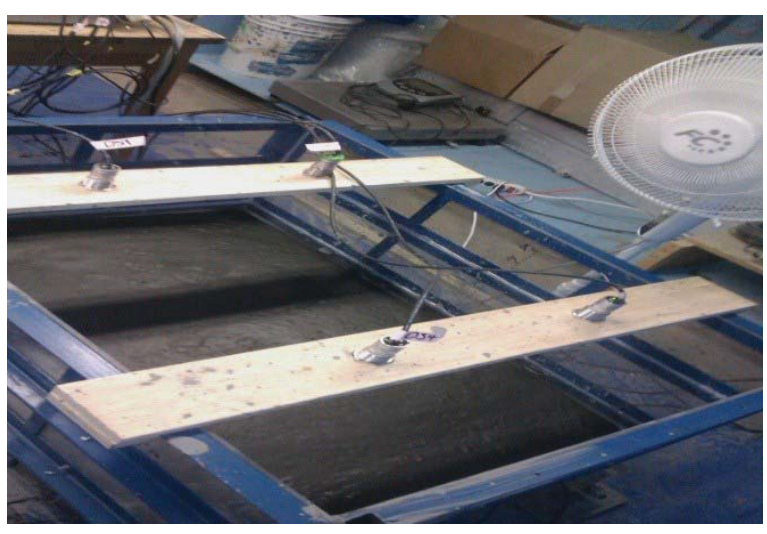

Day 3

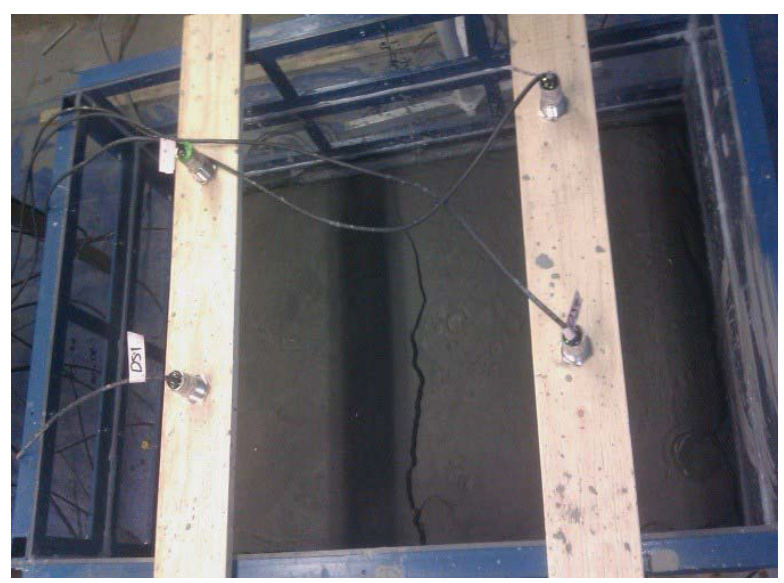

Day 5

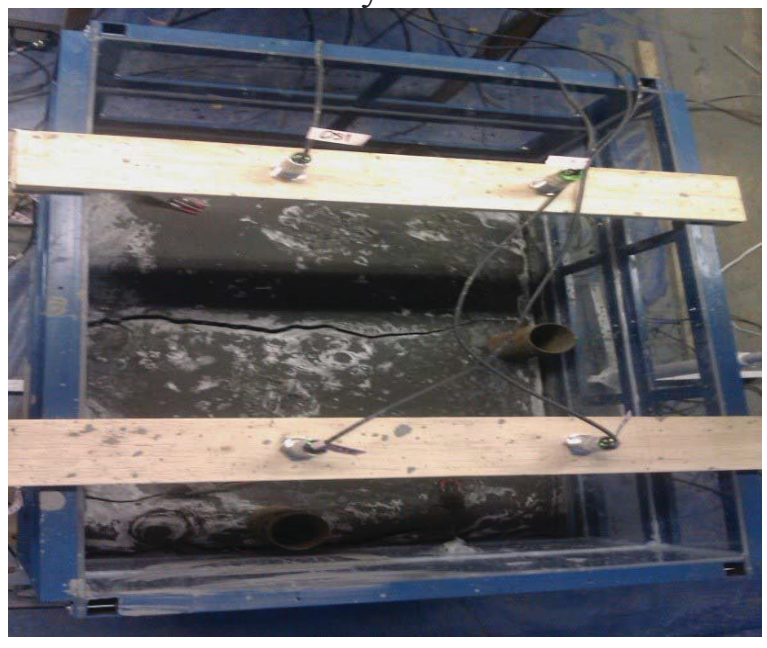

Day 8

Figure 4.7 Desiccation process of the first layer deposition 
attributed not only to increase in matric suction but also increase in osmotic suction because of the appearance of salts. This issue can be confirmed (Figure 4.6b) by measuring the total suction of the high salty area and comparing it with low salty area. It is clear that the total suction value of high salty area is around $3000 \mathrm{kPa}$, which is very higher than the total suction value of low salty area (around $1700 \mathrm{kPa})$.

Figure 4.6 also shows that samples from $1 \mathrm{~mm}$ surface have higher total suction comparing to deeper samples $(3 \mathrm{~mm})$. Figure 4.7 presents pictures of desiccation process of the first layer. It is clear that at day 1 , there was a layer of water on top of the settled tailings. A large crack appeared in the middle of the layer at day 5 . With continuing desiccation to days 7 and 8 , salty areas also appeared on the surface. Tubes prepared for the second layer deposition are also visible at day 8 .

\subsection{Second layer deposition}

A fresh second layer was deposited on top of the desiccated first layer at day 9. Figure 4.8 presents drainage, evaporation and water content variation with time after the deposition of the second layer. Since the second layer was deposited on top of the desiccated first layer, the rate of drainage after deposition is substantially lower than that of the first layer. Figure $4.8 \mathrm{a}$ shows that the rate of drainage decreases with increasing desiccation and stops at day 15 . The rate of evaporation in the first day of deposition of the second layer (day 9) was around $8.1 \mathrm{~mm} / \mathrm{day}$. Figure $4.8 \mathrm{~b}$ shows that the rate of evaporation decreases to $7.8 \mathrm{~mm} /$ day in the second day of deposition (day 10). The rate of evaporation decreases more rapidly in the second day of 

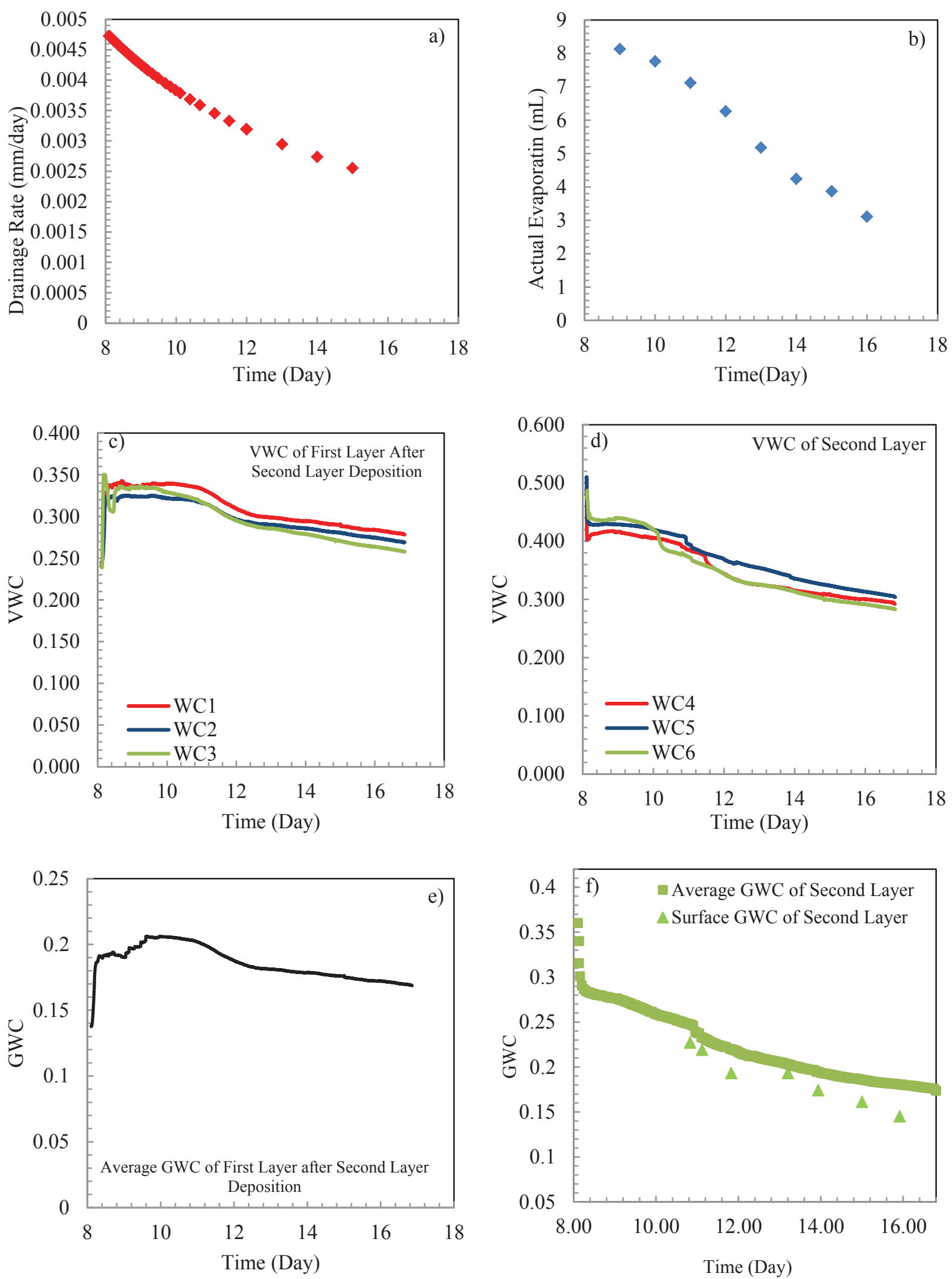

Figure 4.8 Drainage, evaporation and water content results of second layer deposition 
deposition of the second layer in comparison to the second day of deposition of the first layer. This rapid reduction could be attributed to the absorption of the water from the top layer by the desiccated first layer. Figures $4.8 \mathrm{c}$ and $4.8 \mathrm{e}$ present VWC and average GWC of first layer after deposition of the fresh second layer. It is clear that the average GWC of the first layer increases to around $20.6 \%$ after deposition of the second layer. In other words, the GWC of the first layer did not increase to higher value than $20.6 \%$, which means that although the bottom layer got saturated, the void ratio of the desiccated-rewetted first layer does not increase significantly. In fact, the swelling potential of desiccated-rewetted tailings is not remarkable. Figures $4.8 \mathrm{~d}$ and 4.8f present the VWC and average GWC of fresh second layer after deposition of the second layer. It is clear that unlike the first layer, the average GWC of second layer drops rapidly from around $37 \%$ to $29 \%$ after deposition. This rapid reduction also confirms that the desiccated bottom layer quickly absorbs the water from the fresh second layer. It is also shown that reduction of water content to $\mathrm{GWC}=16.9 \%$ takes longer in comparison to the first layer. This longer drying time could be attributed to longer time required to dewater two layers than one layer. Figure $4.8 \mathrm{f}$ also shows the values of surface GWC obtained by taking samples from the surface of the second layer. As expected, the values of surface GWC are $1-2 \%$ lower than the average GWC. However, the trend of reduction is somehow in agreement with average GWC.

Figure 4.9 presents electrical conductivity, temperature and void ratio results after deposition of the second layer. Figure 4.9a shows the variation of temperature in the second layer after deposition. Figure $4.9 \mathrm{~b}$ shows bulk electrical conductivity of the second layer after deposition. It is clear that the bulk electrical conductivity decreases rapidly with decreasing water content after deposition. Figure $4.9 \mathrm{c}$ also shows the bulk electrical conductivity of the first layer after deposition of the second layer. It can be seen that the bulk electrical conductivity of the first 

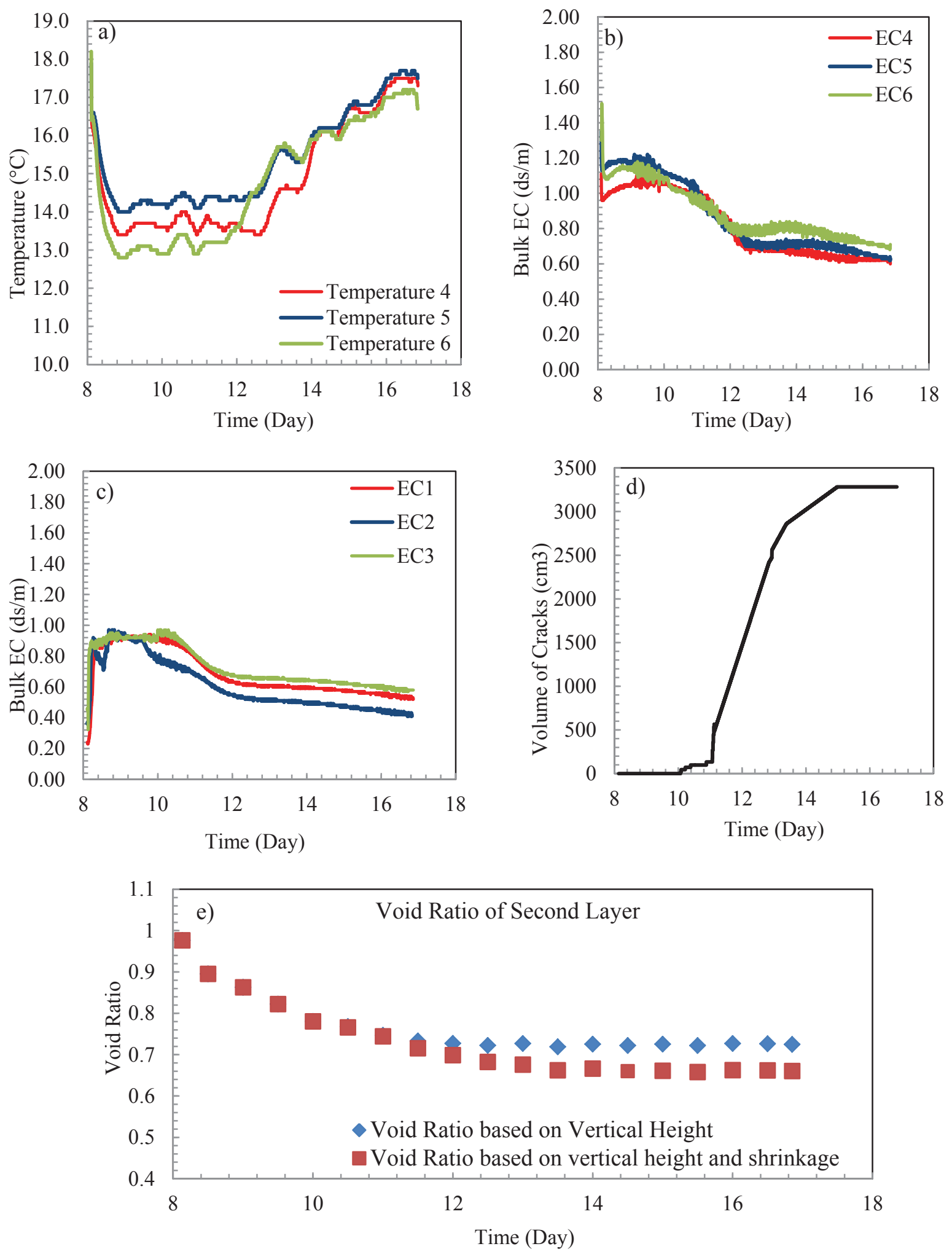

Figure 4.9 Electrical conductivity, temperature and void ratio results after second layer deposition 
desiccated layer increases by rewetting with a fresh layer. The electrical conductivity decreases again during desiccation of the first and second layer. Figure 4.9d shows approximate volume of cracks after second layer deposition. The volume of cracks increased quickly from day 11 to day 13. After passing shrinkage limit, the volume of cracks did increase significantly and remained at a fairly constant value. Figure $4.9 \mathrm{e}$ presents variation of void ratio of the second layer after deposition based on vertical height and both vertical and horizontal shrinkage. Clearly, the void ratio decreases after deposition significantly up to day 11 . After day 11 when cracks appeared, obviously the void ratio calculated using vertical height and shrinkage is different from that based on just vertical height.

Figure 4.10 demonstrates matric and total suction results after deposition of the second layer. Figure 4.10a shows matric suction recorded by the three tensiometers in the first layer after the deposition of the fresh layer. It is shown that matric suction of bottom layer becomes zero or very close to zero. One of the tensiometers showed zero matric suctions and two others exhibited $2.9 \mathrm{kPa}$ and $3.9 \mathrm{kPa}$ after rewetting, which means that the bottom layer becomes fairly saturated after depositing the fresh second layer. Figure 4.10c shows the values of matric suction of the second layer after deposition. It can be seen that matric suction remains zero up to day 10 for two of the tensiometers. From day 10 to day 12 the matric suction increases up to around $100 \mathrm{kPa}$ for tensiometer 5 and $86 \mathrm{kPa}$ for tensiometers 4 and 6 before cavitation. Figure $4.10 \mathrm{~d}$ presents total suction results of the samples obtained from the surface of the second layer and measured by WP-4. It is visible that similar to the first layer; total suction values close to cracks are remarkably higher than other values. It is also shown that with increasing degree of desiccation (reducing water content), the values of total suction increases. It should be noted that the values of water contents correspond to water content of the surface of the second layer after taking 

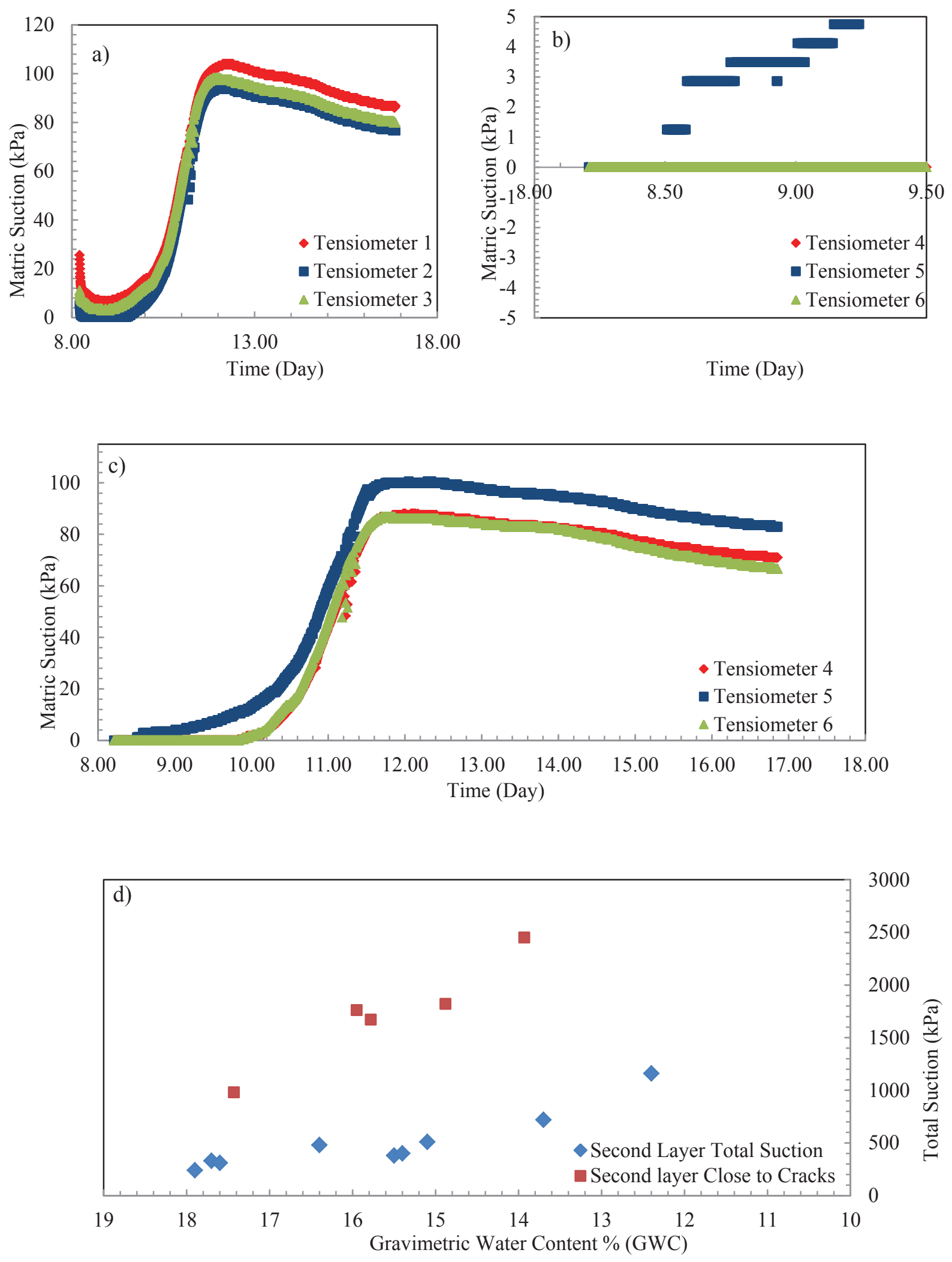

Figure 4.10 Matric and total suction results after deposition of second layer 


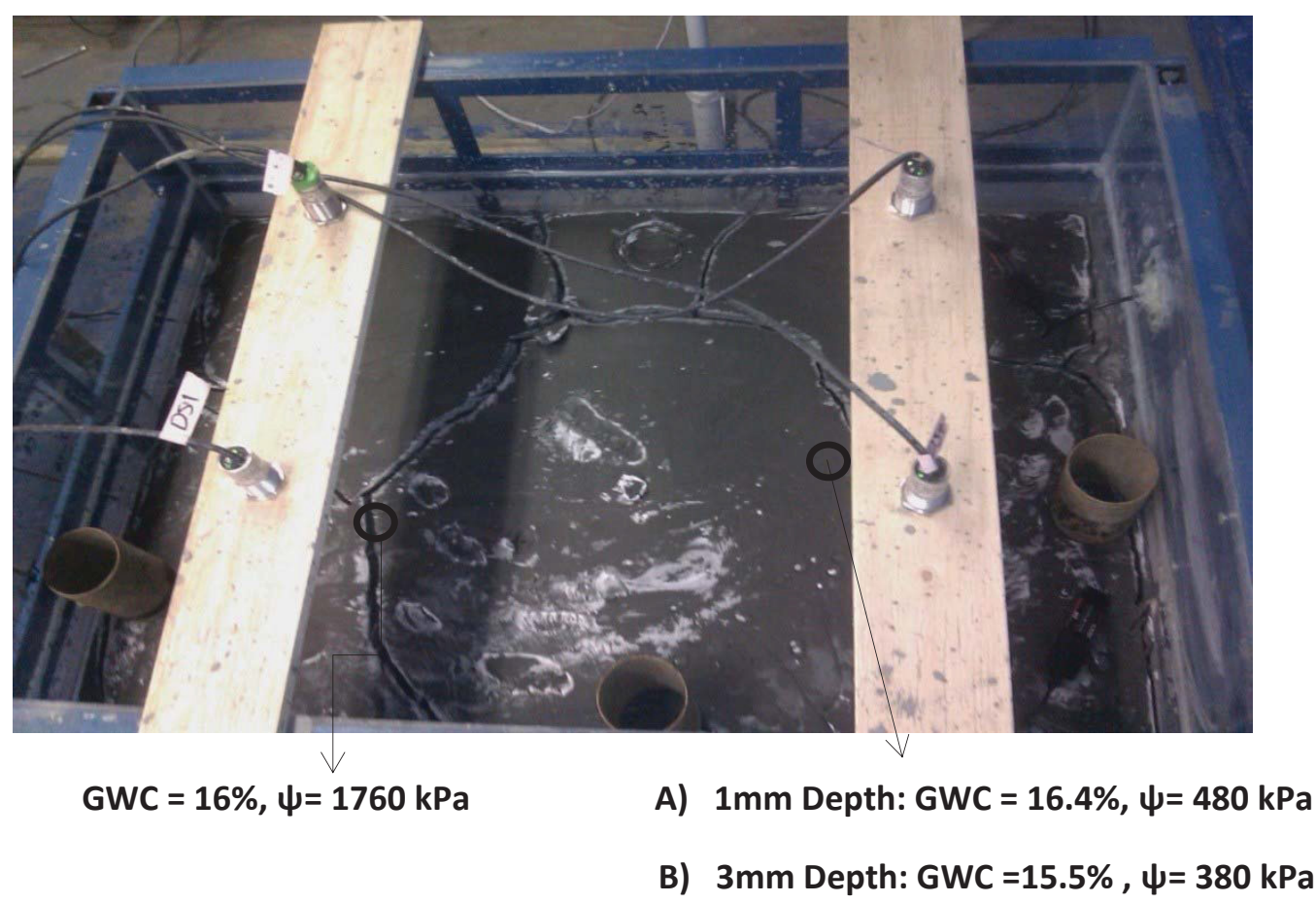

Figure 4.11 Total suction results close and not close to cracks

samples, which are lower than average water contents of the second layer. Figure 4.11 compares the total suction values of samples close to cracks of the surface of the second layer with samples not close to cracks. Similar to the first layer, the total suction values of samples close to cracks are significantly higher than samples not close to cracks.

Figure 4.12 shows the process of desiccation on the surface of the second layer. It is clear that the height of water on top of the desiccated layer did not remain the same as the first layer. This fact is attributed to absorption of the water from the fresh layer by the desiccated first layer. Figure 4.13 also presents the evolution of cracks on the surface of the second layer during desiccation. It can be seen that with increasing degree of desiccation, not only the thickness of cracks increases, but also more salt appeared. 


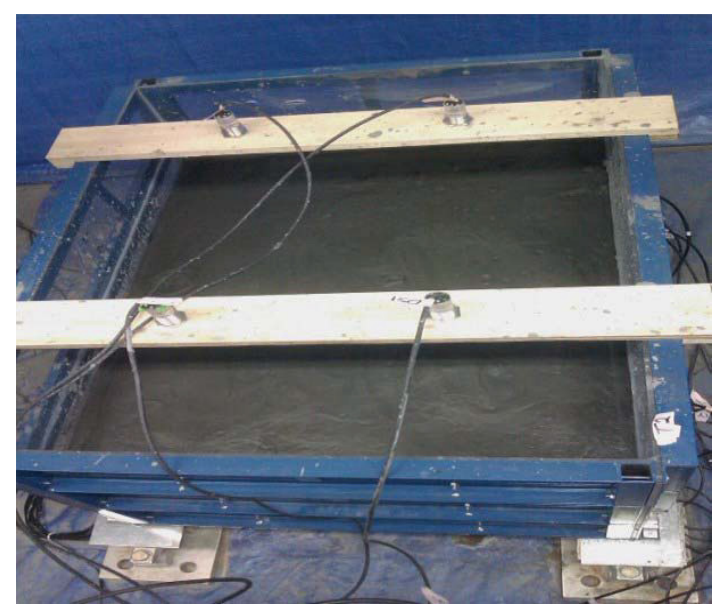

Day 10

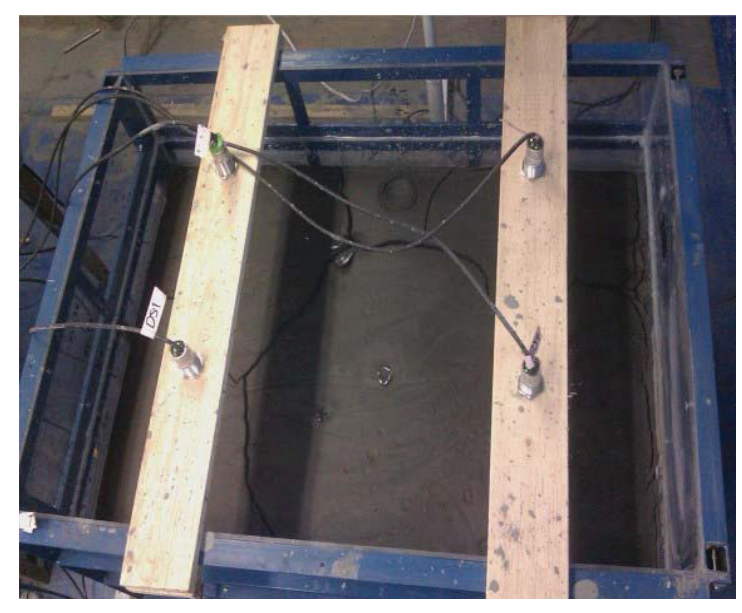

Day 12

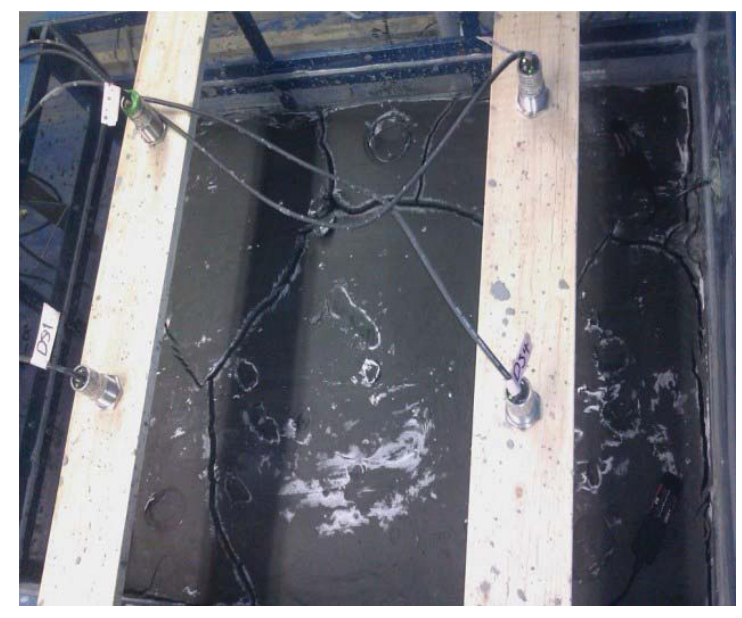

Day 14

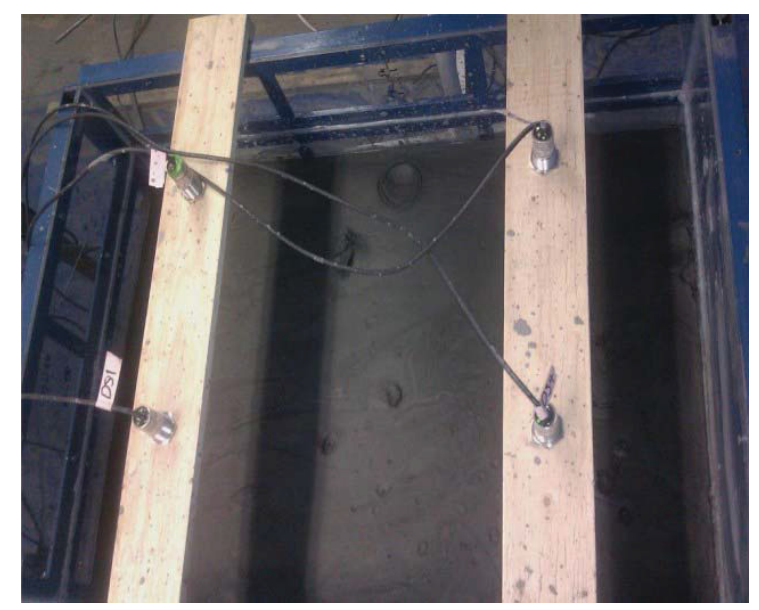

Day 11

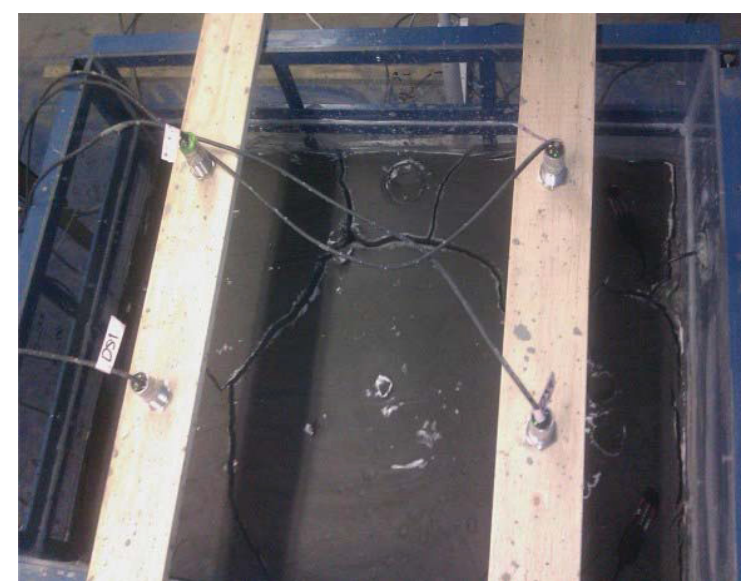

Day 13

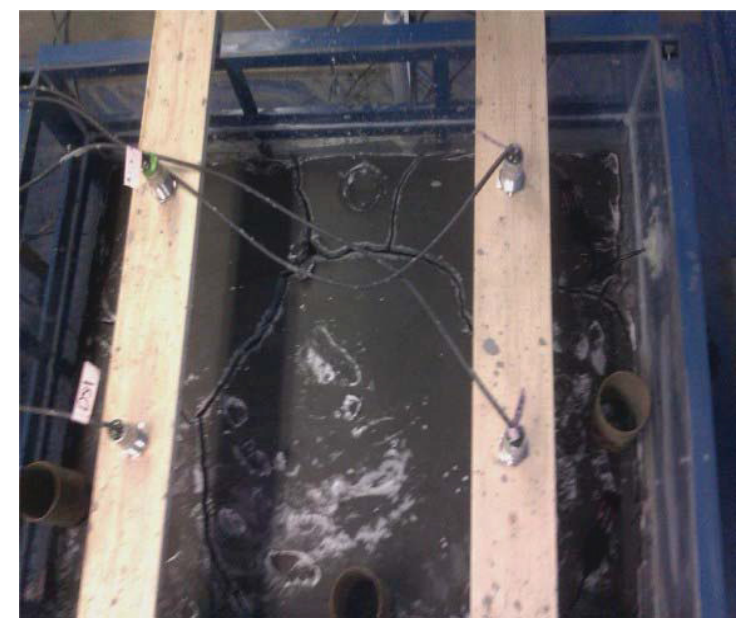

Day 16

Figure 4.12 Desiccation process in the second layer deposition 

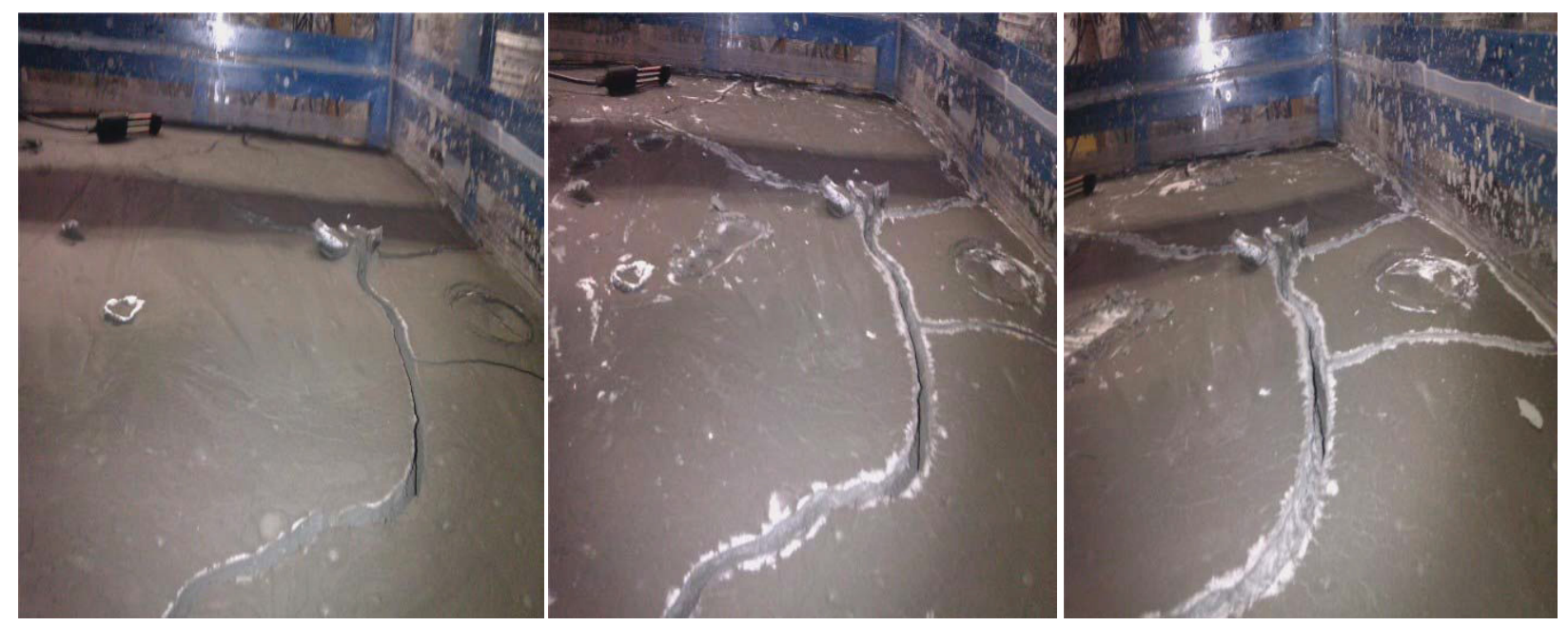

Figure 4.13 Evolution of cracks on the surface of the second layer during desiccation

on the surface of the cracks. The higher concentration of salt results in higher osmotic suction, which finally increases the value of total suction.

\subsection{Third layer deposition}

Third layer was prepared and deposited on top of the desiccated second layer at day 16. Figure 4.14 presents evaporation and water content results after the third layer deposition. The rate of actual evaporation (Figure 4.14a) starts at around $8.5 \mathrm{~mm}$ /day and reached $2.3 \mathrm{~mm} /$ day by the end of the desiccation process. Figure $4.14 \mathrm{~b}$ demonstrates VWC results of the first layer after depositing the fresh third layer. It can be seen that the VWC increases to around $33 \%$ and starts to decrease again at day 19. Figure 4.14c shows the variation of VWC of the fresh deposited third layer during desiccation. GWC results after third layer deposition are shown in Figures 4.14d to 4.14f. Figure 4.14f shows that the GWC of the first layer after depositing fresh third 

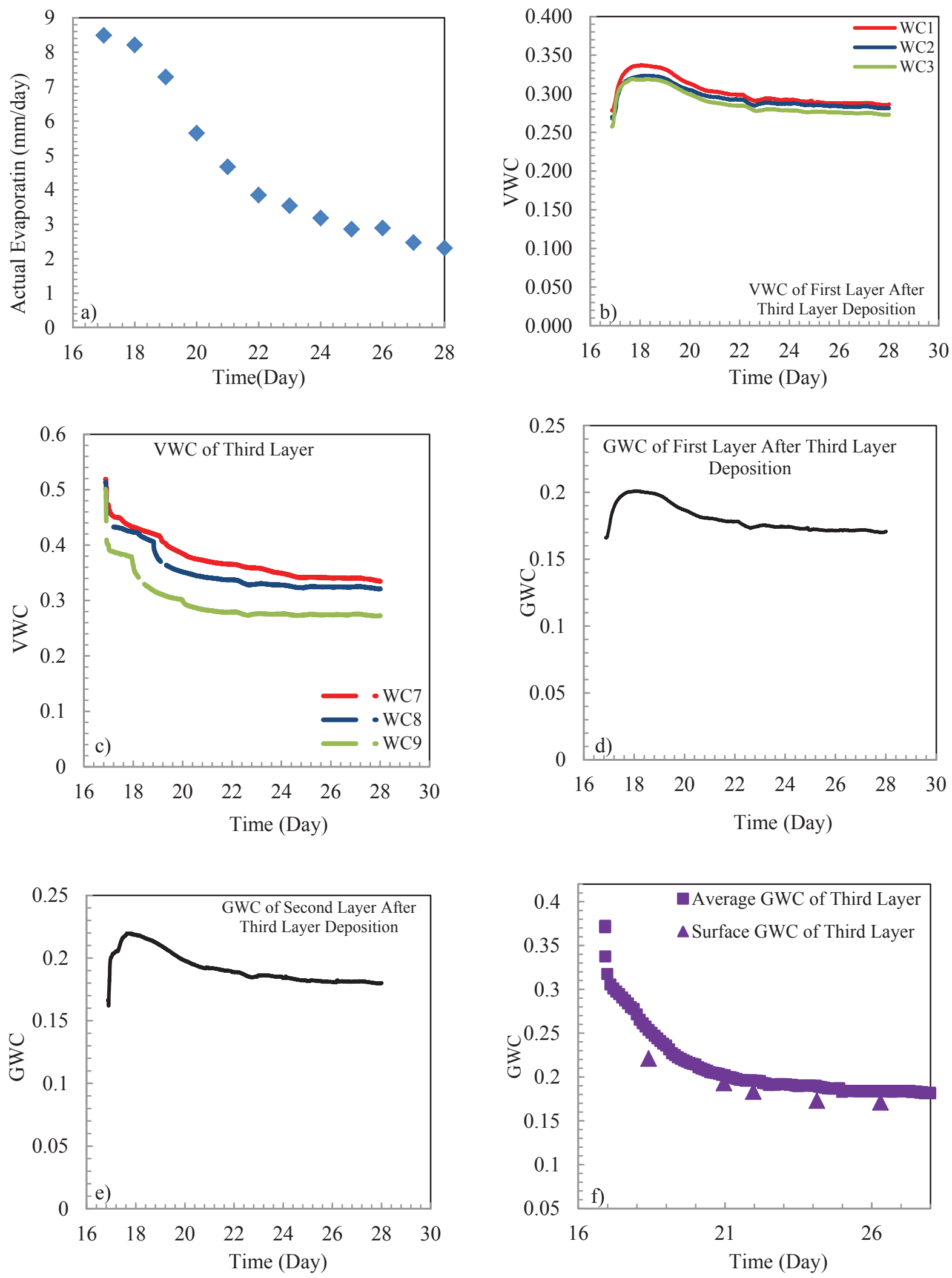

Figure 4.14 Evaporation and water content results after deposition of third layer 
layer increases to around 20\%. The GWC starts to decrease at day 19 and reached around $17.1 \%$ at day 28. The GWC of the second layer after depositing third layer also increased to around $22 \%$. Similar to the second layer deposition, the GWC of the bottom layers after rewetting do not increase beyond 22\%. The average GWC and surface GWC of the fresh third layer are presented in Figure 4.14f. It is clear that the desiccation process of the third layer continued to around $\mathrm{GWC}=18.2 \%$. The GWC of the third layer decreases from $31.5 \%$ at day 17 to $18.2 \%$ at day 28 . The rapid reduction of the water content after deposition is similar to the second layer and is attributed to absorption of water from the fresh layer by desiccated bottom layers. In addition, it is clear that the time required to reach shrinkage limit is higher than first and second deposition. For example, in the first layer deposition, the time required to reach shrinkage limit after deposition was around 5 days. This time was measured around 11 days for the third layer deposition, which is significantly longer than the first layer. Since the thickness of the layers was fairly the same, this difference could be attributed to absorbing water from the bottom layers in the upward direction. More simply, after deposition of the third layer, absorbing water from the fresh layer led to increasing the water content of bottom layers up to GWC $\sim 22 \%$. Continuing desiccation of the third layer led to upward water flux from the bottom layers to the third layer. In fact, as the third layer dewaters, the removed water is substituted by the water coming from the bottom layers. This process makes the drying time of the fresh layer longer. Consequently, in addition to hydrological factors, layer thickness of the fresh layer and numbers of desiccated bottom layers will also govern the drying time required to reach optimum water content.

Temperature, electrical conductivity and void ratio results after deposition of the third layer are shown in Figure 4.15. According to Figure 4.15a, the temperature of the third layer decreased 

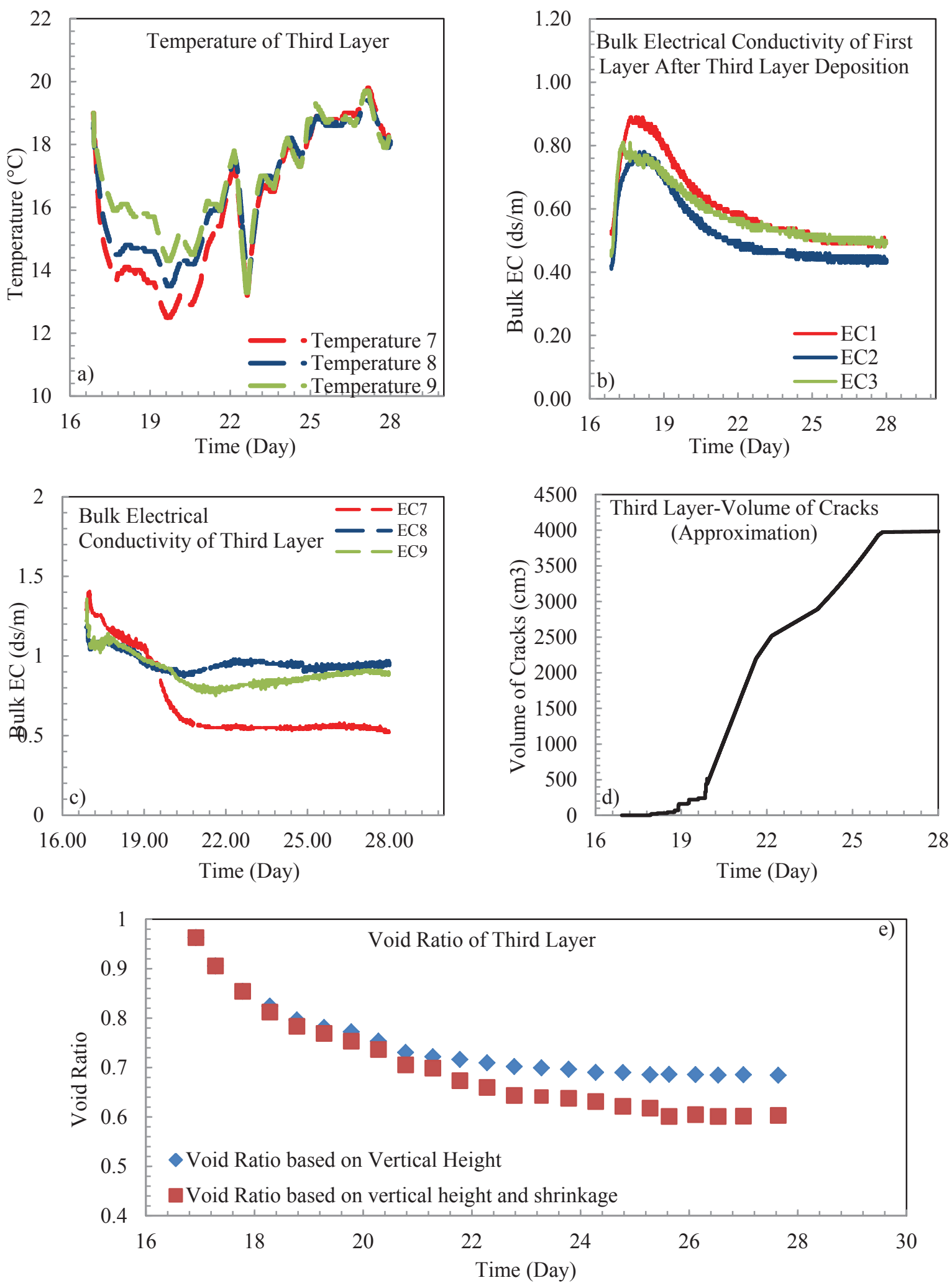

Figure 4.15 Temperature, electrical conductivity and void ratio results after third layer deposition 
after deposition. The fluctuation in the temperature could be attributed to day and night times and higher or lower outside temperature as well. The bulk electrical conductivity of the first layer after deposition of the third layer increases up to day 18 . The electrical conductivity decreases continuously after day 18 until the end of desiccation process. Figure $4.15 \mathrm{c}$ shows that the bulk electrical conductivity of the third layer after deposition continuously decreases during the process of desiccation. Electrical conductivity has inverse correlation with osmotic suction and as expected the osmotic suction increases with desiccation. Figure $4.15 \mathrm{~d}$ demonstrates that cracks appeared at day 18 and the volume of cracks increases up to day 26 when the water content reaches shrinkage limit. Beyond this point, there is no tangible increase in the volume of cracks. Figure $4.15 \mathrm{e}$ also presents the variation of the void ratio with increasing degree of desiccation. It is shown that both void ratio based on vertical height and void ratio based on vertical height and shrinkage are the same up to day 18 when no cracks appeared. After day 18 , the cracks appeared and void ratio based on vertical height and shrinkage becomes lower than void ratio based on vertical height. It is also clear that the time required to reach shrinkage limit is longer in the third layer in comparison to the previous layers, which is associated with slow reduction of water content in the third layer in comparison to the previous layers.

Figure 4.16 presents the matric suction results after the third layer deposition. Figure 4.16a shows the matric suction results of the first layer after deposition of the fresh third layer. It can be seen that just one of the tensiometers reached zero matric suction after rewetting. Two others exhibited reduction in matric suction up to $10 \mathrm{kPa}$ but not lower than this value. Consequently, it is quite possible for a bottom layer not to become saturated after rewetting by the fresh top layer especially if the bottom layer is much deeper than the fresh layer. The matric suction results of 

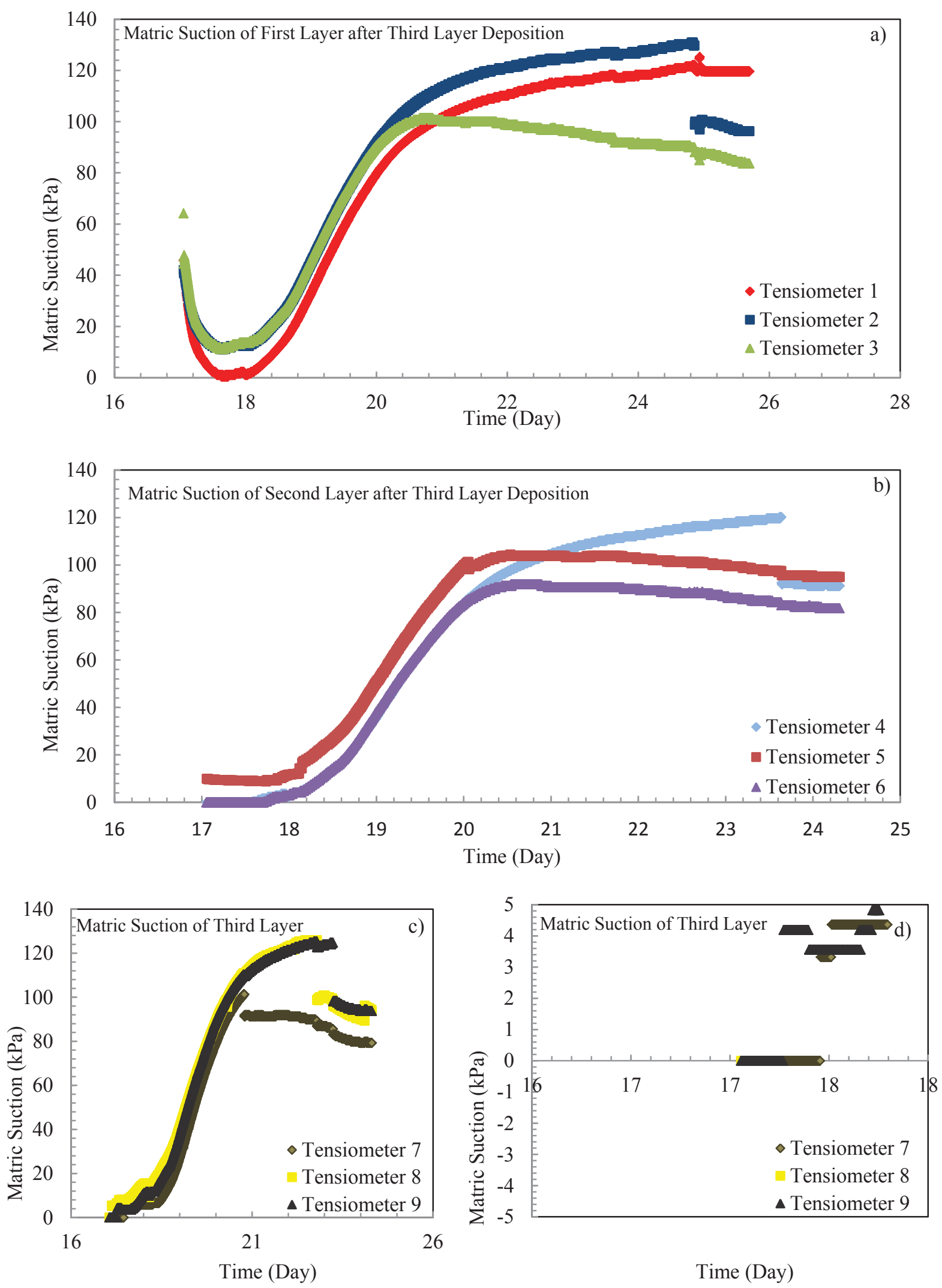

Figure 4.16 Matric suction results after third layer deposition 
the second layer after deposition of the third layer are presented in Figure 4.16b. It can be seen that two of the tensiometers exhibited zero matric suction after rewetting and one of them showed around $10 \mathrm{kPa}$ matric suction. Obviously, the second layer is less deep than the first layer and much closer to the deposited fresh layer. Therefore, the possibility of reaching zero matric suction is higher in the second layer than the first layer. Figure 4.16c presents the values of matric suction of the third layer after deposition. It is clear that all tensiometers required more time to reach the $\mathrm{AEV}$, which confirms the slow desaturation of the third layer in comparison to previous first and second layer deposition. For example, it took only 2 days to reach AEV for the first layer deposition (Figure 4.5a); however, the time required to reach AEV for the third layer was around 3-4 days. Figure 4.17 also presents total suction results of the third layer obtained by WP-4 total suction measurement device. Total suction values of the samples not close to cracks increase to around $500 \mathrm{kPa}$ during desiccation process. Similar to the previous layers, samples close to cracks showed significantly high total suctions than samples not close to cracks, which is attributed to higher osmotic suction due to high salt concentration. However, the difference between total suction values of the samples close to cracks and samples not close to cracks is more significant than previous layers.

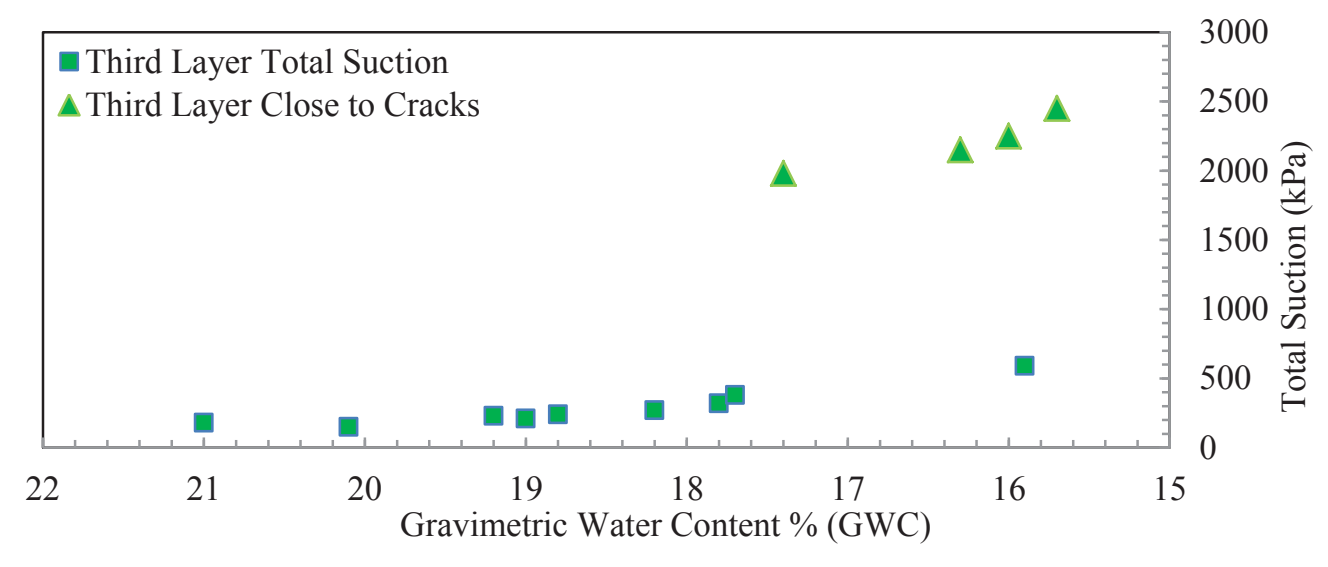

Figure 4.17 Total suction results after third layer deposition 
Figure 4.18 shows the desiccation process of the third layer after deposition. Day 17 shows fresh tailing at 38\% after deposition and before settling. A crack in the middle of the layer appeared in Day 19. Day 28 shows the end of desiccation process, and the tubes that are inserted to be buried by deposition of the fourth layer.

\subsection{Fourth layer deposition}

Fourth layer was prepared at $38 \%$ water content and deposited on top of the desiccated layer at day 28. The thickness of the fourth layer was $140 \mathrm{~mm}$, which was smaller than previous three layers. The thickness of this layer was selected to be lower in order to evaluate the effect of layer thickness on the drying time of the deposited tailings.

Figure 4.19 shows evaporation and water content results after the fourth layer deposition. Figure 4.19a presents the actual evaporation rates of the fourth layer during the desiccation process. The actual evaporation rate started from $7.7 \mathrm{~mm} /$ day at day 29 and decreased to $1.37 \mathrm{~mm} /$ day by the end of the desiccation process. The lower initial actual evaporation in comparison to previous layers is attributed to lower thickness of the fourth layer. Figure 4.19b shows VWC of the fourth layer after deposition. Rapid reduction of water content after deposition is clear for three VWC sensors. VWC of other layers after deposition of fourth layer can be found in appendix I. Figures 4.19c to 4.19f present average GWC results after fourth layer deposition. It is clear that the GWC of all bottom layers increases to $19.5 \%$ to $20.5 \%$ after deposition of the fresh fourth layer.

Obviously, since the first layer is the deepest layer, the GWC of the first layer increases to a lower value (19.5\%). Figure $4.19 \mathrm{f}$ demonstrates the average GWC of the fourth layer after 


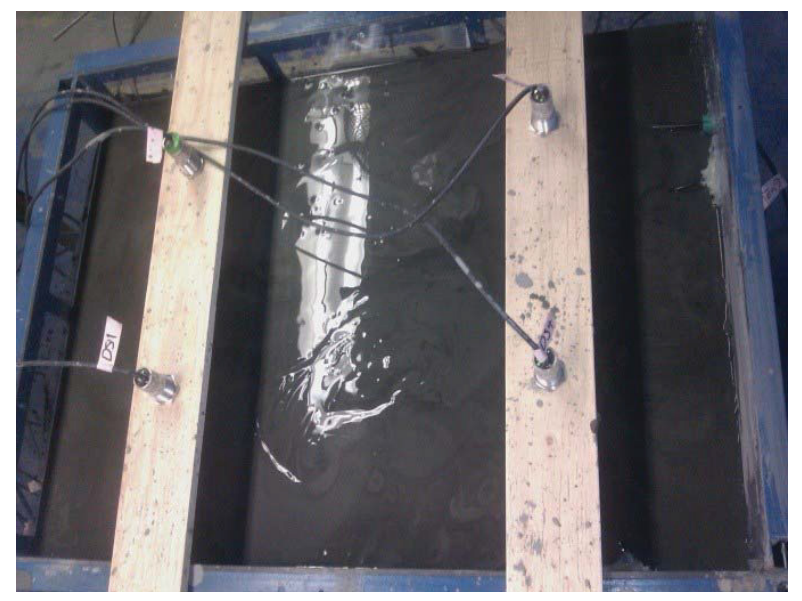

Day 17

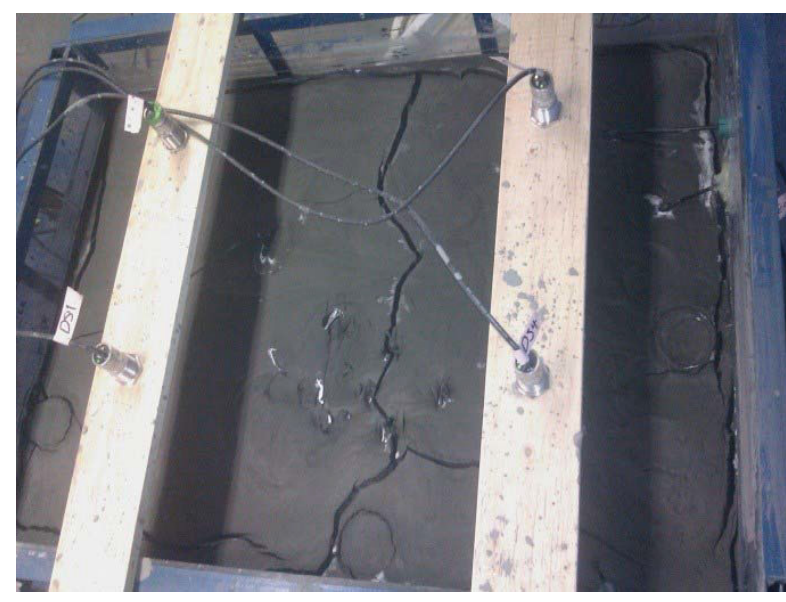

Day 20

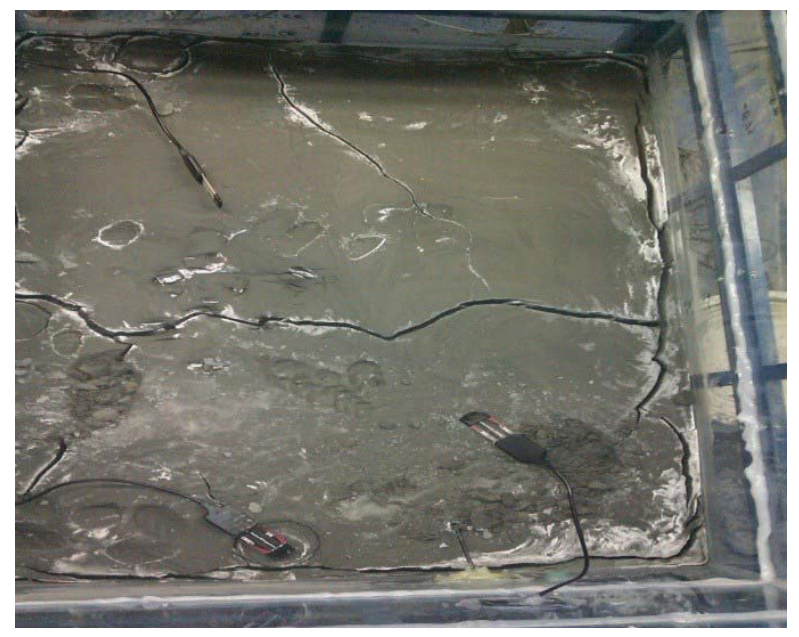

Day 26

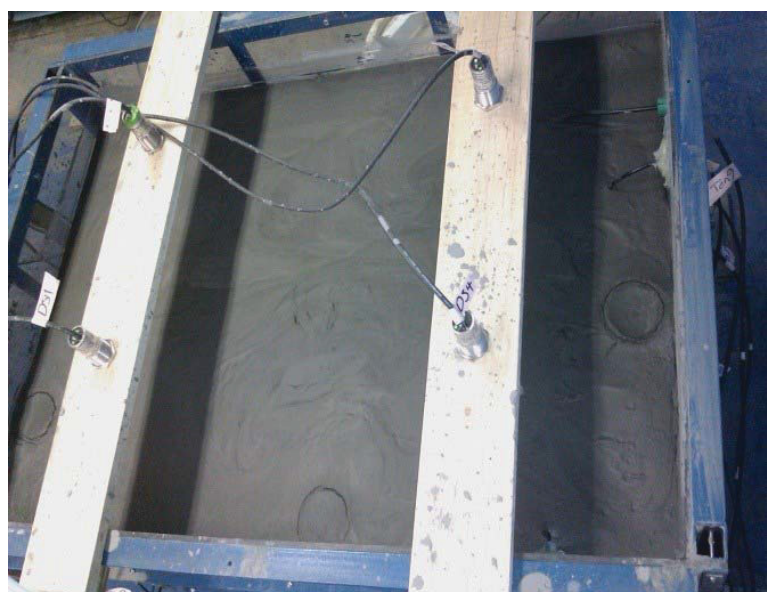

Day 18

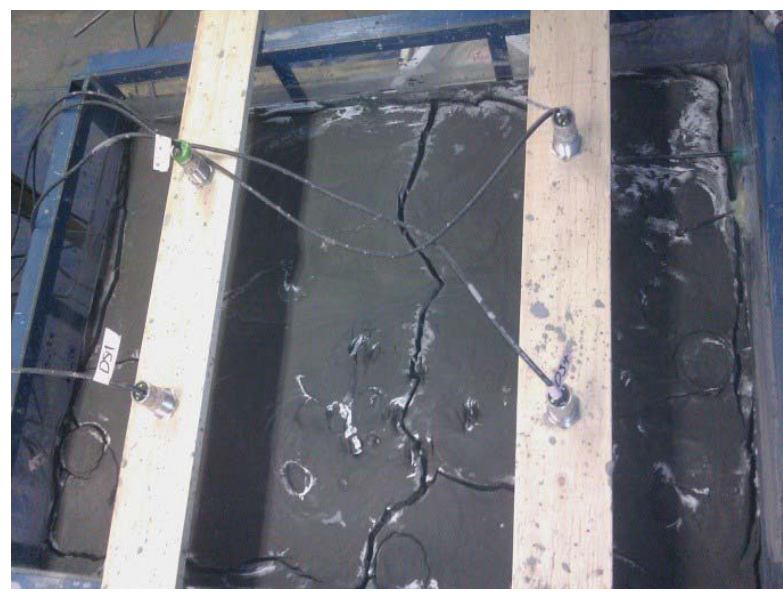

Day 22

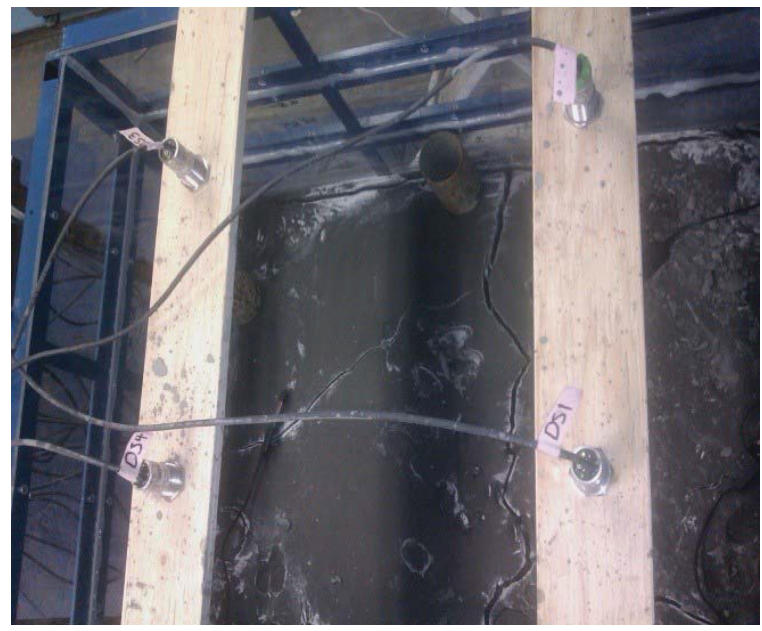

Day 28

Figure 4.18 Desiccation process of the third layer deposition 

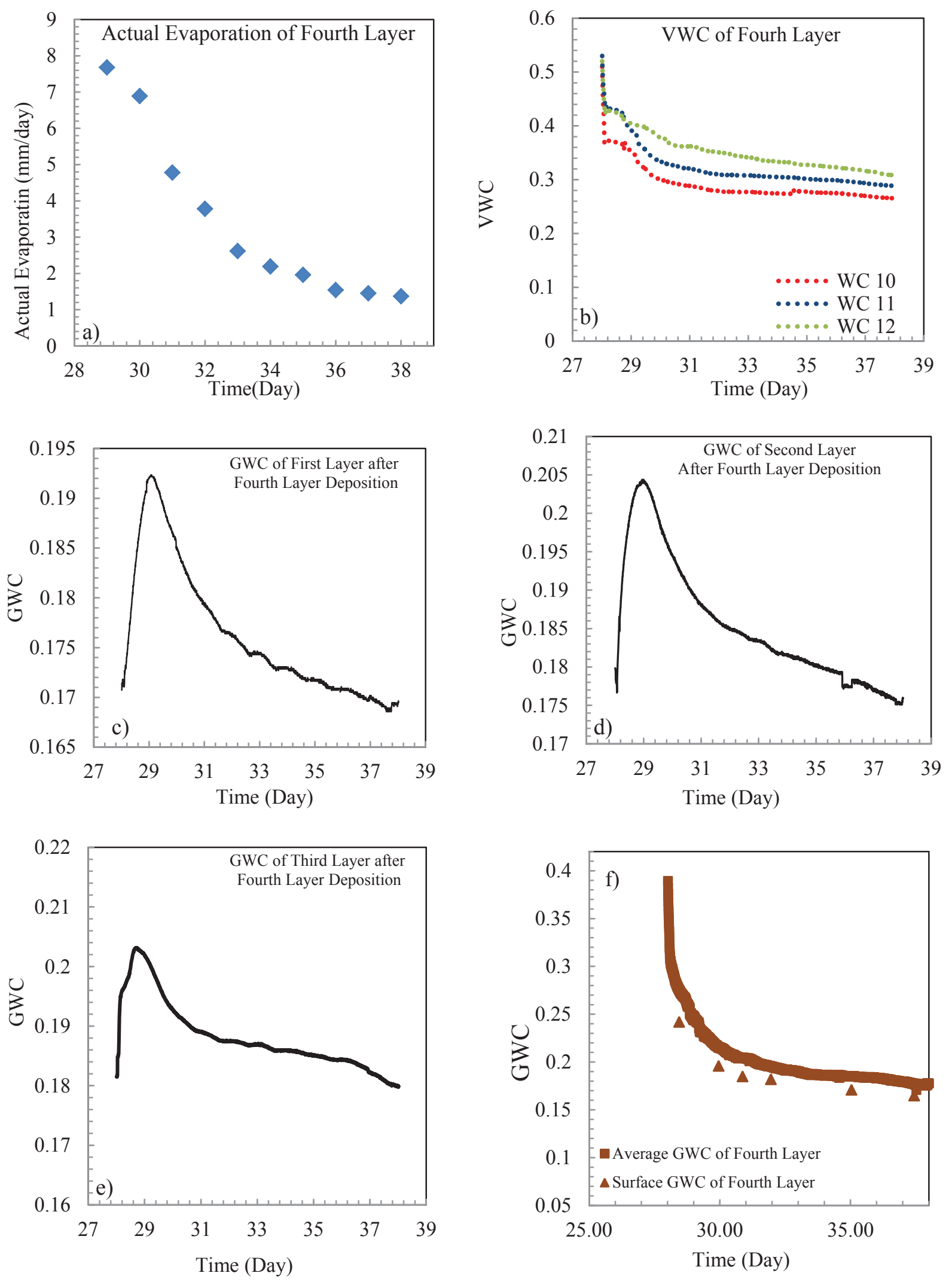

Figure 4.19 Evaporation and water content results after deposition of fourth layer 
deposition. The GWC of the fourth layer decreased quickly from around $38 \%$ to $29 \%$ at day 29 . This rapid reduction is attributed to absorption of water by the three desiccated bottom layers. After day 29, the reduction of GWC became gentler and continued up to $17.7 \%$ GWC at day 38 . Figure 4.19f also shows the GWC of samples obtained from the surface of the fourth layer were lower (1-2\%) than the average GWC; but decreased in a same trend with the average GWC. The time required to reach SL after deposition of the fourth layer was 10 days, which is shorter than the third layer (11 days) and longer than the second (8 days) and first layers (5 days). Although there were more desiccated soils at the bottom for the fourth layer than the third layer, which would be expected to increase the drying time to SL due to absorption of water by the bottom layers, the smaller thickness of the fourth layer made the drying time shorter. On the other hand, this smaller thickness could not alleviate the effect of water absorption by the bottom layers on drying time to SL compared to the first and second layers. In other words, although the fourth layer has smaller thickness, more bottom layers made the drying time longer than first and second layers with larger thickness due to the fact that the bottom layers provided the water for the top layer and made the drying time longer.

Figure 4.20 presents temperature, electrical conductivity and void ratio results after fourth layer deposition. Figure 4.20a shows temperature of the fourth layer after deposition. The temperature decreased after deposition to around $13^{\circ} \mathrm{C}$. Variations of temperature for other layers can be found in Appendix I. Figure 4.20b and 4.20c present variations of bulk electrical conductivity of the first layer and the fourth layer respectively after deposition of the fourth layer. Rewetting the first layer increased the bulk electrical conductivity, which is associated with decreasing osmotic suction. The electrical conductivity decreased during the desiccation process. Figure 4.20d shows 

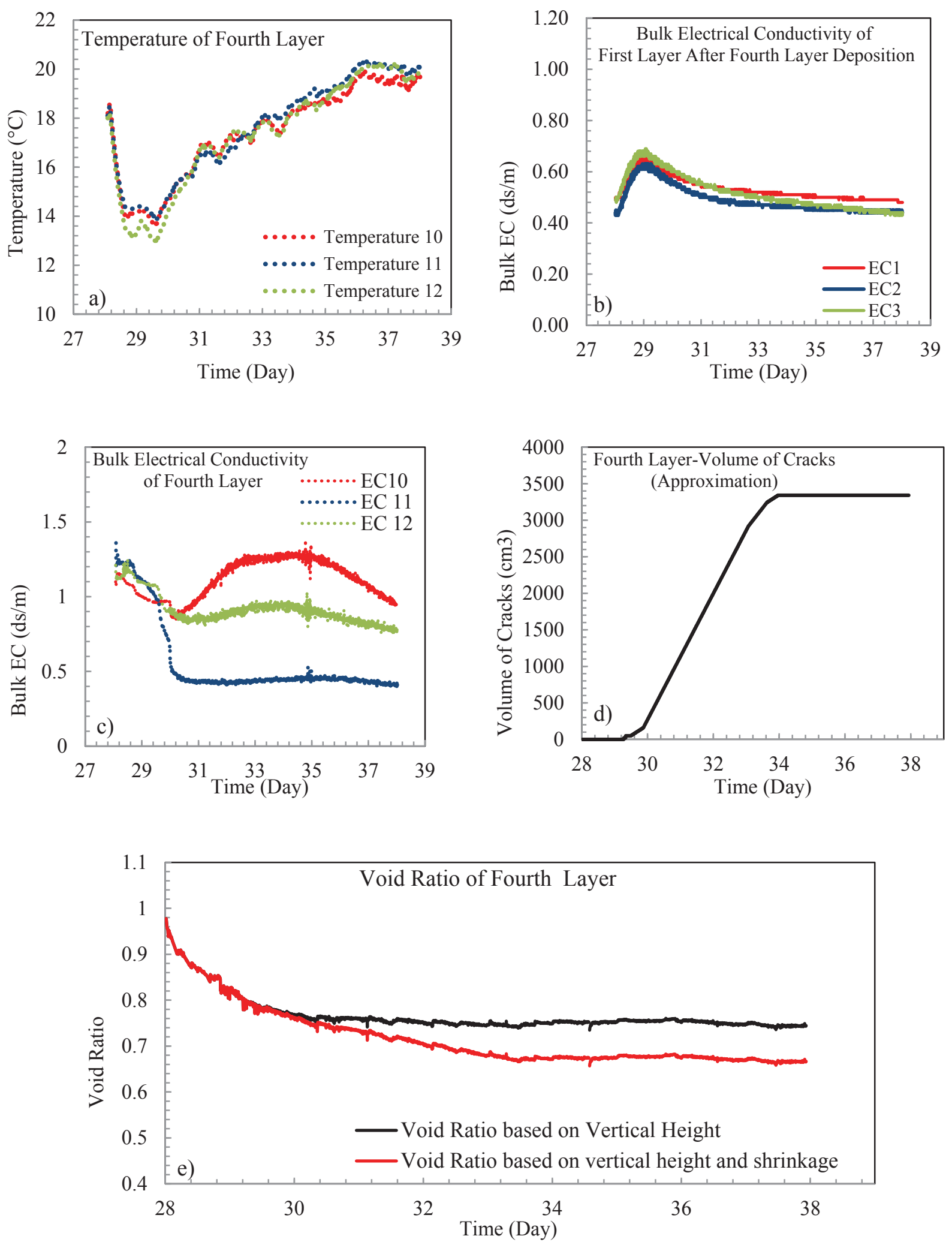

Figure 4.20 Temperature, electrical conductivity and void ratio results after third layer deposition 
the volume of cracks during the desiccation process. Cracks appeared at day 30 and the volume of them increased significantly from day 30 to day 34 . There was no tangible increase in the volume of cracks after day 34 . Figure $4.20 \mathrm{e}$ presents the variation of void ratio during desiccation process. The void ratio based on vertical height and void ratio based on vertical height and shrinkage are the same before appearance of cracks at day 30 . The ultimate value of void ratio at the end of desiccation process obtained around 0.67 considering vertical height and shrinkage.

Figure 4.21 shows matric suction results after fourth layer deposition. Figure 4.21a shows matric suction results of the first layer after deposition of the fourth layer. It is clear that none of the tensiometers reached zero matric suction and lowest matric suction exhibited varied between 15 $\mathrm{kPa}$ and $32 \mathrm{kPa}$. Since the first layer is the deepest layer and is not close to the fresh fourth layer, it did not become completely saturated. Similarly, none of the tensiometers of the second layer showed zero matric suction according to Figure $4.21 \mathrm{~b}$. Therefore, the second layer did not get fully saturated. The tensiometers inside the second layer showed a matric suction value between $10 \mathrm{kPa}$ to $20 \mathrm{kPa}$, which is a lower value than the first layer. In other words, as the layer becomes deeper, the matric suction after rewetting by a fresh layer would be higher. Figure $4.21 \mathrm{c}$ shows the matric suction values of the fourth layer after deposition of the fourth layer. It is also clear that all tensiometers in all layers became cavitated at day 31 or day 32 . Figure 4.22 presents the total suction results of the surface of the fourth layer obtained by the WP-4 device. With decreasing GWC, the total suction values became higher. Similar to previous layers, samples close to cracks exhibited higher total suction values due to high osmotic suction values caused by high concentration of salts close to cracks. Figure 4.23 clearly shows evolution of cracks and high concentration of salt close to them on the surface of the fourth layer. Figure 4.24 also shows 

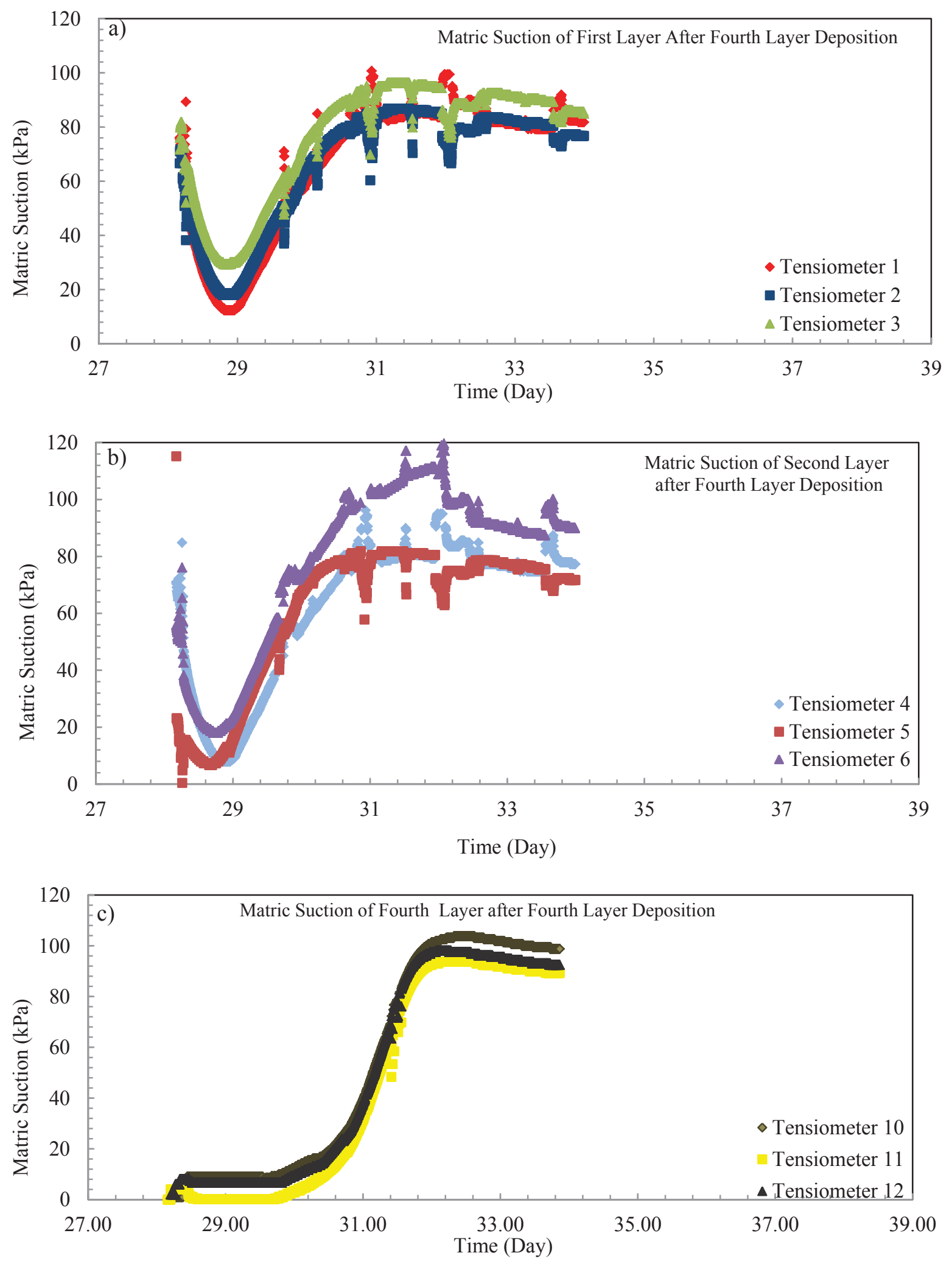

Figure 4.21 Matric suction results after fourth layer deposition 


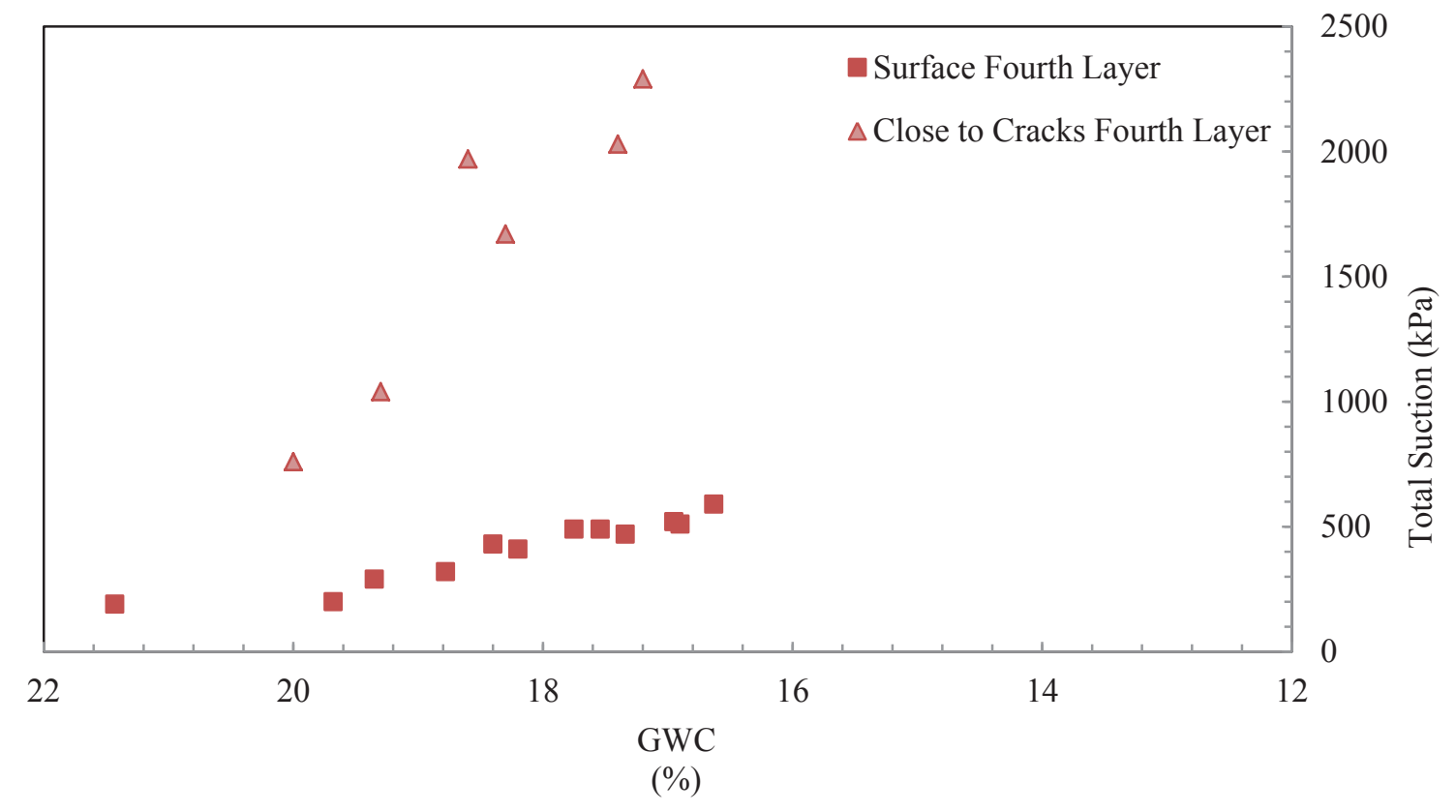

Figure 4.22 Total suction results after fourth layer deposition
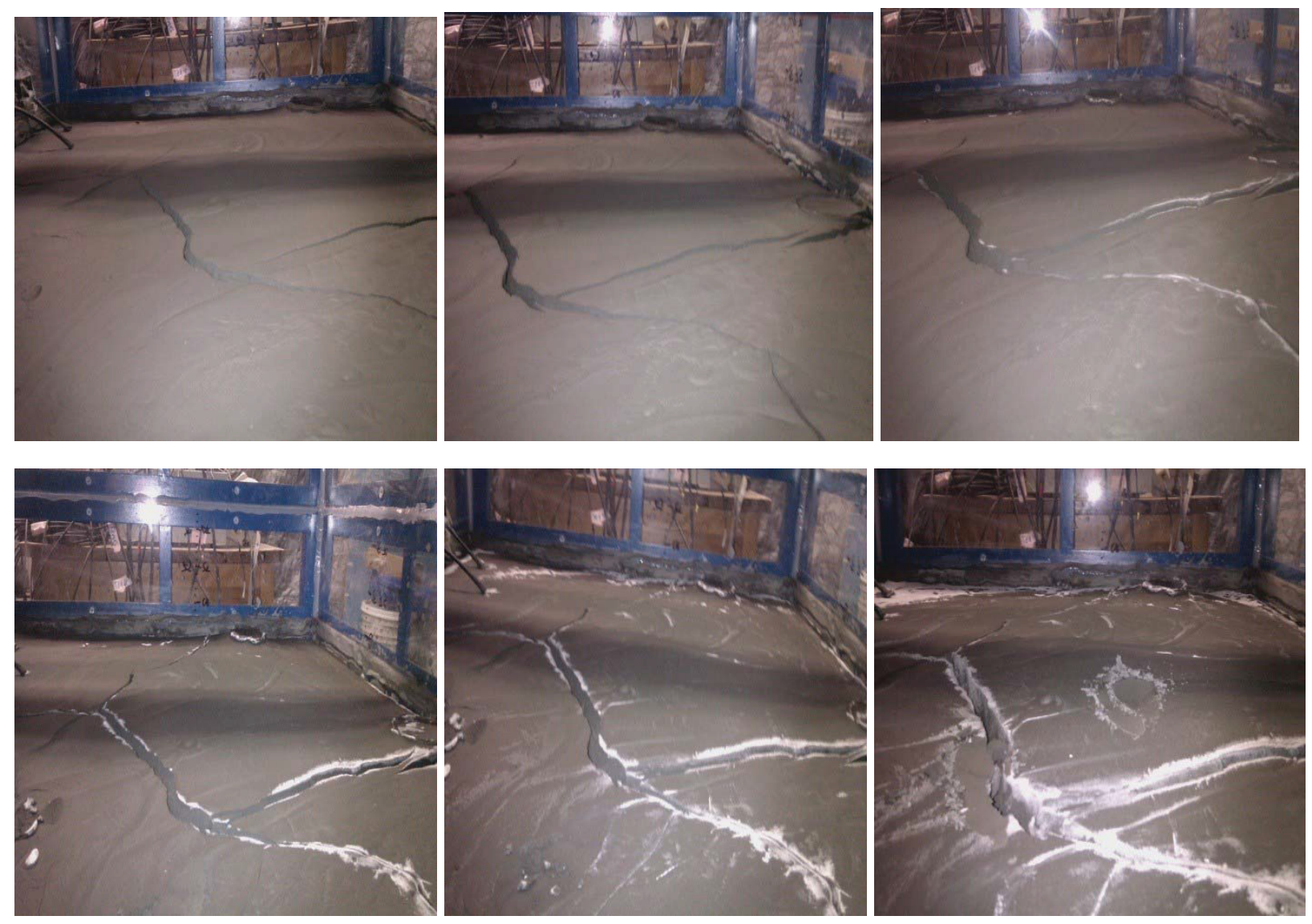

Figure 4.23 Evolution of cracks on the surface of the fourth layer 


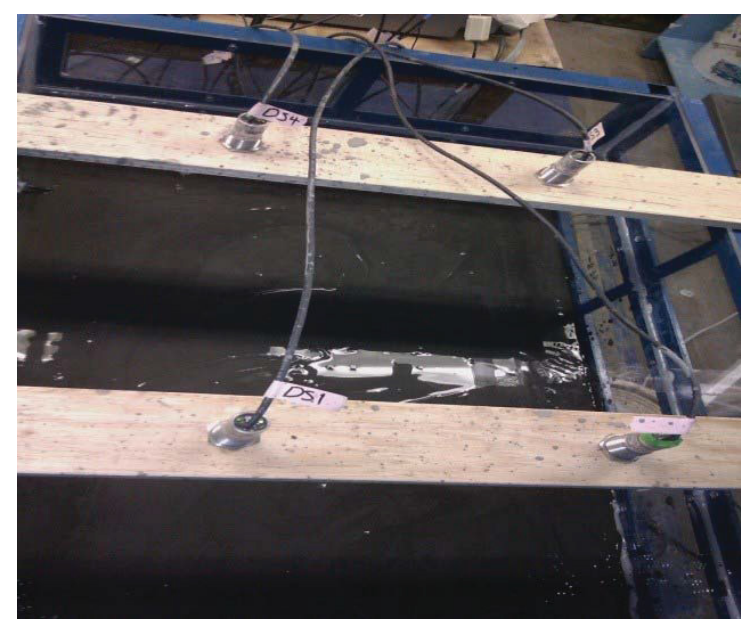

Day 29

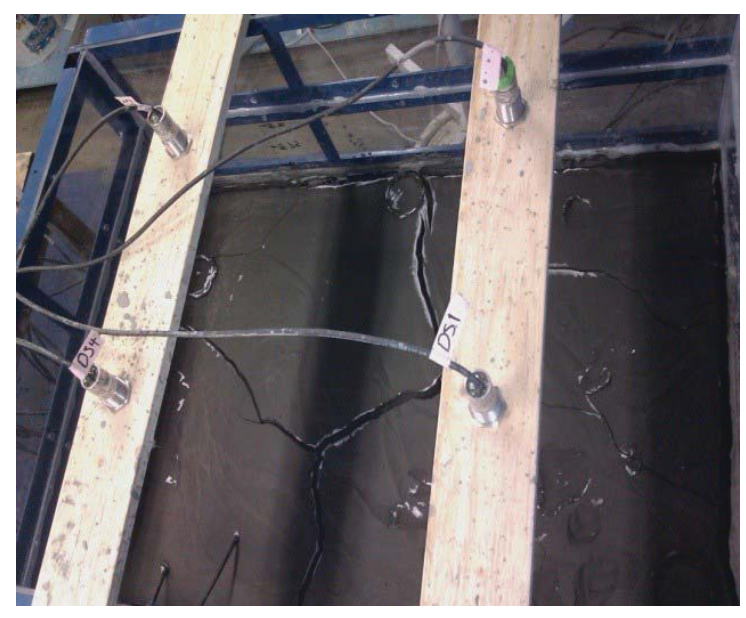

Day 33

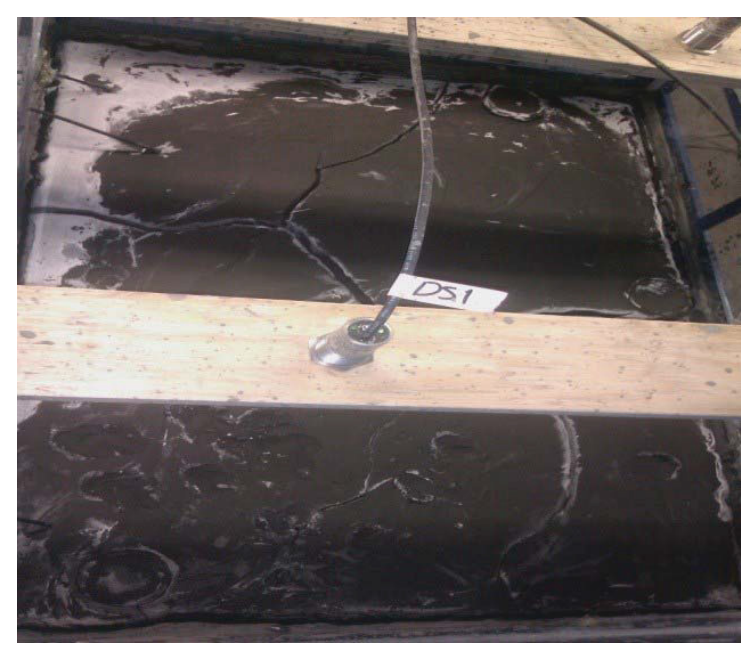

Day 36

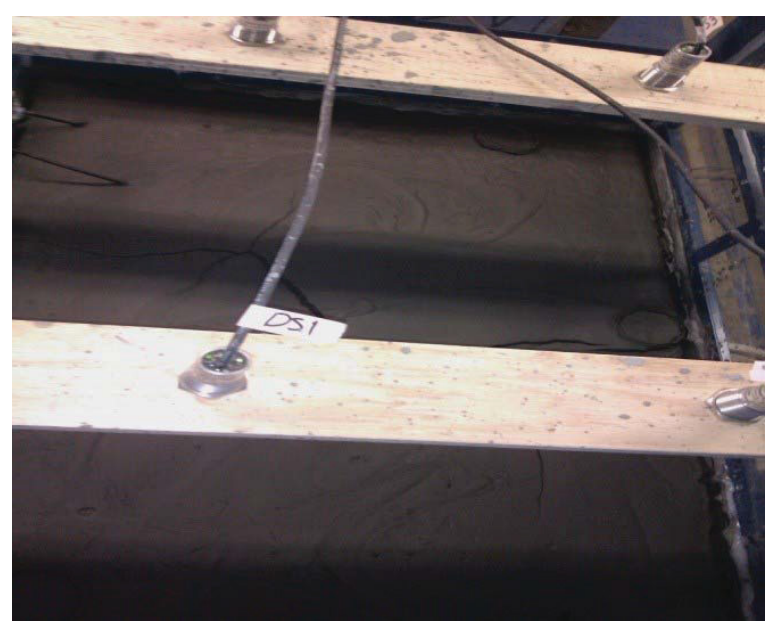

Day 30

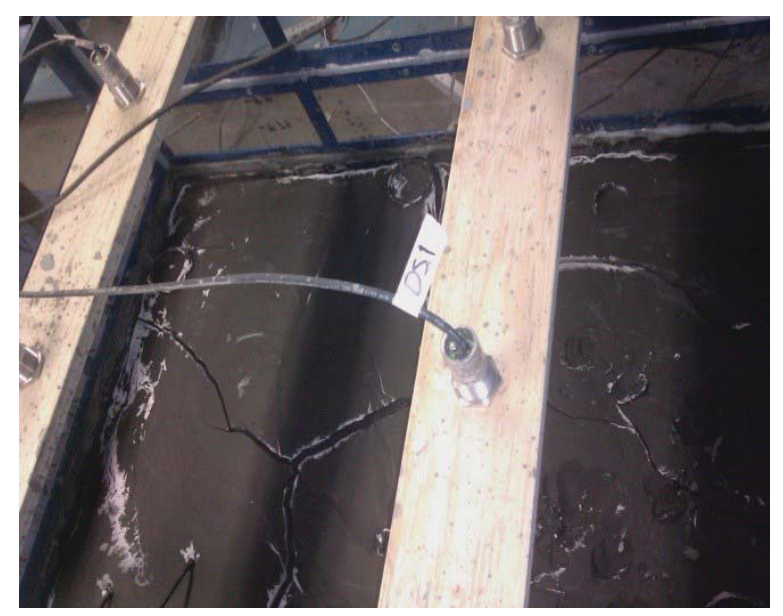

Day 34

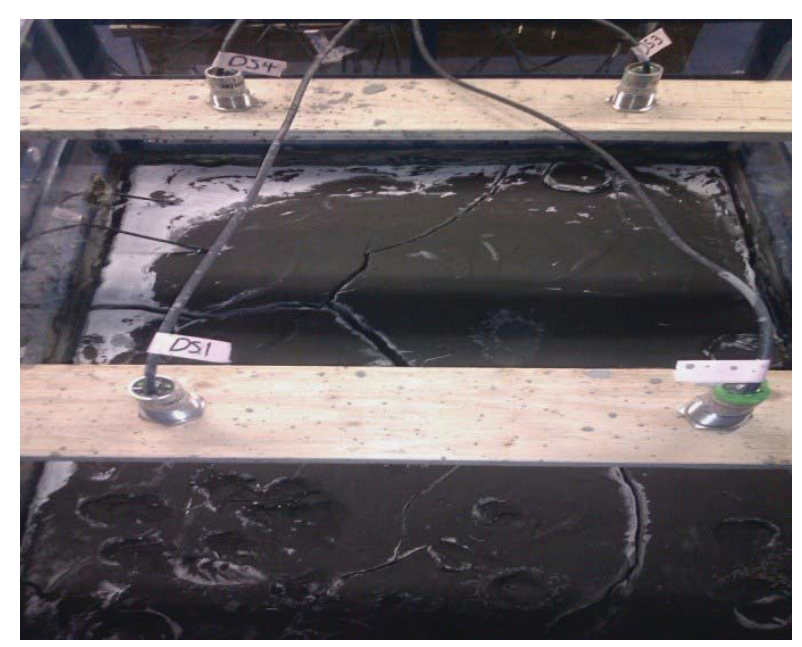

Day 37

Figure 4.24 Desiccation process of the fourth layer deposition 
desiccation process on the surface of the fourth layer.

\subsection{First and second re-saturation}

Two consecutive re-saturations were conducted on top of the desiccated fourth layer. The purpose of two re-saturations was to simulate light and heavy rainfalls. The first rainfall simulation was performed at day 38 by controlling fast re-saturation $(400 \sim 500 \mathrm{~mm} /$ day for 2 hours) to simulate a heavy rainfall. The second re-saturation was applied at day 49 to simulate a light rainfall with the rate of $25 \sim 50 \mathrm{~mm}$ /day for 9 hours). Similar to the first re-saturation, the saturated fourth layer was allowed to desiccate to the shrinkage limit.

Figure 4.25 presents water content results after first and second re-saturations. It is clear that all layers became rewetted and the water content for all layers increased to GWC $=20 \%$ to $22 \%$. The rates of decreasing water content for both re-saturations were fairly similar. Figure 4.26 also shows the rate of actual evaporation for first and second re-saturations. It is clear that in both resaturations, the rate of evaporation starts from $5 \sim 5.2 \mathrm{~mm} /$ day and continued during desiccation up to $1.5 \sim 1.6 \mathrm{~mm} /$ day. Therefore, the evaporation rates for both first and second re-saturations were fairly similar.

The results of first and second re-saturations show no tangible difference in the desiccation process of the first and second re-saturation tests. In other words, as long as the bottom layer becomes saturated (matric suction is alleviated), rate of re-saturation does not affect the desiccation process. 

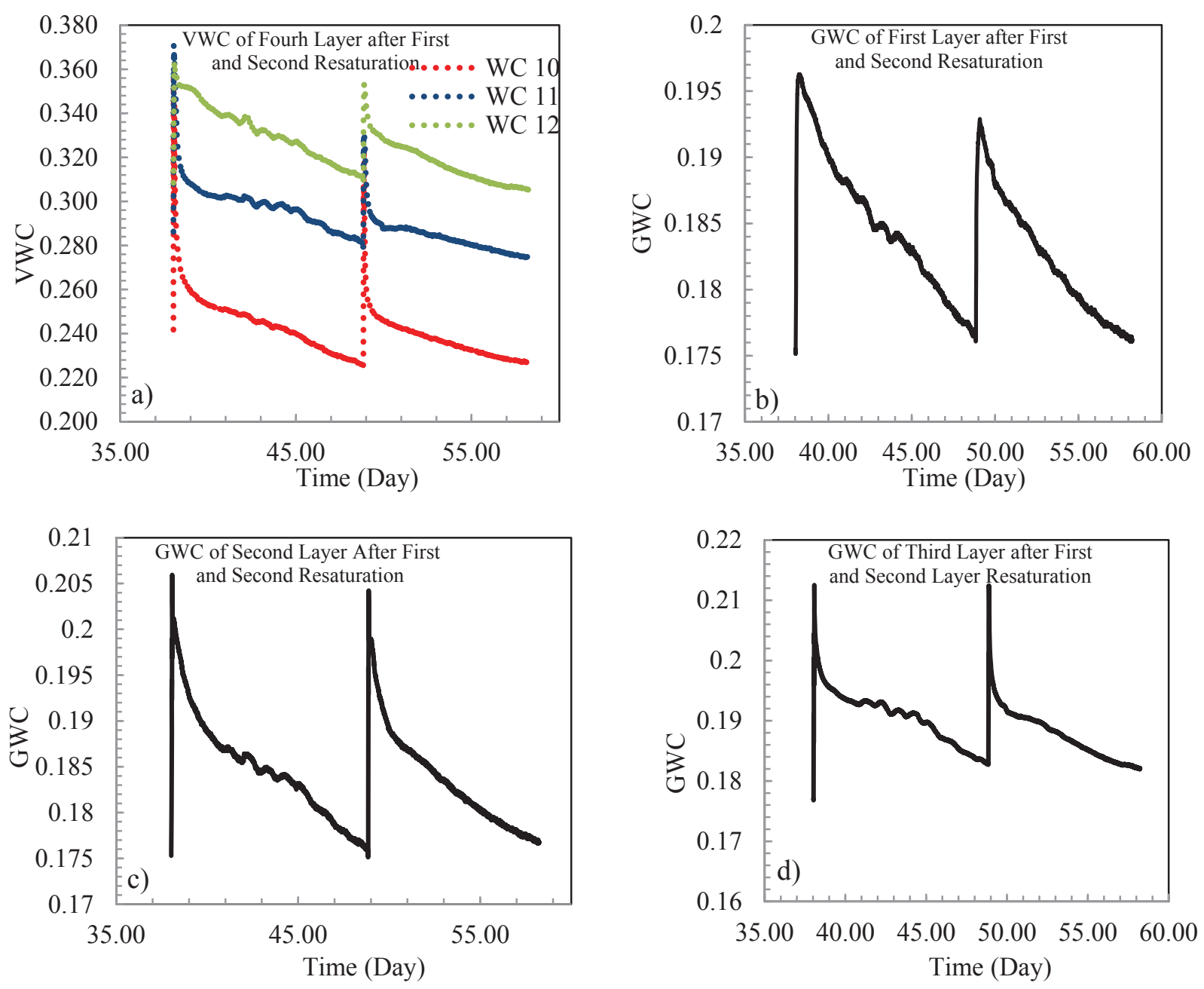

Figure 4.25 Water content results after first and second re-saturations

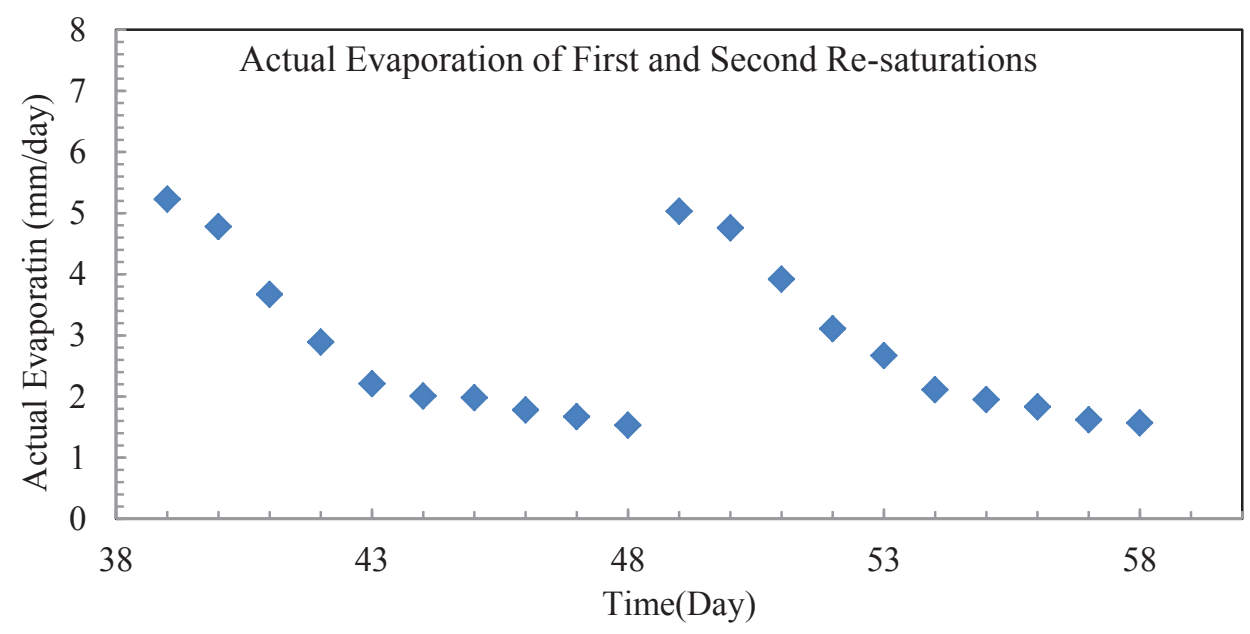

Figure 4.26 Actual evaporation rates of first and second re-saturations 


\subsection{Fifth layer deposition}

Before depositing the fifth layer, the desiccated fourth layer was re-saturated. After re-saturation and confirming that all tensiometers showed zero matric suction, the fifth layer was deposited. Figure 4.27 shows the surface of the fourth layer after re-saturation prior to the fifth layer deposition. The purpose of re-saturation before deposition of the first layer was to prevent absorption of the water from the fresh fifth layer by the bottom layer in order to simulate a deposition after a rainfall. In this case, it was expected that the water content of the fifth layer would not decrease rapidly and the drying time would be longer.

Figure 4.28 presents water content results after the fifth layer deposition. Figure $4.28 \mathrm{a}$ and $4.28 \mathrm{~b}$ show that the average GWC of the fifth layer does not decrease rapidly. As expected, since the bottom layer became re-saturated, the dewatering process of the fresh fifth layer was slower than previous fresh layers (except the first layer). Figure $4.28 \mathrm{~b}$ shows that the average GWC of the fifth layer starts from around $38 \%$ at day 57 and decreases to shrinkage limit at day 72 . It is clear

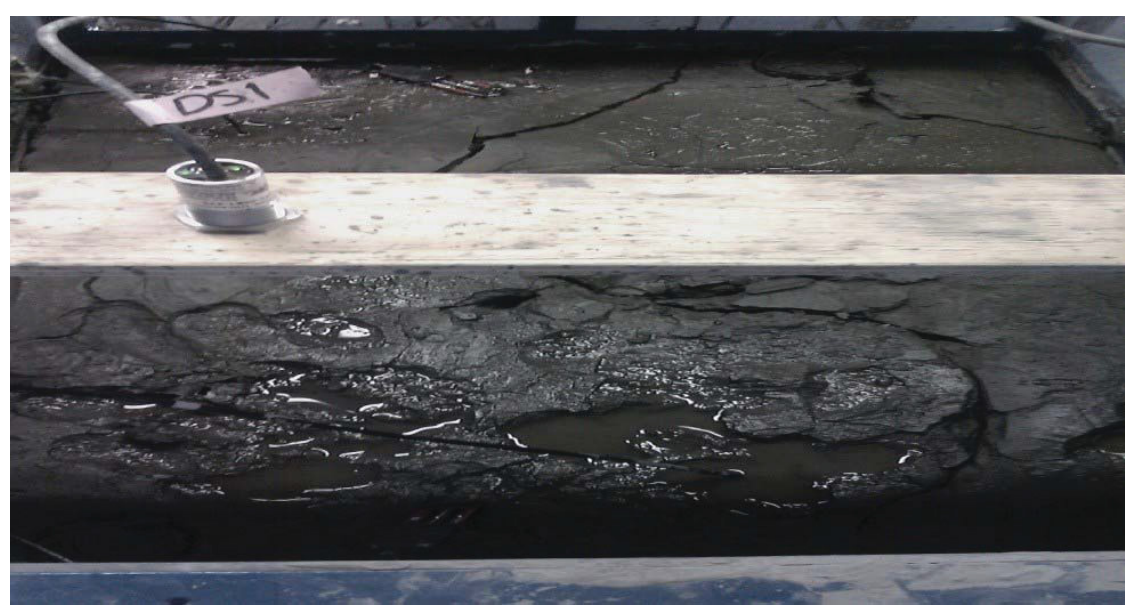

Figure 4.27 The surface of the fourth layer after re-saturation prior deposition of the fifth layer 

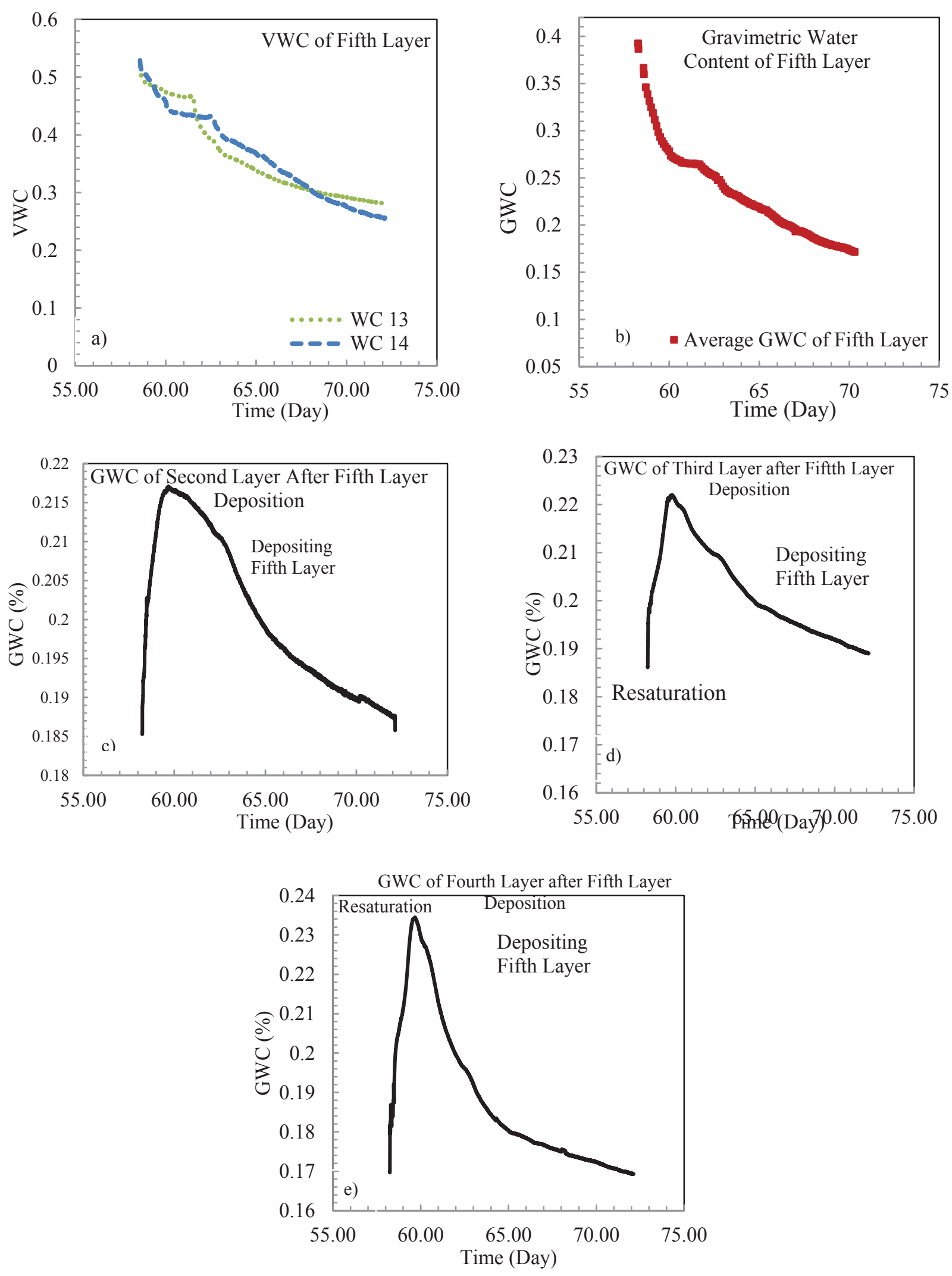

Figure 4.28 Water content results after the fifth layer deposition 
that the drying time to the shrinkage limit of this layer is longer than previous layers due to resaturation before deposition. Figures $4.28 \mathrm{c}$ to $4.28 \mathrm{e}$ present the average GWC of the bottom first to fourth layers during re-saturation and fifth layer deposition. As expected, the water content of all bottom layers increases to $21 \% \sim 23 \%$ during re-saturation. The increase in water content is more tangible in top layers. For example, while the average GWC of the fourth layer increases to $23.5 \%$ (Figure $4.28 \mathrm{c}$ ), the first layer increases to $20.3 \%$ (Figure $4.28 \mathrm{~b}$ ).

Figure 4.29 presents electrical conductivity and temperature results after the fifth layer deposition. It is clear that bulk electrical conductivity of bottom layers increased after resaturation and decreased during desiccation. Figure 4.29a presents bulk electrical conductivity of the fifth layer after deposition. Since the tailings of the fifth layer were re-used from disposed tailings, it was expected that salt precipitation of the fifth layer and therefore electrical conductivity is higher. Figure 4.29 a shows that the bulk electrical conductivity of the fifth layer was significantly higher than bottom layers. Figures $4.29 \mathrm{e}$ and $4.29 \mathrm{f}$ present variation of temperature after deposition of the fifth layer in the first and fifth layers respectively.

Figure 4.30 demonstrates matric suction results obtained from tensiometers after re-saturation and deposition of the fifth layer. Figures $4.30 \mathrm{a}$ to $4.30 \mathrm{c}$ show that most of the tensiometers exhibited zero matric suction during re-saturation prior to deposition. In fact, the re-saturation process was continued until zero matric suction in all layers. According to Figure 4.30d, since the bottom layers were re-saturated, the matric suction of the fresh fifth layer remained zero up to 3 days after deposition. In previous fresh depositions, the bottom layers were desiccated and absorbed water from the fresh layer, which resulted in rapid dewatering of the fresh layer and rapid generation of matric suction. However, in the fifth layer deposition, the bottom layers do 

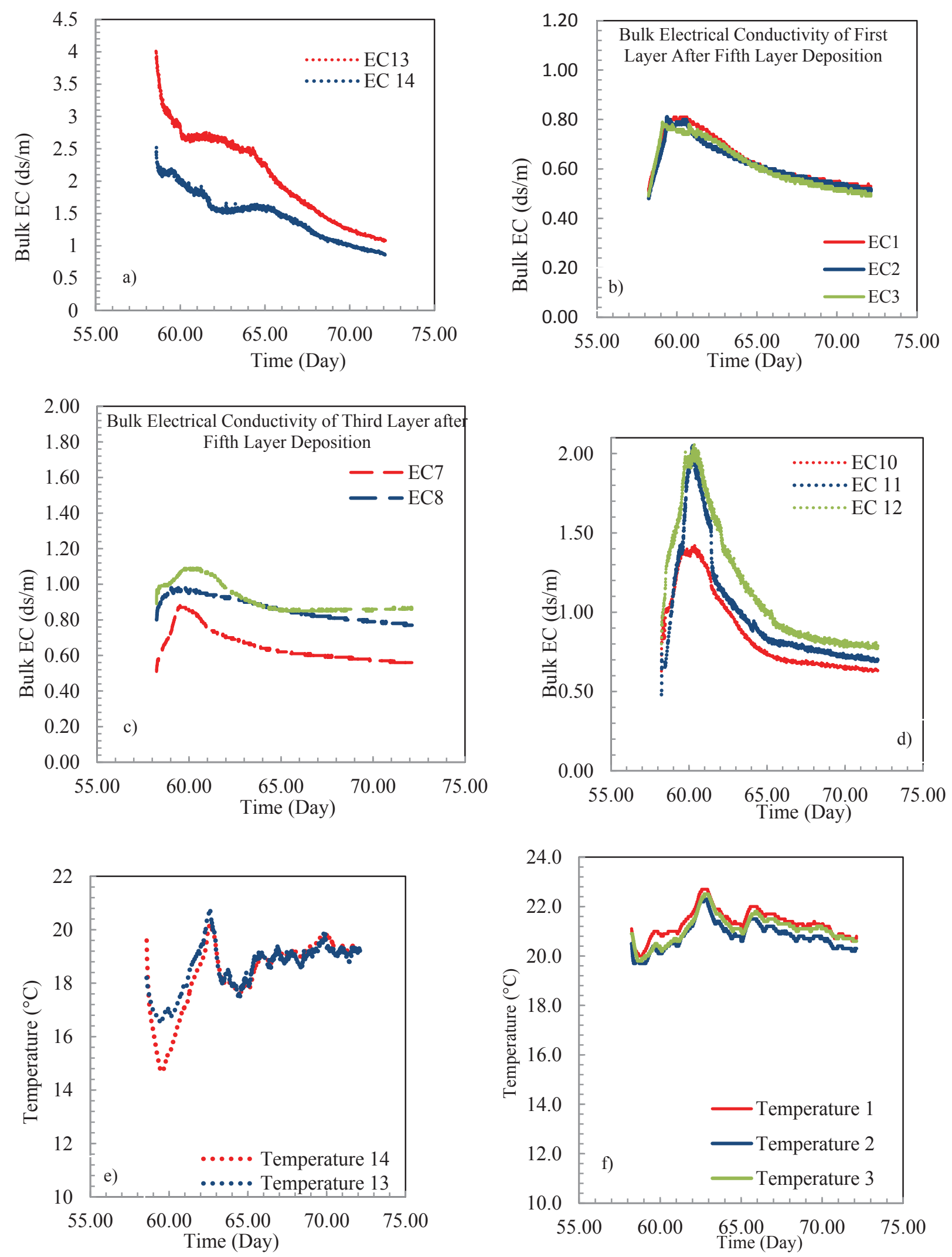

Figure 4.29 Electrical conductivity and temperature results of fifth layer deposition 

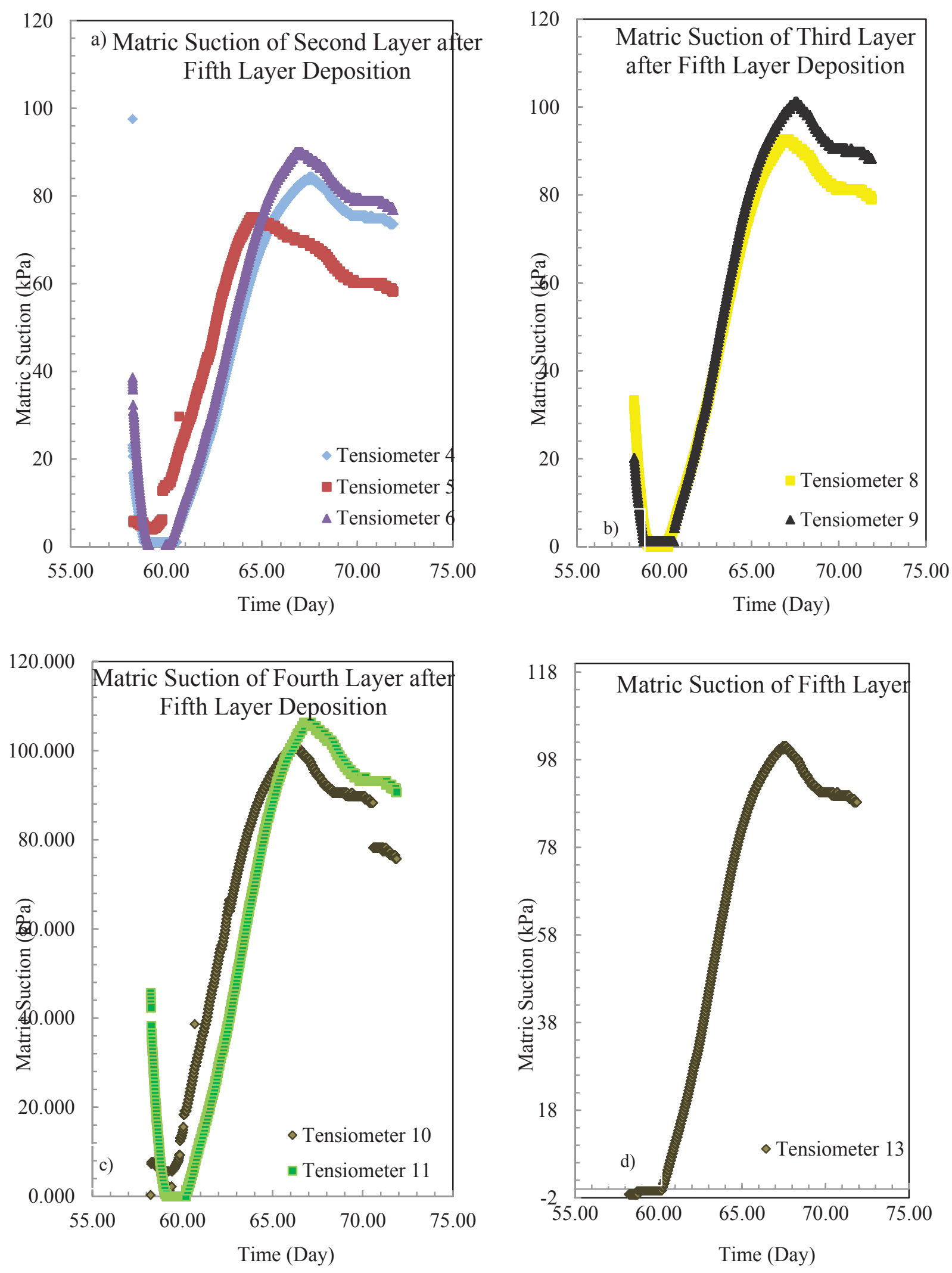

Figure 4.30 matric suction results after fifth layer deposition 
not absorb water from the fresh layer significantly. In addition, during desiccation process, the fresh layer absorbs water from the bottom layers, which makes the drying time longer. According to Figure 4.30, it took around 8 days from appearance of suction to reaching cavitation, which is tangibly longer than previous depositions. Most of tensiometers cavitated at around $100 \mathrm{kPa}$ matric suction at day $67 \sim 68$.

Figure 4.31 presents some photos of the drying after deposition of the fifth layer. Figure 4.31a presents the surface of the fifth layer after deposition. Figure $4.31 \mathrm{~b}$ shows the surface of the fifth layer after at the end of desiccation process. High concentration of salt on top of the desiccated layer confirms high values of electrical conductivity and therefore osmotic suction.
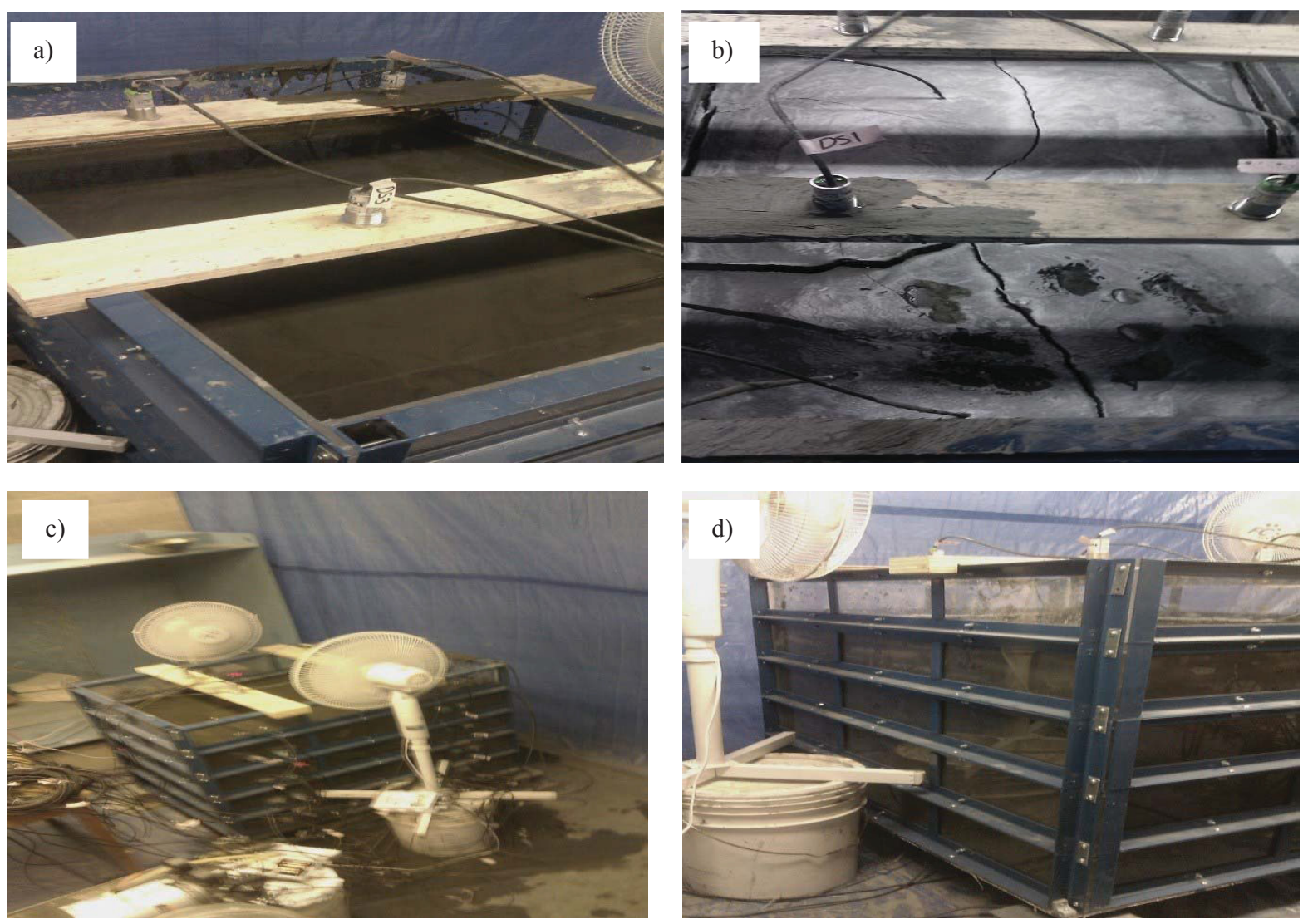

Figure 4.31 Photos of drying box after deposition of the fifth layer 
Figures $4.31 \mathrm{c}$ and $4.31 \mathrm{~d}$ present photos of all layers in the drying box after deposition of the fifth layer.

\subsection{Cumulative Results}

In this section, cumulative results of all depositions are presented. The cumulative results consist of five depositions and three re-saturations occurred in 73 days of the drying box experiment. The cumulative results attempt to simplify investigation on the stress history of all layers, degrees of desiccation, re-saturation processes and etc.

\subsubsection{Water content results}

Figure $4.32 \mathrm{a}$ and $4.32 \mathrm{~b}$ present the VWC (obtained by VWC sensors) of the first and second layers during deposition of fresh layers. Figure 4.32a demonstrates that the VWC of the first layer increases to $30 \% \sim 33 \%$ after rewetting by depositing a fresh layer. However, as additional layers are added (thus increasing the thickness of the tailings between the first layer and the fresh layer) the increase in VWC is lower. For example, by depositing the fresh second layer, the VWC of the first layer increases to around 33\%. However, by depositing the fresh fourth layer, which has more distance to the first layer, the VWC of the first layer increases to around $31 \%$. Figure $4.32 \mathrm{~b}$ also presents the VWC of the second layer after rewetting by depositing fresh top 

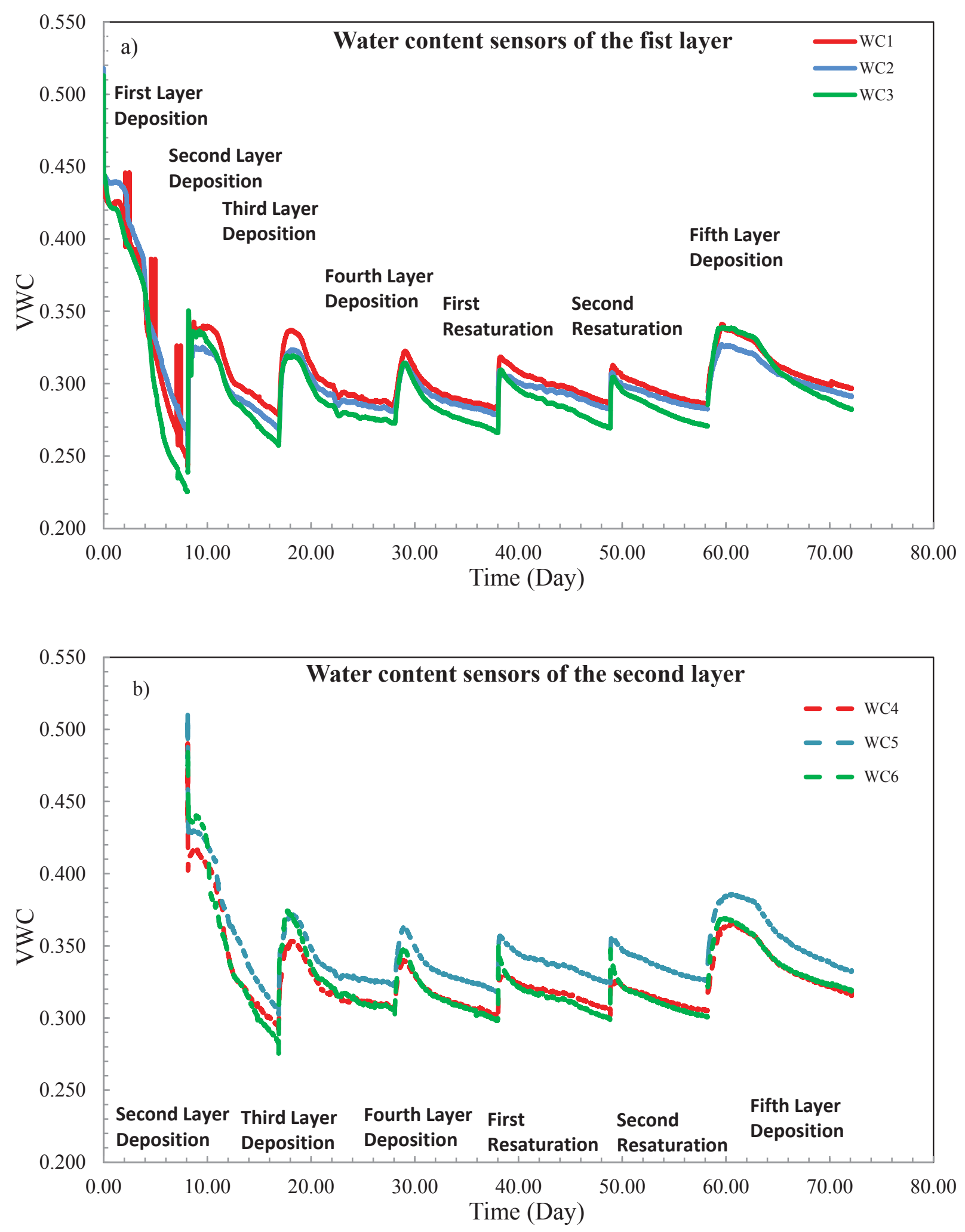

Figure 4.32 VWC of a) first layer and b) second layer during deposition of top fresh layers or resaturations 


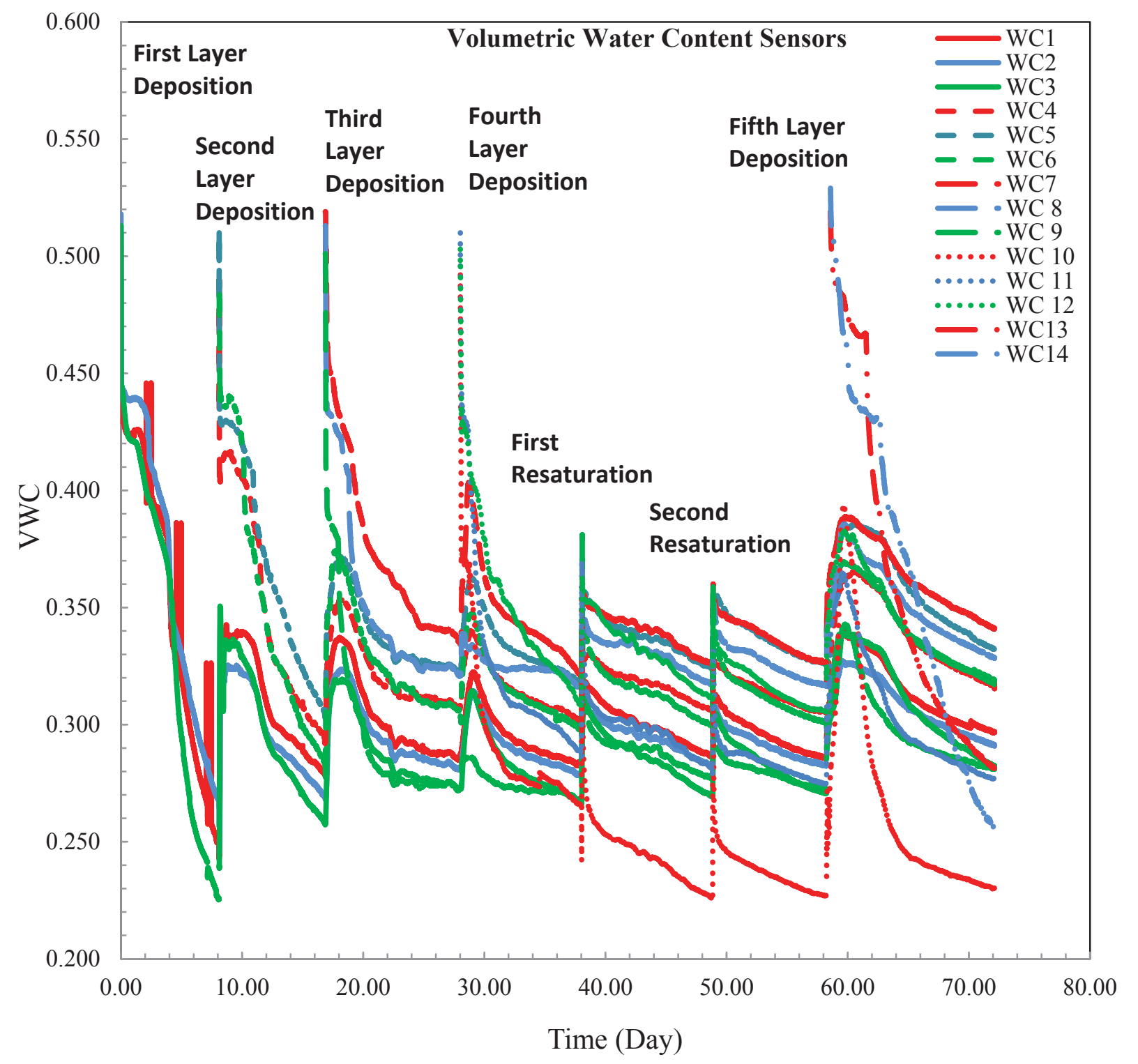

Figure 4.33 VWC of all layers during depositions and re-saturations

layers. The VWC of the second layer increases to around 34\% 36\% after rewetting by fresh layers. Similar to the fresh layer, as additional layers are added (thus increasing the thickness of the tailings between the second layer and the fresh layer) the increment in VWC is lower. Figure 4.33 also presents the VWC of all layers during deposition. It is clear that all layers had a VWC 


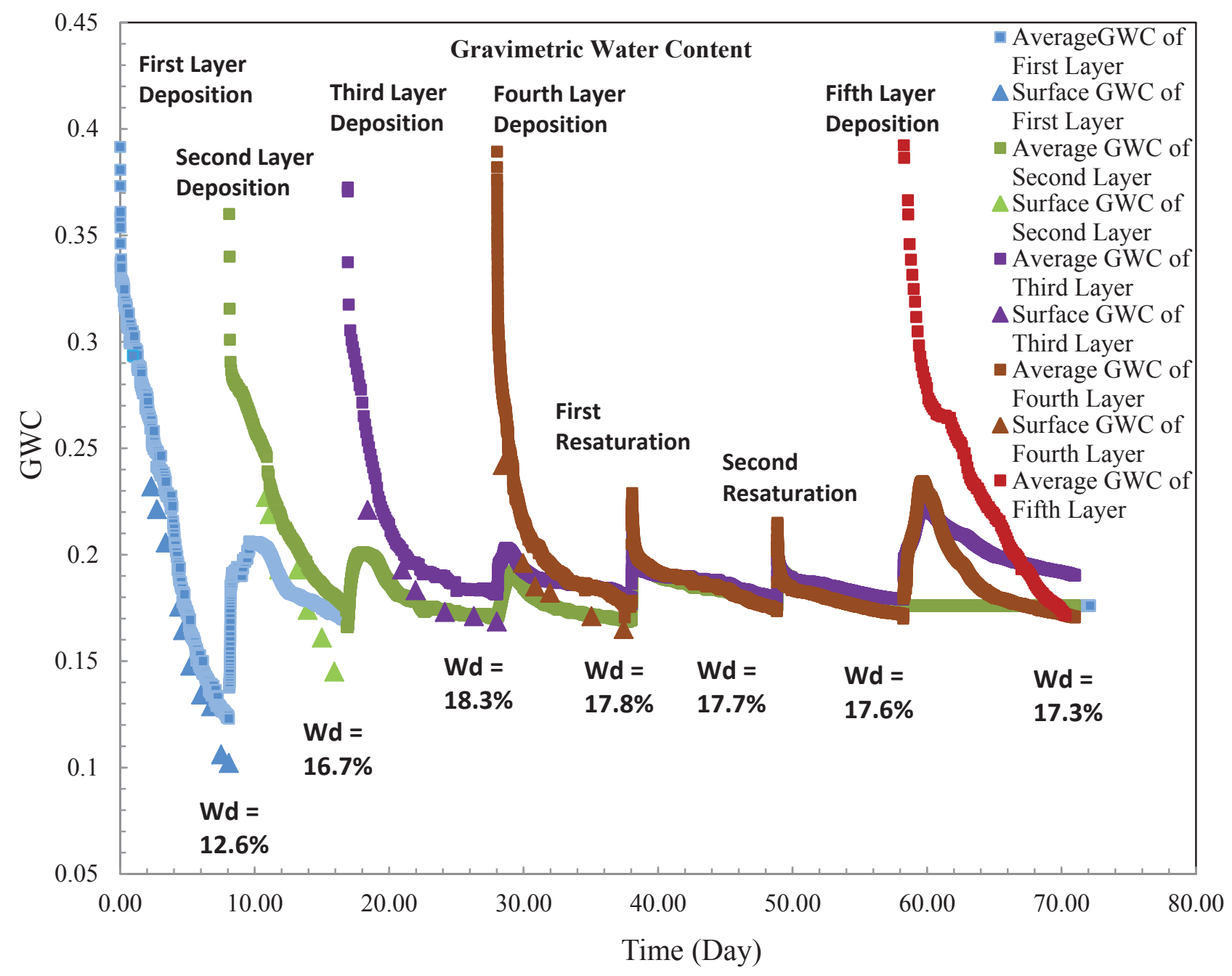

Figure 4.34 Average GWC of all layers during depositions

of around $51 \%$. It should be noted that three volumetric water content sensors were located at different depths of the layer to measure VWC. It was obtained that most of the layers exhibited fairly identical VWC at different depths after rewetting, which confirms the uniformity of desiccated-rewetted layers.

Figure 4.34 also shows average GWC of all layers during depositions. It is clear that all layers had initial GWC of around 35\% 38\% after deposition. Except the first and fifth layers, the 
GWC of other layers rapidly decreased after deposition to around $29 \% \sim 31 \%$. This rapid reduction is attributed to absorption of water from the fresh layer by desiccated bottom layers. Since the bottom layers became re-saturated before depositing the fifth layer, the dewatering process of this layer is slower. Figure 4.34 shows that it took about 3 days for the fifth layer to reach $27 \%$ GWC, while previous layers (two to four) reached 27\% GWC in about 12 hours. Figure 4.34 also shows that the first and second layers were allowed to desiccate to $12.5 \%$ and $16.8 \%$ GWC respectively. The third, fourth and fifth layers were allowed to desiccate to $17.8 \%$, $17.7 \%$ and $17.6 \%$ GWC respectively, which all are close to the shrinkage limit. Therefore, the first layer experienced higher degree of desiccation. In other words, the desiccation history of this layer led to higher matric suctions in comparison to other layers. After the first layer, the second layer exhibited higher degree of desiccation in comparisons to top layers. The third, fourth and fifth layers had similar degree of desiccation. In addition, the first layer experienced more desiccation/rewetting cycles in comparison to other layers. It is also clear that the average GWC of rewetted layers increased to a value of $20 \% \sim 22 \%$. Figure 4.34 also shows that the surface GWC of fresh layers during desiccation was $1 \% \sim 2 \%$ lower than the average values.

\subsubsection{Evaporation, electrical conductivity and temperature results}

Figure 4.35 shows the actual evaporation rates of all depositions and re-saturations. Since a layer of water remained on top of the first layer after deposition, the actual evaporation of the first layer is higher than other layers and closer to the rate of potential evaporation. The rate of evaporation of other layers started from 7 8 $\mathrm{mm} /$ day. 


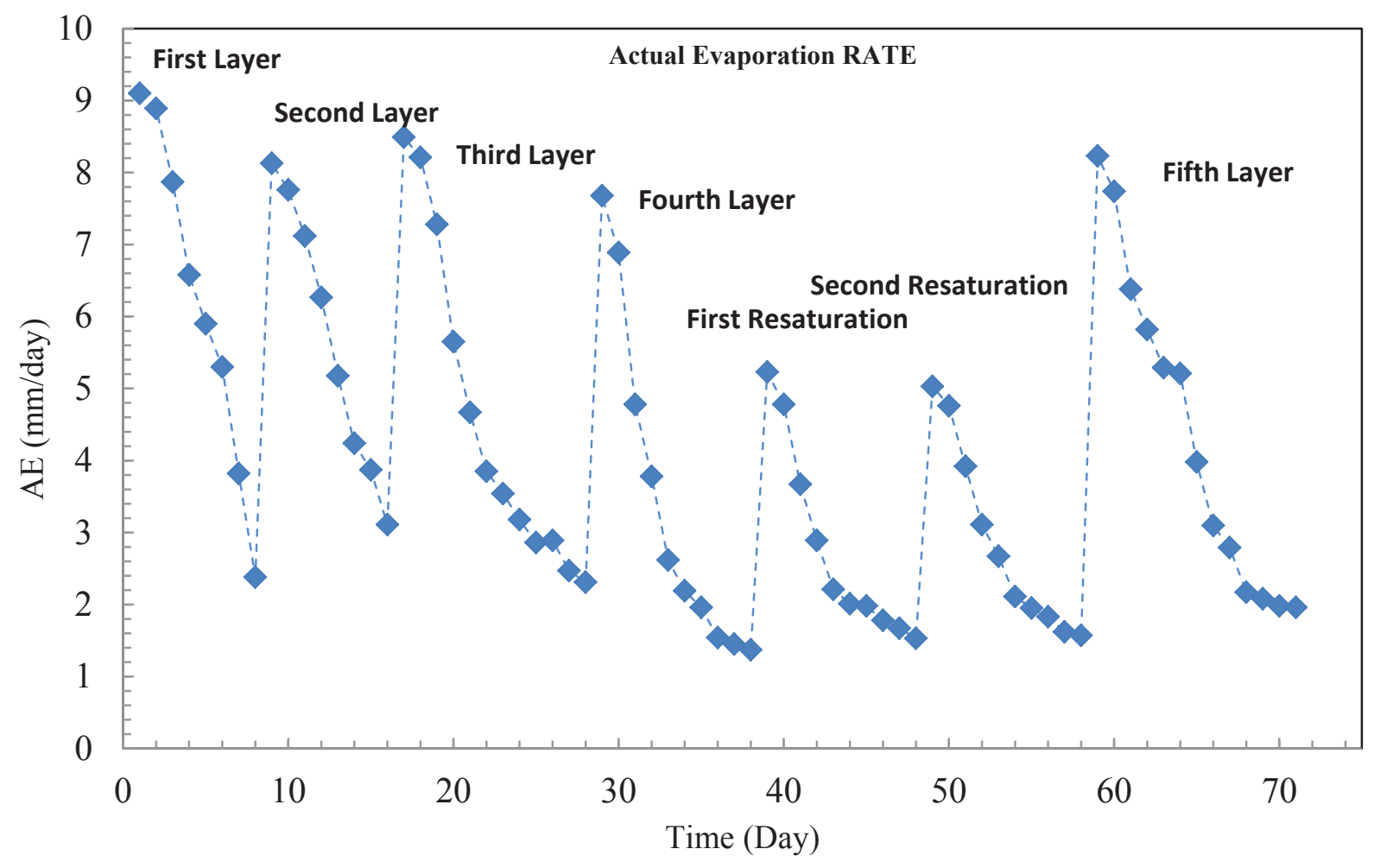

Figure 4.35 Actual evaporation rates of all depositions and re-saturations

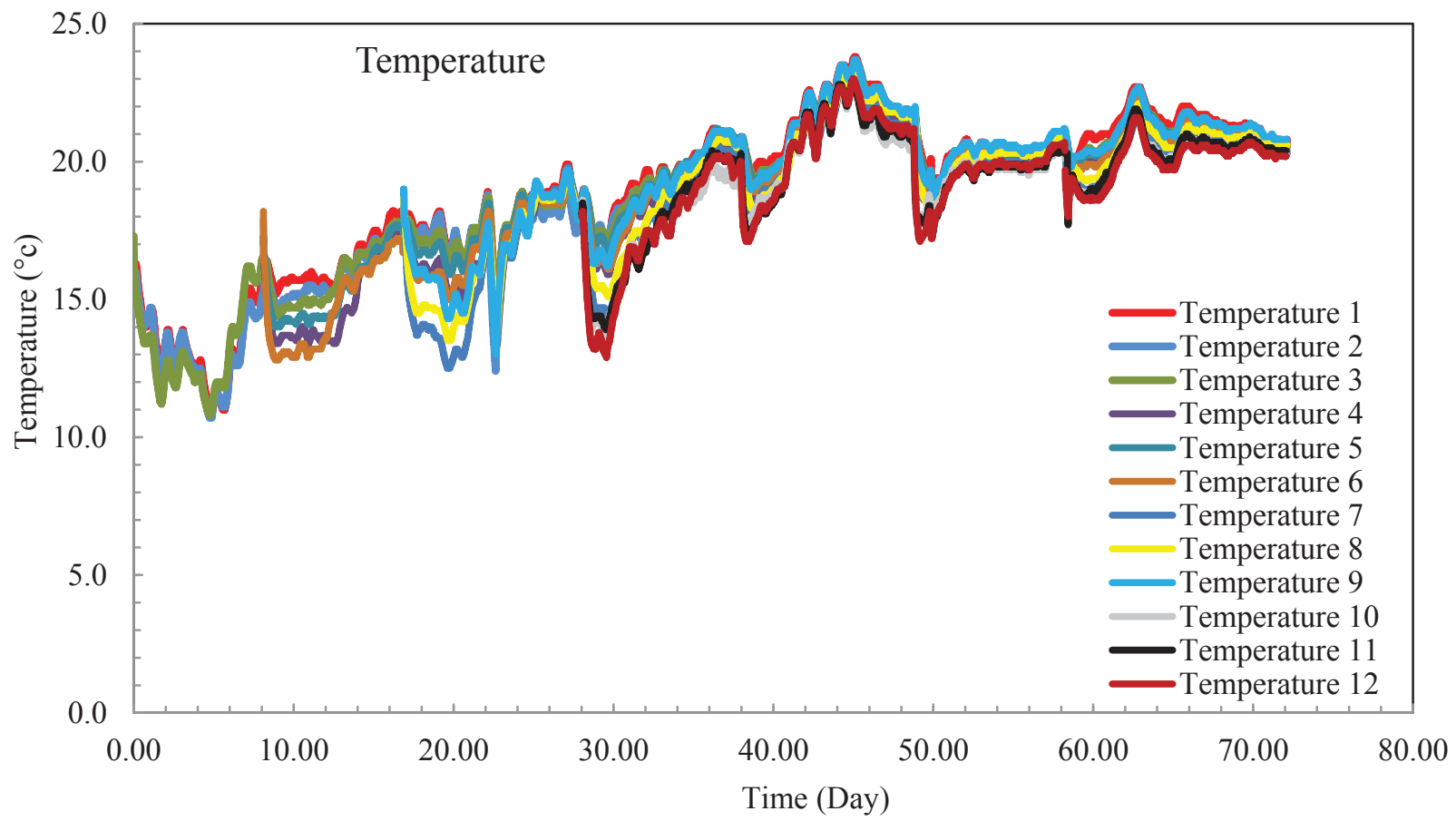

Figure 4.36 Temperature results of all layers during depositions and re-saturations 


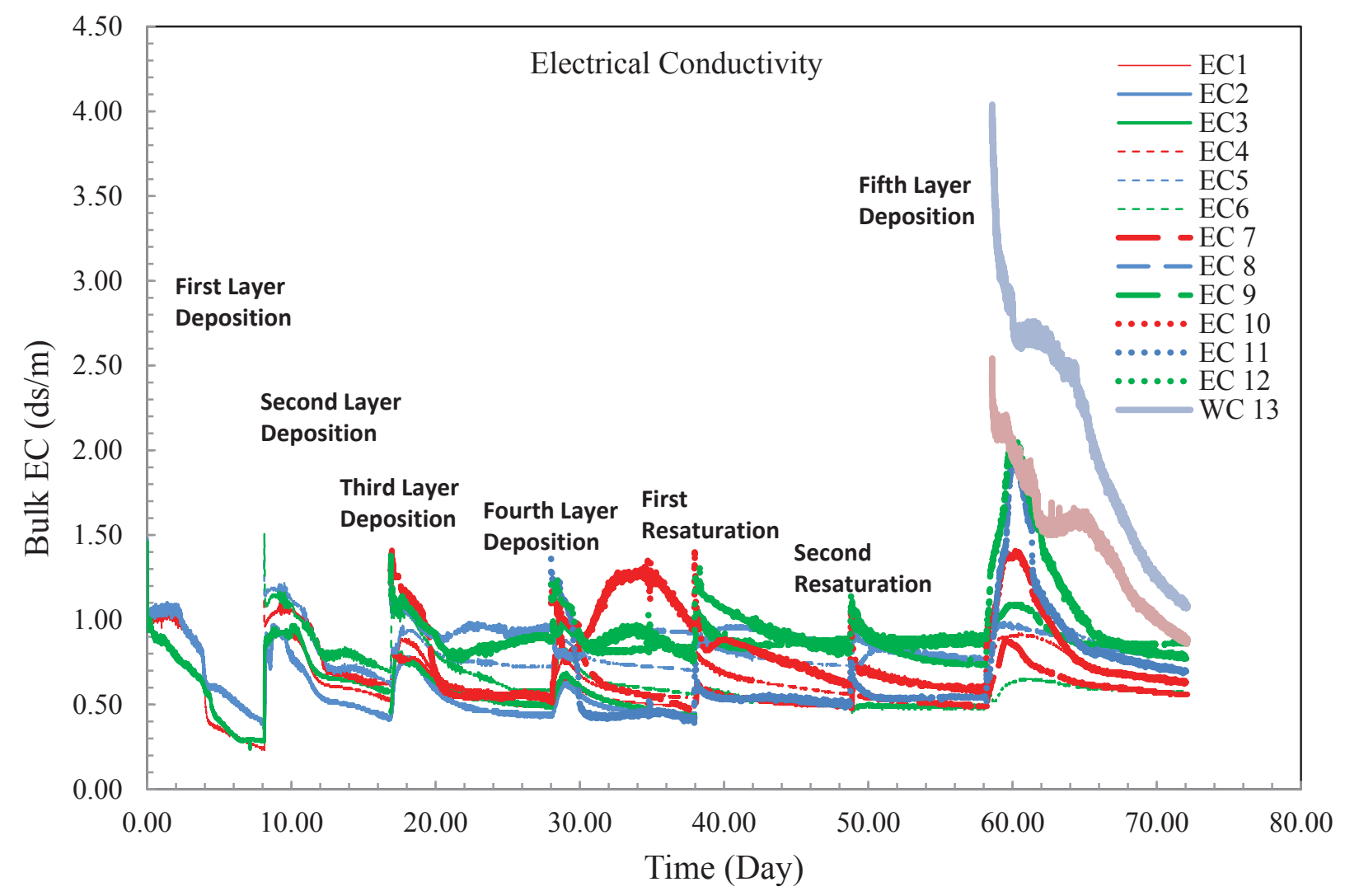

Figure 4.37 Bulk electrical conductivity results of all depositions and re-saturations

Figure 4.36 presents temperature results of all depositions. The temperature inside the tailings varied from $12^{\circ}$ to $23^{\circ}$. It is clear that the temperature of the tailings slightly decreased after each fresh layer deposition, which could be attributed to temperature of bleed water. Figure 4.37 also presents bulk electrical conductivity results of all depositions and re-saturations. Bulk electrical conductivity of layers decreased during desiccation. The fifth layer exhibited very high electrical conductivity, which is associated with high salt concentration of the fifth layer. The fifth layer consisted of re-used tailings from disposal sites. In fact, if the tailings had previously experienced desiccation it could influence the value of current electrical conductivity. 


\subsubsection{Matric and total suction results}

Figure 4.38 presents matric suction (tensiometer) results of first and second layers after deposition of top layers. Figure 4.38a demonstrates the matric suction results of the first layer during deposition of top layers. It is clear that in one of the tensiometers, the matric suction of the first layer after rewetting by the fresh second layer came back to zero (day 10). Two other tensiometers showed a small value of suction $(5-10 \mathrm{kPa})$. As the distance to the first layer from the fresh layer increases, the value of matric suction in the first layer after rewetting increases. For instance, the matric suction of tensiometers in the first layer after depositing the fresh fourth layer came back to around $15 \mathrm{kPa} \sim 30 \mathrm{kPa}$. It is also clear that all tensiometers cavitated after around $100 \mathrm{kPa}$ matric suction. Figure 38a also demonstrates that the time required for tensiometers inside the fifth layer to reach cavitation is longer than previous layers. This longer time is associated with re-saturation before depositing the fresh fifth layer, which avoided rapid absorption of water from the fresh layer. Figure $4.38 \mathrm{~b}$ presents matric suction of the second layer after deposition of top fresh layers. Two of the tensiometers inside the second layer measured zero suction after depositing the fresh third layer (day 18). However, one tensiometer did not go back to zero and measured around $12 \mathrm{kPa}$ matric suction. The longer drying time of the fifth layer is also obvious in the matric suction values of the second layer after deposition of the fresh fifth layer. Figure 4.39 presents matric suction results of the fresh deposited layers. It is clear that most of the tensiometers reached cavitation around $100 \mathrm{kPa}$. As mentioned before, the time required for the tensiometers inside the fifth layer to reach cavitation is longer than other layers due to a re-saturation before depositing the fifth layer. 

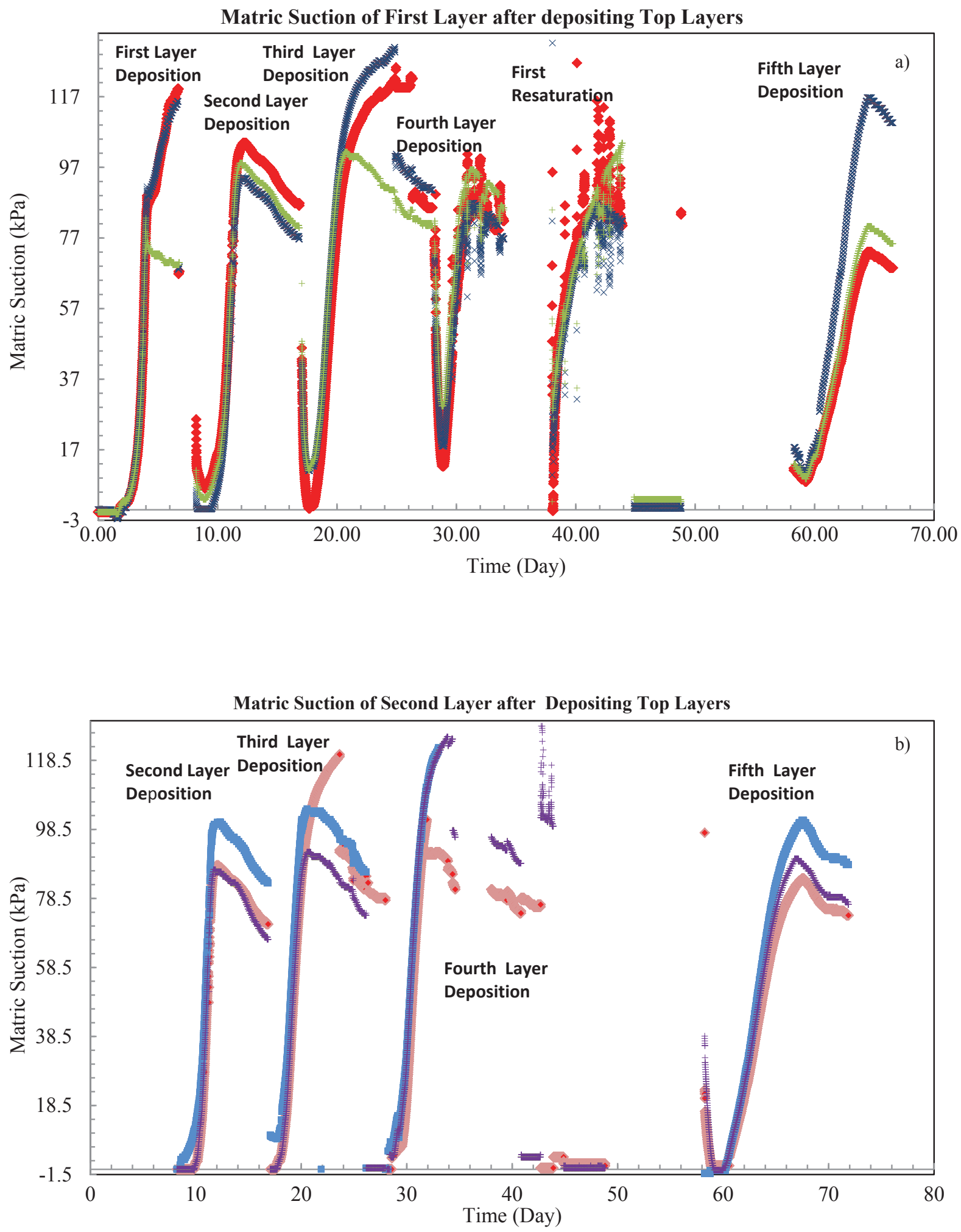

Figure 4.38 Matric suction of a) first layer and b) second layer after deposition of top layers 
Figure 4.40 presents total suction results of samples obtained from the surface of deposited layers and measured by WP-4 total suction measurement device. It is clear that in each deposition, continuing desiccation resulted in increasing total suction values. Total suction values of the samples obtained from areas close to the cracks are significantly higher than other samples due to high salt concentration of areas close to cracks. As expected, total suction values of samples obtained from the fifth layer were significantly higher than other layers due to higher osmotic suctions. Figure 4.41 also presents variation of total suction during desiccation process for all layers. Clearly, as water content decreases, total suction values increases. For instance, samples desiccated to the shrinkage limit exhibited a total suction value around $300 \mathrm{kPa} \sim 400$ $\mathrm{kPa}$ (Sample not close to cracks). With decreasing water content to $12 \%$ (increasing degree of desiccation), the value of total suction became around $1100 \mathrm{kPa} \sim 1200 \mathrm{kPa}$. The first layer that experienced higher degree of desiccation generated higher total suctions. The total suction values of samples close to cracks are higher than other samples due to high osmotic suction values.

Figure 4.42 presents matric suction and total suction results versus GWC in order to create SWCC of the tailings. Matric suction results were obtained from two tensiometers in the deposition of the first layer. Total suction results consisted of the results from surface of different layers of the drying box and also samples obtained from a bucket at different degrees of desiccation. In fact, Figure 4.42 attempts to visualize SWCC of the tailings based on matric and total suction results. Obviously, the difference between matric and total suction is attributed to osmotic suction.

Figure 4.43 presents variations of matric suction and total suction with evaporation rate for all depositions and re-saturations. It is clear that as matric suction increases during the desiccation 


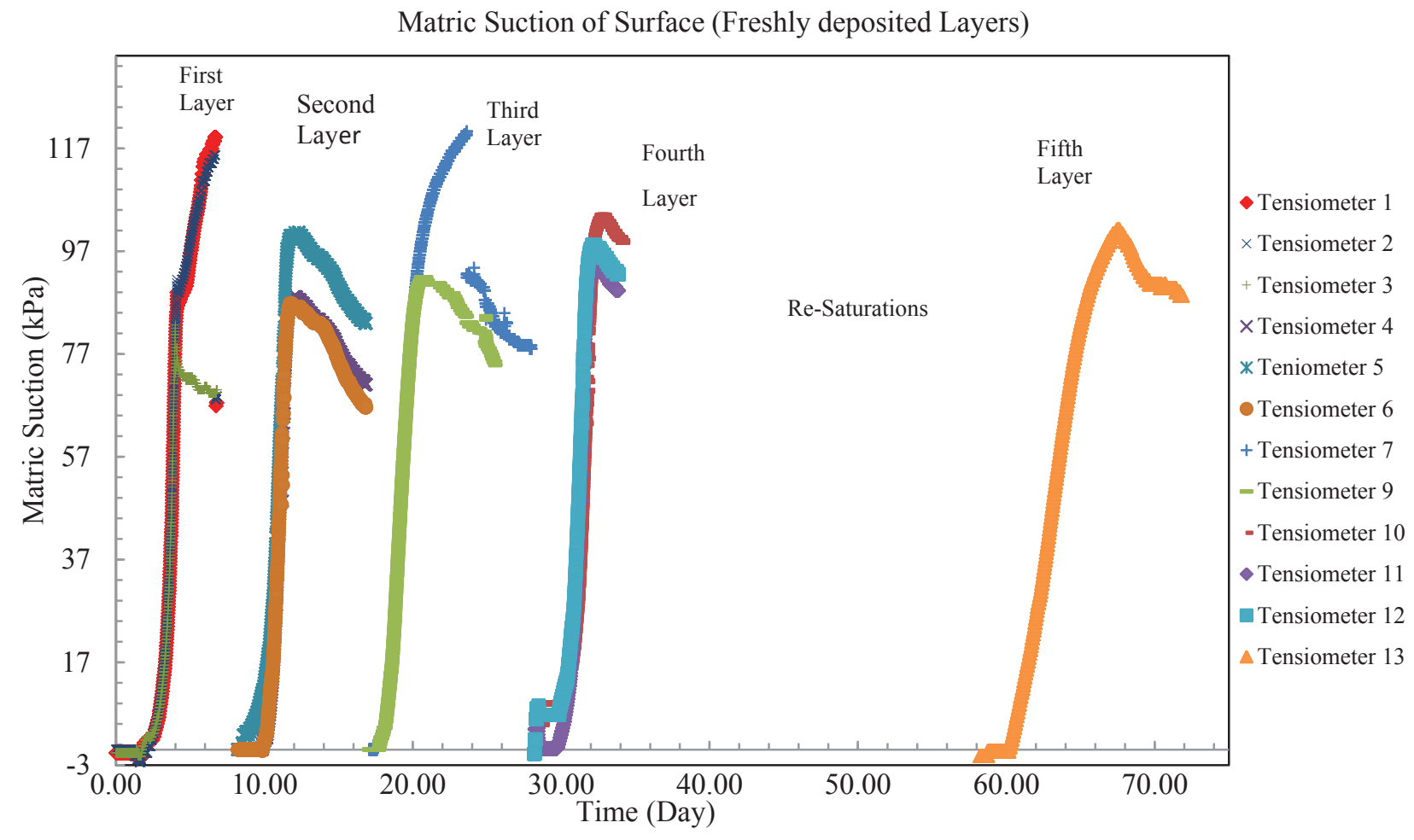

Figure 4.39 Matric suction of the deposited fresh layers

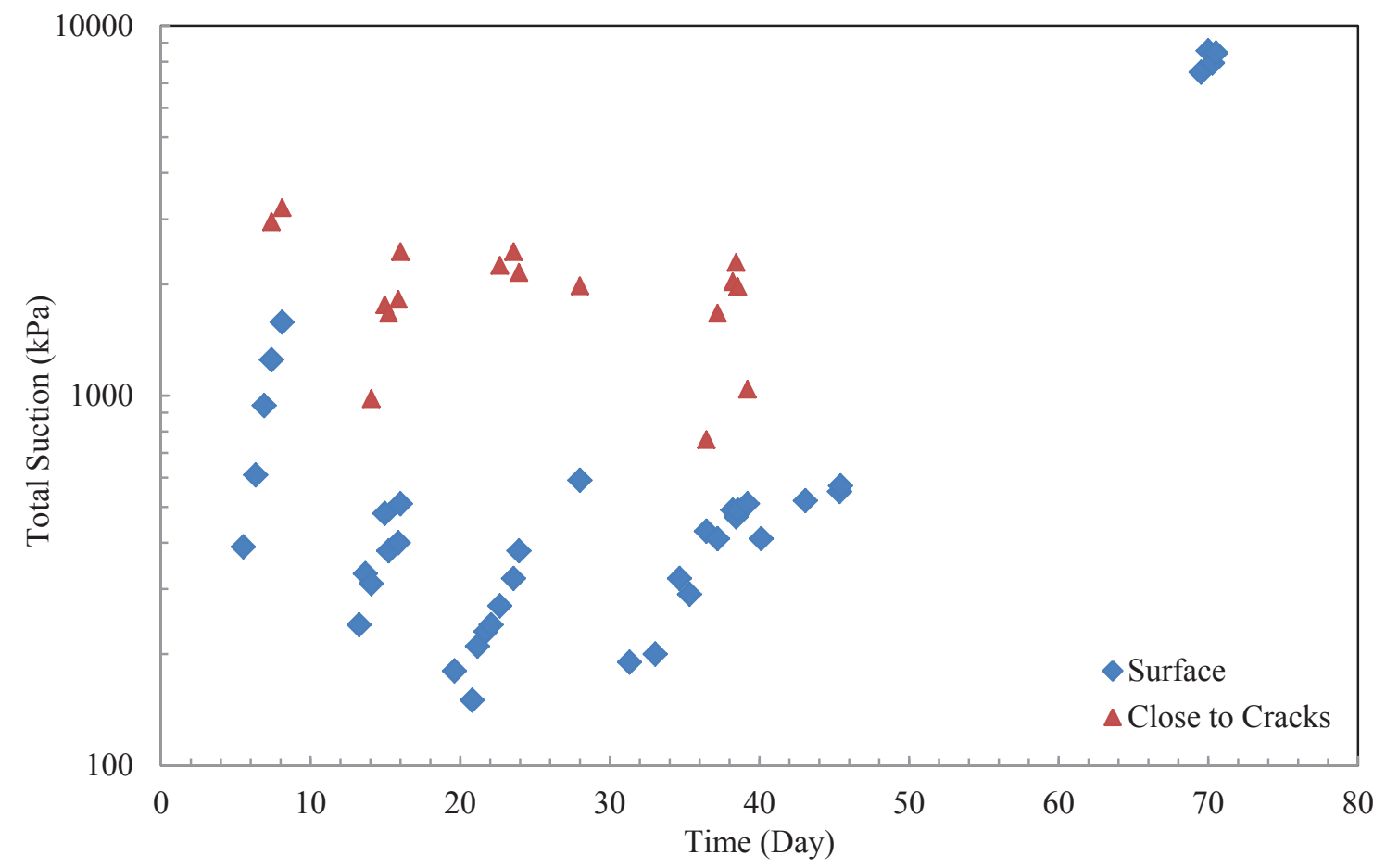

Figure 4.40 Surface total suction results of all depositions 


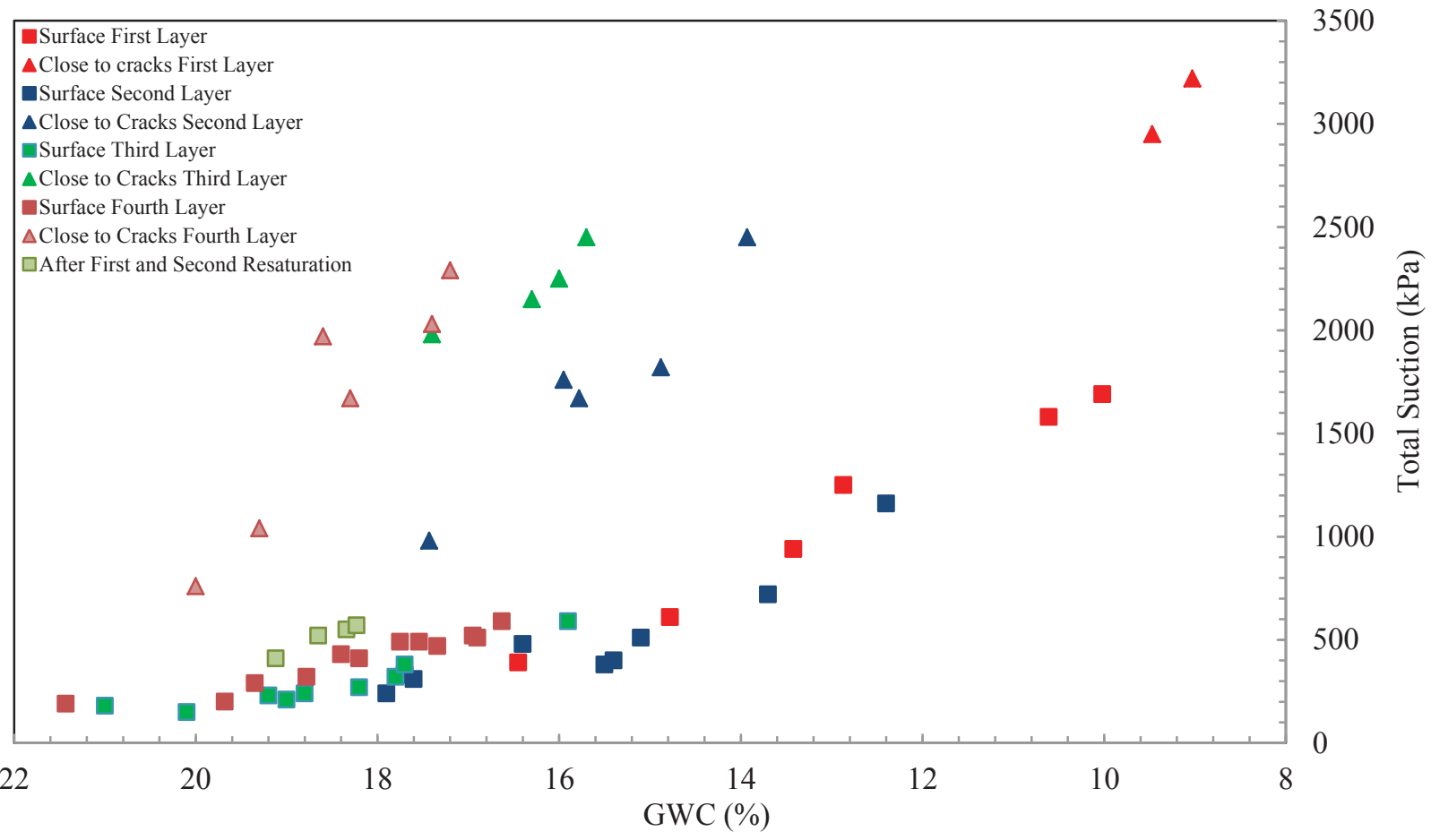

Figure 4.41 Variation of surface total suction during dewatering process of all layers

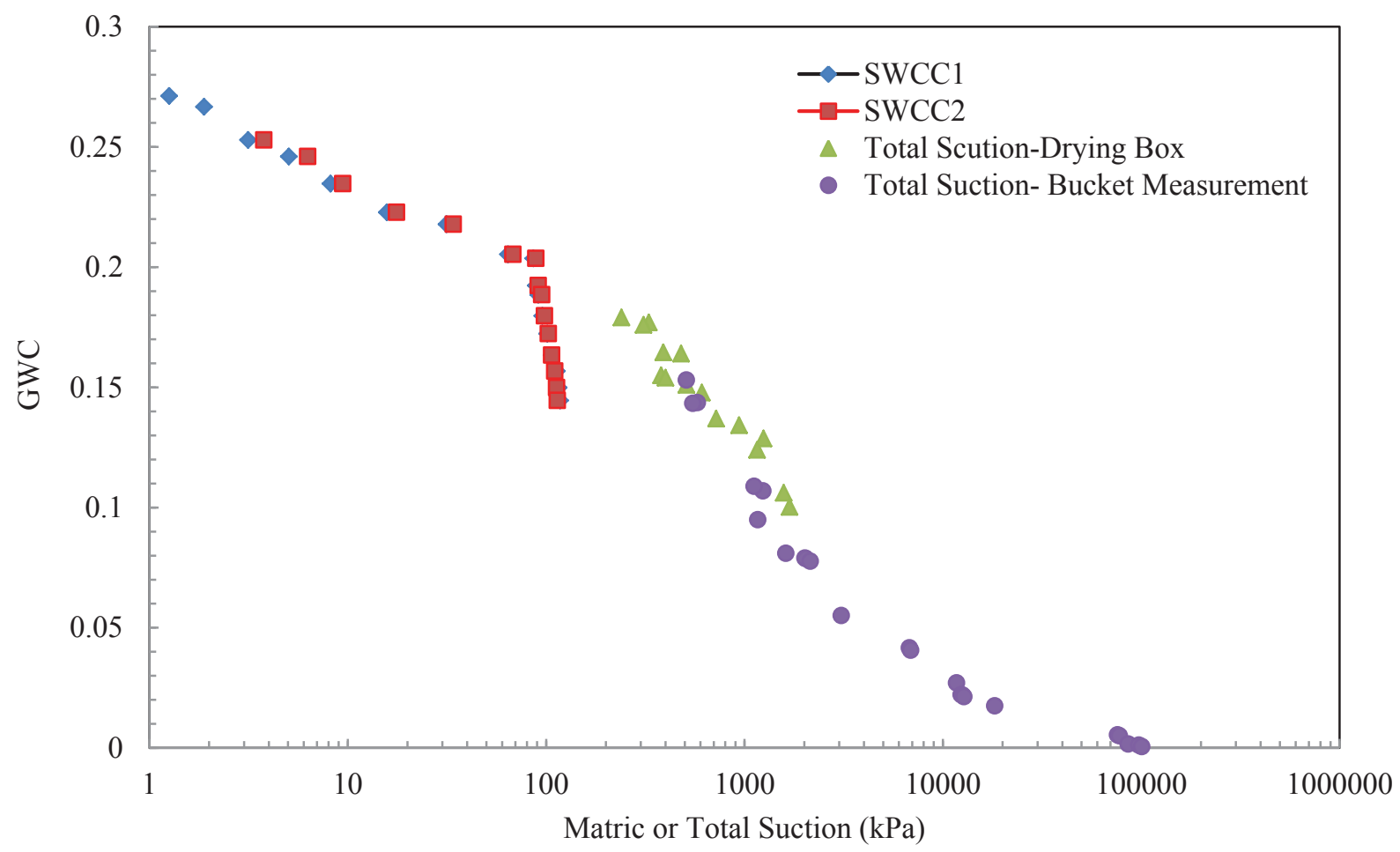

Figure 4.42 SWCC of tailings based on matric and total suction results 

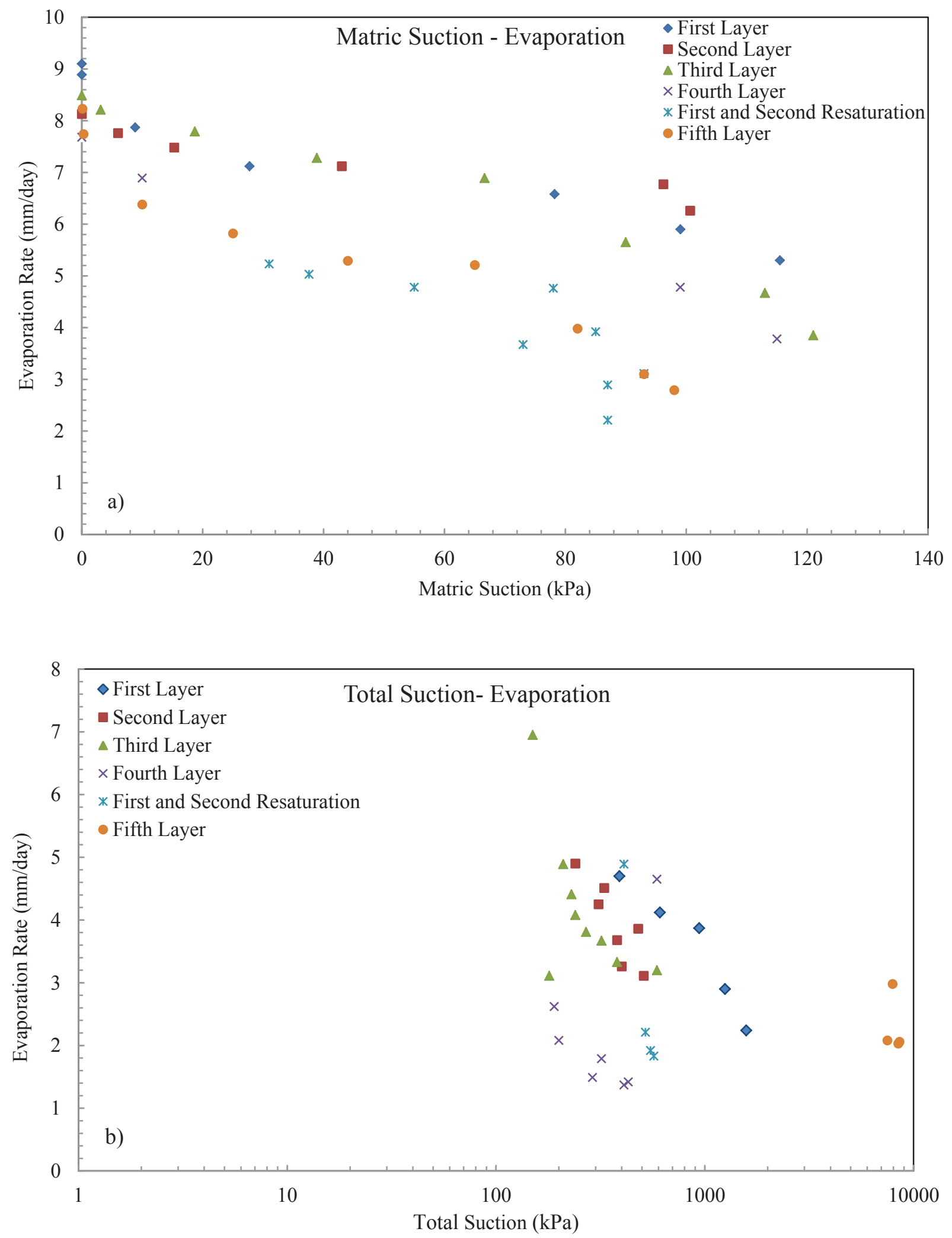

Figure 4.43 Variations of a) matric suction, b) total suction with evaporation rate 
process, the evaporation rate decreases. Figure $4.43 \mathrm{a}$ demonstrates that after passing the AEV $(84 \mathrm{kPa})$, the evaporation rate decreases more tangibly.

\subsection{Tube extraction methods}

After finishing the desiccation process of the fifth layer, the box was re-saturated and after reaching zero suction for most of the tensiometers, tubes from different layers were extracted. Two tube extraction methods were employed in this study to extract samples from different layers of the drying box. The first method consisted of extracting tubes that had been previously buried before deposition of fresh layers. The second method consisted of sampling by thinwalled tubes pushed inside the tailings by a hydraulic jack. Details of tube extraction methods have been explained in the third chapter (Section 3.4.2.4). Samples from different layers were prepared from extracted tubes and placed in the simple shear device for shear strength measurement.

\subsection{Discussion}

Multilayer deposition of the thickened tailings layers in a drying box was performed in order to simulate multilayer field deposition of thickened tailings. This method is a complementary experimental method to the small scale deposition of thickened tailings in a bucket. The main purpose of this experiment was to simulate field deposition of thickened tailings and prepare 
samples that experienced different stress histories (desiccation histories) for the simple shear test. Investigating the influence of desiccation history on final monotonic and cyclic strength is an important goal of this multilayer deposition test. Five thickened tailings layers were prepared at pumping water content and deposited consecutively. Layers were allowed to dewater to different values of water content under controlled desiccation. The first layer experienced higher degree of desiccation. The degree of desiccation was alleviated for the second and third layers. Meanwhile, the third, fourth and fifth layers were allowed to desiccate to the same water content (shrinkage limit) but under different conditions. In addition to different degrees of desiccation, factors affecting the required drying time of layers were also investigated. The fourth layer had smaller thickness and the fifth layer had a re-saturation prior to deposition. Moreover, two re-saturations were also conducted to simulate heavy and light rainfalls.

In addition to the results and conclusions of simple shear strength tests on extracted tubes which will be discussed in the next chapter, the following outcomes were also obtained by simulation of multilayer deposition in the drying box:

- The thickness of the deposited thickened tailings layer does affect the drying time of the layer. Shorter drying time of the fourth layer, which had smaller thickness, confirms this outcome.

- The swelling potential of tailings after rewetting is not remarkable, which did not lead to GWC of desiccated-rewetted layers to become more than $22 \% \sim 23 \%$ after rewetting. 
- As the degree of desiccation of the bottom desiccated layers is higher, the dewatering process of the fresh top layer is quicker after deposition. Rapid reduction in water content of the second to fourth layers in comparison to the fifth layer confirms this phenomenon.

- Matric suction of desiccated bottom layers after re-wetting by a fresh layer does not necessarily reach zero value especially if the layers are deeper and have more distance to the fresh layer.

- Air entry value and shrinkage limit of tailings are $84 \mathrm{kPa}$ and $17 \% \sim 18 \%$ respectively.

- Re-saturation of desiccated bottom layers by rainfall before depositing a fresh layer led to longer drying time of the fresh layer. 


\section{Chapter 5: Applicability of constant volume simple shear tests for sand and silty materials in partially-saturated condition}

\subsection{Introduction}

As previously mentioned, simulating the stress history of thickened gold tailings deposition by laboratory methods and evaluating the strength of the desiccated-rewetted tailings are the main goals of this study. Desiccated-rewetted Bulyanhulu tailings are expected to contain low or zero suction after rewetting. However, the degree of saturation may be as low as $85 \%$ in the rewet tailings. Monotonic and cyclic shear strength of desiccated-rewetted gold tailings were measured by constant volume simple shear tests. The identical response of saturated constant volume test and undrained test was verified by Dyvik et al. (1987). Since the desiccated-rewetted tailings are expected to have low or zero values of suction, if the equivalence of the responses of constant volume simple shear tests on partial saturated fine silts (at low suctions) is verified with the response of dry fine silt specimens, the applicability of constant volume simple shear tests for desiccated-rewetted gold tailings can be also evaluated. 
The NGI simple shear test was employed in this study to measure the monotonic and cyclic shear strength of thickened gold tailings. The purpose of this laboratory shear testing was to simulate the in-situ loading conditions in an element test. In the simple shear device, the specimens were initially consolidated under one-dimensional vertical load followed by horizontal shear loading. Direct shear tests are typically performed to determine the soil strength parameters. But the drainage state is undefined, cannot be controlled, and the failure surface is forced to be more or less horizontal in direct shear tests (Dyvik et al., 1987; Prevost and Høeg 1970). Reaching a truly undrained condition is difficult in most laboratory tests on account of system compliance. Constant volume test is a convenient alternative in the simple shear device to simulate the truly undrained condition (Bjerrum and Landva, 1966). Since the simple shear device does not allow lateral deformation $\left(\varepsilon_{x}=\varepsilon_{y}=0\right)$, only the height should be kept constant to achieve constant volume condition. The constant height condition can be applied by clamping the bottom loading cap against vertical movement (the top cap is vertically fixed in the CU simple shear device). In a constant volume test, it is assumed that the decrease (or increase) of vertical stress is essentially identical to the increase (or decrease) of excess pore water pressure that would have developed in an undrained simple shear test conducted on the saturated specimen (Bjerrum and Landva, 1966; Finn et al. 1978; Dyvik et al. 1987). Dyvik et al. (1987) designed a chamber for NGI simple shear device that enables performing a truly undrained test with pore water pressure measurement. They performed four parallel undrained and constant volume simple shear tests on consolidated Drammen clay to verify the validity of the assumption. Figure 2.14 in chapter 2 shows a comparison of the truly undrained and constant volume responses. Based on these results, Dyvik et al. (1987) reached the following conclusion: "The assumption that the change in applied vertical stress is equal to the pore pressure which would have developed in an 
undrained test is valid. This conclusion applies to saturated soils". Finn et al. (1978) have demonstrated that the constant volume behavior of both saturated and completely dry sands is similar.

As discussed in the previous paragraph, the assumption of identical response of undrained test and constant volume test was verified for saturated soil. Since the desiccated/rewetted tailings samples in this study were not fully saturated and contained a degree of saturation close to $\mathrm{S}_{\mathrm{r}}=$ 0.9 , the applicability of simple shear test for fine silt materials with low values of suction and high degrees of saturation (but not 1) is of significant importance. In other words, it should be verified if the constant volume simple shear test can achieve truly undrained condition for unsaturated tailings which has low values of suction $(<20 \mathrm{kPa})$.

This chapter aims to verify the similarity of the response of constant volume tests on dry and partially saturated coarse and fine grained materials, and investigate the effect of degree of saturation on the response of constant volume tests performed on fine grained soils that generate low values of suction in unsaturated condition.

\subsection{Test equipment and methodology}

The apparatus employed in this study was a NGI type simple shear device located in the geotechnical research laboratory at Carleton University. The constant volume condition was obtained during shear loading by keeping the height of the sample constant using a clamping mechanism. In order to investigate the influence of degree of saturation on the response of constant volume tests, samples with different degrees of saturation (from saturated $S_{r}=1$ to dry $\mathrm{S}_{\mathrm{r}}=0$ ) should be prepared under similar conditions i.e. density, fabric and consolidation 
pressures. Both coarse and fine grained materials were tested in this study. All specimens were initially deposited by the air pluviation method. When partially saturated samples were required, the initially dry material was placed under simple shear device and slowly saturated using a reservoir of water. The water in the reservoir was adjusted to be one or two centimeter higher (depending on the grain size of the sample) than the top of the sample in the simple shear device. This adjustment resulted in hydraulic gradient equal to $0.4 \sim 0.8$. Thus, a very slow flow was circulated from the bottom to the top of the sample. The reason for applying upward direction of the flow was to improve the uniformity of the moisture distribution within the sample. The height of the sample was monitored during the test and the maximum height change was 0.015 $\mathrm{mm}$, which does not make a significant change in void ratio. Water flowing out from the top of the sample was collected in a container and once the collected water was around $400 \mathrm{~mL}$, the sample was assumed to be reasonably saturated, the drainage valve was closed and the height was fixed to be ready for a constant volume test. For partial saturated samples, the dry sample was saturated to reach appropriate water content (degree of saturation). As a result, responses of constant volume tests with same fabric, density and consolidation pressure but different degrees of saturation are comparable in this method. Samples were consolidated under $50 \mathrm{kPa}$ or $100 \mathrm{kPa}$ before shearing. Figure 5.1 presents the method of saturation used in this study.

\subsection{Materials}

The specimens studied in this research were reconstituted using three different materials. It was attempted to select various materials to represent different particle size, mineralogy and nature. 


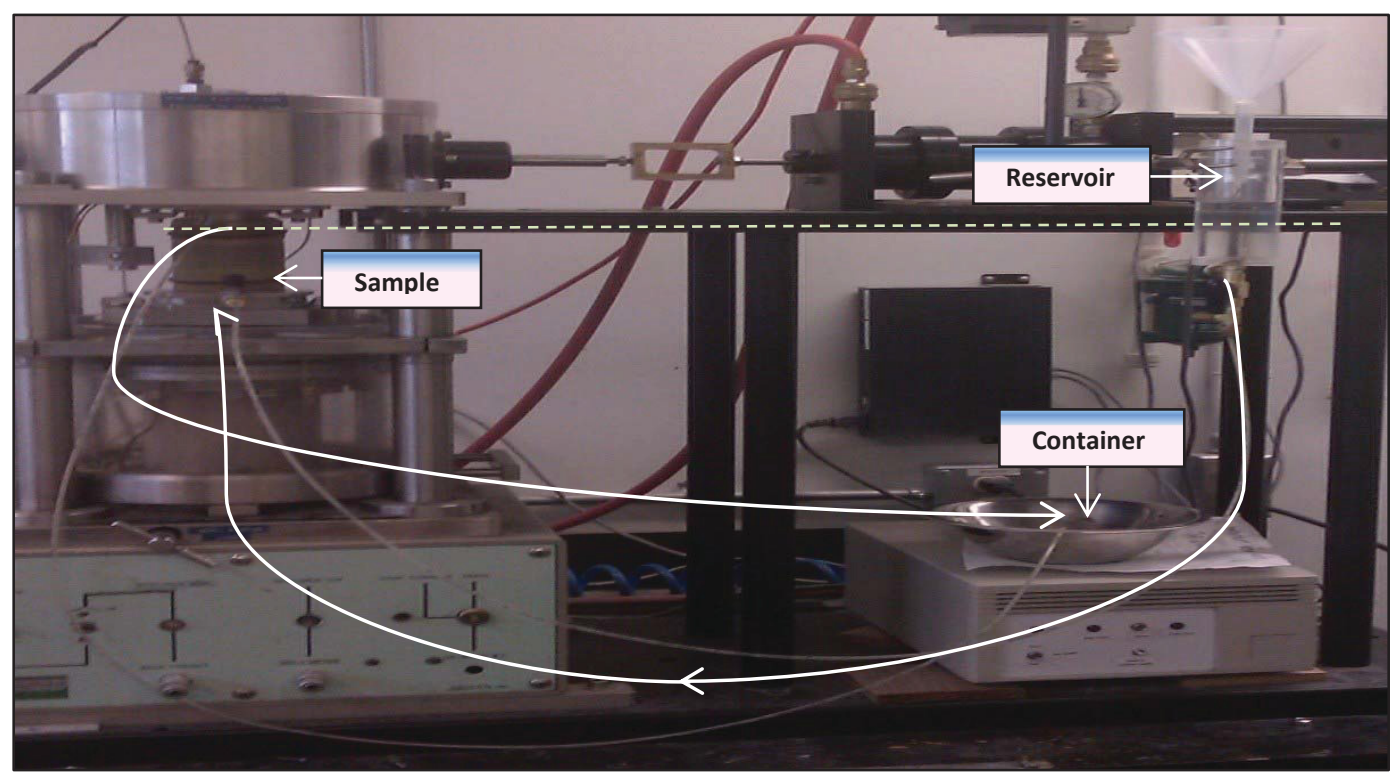

Figure 5.1 Method of saturation used in this study

Two coarse grained and one fine grained material were selected. Two of the selected materials were synthetic glass beads.

\subsubsection{Silica sand}

The Silica sand used in the study is commercially available and meets the ASTM C778 designation. It is poorly graded with a mean particle size of about $0.4 \mathrm{~mm}$ (Sivathayalan and Logeswaran, 2007). The maximum and the minimum void ratios determined using the ASTM standard test methods (ASTM 2001a, 2001b) were $\mathrm{e}_{\max }=0.723$ and $\mathrm{e}_{\min }=0.478$, respectively. Silica sand particles are sub-rounded and the coefficient of uniformity is 1.68 (Sivathayalan and Logeswaran, 2007). Table 5.1 shows other geotechnical properties and Figure 5.2a shows the particle size distribution of Silica Sand. 


\subsubsection{Glass beads}

The glass beads used in this study were sand-sized spherical glass micro-beads with geotechnical properties as shown in Table 5.1. Figure 5.2b shows the grain size distribution of glass beads.

\subsubsection{Synthetic Silt}

The synthetic material used in this study was silt-sized spherical glass micro-beads with geotechnical properties as shown in Table 5.1. The synthetic silt material was adopted since the material is a silty sized soil and also produces low values of suction when partially saturated. Therefore, this material can be expected to simulate the desiccated-rewetted tailings condition. In order to distinguish this material from the sandy sized glass beads and avoid misunderstanding, they are named synthetic silt in this study. The particle size distribution, as determined by the hydrometer method (ASTM D422) is presented in Figure 5.2c.

Table 5.1 Geotechnical properties of materials used in this study

\begin{tabular}{ccccccc}
\hline Material & $\begin{array}{c}\text { Specific gravity } \\
(\mathrm{Gs})\end{array}$ & $\mathrm{D}_{10}(\mathrm{~mm})$ & $\mathrm{D}_{60}(\mathrm{~mm})$ & $\mathrm{Cu}$ & $\begin{array}{c}\text { Liquid Limit } \\
(\%)\end{array}$ & $\begin{array}{c}\text { Plastic Limit } \\
(\%)\end{array}$ \\
\hline Silica Sand & 2.66 & 0.25 & 0.42 & 1.68 & NA & NA \\
Glass Beads & 2.53 & 0.31 & 0.55 & 1.77 & NA & NA \\
Synthetic Silt & 2.48 & 0.001 & 0.039 & 39 & 19 & 13 \\
\hline
\end{tabular}

Figure 5.3 shows the three materials in the simple shear mould. Figure 5.4 presents measured soil water characteristics curve (SWCC) of fine grained synthetic silt used in this study and its 
comparison with gold tailings. It is clear that the amount of suction generated during desiccation in gold mine tailings is significantly higher than synthetic silt. The maximum value of matric suction in synthetic silt material is $100 \mathrm{kPa}$ (for very low water contents). Meanwhile, the amount of matric suction in synthetic silt material never goes beyond $15 \mathrm{kPa}$ for desiccation to $10 \%$ water content, which is a low value of suction. On the other hand, desiccation of gold tailings leads to high values of matric suction. The air entry value (AEV) of the SWCC of gold tailings was obtained $80 \mathrm{kPa}$, while the AEV of the SWCC of synthetic silt is around $10 \mathrm{kPa}$. The difference between the SWCCs of synthetic silt and gold tailings could be attributed to the particle size and fabric differences.

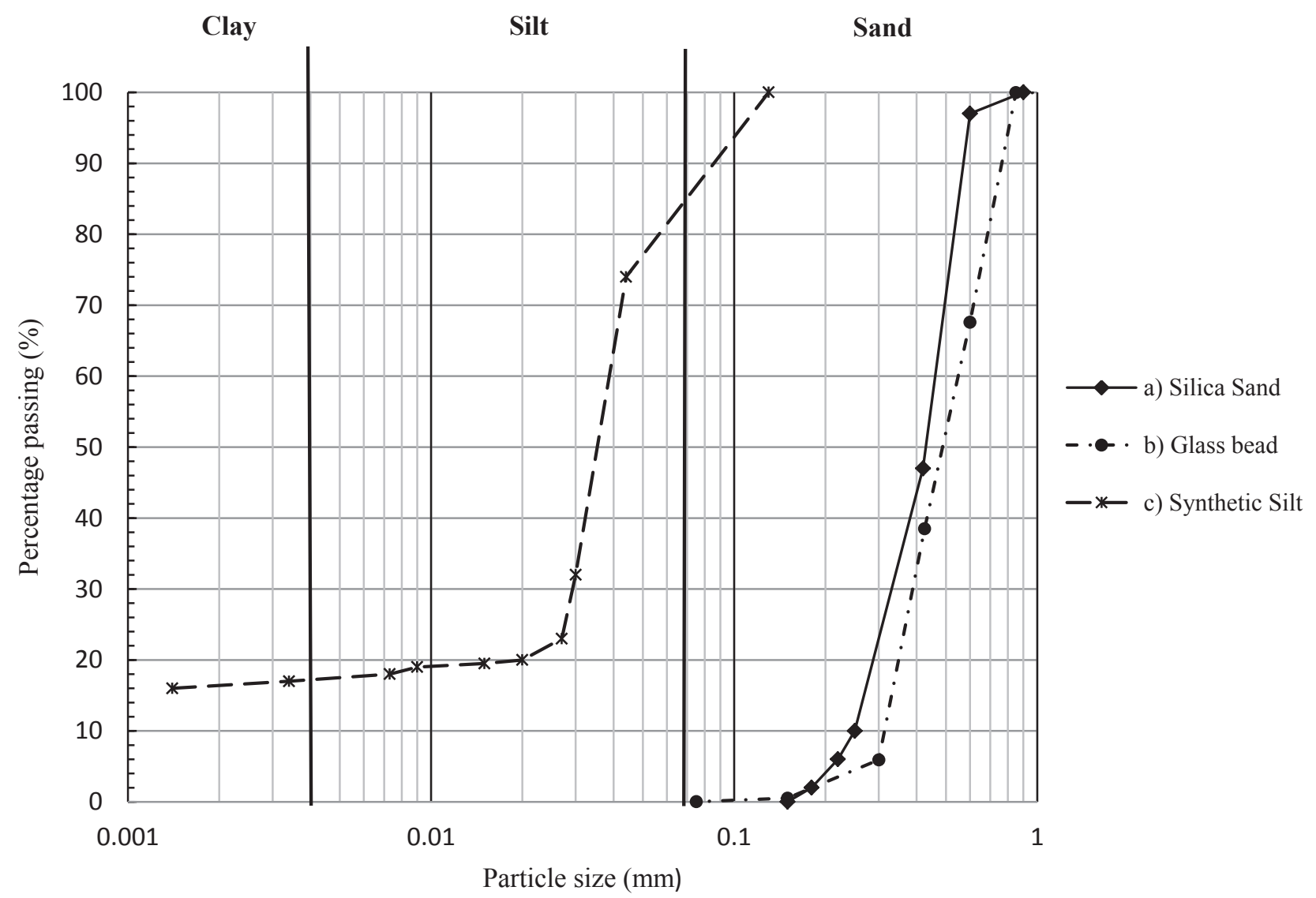

Figure 5.2 Particle size distributions of all tested materials 


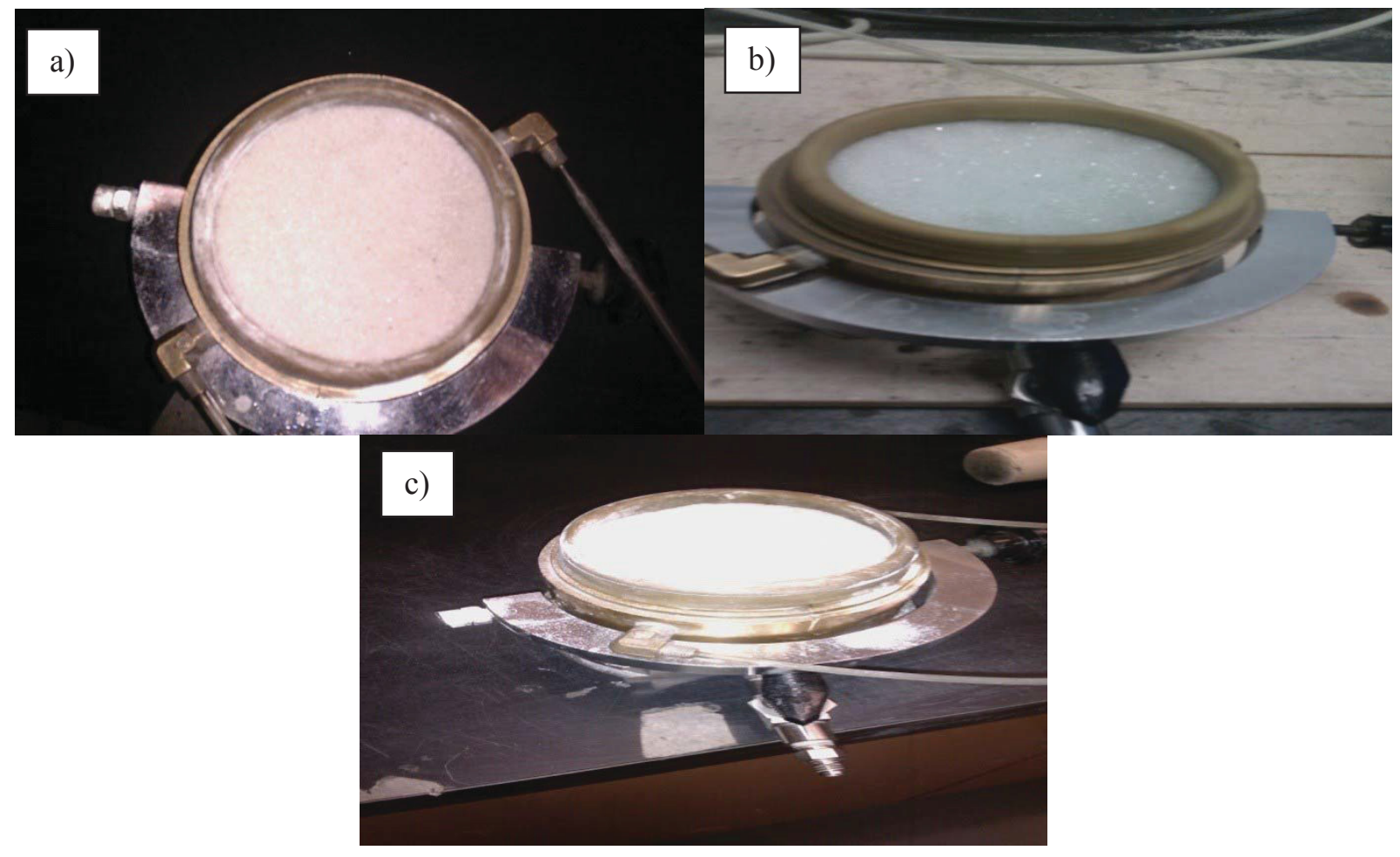

Figure 5.3 Figures of tested materials in the simple shear mould

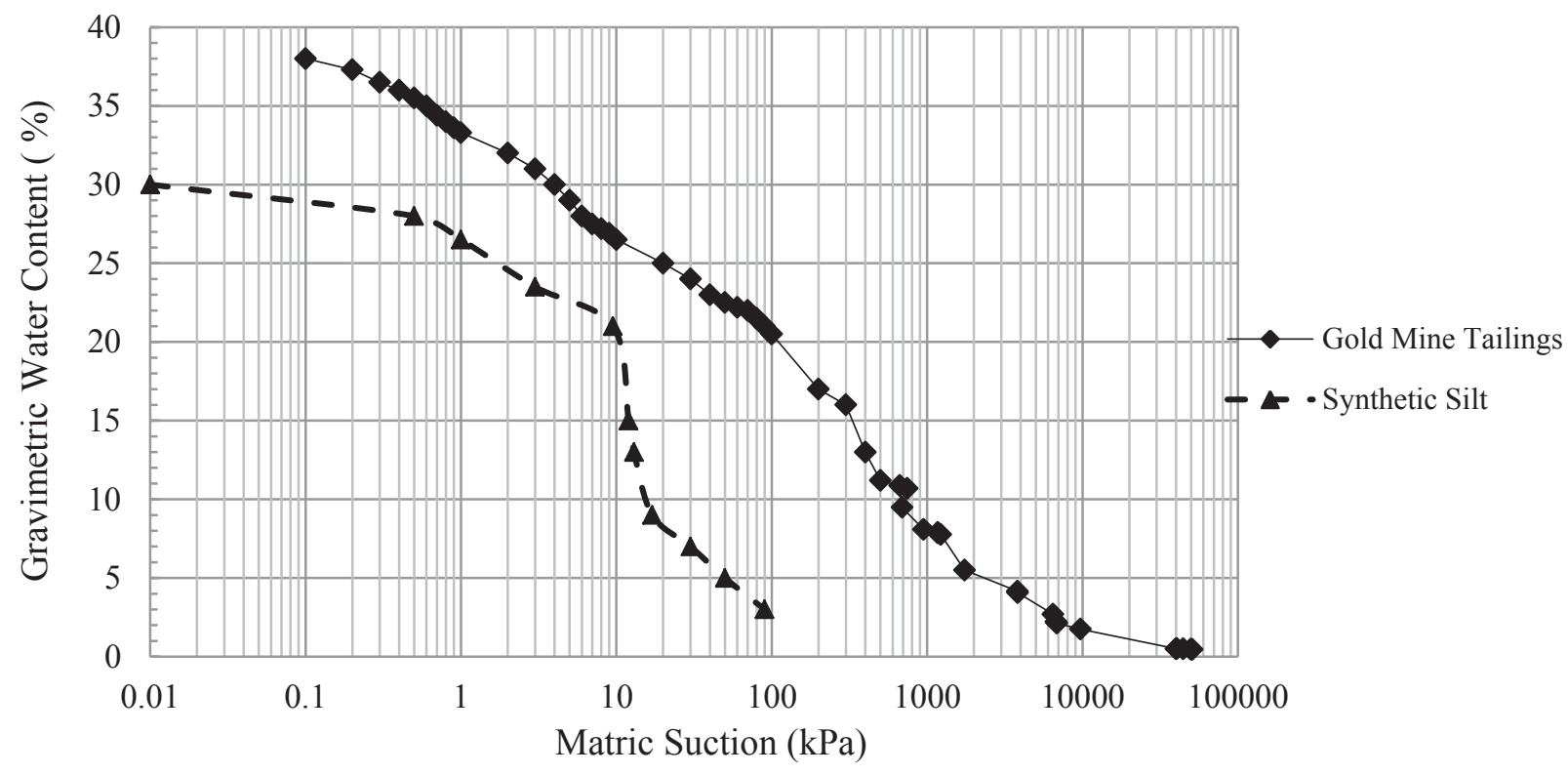

Figure 5.4 SWCCs of fine grained synthetic silts and its comparison with gold tailings 


\subsection{Results}

Monotonic simple shear responses of all materials under different degrees of saturation but at the same density are presented in this section. All materials were air pluviated and gradually saturated using a water reservoir. Water was flushed through the specimens at a very small hydraulic head (and thus slow seepage velocity) to prevent any change in the height of the specimen during saturation. All specimens were consolidated to $50 \mathrm{kPa}$ or $100 \mathrm{kPa}$ vertical effective consolidation pressure $\left(\sigma_{v c}^{\prime}\right)$. Horizontal load were applied using a constant speed motor drive with the rate of $50 \%$ / hr. All tests were continued up to $11 \%$ shear strain.

\subsubsection{Silica Sand Results}

Five different simple shear tests were performed on Silica Sand specimens under different saturation and consolidation conditions. Two specimens were consolidated under $50 \mathrm{kPa}$ effective consolidation pressure $\left(\sigma_{v c}^{\prime}\right)$ and others were consolidated under $100 \mathrm{kPa}$. Three tests were air pluviated and performed under dry condition but at the same void ratio. Two others were air pluviated and gradually saturated to reach a degree of saturation close to 0.9 . Table 5.2 shows the characteristics of tests conducted on Silica Sand.

\subsubsection{Silica sand samples consolidated to $50 \mathrm{kPa}$}


Table 5.2 Constant volume simple shear tests on Silica Sand

\begin{tabular}{cccccc}
\hline Test No. & $\sigma^{\prime}{ }_{\mathrm{vc}}(\mathrm{kPa})^{1}$ & $\mathrm{ec}^{2}$ & $\mathrm{~W}(\%)^{3}$ & $\mathrm{~S}_{\mathrm{r}}(\%)^{4}$ & $\operatorname{Dr}(\%)^{5}$ \\
\hline 1 & 50 & 0.698 & Dry & Dry & 10.2 \\
2 & 50 & 0.694 & 23 & 88 & 11.8 \\
3 & 100 & 0.692 & Dry & Dry & 12.7 \\
4 & 100 & 0.699 & Dry & Dry & 9.8 \\
5 & 100 & 0.693 & 24 & 92 & 12.2 \\
\hline
\end{tabular}

${ }^{1} \sigma^{\prime}$ vc : Effective Consolidation Pressure, ${ }^{2} e_{\mathrm{c}}$ : Void ratio after consolidation, ${ }^{3} \mathrm{~W}$ : Water content after consolidation, ${ }^{4}$ $\mathrm{S}_{\mathrm{r}}$ : Degree of saturation, ${ }^{5}$ Dr: Relative density

Measured monotonic stress-strain response of silica sand samples consolidated to $50 \mathrm{kPa}$ is shown in Figure 5.5. Figure 5.5a clearly shows that both the dry and partially saturated samples exhibited limited liquefaction response with a gentle peak and a phase transformation point after the peak. Figure 5.5c shows that the excess pore pressure in both samples increases up to about 3\% shear strain. The contractive tendency transforms to dilation (decreasing the excess pore pressure) after 3\% shear strain. The excess pore pressure of the both samples continuously decreases until the end of the test. It is also shown that both dry sand and partially saturated sands (with $S_{r}=0.88$ ) have fairly similar response under the same void ratio, fabric and consolidation pressure. In other words, the degree of saturation does not affect the monotonic response of silica sand under same void ratio (relative density), fabric and consolidation pressure. Figure $5.5 \mathrm{~b}$ illustrates the effective stress paths, evaluated using the presumption that the drop in vertical stress would be equivalent to the generated excess pore water pressure. Essentially identical responses noted at both stress levels indicate that the degree of saturation does not affect the constant volume behaviour of silica sands in simple shear. 


\subsubsection{Silica sand samples consolidated to $100 \mathrm{kPa}$}

Figure 5.6 presents the monotonic response of silica sand samples consolidated to $100 \mathrm{kPa}$. Two dried samples are compared to a partial saturated sample with $\mathrm{S}_{\mathrm{r}}=0.92$. Figure 5.6a shows that all samples exhibited limited liquefaction response with a gentle peak and a phase transformation point after the peak. According to Figure 5.6c, similar to samples consolidated to $50 \mathrm{kPa}$, all three samples exhibited contractive response by increasing excess pore water pressure up to around $32 \mathrm{kPa}$. After about 3\% shear strain at the phase transformation point, the contractive response changed into dilative response. The excess pore water pressure continuously decreased from this instant until the end of the test. It is shown that both dry sand samples have fairly similar response compared to the partially saturated sand sample. This indicates that under the same void ratio, fabric and consolidation pressure the degree of saturation does not influence the monotonic response of silica sand samples.

\subsubsection{Glass Beads results}

Figure 5.7 shows the monotonic responses of glass beads materials. Three tests were performed with different degrees of saturation (i.e. $\mathrm{S}_{\mathrm{r}}=0, \mathrm{~S}_{\mathrm{r}}=0.74, \mathrm{~S}_{\mathrm{r}}=0.93$ ) but at fairly similar void ratios. All samples were consolidated under $100 \mathrm{kPa}$ vertical effective stress. The response of one dry specimen was compared to the response of two partial saturated specimens. Figure 5.7 presents that all three glass bead specimens exhibited quite dilative (strain-hardening) response. 

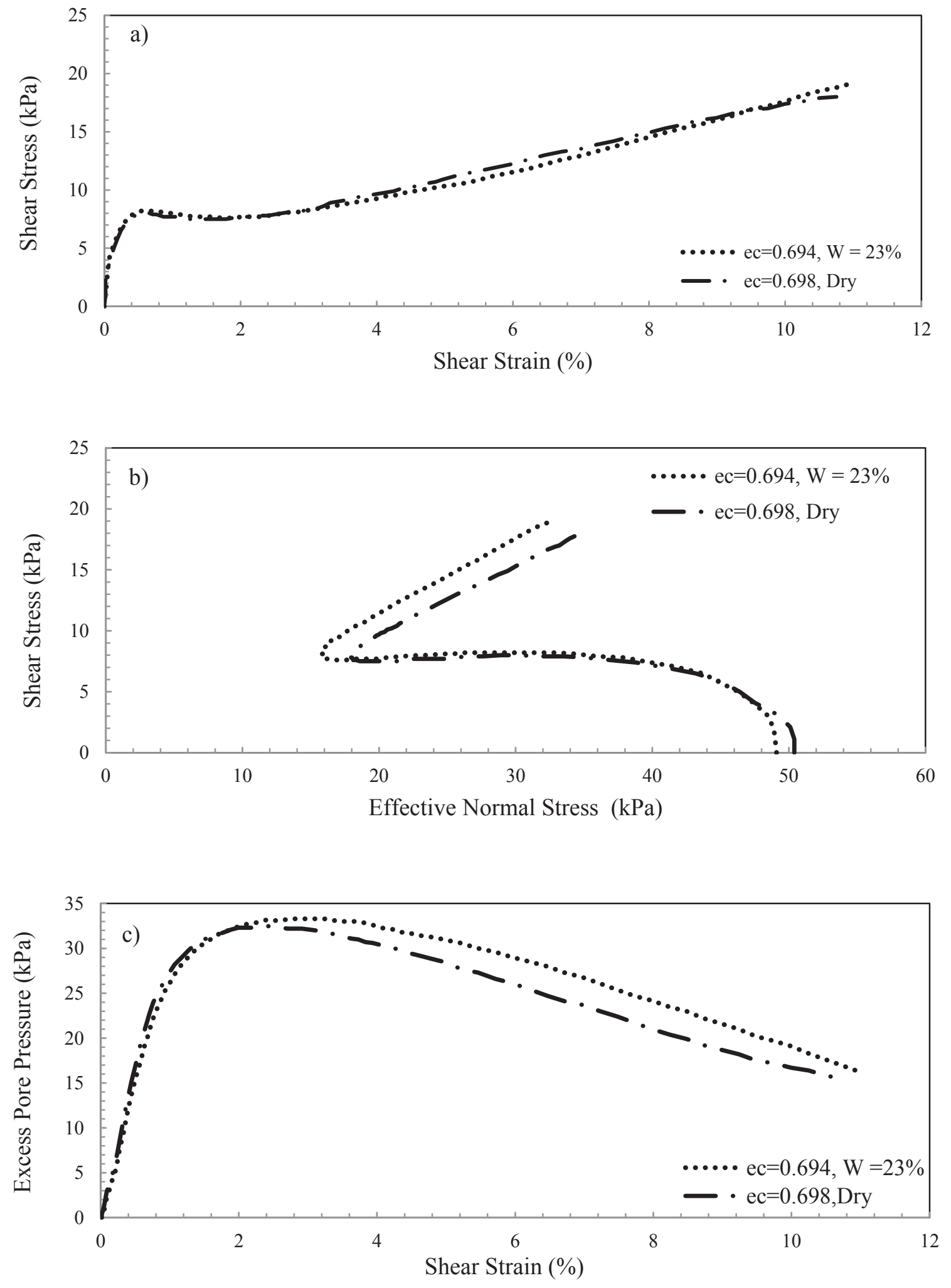

Figure 5.5 Monotonic responses of Silica Sand samples consolidated to $50 \mathrm{kPa}$ 

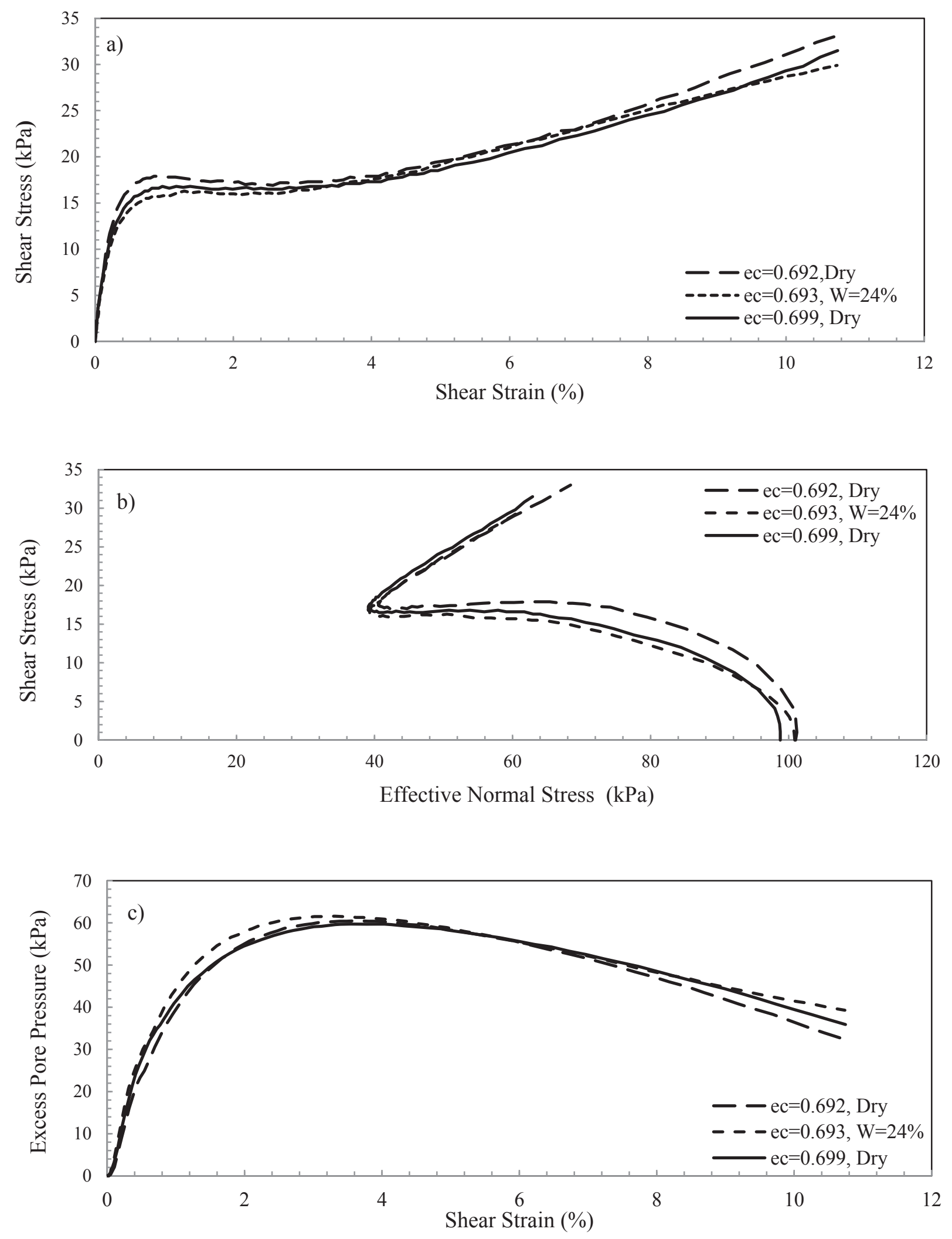

Figure 5.6 Monotonic responses of Silica Sand samples consolidated to $100 \mathrm{kPa}$ 

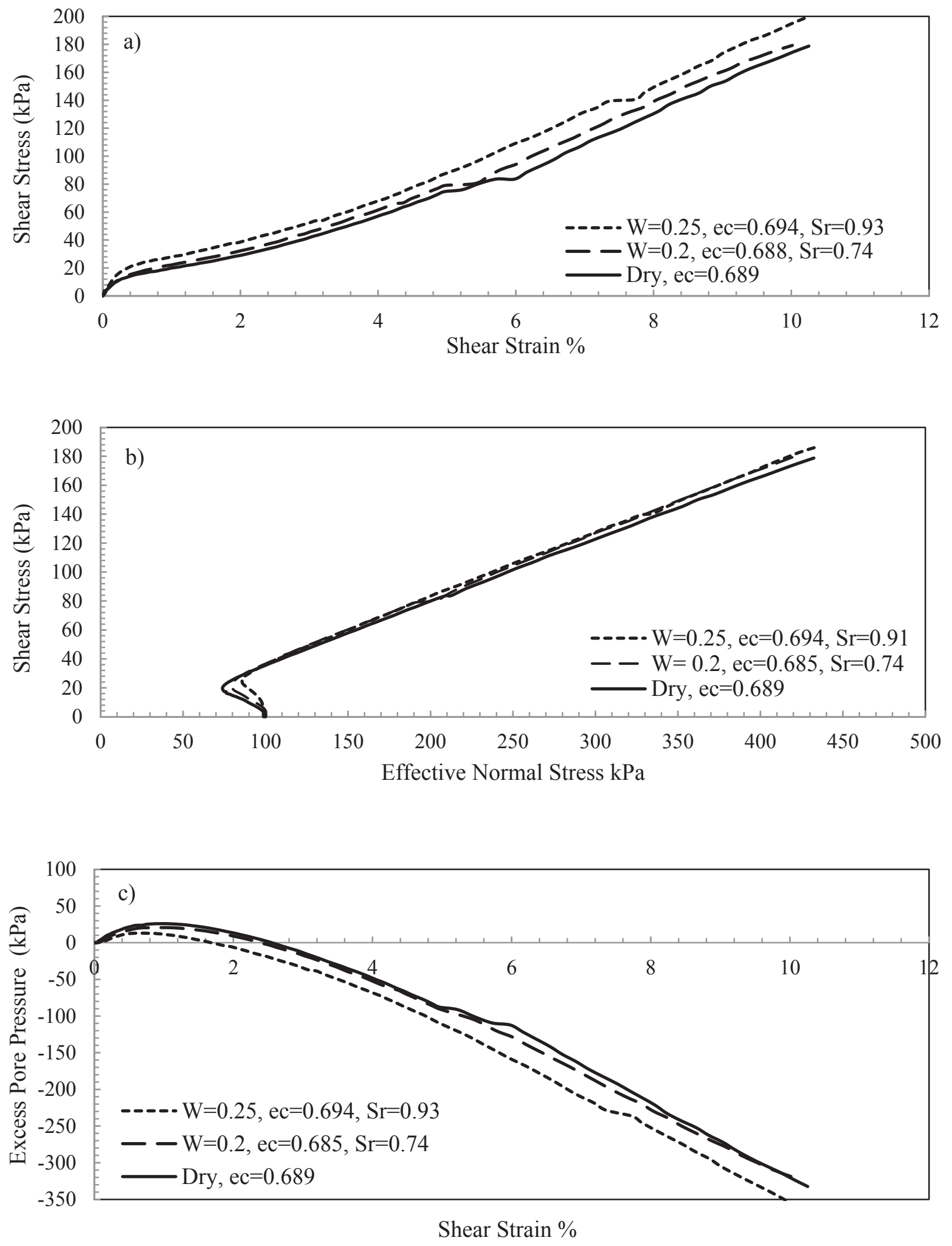

Figure 5.7 Monotonic response of glass bead material under different saturation condition 
The phase transformation point appeared at around $1 \%$ shear strain for all samples, which shows that the phase transformation occurred at a much smaller strain level than the silica sand where the phase transformation occurred at $3 \%$ shear strain. The excess pore pressure significantly decreased until the end of the test, which shows a strong dilative response after the phase transformation point. It is shown that the response of the dry specimen has negligible difference compared to the response of two partial saturated specimens under the same void ratio $(0.68 \pm$ 0.01). In fact, change in the degree of saturation under same void ratio (density) and fabric does not affect the response of glass beads materials significantly.

\subsubsection{Synthetic Silt results}

Synthetic silts were air pluviated, partially saturated using the water reservoir and consolidated to $50 \mathrm{kPa}$ effective consolidation pressure. Eighteen constant volume simple shear tests were conducted on synthetic silts specimens. Table 5.3 presents the details of all conducted tests. It was attempted to investigate the effect of increasing degree of saturation (from $\mathrm{S}=0 \%$ to $\mathrm{S}=$ $73 \%$ ) on monotonic response of constant volume tests under two fixed void ratio states (i.e. 0.66 $\&$ 0.78). Some complementary tests were also prepared at other void ratios (e.g. $0.64,0.68,0.74$, 0.81 ) in order to calculate the phase transformation and failure lines. Tests 1 to 3 were prepared under dry condition. 
Table 5.3 Characteristics of performed constant volume simple shear tests on synthetic silt samples

\begin{tabular}{|c|c|c|c|c|c|c|c|}
\hline Test No. & $\mathrm{W} \%{ }^{1}$ & $\mathrm{Sr} \%{ }^{2}$ & $e c^{3}$ & Test No. & W \% & $\mathrm{Sr} \%$ & $e_{c}$ \\
\hline Test 1 & Dry & Dry & 0.64 & Test 9 & 13.71 & 50 & 0.68 \\
\hline Test 2 & Dry & Dry & 0.7 & Test 10 & 13.63 & 49 & 0.69 \\
\hline Test 3 & Dry & Dry & 0.78 & Test 11 & 13.68 & 48.5 & 0.7 \\
\hline Test 4 & 9.84 & 37 & 0.66 & Test 12 & 13.72 & 46 & 0.74 \\
\hline Test 5 & 9.73 & 35.5 & 0.68 & Test 13 & 13.68 & 43.5 & 0.78 \\
\hline Test 6 & 9.73 & 34.5 & 0.7 & Test 14 & 13.88 & 42.5 & 0.81 \\
\hline Test 7 & 9.75 & 31 & 0.78 & Test 15 & 19.43 & 73 & 0.66 \\
\hline \multirow[t]{2}{*}{ Test 8} & 13.97 & 52.5 & 0.66 & Test 16 & 19.75 & 70 & 0.7 \\
\hline & & & & Test 17 & 19.5 & 62 & 0.78 \\
\hline
\end{tabular}

${ }^{1} \mathrm{~W}$ : Water content after consolidation

${ }^{2} \mathrm{Sr}$ : Degree of Saturation $( \pm 0.5)$

${ }^{3} \mathrm{e}_{\mathrm{c}}$ : Void Ratio $( \pm 0.01)$

\subsubsection{Dry tests}

Figure 5.8 presents the monotonic response of synthetic silts in dry condition and different void ratios (densities) consolidated to the same stress level of $50 \mathrm{kPa}$. It is clearly noted that 

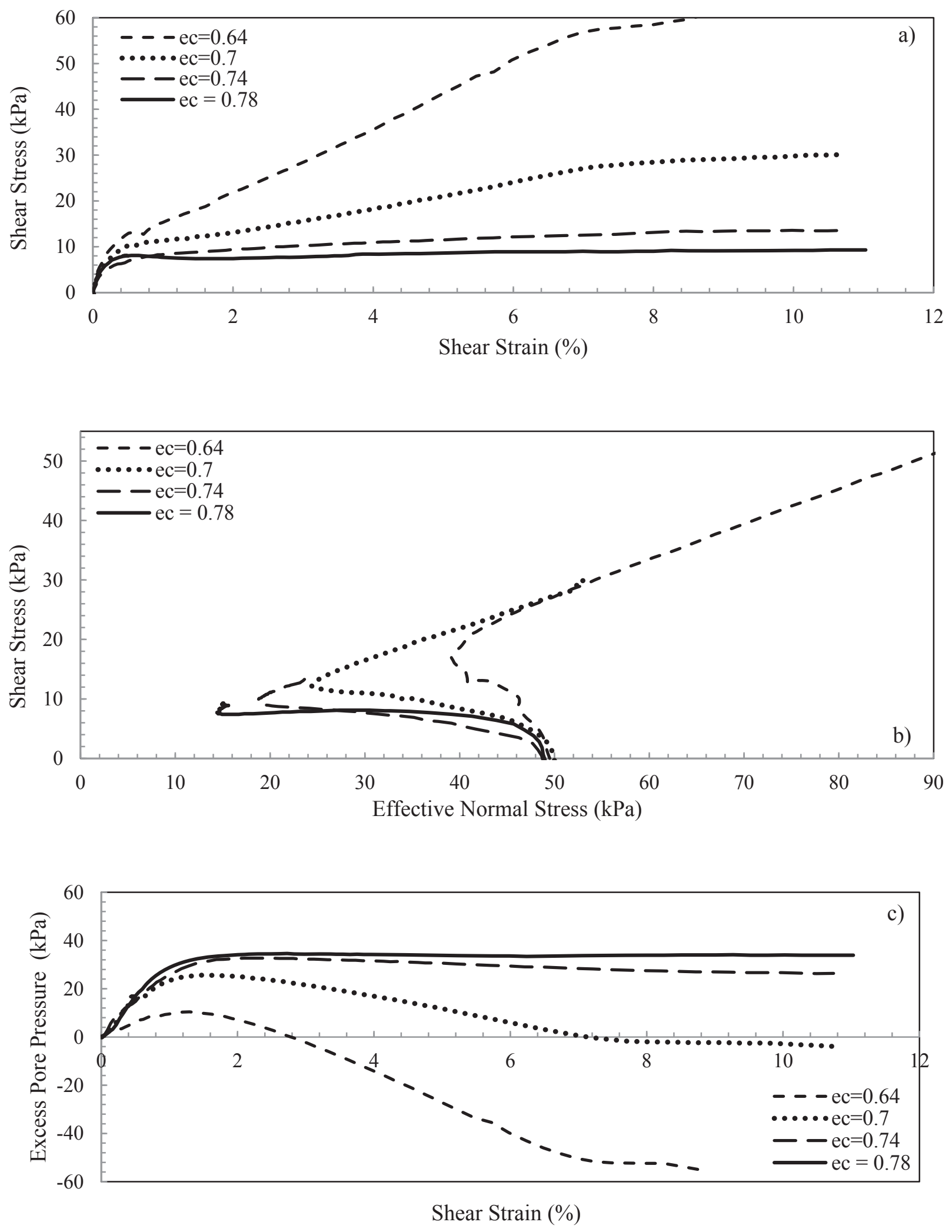

Figure 5.8 Monotonic response of constant volume tests on dry synthetic silt under different void ratios 
decreasing void ratio (increasing density) from $\mathrm{e}_{\mathrm{c}}=0.78$ to $\mathrm{e}_{\mathrm{c}}=0.64$ increases the strainhardening response. At higher density $\left(\mathrm{e}_{\mathrm{c}}=0.64\right)$ the sample exhibited significant strain hardening response. Increasing void ratio (decreasing density) to $\mathrm{e}_{\mathrm{c}}=0.7$ and $\mathrm{e}_{\mathrm{c}}=0.74$ alleviate the dilative response and the sample carried lower shear stress in Figure 5.8a in comparison to the sample with lower void ratio $\left(\mathrm{e}_{\mathrm{c}}=0.64\right)$. Regardless of the density, the dilative behavior starts from around $1.5 \%$ shear strain for samples with $\mathrm{e}_{\mathrm{c}}=0.64$ to $\mathrm{e}_{\mathrm{c}}=0.74$. Increasing the void ratio to $\mathrm{e}_{\mathrm{c}}=0.78$ resulted in lower shear strength and the response is more close to limited liquefaction response. Figure $5.8 \mathrm{c}$ shows that the excess pore pressure of the sample with $\mathrm{e}_{\mathrm{c}}=$ 0.78 significantly increased up to around $2 \%$ shear strain and remains fairly constant after the phase transformation point. Figure 5.8 demonstrates that for the dry specimens, the level of dilation is a function of the density of the sample. In other words, the monotonic stress-strain response of the specimens is highly dependent on the density (void ratio) of the specimens at the same fabric and vertical effective consolidation pressure.

\subsubsection{Samples with $10 \%$ water content $(W=10 \%)$}

Figure 5.9 presents monotonic simple shear responses of partial saturated synthetic silts with $10 \%$ water content but different void ratios. The degree of saturation varied from $\mathrm{S}_{\mathrm{r}}=30 \%$ to $\mathrm{S}_{\mathrm{r}}$ $=37 \%$ in these tests. Figure 5.9 shows that samples with lower void ratios exhibit higher shear stresses during shearing. It is also shown that increasing void ratio (decreasing density) significantly alleviates the dilative response. For example, the sample with $\mathrm{e}_{\mathrm{c}}=0.66$ reached phase transformation point more rapidly than other void ratios. In other words, the point where the response changes from contractive behaviour to dilative behaviour appears more rapidly in 
the sample with higher density $\left(\mathrm{e}_{\mathrm{c}}=0.66\right)$ than other samples. Figure $5.9 \mathrm{~b}$ shows that the effective normal stress of the sample with $e_{c}=0.78$ increases intangibly after the phase transformation point, which confirms that the dilative response after the phase transformation point is not significant. It is obtained that the change in void ratio (density) under the same water content, fabric and vertical effective consolidation pressure does affect the monotonic response of the partial saturated synthetic silt samples.

\subsubsection{Samples with $14 \%$ water content $(W=14 \%)$}

Figures 5.10 to 5.12 present monotonic simple shear response of partially saturated synthetic silts with $14 \%$ water content but different void ratios. The degree of saturation varied from $\mathrm{S}_{\mathrm{r}}=$ $42.5 \%$ to $S_{r}=50 \%$ in these tests. Sample with high density $\left(e_{c}=0.68\right)$ exhibited higher dilation in the monotonic response. Figure 5.12 shows that the excess pore pressure for the sample with $\mathrm{e}_{\mathrm{c}}=0.68$ increased up to around $24 \mathrm{kPa}$ at $2 \%$ shear strain, which corresponds to contractive response up to $2 \%$ shear strain. The excess pore pressure decreases after this peak, which corresponds to dilative response. The gradual decrease of excess pore pressure continued until the end of the test. Figure 5.11 clearly shows that with increasing void ratio (decreasing density) the value of shear stress at phase transformation decreases tangibly. For instance, the shear stress at phase transformation decreases from $14.6 \mathrm{kPa}$ for $\mathrm{e}_{\mathrm{c}}=0.68$ to $8.2 \mathrm{kPa}$ for $\mathrm{e}_{\mathrm{c}}=0.78$. The monotonic response of the sample with the loosest void ratio $\left(\mathrm{e}_{\mathrm{c}}=0.81\right)$ exhibited a truly strain softening response. The stress reached a peak value of about $8 \mathrm{kPa}$, and decreased after the peak to reach an essentially constant value of about $7 \mathrm{kPa}$ at large strains. In fact, samples with void ratios from $\mathrm{e}_{\mathrm{c}}=0.68$ to $\mathrm{e}_{\mathrm{c}}=0.74$ exhibited initial contraction followed by significant dilation (strain-hardening response). Figure 5.10 to 5.12 confirms that the monotonic response of 

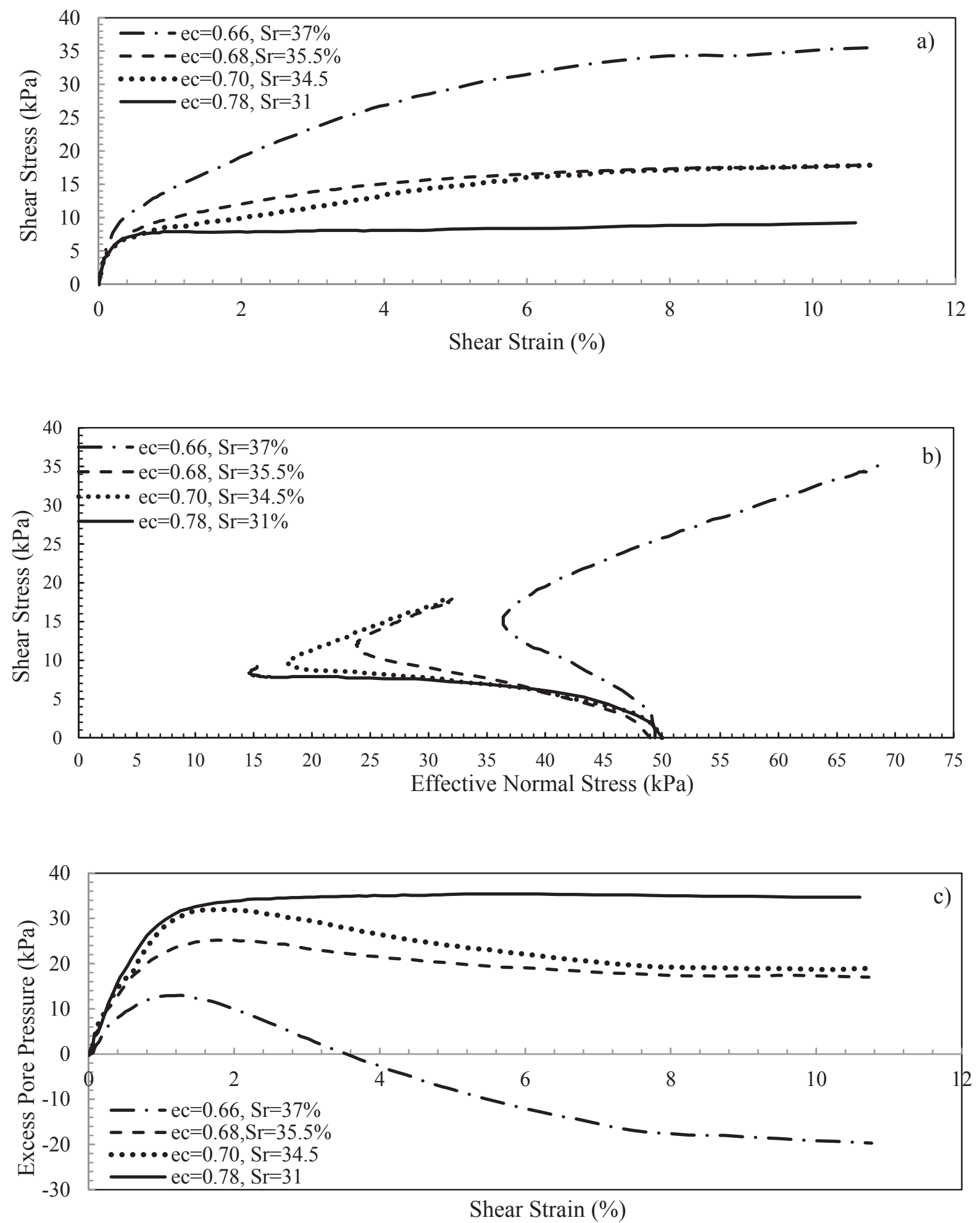

Figure 5.9 Monotonic response of constant volume tests on partial saturated synthetic silt samples $(\mathrm{W}=10 \%)$ under different void ratios 


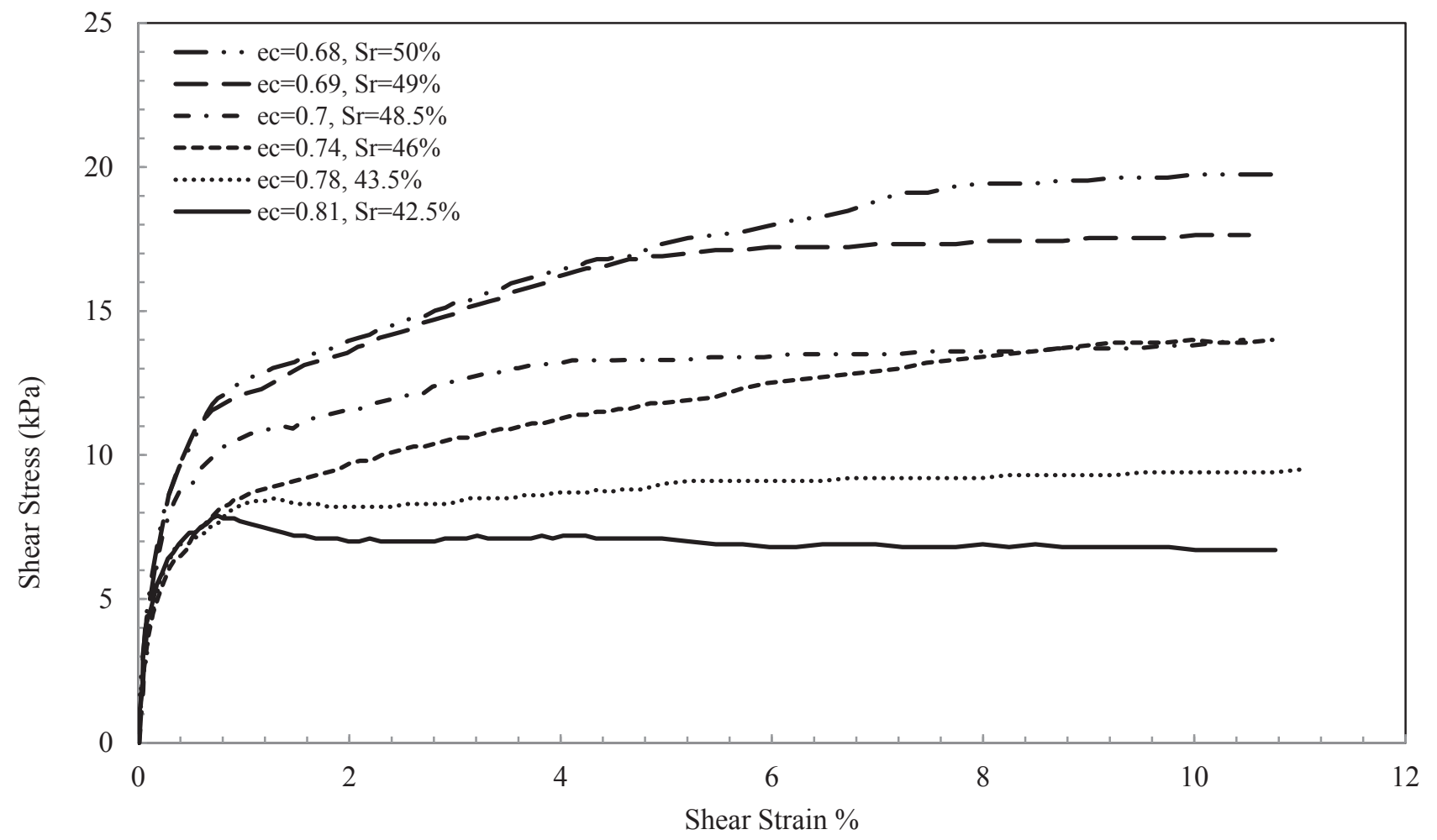

Figure 5.10 Stress-strain monotonic response of partial saturated synthetic silts samples $(\mathrm{W}=14 \%)$ under different void ratios

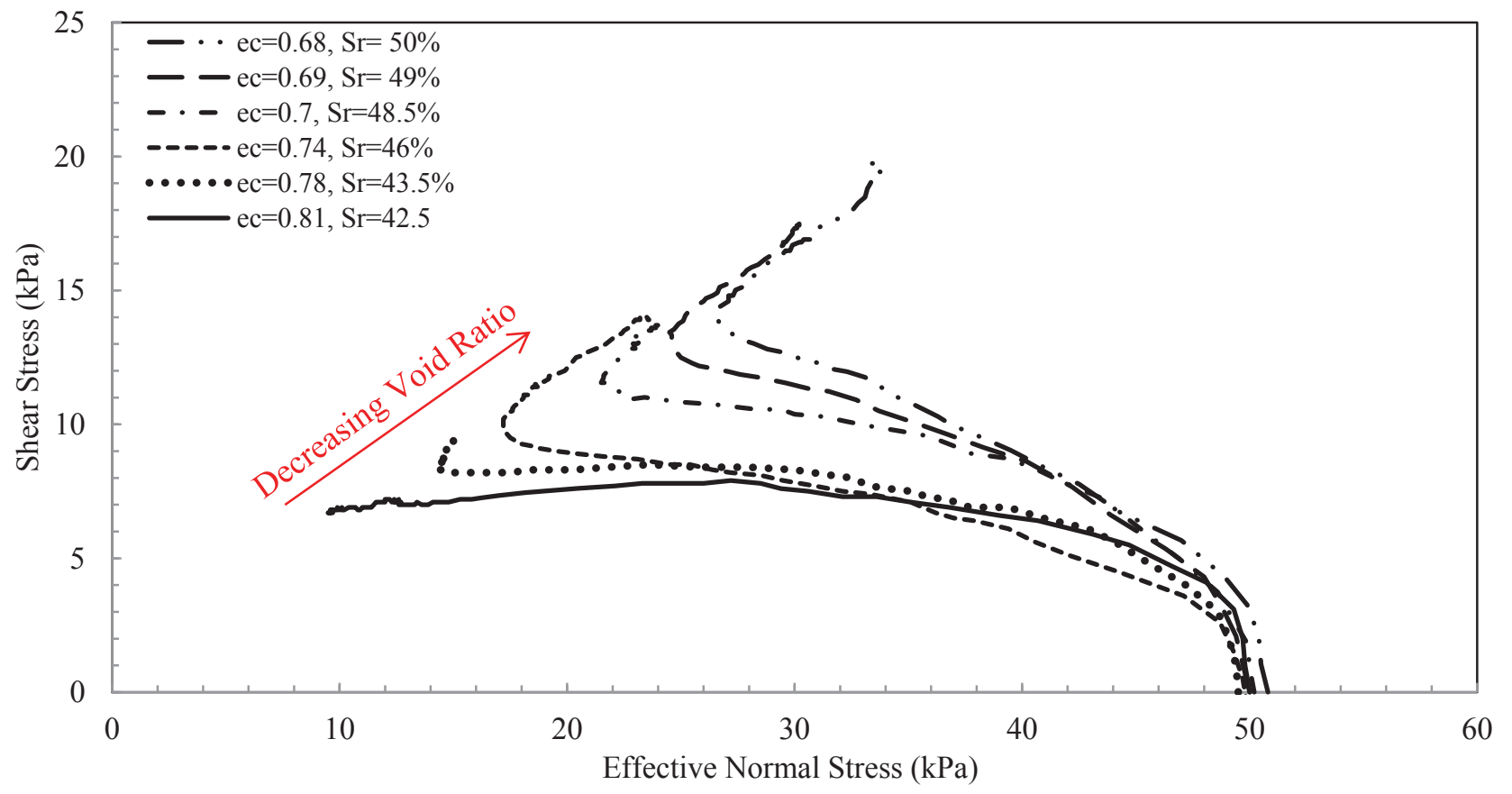

Figure 5.11 Stress path monotonic response of partial saturated synthetic silts samples (W=14\%) under different void ratios 


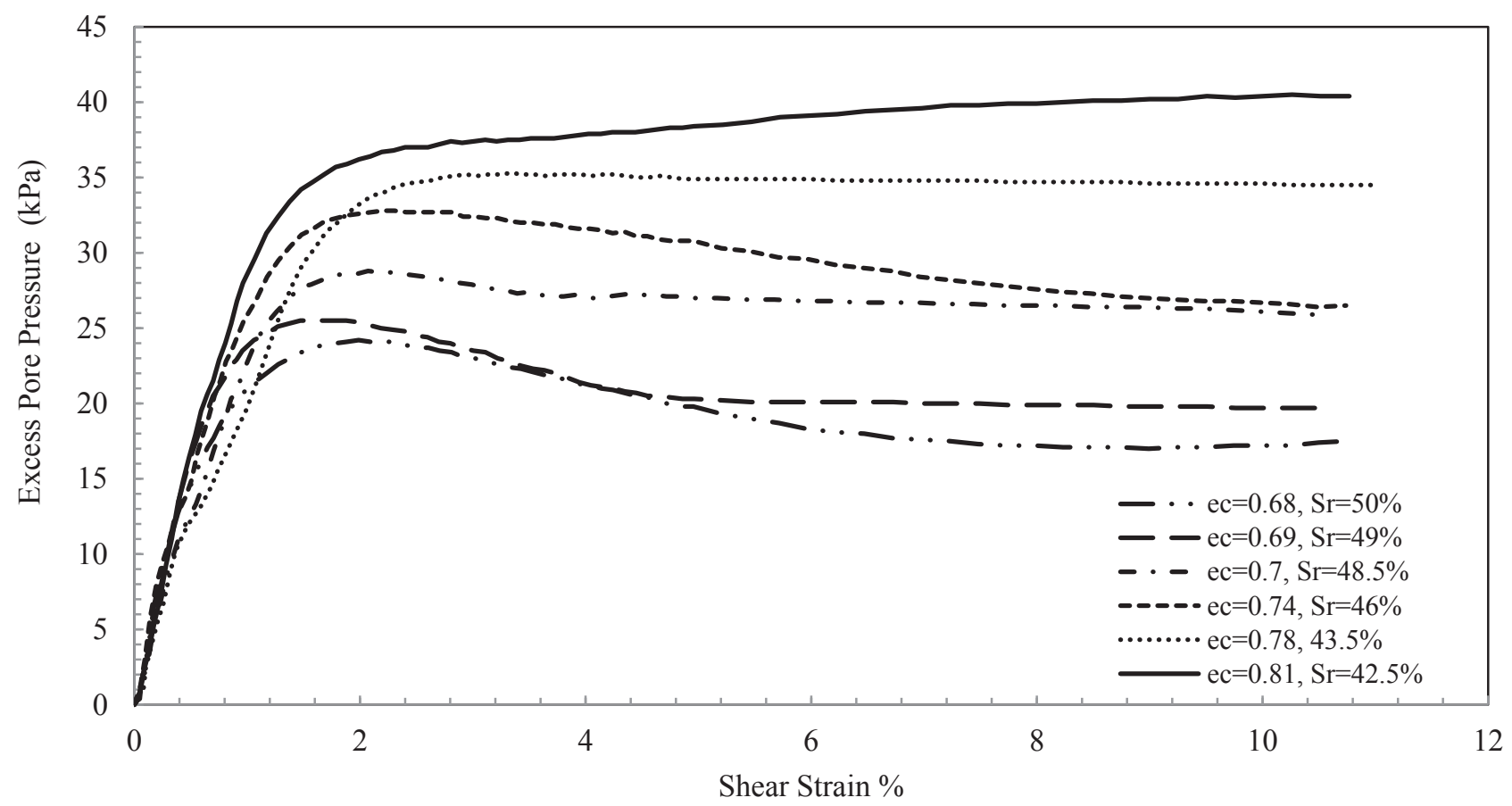

Figure 5.12 Excess pore pressure - shear strain monotonic response of partial saturated synthetic silts samples $(\mathrm{W}=14 \%)$ under different void ratios

synthetic silts is highly dependent on the density of the samples under the same fabric and consolidation conditions.

\subsubsection{Samples with $20 \%$ water content $(W=20 \%)$}

Figure 5.13 presents monotonic constant volume simple shear response under different void ratios at $20 \%$ water content, but with degree of saturation ranging from $\mathrm{S}_{\mathrm{r}}=62 \%$ to $\mathrm{S}_{\mathrm{r}}=73 \%$. Figure 5.13 shows that the sample with $\mathrm{e}_{\mathrm{c}}=0.66$ exhibited significant strain hardening response. The excess pore pressure of this significantly decreased to negative values after a small increase during initial contraction. The phase transformation occurred at around 1.5\% shear strain. 

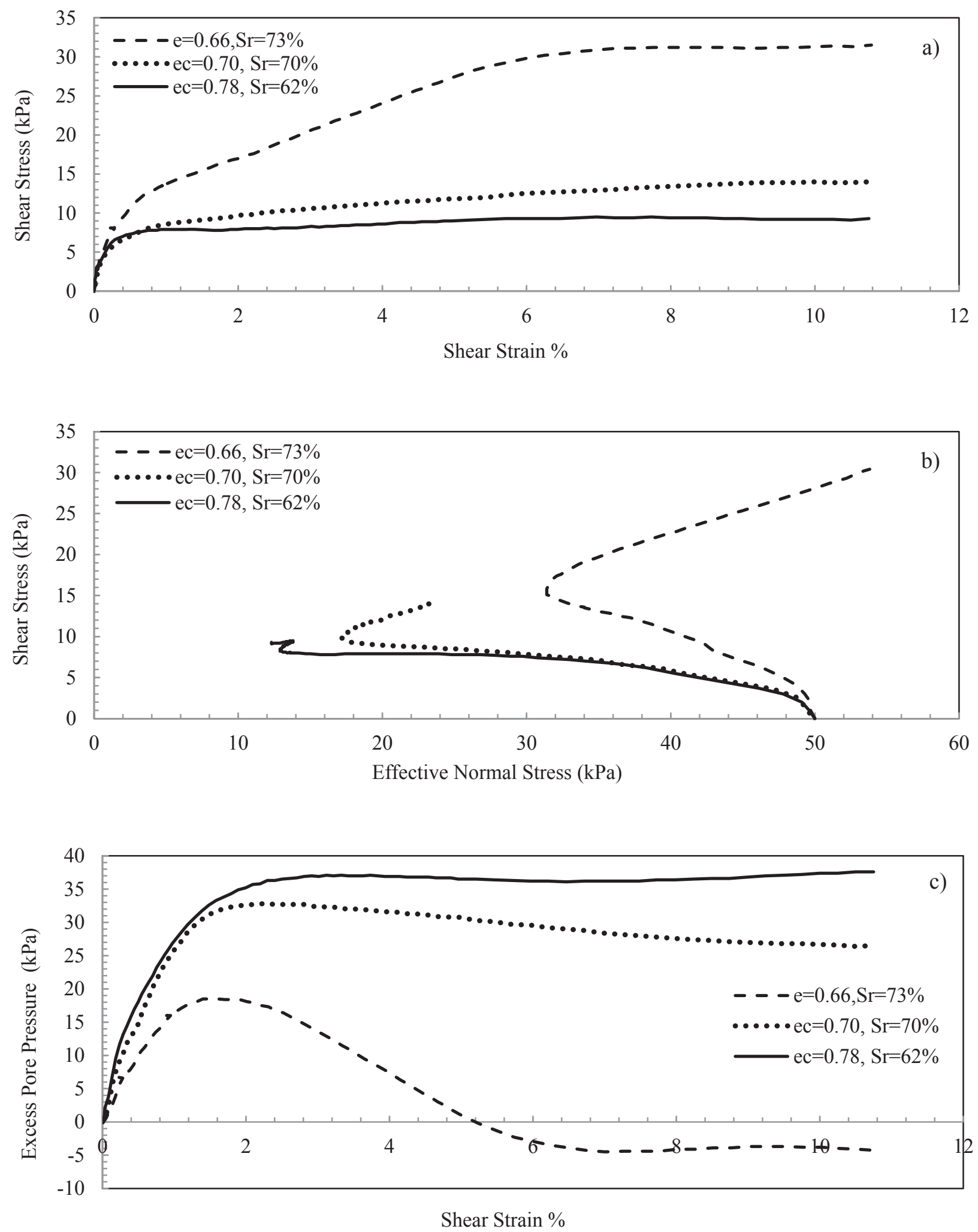

Figure 5.13 Monotonic response of constant volume tests on partial saturated synthetic silt samples $(\mathrm{W}=20 \%)$ under different void ratios 
Figure 5.13 shows that similar to samples with lower degrees of saturation, increasing void ratio to $\mathrm{e}_{\mathrm{c}}=0.7$ and $\mathrm{e}_{\mathrm{c}}=0.74$ alleviates the strain-hardening response.

\subsubsection{Comparisons of monotonic responses at same void ratios}

Monotonic responses of constant volume tests at $e_{c}=0.78$ and different degrees of saturation (derived from the data presented previously) are presented in Figure 5.14. It is clear that all samples exhibit limited liquefaction response with a mild peak at this void ratio. Figure 5.14c shows that in all samples the excess pore pressure increased significantly to around $35 \mathrm{kPa}$ at $2 \%$ shear strain. The excess pore pressure remains fairly constant until the end of the tests. All samples exhibited significant contractive response followed by a small dilation. It is also noted that even though the degree of saturation varied from $\mathrm{Sr}=0 \% \mathrm{Sr}=62 \%$ there is no tangible change in the response. This indicates that the degree of saturation does not influence the constant volume monotonic response of the synthetic silts at $e_{c}=0.78$.

Figure 5.15 presents the comparison of monotonic response of constant volume tests on synthetic silts at same void ratio $\left(\mathrm{e}_{\mathrm{c}}=0.66\right)$ but different degrees of saturation. It is clear that all responses are strain-hardening with a phase transformation point at around $16 \mathrm{kPa}$ shear stress. It is also demonstrated that the responses of tests at same void ratio but different degrees of saturation are fairly the same. In other words, the degree of saturation does not affect the response of the material at a constant void ratio. Consequently, the response of dried constant volume test and partially saturated or saturated test at same void ratio would be identical. It should be noted that although matric suction is produced in synthetic silt materials at $\mathrm{Sr}<1$, the effect of suction is 
negligible and not influential since the matric suction values are not high (Figure 5.4). It should be mentioned that according to hysteresis effect, the values of matric suction in SWCC during rewetting are significantly lower than the values during drying (Pham et al., 2005). As a result, not
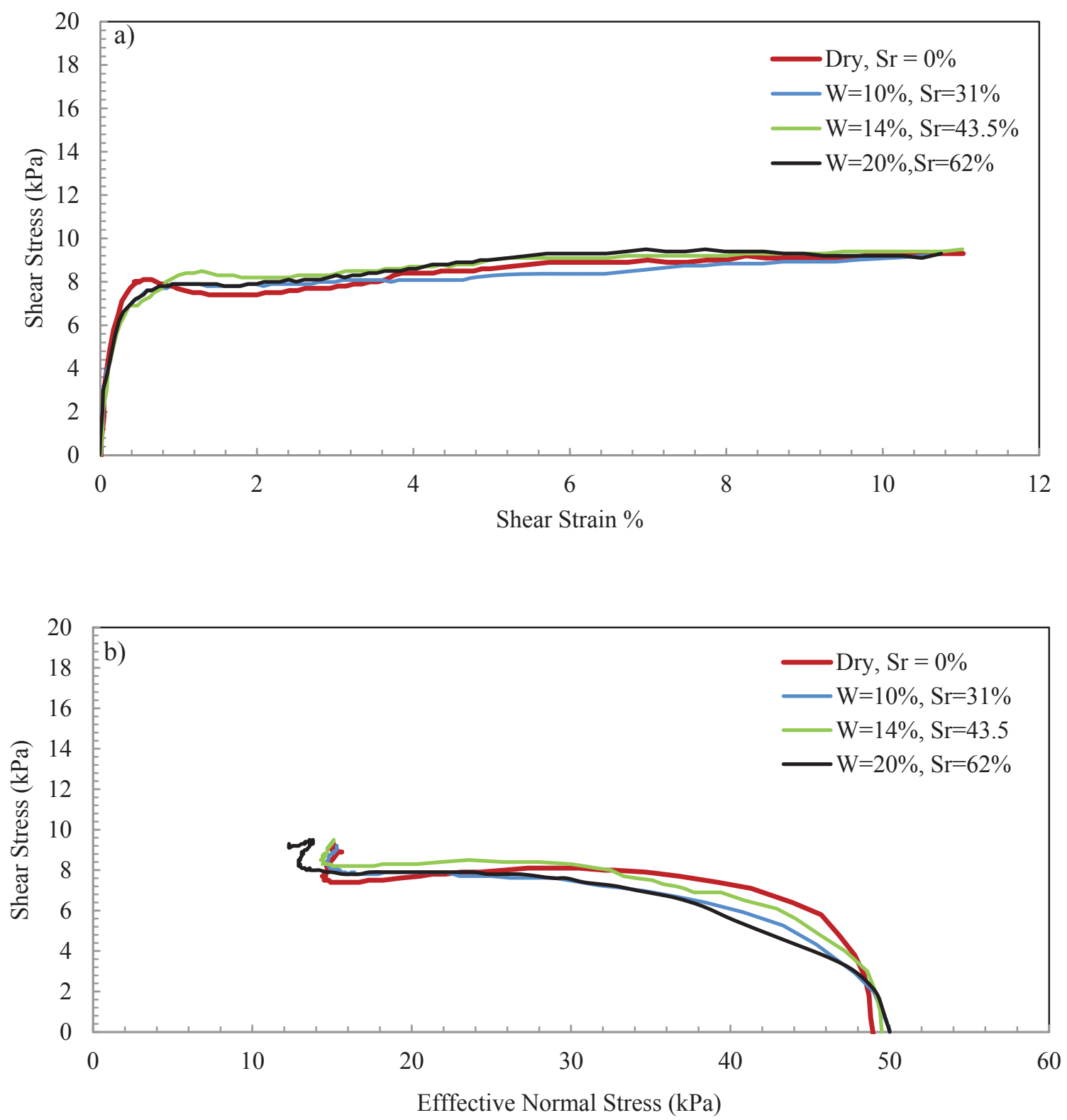

Figure 5.14 Comparison of monotonic response of constant volume tests on synthetic silts under same void ratio $\left(\mathrm{e}_{\mathrm{c}}=0.78 \pm 0.01\right)$ but different degrees of saturation 
only the values of matric suction are low in synthetic silt materials, but also the values would be highly reduced than values presented in Figure 5.4 and therefore cannot affect the response of constant volume tests.
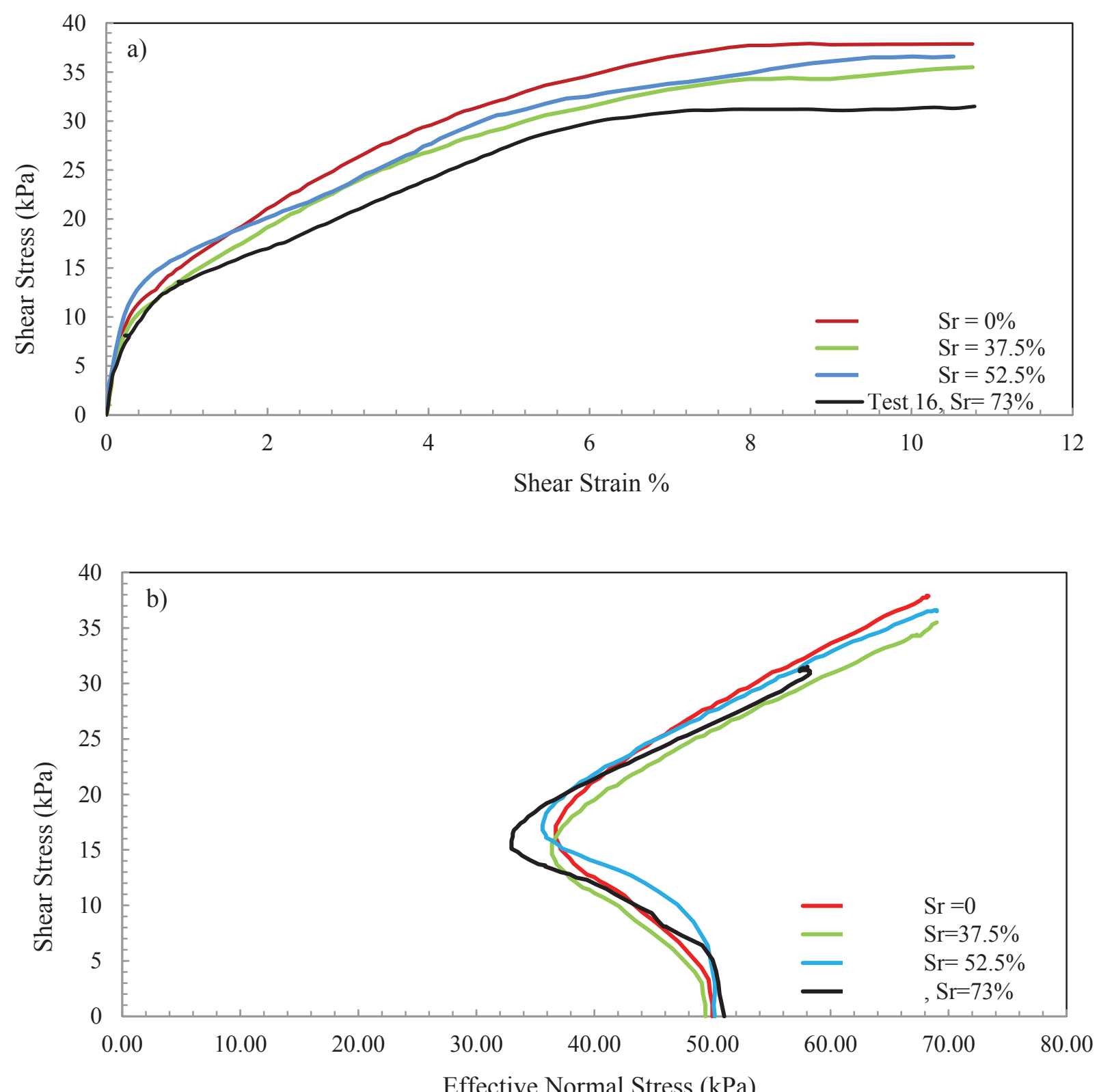

Figure 5.15 Comparison of monotonic response of constant volume tests on synthetic silts under same void ratio $\left(\mathrm{e}_{\mathrm{c}}=0.66 \pm 0.01\right)$ but different degrees of saturation 


\subsubsection{Phase transformation and failure angles}

Figure 5.16 shows a plot of the stress states at phase transformation and failure for all synthetic silt tests. The data points fall within a narrow band, and the best fir regression line yields a

friction angle at phase transformation $\phi_{P T}^{\prime}=25.4^{\circ}$ and at failure $\phi_{\text {Failure }}^{\prime}=29.3^{\circ}$. Figure 5.16a also shows that the phase transformation line for tests at different degrees of saturation is fairly similar. Similarly, Figure 5.16b presents that the failure line for tests at different degrees of saturation is also fairly similar. Therefore, the tests confirm the uniqueness of the phase transformation and failure friction angles irrespective of the degree of saturation. Such uniqueness of the friction angle at phase transformation state has been reported by several other researchers in various granular materials (e.g., Vaid and Chern, 1985; Vaid and Sivathayalan, 1996)

\subsection{Summary and Conclusion}

Three different materials have been tested to investigate the effect of degree of saturation on the monotonic response in constant volume tests. The almost identical response of saturated constant volume test and undrained test was verified by Dyvik et al. (1987). Similarly, Finn et al (1978) have demonstrated equivalence between dry and fully saturated sands in simple shear. However, no attempts have been made until this study to evaluate the potential equivalence of undrained and constant volume behaviour in partially saturated soils. The research reported in this chapter demonstrates that the constant volume behaviour is not dependent on the degree of saturation for the materials tested. The results of dry constant volume test and high degrees of saturation 

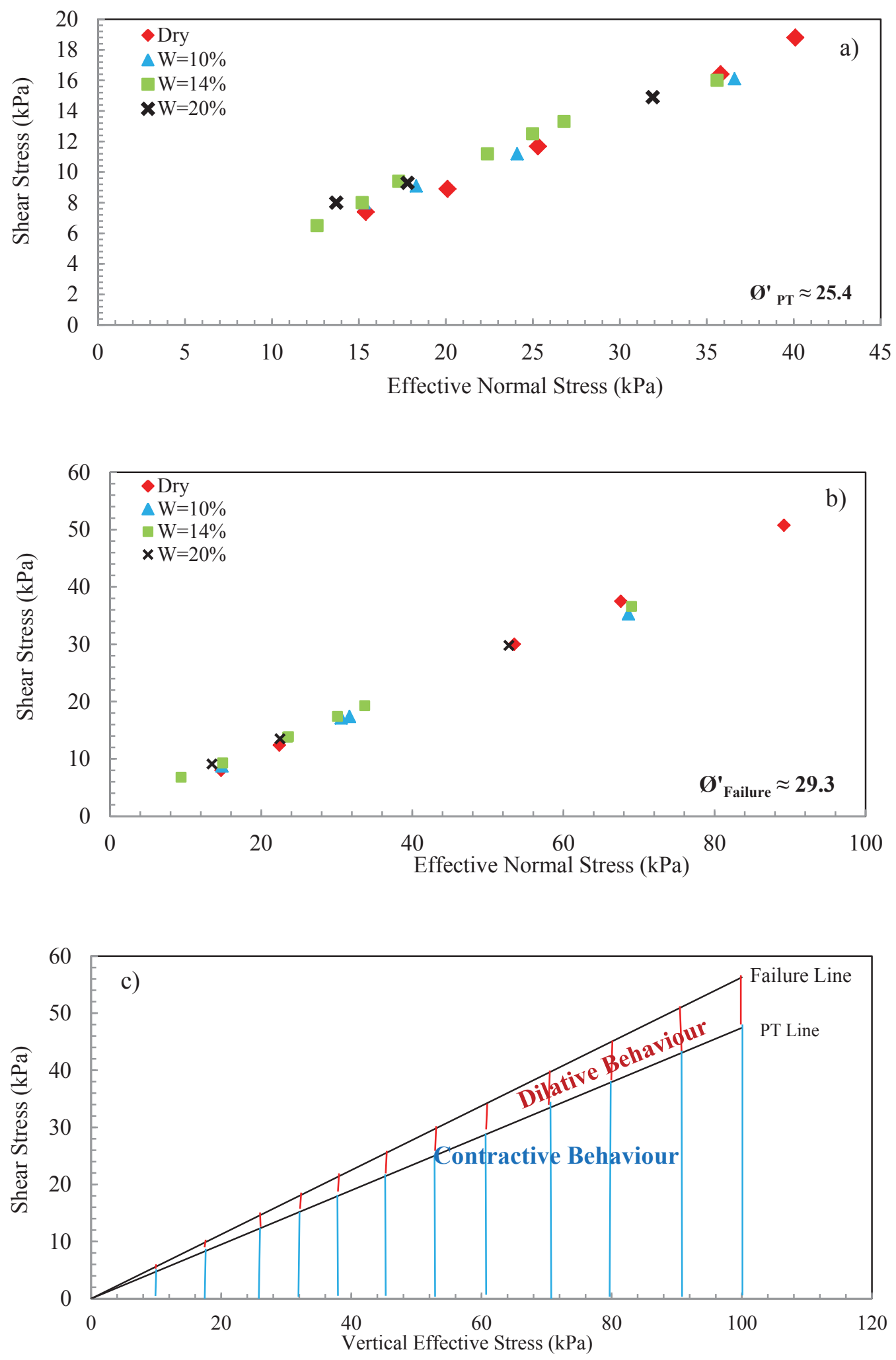

Figure 5.16 Phase Transformation and failure angles of synthetic silt constant volume simple shear tests 
constant volume test on Silica Sand and glass beads were found to be identical. Similarly, the degree of saturation in the synthetic silt did not influence the constant volume simple shear behaviour. As noted earlier, the synthetic silt was adopted due to its similar characteristics compared to the fine grained Bulyanhulu gold tailings. The synthetic silt material generates low values of suction during desiccation in comparison to the gold tailings. Since the desiccatedrewetted tailings also have low or zero values of suction, the observed constant volume behaviour of partially saturated synthetic silt can be expected to be similar to that of desiccatedrewetted gold tailings. These results suggest that the degree of saturation will not influence the monotonic simple shear response (in constant volume simple shear tests) in coarse grained materials as well as fine grained materials that generates low suctions $(<20 \mathrm{kPa})$ during desiccation. The findings reported in this chapter can be summarized as:

- The monotonic response of dry and partially saturated coarse grained materials (at a given density/void ratio, effective consolidation stress and fabric) under constant volume simple shear is essentially identical.

- Under constant volume condition, degree of saturation does not affect the response of fine grained soils that generate low values of suction $(<20 \mathrm{kPa})$ during desiccation. 


\section{Chapter 6: Simple shear, triaxial and vane shear test results}

\subsection{Introduction}

Monotonic and cyclic simple shear and triaxial test results and results of vane shear tests on Bulyanhulu tailings are presented and discussed in this chapter. In the first section, simple shear tests performed on samples prepared by small scale and drying box laboratory methods are presented. Modified and conventional triaxial tests are presented in the next section. The final section presents results of vane shear tests.

\subsection{Results of simple shear tests}

Monotonic and cyclic simple shear tests were performed on desiccated-rewetted samples at different consolidation stresses and water contents. Tests were conducted on specimens extruded from the small scale laboratory method, the drying box and those directly prepared in the simple 
shear mould. Results of tests performed on desiccated samples and mechanically overconsolidated samples are also presented in addition to those on desiccated-rewetted samples.

\subsubsection{Monotonic results of small scale laboratory tests}

Simple shear tests performed on desiccated-rewetted samples obtained from small scale laboratory method consisted of consolidation prior to shearing under 50, 100, 200 and $400 \mathrm{kPa}$. All desiccated samples were consolidated under $400 \mathrm{kPa}$. As mentioned in chapter 3, the small scale method contained controlled desiccation followed by re-wetting and mechanical consolidation. The method consisted of successively depositing two layers of tailings to a thickness of about $100 \mathrm{~mm}$ each in a cylindrical column with $250 \mathrm{~mm}$ diameter. Thickened tailings were deposited in the column at pumping water content $(38 \%)$ and allowed to desiccate to different values of water content, above and below the shrinkage limit ( $\mathrm{W}=30,28,25,23,19$, 17, 12 and 4\%). Table 6.1 presents test characteristics of monotonic simple shear tests performed on desiccated-rewetted samples prepared by small scale laboratory method. The water content (Wd) corresponds to final water content of the sample during desiccation and before rewetting. Current water content of samples desiccated beyond the SL returns to a value between $21 \%$ and

$22 \%$. Current water content of low desiccated tailings $(\mathrm{Wd}=30 \%-23 \%)$ becomes $1 \%-2 \%$ higher after rewetting.

\subsubsection{Monotonic results under $50 \mathrm{kPa}$ vertical effective consolidation pressure}


Figure 6.1 shows the experimental results of the volume change vs. stress history of samples desiccated to $\mathrm{Wd}=23 \%$ and $\mathrm{Wd}=12 \%$ respectively. Lu and Likos (2006)'s equation was used to estimate the equivalent effective stress during desiccation. According to Figure 6.1a, the thickened tailings layer initially has a reduction in volume during settling. With increasing desiccation to $\mathrm{Wd}=23 \%$, a suction stress equal to $34 \mathrm{kPa}$ is generated while the void ratio decreased to $\mathrm{e}=0.82$. Re-wetting caused a marginal increase in void ratio, and subsequent consolidation under $50 \mathrm{kPa}$ resulted in a final void ratio of $\mathrm{e}_{\mathrm{c}}=0.60$. Figure $6.1 \mathrm{~b}$ shows that for samples desiccated to $\mathrm{Wd}=12 \%$, the suction stress around $205 \mathrm{kPa}$ is generated during desiccation. Final void ratio of the sample after rewetting and consolidation is $\mathrm{e}_{\mathrm{c}}=0.63$.

Figure 6.2 presents monotonic results of the desiccated and rewetted samples under $50 \mathrm{kPa}$ consolidation pressure after extraction. The sample at $\mathrm{Wd}=30 \%$, which consisted of tailings that have been settled from the deposition water content (38\%) but did not experience any desiccation exhibits a strain-softening response. A dilative phase in the behavior (noted by the presence of strain hardening, or the phase transformation point in stress path) starts to appear for tailings dried to $\mathrm{Wd}=28 \%$ and lower, and the dilative response is increased with continuing desiccation. The strain hardeing tendency and the shear stress at phase transformation increases as the desiccated water content varies from $\mathrm{Wd}=28 \%$ down to $\mathrm{Wd}=17 \%$. The increase in strain hardening between samples desiccated to $17 \%$ and $12 \%$ is quite small, and the strength at maximum strain for the sample desiccated to $4 \%$ is somewhat smaller than the strength at $17 \%$. It is also clear that samples that experienced higher degree of desiccation reached higher void ratio after consolidation. Nonetheless, this higher void ratio does not lead to lower shear strength. In other words, the effect of stress history makes the shear strength higher despite the effect of lower density (higher void ratio). 
Table 6.1 Monotonic simple shear tests performed on samples prepared with small scale deposition method

\begin{tabular}{|c|c|c|c|c|}
\hline $\begin{array}{l}\text { Test } \\
\text { No. }\end{array}$ & $\begin{array}{c}\text { Sample } \\
\text { Characteristics }\end{array}$ & $\begin{array}{c}\text { Water content } \\
\text { before rewetting } \\
\text { Wd }(\%)\end{array}$ & $\begin{array}{c}\text { Consolidation } \\
\text { Pressure }\left(\sigma_{v c}^{\prime}\right) \\
\mathrm{kPa} \\
\end{array}$ & $\begin{array}{l}\text { Void ratio after } \\
\text { consolidation }\left(\mathrm{e}_{\mathrm{c}}\right)\end{array}$ \\
\hline 1 & Settled & 30 & 50 & 0.520 \\
\hline 2 & Desiccated/rewetted & 28 & 50 & 0.580 \\
\hline 3 & Desiccated/rewetted & 23 & 50 & 0.604 \\
\hline 4 & Desiccated/rewetted & 18 & 50 & 0.619 \\
\hline 5 & Desiccated/rewetted & 17 & 50 & 0.623 \\
\hline 6 & Desiccated/rewetted & 12 & 50 & 0.628 \\
\hline 7 & Desiccated/rewetted & 4 & 50 & 0.636 \\
\hline 8 & Settled & 31 & 100 & 0.533 \\
\hline 9 & Settled & 29 & 100 & 0.548 \\
\hline 10 & Desiccated/rewetted & 26 & 100 & 0.573 \\
\hline 11 & Desiccated/rewetted & 25 & 100 & 0.604 \\
\hline 12 & Desiccated/rewetted & 18 & 100 & 0.606 \\
\hline 13 & Desiccated/rewetted & 17 & 100 & 0.619 \\
\hline 14 & Desiccated/rewetted & 12 & 100 & 0.628 \\
\hline 15 & Desiccated/rewetted & 28 & 400 & 0.593 \\
\hline 16 & Desiccated/rewetted & 27.5 & 400 & 0.581 \\
\hline 17 & Desiccated/rewetted & 26.5 & 400 & 0.578 \\
\hline 18 & Desiccated/rewetted & 25.5 & 400 & 0.548 \\
\hline 19 & Desiccated/rewetted & 20 & 400 & 0.601 \\
\hline 20 & Desiccated/rewetted & 19 & 400 & 0.622 \\
\hline 21 & Desiccated/rewetted & 18.5 & 400 & 0.606 \\
\hline 22 & Desiccated/rewetted & 18 & 400 & 0.615 \\
\hline 23 & Desiccated/rewetted & 17.5 & 400 & 0.612 \\
\hline 24 & Desiccated/rewetted & 13 & 400 & 0.617 \\
\hline 25 & Desiccated/rewetted & 12 & 400 & 0.621 \\
\hline 26 & Des/Con/Rewetted ${ }^{1}$ & 26 & 400 & 0.579 \\
\hline 27 & Des/Con/Rewetted & 20 & 400 & 0.588 \\
\hline 28 & Des/Con/Rewetted & 19.5 & 400 & 0.574 \\
\hline 29 & Des/Con/Rewetted & 19 & 400 & 0.573 \\
\hline 30 & Des/Con/Rewetted & 18.5 & 400 & 0.577 \\
\hline 31 & Des/Con/Rewetted & 18 & 400 & 0.591 \\
\hline 32 & Des/Con/Rewetted & 16.5 & 400 & 0.584 \\
\hline 33 & Des/Con/Rewetted & 15.5 & 400 & 0.581 \\
\hline 34 & Des/Con/Rewetted & 14.5 & 400 & 0.592 \\
\hline
\end{tabular}

${ }^{1}$ Samples desiccated, consolidated and rewetted (Rewetting performed after consolidation) 

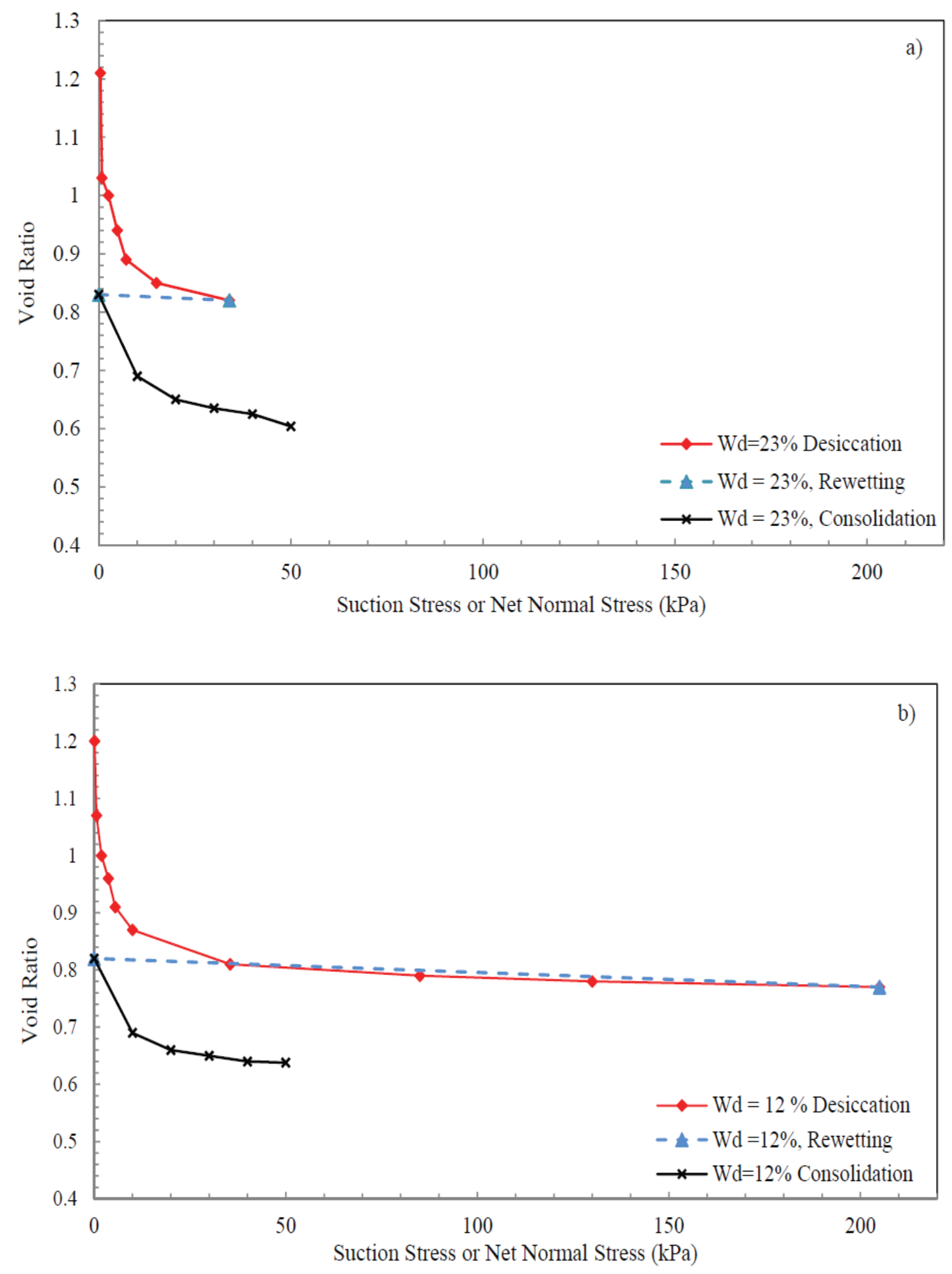

Figure 6.1 Volume change stress history of samples desiccated to a) $\mathrm{W}=23 \%$, b) $\mathrm{W}=12 \%$, rewetted and consolidated to $50 \mathrm{kPa}$ 

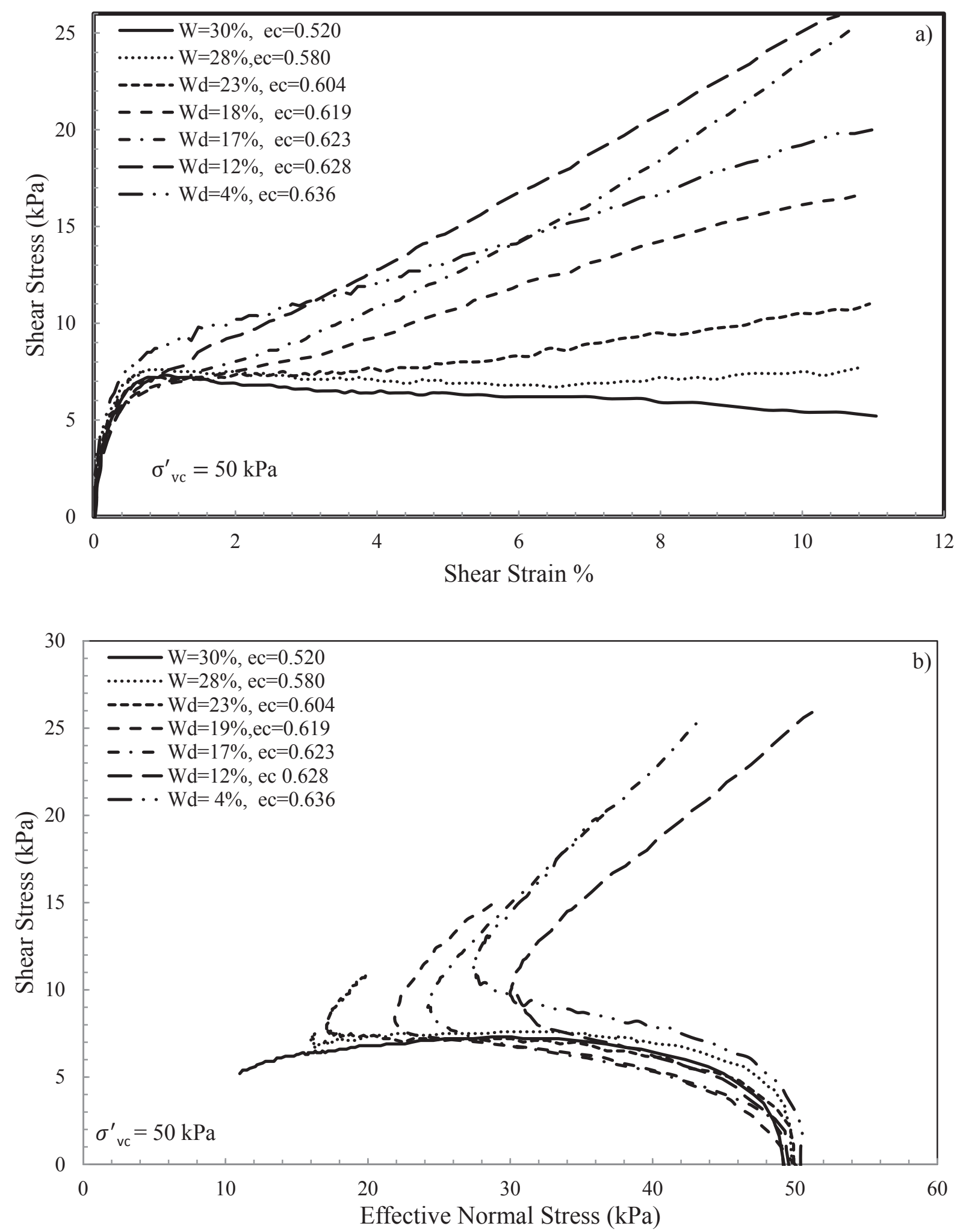

Figure 6.2 Monotonic results of desiccated and rewetted samples prepared by small scale method under $50 \mathrm{kPa}$ 


\subsubsection{Monotonic results under $100 \mathrm{kPa}$ vertical effective consolidation pressure}

Figure 6.3 presents monotonic shear responses of desiccated-rewetted tailings prepared by small scale deposition method and consolidated to $100 \mathrm{kPa}$. Figure 6.3 clearly shows that samples settled $(\mathrm{W}=31 \%$ and $\mathrm{W}=29 \%)$ and consolidated to $100 \mathrm{kPa}$ exhibited strain-softening response. The water content noted corresponds to the water content of the sample after settling and before consolidation. Figure 6.3 also demonstrates that a little degree of desiccation to $\mathrm{Wd}=$ $26 \%$, changes the response to strain-hardening with a clearly identifiable phase transformation point. With further increasing desiccation history to the shrinkage limit, the response becomes more dilative and the shear strength increases significantly. Excessive desiccation beyond the shrinkage limit $(\mathrm{W}=12 \%)$ slightly increases the shear strength. However, the effect of desiccation beyond the shrinkage limit on shear strength is not as beneficial as desiccation to the shrinkage limit.

Figure 6.3a shows that samples that experienced no desiccation reached an essential steady state at large shear strain, even though the void ratio of these samples is quite lower compared to samples that experienced desiccation $(0.533,0.548$ vs. 0.573 to 0.628$)$. The lower void ratio was realized on account of the relatively high compressibility of the tailings with no desiccation history. When samples experience desiccation, they become fairly stiff on account of suction, and the stiffness increases significantly once they are dried to the SL or below. Clearly, the much stronger response of samples at looser void ratio is due to the effect of desiccation history. Samples consolidated after settling $(\mathrm{W}=31 \%, \mathrm{~W}=29 \%)$ have continuous increase in excess pore water pressure, leading to a maximum excess pore water pressure ratio of about $70 \%$ compared to a maximum excess pore water pressure ratio of about $40 \%$ in the sample desiccated 

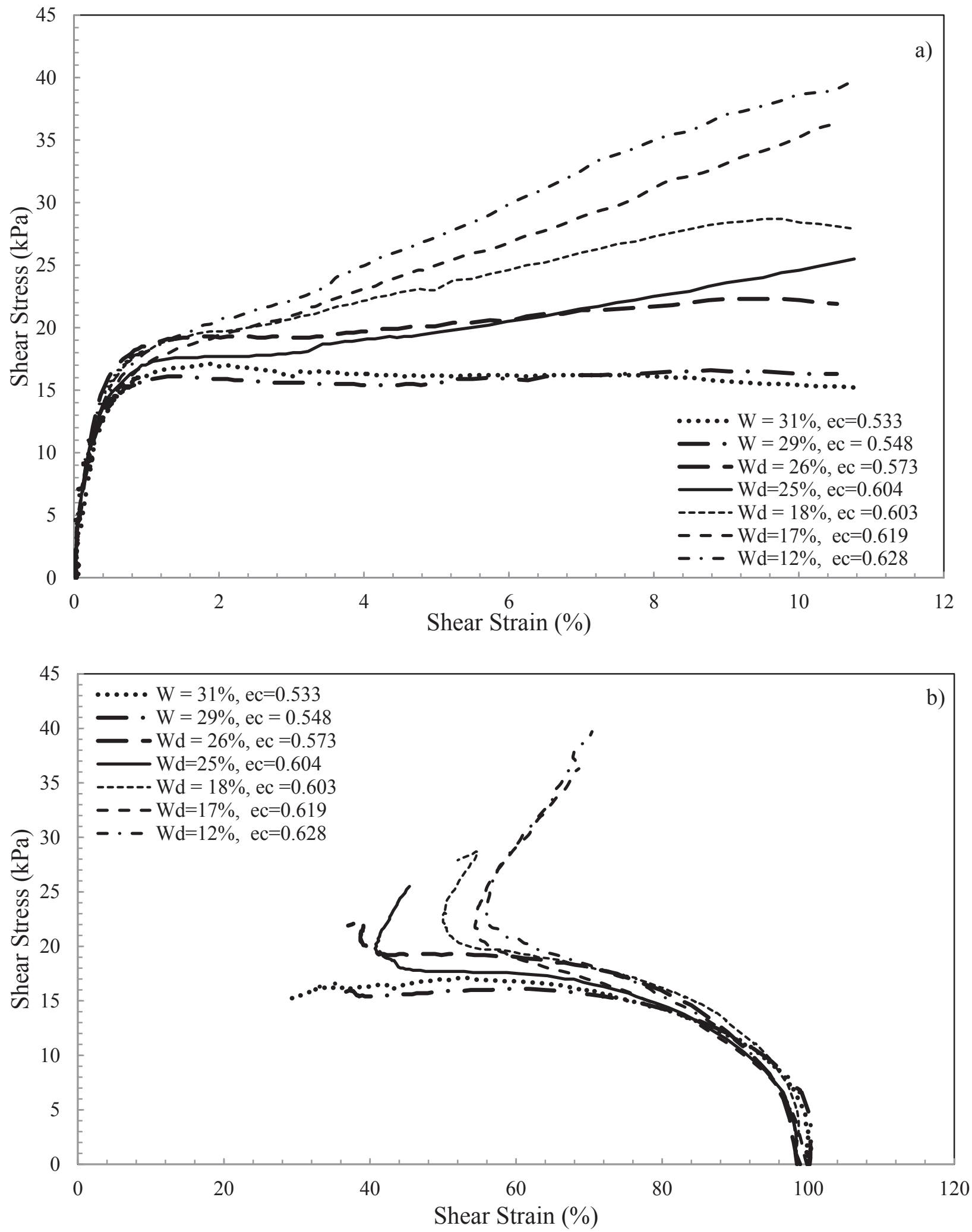

Figure 6.3 Monotonic results of desiccated and rewetted samples prepared by small scale method under $100 \mathrm{kPa}$ 
to $\mathrm{Wd}=12 \%$. The results of excess pore pressure generation can be found in Appendix III.

\subsubsection{Monotonic results under $400 \mathrm{kPa}$ vertical effective consolidation pressure}

\subsection{Desiccated-rewetted-consolidated samples}

Figures 6.4 and 6.5 present monotonic simple shear response of desiccated-rewetted samples consolidated under $400 \mathrm{kPa}$ vertical effective consolidation pressure. A higher consolidation pressure was applied to ensure that the consolidation pressure is higher than equivalent effective stress (suction stress) during desiccation. Similar to $50 \mathrm{kPa}$ and $100 \mathrm{kPa}$ responses, a little degree of desiccation to $\mathrm{W}=28 \%$ resulted in strain-hardening behavior. Figure 6.4 shows that with continuing desiccation from $\mathrm{W}=26 \%$ to $\mathrm{W}=20 \%$, there is some increase in strength (from 97 $\mathrm{kPa}$ to $107 \mathrm{kPa}$ ). Figure 6.5 shows the influence of increasing degree of desiccation from $\mathrm{W}=$ $26 \%$ to $\mathrm{W}=20 \%$ on the degree of dilation. It is clear that continuing desiccation up to $\mathrm{W}=20 \%$ decreases the excess pore water pressure at the end of the test significantly, which is a reflection of increasing dilation. As noted earlier, these tests also demonstrate that continuing desiccation up to the shrinkage limit and beyond slightly increases the shear strength. However, the level of increase is not as beneficial as desiccation to the shrinkage limit of about $\mathrm{W}=20 \%$.

\subsection{Desiccated-consolidated-rewetted samples}


Figure 6.6 presents monotonic simple shear responses of samples desiccated-consolidated under $400 \mathrm{kPa}$ and then rewetted (under the consolidation pressure). The reason for performing these types of tests is to investigate the influence of suction and rewetting on the simple shear response. Figure 6.6 shows that there is significant increase in shear strength with increasing degree of desiccation from $\mathrm{W}=27 \%$ to $\mathrm{W}=19.5 \%$. Shear stress at phase transformation point increases from $87 \mathrm{kPa}$ at $\mathrm{W}=27 \%$ to $103 \mathrm{kPa}$ at $\mathrm{W}=19.5 \%$. Similar to previous tests, desiccation beyond the shrinkage limit only slightly increases the shear strength of the samples. Comparisons with the previous series of tests indicate that the shear strength of desiccatedconsolidated- rewetted samples is slightly higher than desiccated-rewetted-consolidated samples. This phenomenon could be attributed to lower void ratios of desiccated-consolidated-rewetted samples. Figure 6.6 also shows that the maximum water content of desiccated-consolidated-

rewetted samples is $18.7 \%$ whereas the maximum water content in desiccated-rewettedconsolidated samples is $22.7 \%$. Similarly, the loosest void ratio in this series of tests (0.592) is about 0.04 denser than the loosest void ratio in desiccated- rewetted- consolidated tests. It seems that rewetting samples under $400 \mathrm{kPa}$ consolidation pressure limits the amount of water intake and volumetric rebound.

\subsubsection{Monotonic results of desiccated-consolidated samples}

Desiccated samples were prepared using the method explained in 3.5.1.3 section. Table 6.2 presents the parameters of the tests performed on desiccated tailings (with no rewetting). All tests were conducted on specimens consolidated under $400 \mathrm{kPa}$ vertical effective stress. There 

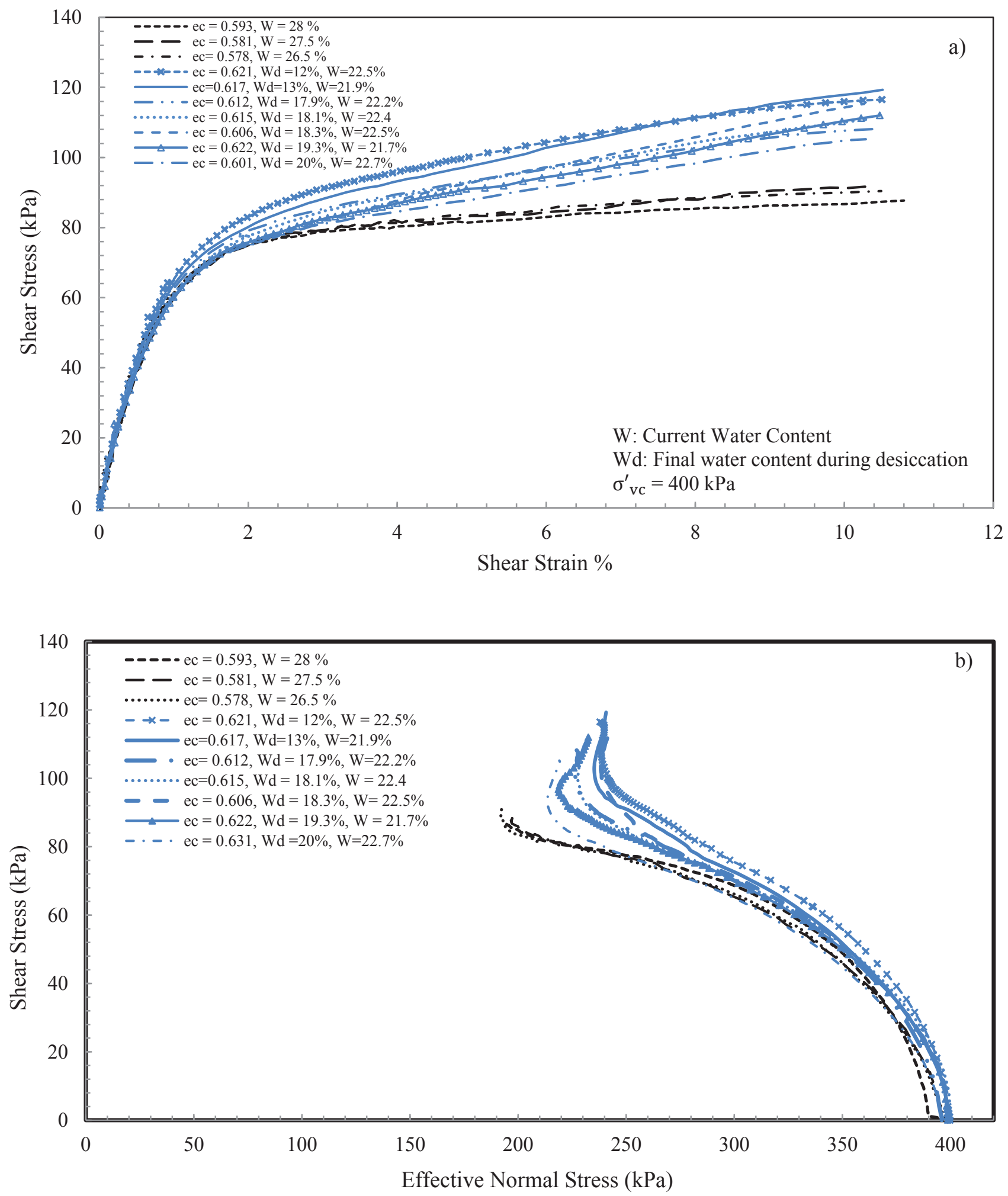

Figure 6.4 Monotonic shear response of desiccated and rewetted samples prepared by small scale method under $400 \mathrm{kPa}$ 
was no suction measurement device in the simple shear mould to measure matric suction, and the matric suction of desiccated samples before consolidation are presented based on the SWCC.

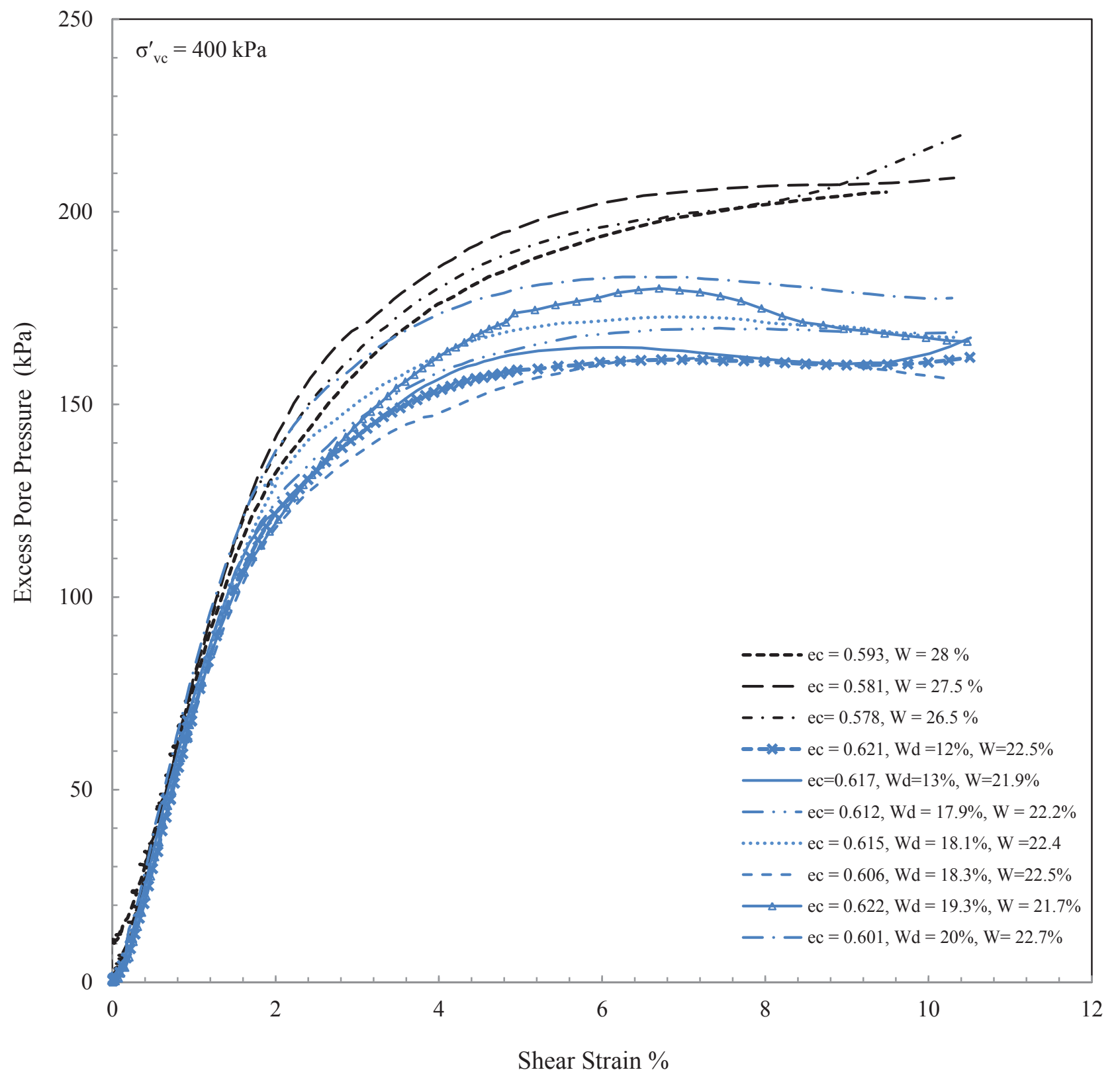

Figure 6.5 Excess pore water pressure generation in desiccated and rewetted samples prepared by small scale method under $400 \mathrm{kPa}$ 

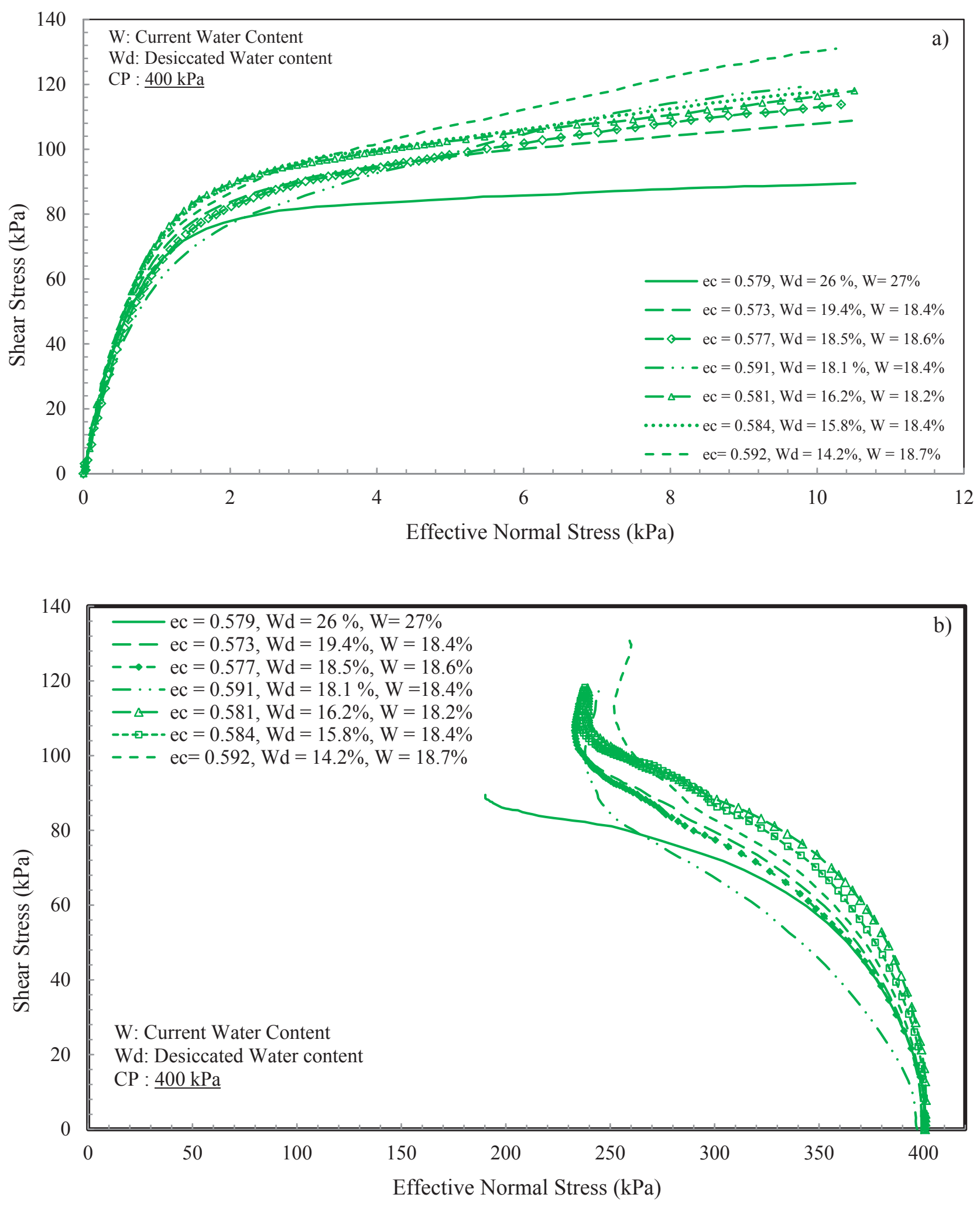

Figure 6.6 Monotonic results of desiccated-consolidated-rewetted samples prepared under 400 $\mathrm{kPa}$ 
Figure 6.7 presents stress-strain response of desiccated tailings. It is clear that samples with low value of matric suction ${ }^{1}$ (around $10 \mathrm{kPa}$ ) have lower shear strength in comparison to highly desiccated samples. Increasing degree of desiccation to $\mathrm{W}=19.5 \%$, which generates $105 \mathrm{kPa}$ matric suction before consolidation, significantly increases shear strength. Increasing degree of desiccation to lower water contents (resulting in higher matric suctions) caused an increase in shear strength. The rate of increasing shear strength with increasing degree of desiccation becomes marginal after passing the shrinkage limit during desiccation. It is also noted that samples with low degree of desiccation $(\psi \sim 10 \mathrm{kPa})$ reached lower void ratios on account of their relative high compressibility.

Table 6.2 Monotonic simple shear tests performed on desiccated samples under $400 \mathrm{kPa}$ consolidation pressure

\begin{tabular}{|c|c|c|c|c|}
\hline $\begin{array}{c}\text { Test } \\
\text { No. }\end{array}$ & $\begin{array}{c}\text { Sample } \\
\text { Characteristics }\end{array}$ & $\begin{array}{c}\text { Water content } \\
\text { W }(\%)\end{array}$ & $\begin{array}{c}\text { Matric Suction based on } \\
\text { SWCC (before consolidation } \\
\text { and shearing) } \psi_{\mathrm{d}}(\mathrm{kPa})\end{array}$ & $\begin{array}{c}\text { Void ratio after } \\
\text { consolidation }\left(\mathrm{e}_{\mathrm{c}}\right)\end{array}$ \\
\hline 1 & Desiccated & 28 & 7 & 0.593 \\
2 & Desiccated & 27.5 & 10 & 0.581 \\
3 & Desiccated & 26.5 & 11 & 0.578 \\
4 & Desiccated & 19.5 & 105 & 0.668 \\
5 & Desiccated & 19.2 & 110 & 0.673 \\
6 & Desiccated & 19 & 115 & 0.669 \\
7 & Desiccated & 18.5 & 140 & 0.678 \\
8 & Desiccated & 18.3 & 150 & 0.662 \\
9 & Desiccated & 18.1 & 160 & 0.643 \\
10 & Desiccated & 18 & 180 & 0.656 \\
11 & Desiccated & 16.7 & 250 & 0.652 \\
12 & Desiccated & 15.9 & 400 & 0.651 \\
\hline
\end{tabular}

\footnotetext{
${ }^{1}$ This matric suction corresponds to matric suction before consolidation based on SWCC
} 


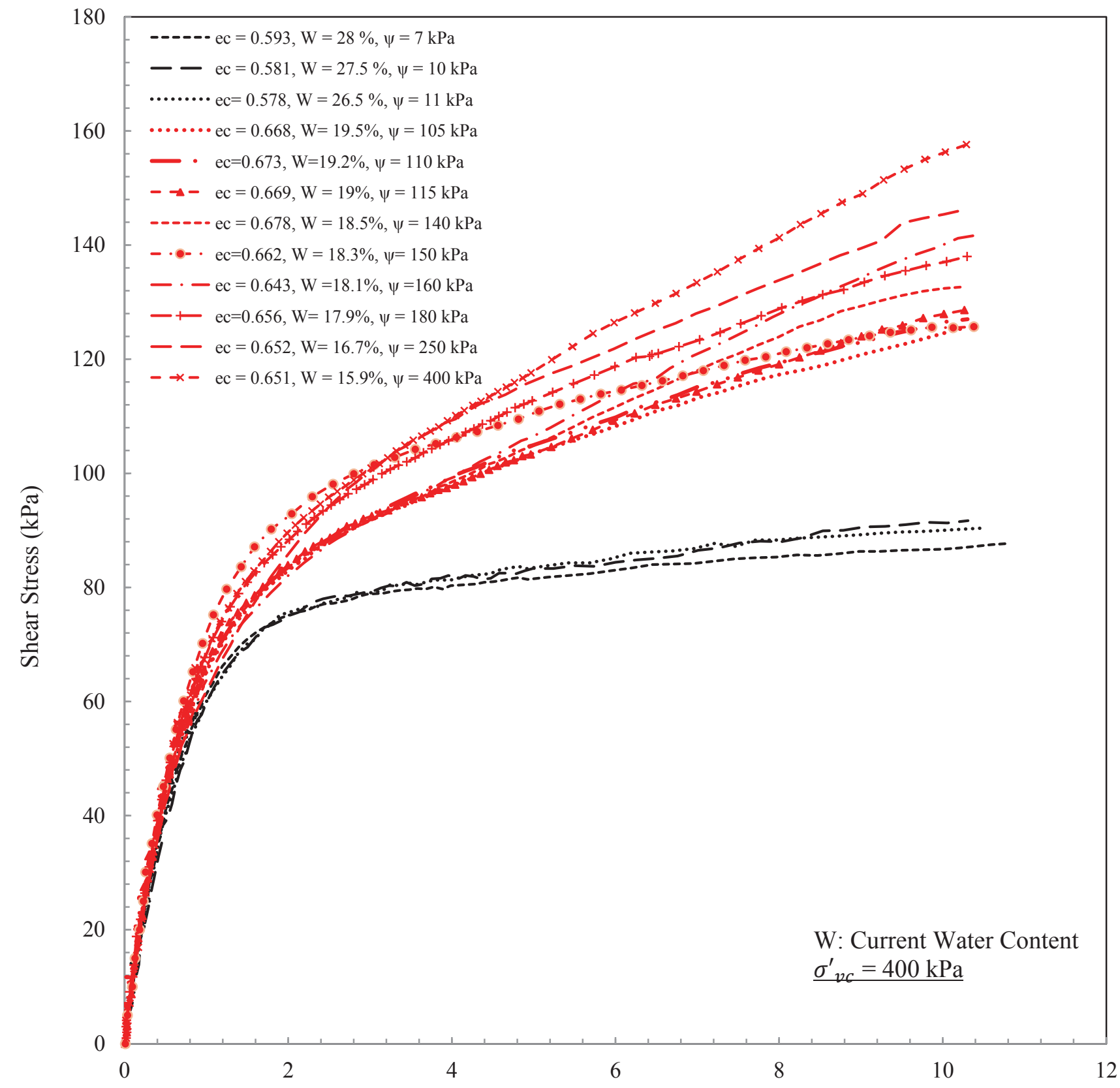

Shear Strain \%

Figure 6.7 Monotonic shear responses of desiccated samples under $400 \mathrm{kPa}$

\subsubsection{Discussion on monotonic results of small scale method}



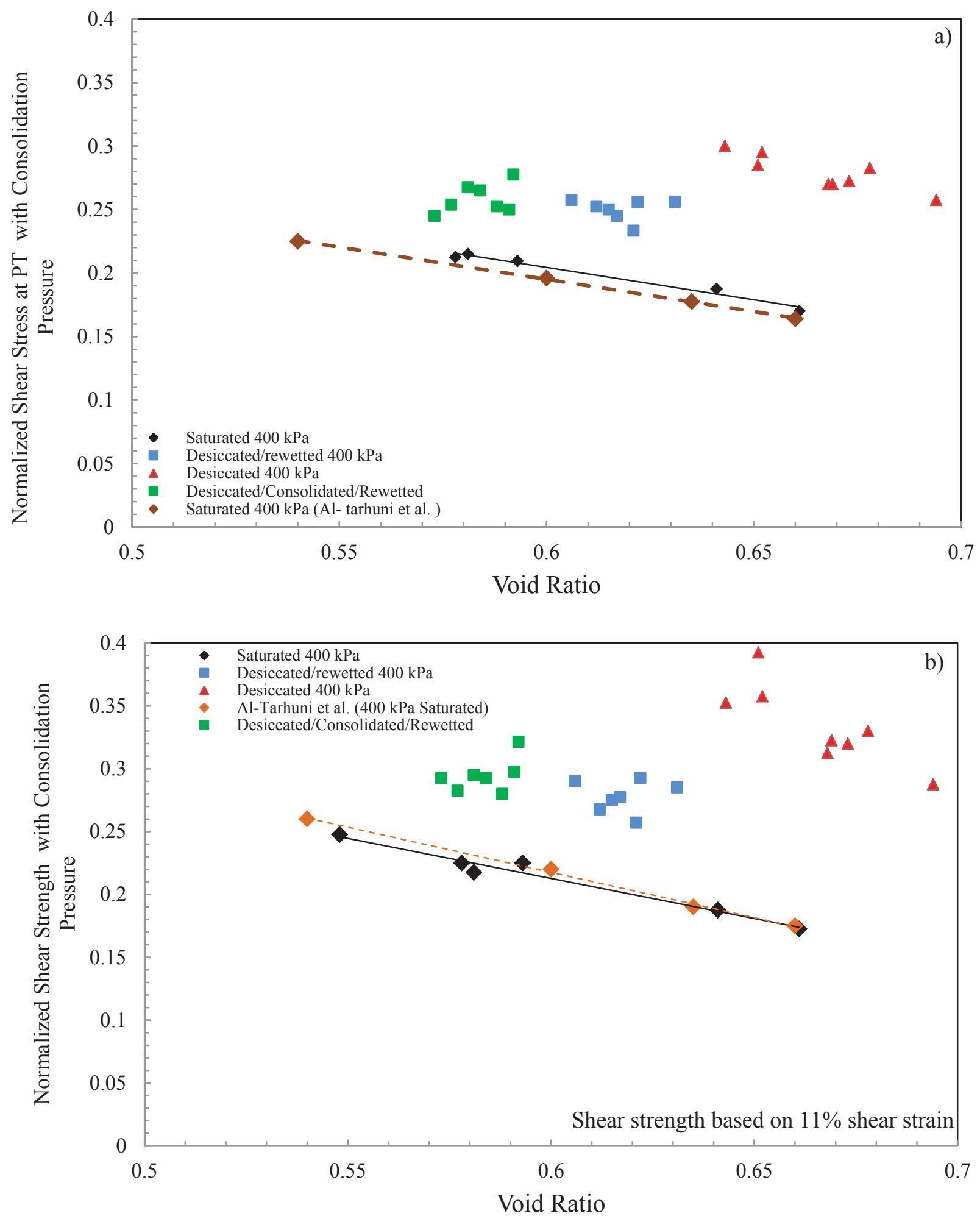

Figure 6.8 Normalized shear stress at PT and shear strength versus void ratio of samples consolidated under $400 \mathrm{kPa}$ 
Figure 6.8 presents normalized shear stress at phase transformation and normalized shear strength versus void ratio for samples consolidated under $400 \mathrm{kPa}$. As noted earlier, the shear stress at the end of tests (approximately 11\% shear strain) was considered indicative of the strength of the material. The true shear strength of the material may be somewhat higher. It is clear that for saturated samples and samples experienced low values of desiccation, there is a fairly linear relationship between shear stress at PT and void ratio. Both, the shear stress at PT and shear strength decrease almost linearly with increasing void ratio. Desiccated-rewetted samples exhibited higher strength at the same void ratios in comparison to saturated samples. Desiccated-rewetted samples exhibited higher strength than saturated samples even at looser void ratios. It is also clear that desiccated-consolidated-rewetted samples realized lower void ratios than desiccated-rewetted-consolidated, which leads to higher shear strength for desiccatedconsolidated-rewetted. As expected, desiccated samples (with no rewetting) exhibited higher shear strength in spite of having looser void ratios. The shear strength of desiccated samples also decreased with increasing void ratio, but the normalized strength ratio of these samples was much higher compared to the virgin tailings. Figure 6.9 presents consolidation results of saturated, desiccated and desiccated-rewetted samples prepared by small scale deposition and consolidated under $400 \mathrm{kPa}$. The starting point of consolidation curves corresponds to the void ratio after placing simple shear top cap, which applies around $5 \mathrm{kPa}$ consolidation pressure to the samples. Figure 6.9 shows that saturated or settled samples, which has higher void ratio at the beginning of the test, reached lower values of void ratio at the end of consolidation. In other words, saturated samples are more compressible than desiccated and desiccated-rewetted samples. These results indicate that the void ratio change when tailings are consolidated to 400 $\mathrm{kPa}$ (from $\sim 5 \mathrm{kPa}$ ) can vary anywhere from 0.03 (desiccated) to 0.25 (virgin) tailings. 


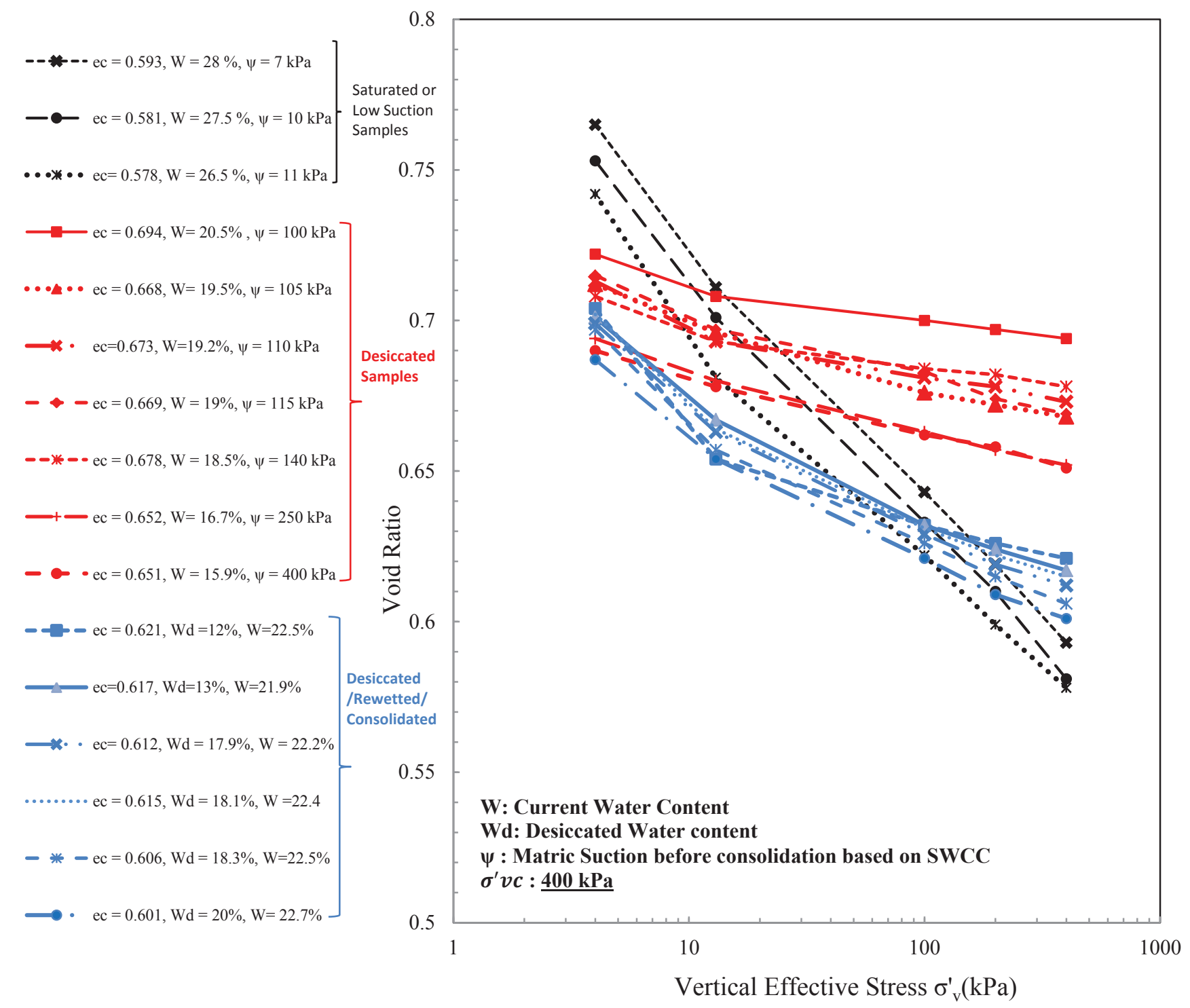

Figure 6.9 Consolidation results of samples prepared based on small scale deposition method and consolidated under $400 \mathrm{kPa}$

Compression index $\left(\mathrm{C}_{\mathrm{c}}\right)$ for data presented in Figure 6.9 is $0.11,0.05$ and 0.015 for saturated, desiccated-rewetted and desiccated samples respectively. Desiccated tailings have low potential of compressibility because of the high values of suction that are generated in desiccated tailings. Desiccated-rewetted samples exhibited less compressibility than saturated tailings and more 
compressibility than desiccated tailing. Process of rewetting obviously makes the tailings less stiff than desiccated samples.

Figure 6.10 presents effect of rewetting for samples consolidated under $400 \mathrm{kPa}$. It should be noted that the values of matric suction correspond to matric suction based on SWCC at the end of the desiccation process and before consolidation. It is clear that rewetting desiccated samples led to lower shear strength. Moreover, desiccated samples have looser void ratio than desiccatedrewetted samples, and yet, they have higher strength due to generation of matric suction. Figure 6.11 shows the results of simple shear tests to illustrate the variation of the shear stress at phase transformation state with void ratio. Results reported by Al-Tarhouni et al. (2008) on the same material at saturated conditions are also included in Figure 6.11. It is shown that saturated samples (with no desiccation or low desiccation) have lower shear stress at PT even though they have very low void ratios $(0.52 \sim 0.53)$. Though desiccated samples had higher void ratios (0.65 0.7), they exhibited very high shear stress at PT. Rewetting process reduced the void ratio of desiccated samples to $0.57 \sim 0.64$. However, this reduction did not lead to higher strength and due to reducing matric suction to zero, the shear stress at PT of desiccated-rewetted samples are lower than desiccated samples.

Figure 6.12 compares normalized shear stress at PT of all monotonic simple shear tests versus desiccated water content (Wd). The water content (Wd) corresponds to final water content of samples during desiccation and before rewetting. It is shown that a little degree of desiccation from $\mathrm{Wd}=30 \%$ to $\mathrm{Wd}=25 \%$ significantly increases the shear stress at PT and is quite helpful in terms of strength. Continuing desiccation up to the shrinkage limit increases the shear stress at PT. Excessive desiccation beyond the SL slightly increases the shear stress at PT. In chapter 4, it was found that due to absorption of water from underlying layers, desiccation of fresh top layers 
to SL is time consuming. Excessive desiccation could also increase the risk of ARD. Figure 6.12 shows that increasing degree of desiccation from $\mathrm{Wd}=30 \%$ to $\mathrm{Wd}=25 \%$ is significantly beneficial in terms of increasing shear strength in comparison to continuing desiccation from $\mathrm{Wd}$

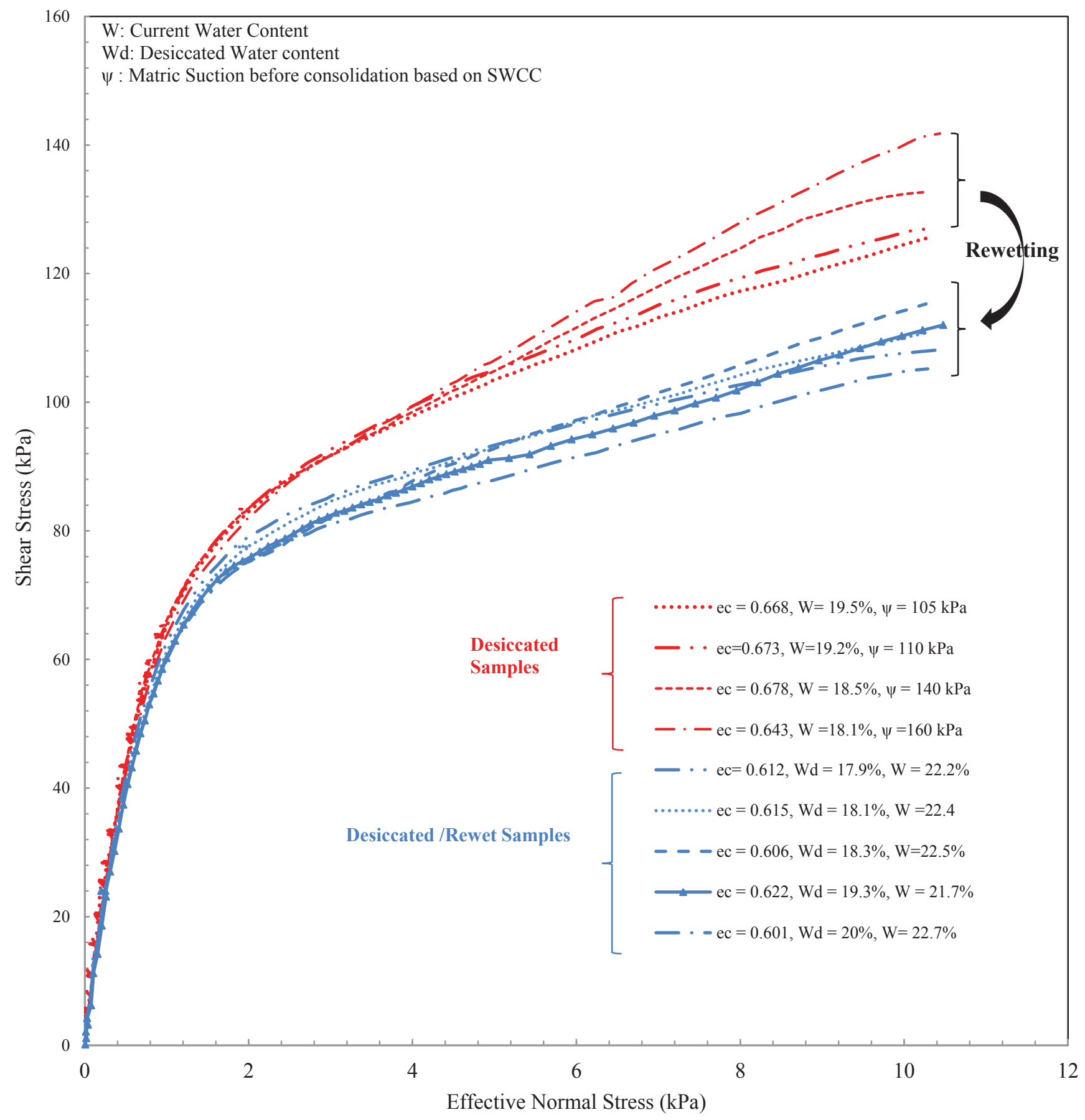

Figure 6.10 Effect of rewetting on stress-strain response of tailings consolidated at $400 \mathrm{kPa}$ 
- Saturated $400 \mathrm{kPa}$

Desiccated/rewetted $400 \mathrm{kPa}$

$\triangle$ Desiccated $400 \mathrm{kPa}$

- Desiccated/Consolidated/Rewetted $400 \mathrm{kPa}$

- Saturated $400 \mathrm{kPa}$ (Al- tarhuni et al.)

- Saturated $50 \mathrm{kPa}$

Desiccated/Rewetted $50 \mathrm{kPa}$

Desiccated/Rewet $200 \mathrm{kPa}$

- Saturated $200 \mathrm{kPa}$ (Al-Tarhuni et al)

- Saturated $100 \mathrm{kPa}$

- Desiccated/Rewetted $100 \mathrm{kPa}$

- Saturated $100 \mathrm{kPa}$ (Al-Tarhuni)

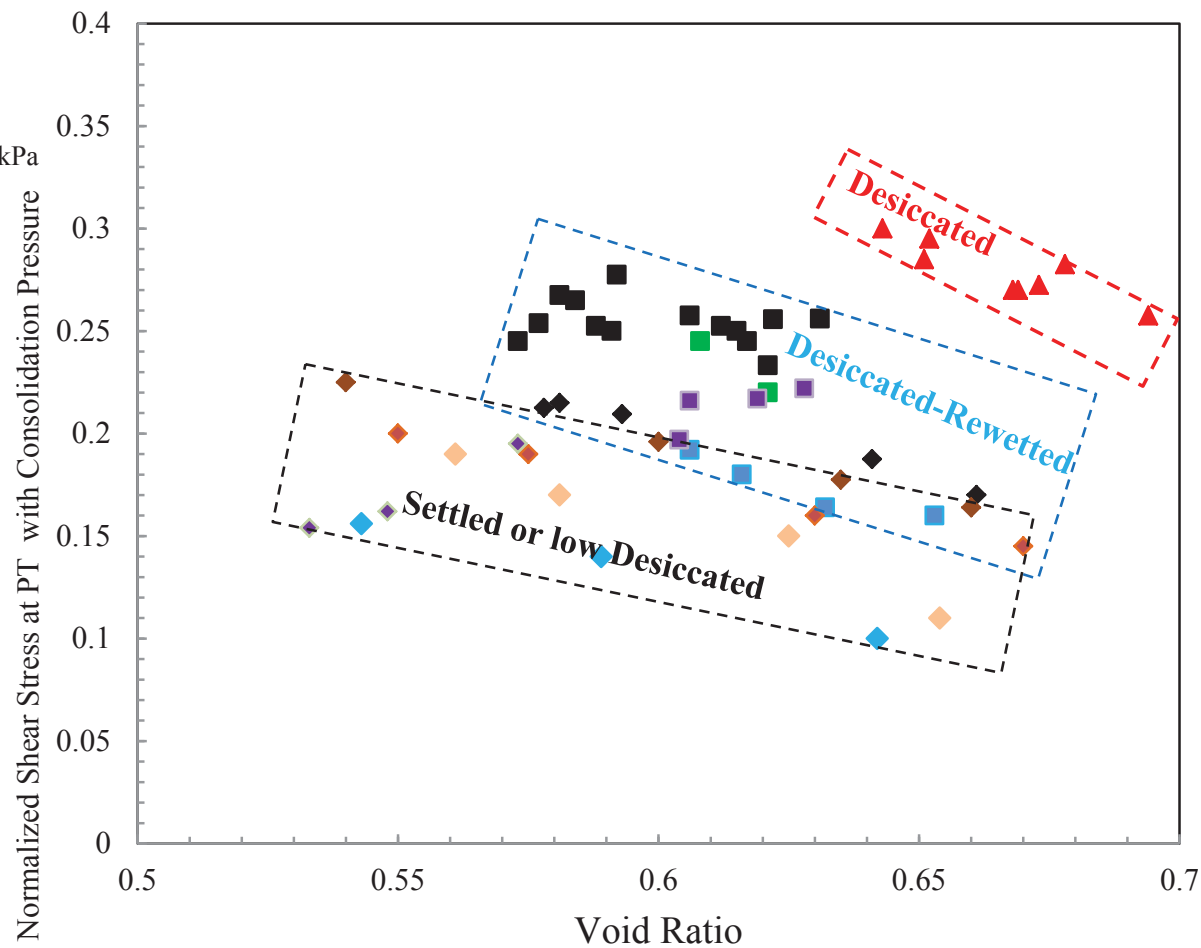

Figure 6.11 Normalized shear stress at PT versus void ratio for all monotonic simple shear tests

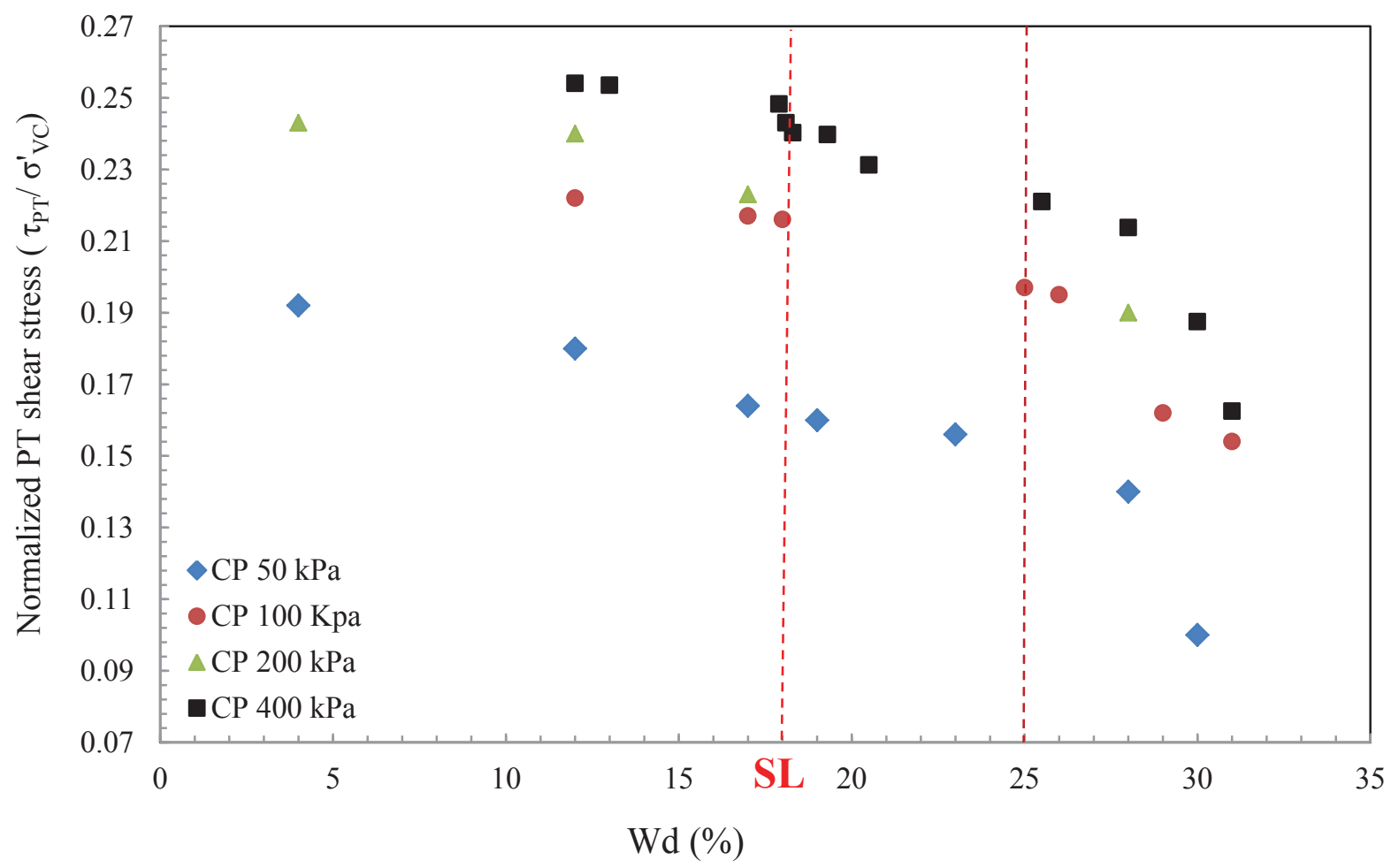

Figure 6.12 Normalized shear stress at PT versus water content during desiccation 
$=25 \%$ to SL. It can be concluded that most benefits are realized at relatively little degree of desiccation, and desiccation to $\mathrm{Wd} \sim 25 \%$ is quite helpful to increase the shear strength of deposited tailings. Continuing desiccation to SL is also helpful in terms of strength; however, the level of increase in strength is not so remarkable that can satisfy negative effects of long drying time and risk of ARD.

\subsubsection{Monotonic Results of Mechanically over consolidated samples}

Figure 6.13 presents monotonic response of samples with mechanical overconsolidation ratio $O C R_{M}=2$ or 4 under $100 \mathrm{kPa}$ vertical effective stress consolidation pressure. Mechanically overconsolidated samples were prepared at the pumping water content, and consolidated to 200 $\mathrm{kPa}$, and $400 \mathrm{kPa}$ in the simple shear device, and then unloaded to $100 \mathrm{kPa}$ before shearing.

It is clear that all mechanically overconsolidated samples exhibited strain-hardening response. Increasing $\mathrm{OCR}_{\mathrm{M}}$ has a significant effect on the stress path and stress strain responses, and the behavior transforms to be more dilative as OCR increases. Figure 6.14 and 6.15 compare monotonic simple shear response of mechanically overconsolidated samples with desiccatedrewetted samples. An important qualitative difference in the behaviour is that while mechanically overconsolidated tailings exhibit a substantial increase in peak strength with increasing OCR, the desiccated tailings exhibit a much smaller increase in the peak strength at PT, but show somewhat higher degree of strain hardening past the phase transformation point. 

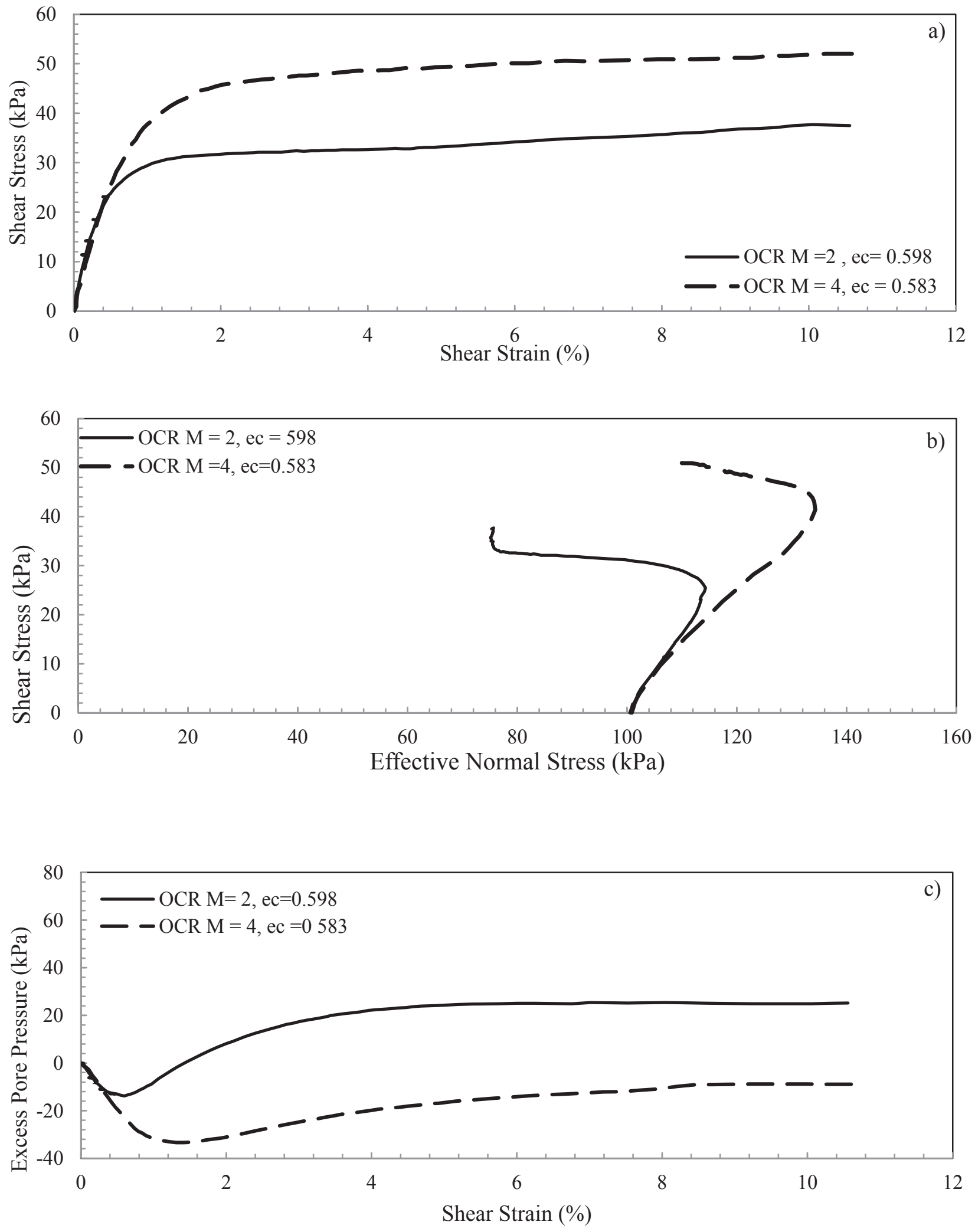

Figure 6.13 Monotonic simple shear response of mechanically over consolidated samples under $100 \mathrm{kPa}$ 

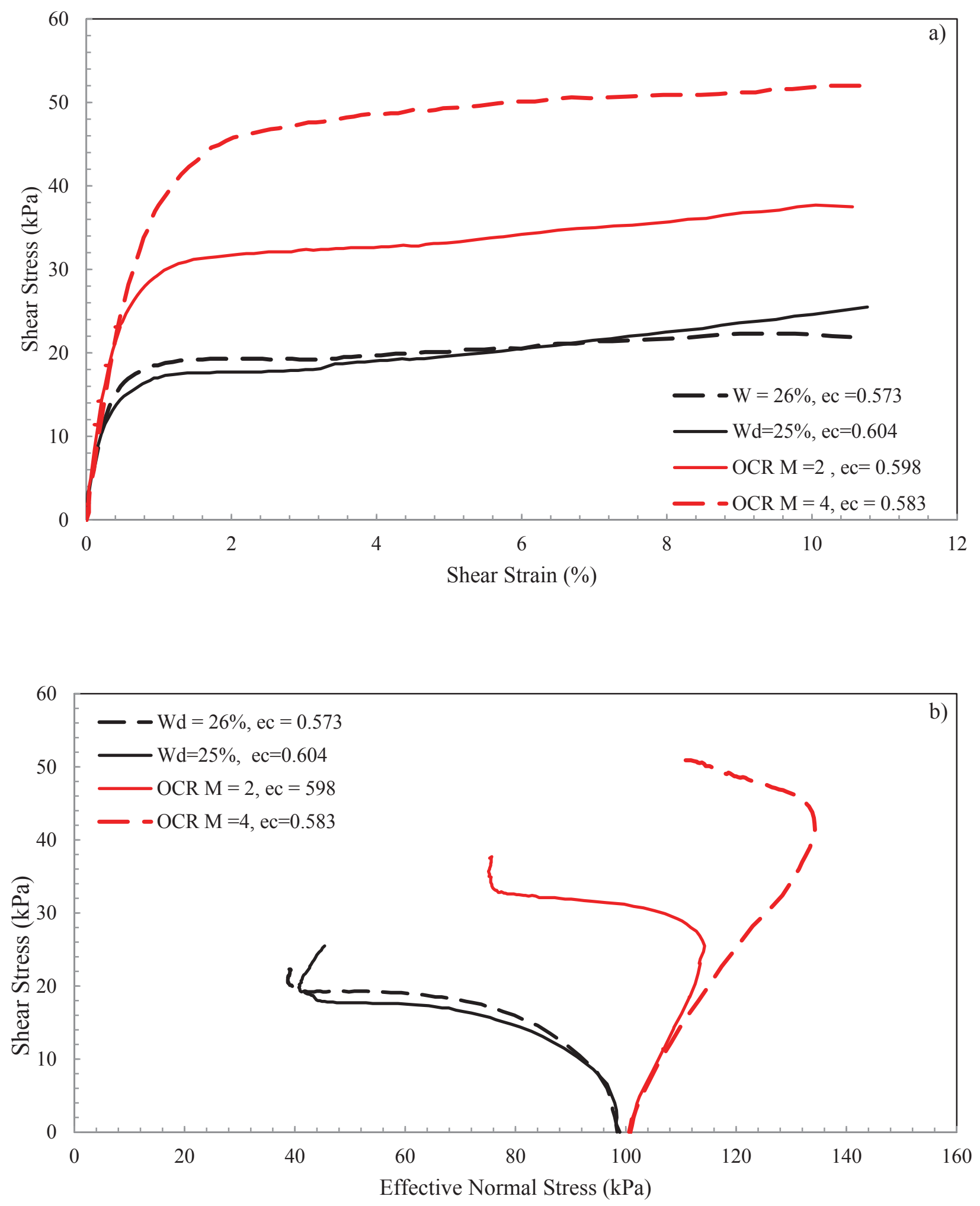

Figure 6.14 Comparison of Monotonic simple shear response of mechanically over consolidated samples and desiccated-rewetted samples under $100 \mathrm{kPa}$ 


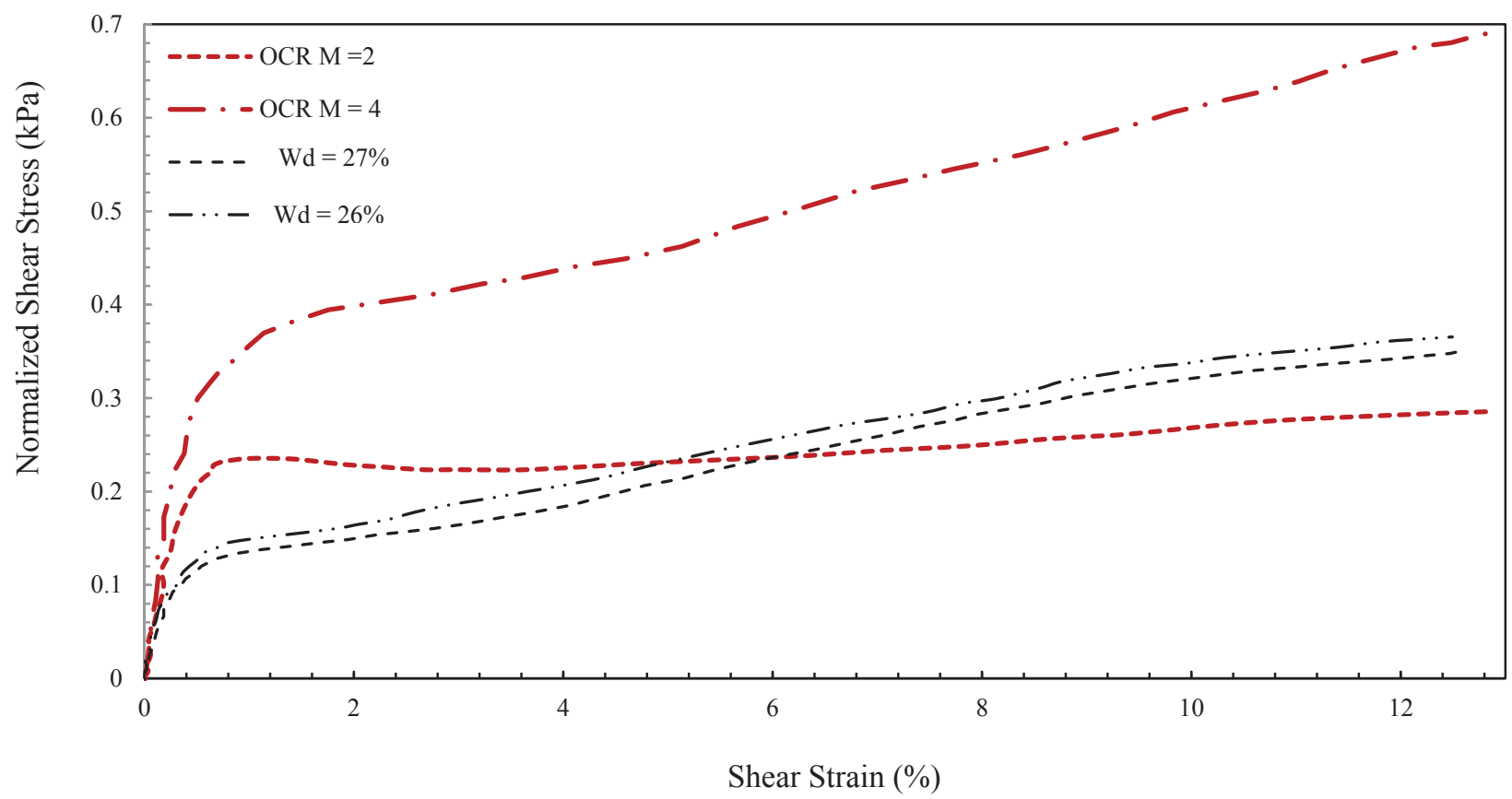

Figure 6.15 Comparison of Monotonic simple shear response of mechanically over consolidated samples and desiccated-rewetted samples under $50 \mathrm{kPa}$

\subsubsection{Cyclic Results of small scale laboratory tests}

Cyclic simple shear tests were performed on samples prepared by small scale laboratory methods under different cyclic stress ratios (CSRs) and different desiccation histories. Samples after settling and no desiccation had $32 \%$ water content before consolidation. Other samples were desiccated to $\mathrm{Wd}=26 \%, 20 \%, 17 \%, 12 \%$ and $10 \%$, and rewetted. Samples desiccated to $\mathrm{Wd}=$ $20 \%, 17 \%, 12 \%$ and $10 \%$ reached a water content value of around $20 \% \sim 22 \%$ following rewetting. All samples were consolidated under $50 \mathrm{kPa}$ consolidation pressure before cyclic loading. Cyclic shear load was applied in the form of a sinusoidal wave with a frequency of 0.1 $\mathrm{Hz}$, and the samples were deemed to have liquefied by adopting a strain based criterion of liquefaction. US National Research Council (NRC 1985) suggested that the development of 
$3.75 \%$ single amplitude shear strain is considered as the triggering of liquefaction under cyclic loading. This shear strain might correspond to the development of $100 \%$ pore pressure ratio for contractive sands when no static shear stress exists on the sample, but criterion is not dependent on the level of excess pore pressure. This criterion has been used in the literature to investigate different types of fine-grained mine tailings under simple shear (Wijewickreme et al., 2005; AlTarhouni et al 2010). It should be mentioned that for fine grained soils including tailings, the development of $100 \%$ pore pressure ratio might not occur even at high shear strain levels, and thus a liquefaction criteria based on the $100 \%$ excess pore water pressure ratio would not be appropriate (Singh, 1996). The use of a shear strain level based criterion provides a consistent means of evaluating the triggering of liquefaction, and allows for a rational comparison of cyclic shear resistance data. Cyclic shear stress ratio (CSR) was defined by the ratio of maximum cyclic shear stress over consolidation confining pressure CSR $=\left(\frac{\tau_{c y c}}{\sigma_{v c}^{\prime}}\right)$. The value of CSR used in this study ranges from 0.05 to 0.15 for desiccated-rewetted tailings.

Table 6.3 presents the test parameters for the cyclic simple shear tests performed on samples prepared by small scale deposition method. All samples were consolidated under $50 \mathrm{kPa}$ vertical effective consolidation pressure. Figures 6.16 to 6.19 present cyclic simple shear results of samples with no desiccation $(\mathrm{Wd}=32 \%)$ under different cyclic stress ratios (CSRs). The sample subjected to a CSR $=0.05$ liquefied in 84 cycles, with an excess pore pressure ratio of 0.88 at the instant liquefaction was triggered. Figure 6.17 presents cyclic simple shear response of tailings subjected to $\mathrm{CSR}=0.065$. As expected, increasing $\mathrm{CSR}$ from $\mathrm{CSR}=0.05$ to $\mathrm{CSR}=0.065$ significantly accelerated the time to reach liquefaction and the sample liquefied in 32 cycles. The 
Table 6.3 Characteristics of cyclic simple shear tests performed on samples prepared by small scale deposition method

\begin{tabular}{|c|c|c|c|c|c|c|}
\hline $\begin{array}{l}\text { Test } \\
\text { No. }\end{array}$ & Sample Characteristics & $\mathrm{Wd}(\%)$ & $\begin{array}{c}\text { Consolidation } \\
\text { Pressure } \\
\left(\sigma_{v c}^{\prime}\right) \mathrm{kPa} \\
\end{array}$ & $\mathrm{e}_{\mathrm{c}}$ & CSR & $\mathrm{N}_{\mathrm{L}}$ \\
\hline 1 & Settled & 32 & 50 & 0.585 & 0.125 & 3 \\
\hline 2 & Settled & 32 & 50 & 0.596 & 0.09 & 8 \\
\hline 3 & Settled & 32 & 50 & 0.582 & 0.075 & 18 \\
\hline 4 & Settled & 32 & 50 & 0.604 & 0.065 & 32 \\
\hline 5 & Settled & 32 & 50 & 0.594 & 0.05 & 84 \\
\hline 6 & Desiccated/rewetted & 26 & 50 & 0.624 & 0.125 & 4 \\
\hline 7 & Desiccated/rewetted & 26 & 50 & 0.635 & 0.11 & 6 \\
\hline 8 & Desiccated/rewetted & 26 & 50 & 0.655 & 0.1 & 11 \\
\hline 9 & Desiccated/rewetted & 26 & 50 & 0.642 & 0.075 & 40 \\
\hline 10 & Desiccated/rewetted & 26 & 50 & 0.629 & 0.05 & 304 \\
\hline 11 & Desiccated/rewetted & 20 & 50 & 0.633 & 0.125 & 4 \\
\hline 12 & Desiccated/rewetted & 20 & 50 & 0.648 & 0.1 & 12 \\
\hline 13 & Desiccated/rewetted & 20 & 50 & 0.655 & 0.09 & 24 \\
\hline 14 & Desiccated/rewetted & 20 & 50 & 0.632 & 0.075 & 70 \\
\hline 15 & Desiccated/rewetted & 20 & 50 & 0.641 & 0.065 & 119 \\
\hline 16 & Desiccated/rewetted & 17 & 50 & 0.656 & 0.14 & 4 \\
\hline 17 & Desiccated/rewetted & 17 & 50 & 0.643 & 0.125 & 6 \\
\hline 18 & Desiccated/rewetted & 17 & 50 & 0.655 & 0.1 & 14 \\
\hline 19 & Desiccated/rewetted & 17 & 50 & 0.667 & 0.075 & 47 \\
\hline 20 & Desiccated/rewetted & 17 & 50 & 0.651 & 0.065 & 69 \\
\hline 21 & Desiccated/rewetted & 12 & 50 & 0.675 & 0.15 & 3 \\
\hline 22 & Desiccated/rewetted & 12 & 50 & 0.689 & 0.115 & 11 \\
\hline 23 & Desiccated/rewetted & 12 & 50 & 0.68 & 0.1 & 16 \\
\hline 24 & Desiccated/rewetted & 12 & 50 & 0.671 & 0.075 & 41 \\
\hline 25 & Desiccated/rewetted & 12 & 50 & 0.668 & 0.065 & 83 \\
\hline 26 & Desiccated/rewetted & 10 & 50 & 0.681 & 0.075 & 50 \\
\hline 27 & Desiccated/rewetted & 10 & 50 & 0.682 & 0.13 & 6 \\
\hline 28 & Desiccated/rewetted & 10 & 50 & 0.695 & 0.1 & 16 \\
\hline 29 & Desiccated/rewetted & 10 & 50 & 0.684 & 0.062 & 120 \\
\hline 30 & Desiccated/rewetted & 10 & 50 & 0.687 & 0.12 & 8 \\
\hline
\end{tabular}



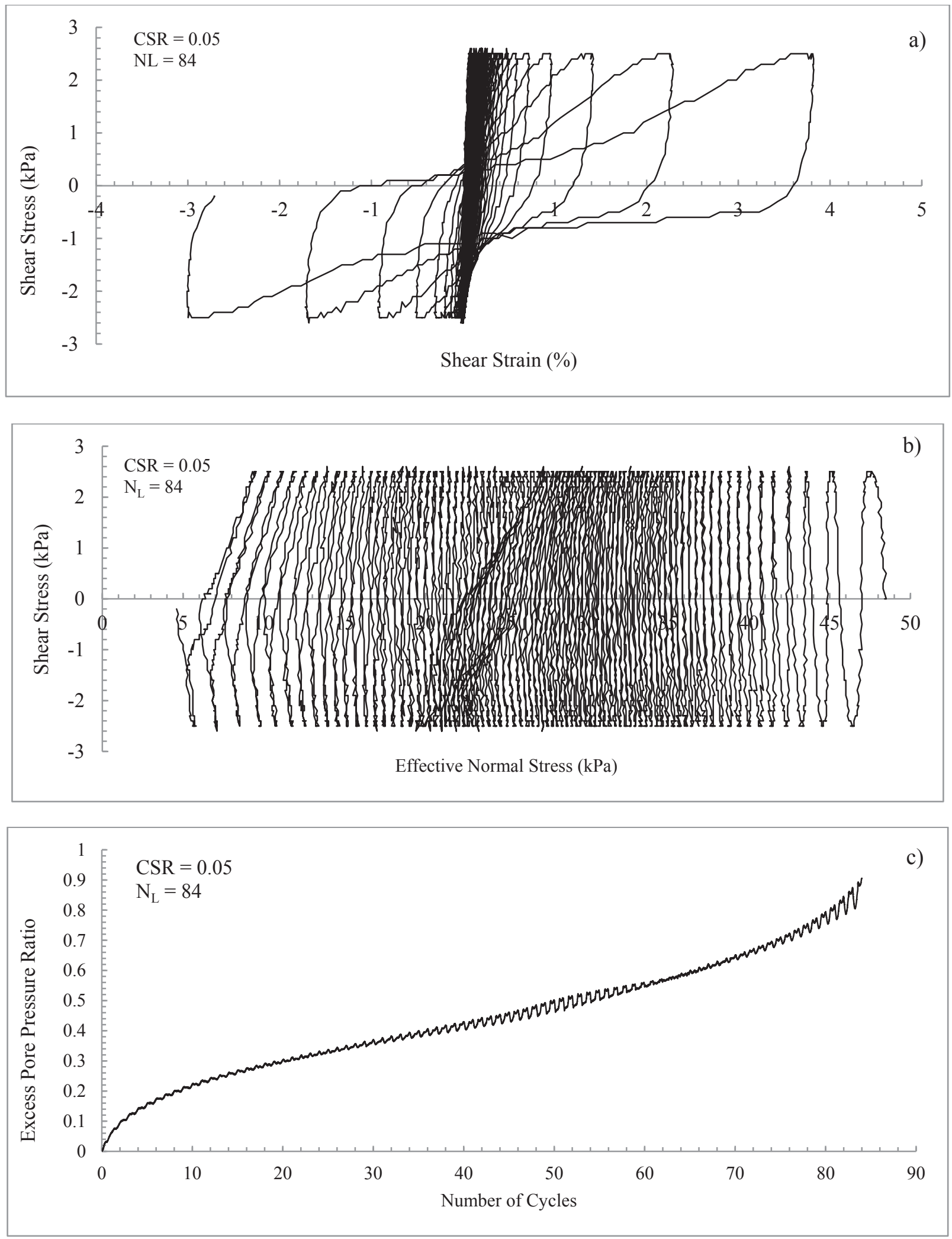

Figure 6.16 Cyclic simple shear response of settled sample $(\mathrm{Wd}=32 \%)$ under $\mathrm{CSR}=0.05$ 

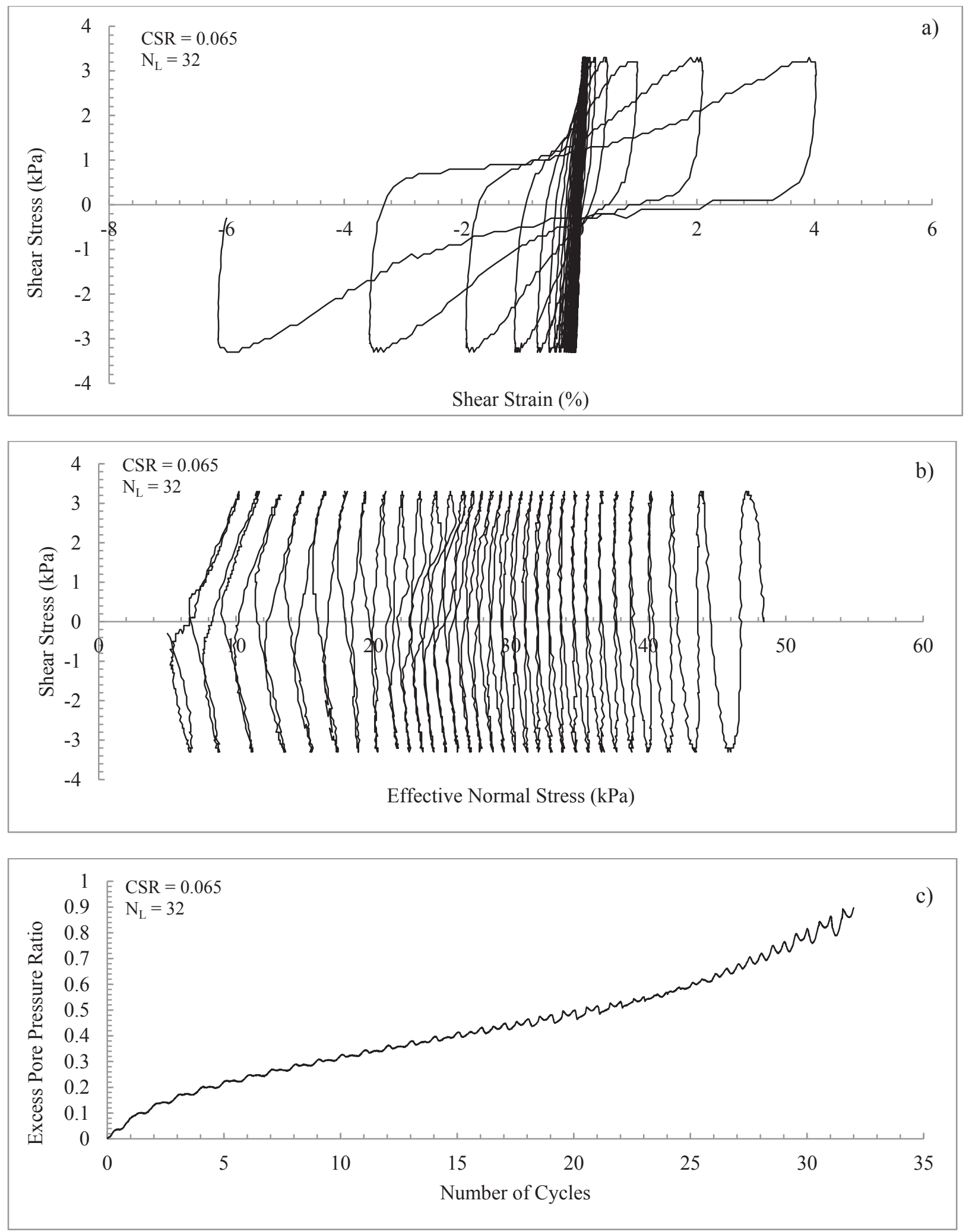

Figure 6.17 Cyclic simple shear response of settled sample $(\mathrm{Wd}=32 \%)$ under $\mathrm{CSR}=0.065$ 

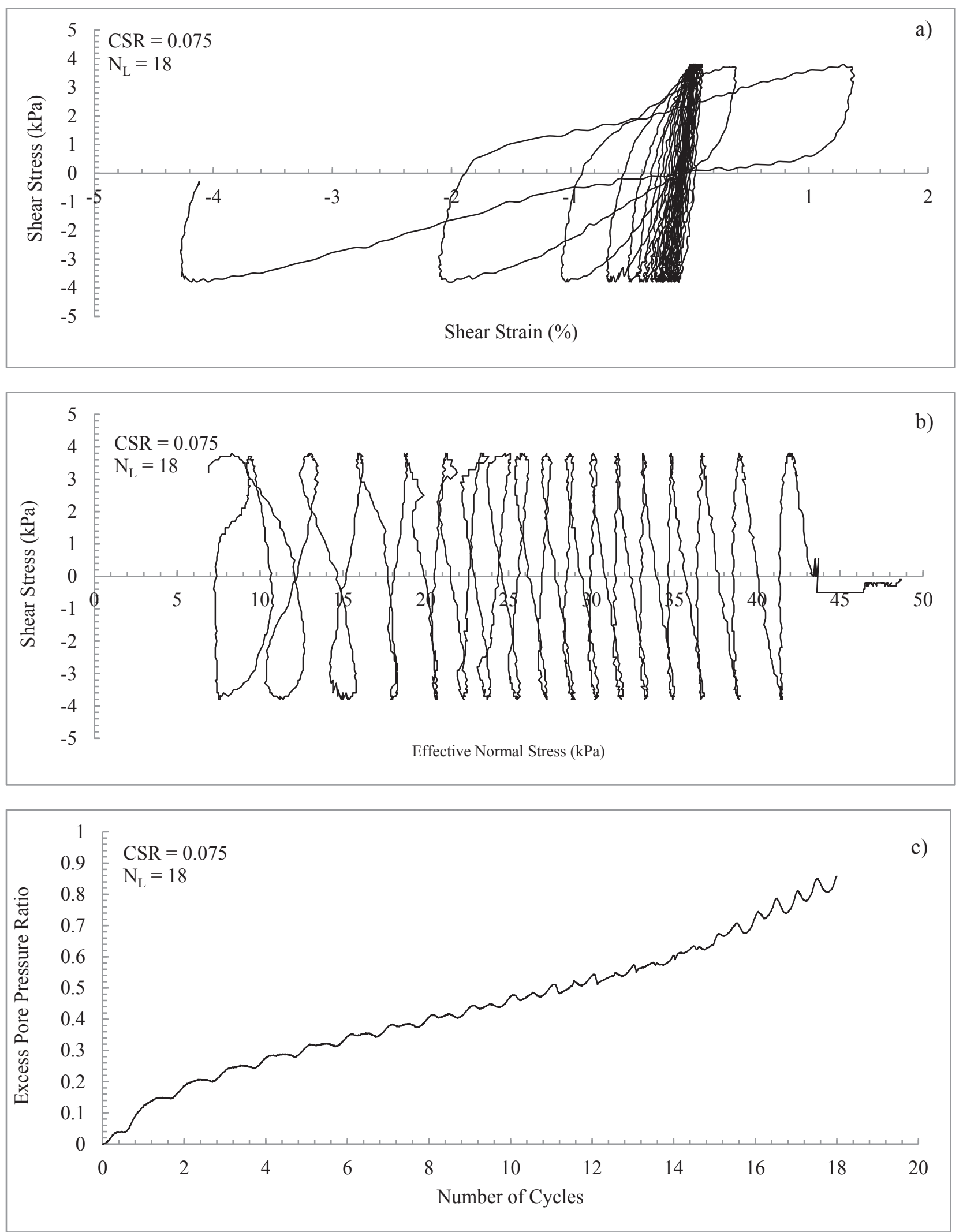

Figure 6.18 Cyclic simple shear response of settled sample $(\mathrm{Wd}=32 \%)$ under $\mathrm{CSR}=0.075$ 

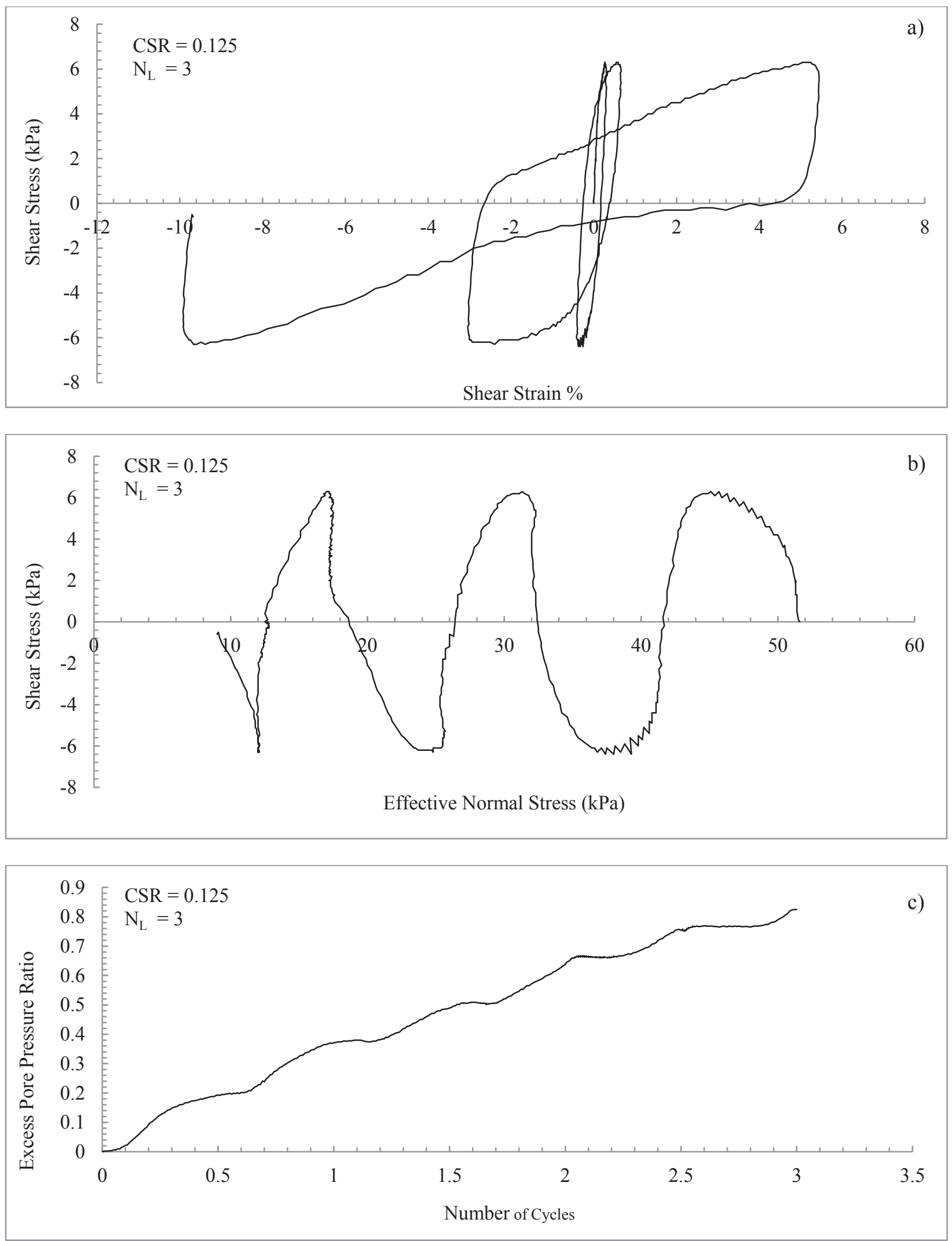

Figure 6.19 Cyclic simple shear response of settled sample $(\mathrm{Wd}=32 \%)$ under $\mathrm{CSR}=0.125$ 
excess pore pressure at the triggering of liquefaction ( 0.87 vs. 0.88$)$ was not dependent on the applied CSR. The behavior of the tailing at even higher CSR values was evaluated as shown in Figures 6.18 and 6.19. These results clearly demonstrated that the numbers of cycles to liquefaction decreases systematically with increasing CSR, and the tailings liquefied in just 3 cycles at $\mathrm{CSR}=0.125$. The excess pore pressure ratio varied between about 0.80 and 0.88 in these tests.

Figures 6.20 to 6.24 present cyclic simple shear response of tailings desiccated to $\mathrm{Wd}=26 \%$ and rewetted under different CSRs. Unlike the earlier case where CSR $=0.05$ caused liquefaction in 84 cycles, the desiccated-rewetted sample withstood 304 cycles prior to liquefaction. The excess pore pressure ratio was about 0.97 in this test, which is fairly close to a state of zero effective stress. A small increase in CSR (from 0.05) to 0.075 significantly reduces numbers of cycles to reach liquefaction to $\mathrm{N}_{\mathrm{L}}=40$. The results presented herein clearly show that samples deemed liquefied as per a strain criteria does not necessarily reach a state of zero effective stress. The excess pore pressure generated upon exceeding 3.75\% shear strain varied between about $80 \%$ to 97\% in the tailings. However, Seed (1983)'s observation that large strain under cyclic loading mobilize upon exceeding an excess pore pressure of about $60 \%$ seems to apply to the tailings as well.

Figure 6.25 and 6.26 present cyclic simple shear response of desiccated-rewetted tailings with $\mathrm{Wd}=20 \%$. Figure 6.25 demonstrates that it requires 119 numbers of cycles to reach liquefaction under CSR $=0.065$ for desiccated-rewetted samples with $\mathrm{Wd}=20 \%$. Comparing the numbers of cycles of settled samples under CSR $=0.065$ (Figure 6.17) with $\mathrm{Wd}=20 \%$ samples, it is clear that desiccation to $\mathrm{Wd}=20 \%$, increased the $\mathrm{N}_{\mathrm{L}}$ from 32 to 119 . It is clear that samples which experienced desiccation have higher cyclic strength than settled samples. 

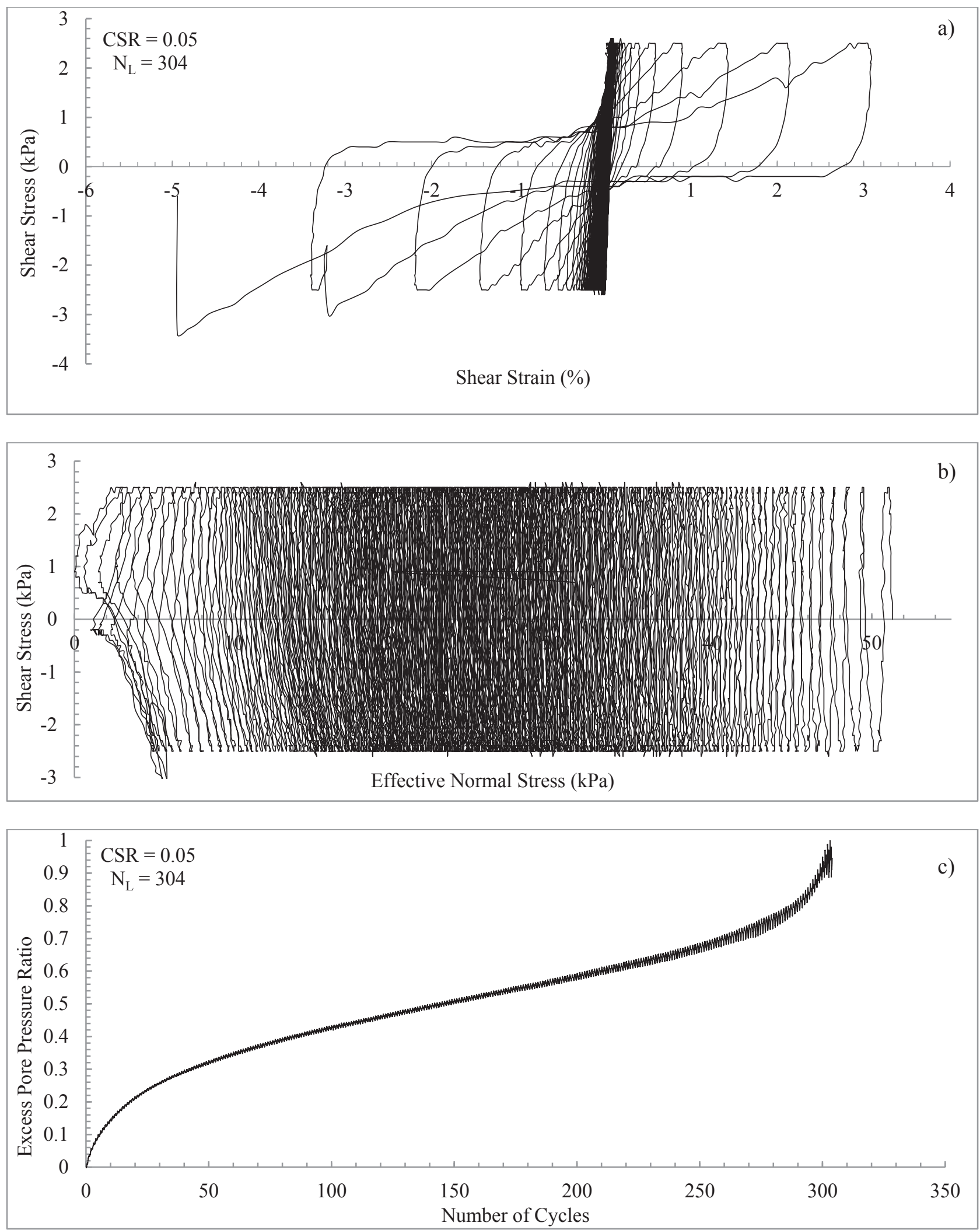

Figure 6.20 Cyclic simple shear response of desiccated-rewetted sample $(\mathrm{Wd}=26 \%)$ under CSR $=0.05$ 

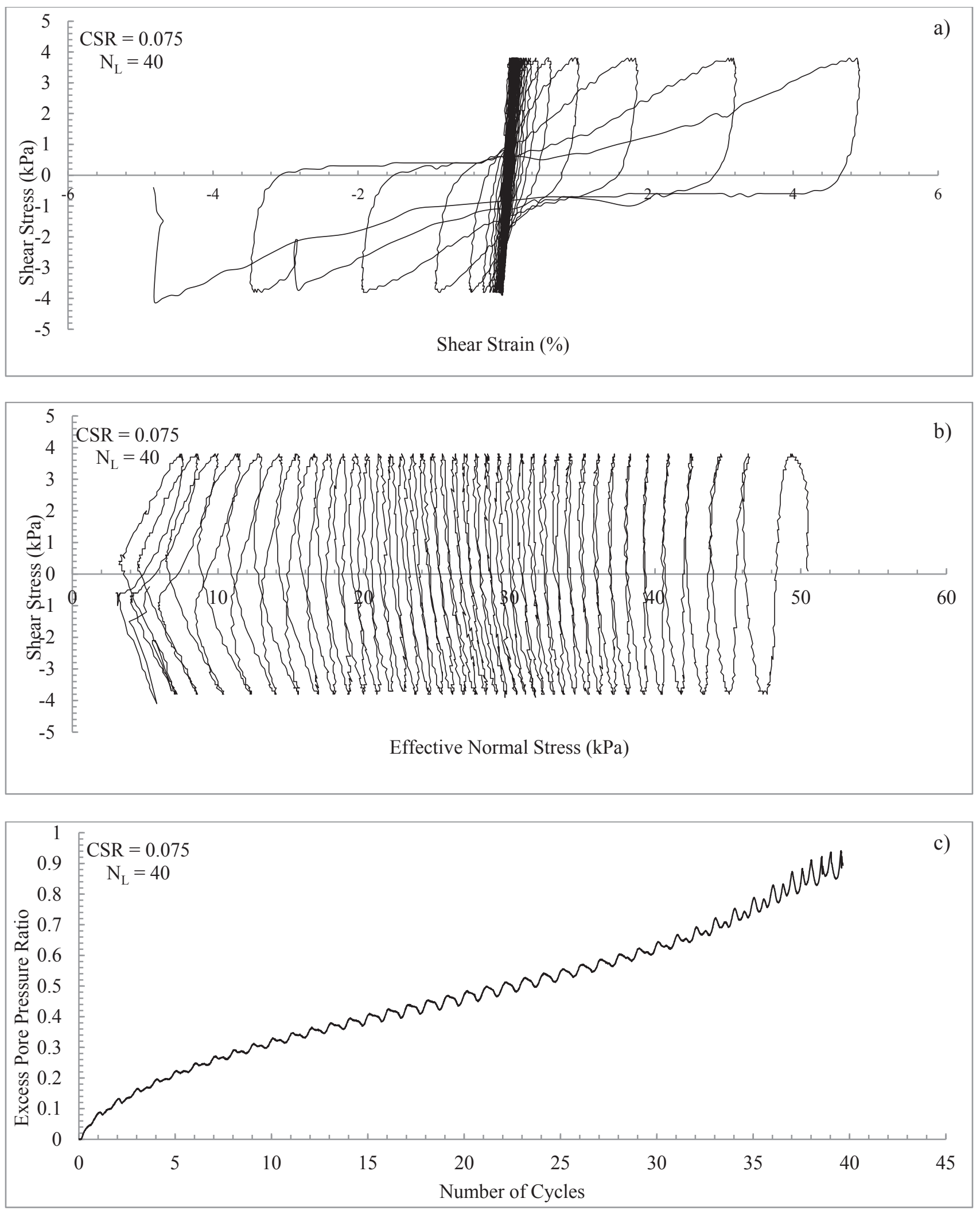

Figure 6.21 Cyclic simple shear response of desiccated-rewetted sample $(\mathrm{Wd}=26 \%)$ under CSR $=0.075$ 

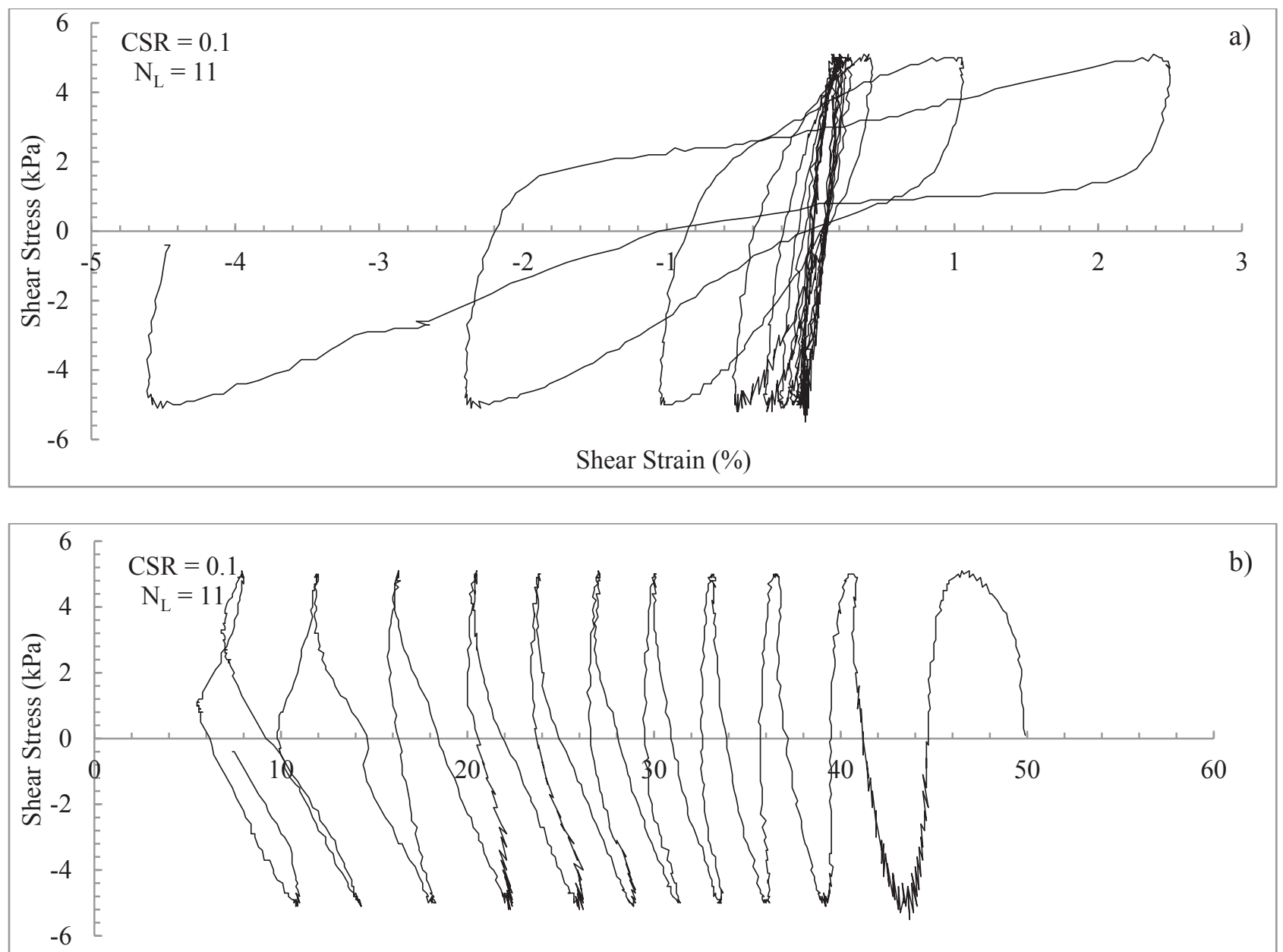

b)

Effective Normal Stress (kPa)

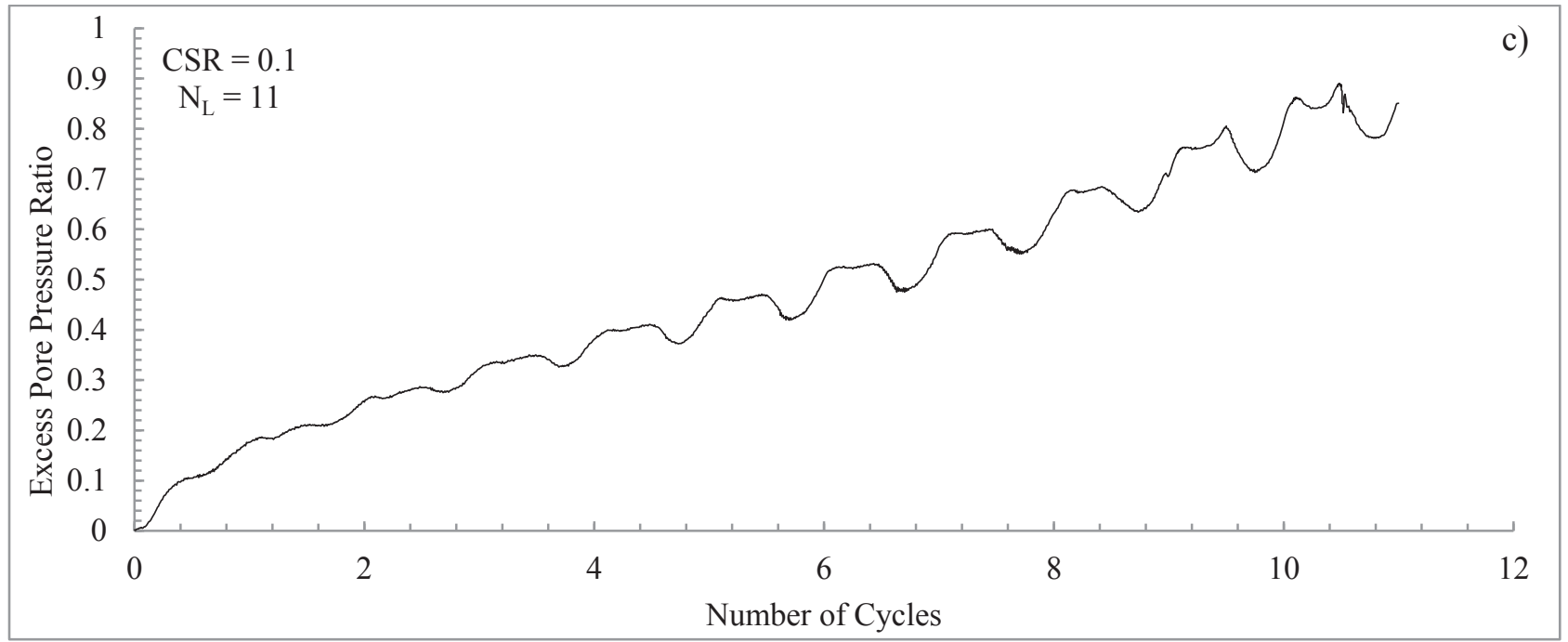

Figure 6.22 Cyclic simple shear response of desiccated-rewetted sample $(\mathrm{Wd}=26 \%)$ under CSR $=0.1$ 

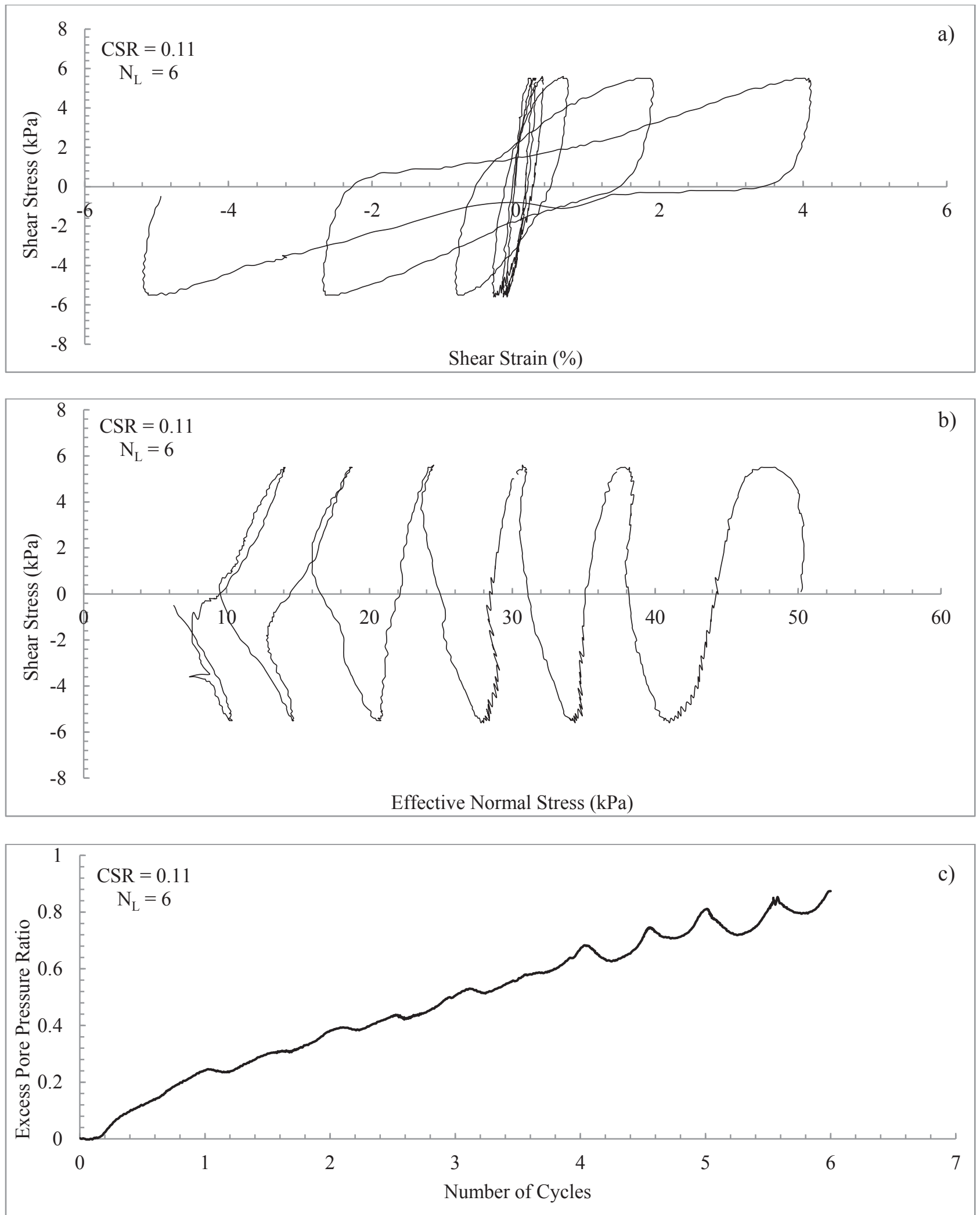

Figure 6.23 Cyclic simple shear response of desiccated-rewetted sample $(\mathrm{Wd}=26 \%)$ under CSR $\mathrm{CSR}=0.11$ 

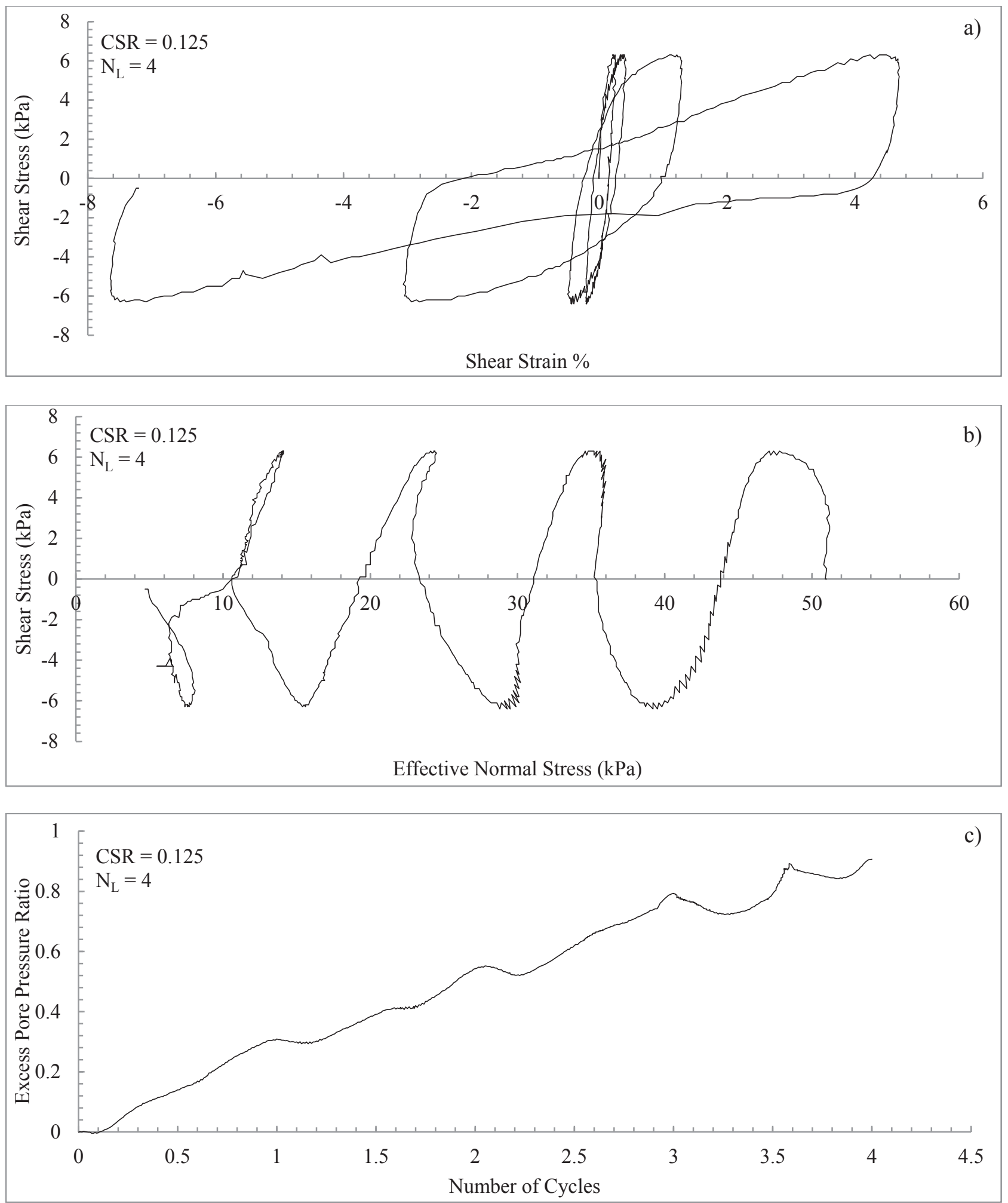

Figure 6.24 Cyclic simple shear response of desiccated-rewetted sample $(\mathrm{Wd}=26 \%)$ under CSR $=0.125$ 
Figure $6.25 \mathrm{~b}$ presents the excess pore pressure generation under $\mathrm{CSR}=0.065$, which shows that the maximum $r_{u}$ on the test is about 0.90 . Increasing CSR from 0.065 to 0.075 reduced the numbers of cycles to $\mathrm{N}_{\mathrm{L}}=70$ and the maximum excess pore pressure ratio to $\mathrm{r}_{\mathrm{u}}=0.94$ (Figure $6.26 \mathrm{c})$.
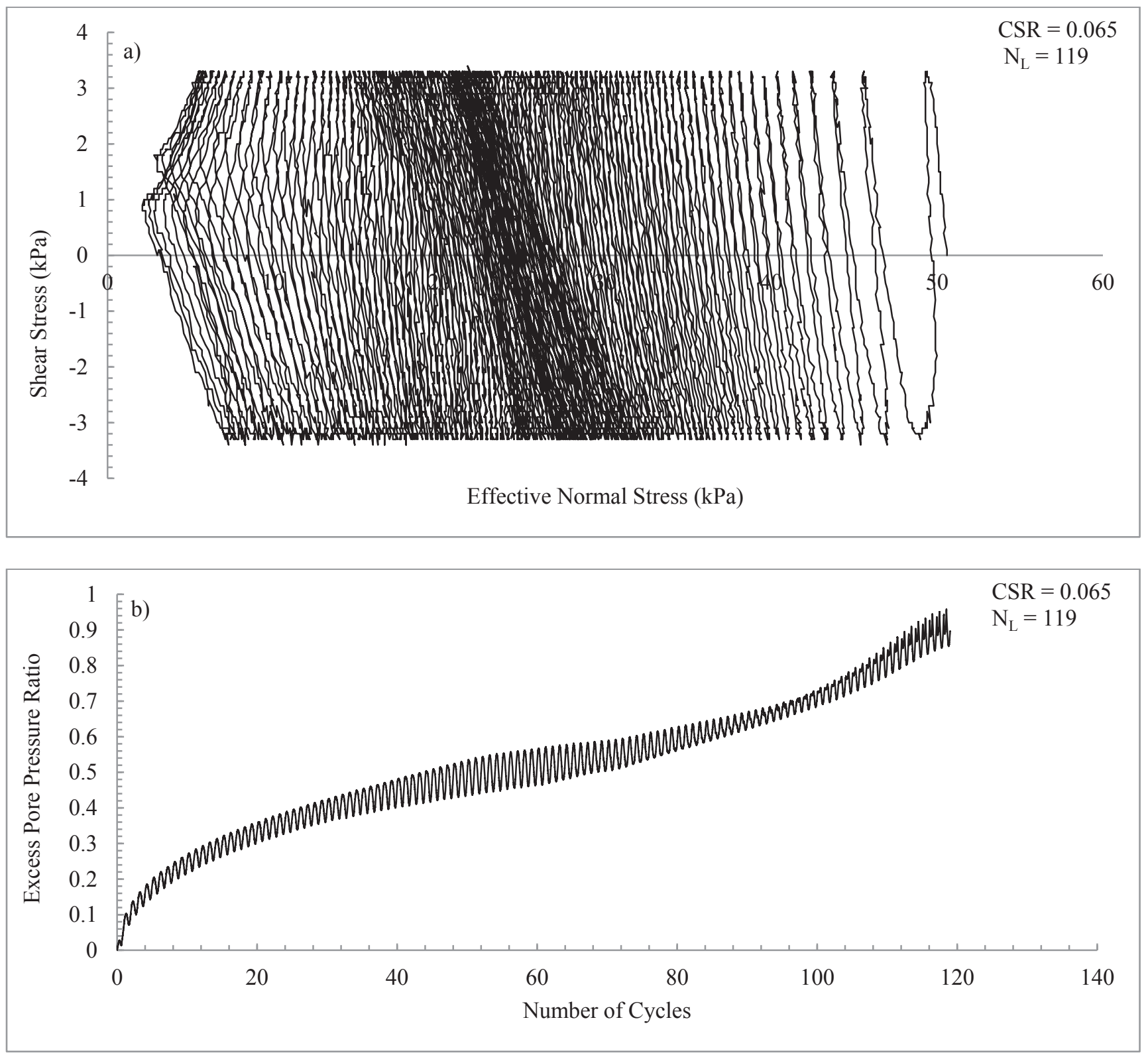

Figure 6.25 Cyclic simple shear response of desiccated-rewetted sample $(\mathrm{Wd}=20 \%)$ under $\mathrm{CSR}=0.065$ 

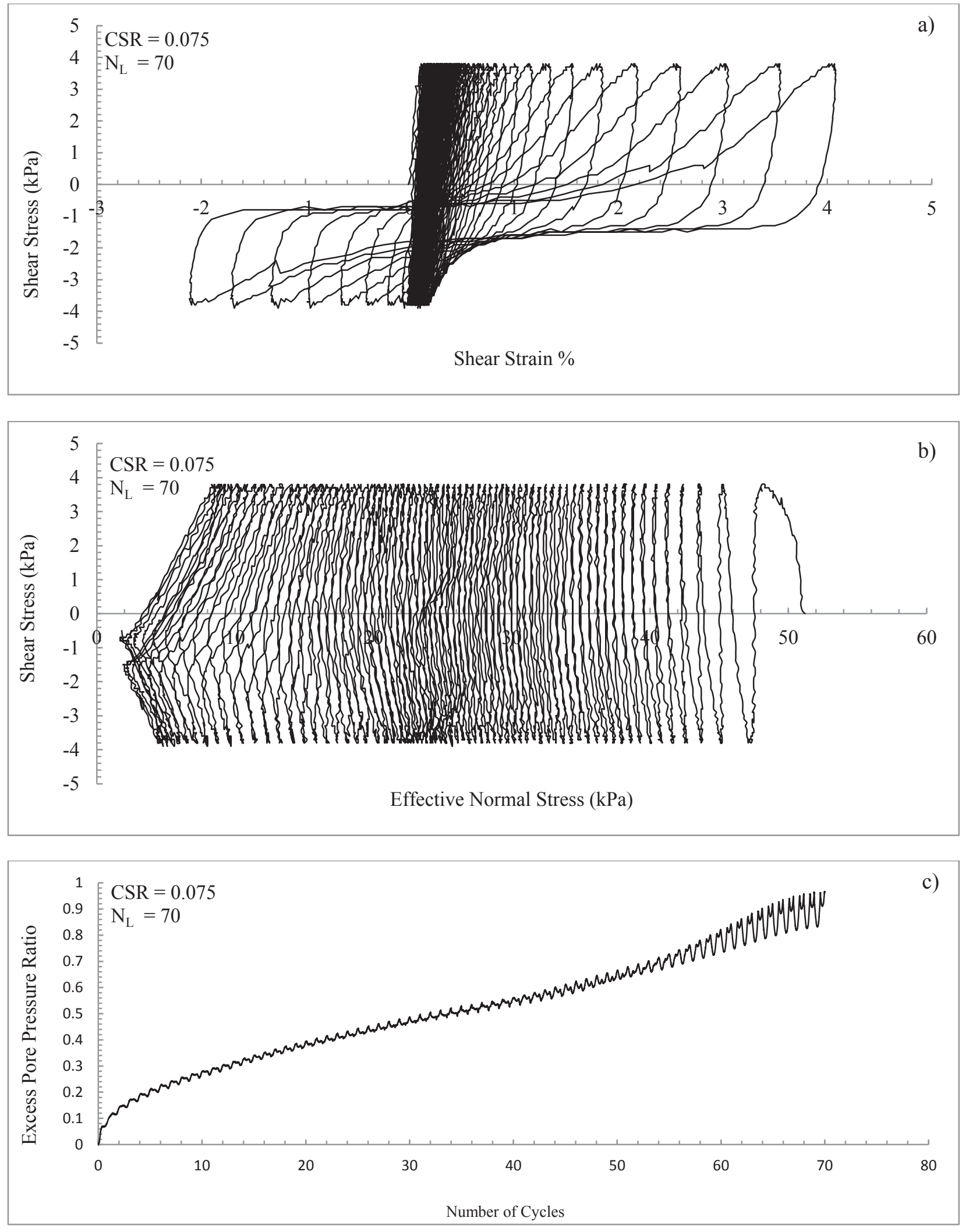

Figure 6.26 Cyclic simple shear response of desiccated-rewetted sample $(\mathrm{Wd}=20 \%)$ under CSR $=0.075$ 
Figures 6.27 and 6.28 present cyclic simple shear response of desiccated-rewetted tailings with $\mathrm{Wd}=12 \%$. Figure 6.27 shows that 83 cycles were required to reach liquefaction under $\mathrm{CSR}=$ 0.065. The final excess pore pressure ratio at the end of the test is equal to around $r_{u}=0.88$. Figure $6.28 \mathrm{~b}$ shows that the effective stress starts at around $50 \mathrm{kPa}$ effective normal stress and reached around $5 \mathrm{kPa}$ at the end of the test. It is shown that zero effective stress is not obtained at shear strain $=3.75 \%$. Increasing cyclic stress ratio to $\mathrm{CSR}=0.075$ reduced the numbers of cycles to reach liquefaction to $\mathrm{N}_{\mathrm{L}}=41$ at this $\mathrm{Wd}$.

\subsubsection{Discussion on cyclic results of small scale method}

Cyclic simple shear tests at different CSRs were conducted on samples prepared based on small scale laboratory method on specimens with different desiccation histories. Figure 6.29 compares the cyclic behavior of the tailings subjected to the same CSR $=0.125$ following consolidation to $50 \mathrm{kPa}$, but dried to different levels of desiccation to highlight the effect of desiccation history on the cyclic simple shear response of tailings. The first test (Figure 6.29a) was conducted on a sample with no desiccation, and it liquefied in just 3 cycles. The sample desiccated to $\mathrm{Wd}=26 \%$, liquefied in 4 cycles, and that desiccated to $\mathrm{Wd}=17 \%$ required 6 cycles to reach liquefaction. Desiccation to $\mathrm{Wd}=17 \%$ has doubled the number of cycles required to trigger liquefaction compared to the sample that underwent no desiccation.

Figure 6.30 compares the cyclic simple shear response of a sample with no desiccation $(\mathrm{Wd}=$ $32 \%$ ) with a sample desiccated to $\mathrm{Wd}=20 \%$ and rewetted at $\mathrm{CSR}=0.065$. The number of cycles to liquefaction increase from 32 (in the case of sample with no desiccation) to 119 when 

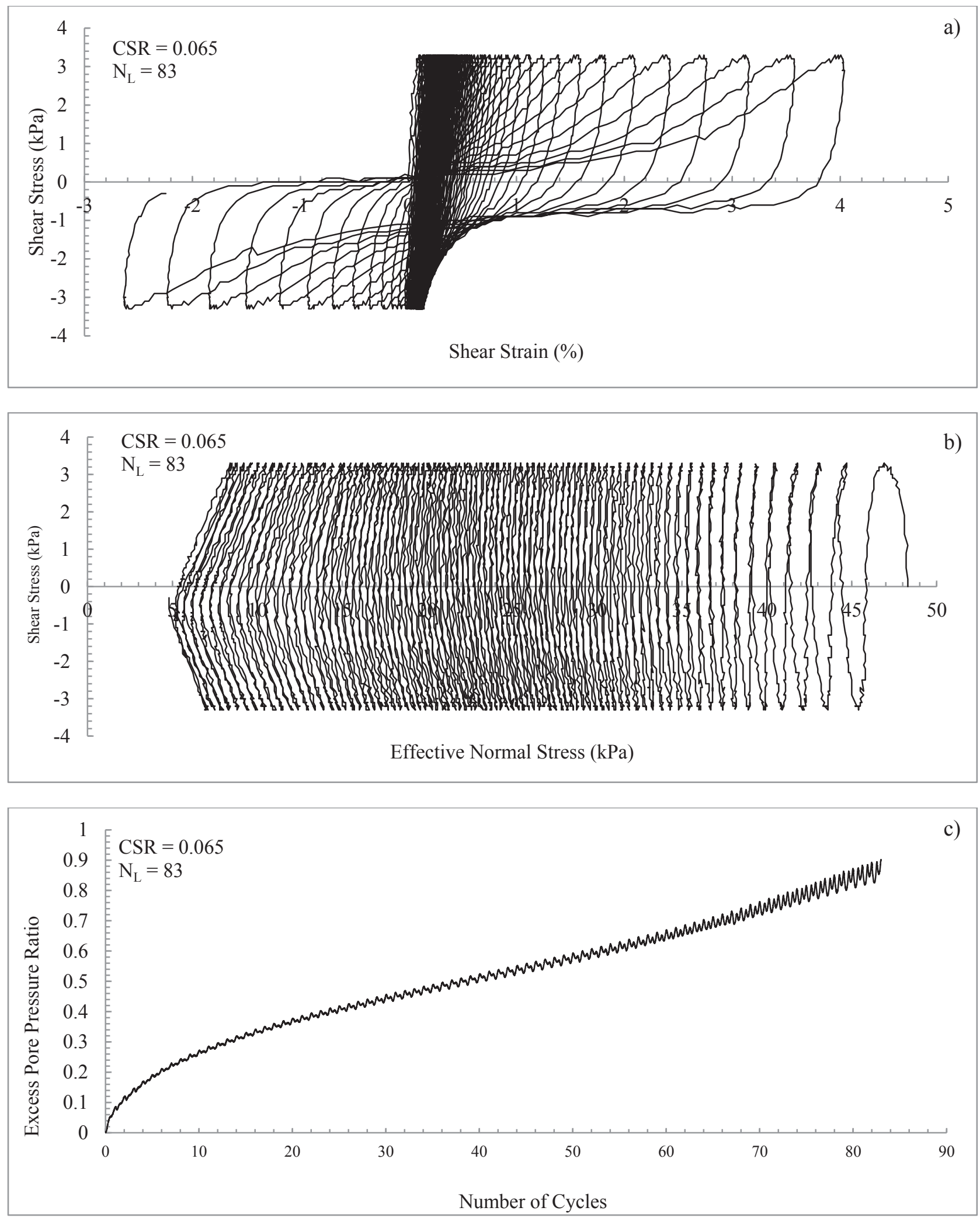

Figure 6.27 Cyclic simple shear response of desiccated-rewetted sample $(\mathrm{Wd}=12 \%)$ under CSR $=0.065$ 

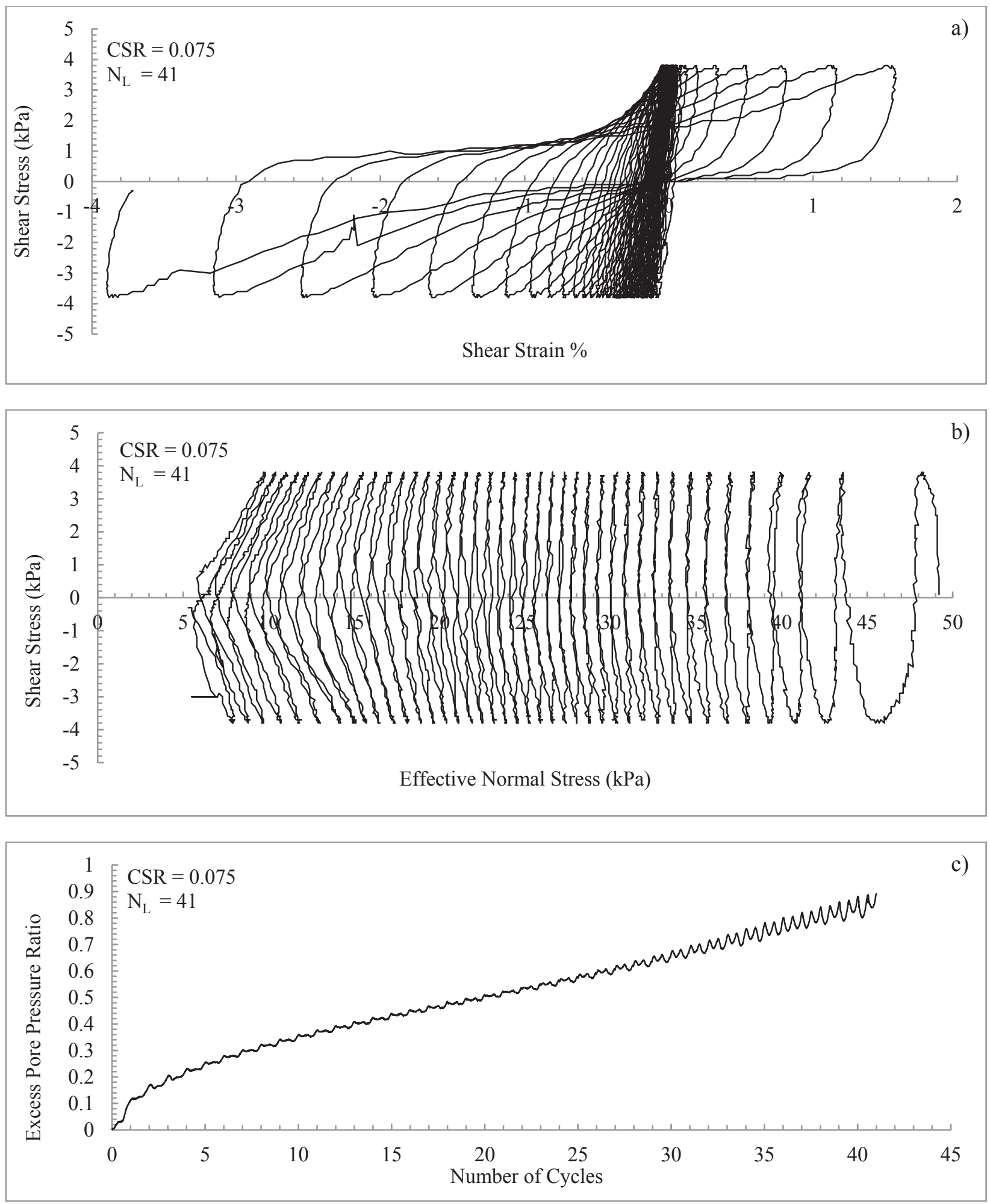

Figure 6.28 Cyclic simple shear response of desiccated-rewetted sample $(\mathrm{Wd}=12 \%)$ under CSR $=0.075$ 
the sample underwent a desiccation and rewet history. This represents a significant difference in the cyclic strength of the material.

Figure 6.31 compares the rate of excess pore pressure generation in samples with different desiccation histories and subjected CSR values of $0.065,0.075$ and 0.125 . One sample was tested with no desiccation history, and the other two samples were rewetted after desiccation to $\mathrm{Wd}=$ $17 \%$ and $12 \%$ respectively. As reflected by the number of cycles to liquefaction, the rate of excess pore pressure generation is significantly faster in the sample with no desiccation than desiccated-rewetted sample. Increasing desiccation history to $\mathrm{Wd}=17 \%$ remarkably slowed the rate of excess pore pressure generation. Higher degree of desiccation $(\mathrm{Wd}=12 \%)$ prior to rewetting further slowed the rate of pore pressure generation but only marginally. It was beneficial in terms of increasing liquefaction resistance; however, the increase in cyclic strength for desiccation from $\mathrm{Wd}=17 \%$ to $\mathrm{Wd}=12 \%$ is not as significant as desiccation from $\mathrm{Wd}=32 \%$ to $\mathrm{Wd}=17 \%$. Similar observations are noted in the tests at the other two CSR values as well. These findings clearly indicate that while desiccation is highly beneficial the 'rate of return' diminishes quite dramatically as sample desiccate below the shrinkage limit.

Figure 6.32 presents cyclic stress ratio (CSR) versus numbers of cycles to reach liquefaction $\left(\mathrm{N}_{\mathrm{L}}\right)$ for all cyclic tests performed on samples prepared by the small scale deposition method. It is clear that settled samples with no desiccation history $(\mathrm{Wd}=32 \%)$ required lowest numbers of cycles to reach liquefaction at a specific CSR, which is evidence of their lower cyclic resistance. Increasing desiccation history to $\mathrm{Wd}=26 \%$ significantly increased the numbers of cycles to reach liquefaction at specific CSR. Increasing desiccation history to $\mathrm{Wd}=17 \%$ is also beneficial in increasing cyclic resistance especially at higher CSRs (0.12-0.15). The difference between cyclic resistance of samples with $\mathrm{Wd}=26 \%$ and $\mathrm{Wd}=17 \%$ at lower CSRs $(0.065-0.075)$ is less 

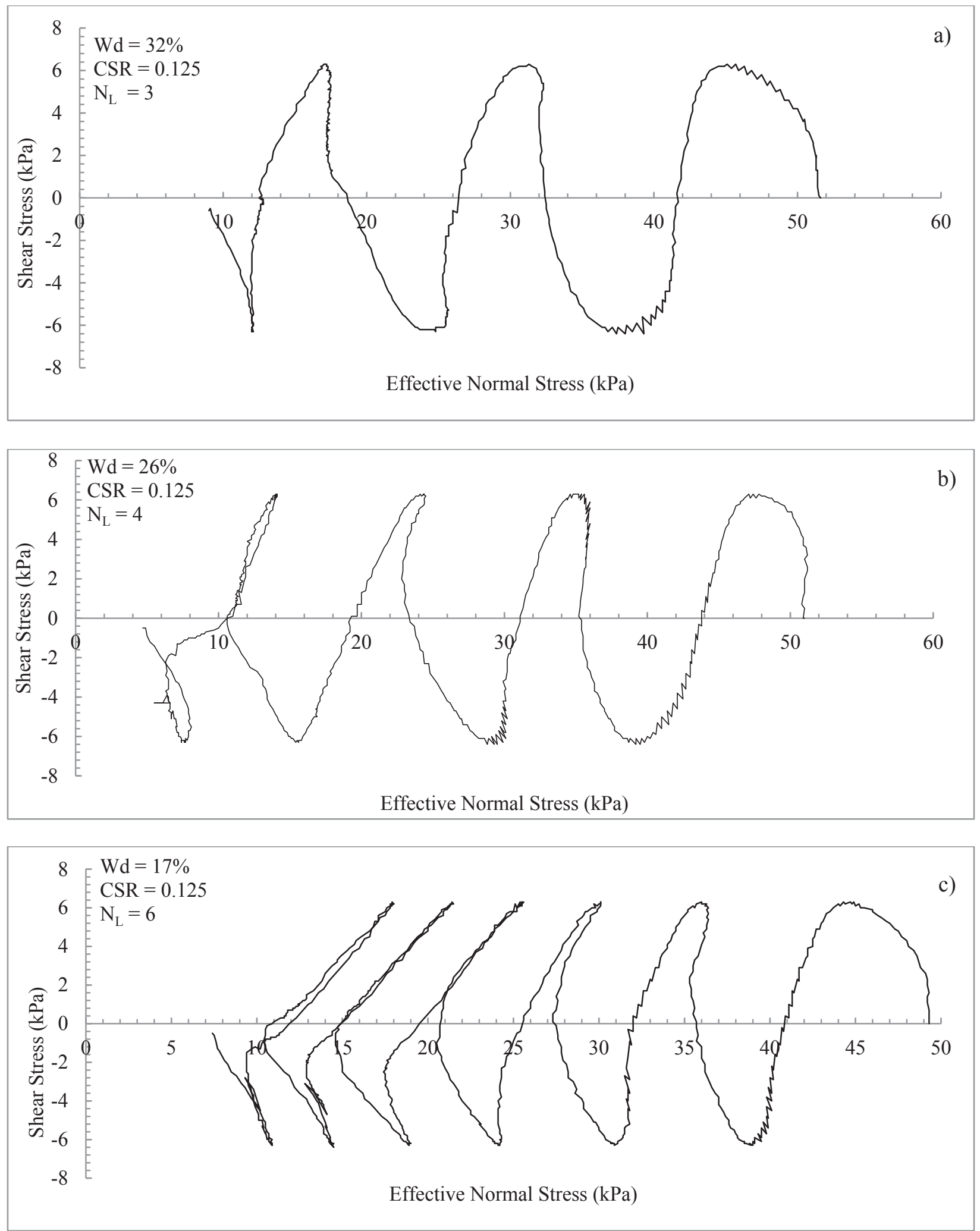

Figure 6.29 Comparison of cyclic simple shear response of samples with different desiccation history at $\mathrm{CSR}=0.125$ 

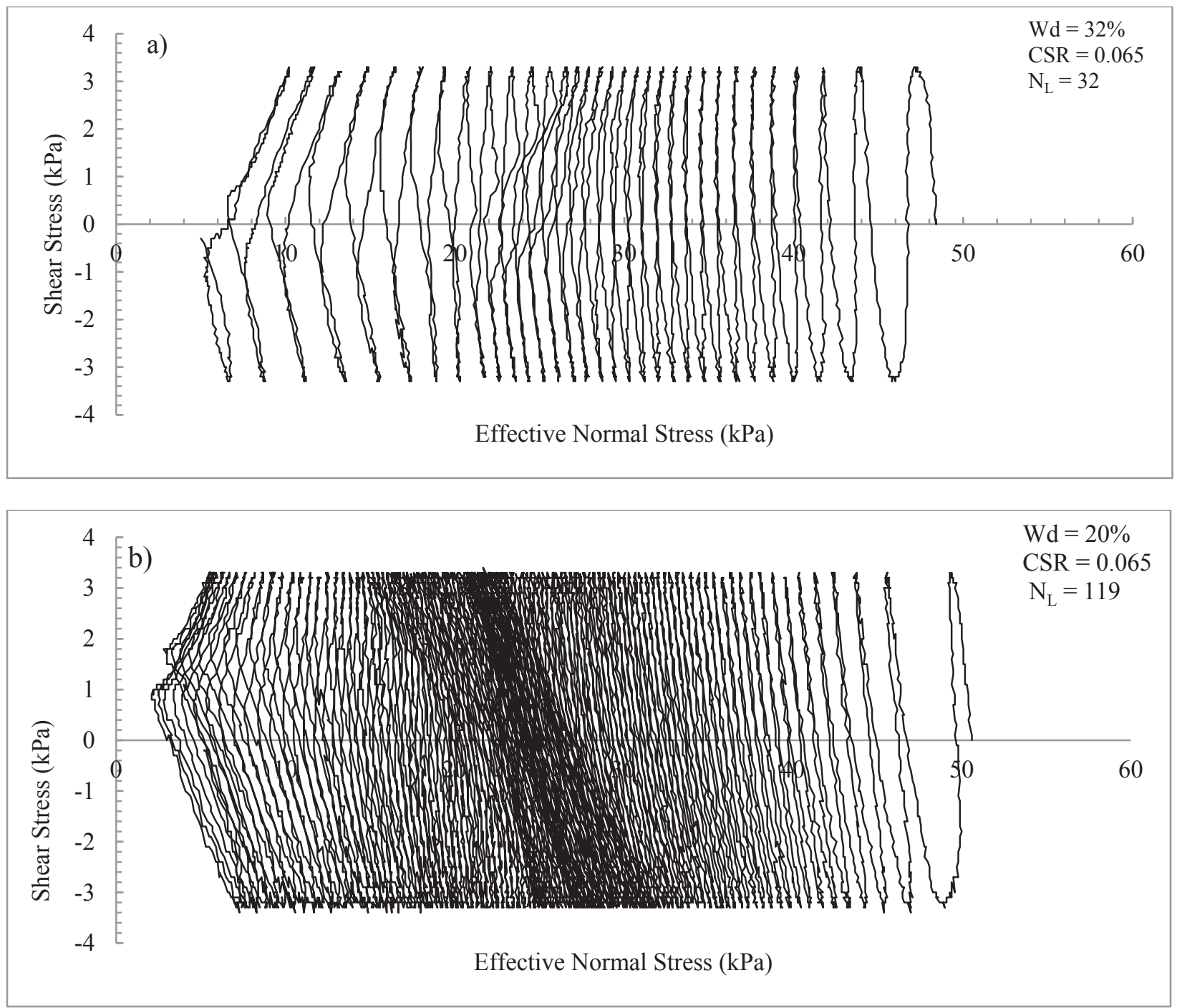

Figure 6.30 Comparison of cyclic simple shear response of samples with different desiccation history at $\mathrm{CSR}=0.065$

tangible than higher CSRs. Increasing desiccation history to lower water contents $(\mathrm{Wd}=12 \%$ and $\mathrm{Wd}=10 \%)$ resulted in higher cyclic resistance at higher CSRs $(0.12-0.15)$. However, at lower CSRs, the cyclic resistance decreased with increasing degree of desiccation to $\mathrm{Wd}=12 \%$ and $\mathrm{Wd}=10 \%$. It is not clear whether this is actual material behavior or scatter in the data as the difference are relatively minor. The data presented in Fig 6.32 indicates that the cyclic resistance 

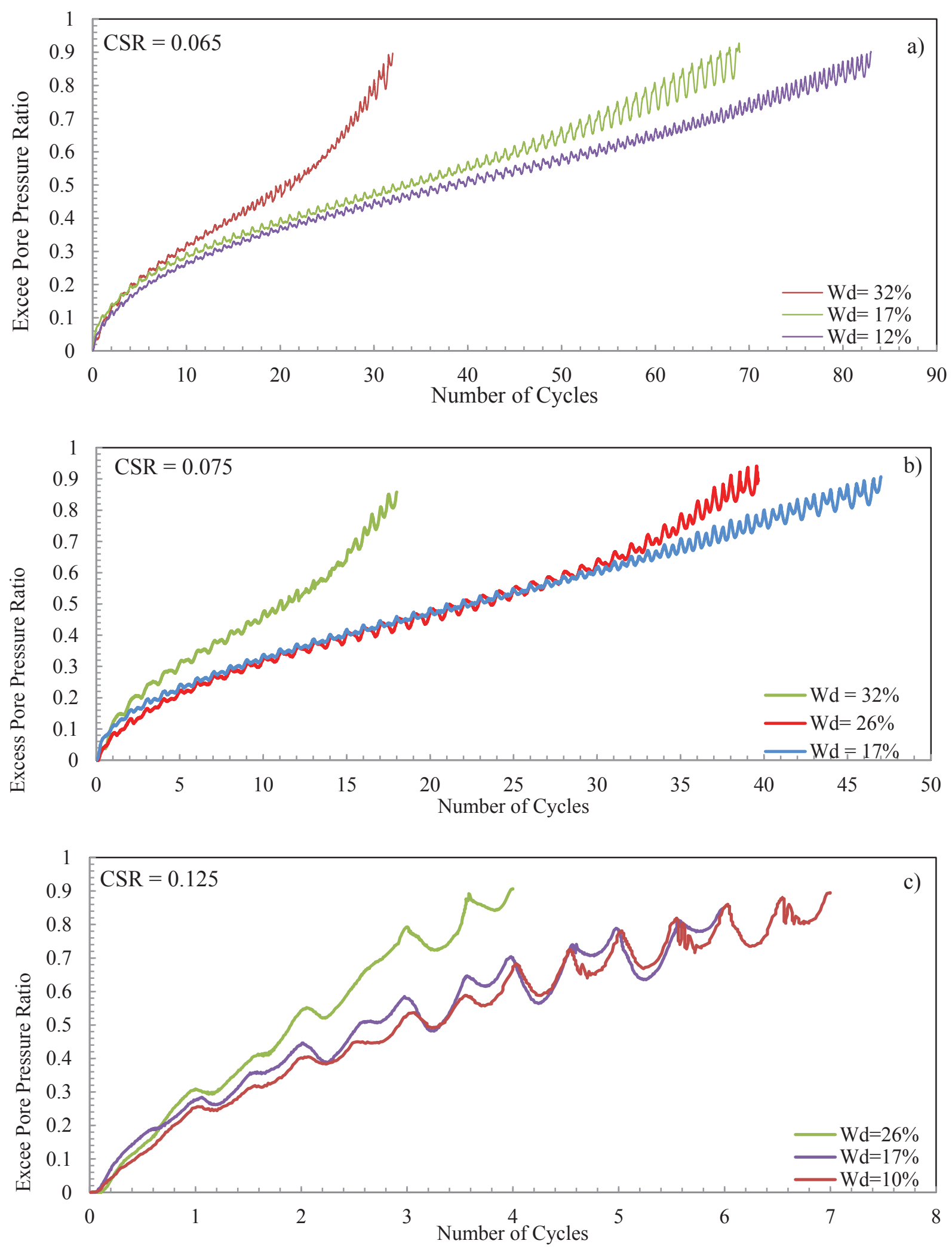

Figure 6.31 Comparison of excess pore pressure generation of samples with different desiccation histories at $\mathrm{CSR}=0.065,0.075$ and 0.1 


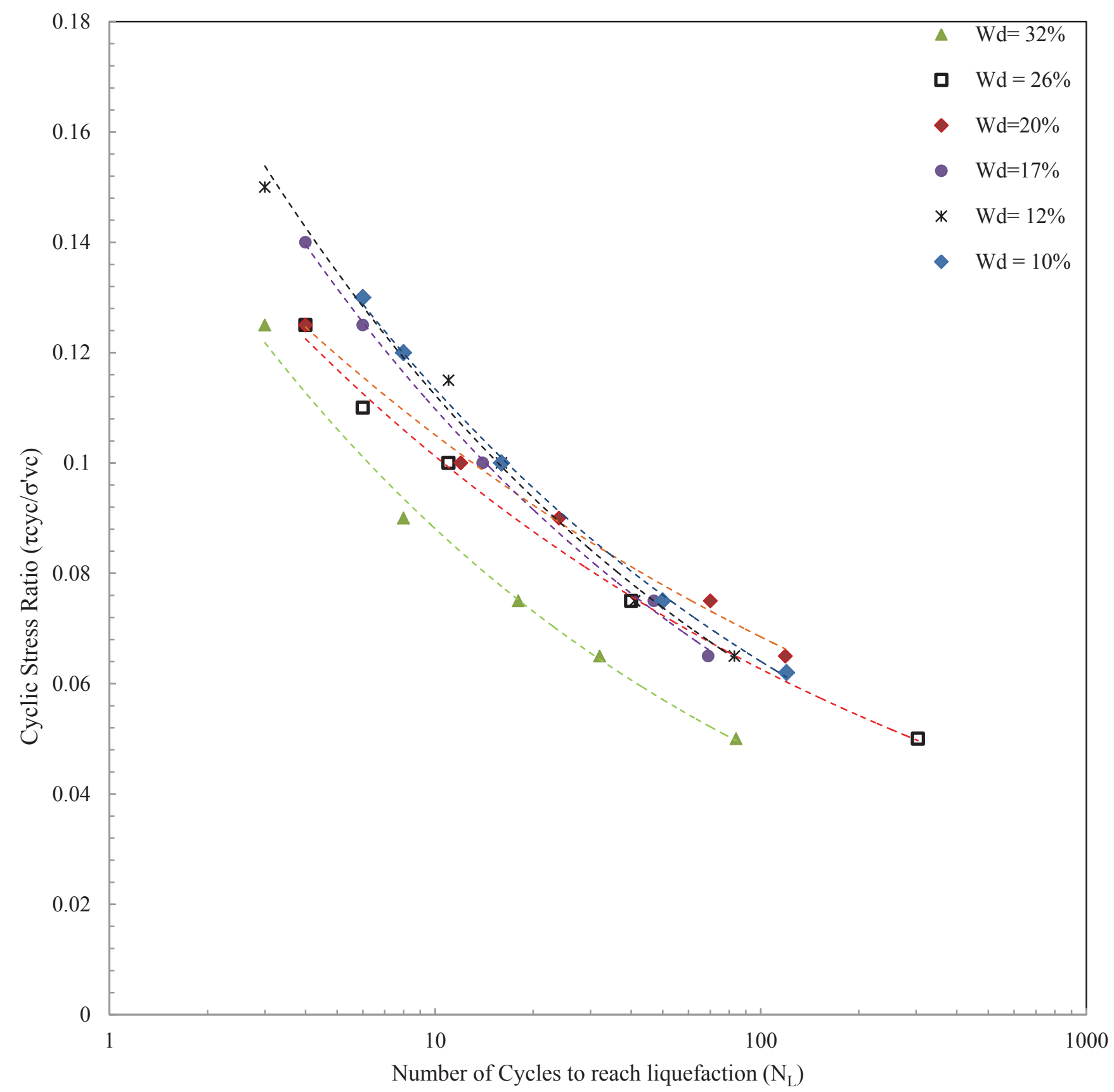

Figure 6.32 Cyclic stress ratio (CSR) versus numbers of cycles to reach liquefaction $\left(\mathrm{N}_{\mathrm{L}}\right)$ for all cyclic simple shear tests performed on samples prepared by small scale deposition method

of the tailings can be increased at all CSR levels by desiccating the tailings to a water content just below the shrinkage limit prior to rewetting. 

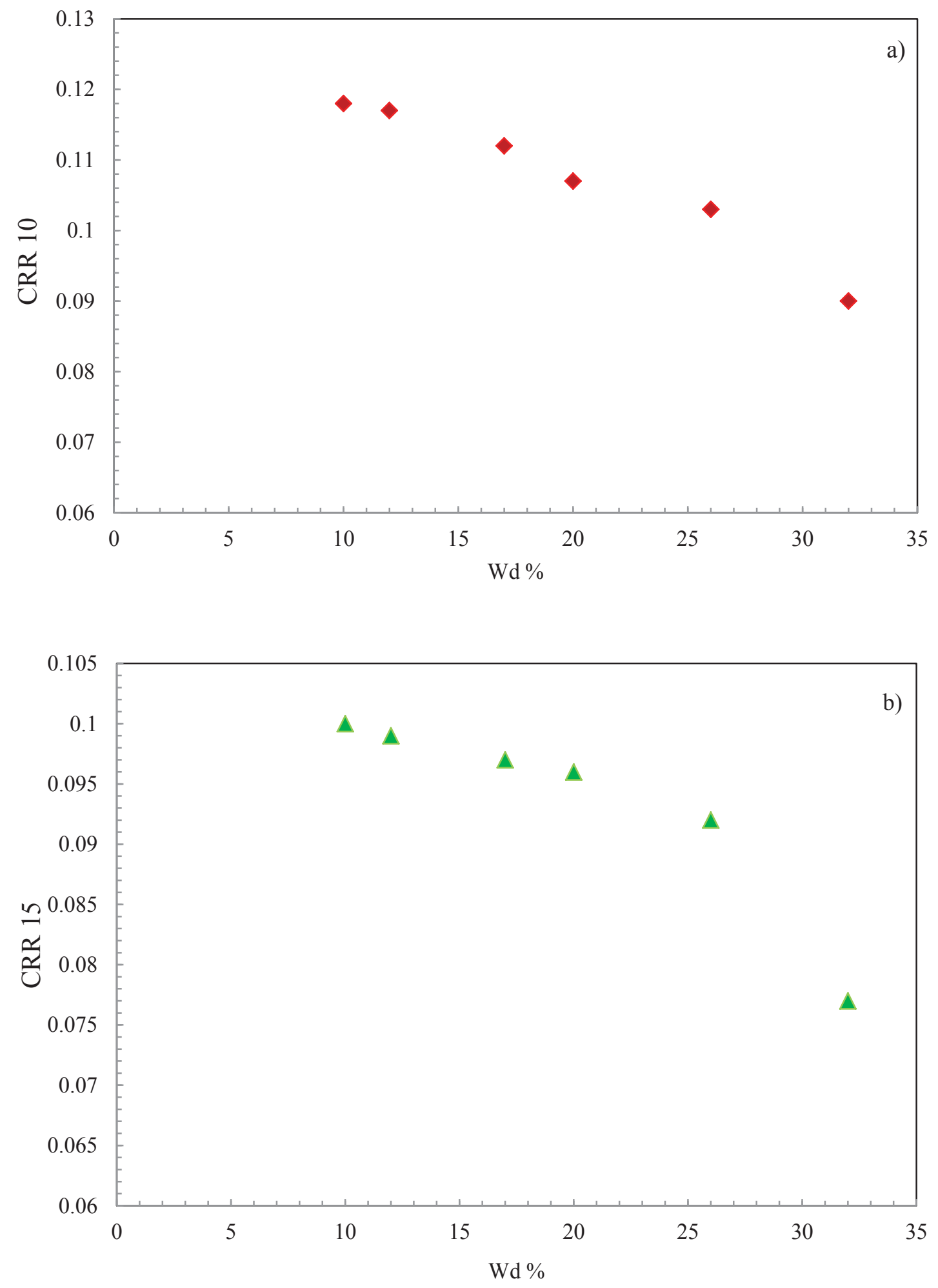

Figure 6.33 The effect of desiccation history on cyclic resistance ratio of small scale prepared samples a) CRR10, b) CRR15 
The cyclic resistance ratio of the tailings, derived from the data shown in Fig 6.32 is shown in Figure 6.33 to highlight the effect of desiccation history on cyclic resistance ratio of the tailings. The cyclic resistance ratios, CRR10 and CRR15, correspond to earthquakes with $M=6.75$ and $\mathrm{M}=7.5$ respectively. It is clear that a little degree of desiccation to $\mathrm{Wd}=26 \%$ significantly increases the CRR10 and CRR15 compared to samples with no desiccation ( $\mathrm{Wd}=32 \%)$. This increase in CRR is more tangible at CRR15, which corresponds to an earthquake with higher magnitude. Increasing desiccation history to shrinkage limit and beyond that slightly increases both CRR10 and CRR15. However, the increase in cyclic resistance is not as significant as desiccation from $\mathrm{Wd}=32 \%$ to $\mathrm{Wd}=26 \%$.

\subsubsection{Monotonic simple shear results of samples extracted from the drying box}

The monotonic simple shear behavior of tailings obtained from the drying box are presented in this section. The details of the deposition and desiccation histories of the drying box layers were discussed in chapter 4 . As discussed in chapter 3, two different methods were employed to obtain samples from the drying box: i) buried tubes before deposition, ii) sampling with thin wall tubes. Extracted samples were consolidated under $50 \mathrm{kPa}$ or $100 \mathrm{kPa}$ consolidation pressure. The monotonic response of samples obtained from buried tubes are compared with monotonic response of samples obtained from thin wall sampling under $50 \mathrm{kPa}$ consolidation pressure. Table 6.4 presents the details of the monotonic simple shear tests performed on samples obtained

from the drying box samples. Figure 6.34 presents results of monotonic simple shear tests performed on buried tubes extracted from different layers under $50 \mathrm{kPa}$ vertical effective stress. 
All samples exhibited strain hardening response with a phase transformation state clearly noticeable in the stress path.

Table 6.4 Monotonic simple shear tests performed on samples obtained from Drying box samples

\begin{tabular}{|c|c|c|c|c|c|}
\hline $\begin{array}{c}\text { Test } \\
\text { No. }\end{array}$ & $\begin{array}{c}\text { Drying Box } \\
\text { Layer }\end{array}$ & $\begin{array}{c}\text { Water content } \\
\text { before rewetting } \\
\text { Wd }(\%)\end{array}$ & $\begin{array}{c}\text { Method of } \\
\text { Sampling }\end{array}$ & $\begin{array}{c}\text { Consolidation } \\
\text { Pressure }\left(\sigma_{v c}^{\prime}\right) \\
\mathrm{kPa}\end{array}$ & $\begin{array}{c}\text { Void ratio after } \\
\text { consolidation } \\
\left(\mathrm{e}_{\mathrm{c}}\right)\end{array}$ \\
\hline 1 & One & 12.5 & Buried & 50 & 0.625 \\
2 & Two & 16.9 & Buried & 50 & 0.633 \\
3 & Three & 18.2 & Buried & 50 & 0.623 \\
4 & Four & 17.8 & Buried & 50 & 0.619 \\
5 & One & 12.5 & Thin Wall & 50 & 0.628 \\
6 & Two & 16.9 & Thin Wall & 50 & 0.617 \\
7 & Three & 18.2 & Thin Wall & 50 & 0.616 \\
8 & Four & 17.8 & Thin Wall & 50 & 0.626 \\
9 & One & 12.5 & Buried & 100 & 0.616 \\
10 & Two & 16.9 & Buried & 100 & 0.622 \\
11 & Three & 18.2 & Buried & 100 & 0.624 \\
12 & Four & 17.8 & Buried & 100 & 0.618 \\
\hline
\end{tabular}

It is shown that the first layer exhibited relatively stronger behavior than the other layers. Layers 2, 3 and 4 exhibited almost identical response. The void ratio of all four samples were fairly similar at $0.626 \pm 0.007$. At phase transformation (PT), layers 2,3 and 4 exhibited about 10.5 $\mathrm{kPa}$ shear stress; while the shear stress of the PT of the first layer was around $12.5 \mathrm{kPa}$. Results of chapter 4 showed that the first layer not only experienced higher degree of desiccation, but also experienced more desiccation-rewet cycles. At this time, it is not clear how much of the higher strength of the first layer is attributable to higher degree of desiccation, and how much to the desiccation-rewet cycles. 
Figure 6.35 presents monotonic results of tests performed on buried tubes under $100 \mathrm{kPa}$ vertical effective stresses and at a void ratio of $0.62 \pm 0.004$. It was noted that samples extracted from the drying box exhibited a somewhat lower void ratio $(0.02 \sim 0.03)$ compared to samples prepared by the small scale laboratory method. The reason could be attributed to higher number of desiccation-rewetting cycles in the drying box compared to the small scale method.

Similar to the tests under $50 \mathrm{kPa}$, the first layer exhibited somewhat stronger response. Unlike the results under $50 \mathrm{kPa}$ consolidation pressure, the second layer exhibited marginally stronger response than the third and fourth layers, which can be attributed to the higher degree of desiccation of the second layer compared to the third and fourth layers. The phase transformation shear strength under $100 \mathrm{kPa}$ was $25.1 \mathrm{kPa}$ for the first layer, $23.7 \mathrm{kPa}$ for the second layer and about 21.5 for the third and fourth layers. These values indicate that the phase transformation strength normalises fairly well with the consolidation pressure, and yield $\frac{S_{P T}}{\sigma_{v c}^{\prime}}=0.25$ for the first layer, and about 0.21 for the third and fourth layers.

Figure 6.36 presents results of monotonic simple shear tests performed on samples obtained by using thin-wall tubes. It is shown that similar to the samples obtained from the buried tubes under $50 \mathrm{kPa}$, all samples exhibited strain hardening response. The first layer exhibited stronger behavior than other layers. Layers 2 and 3 exhibit relatively stronger behavior than layer 4 at the early stages of the loading, but layer 4 exhibited more dilative tendency following phase transformation. The differences, however, are minor.

In order to investigate the potential effect of sampling methods on the response of the tailings, Figure 6.37 compares the responses of tests performed on samples obtained from buried tubes and thin-walled sampler from layers 1 and 3 and consolidated under $50 \mathrm{kPa}$ consolidation 
pressure. The behavior is essentially identical in both cases, and these results clearly show that the sampling method did not influence the monotonic behavior in these samples. A comparison of the behavior of the samples obtained from the $4^{\text {th }}$ layer would not be as similar, and suggest that the insertion effects were somewhat serious in the top layer, but still the differences are quite minor.
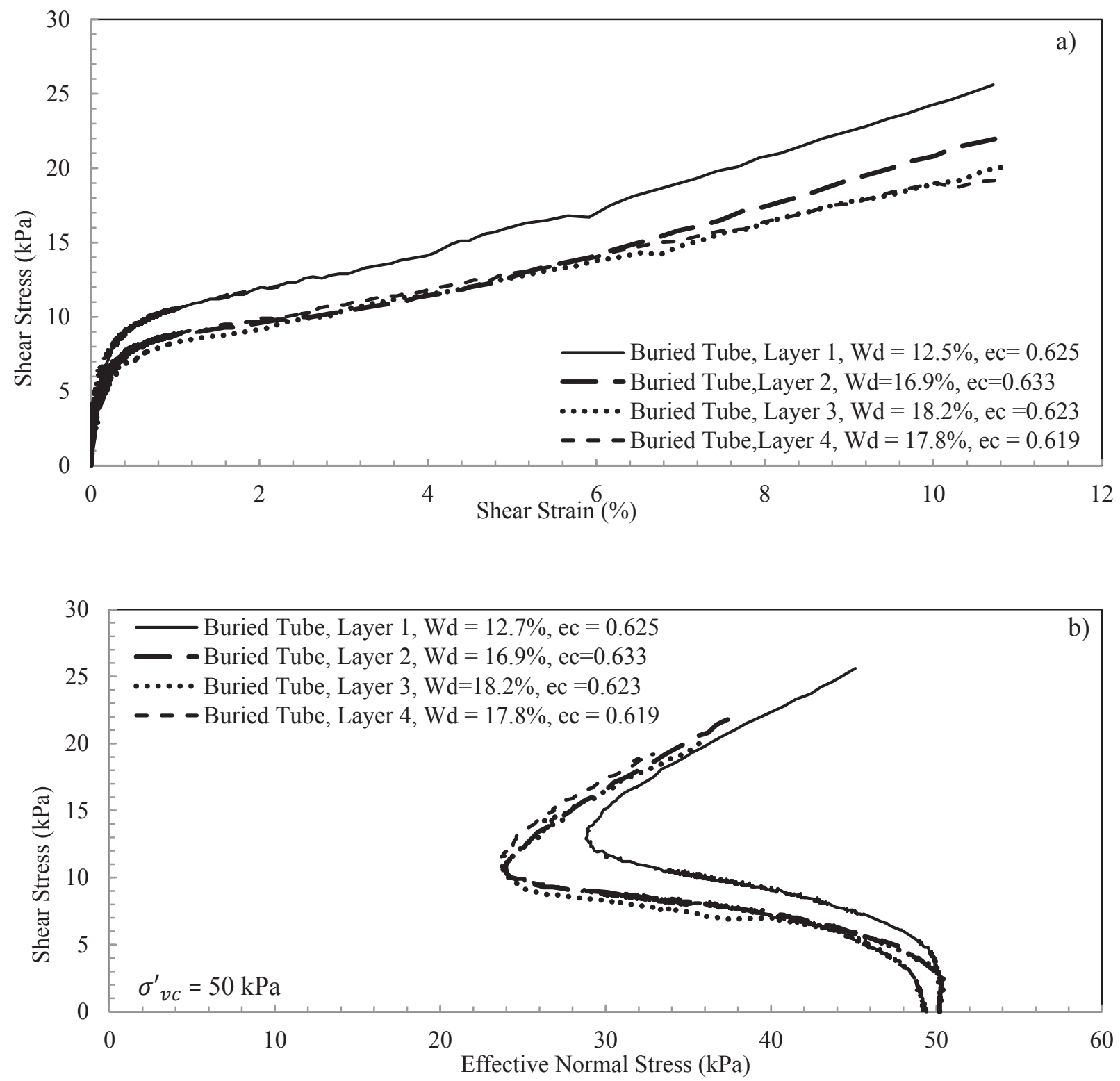

Figure 6.34 Monotonic simple shear response of tests performed on buried tubes under $50 \mathrm{kPa}$ vertical effective stress 

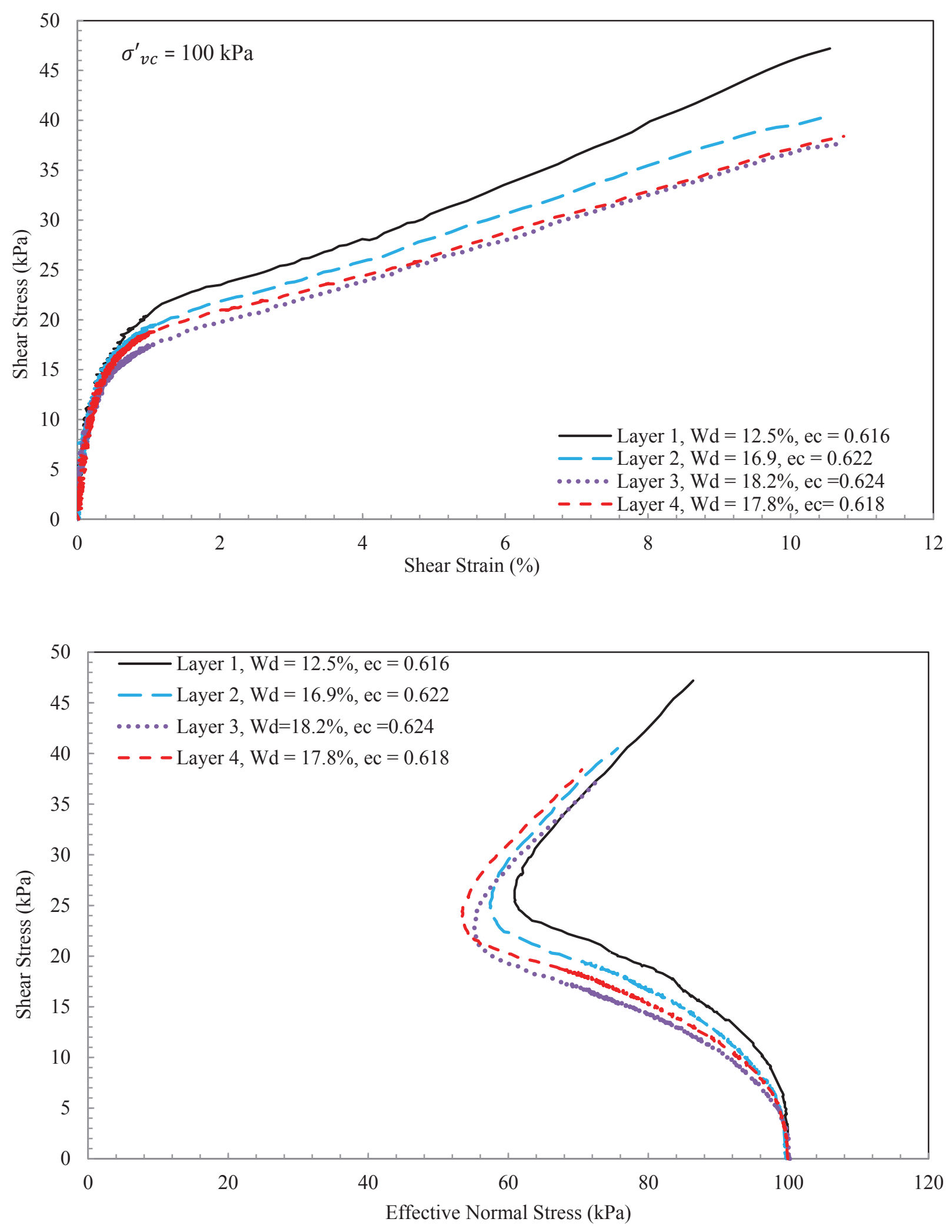

Figure 6.35 Results of simple shear tests on buried tubes under $100 \mathrm{kPa}$ vertical effective stress 

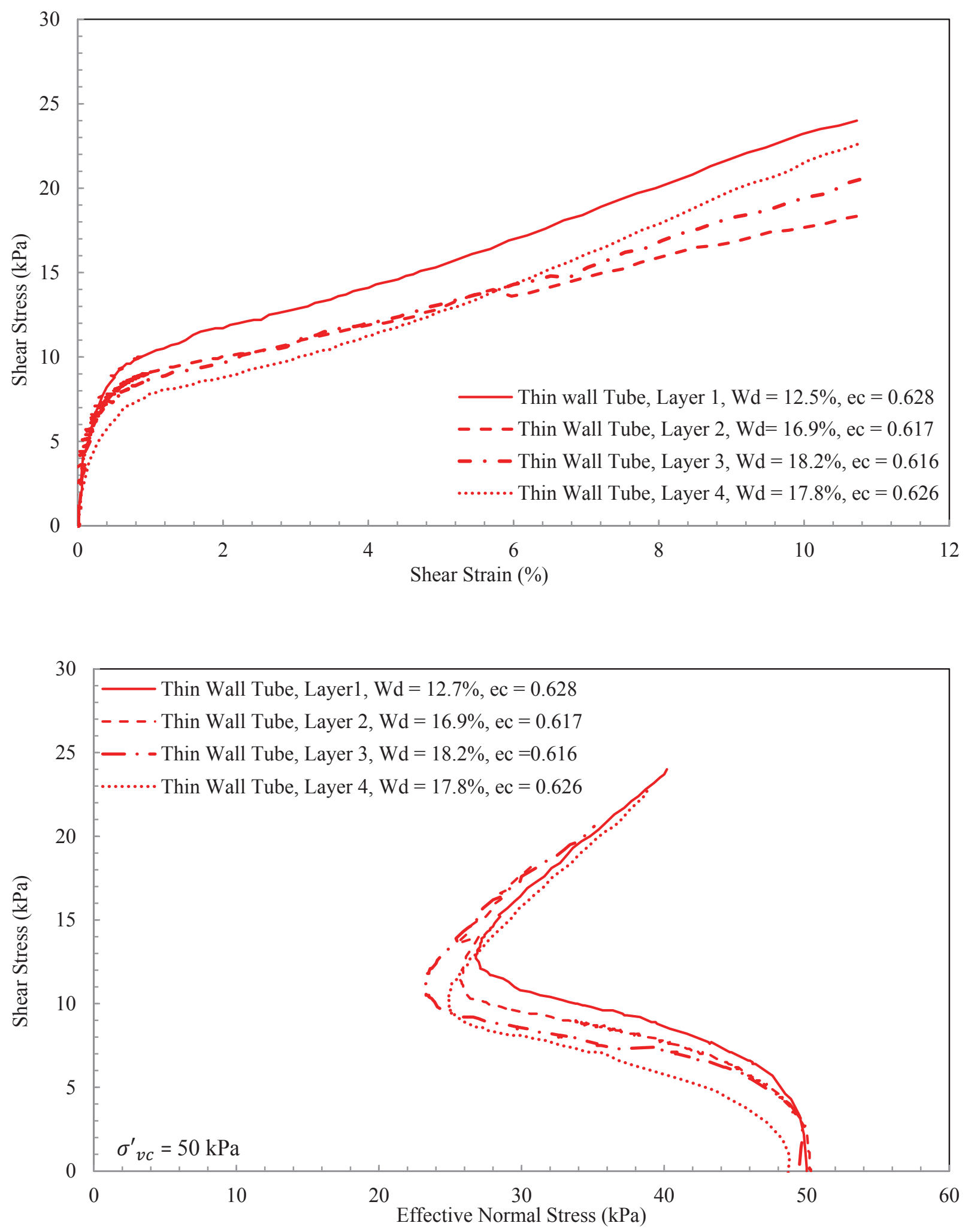

Figure 6.36 Monotonic simple shear response of tests performed on samples prepared by sampling with thin wall tubes under $50 \mathrm{kPa}$ vertical effective stress 


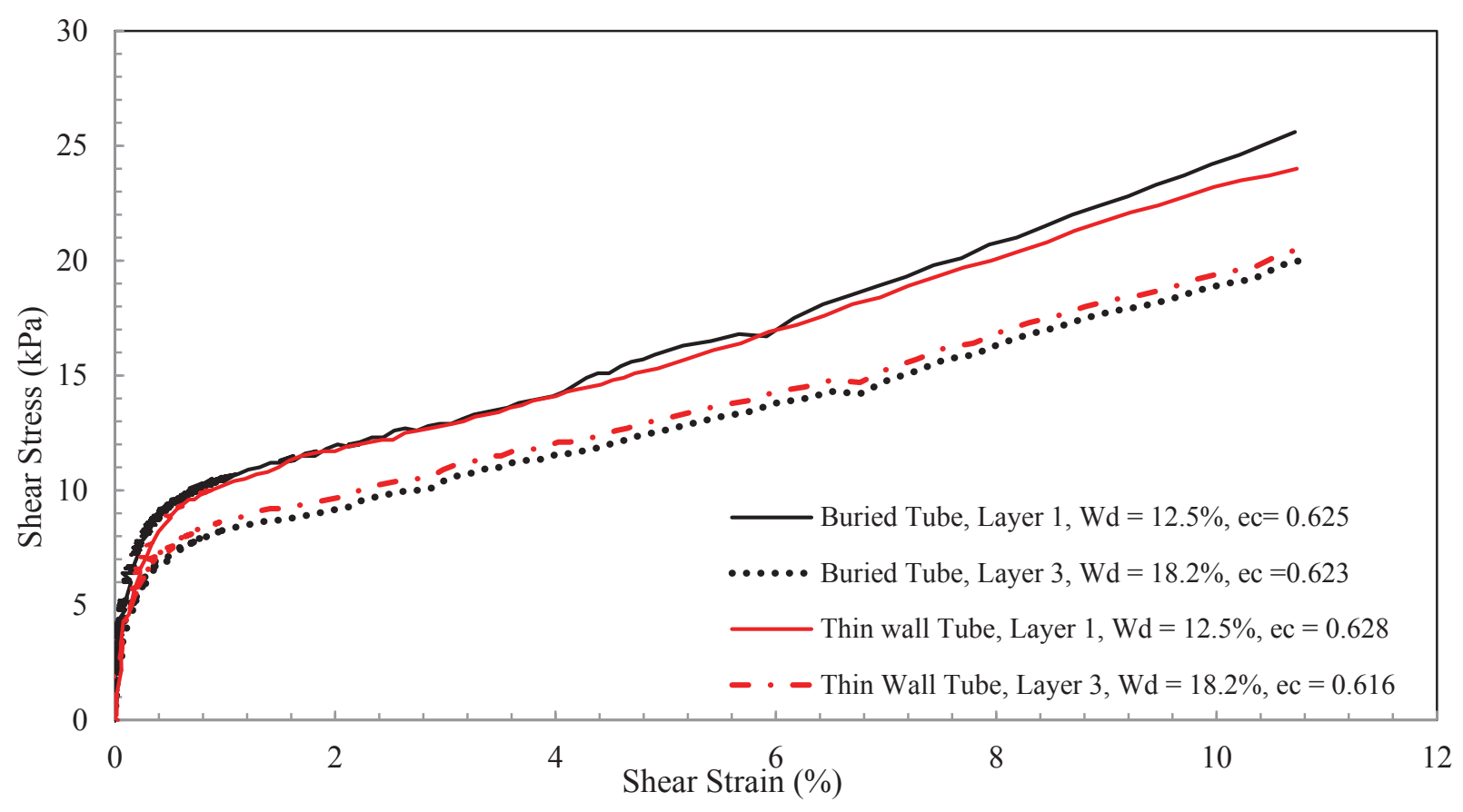

Figure 6.37 Difference between the responses of samples obtained from buried tubes and thin wall tubes extracted by sampling methods

\subsubsection{Cyclic simple shear results of samples extracted from the drying box}

Cyclic simple shear tests were performed on samples obtained from different layers of the drying box. All samples were obtained from buried tubes and consolidated to $100 \mathrm{kPa}$ consolidation pressure before the application of $0.1 \mathrm{~Hz}$ sinusoidal cyclic load. As noted earlier, a strain criterion (NRC 1985) was used to define the triggering of liquefaction. Constant volume cyclic tests were conducted over a range of CSR value from 0.075 to 0.20 .

Table 6.5 presents the sampling locations and test parameters for the cyclic simple shear tests performed on samples extracted from the drying box. Figure 6.38 to 6.40 present cyclic simple shear responses of samples obtained from the first layer of the drying box, which experienced 
Table 6.5 Cyclic simple shear tests performed on samples obtained from Drying box samples

\begin{tabular}{|c|c|c|c|c|c|c|c|}
\hline $\begin{array}{l}\text { Test } \\
\text { No. }\end{array}$ & $\begin{array}{c}\text { Sample } \\
\text { Characteristics }\end{array}$ & $\begin{array}{c}\text { Drying } \\
\text { Box } \\
\text { Layer }\end{array}$ & $\begin{array}{l}\mathrm{Wd} \\
(\%)\end{array}$ & $\begin{array}{c}\text { Consolidation } \\
\text { Pressure } \\
\left(\sigma_{v c}^{\prime}\right) \mathrm{kPa} \\
\end{array}$ & $\mathrm{e}_{\mathrm{c}}$ & CSR & $\mathrm{N}_{\mathrm{L}}$ \\
\hline 1 & Desiccated/rewetted & Layer 1 & 12.7 & 100 & 0.595 & 0.075 & 102 \\
\hline 2 & Desiccated/rewetted & Layer 1 & 12.7 & 100 & 0.633 & 0.1 & 28 \\
\hline 3 & Desiccated/rewetted & Layer 1 & 12.7 & 100 & 0.607 & 0.125 & 12 \\
\hline 4 & Desiccated/rewetted & Layer 1 & 12.7 & 100 & 0.608 & 0.15 & 7 \\
\hline 5 & Desiccated/rewetted & Layer 1 & 12.7 & 100 & 0.603 & 0.175 & 4 \\
\hline 6 & Desiccated/rewetted & Layer 1 & 12.7 & 100 & 0.607 & 0.2 & 4 \\
\hline 7 & Desiccated/rewetted & Layer 2 & 16.9 & 100 & 0.598 & 0.075 & 86 \\
\hline 8 & Desiccated/rewetted & Layer 2 & 16.9 & 100 & 0.606 & 0.1 & 20 \\
\hline 9 & Desiccated/rewetted & Layer 2 & 16.9 & 100 & 0.638 & 0.125 & 9 \\
\hline 10 & Desiccated/rewetted & Layer 2 & 16.9 & 100 & 0.619 & 0.15 & 6 \\
\hline 11 & Desiccated/rewetted & Layer 2 & 16.9 & 100 & 0.611 & 0.175 & 3 \\
\hline 12 & Desiccated/rewetted & Layer 2 & 16.9 & 100 & 0.609 & 0.2 & 2 \\
\hline 13 & Desiccated/rewetted & Layer 3 & 18.2 & 100 & 0.586 & 0.075 & 82 \\
\hline 14 & Desiccated/rewetted & Layer 3 & 18.2 & 100 & 0.589 & 0.1 & 19 \\
\hline 15 & Desiccated/rewetted & Layer 3 & 18.2 & 100 & 0.593 & 0.125 & 9 \\
\hline 16 & Desiccated/rewetted & Layer 3 & 18.2 & 100 & 0.619 & 0.15 & 6 \\
\hline 17 & Desiccated/rewetted & Layer 3 & 18.2 & 100 & 0.614 & 0.175 & 2 \\
\hline 18 & Desiccated/rewetted & Layer 3 & 18.2 & 100 & 0.604 & 0.2 & 2 \\
\hline 19 & Desiccated/rewetted & Layer 4 & 17.8 & 100 & 0.586 & 0.075 & 77 \\
\hline 20 & Desiccated/rewetted & Layer 4 & 17.8 & 100 & 0.623 & 0.1 & 16 \\
\hline 21 & Desiccated/rewetted & Layer 4 & 17.8 & 100 & 0.606 & 0.125 & 7 \\
\hline 22 & Desiccated/rewetted & Layer 4 & 17.8 & 100 & 0.616 & 0.15 & 4 \\
\hline 23 & Desiccated/rewetted & Layer 4 & 17.8 & 100 & 0.606 & 0.175 & 3 \\
\hline 24 & Desiccated/rewetted & Layer 4 & 17.8 & 100 & 0.614 & 0.2 & 2 \\
\hline
\end{tabular}



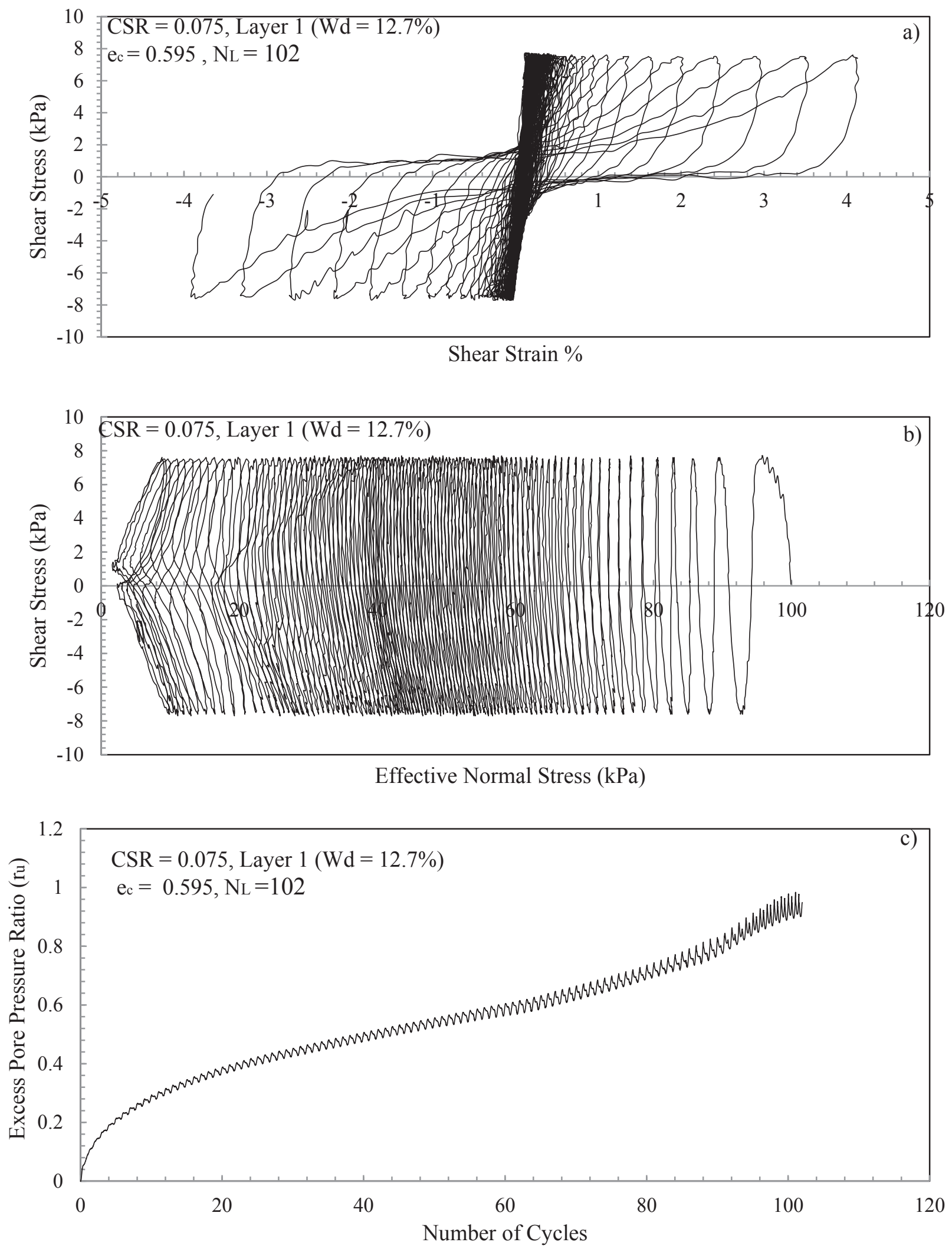

Figure 6.38 Cyclic simple shear response of the sample obtained from Layer 1 at CSR $=0.075$ 

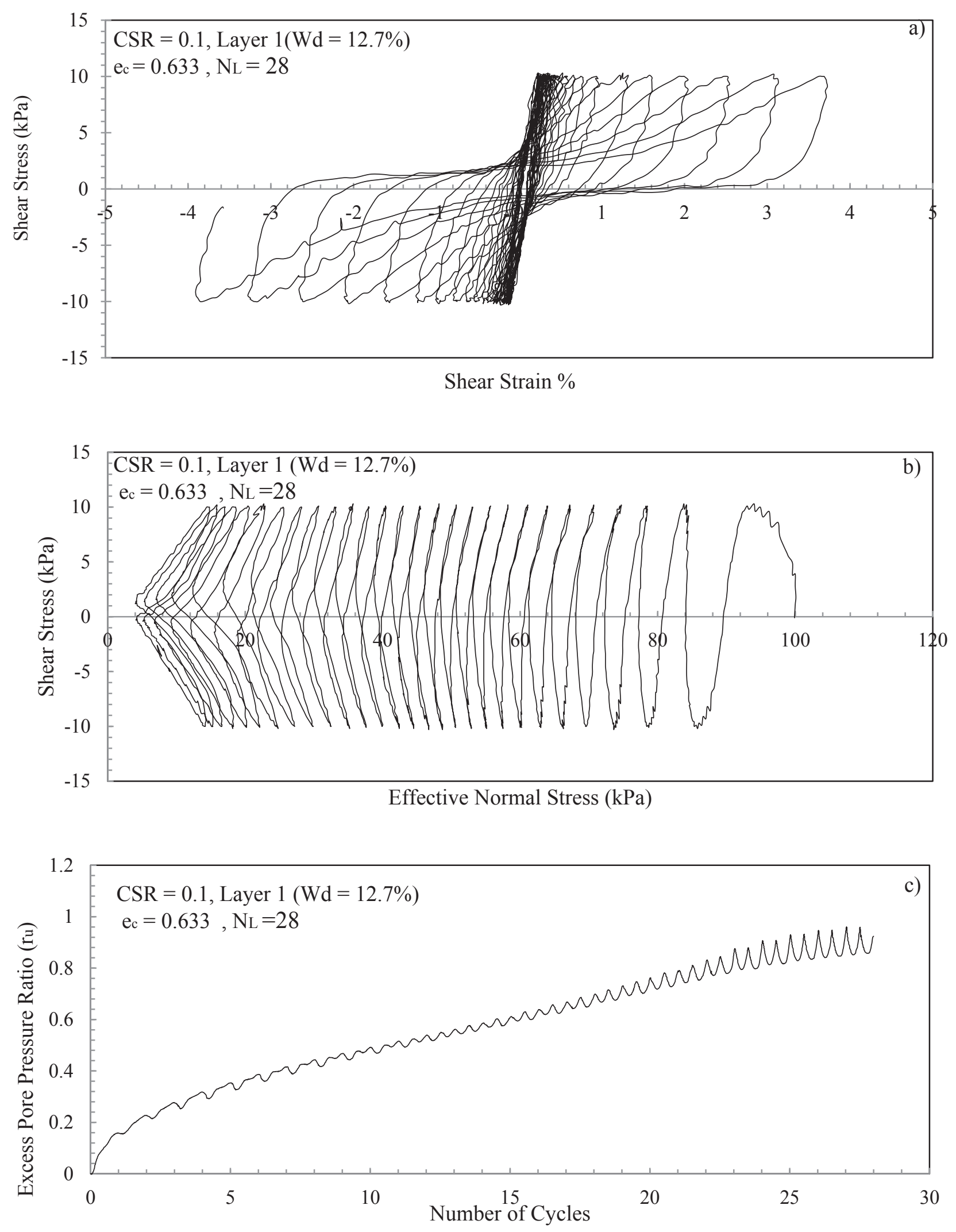

Figure 6.39 Cyclic simple shear response of the sample obtained from Layer 1 at CSR $=0.1$ 

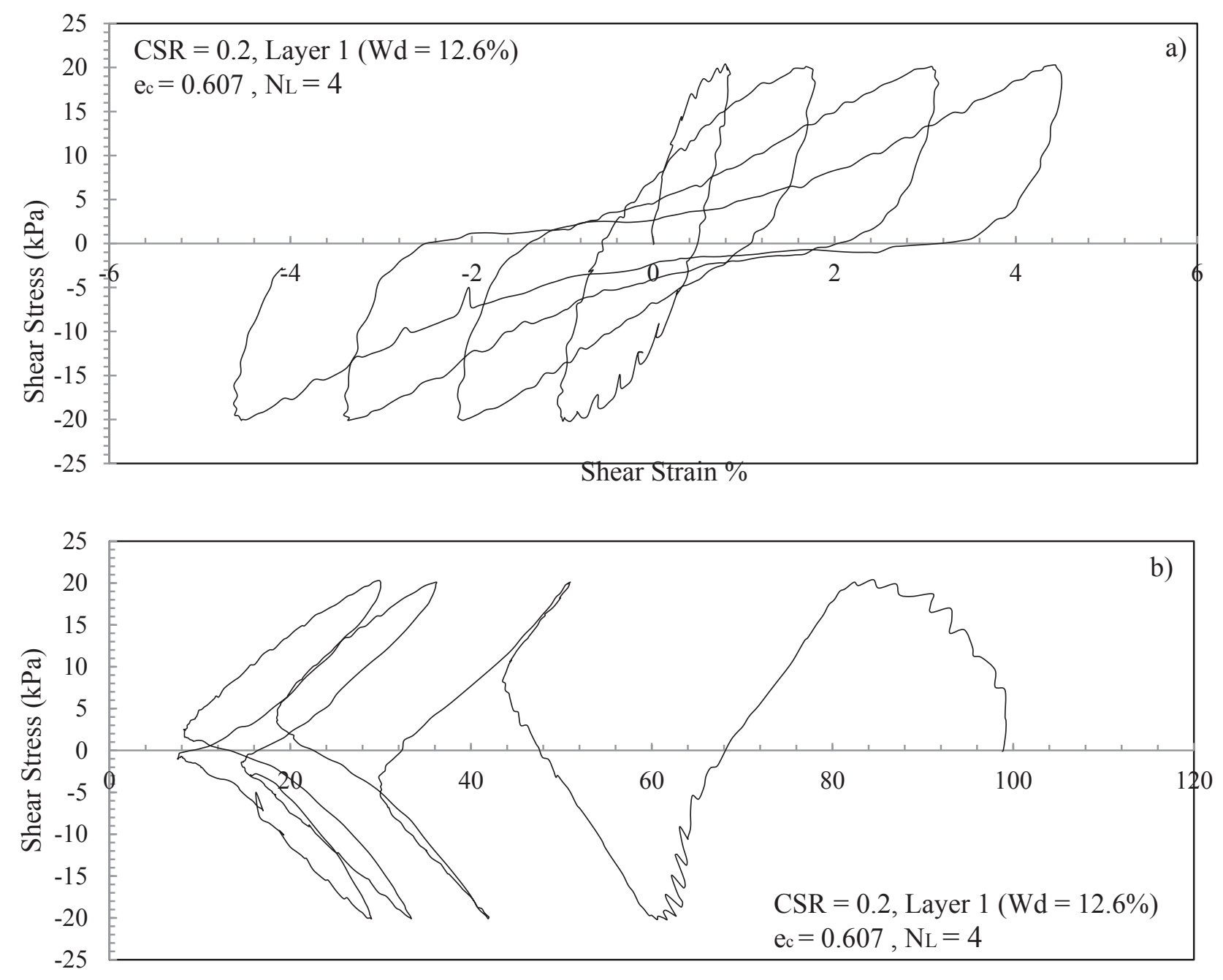

Effective Normal Stress $(\mathrm{kPa})$

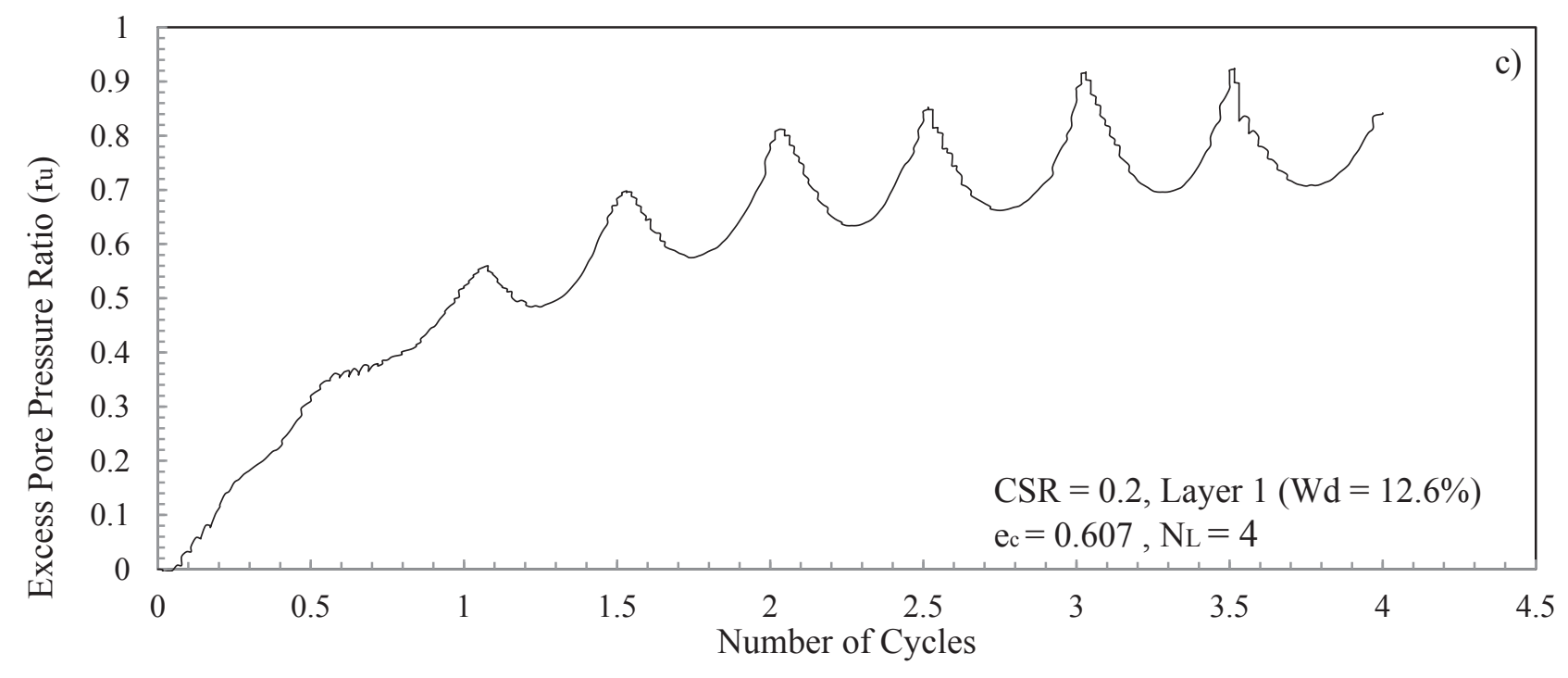

Figure 6.40 Cyclic simple shear response of the sample obtained from Layer 4 at CSR $=0.2$ 
$\mathrm{Wd}=12.6 \%$ desiccation history, under different CSRs. The tailings required 102 cycles to liquefy at a CSR of 0.075 but only 7 cycles at a CSR of 0.15 . As noted earlier, the number of cycles to liquefaction systematically reduced with increasing CSR. The excess pore pressure ratio increased up to $r_{u}=0.98$ in the test with $\operatorname{CSR}=0.075$, and was between 0.90 and 0.95 in the other tests. Similar to the cyclic tests reported earlier on the small scale method, strain development was fairly small until the excess pore pressure ratio reached about $60 \%$ to $70 \%$. Further, the largest excess pore pressure ratio was mobilized in the test with the lowest CSR.
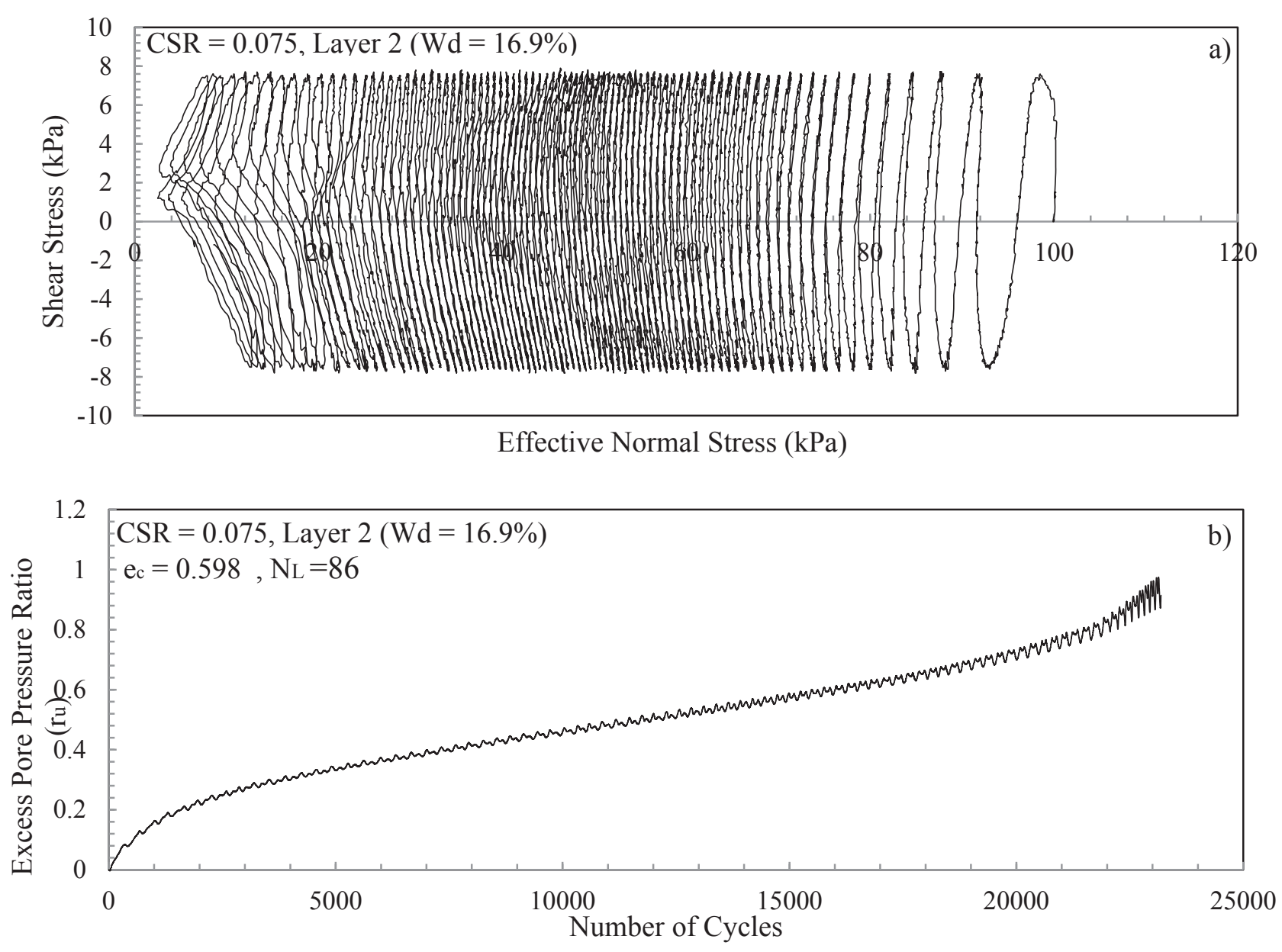

Figure 6.41 Cyclic simple shear response of the sample obtained from Layer 2 at CSR $=0.075$ 

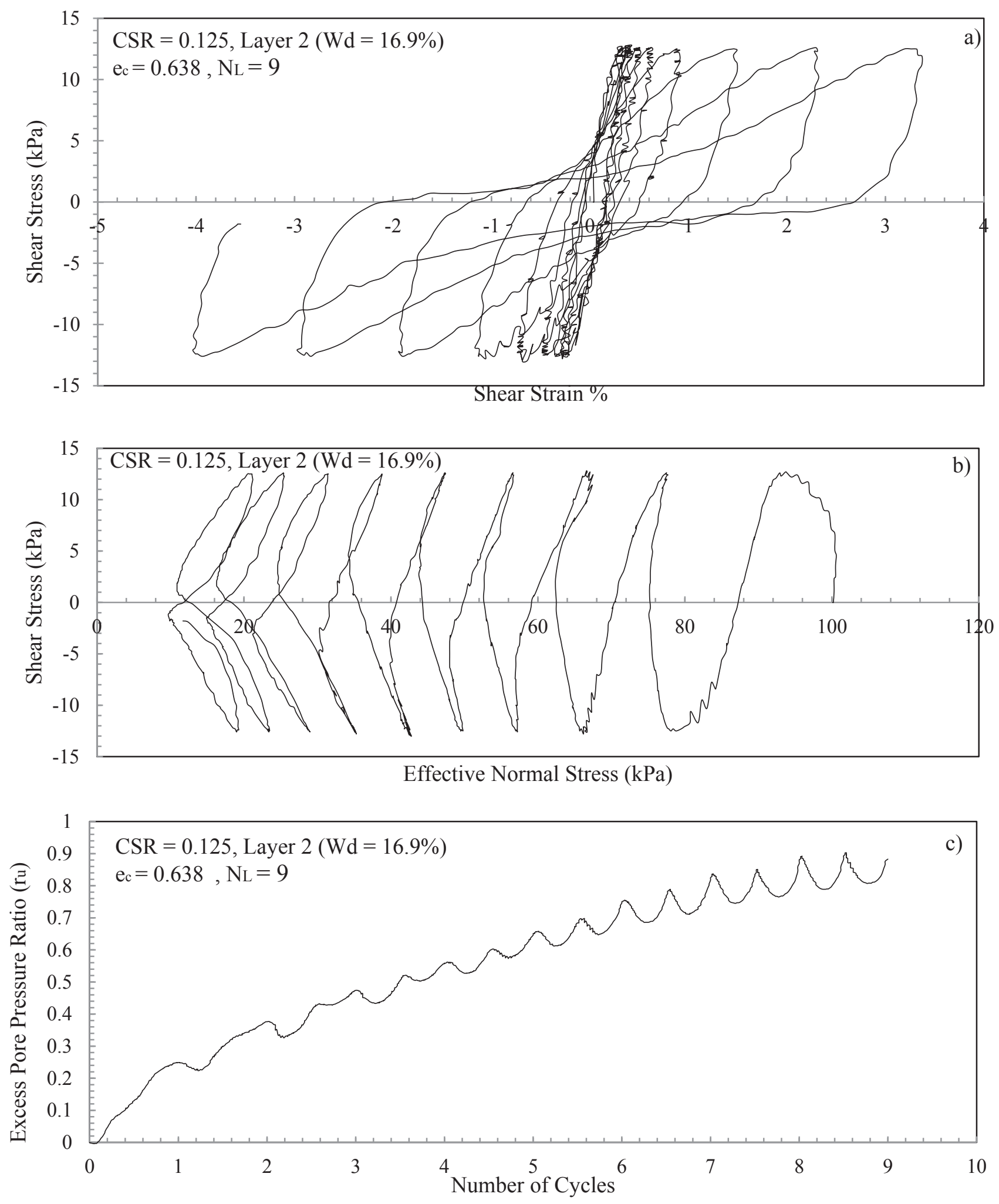

Figure 6.42 Cyclic simple shear response of the sample obtained from Layer 2 at CSR $=0.125$ 

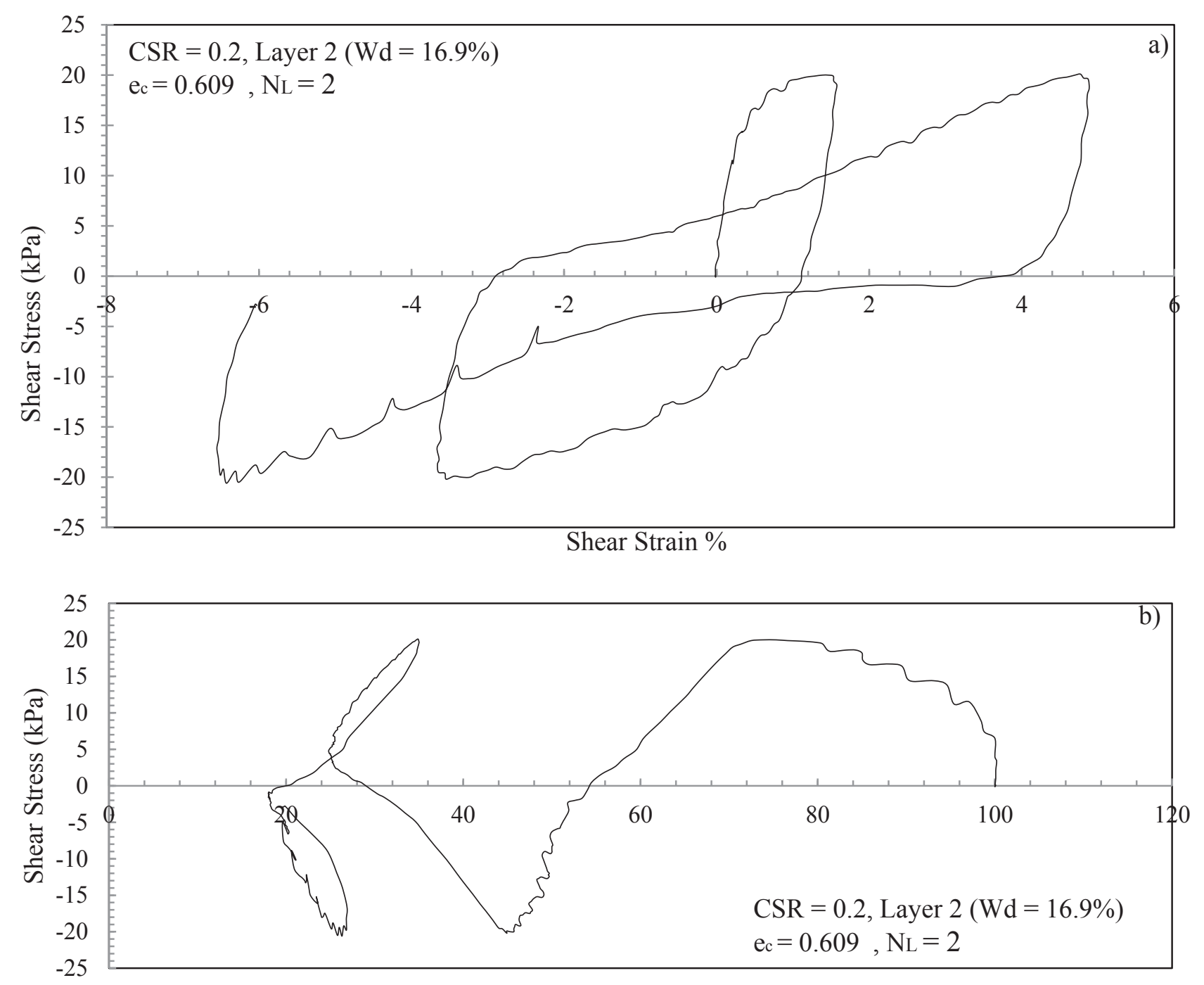

Effective Normal Stress ( $\mathrm{kPa})$

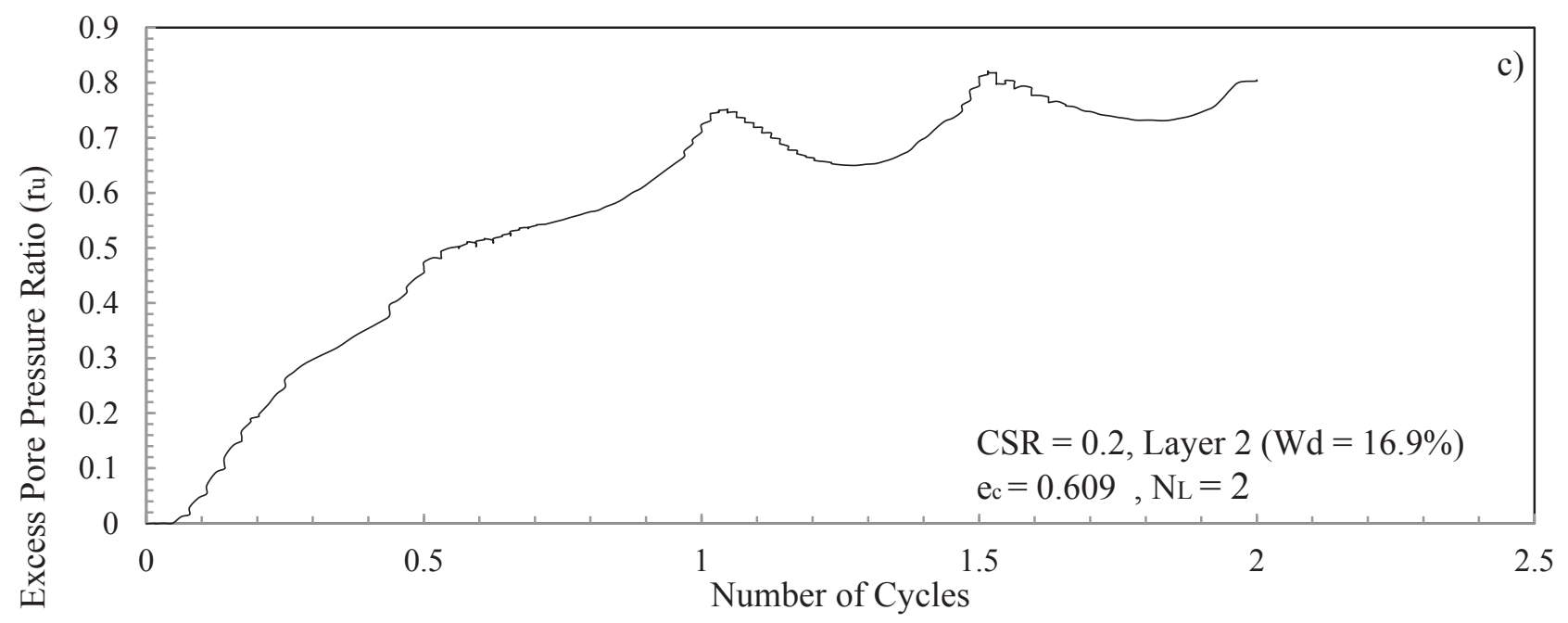

Figure 6.43 Cyclic simple shear response of the sample obtained from Layer 4 at CSR $=0.2$ 
Figures 6.41 to 6.43 present cyclic simple shear response of samples obtained from the second layer of the drying box, which experienced $\mathrm{Wd}=16.9 \%$ desiccation history, at different CSRs. Figure 6.53 shows that it requires 86 numbers of cycles to reach liquefaction at CSR $=0.075$. Comparing with the first layer at the same CSR, the second layer required lower numbers of cycles to reach liquefaction, which shows lower cyclic resistance of the second layer.

Figure 6.44 and 6.45 present cyclic simple shear responses of samples extracted from the third layer, which experienced desiccation history up to $\mathrm{Wd}=18.2 \%$ at different CSRs. Figure 6.44 shows that 82 cycles were required to trigger liquefaction at $\mathrm{CSR}=0.075$. Figure $6.44 \mathrm{~b}$ also shows that the effective normal stress started from $100 \mathrm{kPa}$ at the beginning of the test and became around $2 \mathrm{kPa}$ in the liquefaction stage, which shows that the effective stress is very close to zero effective stress condition. In other words, the NRC liquefaction criterion $(\gamma=3.75 \%)$ resulted in fairly zero effective stress condition in this test. Increasing cyclic stress ratio to CSR $=0.1$ significantly reduced the numbers of cycles to reach liquefaction to $N_{L}=19$. Figure $6.45 \mathrm{c}$ shows that the excess pore pressure ratio in the liquefaction stage becomes around 0.9.

Figure 6.46 to 6.48 present cyclic simple shear responses of samples extracted from the fourth layer at different CSRs. Figure 6.46 presents cyclic simple shear response of a sample extracted from the fourth layer under $\operatorname{CSR}=0.15$. It is shown that it requires just 4 numbers of cycles to reach liquefaction under CSR $=0.15$. The numbers of cycles to reach liquefaction reduced to $\mathrm{N}_{\mathrm{L}}$ $=3$ by applying CSR $=0.175$ in Figure 6.47 . Figure $6.47 \mathrm{~b}$ shows that the sample in the first cycle exhibited contractive behavior. The dilative behavior appeared in the second cycle and alteration in contractive and dilative response continued until the end of the test. Figure 6.48 presents cyclic simple shear response of the sample at CSR $=0.2$. 

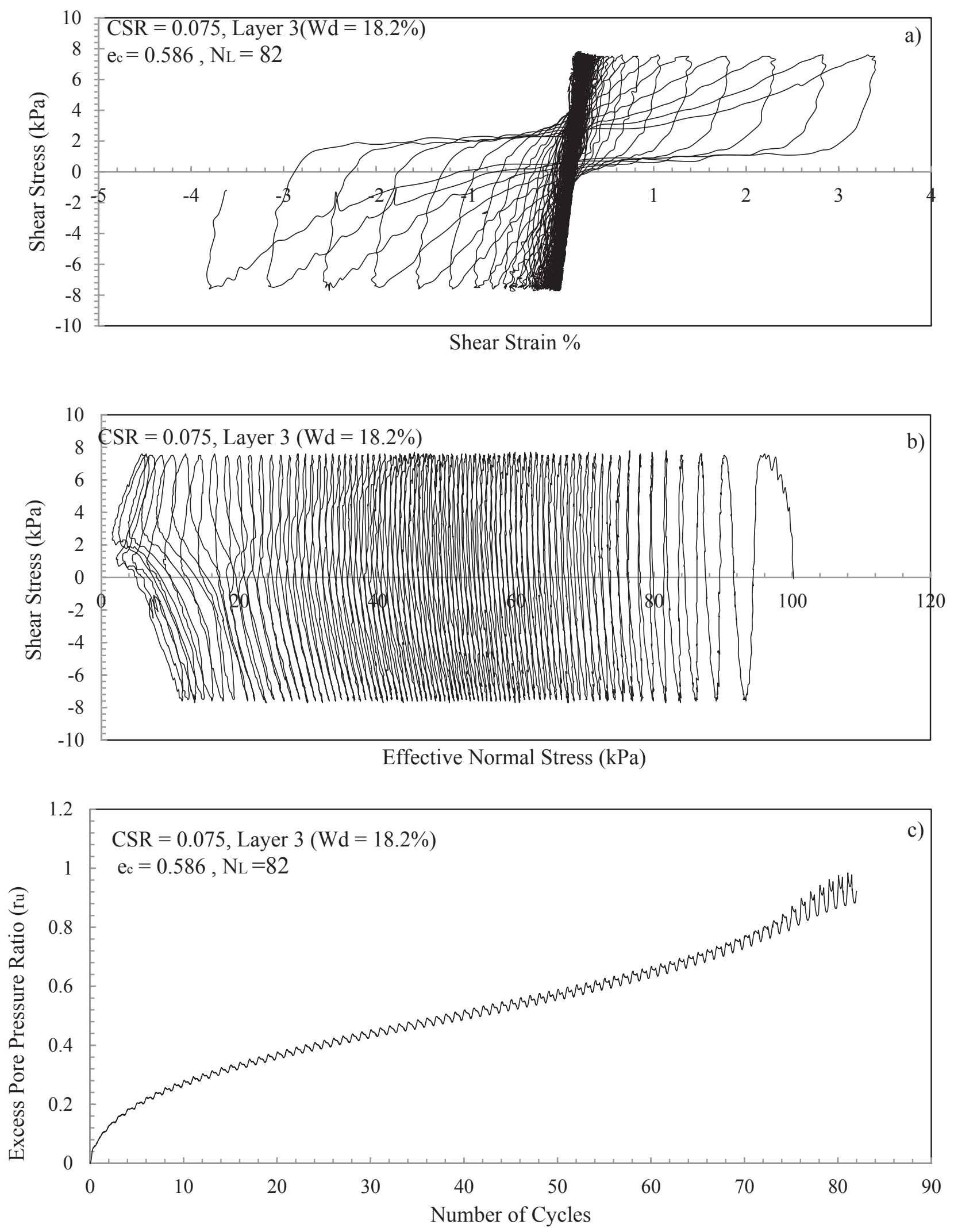

Figure 6.44 Cyclic simple shear response of the sample obtained from Layer 3 at CSR $=0.075$ 

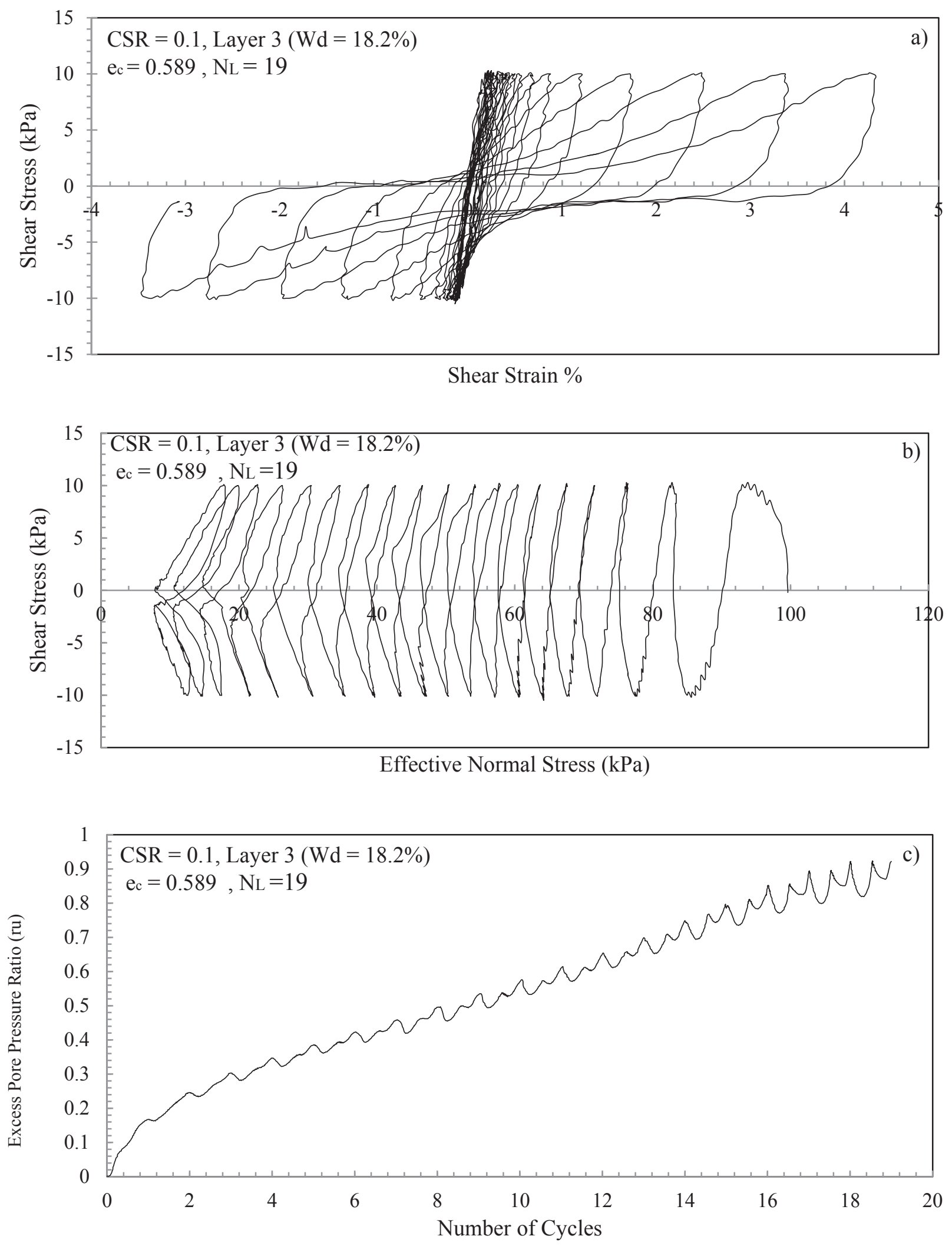

Figure 6.45 Cyclic simple shear response of the sample obtained from Layer 3 at CSR $=0.1$ 

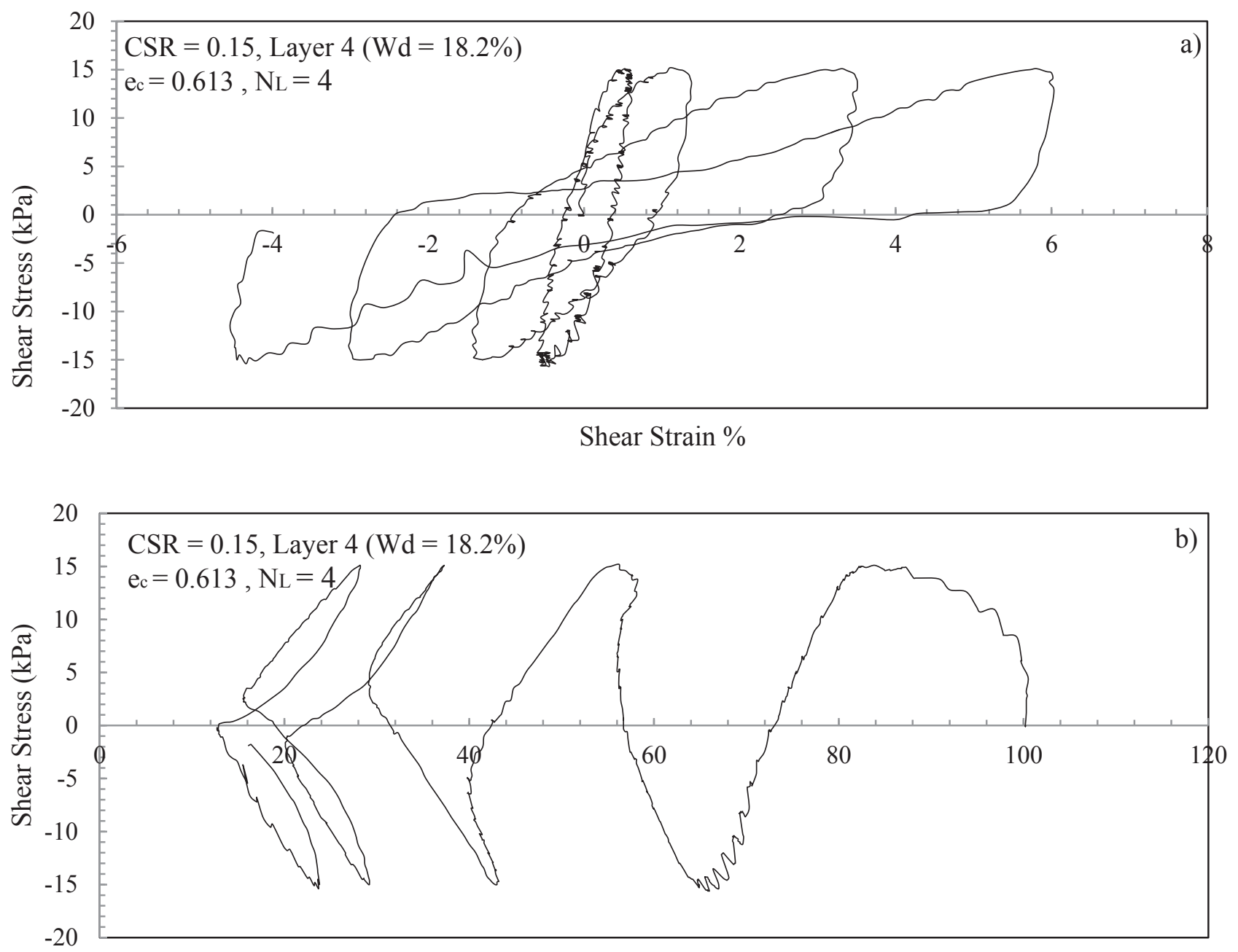

Effective Normal Stress $(\mathrm{kPa})$

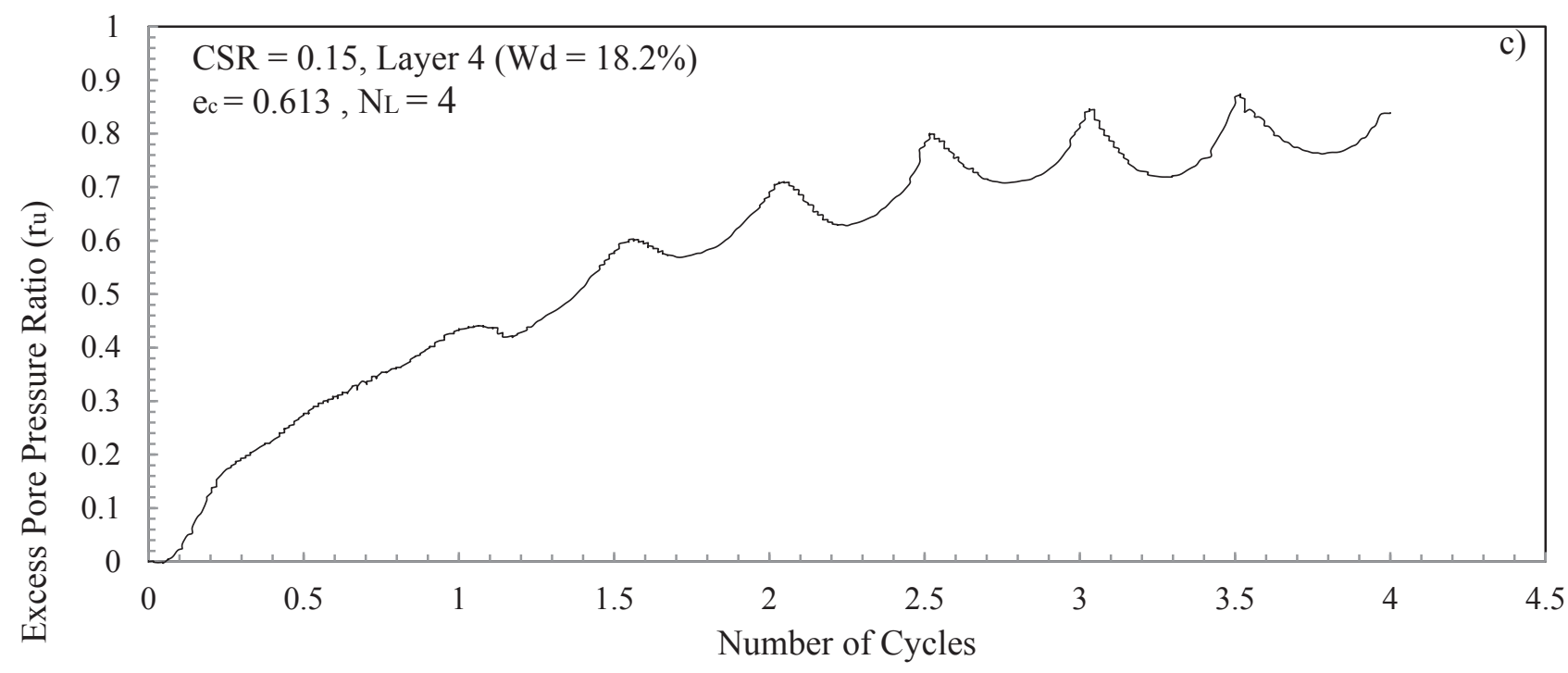

Figure 6.46 Cyclic simple shear response of the sample obtained from Layer 4 at CSR $=0.15$ 

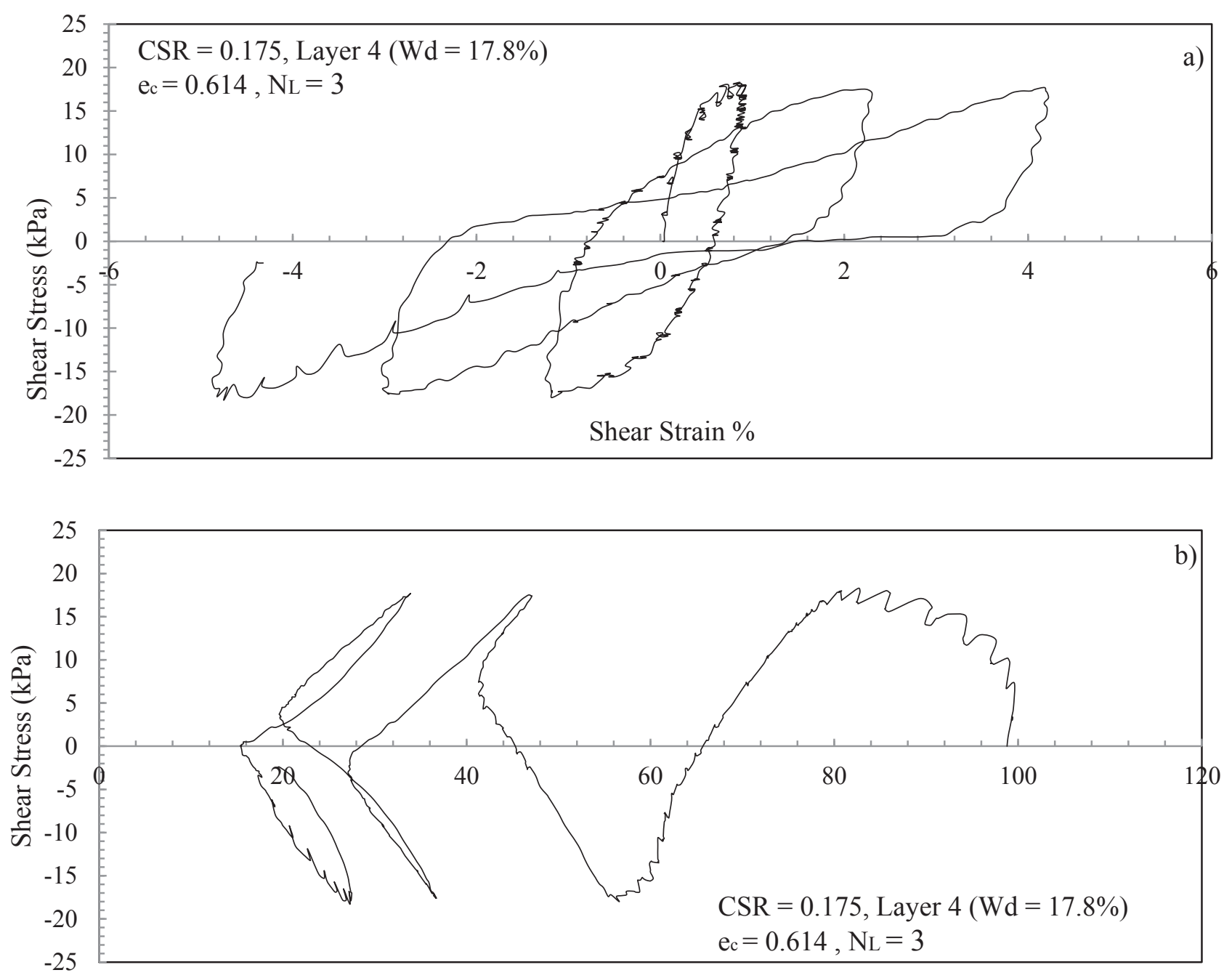

Effective Normal Stress $(\mathrm{kPa})$

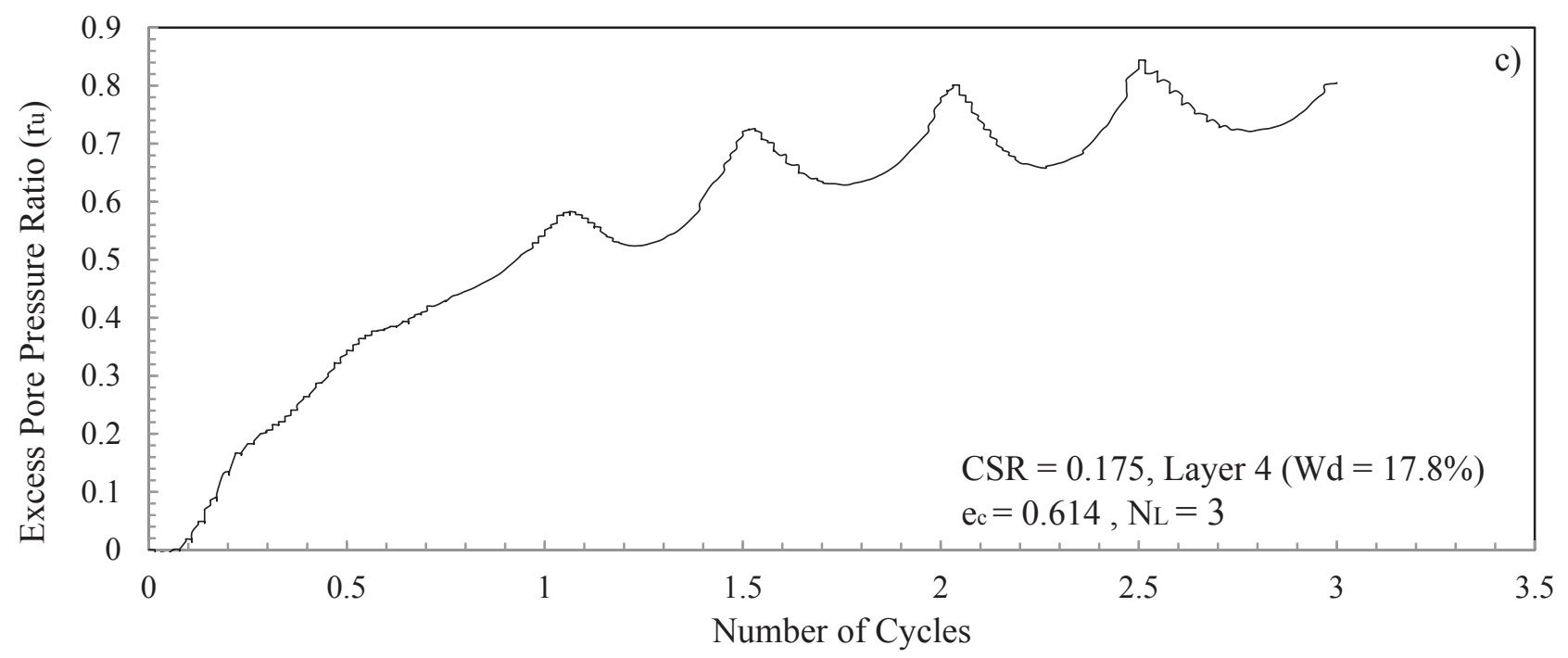

Figure 6.47 Cyclic simple shear response of the sample obtained from Layer 4 at CSR $=0.175$ 

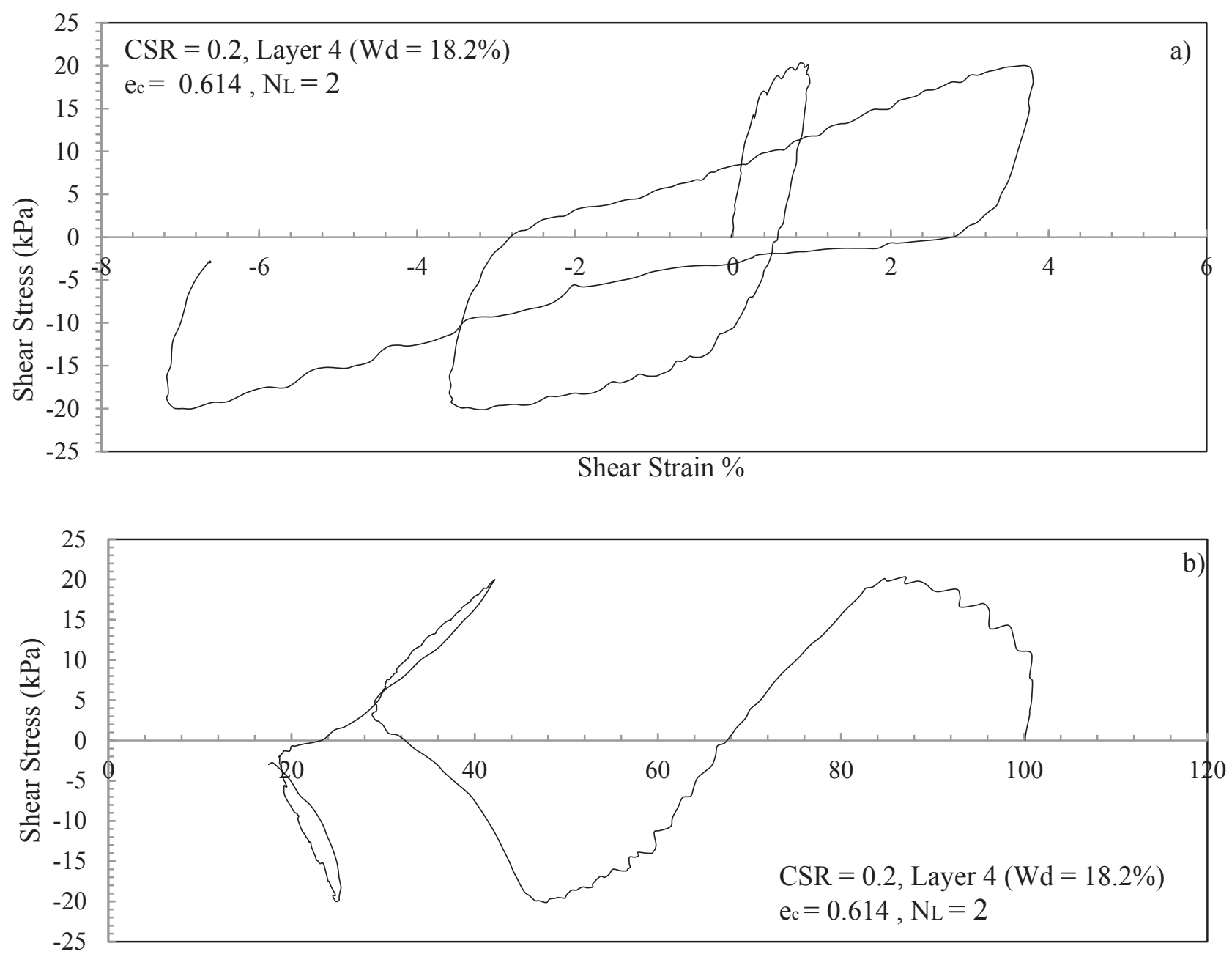

Effective Normal Stress ( $\mathrm{kPa})$

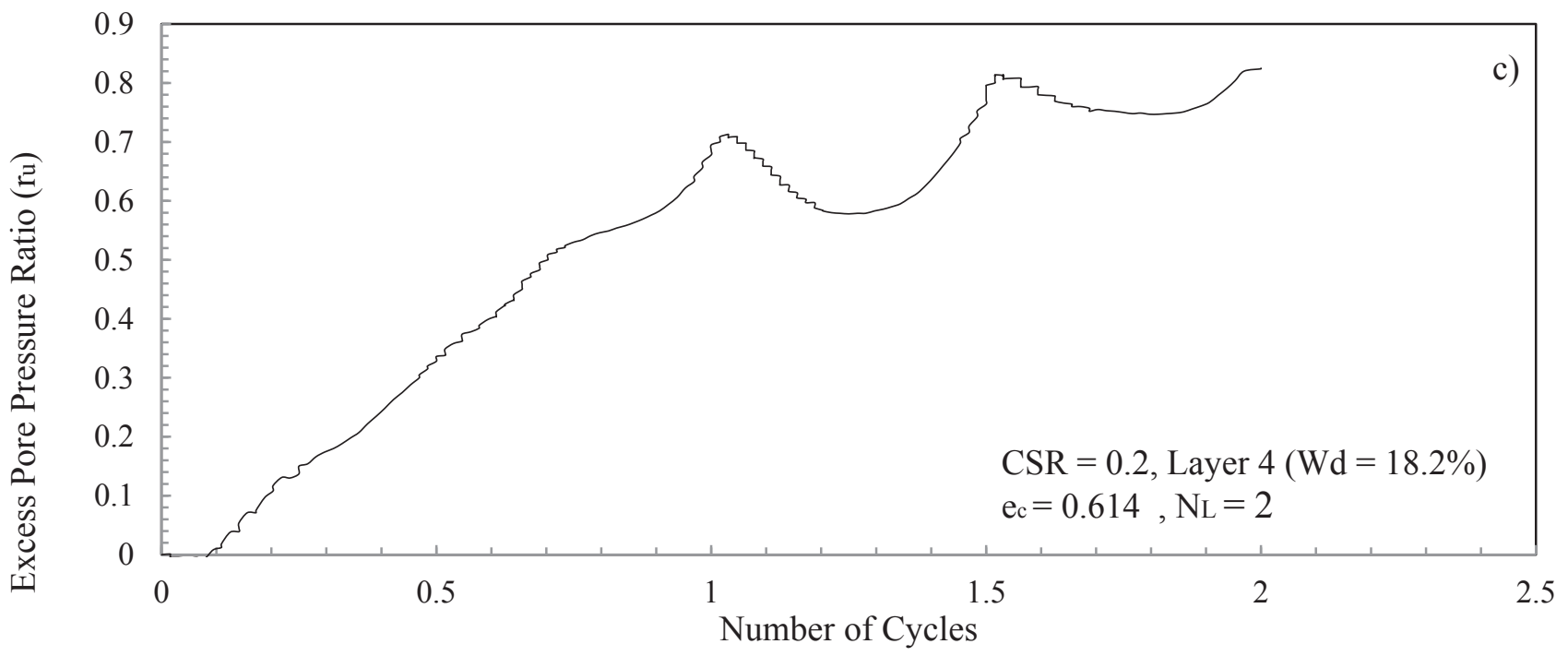

Figure 6.48 Cyclic simple shear response of the sample obtained from Layer 4 at CSR $=0.2$ 


\subsubsection{Discussion on cyclic results of samples obtained from the drying box}

Cyclic simple shear tests were performed on the samples extracted from different layers of the drying box. Each layer experienced a specific desiccation history. Different cyclic stress ratios (CSRs) ranging from 0.075 to 0.2 were applied to the samples. Figures 6.49 and 6.50 compare excess pore pressure generation of samples obtained from different layers and subjected to CSR values of $0.075,0.1,0.125$ and 0.2 . The rate of excess pore pressure generation was the slowest in the first layer, and fastest in the fourth layer at both CSR values, even though the final value of the excess pore pressure ratio was essentially equal among the four tests $(0.95)$. The number of cycles to liquefaction at $\mathrm{CSR}=0.075$ was $102,86,82$ and 77 in layers 1 to 4 respectively. The higher cyclic resistance of the sample obtained from the first layer is attributed to the higher degree of desiccation $(\mathrm{Wd}=12.7 \%)$. In addition to the higher desiccation, this sample went through multiple drying/wetting cycles as well. As noted earlier, the actual impact of the number of drying/wetting cycles is not known at this time. Similar observations can be made from the data presented in Figure $6.49 \mathrm{~b}$ corresponding to $\mathrm{CSR}=0.10$. The maximum excess pore water pressure in the tests with CSR $=0.10$ is about 0.90 as opposed to the 0.95 noted in the tests with $\mathrm{CSR}=0.075$. Tests at other CSR values show similar behavior (Figure 6.50) and a marginally reduction of maximum excess pore pressure ratio as the CSR value increases.

Figure 6.51 presents cyclic stress ratio versus numbers of cycles to reach liquefaction for all tests performed on the drying box layers. It is clear that the first layer, which experienced higher degree of desiccation history $(\mathrm{Wd}=12.7 \%)$ and more desiccation-rewetting cycles, exhibited higher CSRs at a given $\mathrm{N}_{\mathrm{L}}$. Except some tests, layers two, three and four exhibited fairly same cyclic resistance. 

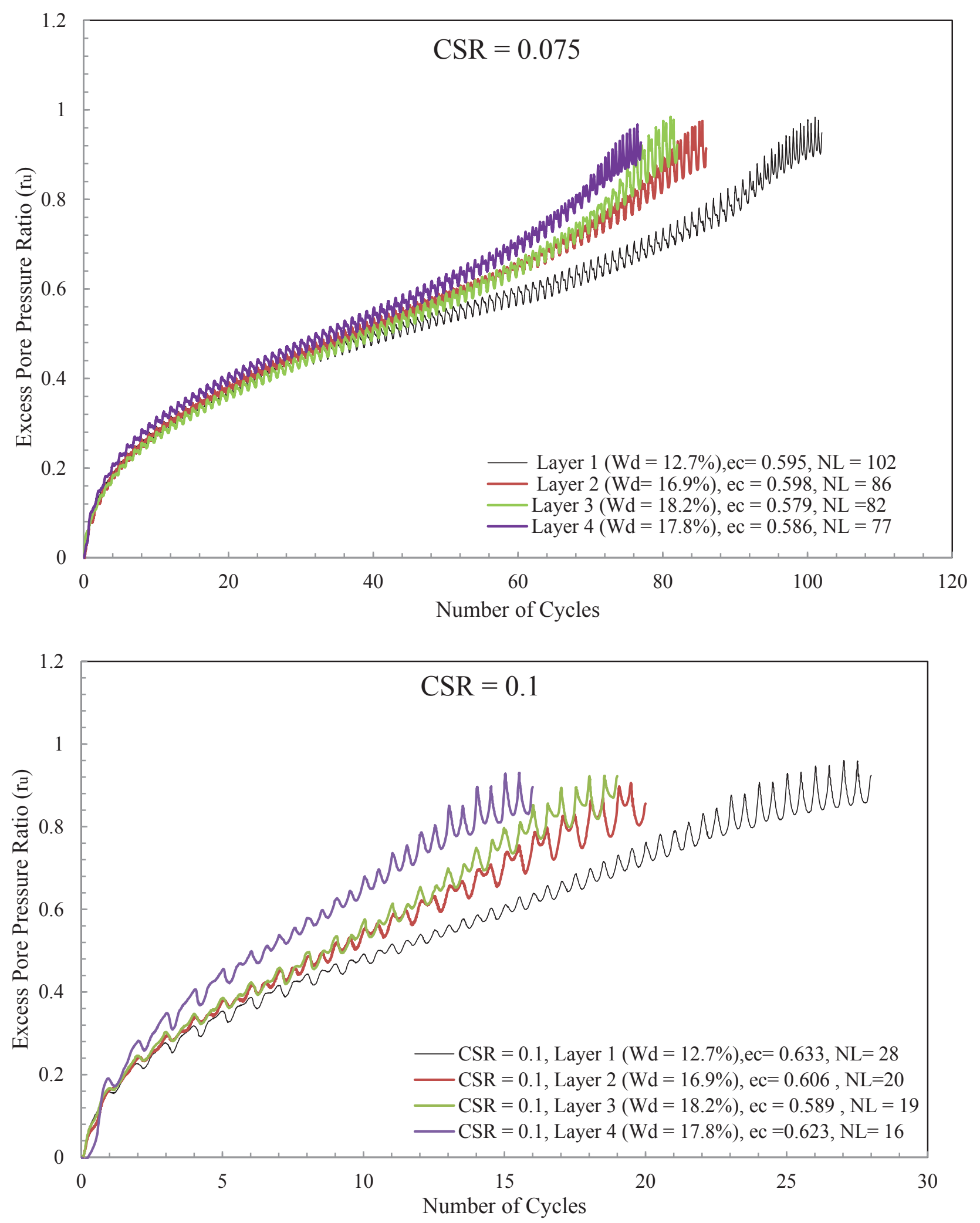

Figure 6.49 Comparison of excess pore pressure generation of samples from different layers at a) $\mathrm{CSR}=$ $0.075, \mathrm{~b}) \mathrm{CSR}=0.1$ 

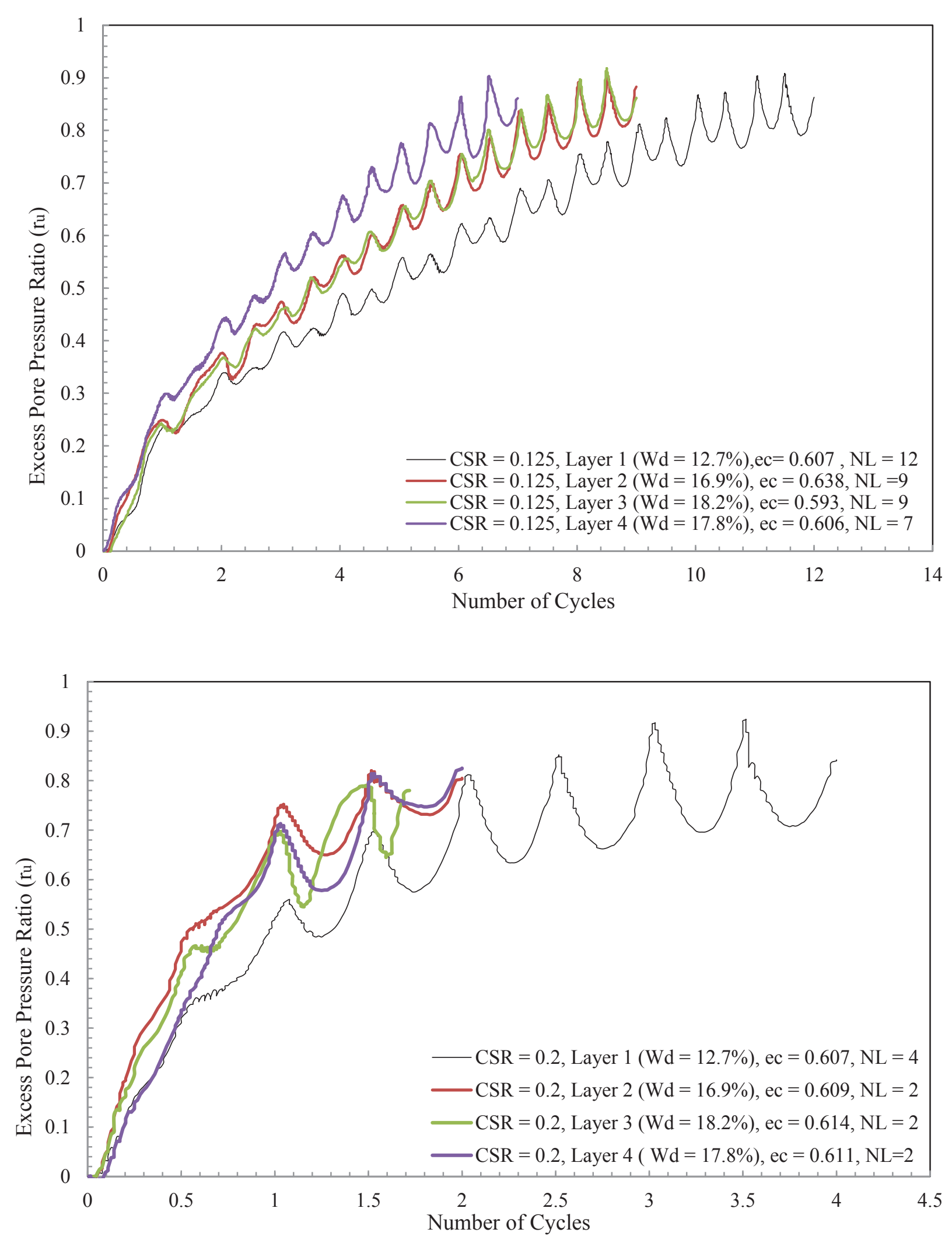

Figure 6.50 Comparison of excess pore pressure generation of samples from different layers at a) $\mathrm{CSR}=$ $0.125, \mathrm{~b}) \mathrm{CSR}=0.2$ 
Figure 6.52 presents the effect of desiccation history on CRR10 and CRR15 of desiccatedrewetted samples. It can be seen that with increasing desiccation history from $\mathrm{W}_{\mathrm{d}}=18 \%$ to $\mathrm{W}_{\mathrm{d}}=$ $12 \%$, the cyclic resistance slightly increases. However, this increase is not as significant as changing desiccation history from settling to the shrinkage limit.

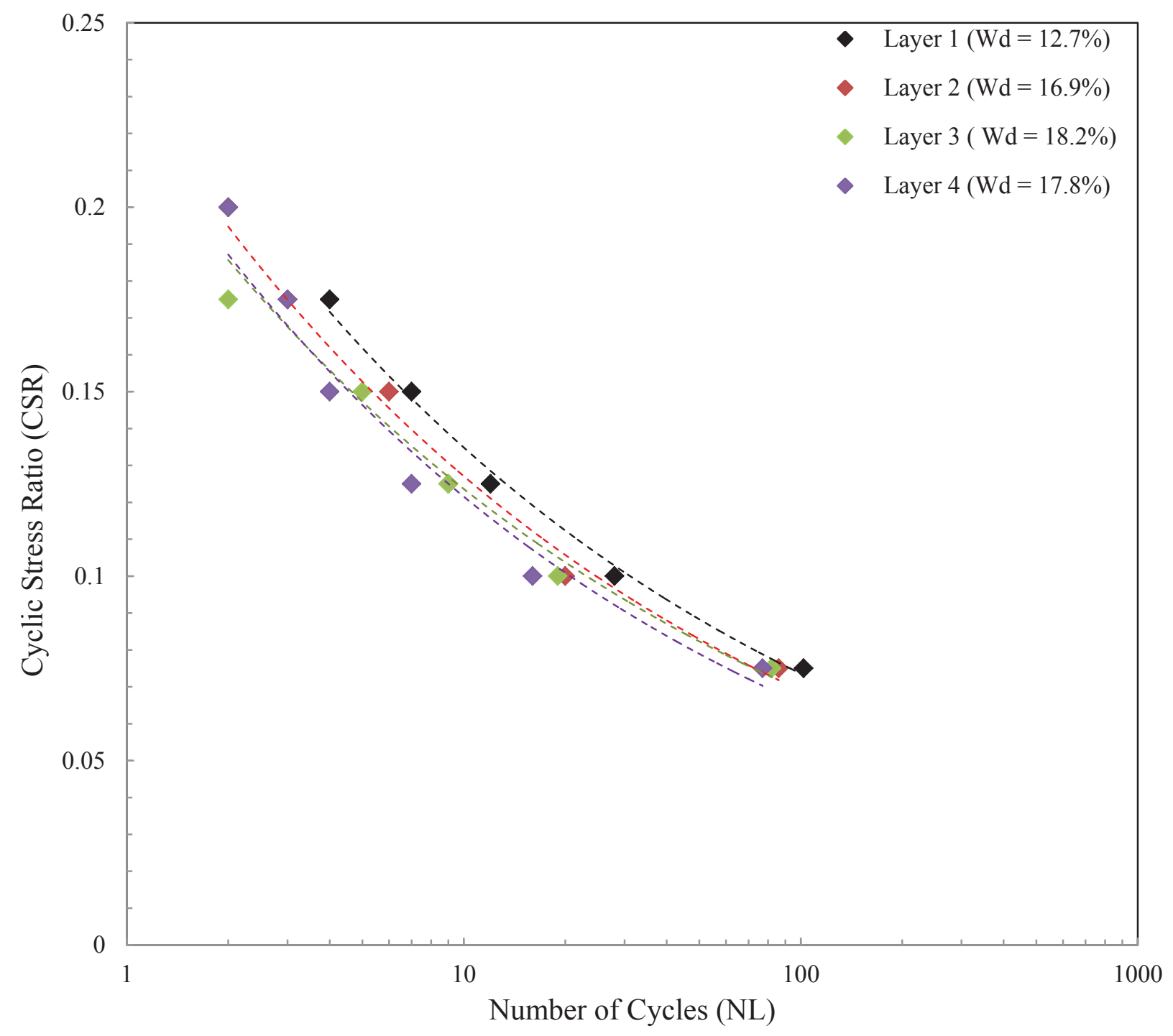

Figure 6.51 Cyclic stress ratio (CSR) versus numbers of cycles to reach liquefaction $\left(\mathrm{N}_{\mathrm{L}}\right)$ for all cyclic simple shear tests performed on samples obtained from different layers of the drying box 


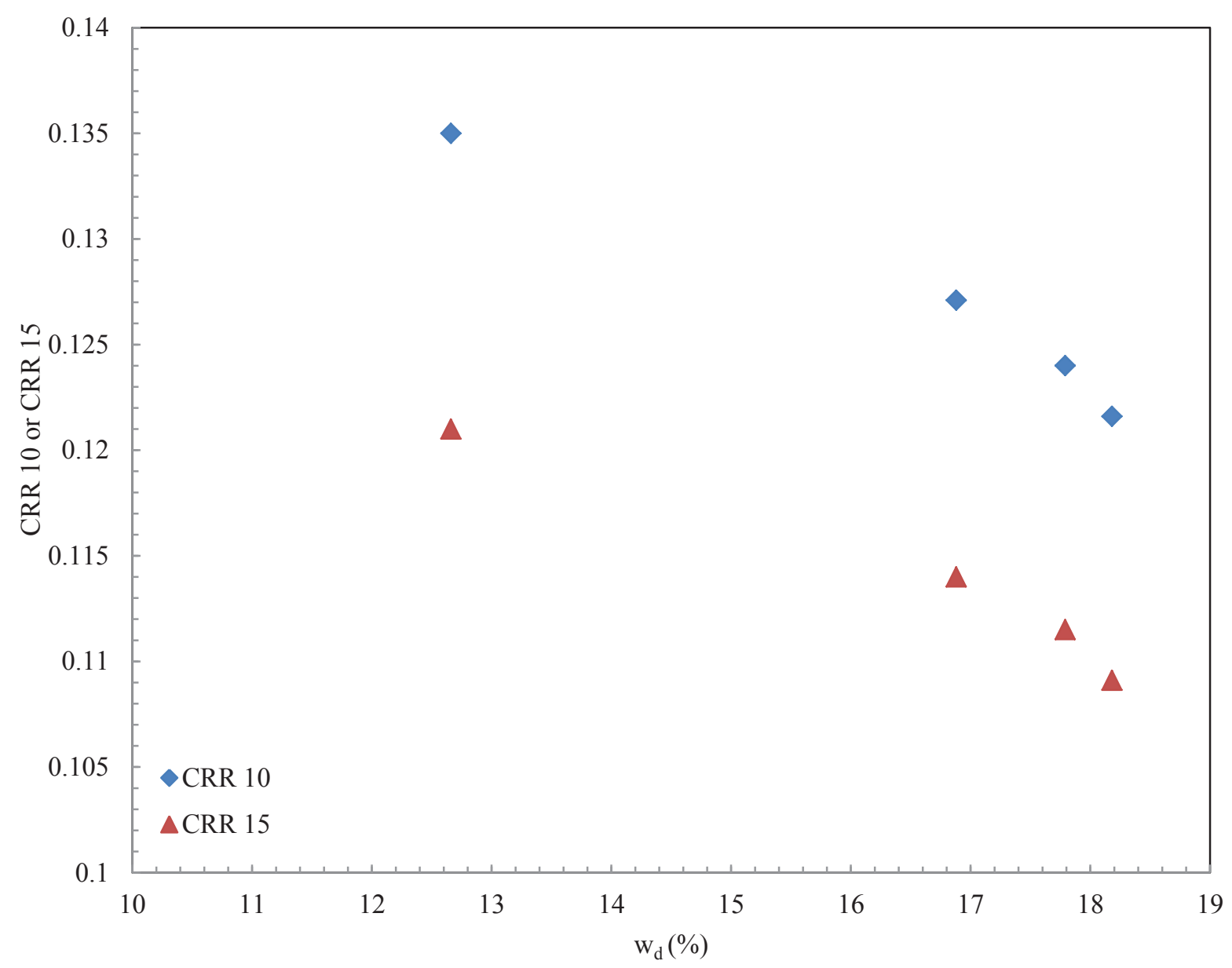

Figure 6.52 The effect of desiccation history on cyclic resistance (CRR10, CRR 15) of samples obtained from different layers of the drying box

\subsection{Results of triaxial test}

In this section, monotonic and cyclic results of modified triaxial tests performed on samples prepared by small scale method are presented. Firstly, results of one conventional triaxial test is presented. Monotonic and cyclic results of modified triaxial tests performed on settled and desiccated-rewetted tailings are presented in the subsequent sections. Finally, results of some triaxial tests performed on desiccated tailings are also presented. 


\subsubsection{Results of conventional monotonic triaxial tests}

A conventional triaxial test was performed on a settled tailings specimen prepared in slurry condition. The sample was consolidated under $50 \mathrm{kPa}$ consolidation pressure $\left(\sigma_{3}\right)$. The saturation process under appropriate back pressure showed that the $\mathrm{B}$ value is greater than 0.95 , which is deemed reasonable for an undrained test at $50 \mathrm{kPa}$ consolidation pressure in this material. Figure 6.53 presents the result of this conventional triaxial test. It is clear that unlike simple shear test, the settled tailings do not exhibit contractive response under triaxial compression test. Figure $6.53 \mathrm{c}$ shows a reduction in excess pore pressure ratio after $\varepsilon_{a} \sim 3 \%$, which represents the strain hardening response. The phase transformation strength under triaxial compression loading was about $18 \mathrm{kPa}$ whereas the peak shear strength in the simple shear test (for the same consolidation stress level of $50 \mathrm{kPa}$ ) was only about $7 \mathrm{kPa}$. The residual strength in the simple shear test was about $5 \mathrm{kPa}$ but the sample did not show a tendency to reach a residual state in the triaxal test as the dilative behavior continued until the end of the test with increasing shear stress.

Figure 6.54 shows a comparison of the results of conventional triaxial and simple shear test on settled tailings (no desiccation). It is clear that the void ratio of the simple shear sample reached a lower value due to 1-D consolidation compared to 3-D consolidation in the triaxial test. It is also shown that the settled tailings exhibited contractive response in the simple shear test while the triaxial test showed a light strain-hardening behavior. The reason could be attributed to the loading mode, applying suction duirng sample preparation of triaxial tests and the normal stronger response of triaxial than simple shear. 

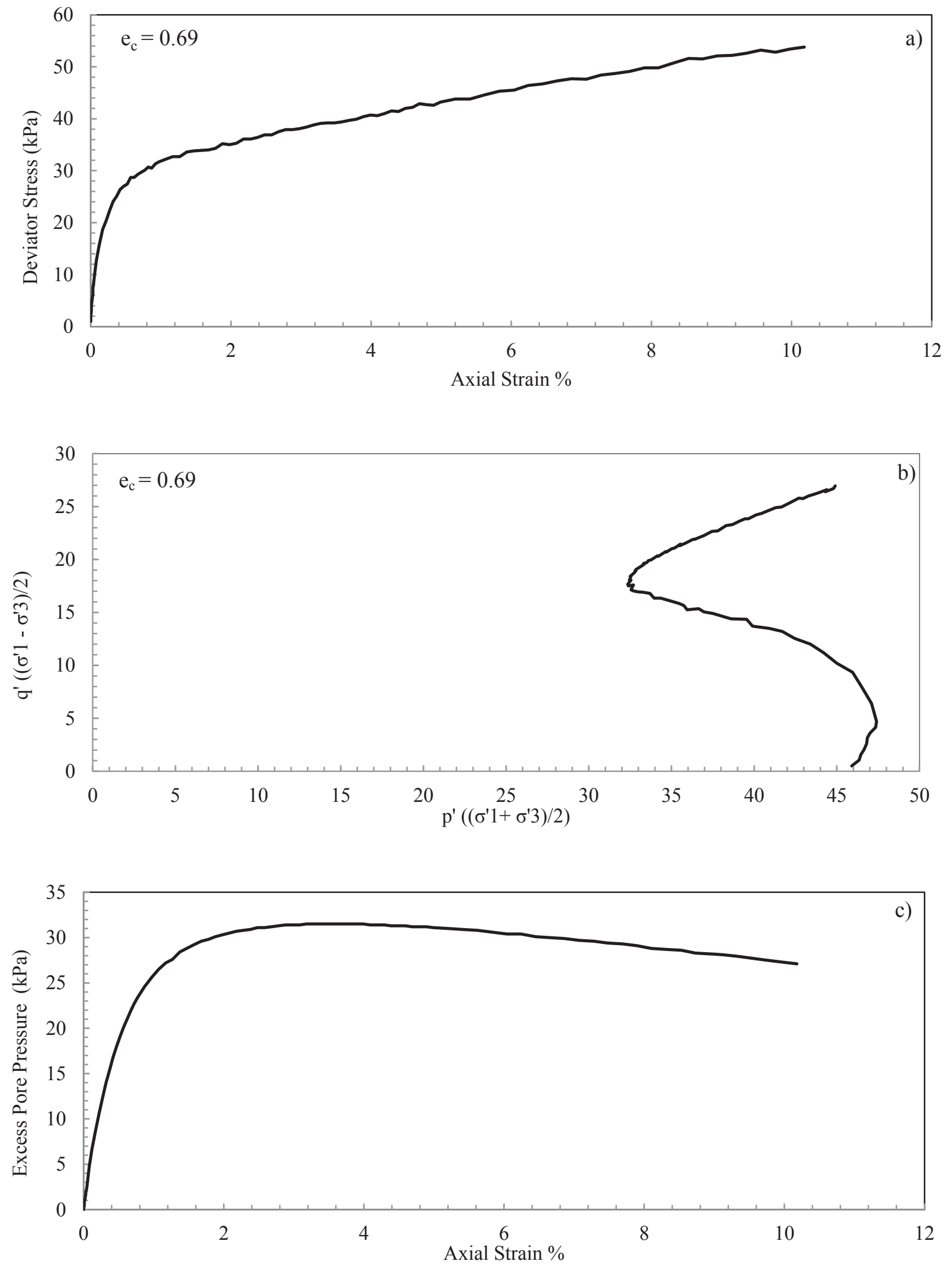

Figure 6.53 Results of conventional triaxial test on saturated tailings 

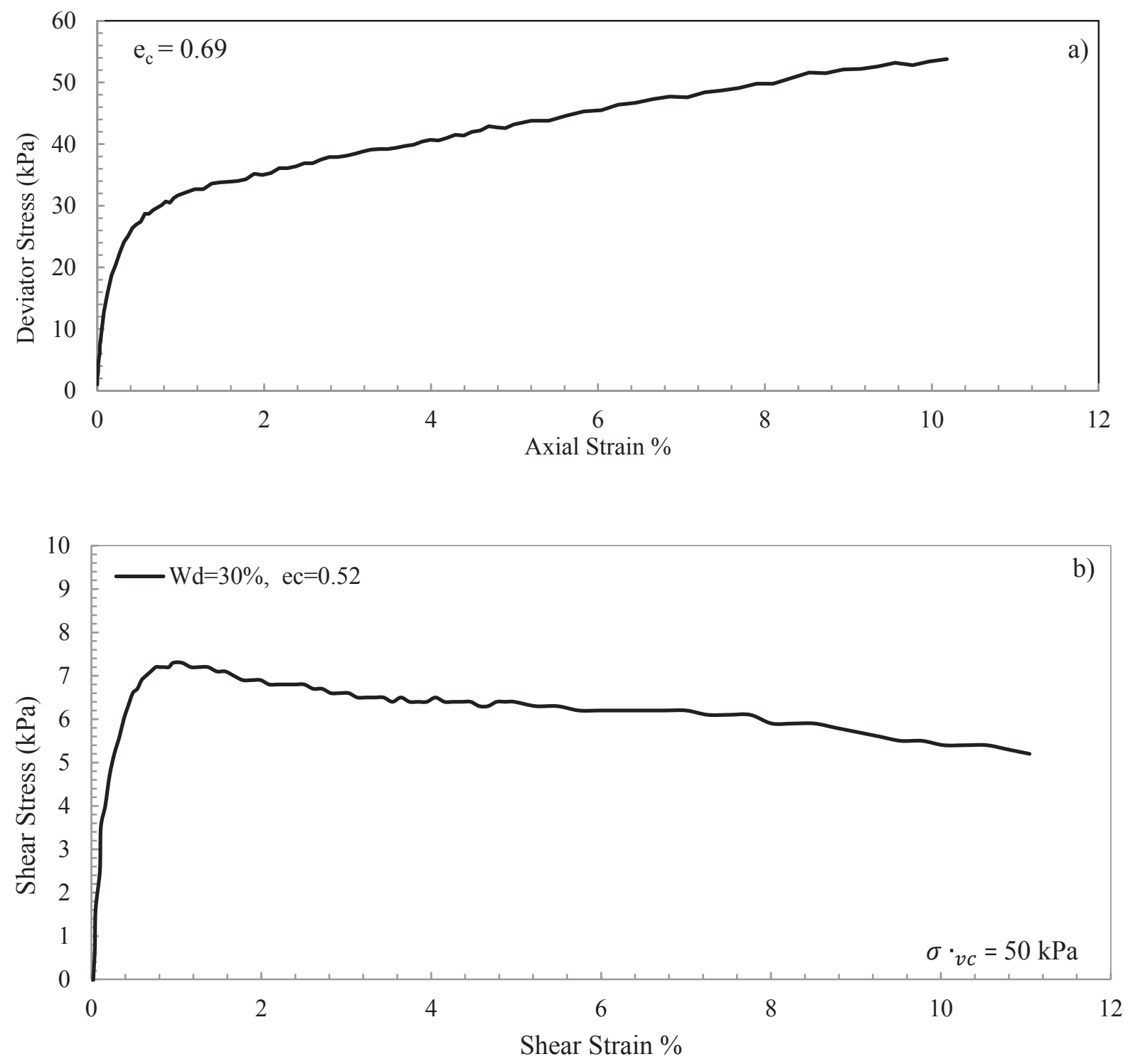

Figure 6.54 Comparison of result of conventional triaxial test and simple shear test on saturated settled tailings

Conventional triaxial tests were not performed on desiccated-rewetted samples due to inability of reaching appropriate B value. Even after applying one line of back pressure, the B value was not reasonable. Consequently, modified triaxial tests were conducted on desiccated-rewetted specimens. 


\subsubsection{Monotonic results of modified triaxial tests}

Modified triaxial tests were performed on settled and desiccated-rewetted samples. Some samples were prepared using small scale method in a bucket and some samples were prepared in the mould under controlled desiccation and rewetting. For samples prepared with the small scale method, desiccation and rewetting were done in a bucket followed by sample extraction. The desiccation and rewetting process of samples prepared in the mould were conducted by controlling water content and matric suction (by the tensiometer). Table 6.6 presents charactristics of modified traxial tests performed on settled and desiccated-rewetted tailings. The actual volume change during consolidation is not exactly known in these unsaturated tests, and the void ratios were mostly estimated assuming that water removal is the same as volume change. Estimating lateral displacement by pictures and caliper were also used for lower degrees of saturation. All tests were conducted on specimens consolidated under $50 \mathrm{kPa}$ cell pressure. Some samples experienced a small degree of desiccation and some samples were desiccated to the shrinkage limit and beyond. Figure 6.55 presents the varitation of matric suction for tests 1,2 and 3 during desiccation and rewetting process. Figure 6.56 presents consolidation results of modified triaxial tests. It is clear that unlike simple shear sepcimens, the void ratio after consolidation of settled samples does not reach low void ratios. This issue is attributed to $3 \mathrm{D}$ consolidation in triaxial tests versus $1 \mathrm{D}$ consolidation in the simple shear device. It is also clear that most of the process of consolidation has occurred in the first hour. Figure 6.57 shows the lateral strain development in a modified triaxial test. It should be noted that the void ratio measurement is highly dependent on accurate recording of water removal (volume change) or 
Table 6.6 Modified triaxial tests performed on settled and desiccated-rewetted samples

\begin{tabular}{|c|c|c|c|c|c|}
\hline $\begin{array}{l}\text { Test } \\
\text { No. }\end{array}$ & $\begin{array}{c}\text { Matric } \\
\begin{array}{c}\text { Suction } \\
\text { before } \\
\text { rewetting } \\
(\mathrm{kPa})\end{array}\end{array}$ & $\begin{array}{c}\text { Water content } \\
\text { before rewetting } \\
\mathrm{w}_{\mathrm{d}}(\%)\end{array}$ & $\begin{array}{c}\text { Method of } \\
\text { Sampling }\end{array}$ & $\begin{array}{c}\text { Consolidation } \\
\text { Pressure }\left(\sigma_{3}\right) \\
\mathrm{kPa}\end{array}$ & $\begin{array}{c}\text { Void ratio after } \\
\text { consolidation } \\
\left(\mathrm{e}_{\mathrm{c}} \pm 0.02-\right. \\
0.03)\end{array}$ \\
\hline 1 & 27 & 26.5 & Mould Preparation & 50 & 0.65 \\
2 & 59 & 23 & Mould Preparation & 50 & 0.62 \\
3 & 20 & 27 & Mould Preparation & 50 & 0.64 \\
5 & $\mathrm{~N} / \mathrm{A}^{1}$ & 24 & Small Scale & 50 & 0.56 \\
6 & $\mathrm{~N} / \mathrm{A}$ & 25 & Small Scale & 50 & 0.55 \\
7 & $\mathrm{~N} / \mathrm{A}^{2}$ & 17 & Mould Preparation & 50 & 0.61 \\
8 & $\mathrm{~N} / \mathrm{A}$ & 16.5 & Mould Preparation & 50 & 0.60 \\
9 & $\mathrm{~N} / \mathrm{A}$ & 14 & Mould Preparation & 50 & 0.60 \\
\hline
\end{tabular}

${ }^{1}$ In the small scale method, there was no suction measurement

${ }^{2}$ For higher degrees of desiccation, the tensiometer caviated during desiccation process

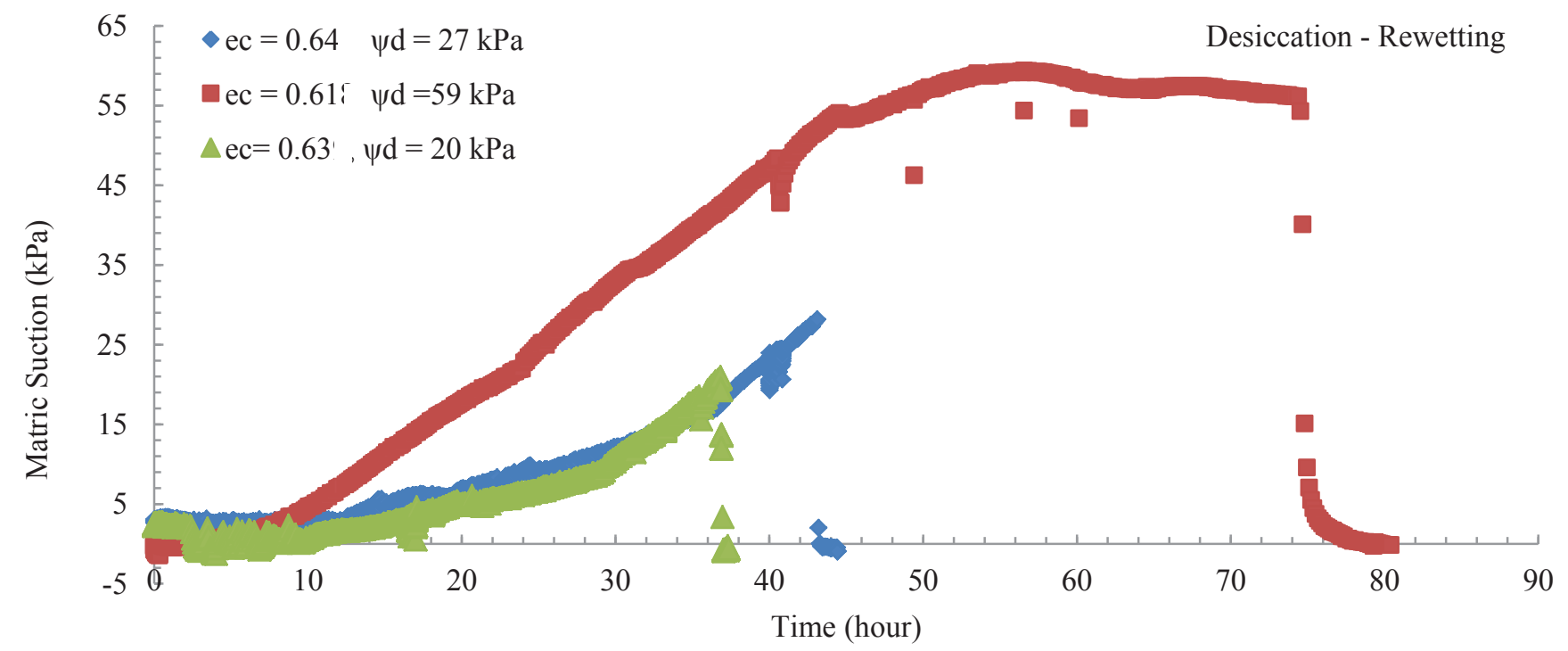

Figure 6.55 Variation of matric suction during desiccation-rewetting process of some triaxial samples 


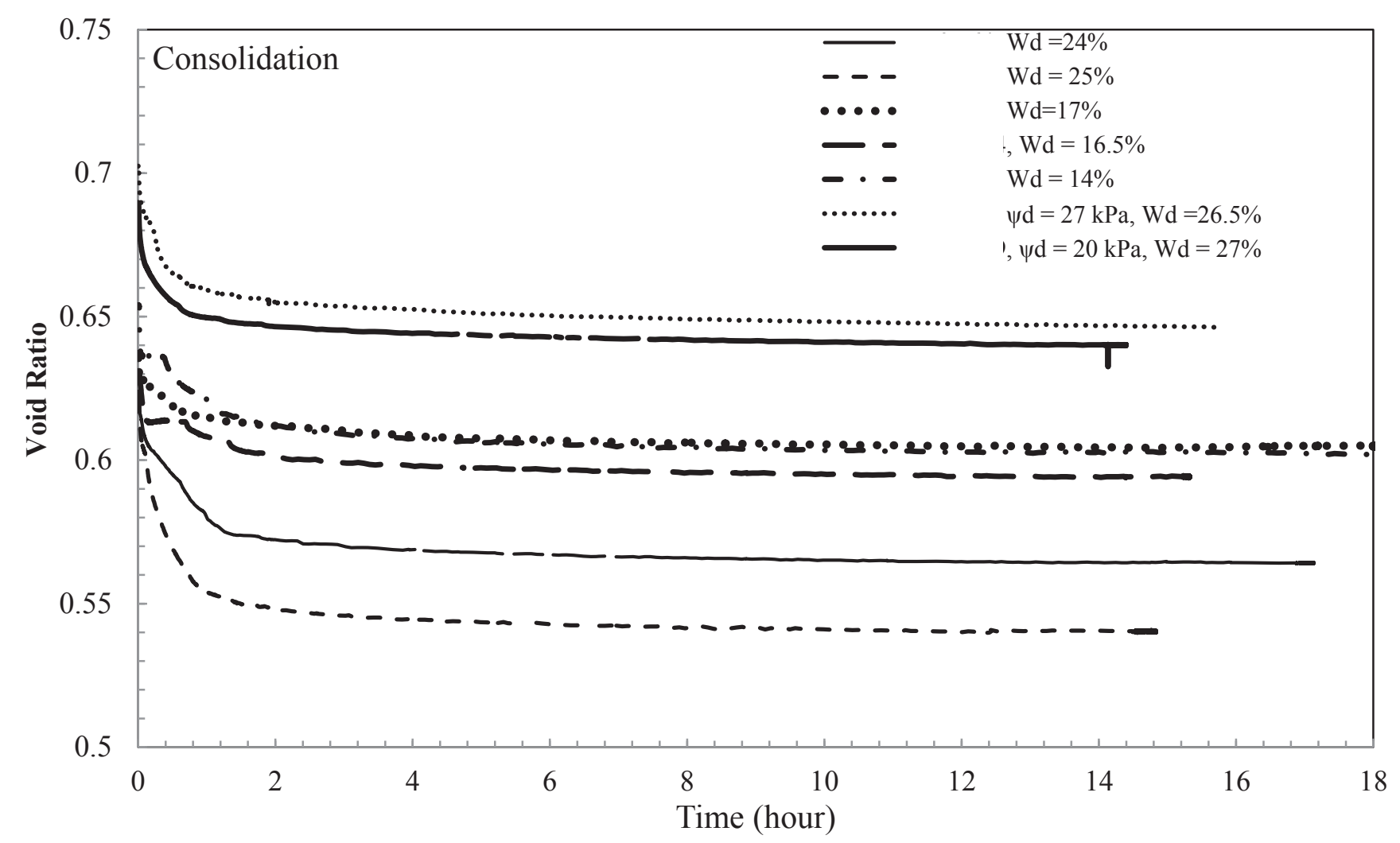

Figure 6.56 Consolidation results of modified triaxial tests

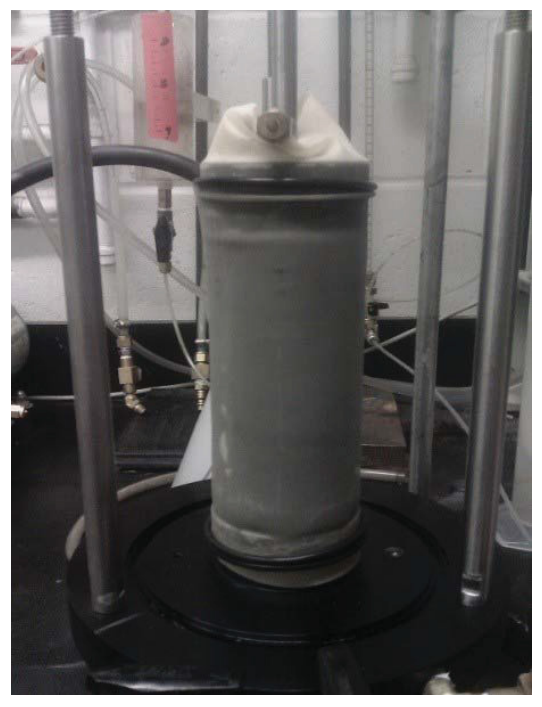

Before Consolidation

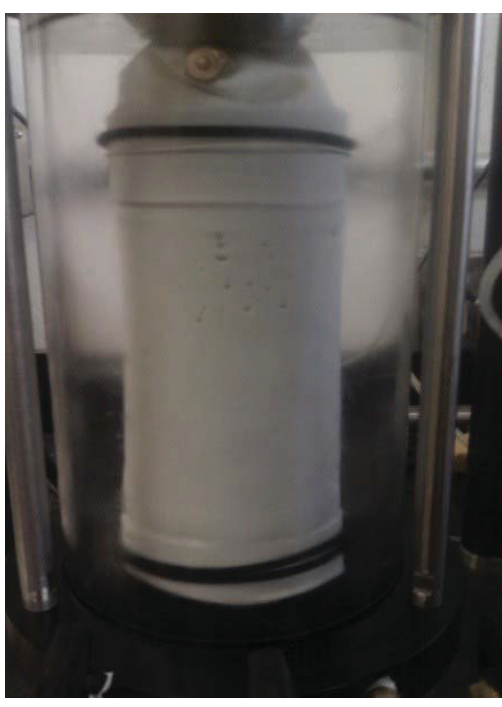

After consolidation

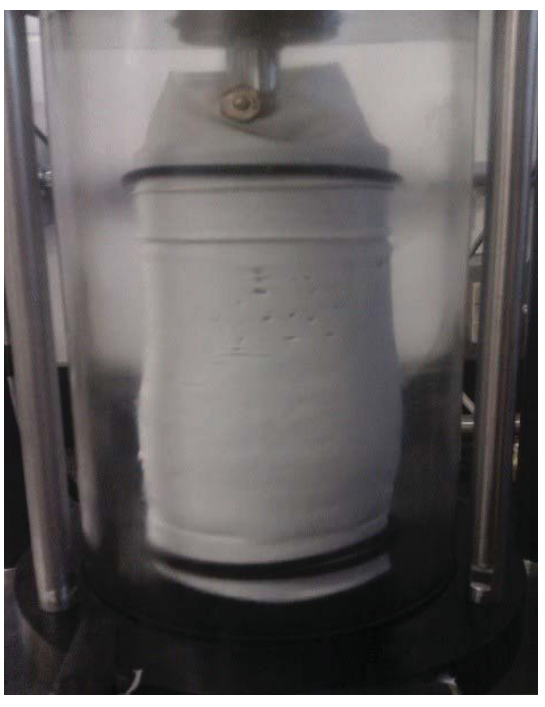

After Failure

Figure 6.57 Pictures of lateral strain in a modified triaxial test 
lateral strain development and since the volume change and lateral displacement measurements were approximate, the void ratio measurement was also approximate $( \pm 0.02 \sim 0.03)$.

Figure 6.58 and 6.59 present monotonic triaxial responses of modifed triaxial tests performed on settled and desiccated-rewetted tailings. It is clear that the sample with low desiccation exhibited the weakest response leading to the lowest shear strength in comparison to desiccated-rewetted samples even though all samples exhibited strain hardening response. A small degree of desiccation to $\mathrm{Wd}=24 \% \sim 25 \%$ significantly increases the shear strength of the sample. Figure 6.59 shows the excess pore water pressure response of the modified triaxial tests. The essentially linear portion of the stress path at large strains (well beyond the phase transformation state) indicates that the material has negligible cohesion. The average value of the friction angle was calculated to be about $34^{\circ}$. It is shown that all samples exhibited contractive response at the beginning of the tests. It is also clear that in all samples, the excess pore pressure generation becomes negative at $\varepsilon_{a}=2 \% \sim 3 \%$ where the phase transformation state is realized. It is also shown that the excess pore pressure of two samples with high degrees of desiccation $(\mathrm{Wd}=17 \%$ , $\mathrm{Wd}=14 \%$ ) becomes negative at the end of the tests, which confirms high degree of dilation in these two tests. To calculate modified effective stress and friction angles, the values of effective stress ( $\mathrm{p}^{\prime}$ and $\mathrm{q}$ ) were modified by considering $\mathrm{Sr}=\chi$ in Bishop's equation. Figure 6.60 presents the effect of desiccation hisotry on shear strength of the samples based on modified triaxial results. Figure 6.60a presents the effect of degree of desiccation on the value of $q$ at the phase transformation points. It can be seen that increasing desiccation history from settling to the shrinkage limit significantly increased values of $q$ at PT. Increasing desiccation history to beyond the shrinkage limit was not beneficial and sometimes resulted in lower $q$. Figure $6.60 \mathrm{~b}$ presents the value of $q$ at the end of the test with respect to degree of desiccation. It is clear that 
increasing degree of desiccation would significantly increase the failure strength of the material. This is found to be the case when the samples are desiccated to and beyond the shrinkage limit.

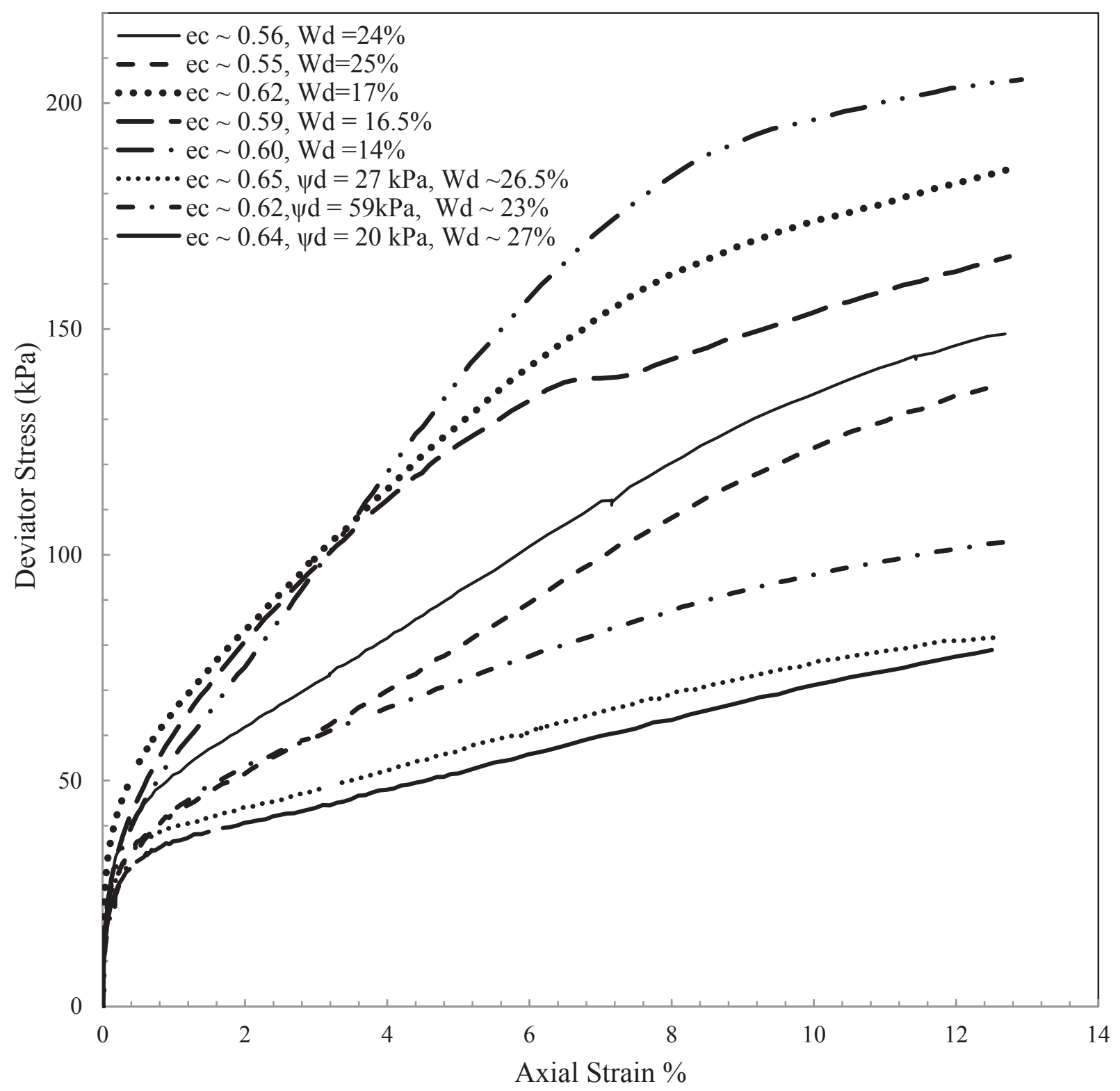

Figure 6.58 Stress-strain responses of modified triaxial tests on settled and desiccated-rewetted tailings 


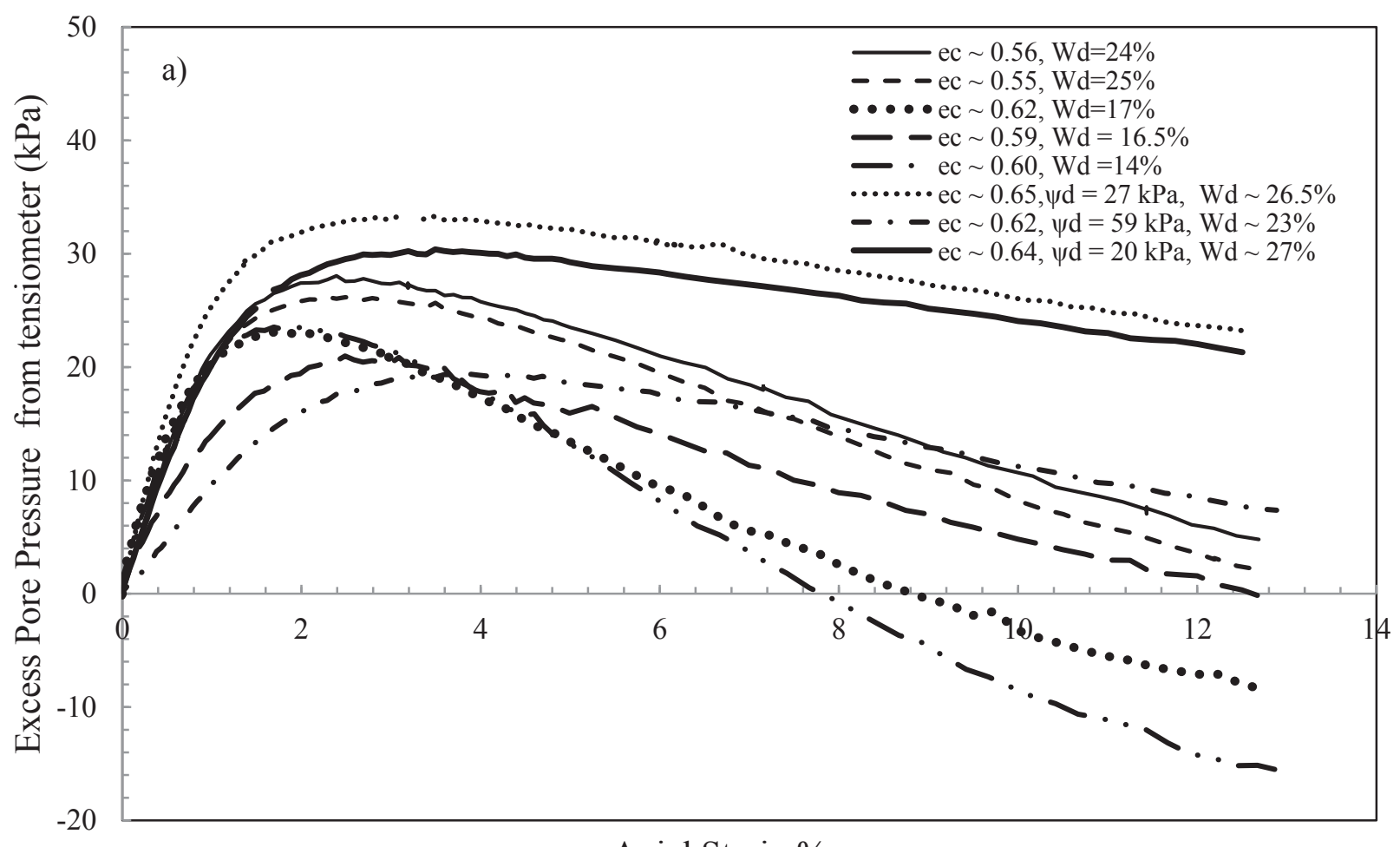

Axial Strain \%

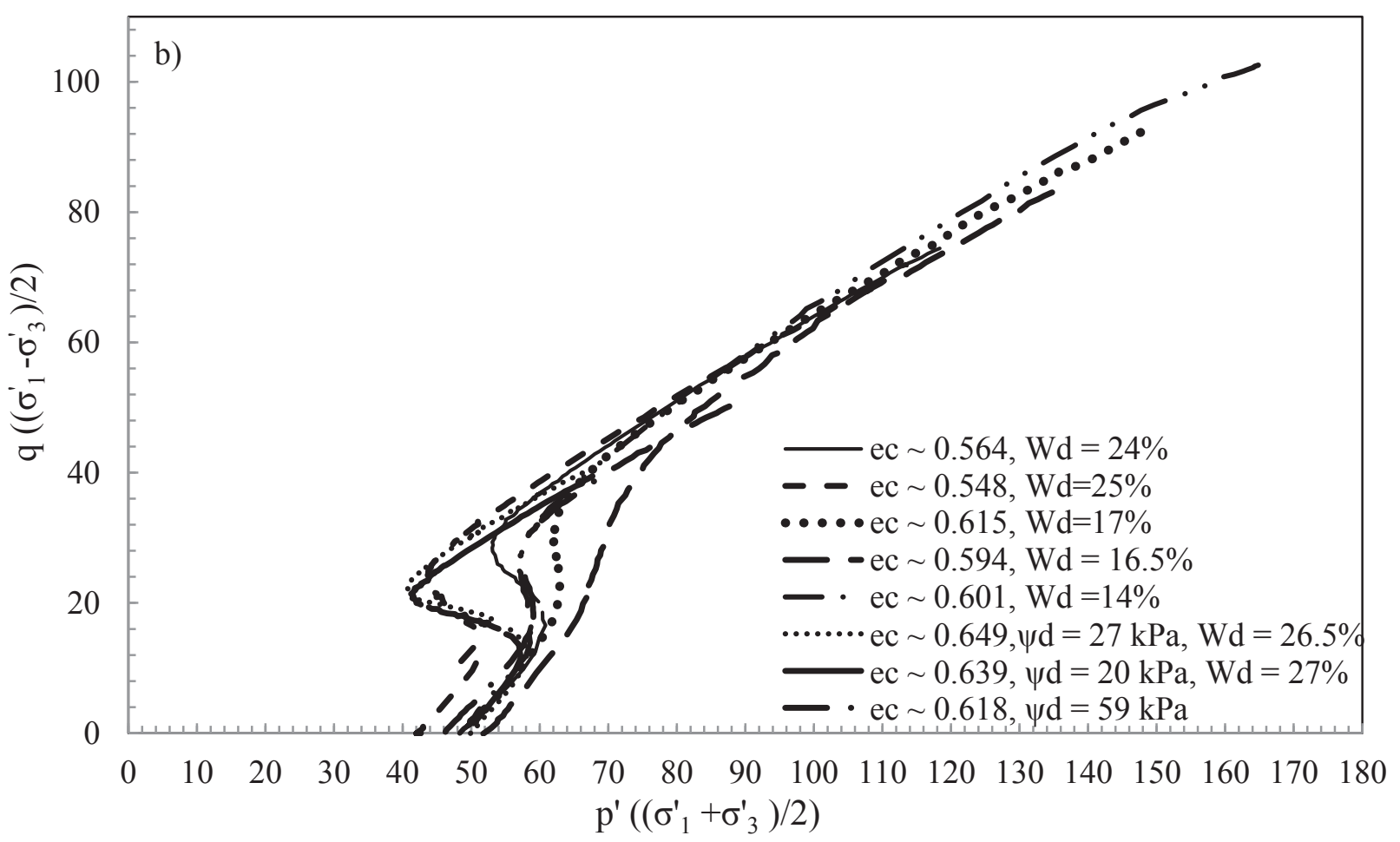

Figure 6.59 Excess pore pressure generation and stress path of modified triaxial tests on settled and desiccated-rewetted tailings 

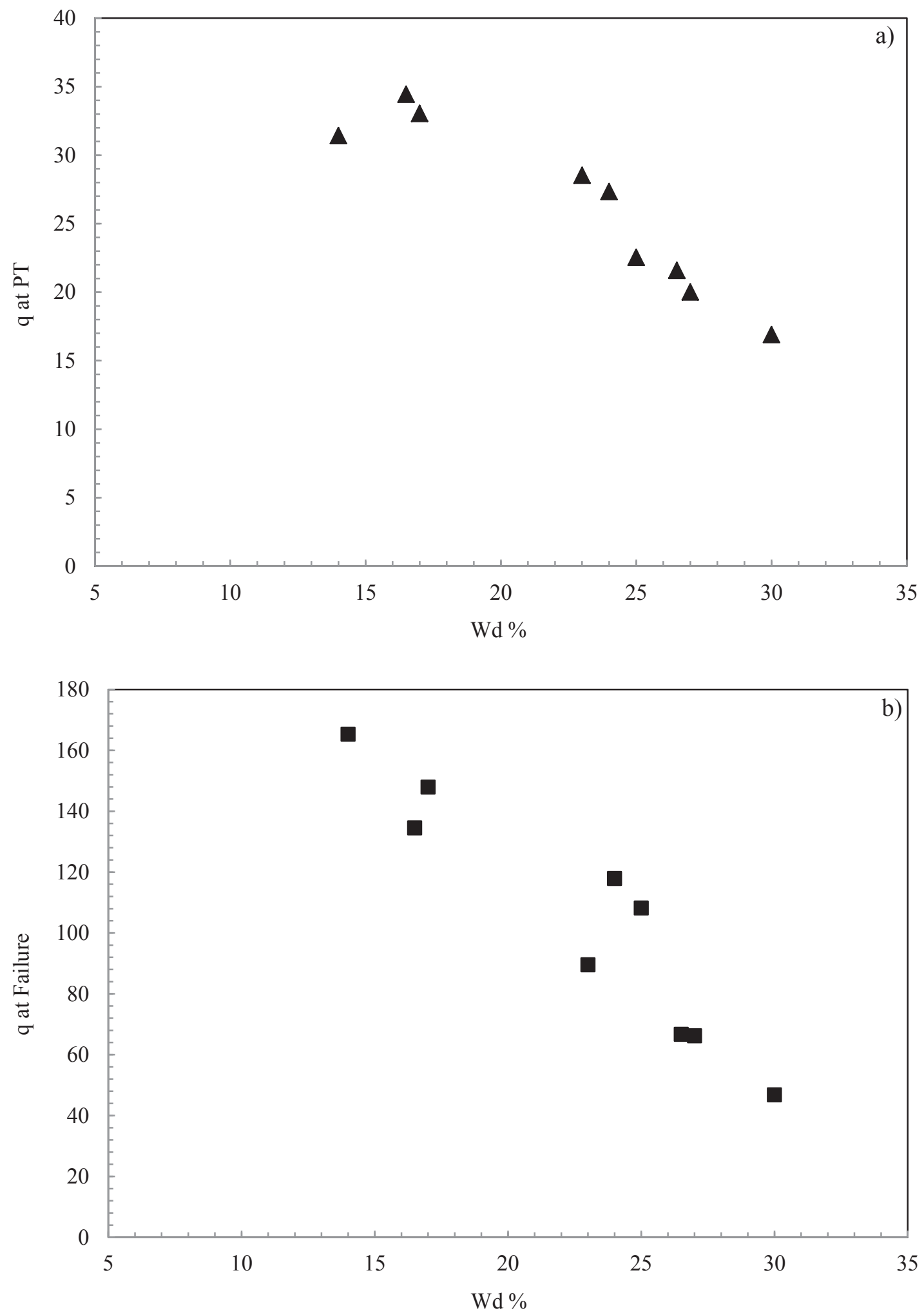

Figure 6.60 Variation of $\mathrm{q}$ at PT and failure versus water content 


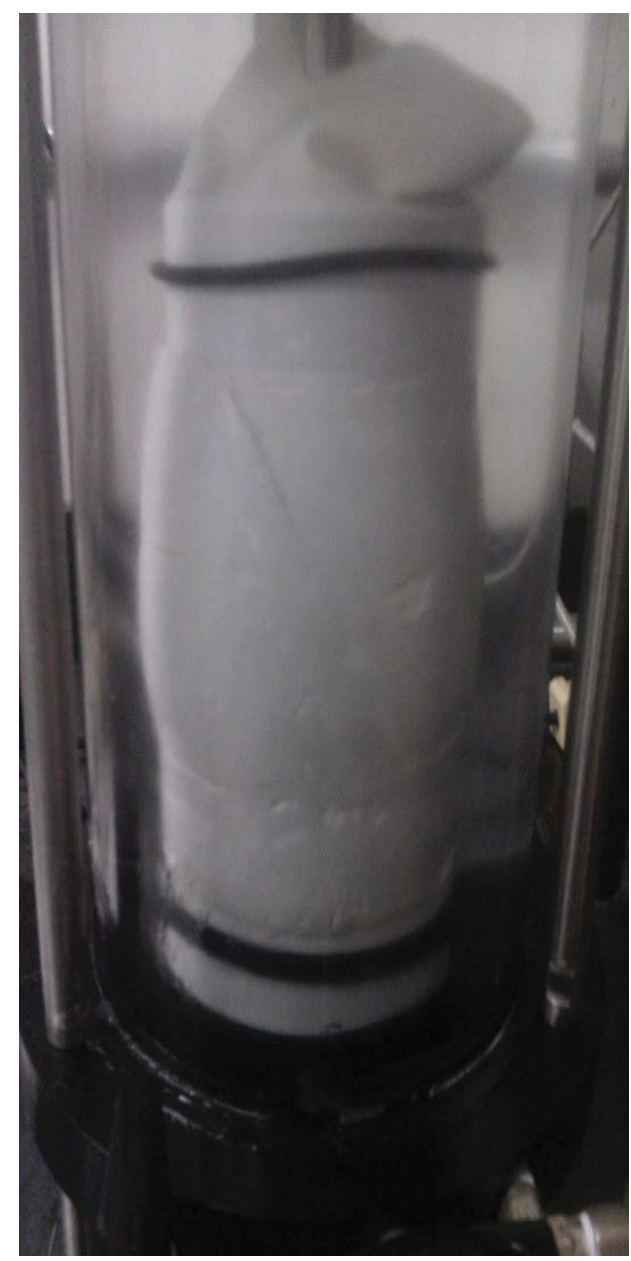

a)

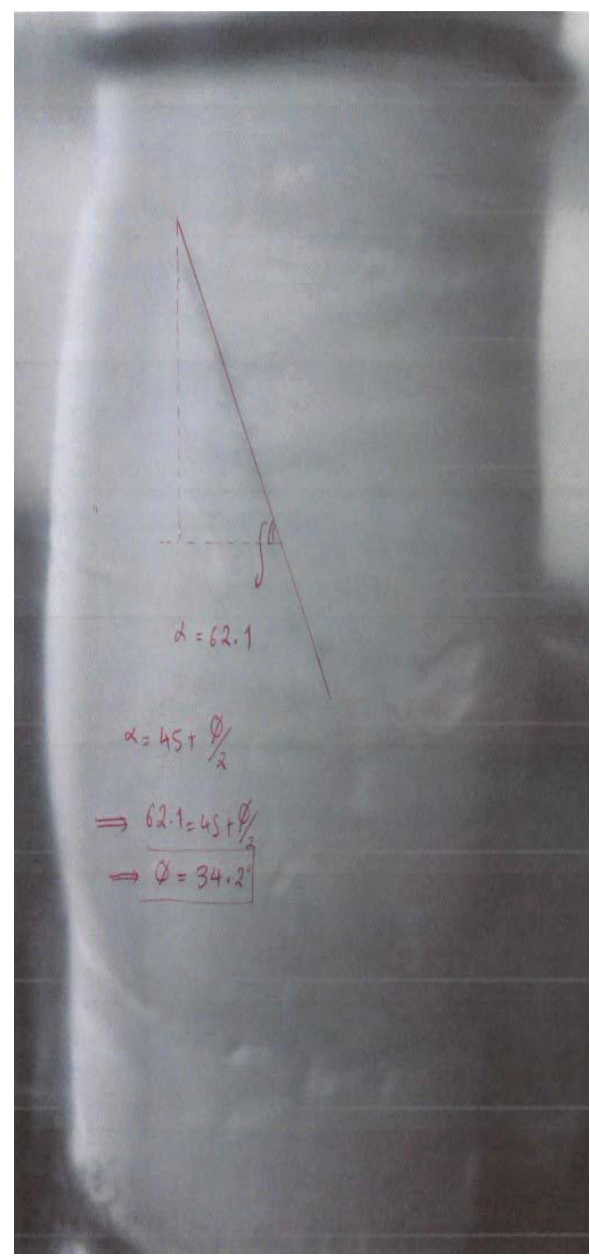

b)

Figure 6.61 Shape of failure surface obtained from a modified triaxial test performed on a desiccated-rewetted sample

Figure 6.61 shows a triaxial sample at the end of the test, which clearly shows the shape of the failure surface from a desiccated-rewetted sample. The inclination of the failure place conforms to a friction angle of $34^{\circ}$ in a material with no cohesion.

Figure 6.62 presents comparison of conventional and modified triaxial tests. The stress-strain response of samples show that the modified test, which has lower void ratio, exhibited higher strength. 


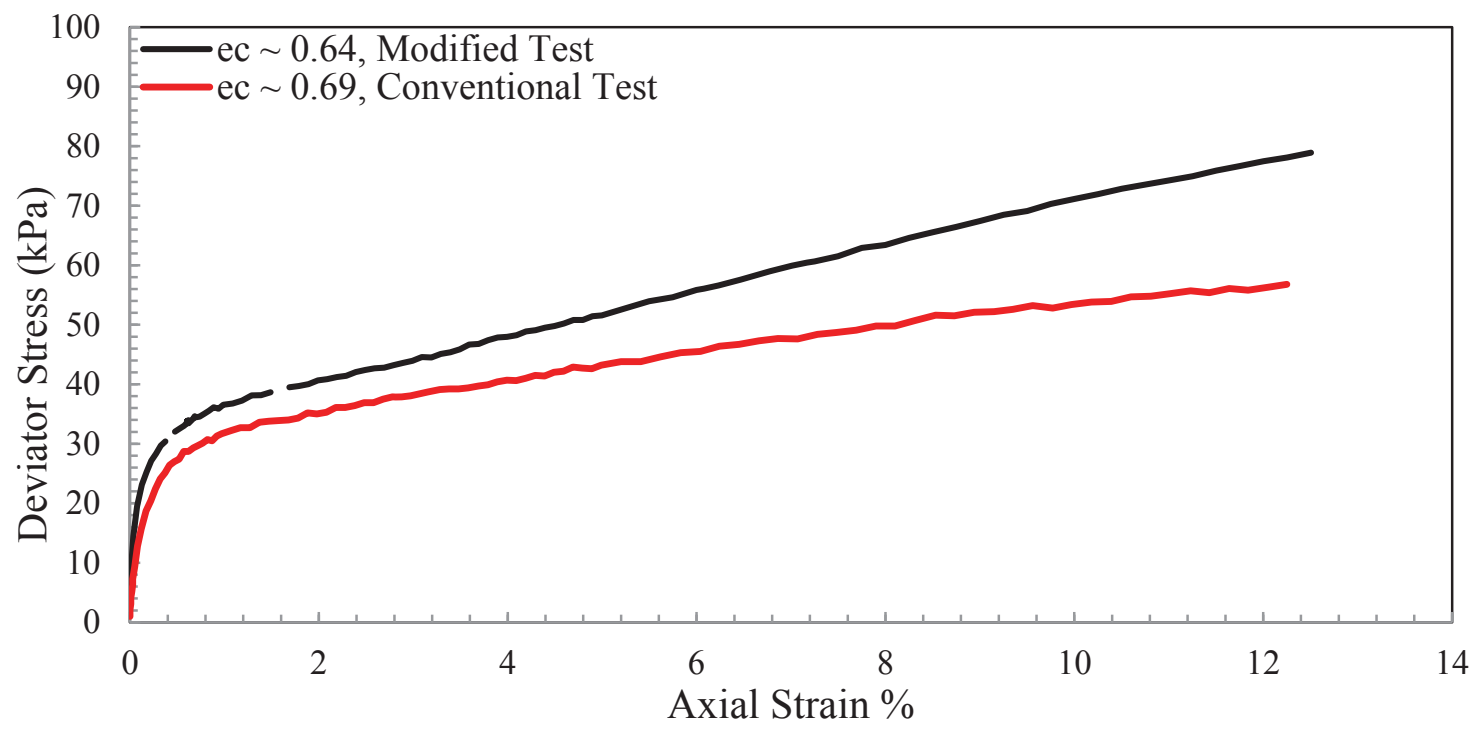

Figure 6.62 Comparison of conventional and modified triaxial tests on settled tailings

\subsubsection{Limitation in results interpretation of modified triaxial tests}

As previously discussed in chapter 3, there were some limitations in modified triaxial device. Conventional triaxial tests were not employed for desiccated-rewetted samples due to the fact that reaching appropriate B value even under back pressure was not successful. Therefore, the modified triaxial cell was designed to monitor the pore water pressure (or matric suction) during the test and avoid the saturation part. However, since the specimen was not fully saturated, there were some approximation in void ratio measurement and stress calculation.

There were two methods to approximately estimate void ratios after consolidation: i) for higher degrees of saturation $(0.95<\mathrm{Sr}<1)$ the volume of removed water during consolidation was assumed to be the same as volume change of the sample and recording the height change during 
consolidation, the area and therefore the diameter were calculated to be used for void ratio measurement; ii) for lower degrees of saturation $(0.89<\mathrm{Sr}<0.95)$ the diameter was approximately estimated during the test by taking pictures or using a caliper. Obviously, due to variation of the diameter in different heights of the sample, the estimation is not expected to be as accurate as the first method.

The diameter was also influential in calculating effective stresses during shearing. The diameter was calculated assuming no volumetric strain during shearing. In other words, considering compressibility of air, the volume was assumed to be constant during shearing. Consequently, the area and the diameter were calculated by recording the height change based on constant volume. However, the constant volume change was checked during shearing by tracking the level of water of the cell. It was obtained that in all modified triaxial tests, there was no tangible change in the level of water of the cell, which can confirm no tangible volume change during shearing. In order to calculate the effective stresses during shearing and calculating friction angles, the effective stresses were also modified by Bishop's equation assuming $\mathrm{Sr}=\chi$ and atmospheric air pressure.

The measurement of suction/pore water pressure was made by inserting a tensiometer from the bottom of the sample. Obviously, the measured matric suction corresponds to the value of matric suction at the bottom of the sample. However, all of the triaxial tests (except three desiccated samples) were conducted on rewetted samples after desiccation, and suction in these specimens was negligible upon rewetting. In fact, these specimens had positive pore water pressure following consolidation to the target effective stress, and thus non-uniformity is not a concern in these tests. However, the three desiccated specimens would have had a gradual change in the water content profile with height. It is expected that the water content of the desiccated tailings is 
lower on the top of the sample and the level of variation from top to the bottom is estimated to be $1 \% \sim 2 \%$ based on the measurements in the drying box. Therefore the measured behaviour is considered representative of material with an average suction value.

\subsubsection{Results of monotonic triaxial tests on desiccated tailings}

Three triaxial tests were conducted under $50 \mathrm{kPa}$ consolidation pressure on desiccated samples with no rewetting. Figure 6.63 presents stress-strain reponse of these three desiccated samples and compares the responses with desiccated-rewetted tailings. As expected, desiccated samples exhibited much higher shear strength due to the generation of suction. Tailings desiccated to lower water content $(\mathrm{W}=16 \%$ ) exhibited a peak value of around $310 \mathrm{kPa}$ deviator stress at $8 \%$ shear strain. As degree of desiccation decreases to higher water contents, the shear strength is reduced and the shape of peak disappears.

Figure 6.64 presents variation of matric suction in the modified triaxial tests on desiccated tailings. It should be noted that the tensiometer was inserted to the sample after finishing consolidation, when it is expected that the matric suction is reduced to be less that cavitation zone. It is shown that the matric suction was mostly reduced during shearing especially at the beginning of the test. For the sample desiccated to $\mathrm{W}=17.5 \%$, the matric suction starts to increase at around 5\% axial strain. A small increment after $4 \%$ axial strain is also visible for the sample desiccated to $\mathrm{W}=18.3 \%$. Fairly linear reduction of matric suction in the sample desiccated to $\mathrm{W}=16 \%$ can be attributed to possibility of cavitation after inserting the tensiometer. However, for two other tests, matric suction was monitored to confirm that the tensiometer is not cavitated after insertion. 


\subsubsection{Cyclic results of modified triaxial tests on settled tailings}

Figure 6.65 and 6.66 present results of cyclic modified triaxial test on a settled specimen at CSR

$=0.1$. The specimen was deemed to have liquefied upon reaching an axial strain $\varepsilon_{a}=2.5 \%$

(consistent with the definition of liquefaction used in simple shear tests). The sample subjected

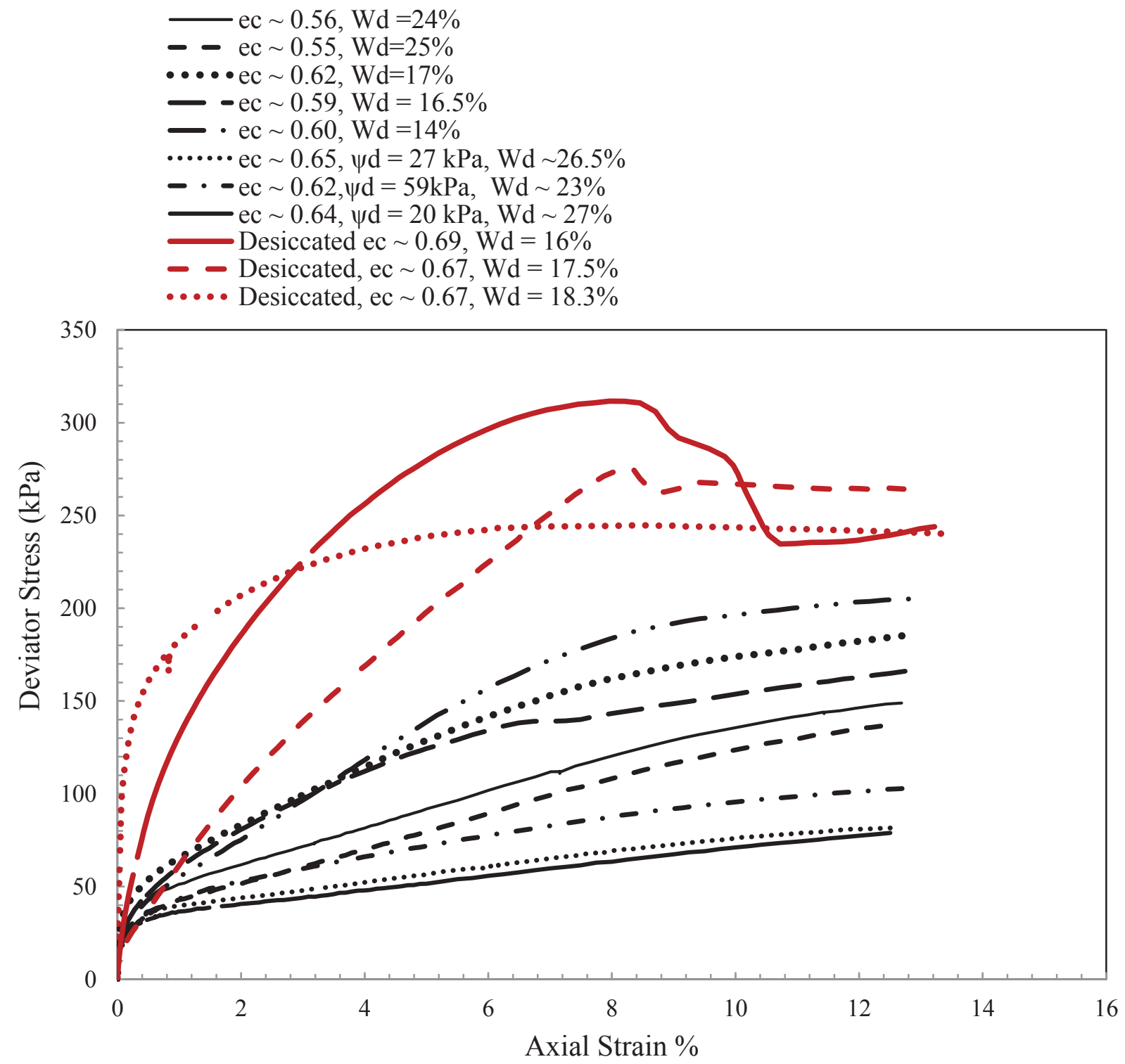

Figure 6.63 Comparison of the results of triaxial tests performed on desiccated tailings with desiccated-rewetted samples 


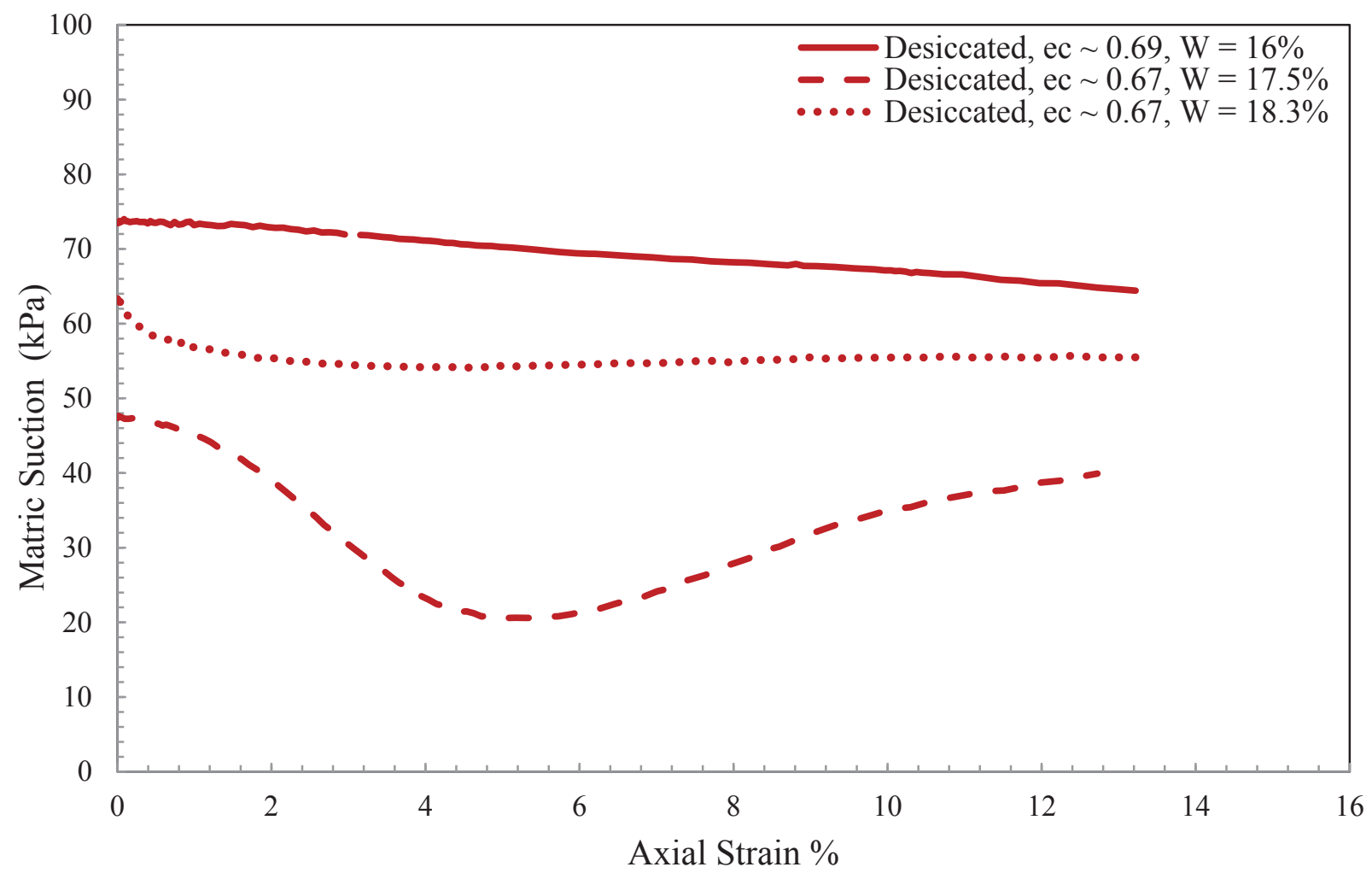

Figure 6.64 Variation of matric suction in modified triaxial tests on desiccated tailings

to a CSR of 0.1 liquefied in 149 cycles with a maximum excess pore pressure ratio at the end of the test $r_{u}=0.77$. Unlike the cyclic simple shear tests where strain development was symmetrical, the strain development in the cyclic triaxial test indicates directional preference. The mobilization of strain shown in Figure 6.66b in an indication of the stress path effect or the anisotropic nature of the sample. The sample mobilized higher axial strain during unloading part (triaxial extension loading) and the liquefaction criterion was met due to large extensional strain. Figure 6.67 showing the cyclic triaxial response at $\mathrm{CSR}=0.15$ also shows weaker response when the major principal stress is aligned with the horizontal direction (extension mode) . The specimen liquefied at 28 cycles, but the maximum compressive strain was less than $0.5 \%$ and the extensional strain about $3 \%$. 

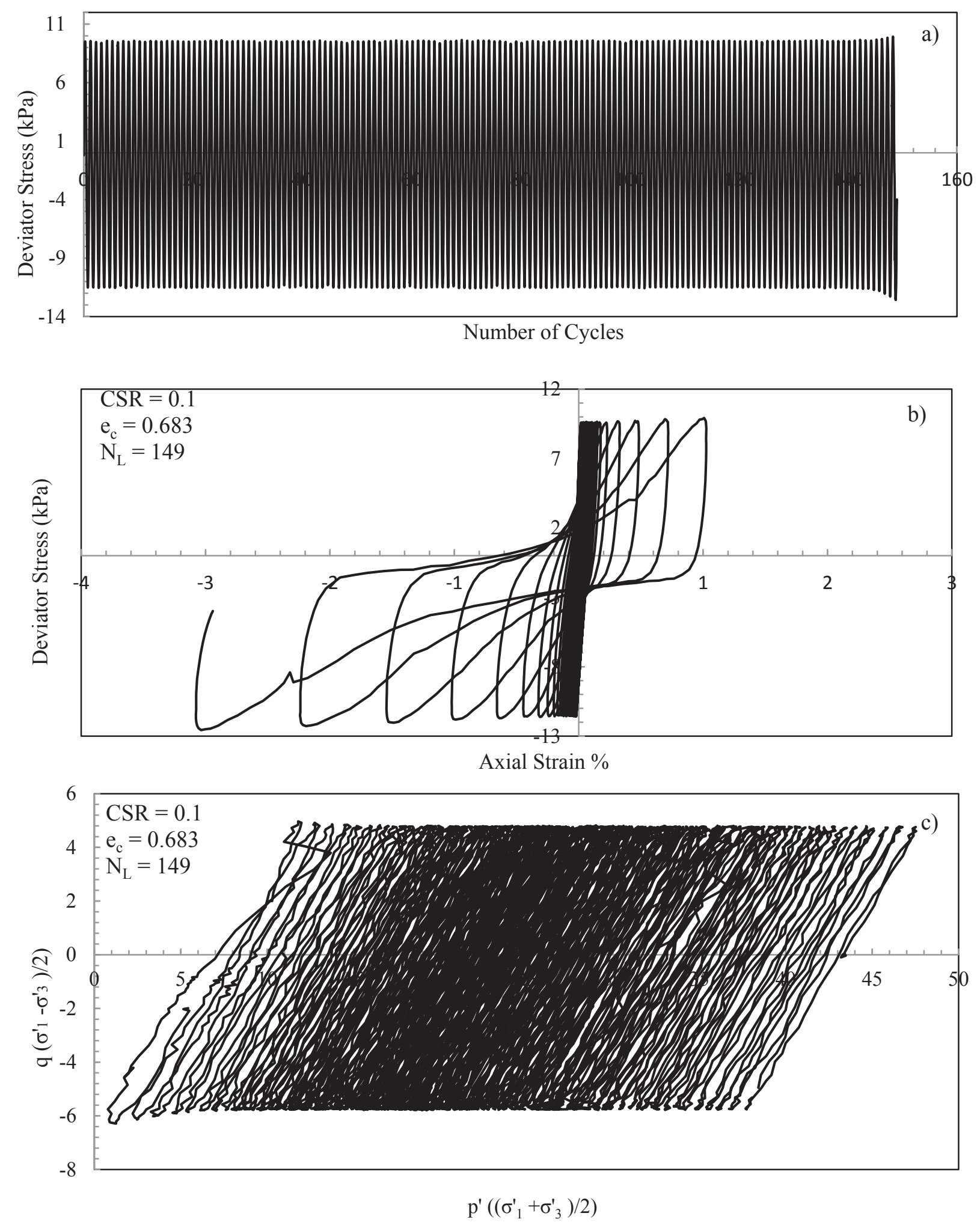

Figure 6.65 Cyclic modified triaxial response of settled tailings at CSR $=0.1$ 

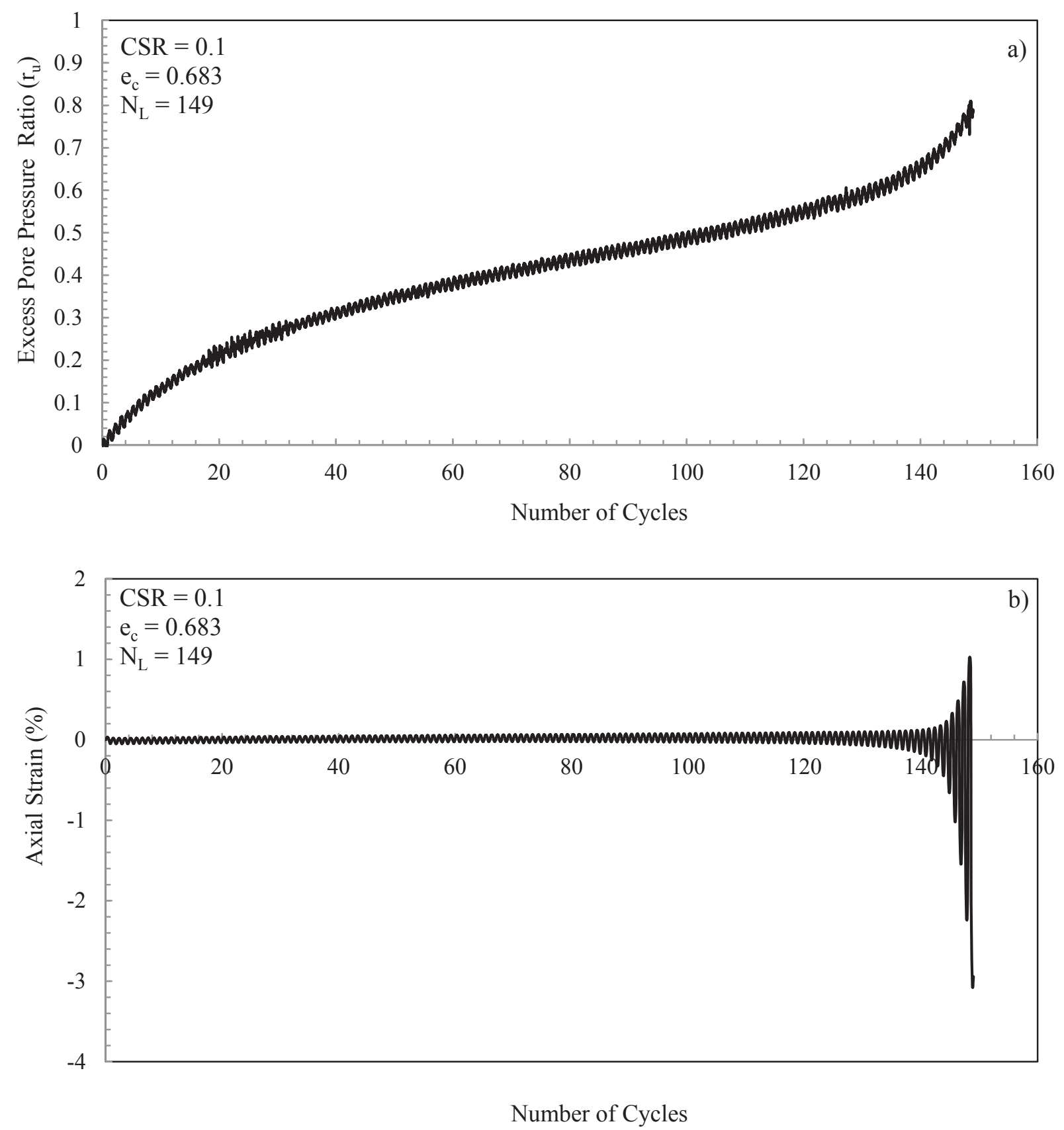

Figure 6.66 Expess pore pressure and axial strain generation of settled tailings at CSR $=0.1$

Figure 6.68 compares the measured cyclic resistance (CSR) versus numbers of cycles to reach liquefaction $\left(\mathrm{N}_{\mathrm{L}}\right)$ under triaxial and simple shear loading modes on tailings that did not undergo any desiccation. 

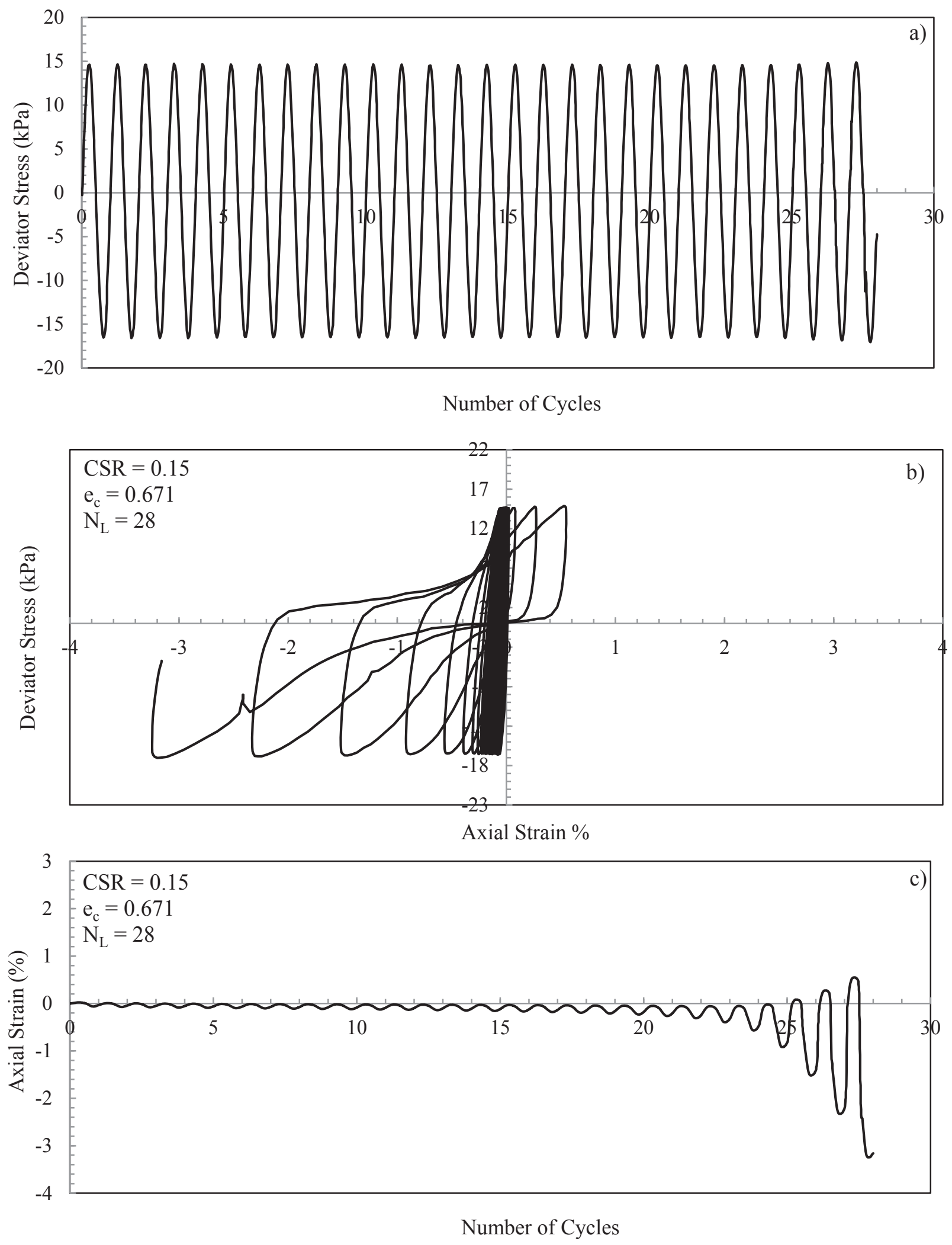

Figure 6.67 Cyclic modified triaxial response of settled tailings at CSR $=0.15$ 


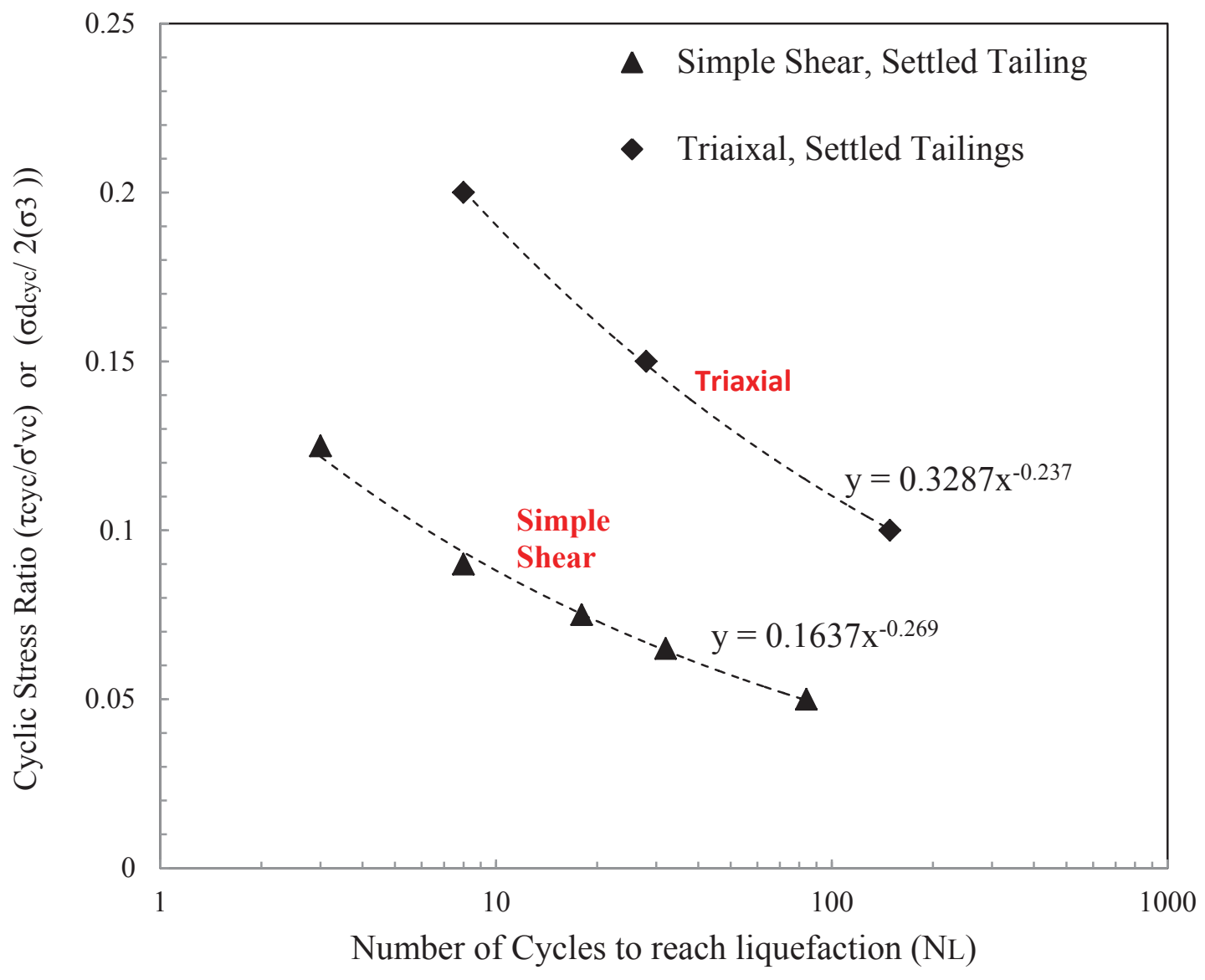

Figure 6.68 Comparison of CSR vs $\mathrm{N}_{\mathrm{L}}$ for modified triaxial and simple shear tests perfromed on settled tailings

The cyclic resistance measured under triaxial loading is significantly larger compared to that in simple shear. Similar behavior in sands have been widely reported in the literature (Vaid and Sivathayalan, 1996) and a correction factor $\mathrm{Cr}$ is sometimes used to convert cyclic resistance measured using triaxial devices to equivalent simple shear resistance. Typically, the $\mathrm{Cr}$ factor is determined for a specific value of CRR corresponding to a $\mathrm{N}_{\mathrm{L}}$ (e.g., $\mathrm{N}_{\mathrm{L}}=10$ or 15) but given the available data, $\mathrm{Cr}$ values as a function of the number of cycles to liquefaction $\mathrm{N}_{\mathrm{L}}$ are computed as:

$$
\mathrm{C}_{\mathrm{r}}=0.498 N_{L}^{-0.032} \quad \text { Equation } 6.1
$$


The $\mathrm{Cr}$ factor for liquefaction due to a $\mathrm{M}=6.75$ earthquake $\left(\mathrm{N}_{\mathrm{L}}=10\right)$ is thus equal to 0.47 in the tailings. In comparison, $\mathrm{Cr}$ values ranging from 0.4 to 0.9 have been reported in sands depending on the density and confining stress level (Vaid and Sivathayalan, 1996).

\subsection{Friction angle at Phase Transformation, Failure states}

Figure 6.69a shows the stress state at the phase transformation state in simple shear tests performed on settled and desiccated-rewetted tailings, and Figure 6.69b shows similar data at $11 \%$ shear strain representing the failure state. Even though there is minor scatter in the data (corresponding to about $\pm 1^{\circ}$ variation in friction angle) the test results suggest that the friction angle at each of these states is essentially a constant value. Such uniqueness of phase transformation, failure friction angles have been reported in sands by several authors. The best fit lines yield a friction angles of $26.5^{\circ}$ and $30^{\circ}$ at phase transformation and failure respectively.

Similar data from the triaxial tests is shown in Figure 6.70 for tests performed on settled and desiccated-rewetted tailings. Considering the scatter of the data and approximation of stresses, which can lead to variation of $\pm 2^{\circ}$, the data presented yields a phase transformation friction angle of $28.5^{\circ}$ and failure friction angle of $37.5^{\circ}$. Vaid and Sivathayalan (1995) reported the PT friction angle of triaxial tests slightly higher than the simple shear tests in Fraser River sands. Miura \& Toki (1982) noted that the failure friction angle is somewhat dependent on the loading mode. The PT friction angles measured in this study are consistent with the findings in the literature $\left(2^{\circ}-3^{\circ}\right.$ higher in triaxial tests compared to simple shear tests). The failure friction angles measured in triaxial tests have been relatively large. Potential systematic errors in the calculated effective stresses and non-uniformities at large strains might be a reason for this. 

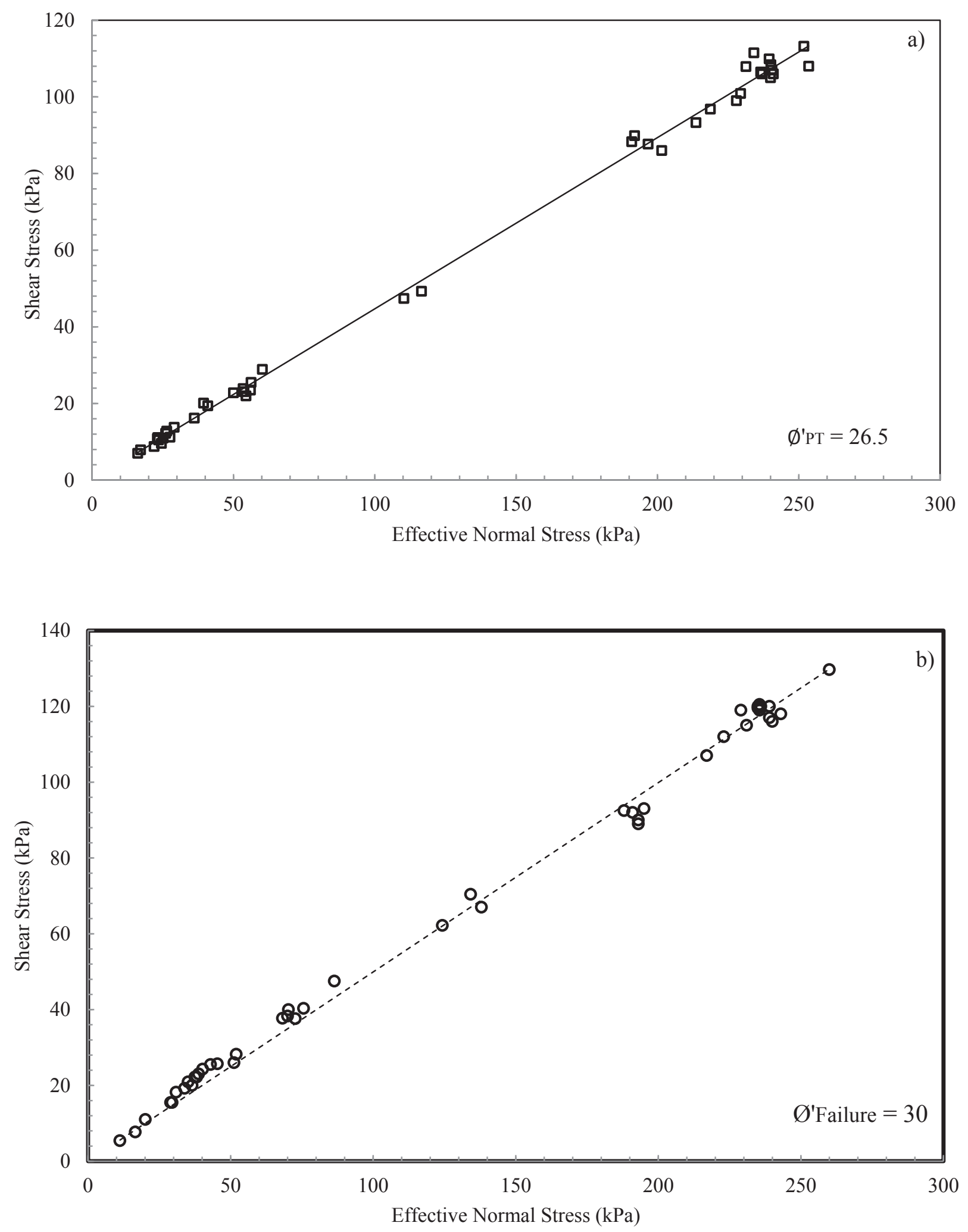

Figure 6.69 Phase transformation and failure friction angles of simple shear tests 

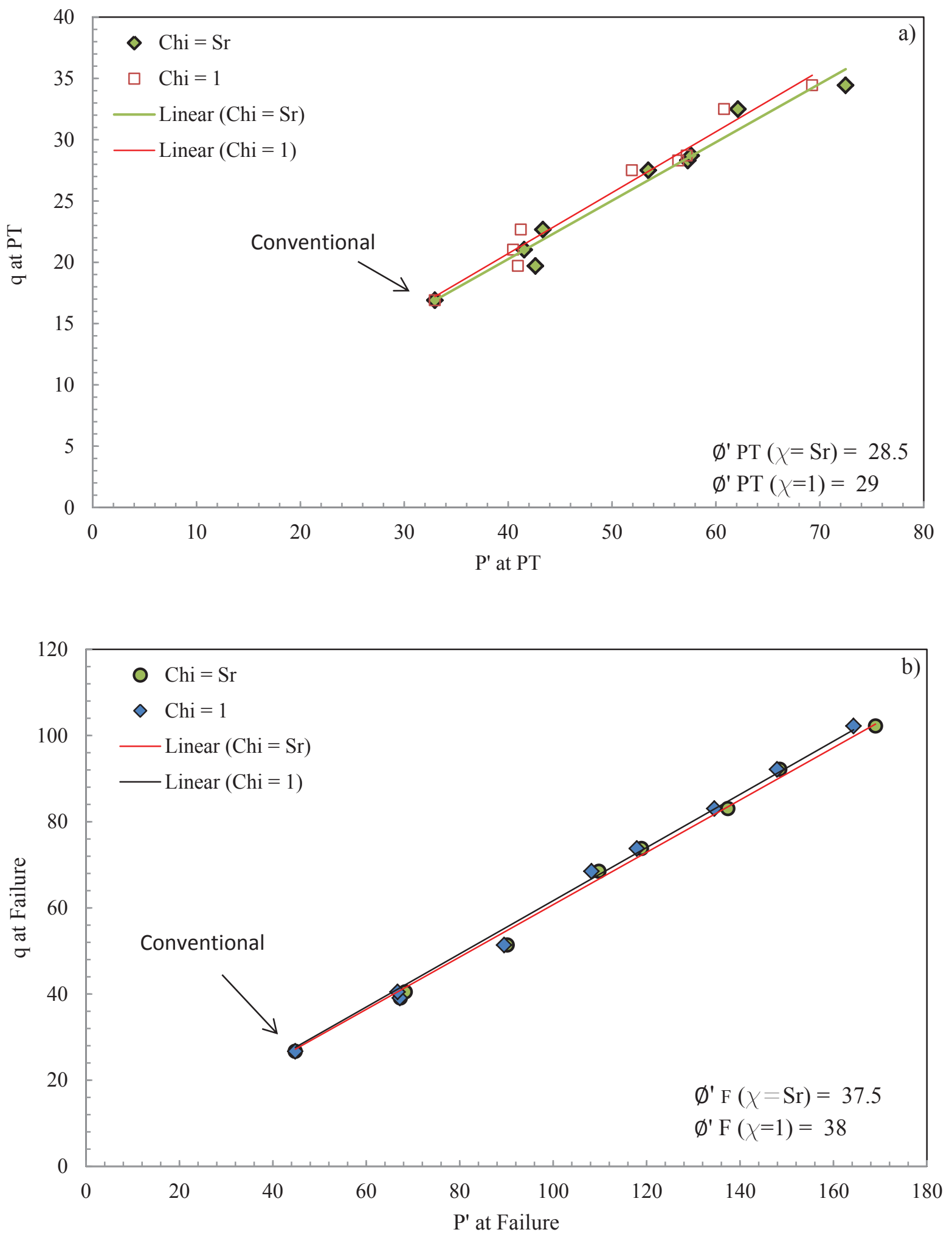

Figure 6.70 Phase transformation and failure friction angles of modified triaxial tests 


\subsection{Results of vane shear tests}

Vane shear tests were performed on samples deposited in a cylindrical bucket and allowed to desiccate to different values of water contents. Some vane shear tests were also conducted on desiccated-rewetted tailings to investigate the effect of rewetting and stress history. Table 6.7 presents details of the vane shear tests performed on settled and desiccated tailings. The matric suction was obtained based on the SWCC of the tailings. The void ratio measurement was approximately done by measuring the height of deposited tailings and thickness of cracks. Figure 6.71 presents vane shear results of tests performed on settled and desiccated samples. It is clear that up to $\mathrm{W}=30 \%$, the undrained shear strength of settled samples is extremely low. A little degree of desiccation to $\mathrm{W}=28 \%$ resulted in $4 \mathrm{kPa}$ undrained shear strength. Desiccation to $\mathrm{W}=$ $26 \%$ significantly increased the undrained shear strength to $18 \mathrm{kPa}$. Continuing desiccation to the shrinkage limit increased the undrained shear strength to around $60 \mathrm{kPa}$. It should be mentioned that vane shear tests were not feasible at lower water contents due to the fact that the vane did not have capacity of being pushed into the tailings with low water contents.

Table 6.8 presents characteristic of vane shear tests performed on settled and desiccated-rewetted tailings. It is clear that for high water contents, rewetting process resulted in increasing the water content by about $1 \%$. For samples that experienced higher degree of desiccation (beyond $\mathrm{W}_{\mathrm{d}}=$ $20 \%$ ) the value of water content upon rewetting is between $21 \% \sim 22 \%$ regardless of the degree of desiccation. Figure 6.72 presents vane shear results of settled and desiccated-rewetted tailings. It is shown that settled samples do not exhibit significant undrained shear strength. Increasing the desiccation history to around $\mathrm{Wd}=26 \%$ led to a jump in undrained shear strength to $14 \mathrm{kPa}$. 
Table 6.7 Characteristics of vane shear tests performed on settled and desiccated samples

\begin{tabular}{|c|c|c|c|c|c|}
\hline $\begin{array}{l}\text { Test } \\
\text { No. }\end{array}$ & $\begin{array}{l}\text { Water content } \\
\text { before rewetting } \\
\left(\mathrm{w}_{\mathrm{d}}\right)\end{array}$ & $\begin{array}{c}\text { Matric Suction } \\
\text { before rewetting } \\
\text { (from SWCC) } \\
(\mathrm{kPa})^{1}\end{array}$ & $\begin{array}{c}\text { Type of } \\
\text { Test }\end{array}$ & $\begin{array}{l}\text { Undrained } \\
\text { Shear } \\
\text { Strength } \\
(\mathrm{kPa})^{2}\end{array}$ & $\begin{array}{l}\text { Void ratio at } \\
\text { the end of } \\
\text { desiccation }\left(\mathrm{e}_{\mathrm{c}}\right. \\
\sim \pm 0.01)^{3}\end{array}$ \\
\hline 1 & 33 & 1 & Settled & 0.5 & 0.92 \\
\hline 2 & 31 & 3 & Settled & 1 & 0.91 \\
\hline 3 & 30 & 5 & Settled & 1.5 & 0.90 \\
\hline 4 & 28 & 6 & Desiccated & 4 & 0.79 \\
\hline 5 & 34.5 & 1 & Settled & 1.5 & 0.93 \\
\hline 6 & 34 & 1 & Settled & 1 & 0.92 \\
\hline 7 & 33 & 2 & Settled & 1.5 & 0.92 \\
\hline 8 & 32 & 2 & Settled & 1 & 0.91 \\
\hline 9 & 28 & 6 & Desiccated & 4 & 0.81 \\
\hline 10 & 25 & 20 & Desiccated & 18 & 0.72 \\
\hline 11 & 23 & 40 & Desiccated & 23 & 0.69 \\
\hline 12 & 22 & 70 & Desiccated & 32 & 0.68 \\
\hline 13 & 21 & 90 & Desiccated & 39 & 0.66 \\
\hline 14 & 19 & 150 & Desiccated & 53 & 0.65 \\
\hline 15 & 18 & 170 & Desiccated & 68 & 0.64 \\
\hline 16 & 17 & 200 & Desiccated & 71 & 0.64 \\
\hline 17 & 26.5 & 9 & Desiccated & 27 & 0.74 \\
\hline 18 & 25 & 20 & Desiccated & 26 & 0.73 \\
\hline 19 & 24 & 32 & Desiccated & 24 & 0.72 \\
\hline 20 & 23 & 40 & Desiccated & 25 & 0.69 \\
\hline 21 & 19.5 & 145 & Desiccated & 22 & 0.67 \\
\hline 22 & 18.5 & 160 & Desiccated & 21 & 0.65 \\
\hline 23 & 17.5 & 190 & Desiccated & 22 & 0.65 \\
\hline
\end{tabular}

\footnotetext{
${ }^{1}$ Matric suction values were obtained from SWCC and not measured directly

${ }^{2}$ Residual undrained shear strength values were measured based on two or three trials

${ }^{3}$ Approximate values of void ratio were measured based on vertical height and horizontal shrinkage
} 


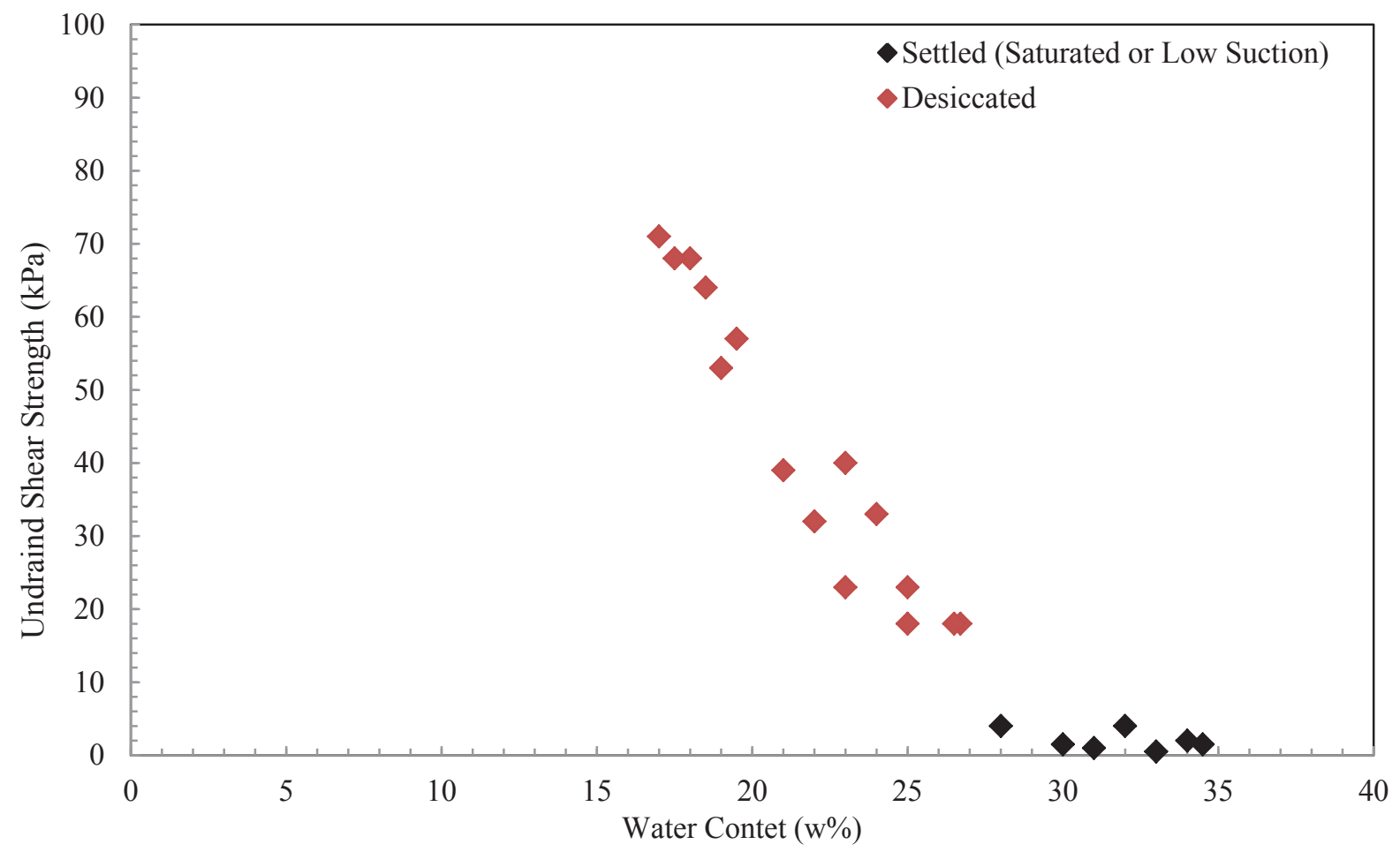

Figure 6.71 Undrained shear strength versus water content for settled and desiccated tailings

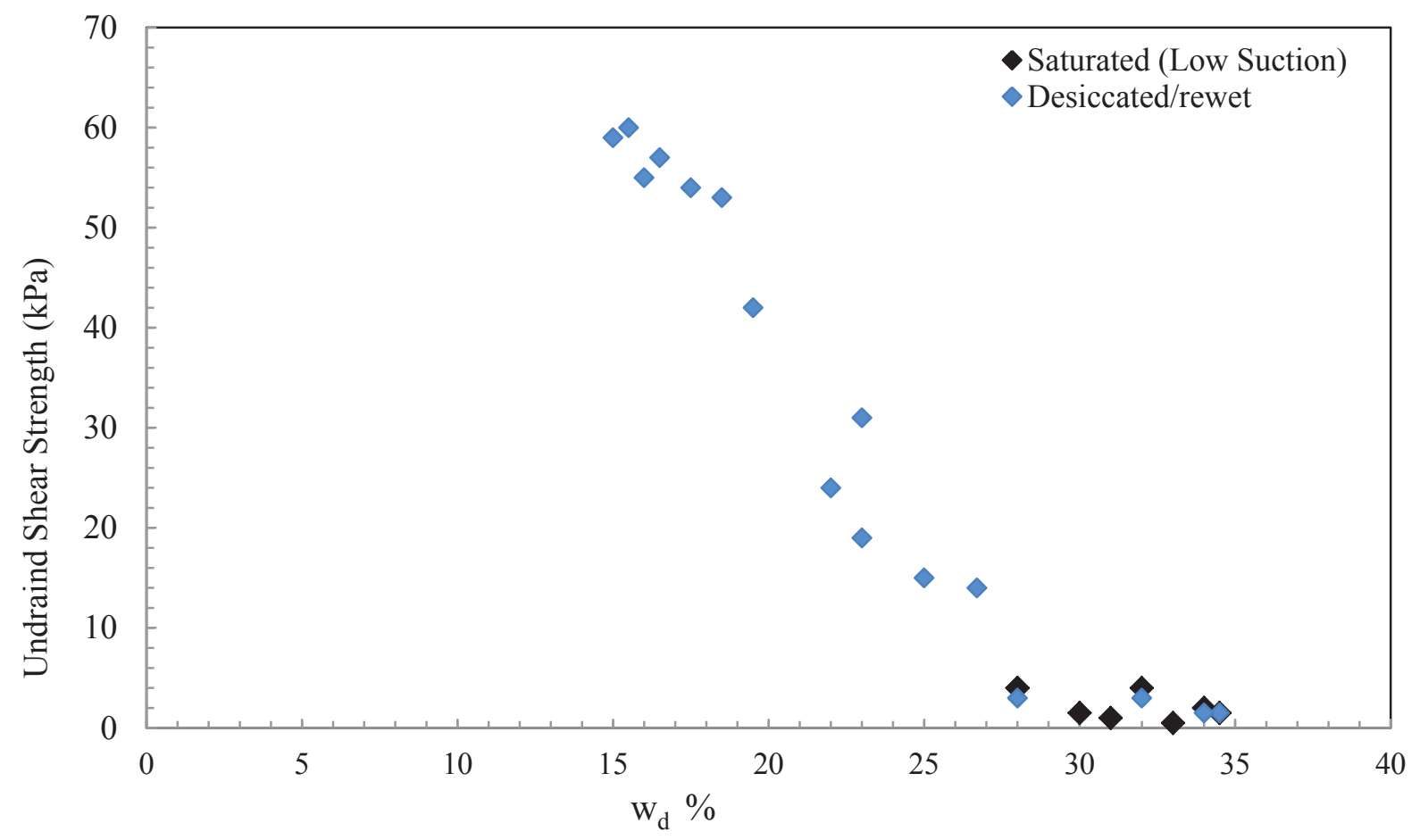

Figure 6.72 Undrained shear strength versus water content for settled and desiccated-rewetted tailings 
Table 6.8 Characteristics of vane shear tests performed on settled and desiccated-rewetted samples

\begin{tabular}{|c|c|c|c|c|c|}
\hline $\begin{array}{l}\text { Test } \\
\text { No. }\end{array}$ & $\begin{array}{l}\text { Water content } \\
\text { before } \\
\text { rewetting }\left(\mathrm{w}_{\mathrm{d}}\right)\end{array}$ & $\begin{array}{l}\text { Water content } \\
\text { after } \\
\text { rewetting (w) }\end{array}$ & $\begin{array}{c}\text { Matric } \\
\text { Suction } \\
\text { before } \\
\text { rewetting } \\
\text { (from SWCC) } \\
(\mathrm{kPa})^{1}\end{array}$ & Type of Test & $\begin{array}{l}\text { Undrained } \\
\text { Shear } \\
\text { Strength } \\
(\mathrm{kPa})^{2}\end{array}$ \\
\hline 1 & 34.5 & - & 1 & Settled & 1.5 \\
\hline 2 & 34 & - & 1 & Settled & 1.5 \\
\hline 3 & 33 & - & 2 & Settled & 2 \\
\hline 4 & 32 & - & 2 & Settled & 3 \\
\hline 5 & 28 & 29 & 6 & Desiccated/Rewetted & 3 \\
\hline 6 & 25 & 26 & 20 & Desiccated/Rewetted & 15 \\
\hline 7 & 23 & 25 & 40 & Desiccated/Rewetted & 19 \\
\hline 8 & 22 & 24 & 70 & Desiccated/Rewetted & 24 \\
\hline 9 & 26.5 & 28 & 9 & Desiccated/Rewetted & 14 \\
\hline 10 & 23 & 24 & 40 & Desiccated/Rewetted & 31 \\
\hline 11 & 19.5 & 21 & 145 & Desiccated/Rewetted & 42 \\
\hline 12 & 18.5 & 21 & 160 & Desiccated/Rewetted & 53 \\
\hline 13 & 17.5 & 21 & 190 & Desiccated/Rewetted & 54 \\
\hline 14 & 16 & 20.5 & 300 & Desiccated/Rewetted & 55 \\
\hline 15 & 15 & 21 & 370 & Desiccated/Rewetted & 59 \\
\hline 16 & 16.5 & 22 & 260 & Desiccated/Rewetted & 57 \\
\hline 17 & 15.5 & 20.5 & 340 & Desiccated/Rewetted & 60 \\
\hline
\end{tabular}

Increasing degree of desiccation history to shrinkage limit remarkably increased the undrained shear strength to around $50 \mathrm{kPa}$. Desiccation history beyond the shrinkage limit slightly increased the undrained shear strength. However, the rate of increase of undrained shear strength for tailings desiccated beyond the shrinkage limit is gentler than desiccated up to the shrinkage limit. Figures 6.73 and 6.74 compares the vane results of settled, desiccated and desiccatedrewetted tailings. It is shown that up to $\mathrm{W}=25 \%$, there is no significant difference between the 


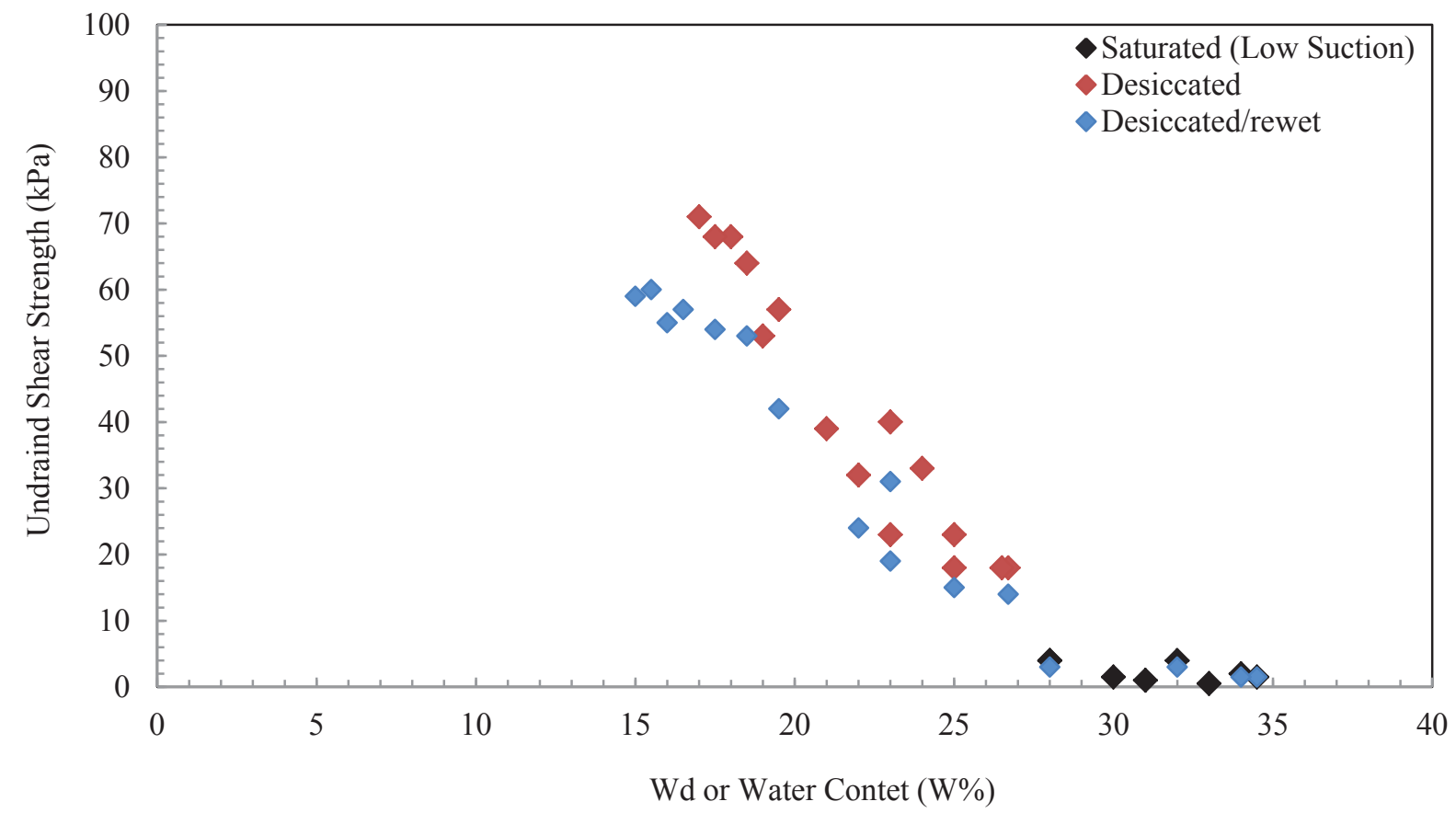

Figure 6.73 Undrained shear strength versus desiccated water content for settled and desiccatedrewetted tailings

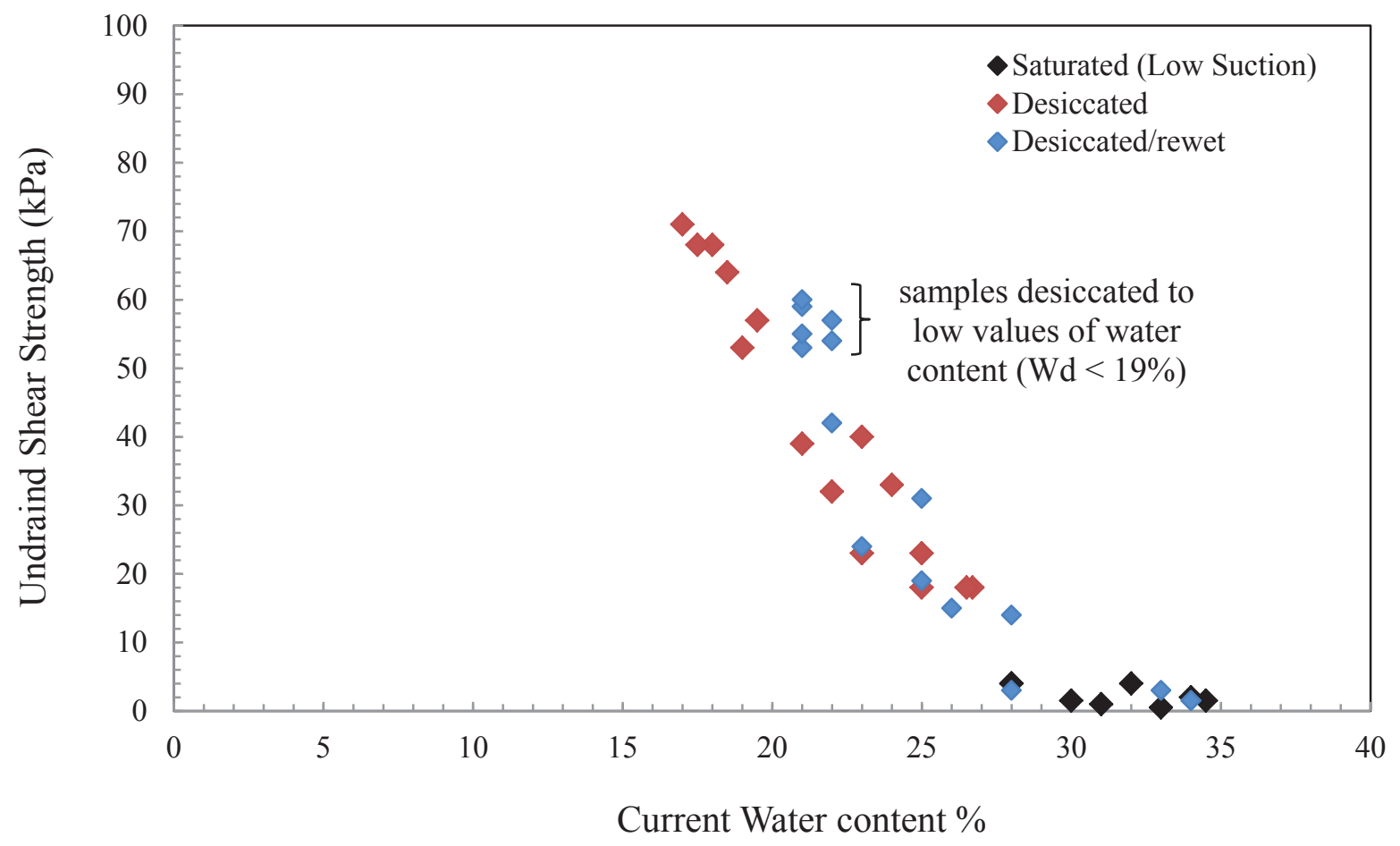

Figure 6.74 Undrained shear strength versus current water content for settled and desiccatedrewetted tailings 
results of desiccated tailings and desiccated-rewetted tailings. The effect of rewetting is more tangible for higher degrees of desiccation. Figure 6.74 presents comparison of undrained shear strength of desiccated tailings and desiccated-rewetted tailings based on current water content. Figures 6.73 and 6.74 clearly indicate that the vane shear strength of desiccated-rewetted tailings is smaller than desiccated tailings. The difference can be attributed to both stress state (contribution of matric suction to effective stress) and fabric (dissipation of suction leading to change in density and / or particle re-arrangement). Due to the presence of suction, the vane strengths measured in the desiccated tailings are not true measures of undrained shear strength. .

\subsection{Effect of sample disturbance}

In all simple shear and triaxial tests, the method of extraction of tubes and transporting samples to the strength measurement devices was performed very carefully to avoid any sample disturbance. However, these processes inevitably cause some disturbance to the soil. The effect of this is not expected to be of concern when comparing the results from this study since a consistent procedure was adopted throughout. Kim (2010) performed monotonic simple shear tests on reconstituted desiccated-rewetted tailings prepared in the simple shear mould (Figure 6.75). Comparing responses of the reconstituted samples prepared by Kim (2010) and the simple shear results of this study, it is found that the small difference between the responses could be attributed to sample disturbance/or density. 


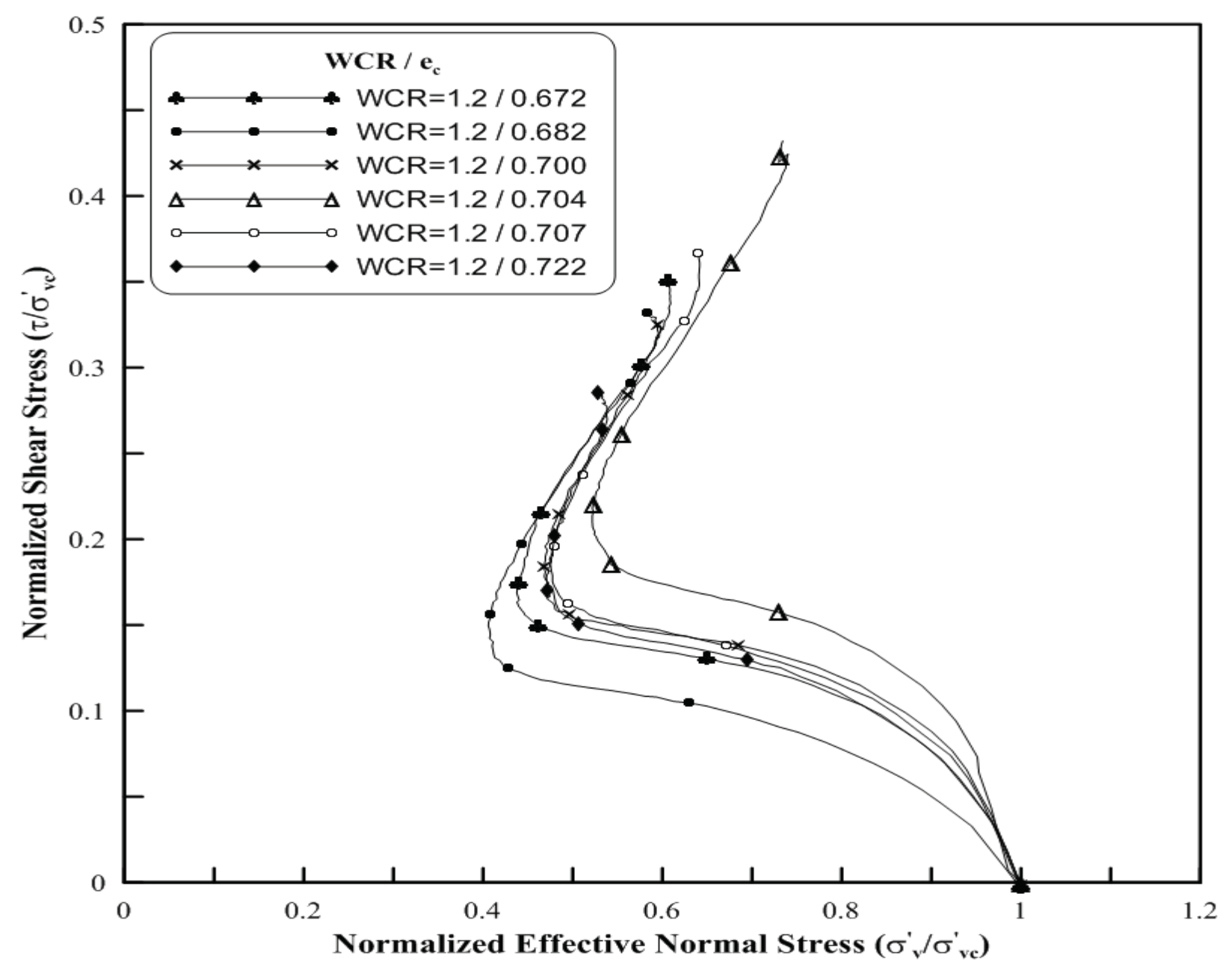

Figure 6.75 The influence of desiccation on monotonic simple shear response of reconstituted gold tailings prepared in the simple shear mould 


\section{Chapter 7: Discussions and conclusions}

This chapter consists of discussions, summary and conclusions. In the first part of the chapter, it is attempted to quantitatively compare the shear behaviour of mechanically over-consolidated and desiccated-rewetted tailings using the suction stress concept of Lu and Likos (2006). The influence of desiccation history on the strength of the tailings is then discussed based on simple shear, triaxial and vane shear results. The shear behaviour is then compared with dewatering behavior to develop guidelines for drying or cycle times for TT stacks with multipoint deposition. Important outcomes and final conclusions are presented in the last part of the chapter.

\subsection{Quantifying overconsolidation derived by desiccation}

As discussed in chapter 2, there are several approaches to quantify the contribution of matric suction to effective stress in partially saturated soils. These can be broadly classified into two groups including: 
1) The modified effective stress approach, proposed by Bishop (1959)

2) The independent stress state variable approach, which is attributed to the work of Fredlund and Morgenstern (1977).

The contribution of matric suction to effective stress $\left(\sigma^{s}\right)$ of unsaturated soils can be derived from equations 2.13 and 2.18 as:

$$
\begin{array}{lll}
\sigma^{s}=-\left(u_{a}-u_{w}\right) \cdot S_{r} & \text { Lu and Likos }(2006,2010) & \text { Equation 7.1 } \\
\sigma^{s}=-\left(u_{a}-u_{w}\right) \cdot S_{r}^{\mathrm{K}} & \text { From Vanapalli (2010) } & \text { Equation 7.2 }
\end{array}
$$

where $\mathrm{S}_{\mathrm{r}}$ is degree of saturation and $\mathrm{K}$ is a fitting parameter. In fact, equation 7.2 has added an empirical correlation factor "K" to Lu and Likos (2010)'s equation in an attempt to further refine this method, which can be calculated by one of equations $2.19,2.20$ or 2.21 .

The term overconsolidation ratio derived by desiccation $\left(\mathrm{OCR}_{\mathrm{D}}\right)$ is defined by the ratio of suction stress achieved during drying, and the maximum effective consolidation stress (applied vertically in the simple shear device) after rewetting:

$$
O C R_{D}=\frac{\text { (Maximum suction stress during desiccation) }}{\text { (Maximum effective consolidation stress, } \left.\sigma_{\mathrm{v}}^{\prime}\right)}=\frac{\sigma_{\mathrm{max}}^{\mathrm{s}}}{\sigma_{\mathrm{v}}^{\prime}}
$$

Equation 7.3

Table 7.1 presents the values of suction stress and $\mathrm{OCR}_{\mathrm{D}}$ for Bulyanhulu tailings at different water contents. The values of suction stresses are calculated based on equations 7.1 and 7.2; values of $\mathrm{OCR}_{\mathrm{D}}$ are calculated based on equation 7.3 and considering $50 \mathrm{kPa}$ vertical effective 
consolidation pressure, which was applied under the simple shear device. Figure 7.1 also compares the SWCC of Bulyanhulu tailings with SSCC curves obtained by equations 7.1 and 7.2. It is clear the applying " $\mathrm{K}$ " factor reduced the values of suction stress especially at higher degrees of desiccation and resulted in more reasonable suction stresses. However, as mentioned in chapter 6, the responses of mechanically overconsolidated samples were qualitatively different from the responses of desiccated-rewetted samples $\left(\mathrm{OCR}_{\mathrm{D}}\right.$ samples). Figures 6.14 and 6.15 present qualitative difference between $\mathrm{OCR}_{\mathrm{D}}$ and $\mathrm{OCR}_{\mathrm{M}}$ samples. Kim et al. (2011) also showed that specimens with higher mechanical overconsolidation are much more resistant to liquefaction under cyclic loading in comparison to samples with lower OCR or normally consolidated specimens. This observation agrees with the observations under monotonic loading.

Table 7.1 Values of suction stress and $\mathrm{OCR}_{\mathrm{D}}$

\begin{tabular}{|c|c|c|c|c|c|c|}
\hline $\begin{array}{c}\text { GWC } \\
(\%)\end{array}$ & $\begin{array}{c}\text { Degree of } \\
\text { Saturation }\end{array}$ & $\begin{array}{c}\text { Matric } \\
\text { Suction }(\mathrm{kPa})\end{array}$ & $\begin{array}{c}\text { Suction } \\
\text { Stress }^{1}(\mathrm{kPa})\end{array}$ & $\begin{array}{c}\text { Suction } \\
\text { Stress }^{2}(\mathrm{kPa})\end{array}$ & OCR $_{\mathrm{D}}{ }^{3}$ & OCR $_{\mathrm{D}}{ }^{4}$ \\
\hline 38 & 0.995 & 0.1 & 0.1 & 0.1 & 1 & 1 \\
34 & 0.967 & 0.8 & 0.8 & 0.8 & 1 & 1 \\
30 & 0.93 & 4 & 3.7 & 3.6 & 1 & 1 \\
25 & 0.88 & 20 & 17.6 & 16.9 & 1 & 1 \\
22 & 0.82 & 70 & 57.4 & 54.0 & 1.15 & 1.08 \\
21 & 0.785 & 90 & 70.7 & 65.6 & 1.41 & 1.31 \\
17 & 0.65 & 200 & 130.0 & 113.9 & 2.60 & 2.28 \\
16 & 0.585 & 300 & 175.5 & 148.9 & 3.51 & 2.98 \\
13 & 0.5 & 400 & 200.0 & 161.8 & 4.00 & 3.24 \\
11 & 0.42 & 500 & 210.0 & 161.3 & 4.20 & 3.23 \\
\hline
\end{tabular}

${ }^{1}$ Suction stress based on Lu and Likos (2006)'s equation

${ }^{2}$ Suction stress based on Vanapalli (2010)'s equation and equation 2.20

${ }^{3} \mathrm{OCR}_{\mathrm{D}}$ based on Lu and Likos (2006)'s equation and $50 \mathrm{kPa}$ vertical effective consolidation pressure

${ }^{4} \mathrm{OCR}_{\mathrm{D}}$ based on Vanapalli (2010)'s equation and $50 \mathrm{kPa}$ vertical effective consolidation pressure 


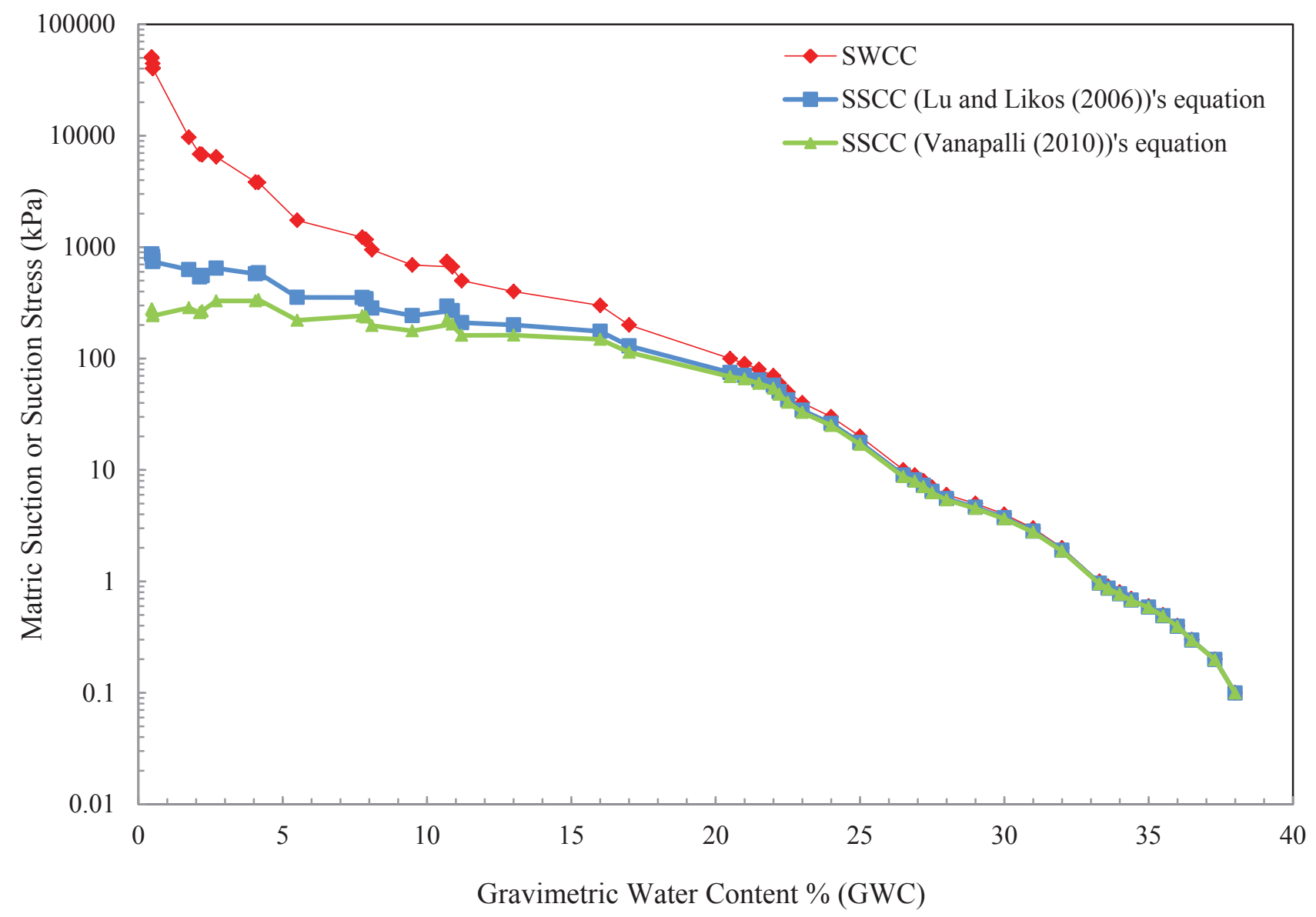

Figure 7.1 SWCC and SSCC of Bulyanhulu tailings

\subsubsection{Validity of suction stress method}

It is recognized that the use of the simple estimate of suction stress $\left(-\left(u_{a}-u_{w}\right) \cdot S_{r}\right)$ or $\left.-\left(u_{a}-u_{w}\right) \cdot S_{r}^{\mathrm{K}}\right)$ may be insufficient to represent the actual equivalent stress imparted to the sample by matric suction. The results of this study indicate that the responses of mechanically overconsolidated samples and desiccated-rewetted samples were qualitatively and quantitatively different. These differences could be attributed to differences in the stress field and the resulting differences in the fabrics. Desiccation generates isotropic internal forces inside the samples. The 
rewetting process dissipates these internal forces; Mechanical over-consolidation in simple shear does not yield isotropic stress state due to the enforced $\mathrm{K}_{0}$ conditions. The influence of OCR on $\mathrm{K}_{0}$ also adds to the complexity. In other words, it is apparent that very different material behavior, qualitatively as well as quantitatively, is generated by preconsolidation by suction rather than by preconsolidation by mechanical loading. Fine grained soils are known to develop different microstructure under consolidation or desiccation (Simms and Yanful 2004, 2005). Figures 7.2 and 7.3 present MIP results on gold tailings under different conditions. Figure 7.2 presents the effect of consolidation on the cumulative intrusion of settled and consolidated tailings. Figure 7.3 compares the cumulative intrusion of dilative and contractive behaviors.

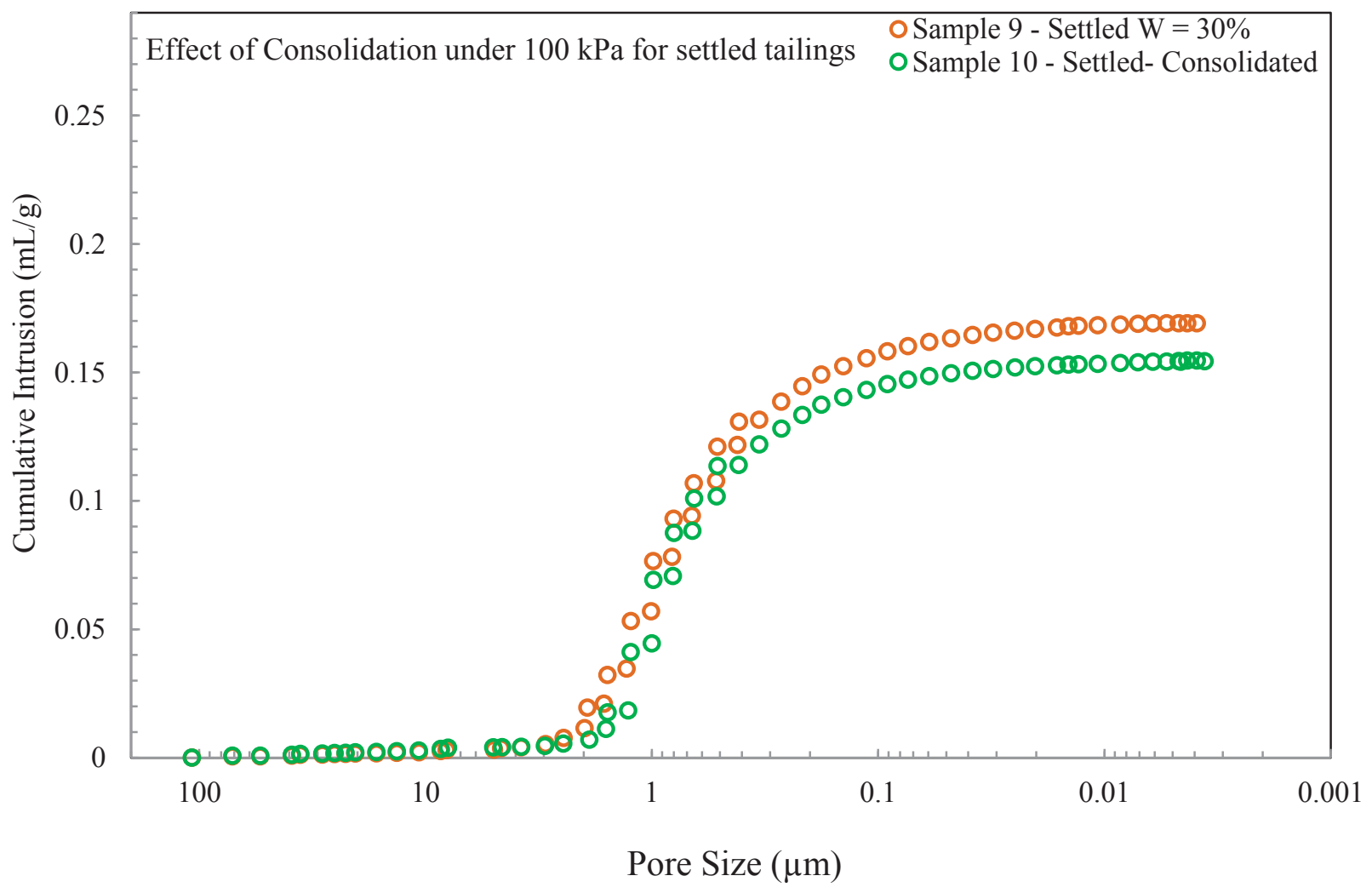

Figure 7.2 The influence of consolidation under $100 \mathrm{kPa}$ on MIP results of thickened gold tailings 


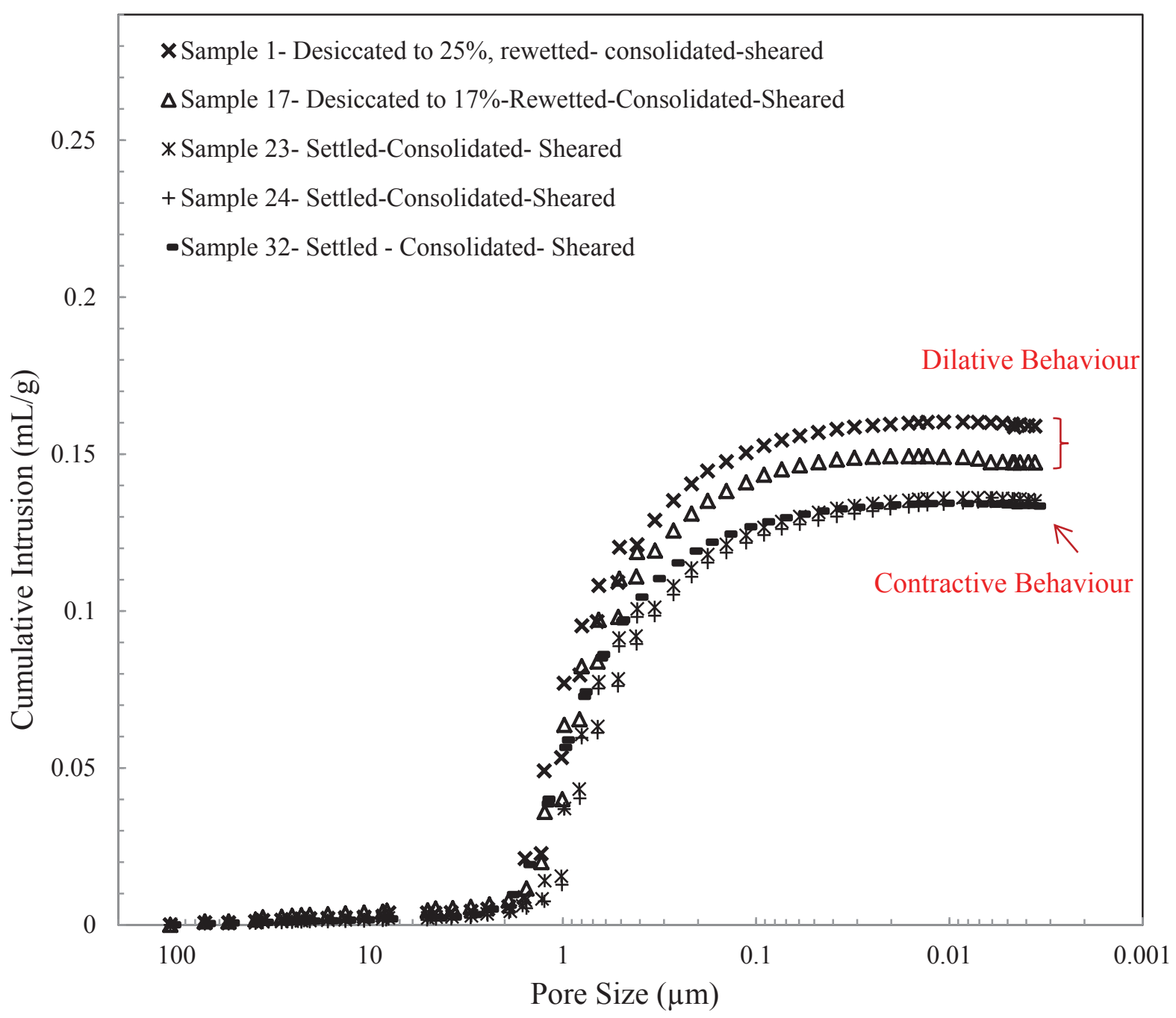

Figure 7.3 The comparison of dilative and contractive simple shear responses under $100 \mathrm{kPa}$ on MIP results of thickened gold tailings

Using Lu and Likos (2006, 2010)'s approach to compare equivalent stress histories, mechanically overconsolidated samples exhibited a much more significant increase in strength in both monotonic and cyclic loading, but exhibited smaller degrees of dilation under monotonic loading. Therefore, simulating desiccation through mechanical overconsolidation is not accurate and would lead to unconservative design. 


\subsection{Comparisons based on the results of simple shear tests}

Figure 7.4 presents shear stress at PT and shear strength of samples desiccated (or desiccatedrewetted) to different values of water content under $400 \mathrm{kPa}$ vertical effective consolidation pressure. It is clear that increasing desiccation or desiccation history up to $26 \%$ significantly increased both shear stress at PT and shear strength. Continuing desiccation or desiccation history to the shrinkage limit further increased the shear stress at PT and shear strength. Desiccation beyond the shrinkage limit resulted in marginal reduction in shear stress at PT in both desiccated and desiccated-rewetted samples. In summary, it is clear that the effect of the degree of desiccation beyond the SL is more influential in increasing shear strength at large strains than increasing shear stress at PT.

Figure 7.5 presents shear stress at PT and shear strength of samples desiccated-rewetted to different values of water content under $50 \mathrm{kPa}$ and $100 \mathrm{kPa}$ vertical effective consolidation pressure. Figure 7.5 shows that both shear stress at PT and shear strength have significantly increased with increasing degree of desiccation from $\mathrm{Wd}=30 \%$ to $\mathrm{Wd}=26 \%$. For samples consolidated to $50 \mathrm{kPa}$, the value of shear stress at PT significantly increased from settling to $\mathrm{Wd}$ $=26 \%$. However, beyond $\mathrm{Wd}=26 \%$, increasing degree of desiccation history did not increase the shear stress at PT significantly. Similarly, under $100 \mathrm{kPa}$ consolidation pressure. Increasing degree of desiccation history from $\mathrm{Wd}=26 \%$ to $\mathrm{Wd}=18 \%$ and beyond that under $100 \mathrm{kPa}$ consolidation pressure slightly increased the shear stress at PT but not as beneficial as desiccation to $\mathrm{Wd}=26 \%$. Conversely, increasing degree of desiccation history continuously led to increasing shear strength at both consolidation pressure levels $(50 \mathrm{kPa}$ and $100 \mathrm{kPa})$. 


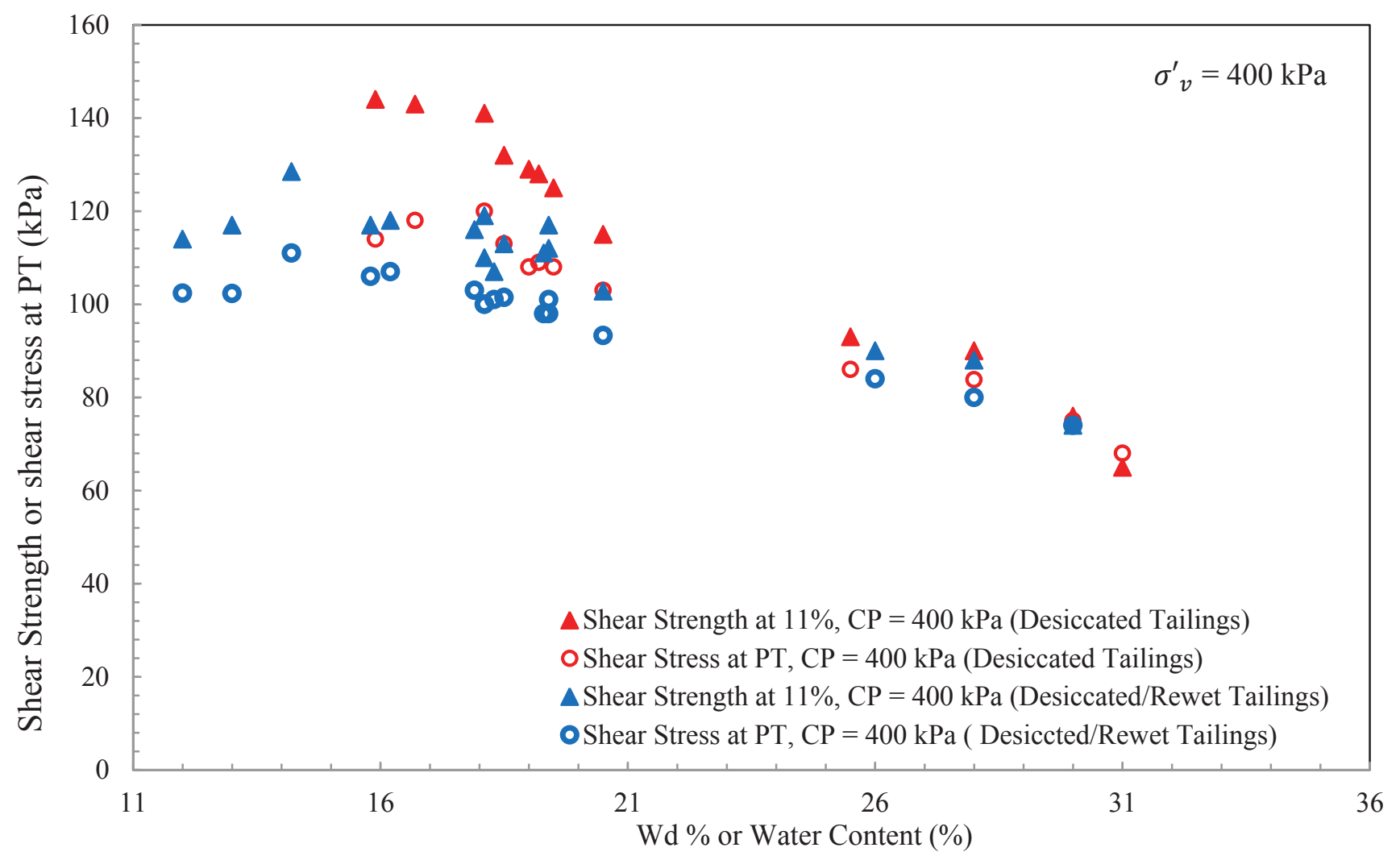

Figure 7.4 Shear stress at PT and shear strength for different degrees of desiccation under 400 $\mathrm{kPa}$ vertical effective consolidation pressure

Continuous increase in shear strength (at 11\% shear strain) can be seen for samples desiccated beyond the shrinkage limit and high degrees of desiccation histories $(\mathrm{Wd}=12 \%)$. Figure 7.6 presents the values of normalized shear stress at PT for different degrees of desiccation history. Normalized values of shear stress at PT are higher for higher consolidation pressures. Figure 7.6 also compares the values of shear stress at PT for samples extracted from the drying box (under $100 \mathrm{kPa}$ ) and samples prepared by small scale method (under $100 \mathrm{kPa}$ ). It is shown that the PT shear stresses of samples obtained from the drying box are higher than samples prepared by small scale methods especially for higher degrees of desiccation. The first layer of the drying box experienced more cycles of desiccation and re-wetting. It is not clear at this point whether the 


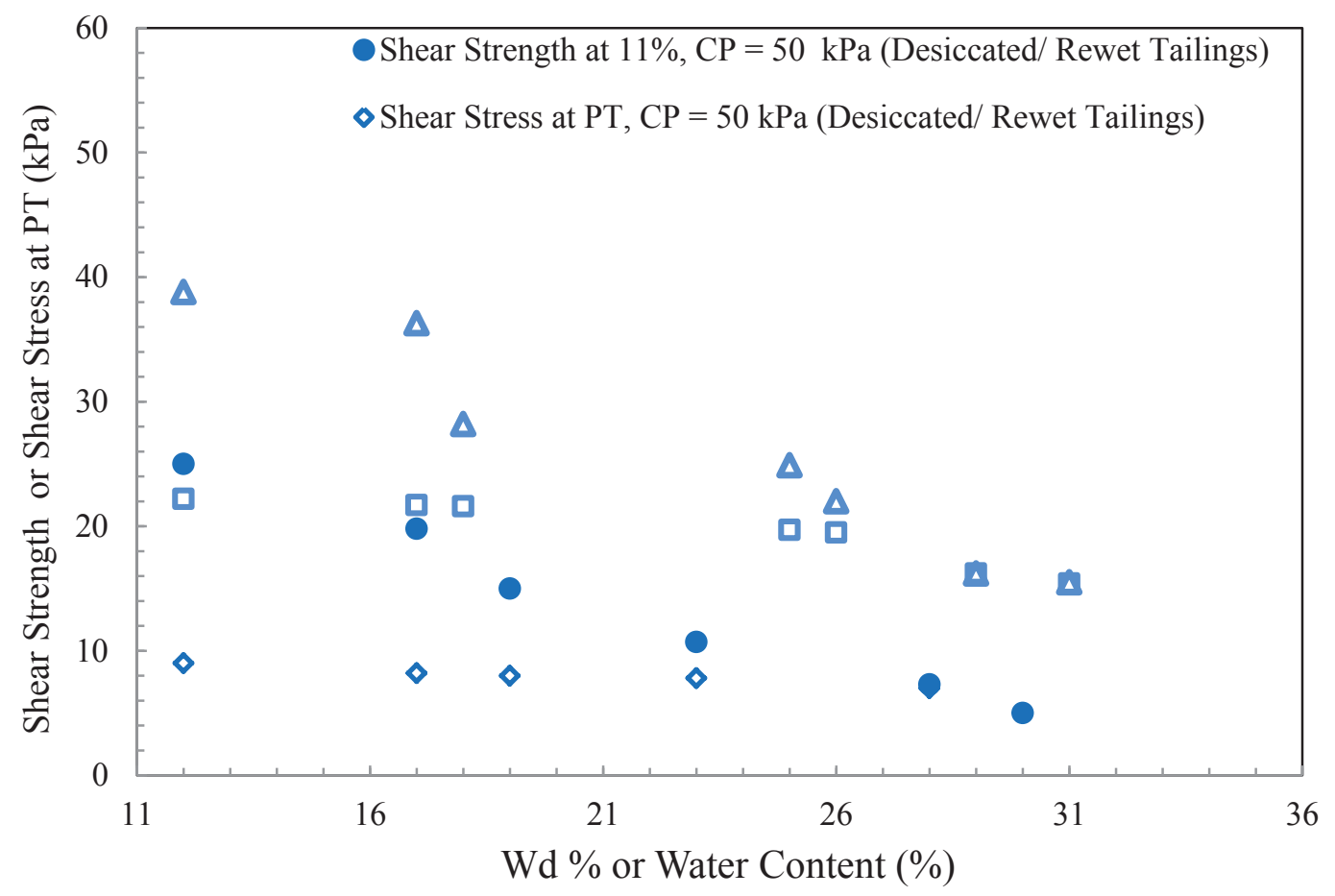

Figure 7.5 Shear stress at PT and shear strength for different degrees of desiccation under $50 \mathrm{kPa}$ and $100 \mathrm{kPa}$ vertical effective consolidation pressure

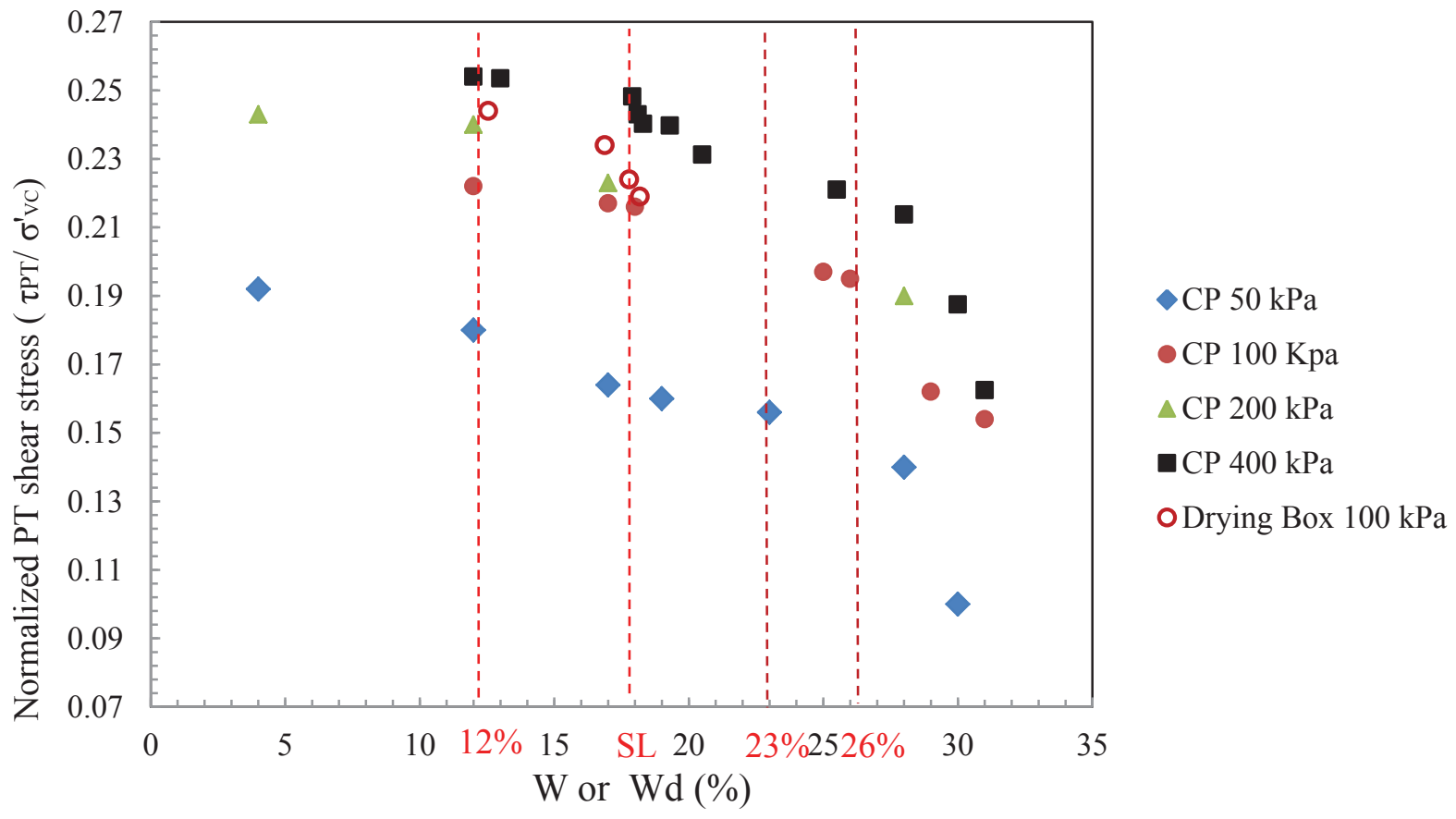

Figure 7.6 Normalized shear stress at PT for different degrees of desiccation 
higher shear stress of this layer is attributed to more cycles of desiccation and rewetting in the drying box samples or any other factors.

\subsubsection{The impact of density on the simple shear results}

Figure 7.7 presents the values of shear stress at PT for all simple shear tests performed on samples prepared by the small scale method. It is clearly shown that desiccated samples exhibited higher shear stress at PT at even at higher void ratios (lower density). This phenomenon is attributed to the generation of suction in the sample during desiccation, which makes the sample stiffer and reduces compression during subsequent consolidation. Likewise, though desiccated- rewetted samples had higher void ratio (lower density) than saturated samples, they exhibited higher value of shear stress at PT than saturated samples. Figure 7.8 shows compressibility zones of desiccated, desiccated-rewetted and saturated samples under 400 $\mathrm{kPa}$ vertical effective consolidation pressure. As mentioned, saturated samples compress more during consolidation than desiccated and desiccated-rewetted samples. The starting points of void ratio of desiccated samples are lower than saturated samples as the desiccated samples have previously reached lower void ratios during desiccation. The stiffness of desiccated-rewetted samples is lower than desiccated samples and they can reach lower void ratios than desiccated samples.

Figure 7.9 presents the effect of degree of desiccation on monotonic and cyclic shear strength of thickened gold tailings. The degree of desiccation is presented based on the division of maximum suction stress during desiccation over the vertical effective consolidation pressure 
$\left(\frac{\sigma_{\max }^{S}}{\sigma^{\prime} v c}\right)$. Values of suction stress are calculated using Lu and Likos (2006)'s equation (equation 7.1). It should be noted that though the accuracy of suction stress method is not clear according to discussions at the beginning of this chapter, this method has been only used to compare the degrees of desiccation. Monotonic and cyclic shear stresses at PT are normalized by the value of shear stress at PT of normally consolidated tailings (no desiccation). Figure 7.9 shows that increasing degree of desiccation up to the AEV significantly increased the normalized shear stress at PT. Continuing desiccation from the AEV to the SL slightly improved the values of shear stress at PT. Continuing desiccation beyond the SL is beneficial for increasing shear stress at PT; however, the rate of improvement is not as beneficial as desiccation to the AEV.

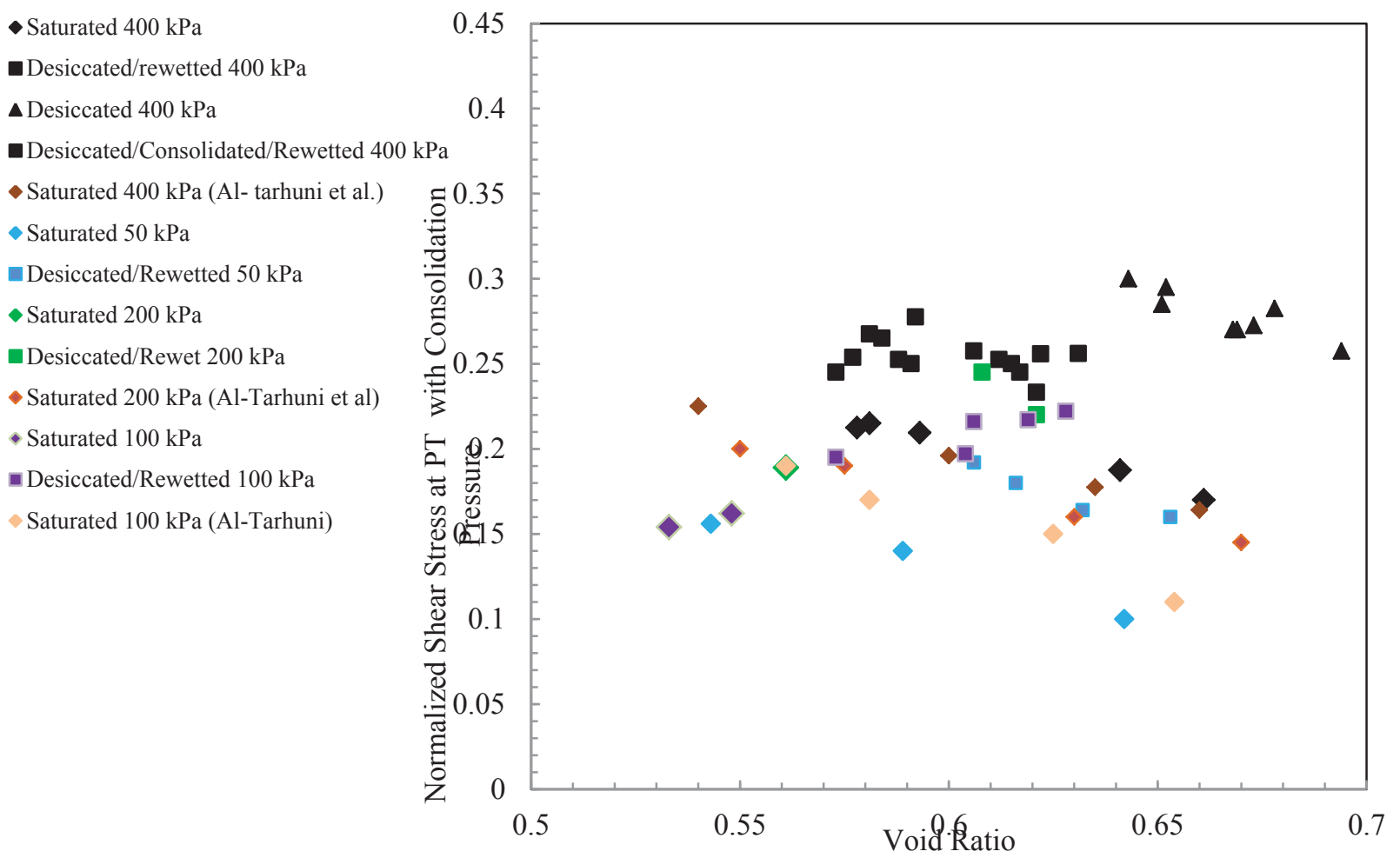

Figure 7.7 Normalized shear stress at PT versus void ratio obtained from the simple shear tests 


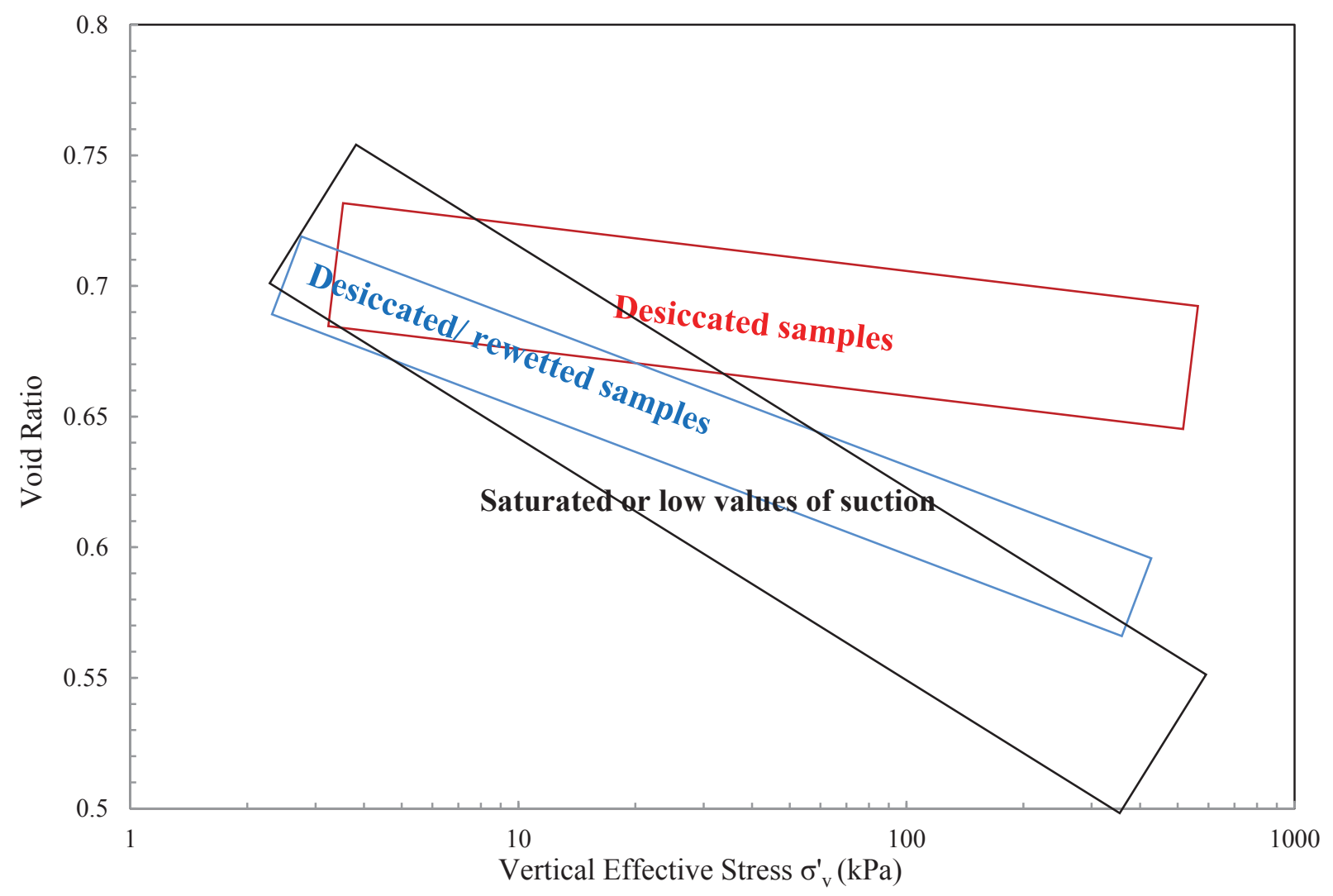

Figure 7.8 Compressibility of saturated, desiccated and desiccated/rewetted samples under 400 $\mathrm{kPa}$ vertical effective consolidation pressures

\subsection{Comparisons based on the results of triaxial tests}

Figure 7.10 presents the values of $q$ at PT and shear strength based on modified triaxial results under $50 \mathrm{kPa}$ consolidation cell pressure. It is shown that the values of $q$ at PT continuously increased with increasing degree of desiccation history up to $\mathrm{Wd}=23 \%$. Continuing degree of desiccation history to the shrinkage limit slightly improved the value of $q$ at PT. Increasing degree of desiccation history beyond the shrinkage limit was not beneficial in terms of increasing $q$ at PT. High degrees of desiccation history $(\mathrm{Wd}=14 \%)$ marginally reduced the value of $q$ at 


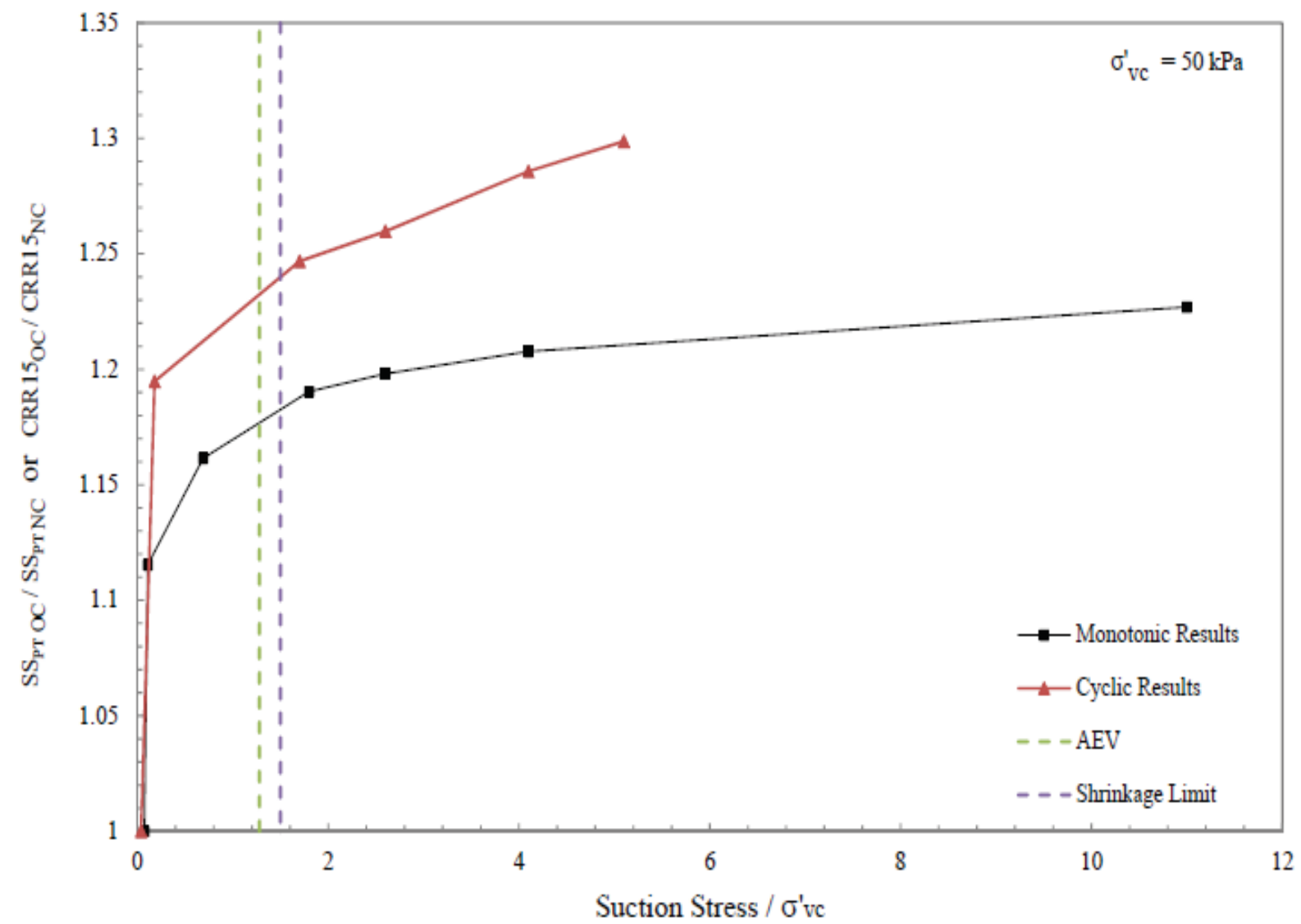

Figure 7.9 The influence of degree of desiccation on monotonic and cyclic shear strength of the tailings

PT. In contrast, the values of shear strength (at $11 \%$ axial strain) continuously increased with increasing degree of desiccation history. Figure 7.11 compares the values of shear stress at PT and shear strength of samples at different degrees of desiccation under simple shear and modified triaxial tests. To approximately compare the shear strength of modified triaxial with simple shear tests, axial strain of $7.3 \%$ is assumed to be equivalent with $11 \%$ shear strain assuming that there is no volumetric strain in the modified undrained triaxial tests. As expected, both values of shear stress at PT and shear strength are significantly higher in triaxial tests compared to simple shear. This phenomenon could be attributed to the following reasons: i) while constant volume simple shear tests assume truly undrained condition, samples of modified triaxial tests are not fully 


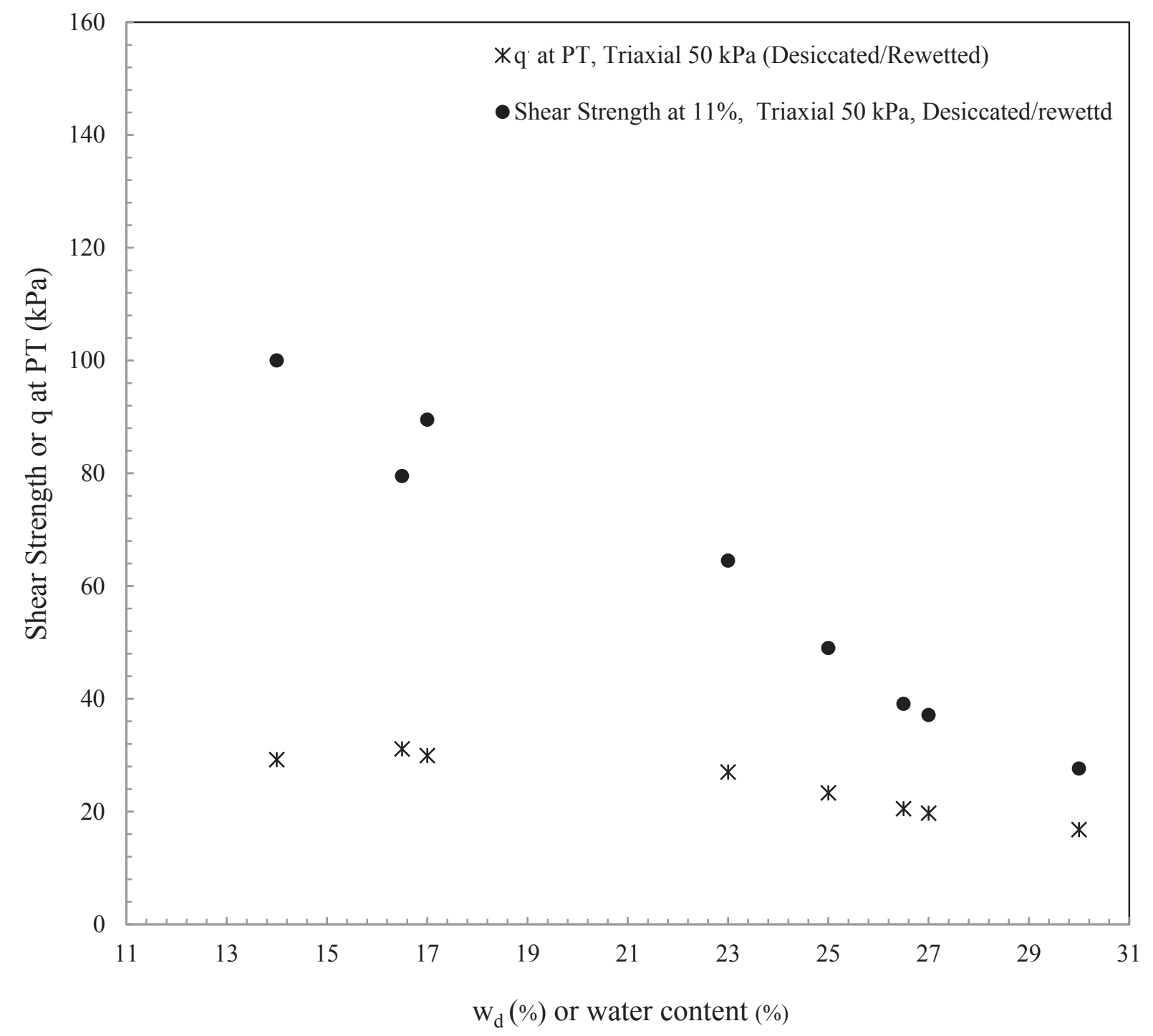

Figure 7.10 shear strength and $q$ at PT for different degrees of desiccation under $50 \mathrm{kPa}$ consolidation cell pressure

undrained because of the specimens are not fully saturated, ii) loading mode and fabric anisotropy effects at constant void ratio, iii) applying $30 \mathrm{kPa}$ suction to stabilize the triaxial samples. Figure 7.12 presents anisotropic behavior of thickened gold tailings under extension and cyclic triaxial tests. Figure 7.13 compares cyclic simple shear and triaxial results of settled tailings. As expected, the cyclic triaxial resistance is much higher than the simple shear resistance. 


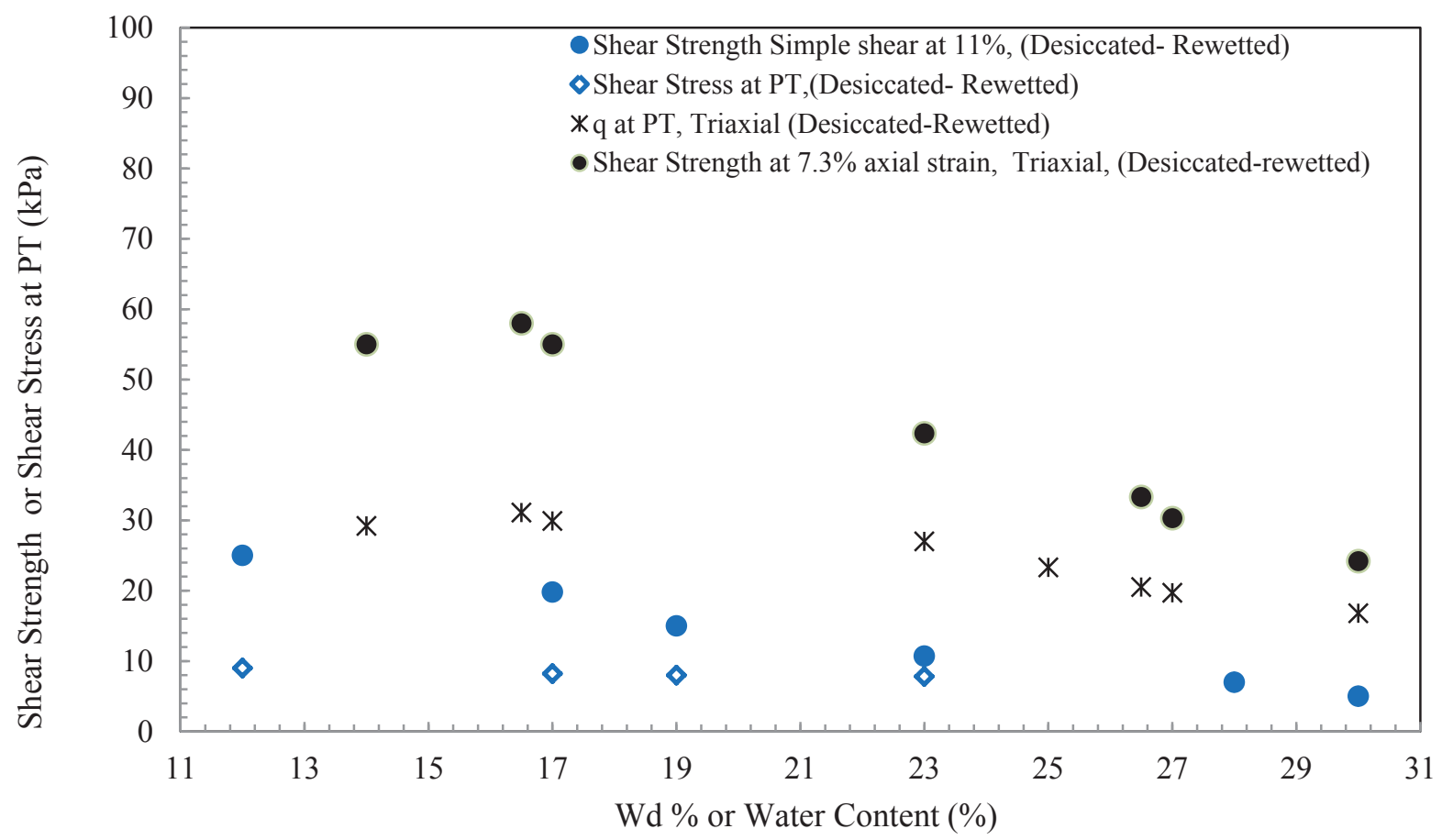

Figure 7.11 Comparison of the monotonic results of simple shear tests and triaxial tests under 50 $\mathrm{kPa}$ consolidation pressure
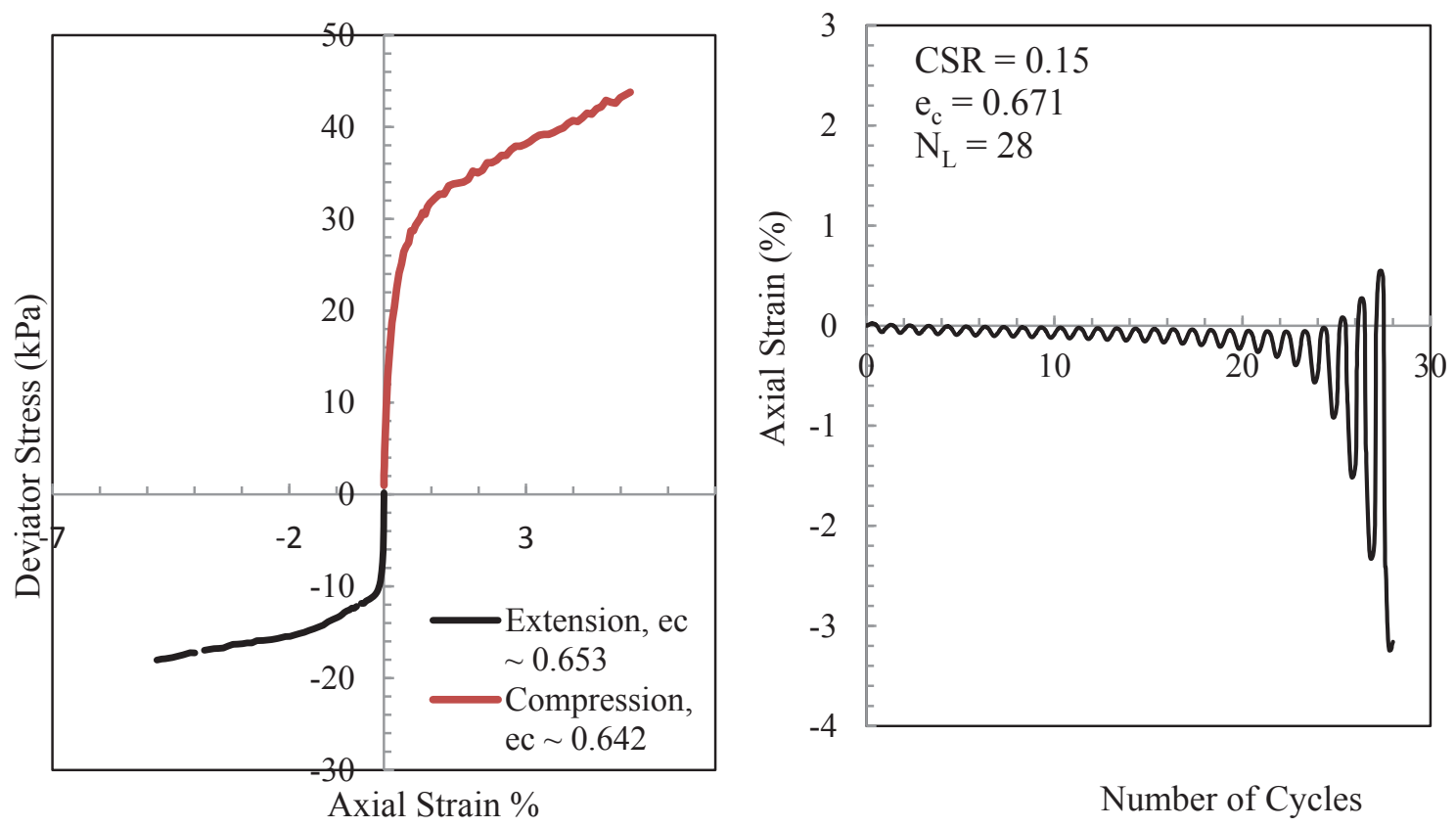

Figure 7.12 Anisotropic behaviour of thickened tailings under extension and cyclic triaxial tests 


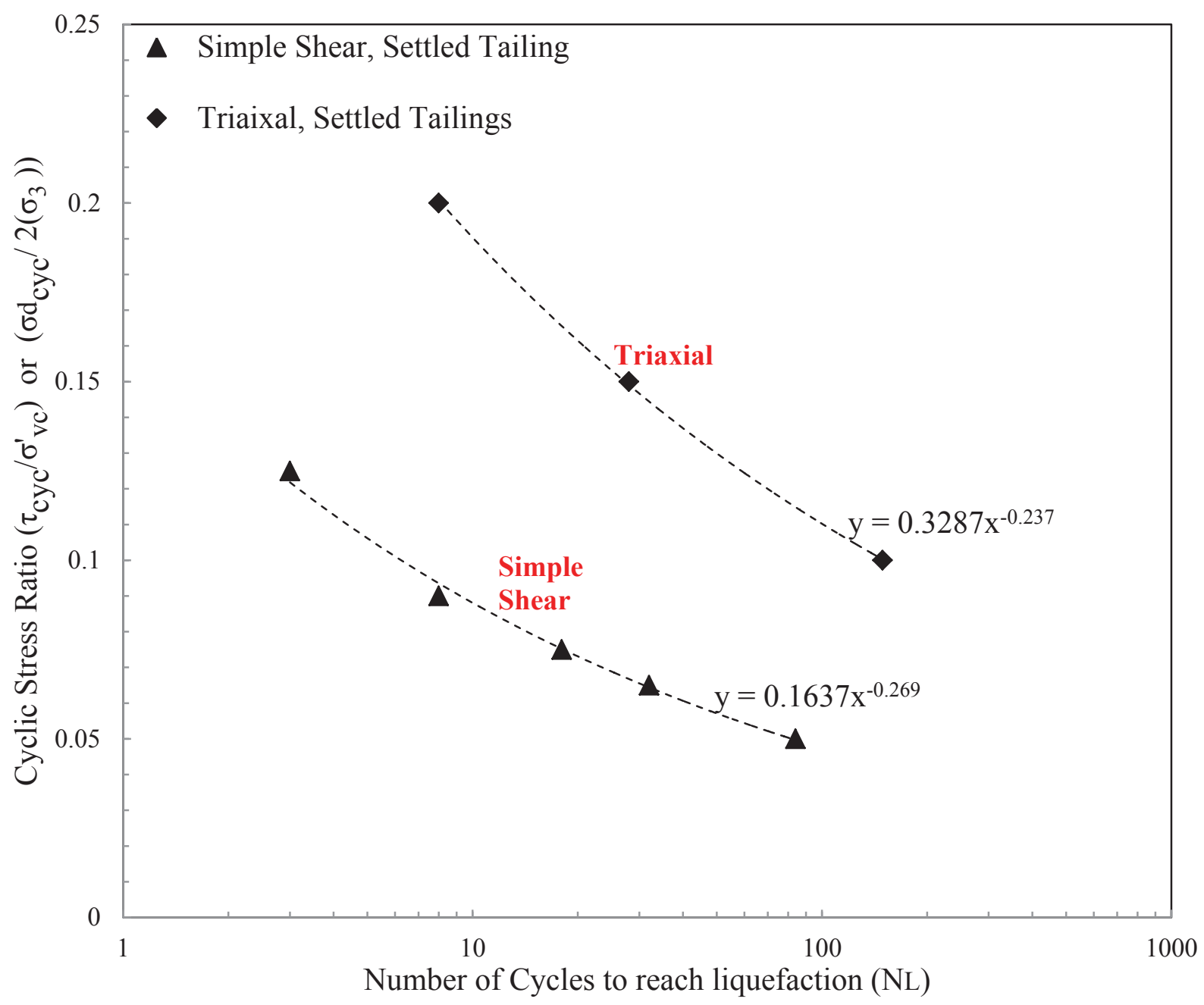

Figure 7.13 Comparison of cyclic simple shear and triaxial results

\subsection{Critical / steady state analysis with respect to desiccation history}

Figure 7.14 presents values of final void ratio after consolidation $\left(e_{c}\right)$ versus normalized shear strength $\left(\frac{S_{u}}{\sigma_{v c}^{\prime}}\right)$ at $11 \%$ shear strain. For most of dilative samples (samples experienced small degree of desiccation up to $\mathrm{Wd} \sim 23 \%$ ), the value of final shear strength or/ effective stress is similar to the critical/steady state strength since the shear stress does not vary significantly with increasing shear strain. However, for samples desiccated beyond the AEV $(\mathrm{Wd}<22 \%)$, the final 
shear stress at failure cannot be the steady state strength since the shear stress might increase after $11 \%$ shear strain. Figure 7.14 a shows that desiccated-rewetted samples exhibited higher normalized shear strength than saturated samples in spite of looser void ratio. This phenomenon is attributed to the potential differences in the fabric due to the effect of desiccation history. In other words, though samples experienced a desiccation history gained more strength in the desiccation-rewetting process, they were stiffer than settled samples to reach denser void ratios. Figure $7.14 \mathrm{~b}$ shows the effect of different degrees of desiccation on the normalized shear strength vs. void ratio. In general, samples with looser void ratio at the same degree of desiccation exhibited lower normalized shear strength, which is in agreement with conventional steady state analyses. However, these results clearly suggest that the steady state strength will be highly dependent on the degree of desiccation prior to shearing. Figure 7.15 compares the results of dilative tests versus the results of contractive tests. It is shown that most of contractive tests consisted of samples with no desiccation or low degrees of desiccation in spite of denser void ratio. Figures 7.14 and 7.15 show that the critical steady state line is not specific for desiccatedrewetted gold tailings. They also show that the final strength of desiccated-rewetted tailings is not uniquely related to void ratio (and thus not consistent with the CSS theories that expect a unique relationship between void ratio and steady state strength).

\subsection{Effect of rewetting}

It was found that desiccated samples exhibited higher shear strength than desiccated-rewetted samples. In order to compare the effect of rewetting, simple shear and vane shear results of desiccated and desiccated-rewetted samples that experienced same degree of desiccation are 

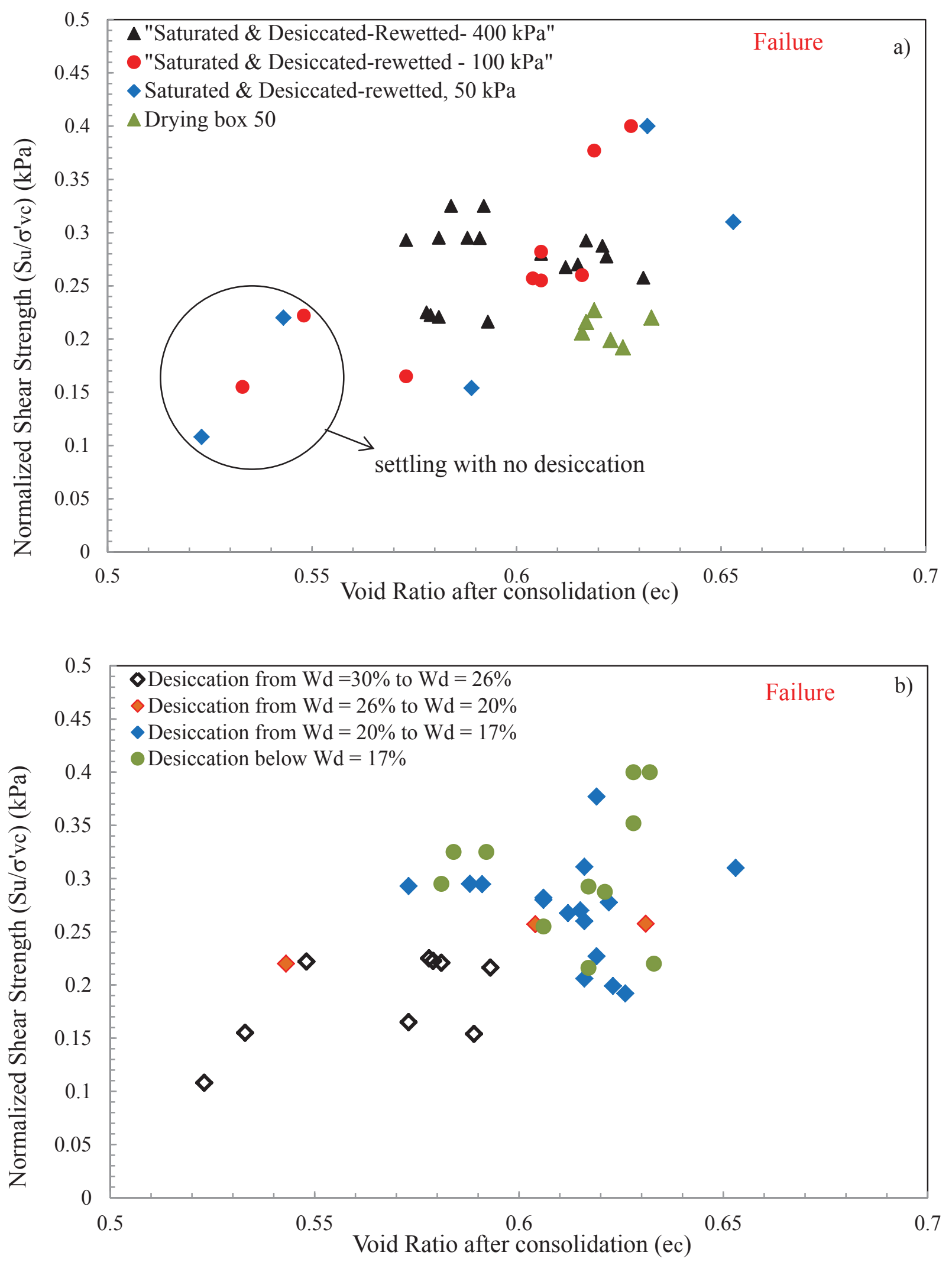

Figure 7.14 Normalized shear strength of simple shear tests vs. void ratio 


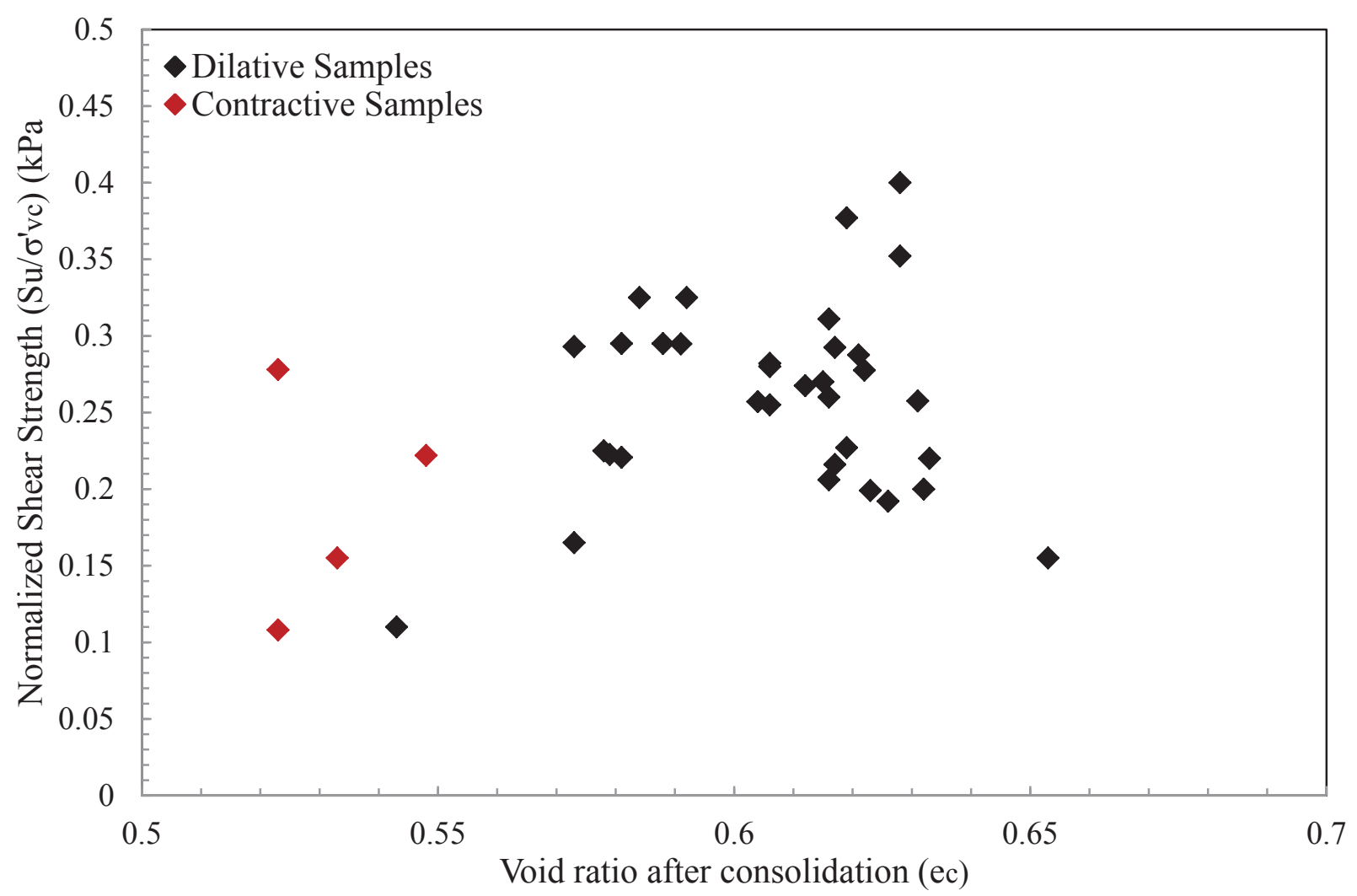

Figure 7.15 Comparisons of dilative and contractive responses

considered. Figure 7.16 presents the simple shear results of desiccated and desiccated-rewetted samples under $400 \mathrm{kPa}$. The value of matric suction noted in the figure corresponds to the matric suction before consolidation and based on the SWCC. It is shown that the current water contents of desiccated samples (W) are fairly the same as water contents of desiccated-rewetted tailings before rewetting $(\mathrm{Wd})$. As seen in Figure 7.16, the void ratio after rewetting reduced from $0.64 \sim$ 0.68 to 0.60 to 0.62 . Consequently, rewetting makes the sample more compressible leading to a lower void ratio after consolidation. Since the matric suction of the rewetted samples returned to zero, desiccated-rewetted samples exhibited lower shear stresses than desiccated samples. Figure 7.17 also shows the effect of rewetting based on the vane shear results. It is clear that up to around $\mathrm{W}=28 \%$, there is no significant difference between undrained shear strength of 
desiccated and desiccated-rewetted samples. With increasing degree of desiccation, the difference between the undrained shear strength of desiccated and desiccated-rewetted samples becomes more significant.

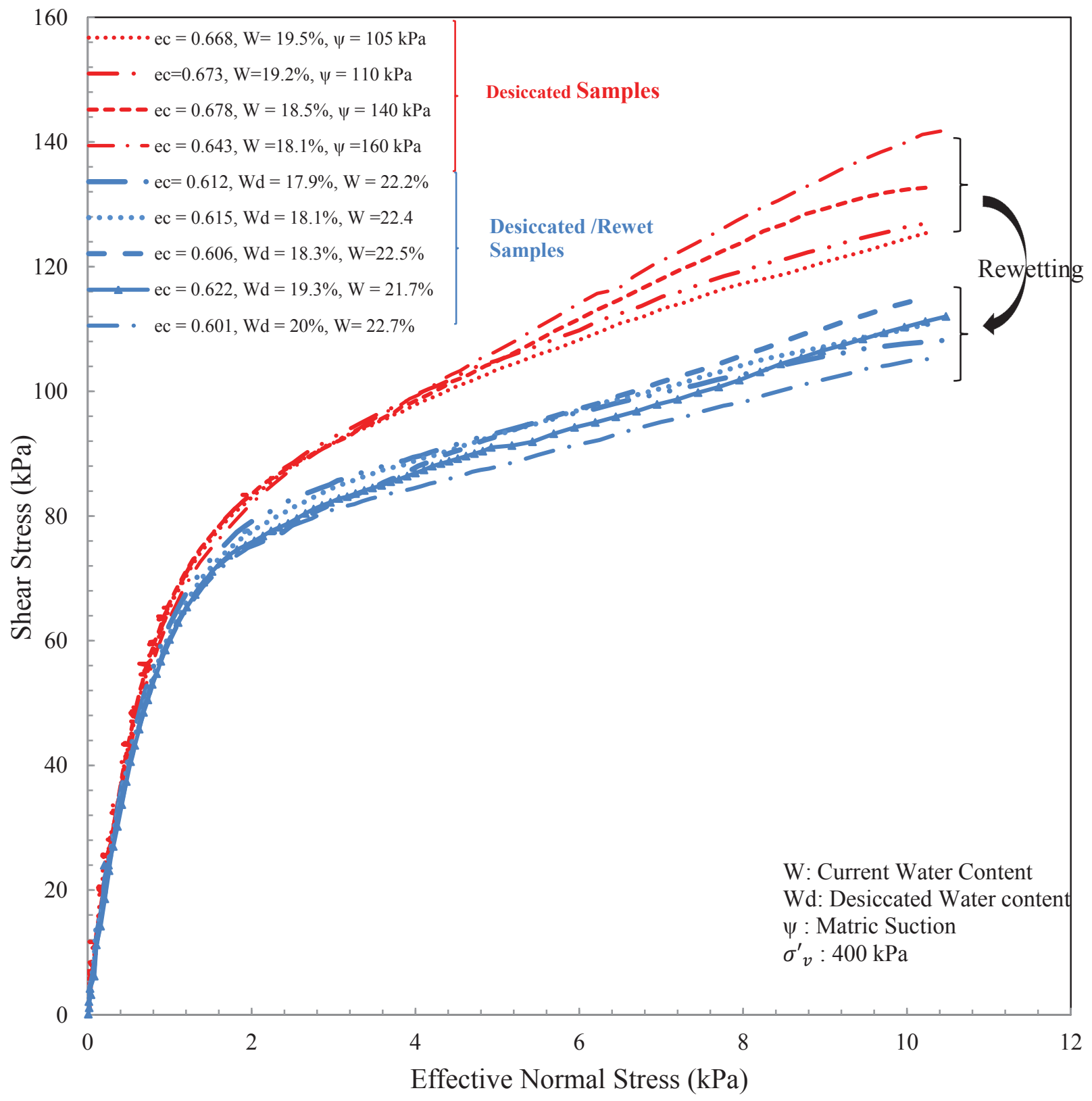

Figure 7.16 Impact of rewetting for simple shear samples consolidated under $400 \mathrm{kPa}$ 

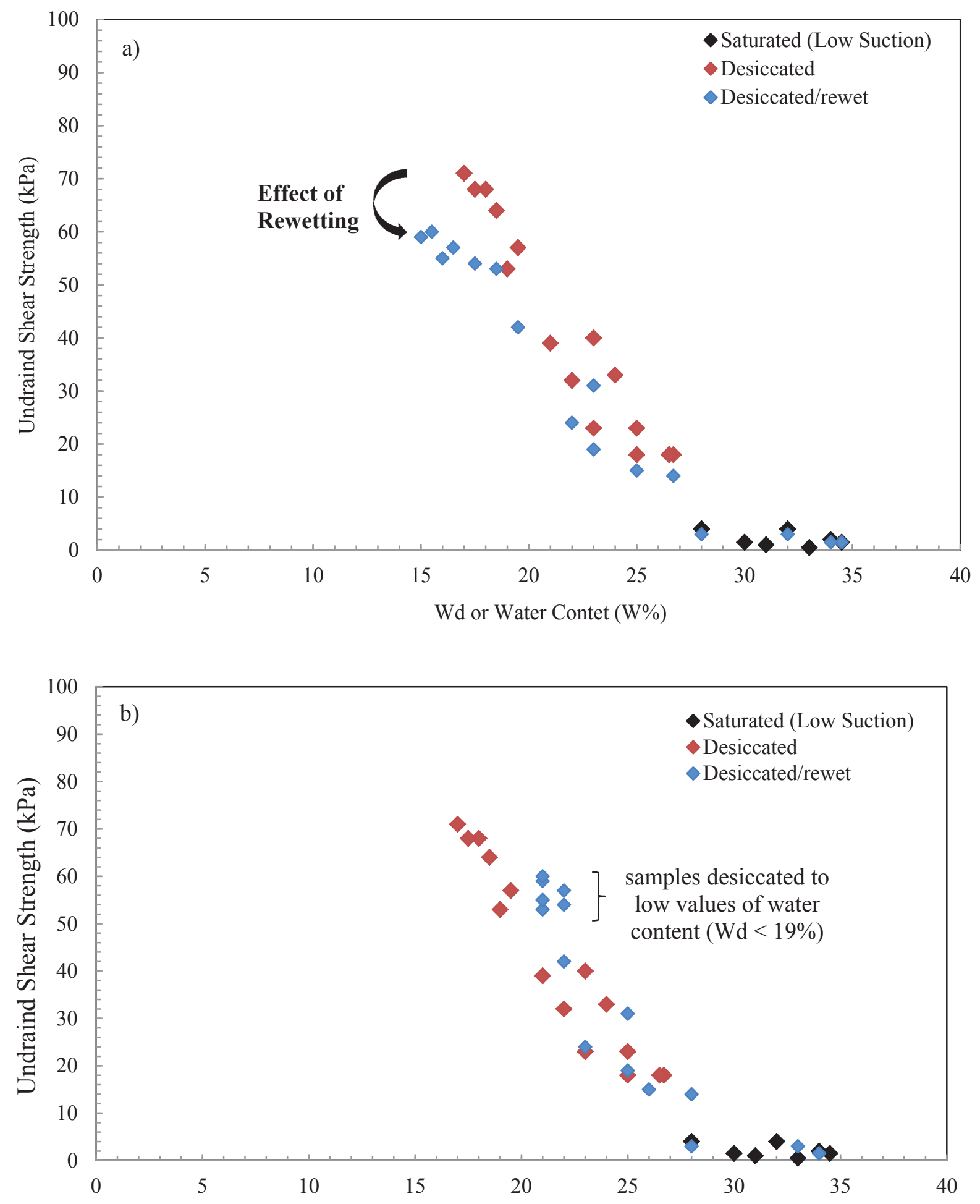

Current Water content \%

Figure 7.17 Impact of rewetting for vane shear tests a) desiccated water content, b) current water content 


\subsection{Applicability of the cyclic results of this study for different earthquakes}

The cyclic resistance ratios determined in this study (CRR15, CRR10) represent the seismic strength of the tailings (i.e., capacity to withstand dynamic loads) when subjected to specific number of uniform load cycles (e.g., 10 or 15). Clearly, the level of loading, generally represented by the cyclic stress ratio, CSR and calculated using PGA in practice, is dependent on the characteristics of the earthquake, nature of the geology and local site conditions. Thus, the equivalent number of cycles for an earthquake of a given magnitude may be affected by the local geology, and the determination of the CSR would become complicated. But, the reported CRR value for a given number of load cycles is unaffected by the geology. The local geology can affect the duration and frequency content of the time history of shaking due to an earthquake. In general, the predominate frequencies of motion vary from about $0.5 \mathrm{~Hz}$ to $5 \mathrm{~Hz}$, and this is a fairly small variation in the frequency from a practical perspective. The effect of such frequency variation on liquefaction susceptibility of soils is fairly minor (Mortezaie and Vucetic, 2013).

\subsection{Influence of desiccation on strength and cost development in a TT stack}

Multilayer deposition of the thickened tailings layers in a drying box was performed in order to simulate multilayer field deposition of thickened tailings. This method was a complementary experimental method to the small scale deposition of thickened tailings in a bucket. The main purpose of this experiment was to simulate the field deposition of thickened tailings and obtain samples with different stress/desiccation histories for the simple shear test. Five thickened

tailings layers were deposited at the pumping water content. Layers were allowed to dewater to different values of water content under controlled desiccation. The first layer experienced higher 
degree of desiccation. The degree of desiccation was lower for the second and third layers. Meanwhile, the third, fourth and fifth layers were allowed to desiccate to the same water content (shrinkage limit) but under different conditions. It was established that the thickness of the deposited thickened tailings layer affects the drying time of the layer. Shorter drying time of the fourth layer, which had smaller thickness, confirms this phenomenon (Simms et al, 2010, 2012). It was also found that the swelling potential of tailings after rewetting is not remarkable and GWC of desiccated-rewetted layers did not exceed 22\% 23\% after rewetting. Matric suction measurement of bottom layers after rewetting showed that the matric suction of bottom layers does not necessarily come back to zero after rewetting by a fresh layer. As the bottom layer is deeper, the value of matric suction is higher after rewetting. However, a heavy rainfall might still reduce the matric suction to zero value. Figure 7.18 and Table 7.2 presents dewatering time required to reach $\mathrm{W}=26 \%, 23 \%$ and shrinkage limit for all five layers. Table 7.2 shows that the drying time required to reach the shrinkage limit is four times longer than the drying time to reach $\mathrm{W}=26 \%$. In fact, the dewatering time to achieve given water content may increase with increasing depth of underlying tailings. This is because water transported to the surface to satisfy evaporative demand can come from tailings deeper than the fresh layer. This might make it challenging, costly or impractical for practitioners to dry the tailings to their shrinkage limit. However, as seen from the simple shear strength data, it may not be necessary to dry the tailings to the shrinkage limit, to achieve the required geotechnical performance. The contribution of desiccation through preconsolidation to strength begins to decrease as the AEV is exceeded. This is expected, as decreasing saturation reduces the contribution of matric suction to the stress field. What is less expected is that even a small amount of desiccation, say to only $26 \%$ water content from the post-settling water content of $30 \%$, makes a significant increase in strength - both in terms of strength at PT and failure; also the behaviour changes to stain hardening from strain softening. 


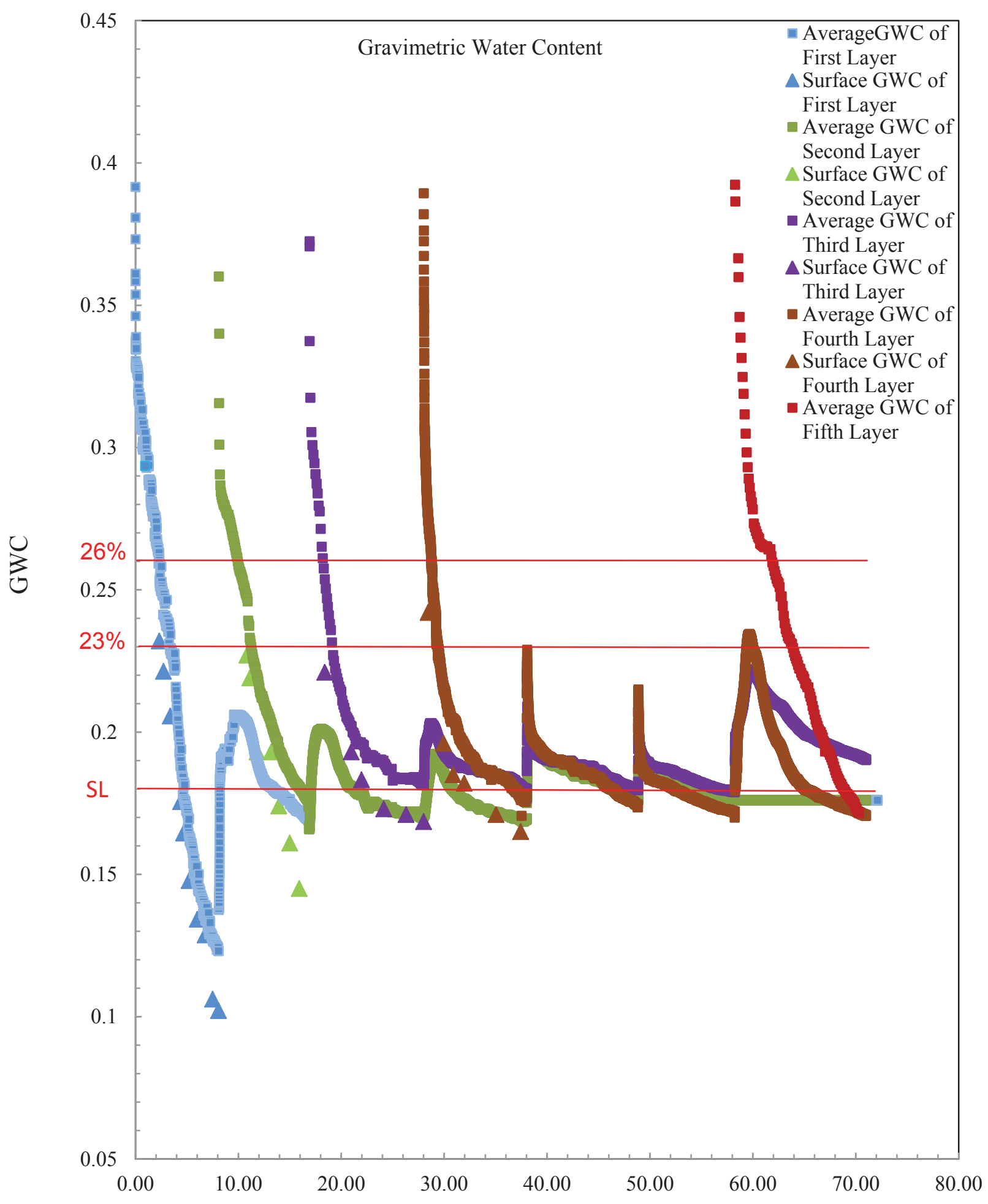

Time (Day)

Figure 7.18 Time required reaching different values of water contents 
Table 7.2 Drying time required to reach different values of water content obtained from the multi-layer deposition in the drying box

\begin{tabular}{cccc}
\hline & $\begin{array}{c}\text { Drying time to GWC } \\
26 \% \text { (days) }\end{array}$ & $\begin{array}{c}\text { Drying time to GWC }= \\
23 \% \text { (days) }\end{array}$ & $\begin{array}{c}\text { Drying time to GWC }= \\
\text { Shrinkage Limit (days) }\end{array}$ \\
\hline Layer 1 & 2.5 & 3.5 & 4.5 \\
Layer 2 & 1.5 & 3 & 10 \\
Layer 3 & 1 & 2 & 9 \\
Layer 4 & 1 & 2 & 11 \\
Layer 5 & 4 & 6 & $\mathbf{4 3}$ \\
Total days & $\mathbf{1 0}$ & $\mathbf{1 6 . 5}$ & 9 \\
\hline
\end{tabular}

This result is important as practitioners often advocate driving the layer to the void ratio at the shrinkage limit by drying. But these results show that geotechnical objectives of thickened tailings stack performance might be achieved with less drying, thereby reducing time required between depositions of different layers, and allowing for greater rates of rise. However, it should be noted that the high degree of desiccation of the first layer might affect the drying time of the second layer. If all the layers were allowed to desiccate to the same values of water content, the comparison would be more accurate.

\subsection{Important outcomes and conclusions}

Based on the analytical and experimental approaches performed in this study, the following conclusions can be reached: 
1. Samples that experience settling but no desiccation exhibit strain-softening (contractive) response under simple shear test, but not under triaxial compression loading. It should be noted that a small suction $(30 \mathrm{kPa})$ was applied to stabilize the triaxial samples before consolidation.

2. A small degree of desiccation was enough to change the response from contractive to dilative in simple shear, and generally the degree of strain hardening increased with the degree of desiccation.

3. Desiccation history decreased the compressibility of the tailings. Tailings with higher degree of desiccation had higher void ratios after consolidation, than tailings with a lower degree of desiccation. This was observed at least up to consolidation pressures of 400 $\mathrm{kPa}$.

4. In a multi-layer deposition of thickened tailings layers, the dewatering time to achieve a given water content may increase with increasing depth of underlying tailings. This is because water transported to the surface to satisfy evaporative demand can come from tailings deeper than the fresh layer.

5. In a multilayer deposition of thickened tailings layers, with significant desaturation of the underlying tailings, the settling of the new layer was greatly accelerated by adsorption of water by the underlying tailings.

6. The swelling potential of these gold tailings after desiccation is negligible. For tailings dried lower than the SL, the rewetted GWC was between $22 \%$ and $23 \%$. 
7. In the multilayer deposition simulation, matric suction of desiccated bottom layers after re-wetting by a fresh layer does not necessarily reach zero.

8. Though excessive desiccation to SL and beyond that improves the shear stress at PT and shear strength, it significantly increases the drying time of multilayers, which might negatively affect cost and efficiency of tailings deposition.

9. The effect of degree of desiccation history beyond the SL is more influential in increasing shear strength at large strains than increasing shear stress at PT.

10. Though re-saturation of desiccated tailings alleviates the effect of desiccation in increasing shear strength, desiccated-rewetted samples even at looser void ratios exhibited more shear strength than saturated samples at denser void ratios. This phenomenon is attributed to the effect of stress history during desiccation.

11. Under constant volume condition, degree of saturation does not affect the simple shear response of constant volume tests on coarse grained soils or fine grained soils that generate low values of suction $(<20 \mathrm{kPa})$ during desiccation. 
12. Mechanical over-consolidation exponentially increases monotonic and cyclic shear strength of thickened tailings, at a much faster rate than overconsolidation due to desiccation. Thus, quantifying stress history using an equivalent effective stress approach (modification of Bishops (1959) equations) is inaccurate for these tailings.

\subsection{Recommendations for future research}

Different variables that affect the mechanical behaviour of tailings were considered in this comprehensive study. However, additional research is required to further clarify the trends noted in the observed behaviour. Some specific tests that could be undertaken to verify and generalize the results found in this work, could include:

1. Comparing mechanical overconsolidation by $3 \mathrm{D}$ consolidation using the triaxial device with overconsolidation by matric suction

2. Improving characterization of fabric changes during different loading modes, by attempting MIP on smaller samples $(<1 \mathrm{~g})$, and using alternative techniques such as SEM or ESEM

3. In simple shear tests for which suctions might be expected, it appears suction need not be factored separately from the vertical load sensed by the device on the fixed plate to describe the stress state. The PT angle of desiccated samples was the same as all others, without accounting for suction separately. Measurement of suction during simple shear might shed some light on this phenomenon 
4. Investigation of the influence of desiccation stress history on finer grained tailings or soils. 


\section{REFERENCES}

- Aachib, M., Aubertin, M., and Mbonimpa, M (2002), "Laboratory measurements and predictive equations for gas diffusion coefficient of unsaturated soils “. In Proceedings of the 55th Canadian Geotechnical Conference and 3rd joint IAH-CNC and CGS Groundwater Specialty Conference, Niagara Falls, Ont., 20-23 October 2002. Edited by A.R.P.D. Stolle and J.J. Crauder. Canadian Geotechnical Society, Richmond, B.C. pp. $163-171$.

- Al-Tarhuni, M., (2008),"Liquefaction and Post-liquefaction behavior of Gold Mine Tailings under simple shear loading", M.Sc. Thesis, Carleton University.

- Al-Tarhouni, M., Simms, P., and Sivathayalan, S., (2011), "Cyclic behaviour of reconstituted and desiccated samples of thickened gold mine tailings", Canadian Geotechnical Journal, Vol. 48, No. 7, pp. 1044-1060.

- Ansell, P. and Brown, S. F., (1978), "A cyclic simple shear apparatus for dry granular materials", Geotechnical Testing Journal, ASTM, Vol. 1, No. 2, pp. 82-92.

- ASTM D4318 - 10, (2000), "Standard Test Methods for Liquid Limit, Plastic Limit, and Plasticity Index of Soils", Annual Book of ASTM Standards, American Society for Testing and Materials, Philadelphia, PA. Vol. 04.08. 
- ASTM D422-63, (2007),"Standard Test Method for Particle-Size Analysis of Soils", Annual Book of ASTM Standards, American Society for Testing and Materials, Philadelphia, PA. Vol. 04.08.

- ASTM D854 - 10, (2000), "Standard Test Methods for Specific Gravity of Soil Solids by Water Pycnometer", Annual Book of ASTM Standards, American Society for Testing and Materials, Philadelphia, PA. Vol. 04.08.

- Aubertin, M., Bussiere, B., and Chapuis, R. P., (1996), "Hydraulic conductivity of homogenized tailings from hard rock mines", Canadian Geotechnical Journal, Vol. 33, pp. $470-482$.

- Baldi G., and Nova R., (1983), "Effetti della penetrazione della membrana in prove di liquefazione R." $15^{\circ}$ Cong Naz. Geotecnica, Spoleto, 1, 3-12.

- Berre, T., (1982), “Triaxial testing at the Norwegian Geotechnical Institute” Geotechnical Testing Journal, ASTM. Vol. 5, No. 1-2, pp. 3-17.

- Bjerrum, L. and Landva, A., (1966), "Direct simple-shear tests on a Norwegian quick clay". Geotechnique, Vol. 16, No. 1, pp. 1-20.

- Bishop, A. W., (1959), “The principle of effective stress.” Tek. Ukeblad, Vol. 106(39), pp. 859-863. 
- Bishop, A.W., and Blight, G.E., (1963), "Some aspects of effective stress in saturated and unsaturated soils", Géotechnique, Vol.13(3), pp. 177-197.

- Bolt, G. H., (1956), "Physicochemical analysis of the compressibility of pure clays." Geotechnique, Vol. 6, pp. 86-93.

- Boulanger, R. W., and Idriss I. M., (2004), "Evaluation the potential for liquefaction or cyclic failure of Silts and Clays", Centre for Geotechnical Modeling, Report No. UCD/CGM-04/01.

- Boulanger, R. W., and Truman, S. P., (1996), "Void redistribution in sand under postearthquake loading." Canadian Geotechnical Journal, 33, pp. 829-834.

- Bussiere, B., (2007), "Hydrogeotechnical properties of hard rock tailings from metal mines and emerging geoenvironmental disposal approaches”, Canadian Geotechnical Journal, Vol. 44(9): 1019-1052

- Casagrande, A., (1976), "Liquefaction and cyclic deformation of sands", A critical review, Harvard Soil Mechanics.,Serial No. 88. 
- Castro, G. (1969), "Liquefaction of sands", PhD Thesis, Harvard University, Cambridge, Massachusetts.

- Castro, G., Poulos, S.J., France, J.W., and Enoso, J.L., (1982),"Liquefaction induced by cyclic loading" Report to National Science Foundation, Washington, DC.

- Collins, K., and McGown, A., (1974), "The form and function of microfabric features in a variety of natural soils", Geotechnique, Vol. 24(2), pp. 223-254.

- Crowder, J. J., (2004), "Deposition, consolidation, and strength of a non-plastic Tailings paste for surface disposal", PhD Thesis, University of Toronto, Toronto.

- Dobry, R., and Alvarez, L., (1967),"'Seismic failure of Chilean tailings dams", Journal of Soil Mechanics and Foundation Division. In proceedings of the ASCE. No. SM6.

- Dyvik, R., Berre, T., Lacasse, S., and Raadim, B., (1987), "Comparison of truly undrained and constant volume direct simple shear tests", Geotechnique, Vol. 37(1), pp.3-10.

- Finn, W. D. L., Vaid, Y. P., and Bhatia, S. K., (1978), "Constant volume simple shear testing”, Proc. $2^{\text {nd }}$ International Conference on Microzonation for Safer Construction Research and Application, San Francisco, CA, pp. 839-851. 
- Fisseha, B., Bryan, R., and Simms, P., (2010), "Evaporation, unsaturated flow, and salt accumulation in multilayer deposits of a paste gold tailings", Journal of Geotechnical and Geoenvironmental Engineering.

- Fredlund, D. G., and Morgenstern, N. R., (1977), "Stress state variables for unsaturated soils.” Journal of Geotechnical Engineering Division, ASCE, 103(5), pp. 447- 466.

- Fredlund, D.G., and Rahardjo, H., (1993), "Soil mechanics for unsaturated soils", John Wiley \& Sons Inc., New York, NY.

- Fredlund, M. D., Wilson, G. W., and Fredlund, D. G., (1997), "Prediction of the soilwater characteristic curve from the grain-size distribution curve.” Proc., 3rd Symp. on Unsaturated Soil, Rio de Janeiro, Brazil, pp. 13-23.

- Fredlund, D. G., and Xing, A., (1994), "Equations for the soil-water characteristic curve", Canadian Geotechnical Journal, Vol. 31: pp. 521-532.

- Fredlund, M. D., Wilson, G. W., and Fredlund, D. G., (1997), "Prediction of the soilwater characteristic curve from the grain-size distribution curve," Proc., 3rd Symp. on Unsaturated Soil, Rio de Janeiro, Brazil, pp. 13-23.

- Garven, E.A., and Vanapalli, S.K., (2006), "Evaluation of empirical procedures for predicting the shear strength of unsaturated soils", Proceedings of 4th International Conf. 
on Unsaturated Soils, 2-6 April 2006, Carefree, Arizona, American Society of Civil Engineers Geotechnical Special Publication, Vol. 147(2), pp. 2570-2581.

- Heidarian, P., (2012), "Effect of initial water content and stress history on waterretention behaviour of mine tailings", M.Sc. Thesis, Carleton University.

- Henderson, A., Newman, P., Landriault, D., and Antoniazzi, P., (1997), "The Cost Advantage of Using Paste as a Backfill”, Proceedings of the 99th CIM Annual General Meeting, Vancouver, Canada.

- Hilf, J.W., (1956), "An investigation of pore-water pressure in compacted cohesive soils", PhD Thesis. Technical Memo No.654, United States Bureau of Reclamation, Denver.

- Holtz, R.D., and Kovacs, W.D., (1981), “An Introduction to Geotechnical Engineering”, Prentice Hall.

- ICOLD and UNEP, (2001), Tailings dams, risk of dangerous occurrences. Bulletin 121: Lessons learnt from practical experiences Paris, 144.

- Innocent, T., (2013), "Evaporation in Oil Sand Thickened Tailings". M. Sc. Thesis, Carleton University. 
- Ishihara, K., (1993). "Liquefaction and flow failure during earthquakes." Geotechnique, Vol. 43(3), pp. 351-415.

- Ishihara, K., Tatsuoka, F., and Yasuda, S. (1975), "Undrained deformation and liquefaction of sand under cyclic stresses", Soils and Foundations, Vol. 15, No. 1, pp 2944.

- James, M., (2009), "The Use of Waste Rock Inclusions to control the Effects of Liquefaction in Tailings Impoundments", PhD Thesis, École Polytechnique de Montréal, Qc, Canada.

- Jennings. J.E., and Burland, J.B., (1962), "Limitations of the use of effective stresses in partly saturated soils." Géotechnique, Vol. 12(2): pp. 125-144.

- Jewell, R.J., (2012), "Putting beach slope predictions into perspective. Proceedings of the15th international seminar on paste and thickened tailings", Sun City, South Africa, pp. 85-93.

- Khalili, N., Geiser, F., and Blight, G. E. (2004), "Effective stress in unsaturated soils, a review with new evidence.” International Journal of Geomechanics, Vol.4 (2), pp. 115126. 
- Kramer, S. L., (1996), “Geotechnical Earthquake Engineering”. Upper Saddle River, NJ: Prentice-Hall Inc.

- Kim, H., (2010), "Effect of overconsolidation and desiccation on the response of a gold mine tailings under simple shear loading”. M. Eng. Report, Carleton University.

- Kim, H., Daliri, F., Simms, P., and Sivathayalan, S., (2011), "The influence of desiccation and over-consolidation on monotonic and cyclic shear response of thickened gold tailings." Proceedings of the 64th Canadian Geotechnical Conference (Pan-Am CGS), Toronto, Canada.

- Kong LW, Guo AG, Zhao YW, and Liu YY, (2005), "Influence of moisture content on porosity features of Red clay.", Proceedings International Symposium on Advanced Experimental Unsaturated Soil Mechanics, Trento, Italy, 27-29 June 2005. In: Tarantino A, Romero E, Cui YJ (eds) Advanced experimental unsaturated soil mechanics. Taylor, Francis Group, London, pp. 419-424.

- Ladd, C. C., (1991). "Stability evaluation during staged construction," Journal of Geotechnical Engineering, ASCE, 117(4), pp. 540-615.

- Ladd, C. C., and Foott, R., (1974), "New design procedure for stability of soft clays." Journal of the Geotechnical Engineering, Div., ASCE, 100(7), pp. 763-786. 
- Lambe, T. W., (1960), "A mechanistic picture of the shear strength of clay.” Proceedings Research Conference on the shear strength of cohesive soils, ASCE, New York, 437

- Leong, E. C., and Rahardjo H., (1997), "Permeability functions for unsaturated soils". Journal of Geotechnical and Geoenvironmental Engineering, ASCE. Vol. 123(2), pp. 1118-1126.

- Lu, N., Godt, J.W., and Wu, D.T., (2010), “A closed-form equation for effective stress in unsaturated soil.” Water resources research, Vol. 46(5), DOI: 10.1029/2009WR008646.

- Lu, N., and Likos, W.J., (2006), "Suction Stress Characteristic Curve for Unsaturated" Soil. Journal of Geotechnical and Geoenvironmental Engineering, ASCE, Vol. 132, No.2, pp. 131-142.

- Marinho, F., (2005), "Nature of Soil - Water Characteristics Curve for Plastic Soils", Journal of Geotechnical and Geoenvironmental Engineering, ASCE, Vol. 131(5), pp. $654-661$.

- Matyas, E.L., and Radhakrishna, H.S., (1968), "Volume change characteristics of partially saturated soils". Géotechnique, Vol. 18(4), pp. 432-448.

- Meilani, I., Rahardjo, H., Leong, E., and Fredlund, D. G., (2002), "Mini suction probe for matric suction measurements", Canadian Geotechnical Journal, Vol. 39, pp. 1427-1432 
- Michael, J., (2009), "The use of waste rock inclusions to control the effects of liquefaction in Tailings impoundments “, PhD Thesis, University of Montreal.

- Mitchell, J. K., (1976). "Fundamentals of soil behavior”, Wiley, New York.

- Mitchell, J.K., and Soga, K., (2005), "Fundamentals of soil behaviour", 3rd edn. John Wiley, Sons, Inc, New Jersey.

- Miura, S., and Toki, S., (1982), “A sample preparation method and its effect on static and cyclic deformation strength properties of sands", Soil and Foundations, Vol. 22, No. 1, pp. $61-77$.

- Moriwaki, Y., Akky, M. R., Ebeling, R., Idriss, I. M., and Ladd, R. S., (1982), "Cyclic strength and properties of tailing slimes.", Proc. Specialty Conference on Dynamic Stability of Tailings Dams, ASCE.

- Mortezaie, A., and Vucetic, M., (2013),"Effect of Frequency and Vertical Stress on Cyclic Degradation and Pore Water Pressure in Clay in the NGI Simple Shear Device.” Journal of Geotechnical and Geoenvironmental Engineering, ASCE, Vol. 139(10), pp. $1727-1737$. 
- National Research Council (NRC), (1985), "Liquefaction of soils during earthquakes", National Academy Press, Washington, D. C.

- Oliveira, O.M., and Marinho, F.A.M., (2003), "Unsaturated shear strength behaviour of a compacted residual soil". Proceeding of Asian Conference on Unsaturated Soils: Unsaturated Soil Geotechnical and Geoenvironmental Issues. Osaka, Japan, pp. 237-242.

- Peacock, W. H., and Seed, H. B. (1968). "Sand liquefaction under cyclic loading simple shear conditions.” Journal of Soil Mechanics and Foundations Div. American Society of Civil Engineers. Vol. 94, SM3, pp. 689-708.

- Pham, Q.H., and Fredlund, D.G., Barbour S.L., (2005), “ A study on the hysteresis models for soil-water characteristic curves" , Canadian Geotechnical Journal 42(6):1548-1568.

- Poulos, S. J. (1981), "The Steady State of Deformation", Journal of Geotechnical Engineering 107.

- Prevost, J. H., and HØeg, K. 1975. Effective stress-strain- strength model for soils. Journal of Geotechnical Engineering Div., ASCE. 101(GT3), pp. 259-278.

- Qiu, Y., and Sego D.C., (2001), "Laboratory properties of mine tailings", Canadian Geotechnical Journal, Vol. 38, pp. 183- 109. 
- Rahardjo H., Guan G., and Choon L., (2010), "Shear Strength Equations for Unsaturated Soils under drying and wetting”, Journal of Geotechnical and Geoenvironmental Engineering, Vol. 136.

- Richards, L.A., (1965) "Physical condition of water in soil” pp. 128-152.

- Ridley, A.M., and Burland, J.B, (1993), “A new instrument for the measurement of soil moisture suction", Ge'otechnique Vol. 43(2), pp. 321-324.

- Ridley, A.M., and Burland, J.B., (1996), "A pore pressure probe for the in situ measurement of a wide range of soil suction", Advances in site investigation practice. Thomas Telford London, pp. 510-520.

- Ridley, A. M., and Wray, W. K., (1996), "State of the art report-Suction measurement: a review of current theory and practices", Proceedings of 1st international conference on unsaturated soils, unsaturated soils, Vol. 3. Paris, pp. 1293-1322.

- Ridley, A. M., Patel, A. R., and Marsland F. ,(1998), “ Tensiometers: their design and use for civil engineering purposes", Geotechnical Site Characterisation. Balkema Rotterdam. 
- Robinsky, E.I., (1975), "Thickened Discharge - A New Approach to Tailings Disposal", Canadian Mining and Metallurgical Bulletin, Vol. 68, pp. 47-53.

- Robinsky, E.I., (1999), “Thickened Tailings Disposal in the Mining Industry”, Toronto, Ontario, Canada, E. I. Robinsky Associates Ltd.

- Romero, S., (1995), “The behavior of silt as clay content is increased", M.Sc. thesis, University of California, Davis.

- Romero, E., and Simms, P.H., (2008), "Microstructure investigation in unsaturated soils: a review with special attention to contribution of mercury intrusion porosimetry and environmental scanning electron microscopy". Geotechnical and Geological Engineering, Vol. 26, No. 6, pp.705-727.

- Sanin, M. V., (2005)," Cyclic Shear loading response of Fraser river delta silts”, M. Sc. Thesis, University of British Columbia.

- Seed, H. B., and Idriss, I. M., (1982). "Ground Motions and Soil Liquefaction during Earthquakes" Berkeley CA: Earthquake Engineering Research Institute. 
- Seed, H. B., (1983), "Earthquake resistant design of earth dams." Proc., Symposium on Seismic Design of Embankments and Caverns, Pennsylvania, ASCE, N.Y., pp. 41-64.

- Seed, H. B., (1987), "Design problems in soil liquefaction." Journal of Geotechnical Engineering, ASCE, Vol. 113(8), pp. 827-845

- Seed, R. B., Cetin, K. O., Moss, R. E. S., Kammerer, A., Wu, J., Pestana, J., Riemer, M., Sancio, R. B., Bray, J. D., Kayen, R. E., and Faris, A., (2003). "Recent advances in soil liquefaction engineering: A unified and consistent framework." Keynote presentation, 26th Annual ASCE Los Angeles Geotechnical Spring Seminar, Long Beach, CA.

- Shuttleworth, J. A., Thomson, B.J., and Wates, J. A., (2005), "Surface disposal at Bulyanhulu-practical lessons learned", In Proc. of the 6th International Conference on Paste and thickened tailings, Santiago, Chile, 20-22- April 2005. Edited by R. Jewel and S. Barerra, pp. 207-216.

- Sidey, R., Strom, J. A., and Pyke, R. M., (1978). "Discussion on Measurement of dynamic soil properties.” Proceeding of Conference of Earthquake Engineering and Soil Dynamic .Pasadena III, pp. 1478-1481. 
- Simms, P., Daliri, F., and Dunmola, A., (2012). "Deposition sequencing or drying time for multi-point deposition of high density tailings", Proceedings of Tailings and Mine Waste 2012 Conference, Colorado, B.C., U.S.A.

- Simms, P., and Grabinsky, M. W. (2004), "A simple method for estimating rates of drying and desaturation of paste tailings during surface deposition”, Lassonde Institute for Engineering Geoscience, University of Toronto, Toronto, Canada.

- Simms, P., Grabinsky M., and Zhan G., (2007), "Modelling evaporation of paste tailings from the Bulyanhulu mine", Canadian Geotechnical Journal, Vol. 44, pp. 1417-1432.

- Simms, P., and Grabinsky, M. W., (2009), "Direct measurement of matric suction in triaxial tests on early age cemented paste backfill" Canadian Geotechnical Journal, Vol. 43, pp. 93-101.

- Simms, P., Dunmola, A., and Bryan, R., 2010. Generic modeling of desiccation for cyclic deposition of thickened tailings to maximize density and to minimize oxidation. In Proceedings of the $13^{\text {th }}$ international seminar on paste and thickened tailings, Toronto, Canada, pp. $293-303$. 
- Simms, P.H., and Yanful, EK (2001), "Measurement and estimation of pore shrinkage and pore distribution in a clayey till during soil-water characteristic curve tests.", Canadian Geotechnical Journal, Vol. 38, pp. 741-754.

- Simms, P.H., and Yanful, EK, (2002), "Predicting soil-water characteristic curves of compacted plastic soils from measured pore-size distributions.", Ge'otechnique, Vol. 52(4), pp. 269-278.

- Simms, P.H., and Yanful, EK (2004), “A discussion of the application of mercury intrusion porosimetry for the investigation of soils, including an evaluation of its use to estimate volume change in compacted clayey soils." Ge'otechnique, Vol. 54(6), pp. 421426

- Simms, P.H., and Yanful, EK, (2005), "A pore-network model for hydromechanical coupling in unsaturated compacted clayey soils.", Canadian Geotechnical Journal, Vol. 42, pp. 499-514.

- Singh, S., (1996), "Liquefaction Characteristics of Silts", Geotechnical and Geological Engineering, Vol. 14, pp. 1-19.

- Sivathayalan, S, and Logeswaran, P., (2007), "Behaviour of sands under generalized drainage boundary conditions", Canadian Geotechnical Journal, Vol. 44, No. 2, pp. 138150. 
- Skempton, A. W., (1960), "Significance of Terzaghi's concept of effective stress." From theory to practice in soil mechanics.

- Sladen J.A., D'Hollander R.D., and Krahn J., (1985), "The liquefaction of sands, a collapse surface approach", Canadian Geotechnical Journal, Vol. 22, pp. 564-578.

- Sofra, F., and Boger, D.V, (2001), "Slope prediction for thickened tailings and pastes" In Tailings and Mine Waste '01: Proceedings of the 8th International Conference, Fort Collins, Colo., 15- 18 January 2001. A.A. Balkema, Rotterdam, The Netherlands pp. 7583.

- Sridharan, A., and Rao, V. G., (1973), "Mechanisms controlling volume change of saturated clays and the role of the effective stress concept." Geotechnique, Vol. 23(3), pp. 359-382.

- Sriskandakumar, S., (2004), "Cyclic Loading Response of Fraser River Sand for Numerical Models Simulating Centrifuge Tests”, M.A.Sc. Thesis, University of British Columbia, Vancouver, B.C.

- Swanson, D. A., Savci, G., and Danziger G., (1999), "Predicting the soil water characteristics of mine soils", Tailings and Mine Waste '99. 
- Tarantino, A., Ridley, A. M., and Toll, D. G., (2008), "Field Measurement of Suction, Water Content, and Water Permeability", Geotechnical and Geological Engineering, Vol. 26, pp. $751-782$.

- Tawil, M. R., (1997), "Engineering properties of iron mine slimes for tailings dam stability", M.Sc. thesis, Massachusetts Institute of Technology.

- Terzaghi, K., (1943). “Theoretical soil mechanics”, Wiley, New York.

- Terzaghi, K., and Peck, R. B. (1967) "Soil Mechanics in Engineering Practice (2nd ed.)".

- Theriault, J., Frostiak, J., and Welch, D., (2003), “ Surface disposal of paste tailings at the Bulyanhulu gold mine, Tanzania", In Proc. of Sudbury 2003, Mining and the Environment, Sudbury, Ont., 26-28 May 2003.

- Thu T. M., Rahardjo H., and Leong E., (2007), "Soil Water Characteristics Curve and consolidation behavior for a compacted silt”, Canadian Geotechnical Journal, Vol. 24, pp. 266-275.

- Vaid, Y. P., and Chern, J.C. (1983), "Effect of static on resistance of liquefaction”, Soils and Foundations, pp. 47-60. 
- Vaid, Y. P., and Chern, J.C. (1985), "Cyclic and monotonic undrained response of saturated sands", Proc. Advances in the art of testing soils under cyclic loading conditions, Detroit, pp. 120-147.

- Vaid, Y. P., and Thomas, J., (1994), "Post liquefaction behaviour of sand", Proc. Thirteenth International Conference on Soil Mechanics and Foundation Engineering, New Delhi, India, pp. 1305-1310.

- Vaid, Y. P., Stedman, J. D., and Sivathayalan S., (2001), "Confining stress and static shear effects in cyclic liquefaction", Canadian Geotechnical Journal, Vol: 38, pp. 580592.

- Vaid Y. P., and Sivathayalan S., (1996), "Static and Cyclic Liquefaction potential of Frase Delta and in simple shear and triaxial tests." Canadian Geotechnical Journal. Vol. 33: pp. 281-289.

- Van Genuchten, and M. T., (1980), "A closed form equation for predicting the hydraulic conductivity of unsaturated soils.” Soil Sci. Soc. Am. J. Vol. 44, pp. 892-898. 
- Vanapalli, S.K., Fredlund, D. G., and Pufahl, D. E., and Clifton, A. W., (1996), “ Model for the prediction of shear strength with respect to soil suction", Canadian Geotechnical Journal, Vol. 33, No. 3, pp. 379-392.

- Vanapalli, S.K., Fredlund, D.G., and Pufahl, D.E., (1996), “The relationship between the soil-water characteristic curve and the shear strength of a compacted glacial till", Geotechnical Testing Journal, Vol. 19, No. 3 pp. 259-268.

- Vanapalli, S.K., and Fredlund, D.G. 2000. "Comparison of empirical procedures to predict the shear strength of unsaturated soils uses the soil-water characteristic curve.", Geo-Denver 2000, American Society of Civil Engineers, Special Publication, Vol. 99, pp. 195-209.

- Vanapalli, S.K., Pufahl, D.E., and Fredlund, D. G., (1998), "The meaning and relevance of residual water content to unsaturated soils." $51^{\text {st }}$ Canadian Geotechnical Conference, 1998, Edmonton, pp. 101- 108.

- Vanapalli, S.K., (2009), "Shear strength of unsaturated soils and its applications in geotechnical engineering practice", Proceedings of the $4^{\text {th }}$ Asia-Pacific Conference on Unsaturated Soils. New Castle, Australia. Nov. 23-25. Edited by O. Buzzi, S. Fityus and D. Sheng. 2010 Taylor \& Francis Group, London, ISBN 978-0-415-80480-6. pp. 579598. 
- Vick, S. G., and Wiley, J., (1983), "Planning, Design, and Analysis of Tailings Dams", New York, 1983, 369 p., ISBN 0-471-89829-5.

- Vick, S. G., (1990), "Planning, Design and Analysis of Tailings Dams", Vancouver, BC, BiTech Publishers Ltd.

- Vucetic, M., and Lacasse, S. (1983), "Specimen size effect in simple shear test: closure." Journal of Geotechnical Engineering Division, American Society of Civil Engineers. Vol. 110, GT4, pp.447-453.

- Washburn, E. W., (1921), “The Dynamics of Capillary Flow. Physical Review, 17, 273.

- Wijewickreme, D., and Sanin, M., (2004). "Cyclic shear loading response of Fraser river delta silt", Proceedings of 13th World Conference on Earthquake Engineering Vancouver, B.C., Canada, paper No. 499.

- Wijewickreme, D., and Sanin, M. V. and Greenaway, G. R., (2005), "Cyclic shear response of fine-grained mine tailings", Canadian Geotechnical Journal, Vol. 42, pp $1408-1421$.

- Yanful, E. K. (1993), "Oxygen diffusion through soil covers on sulphidic mine tailings", Journal of Geotechnical and Geoenvironmental Engineering, 119: 1207-1211. 
- Youd, T. L., and Idriss, I.M., (2001), "Liquefaction resistance of soils: Summary report from the 1996 NCEER and 1998 NCEER/NSF workshops of evaluation of liquefaction resistance of soils." Journal of Geotechnical and Geoenvironmental Engineering, ASCE, Vol. 127, No. 4, pp. 297-313.

- Zergoun, M., and Vaid, Y. P., (1994),"Effective stress response of clay to undrained cyclic loading," Canadian Geotechnical Journal, Vol. 31, pp. 714-727. 


\section{APPENDIX I: Drying box results}

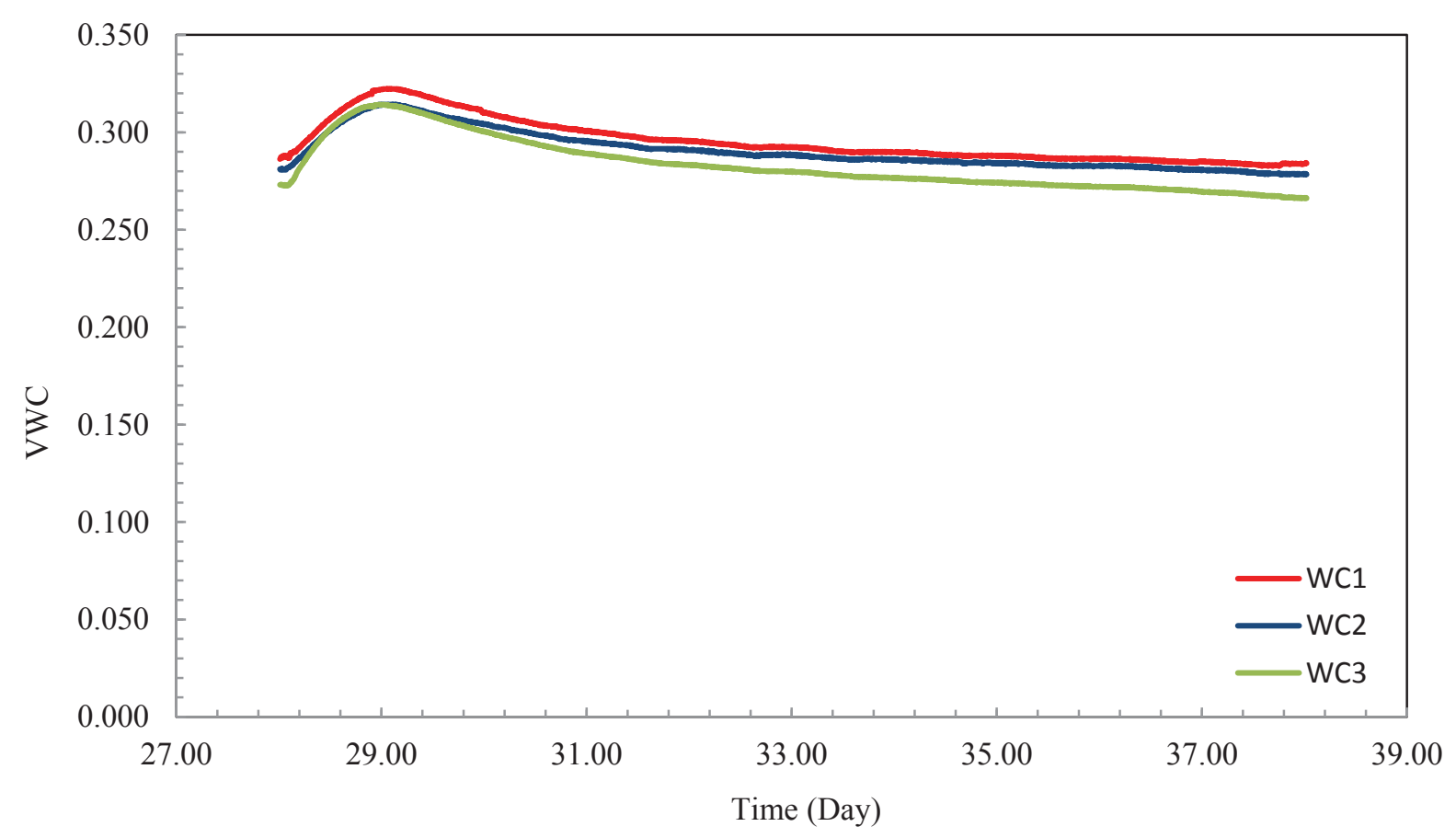

Figure AI1. VWC of first layer after fourth layer deposition

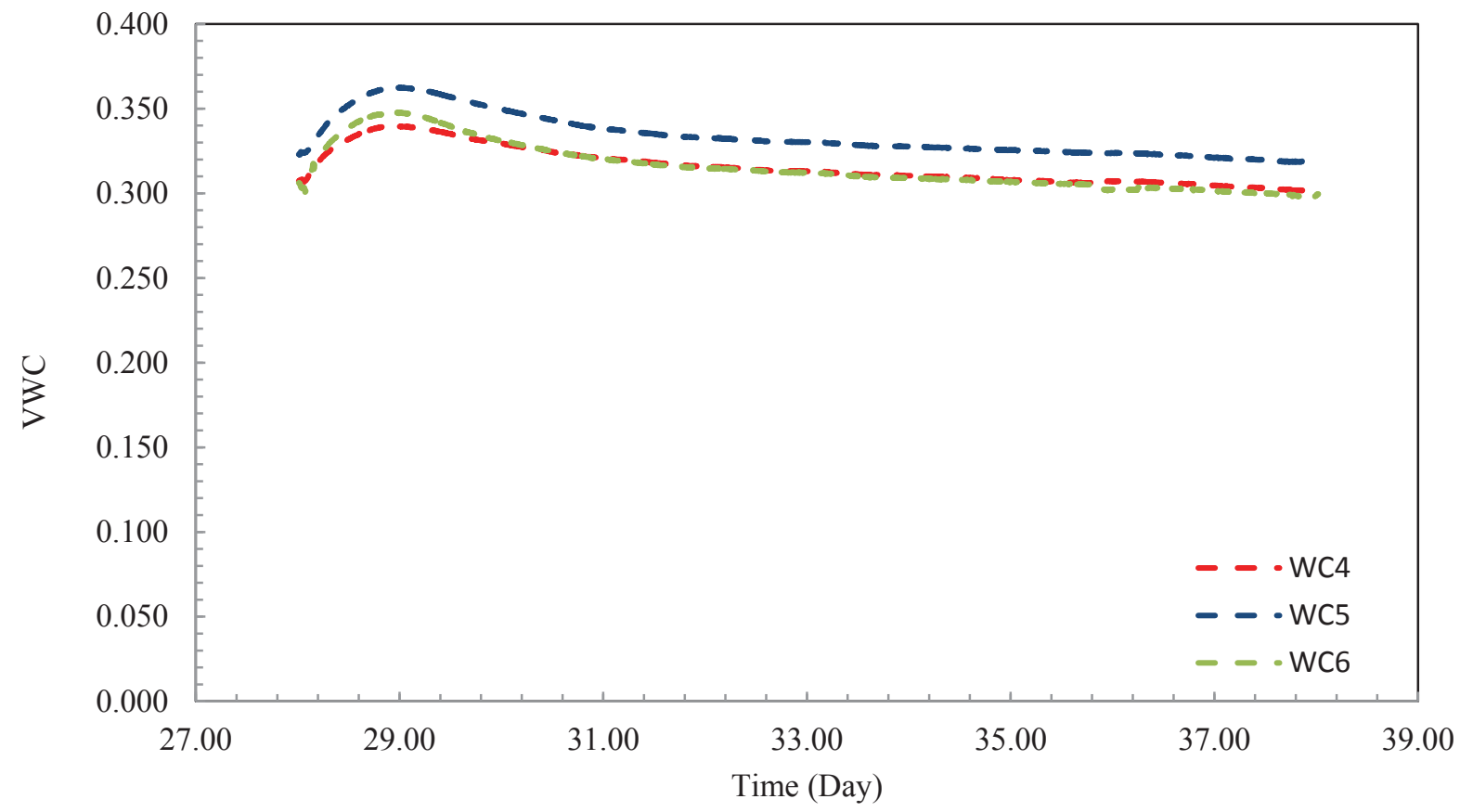

Figure AI2. VWC of second layer after fourth layer deposition 


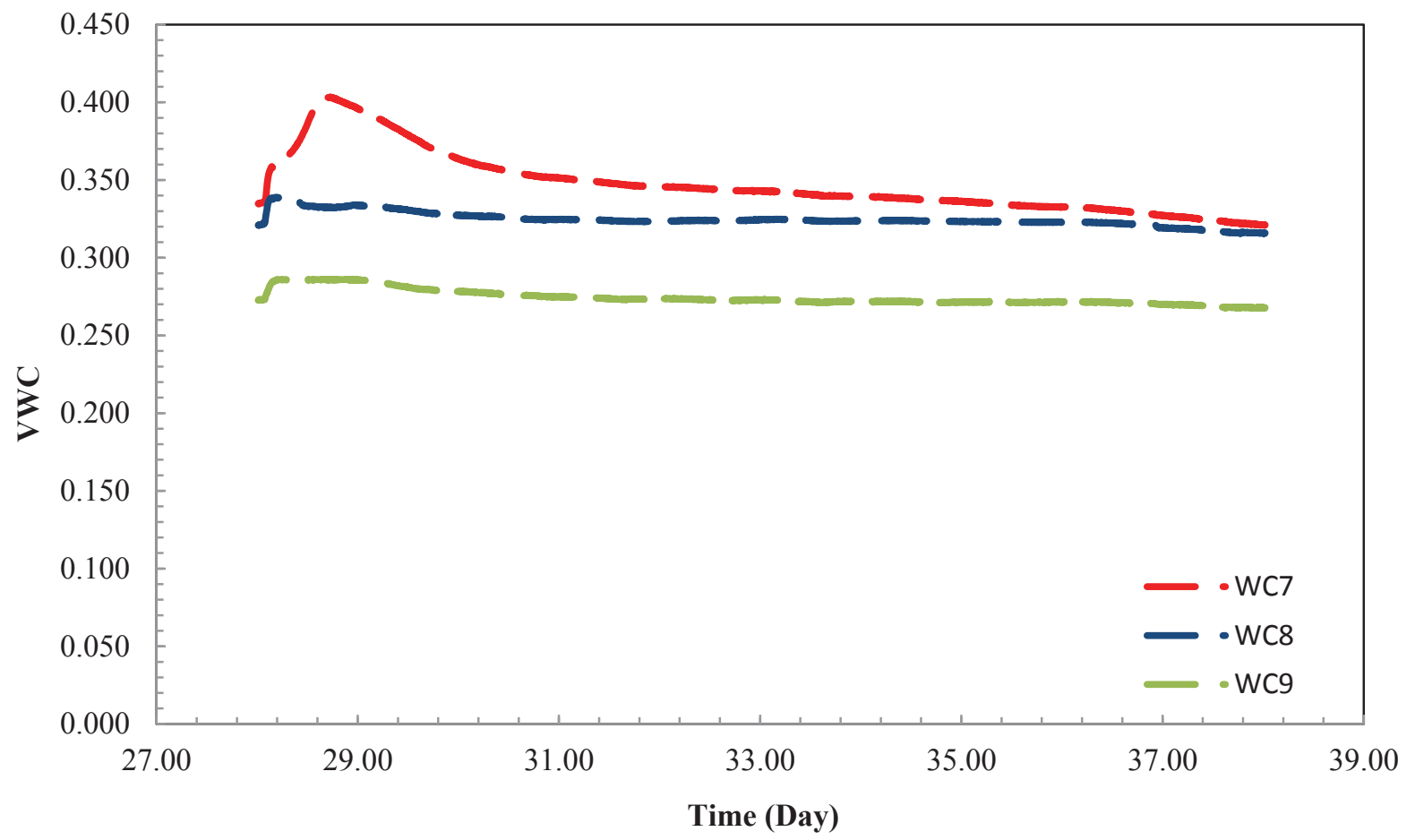

Figure AI3. VWC of third layer after fourh layer deposition

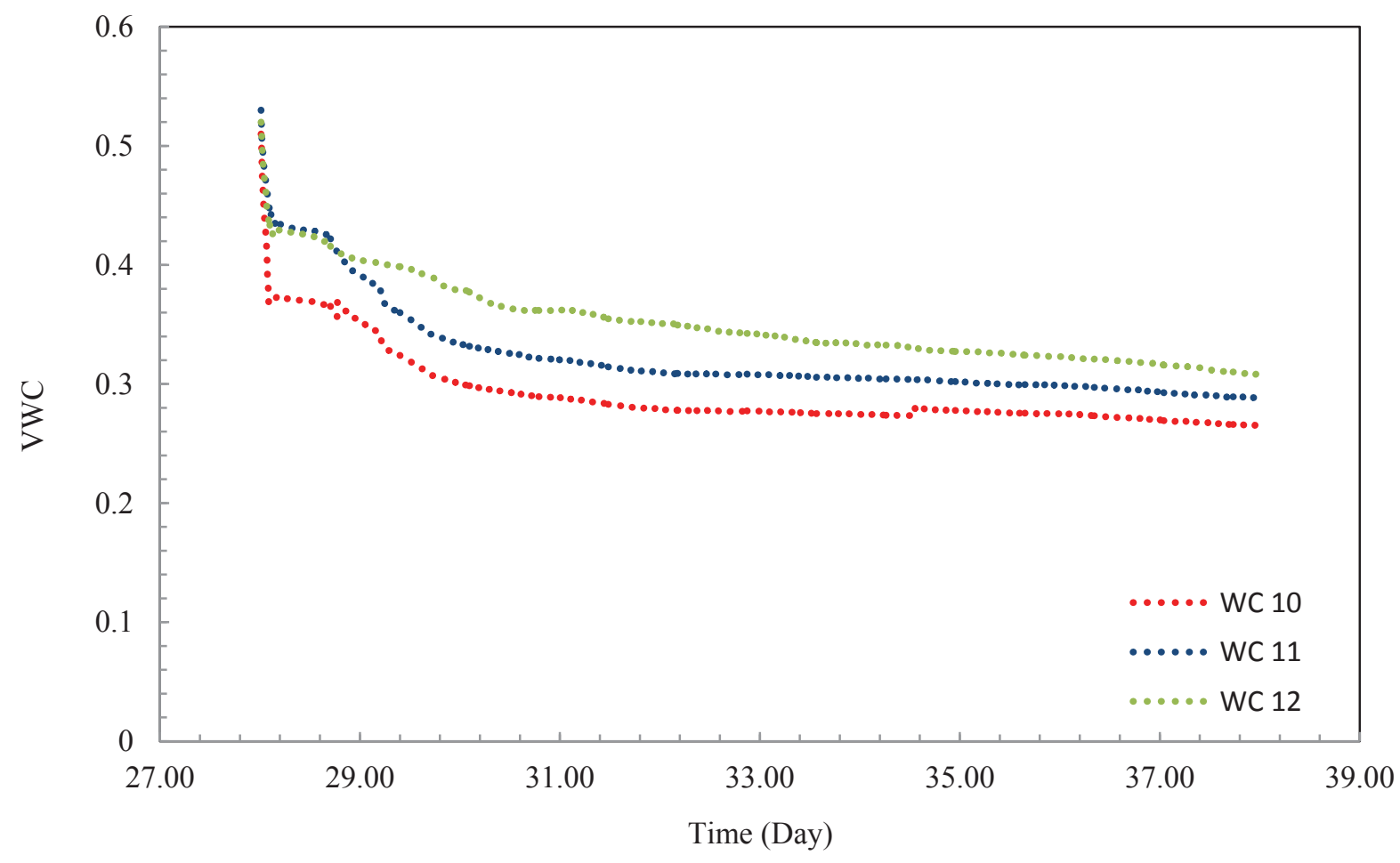

Figure AI4. VWC of the fourth layer 


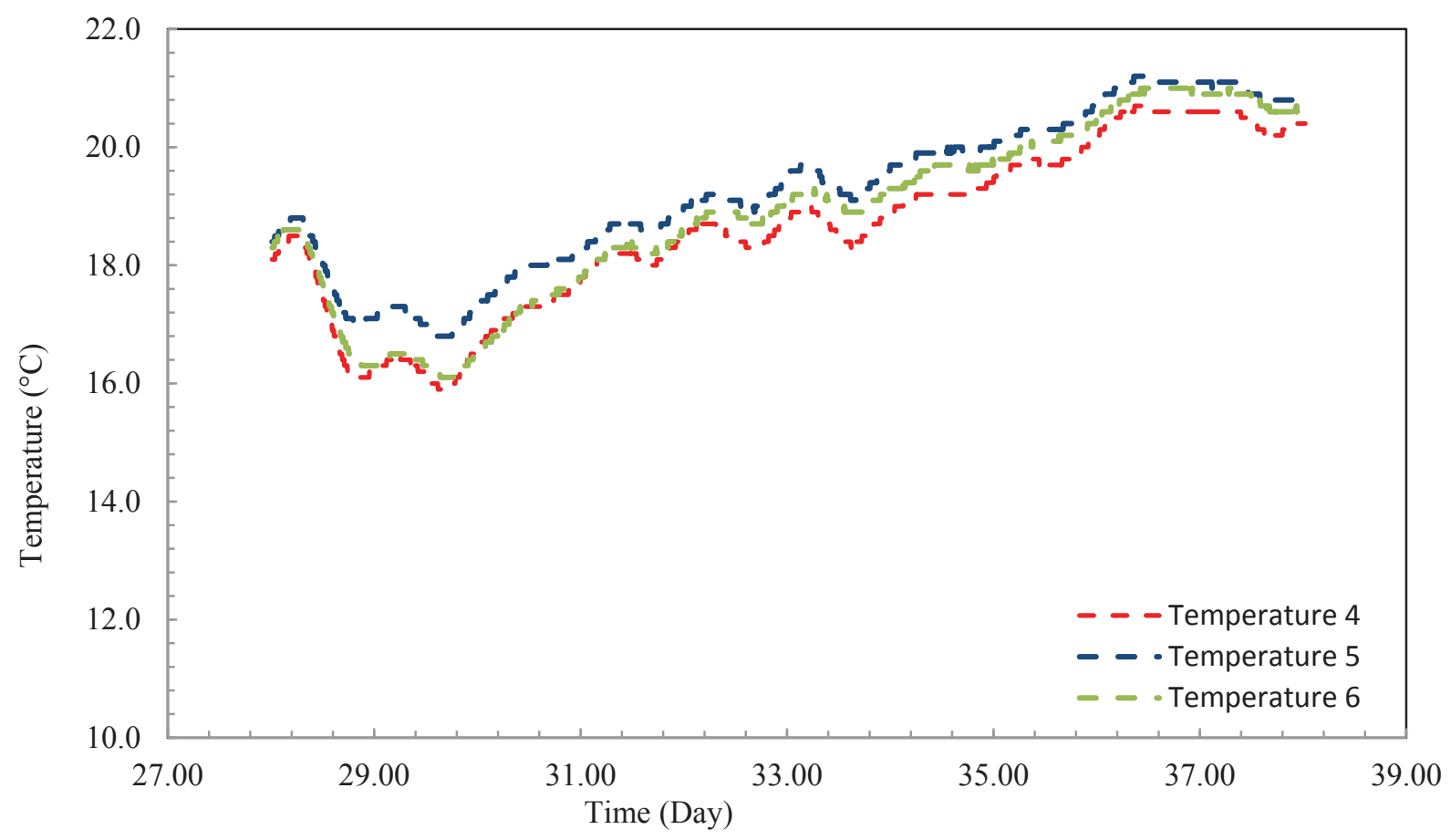

Figure AI5. Temperature of second layer after fourth layer deposition

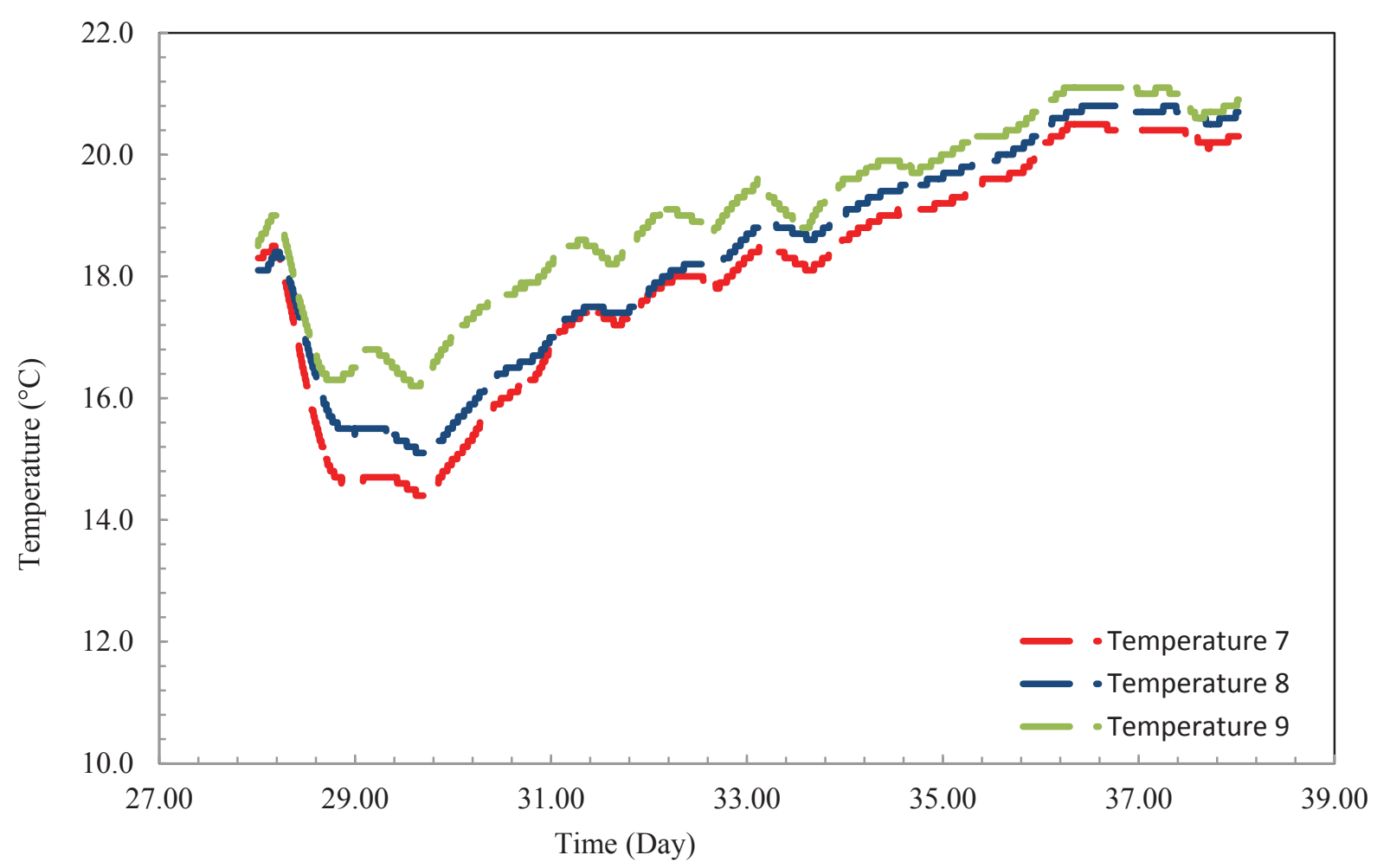

Figure AI6. Temperature of the third layer after fourth layer deposition 


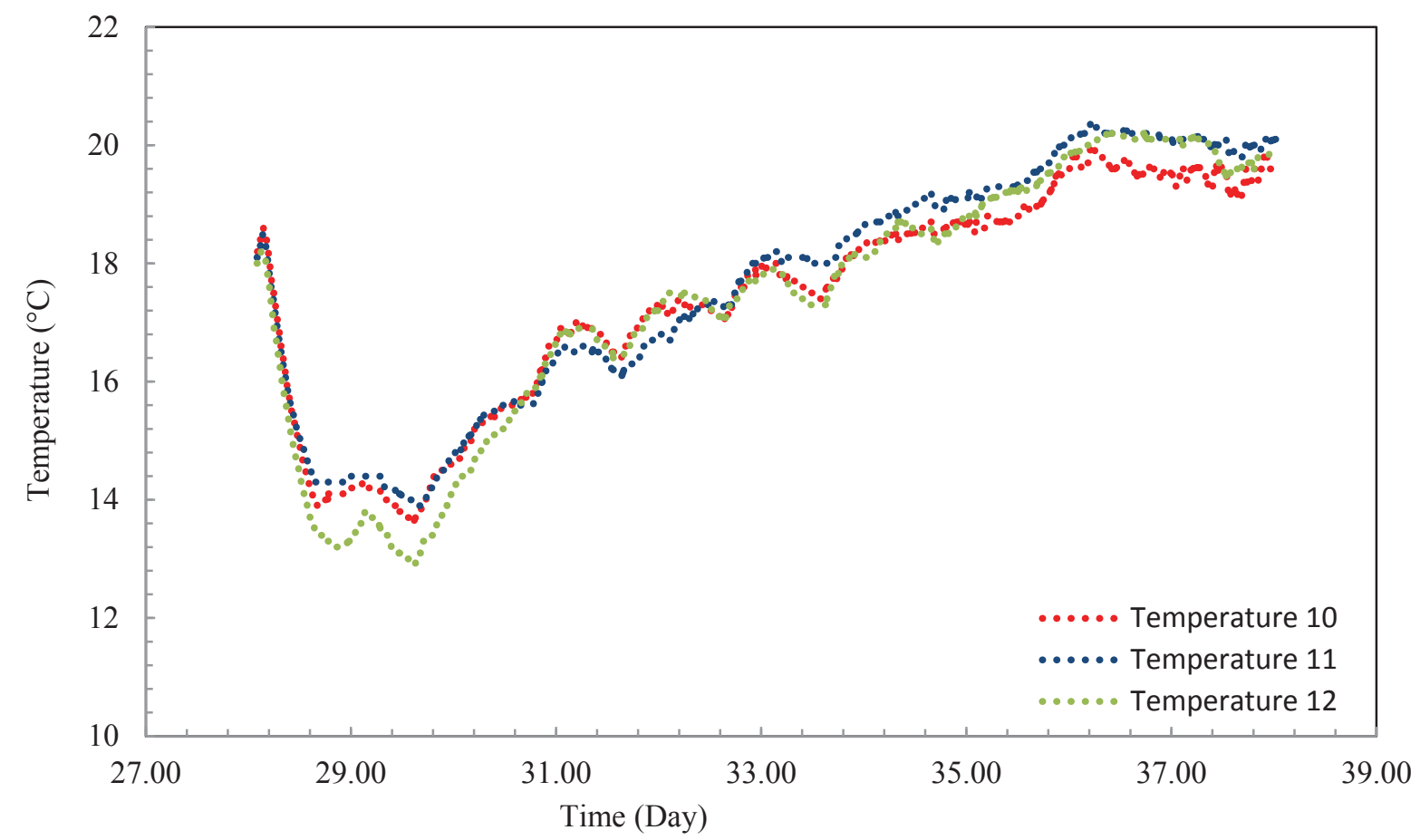

Figure AI7. Temperature of the fourth layer

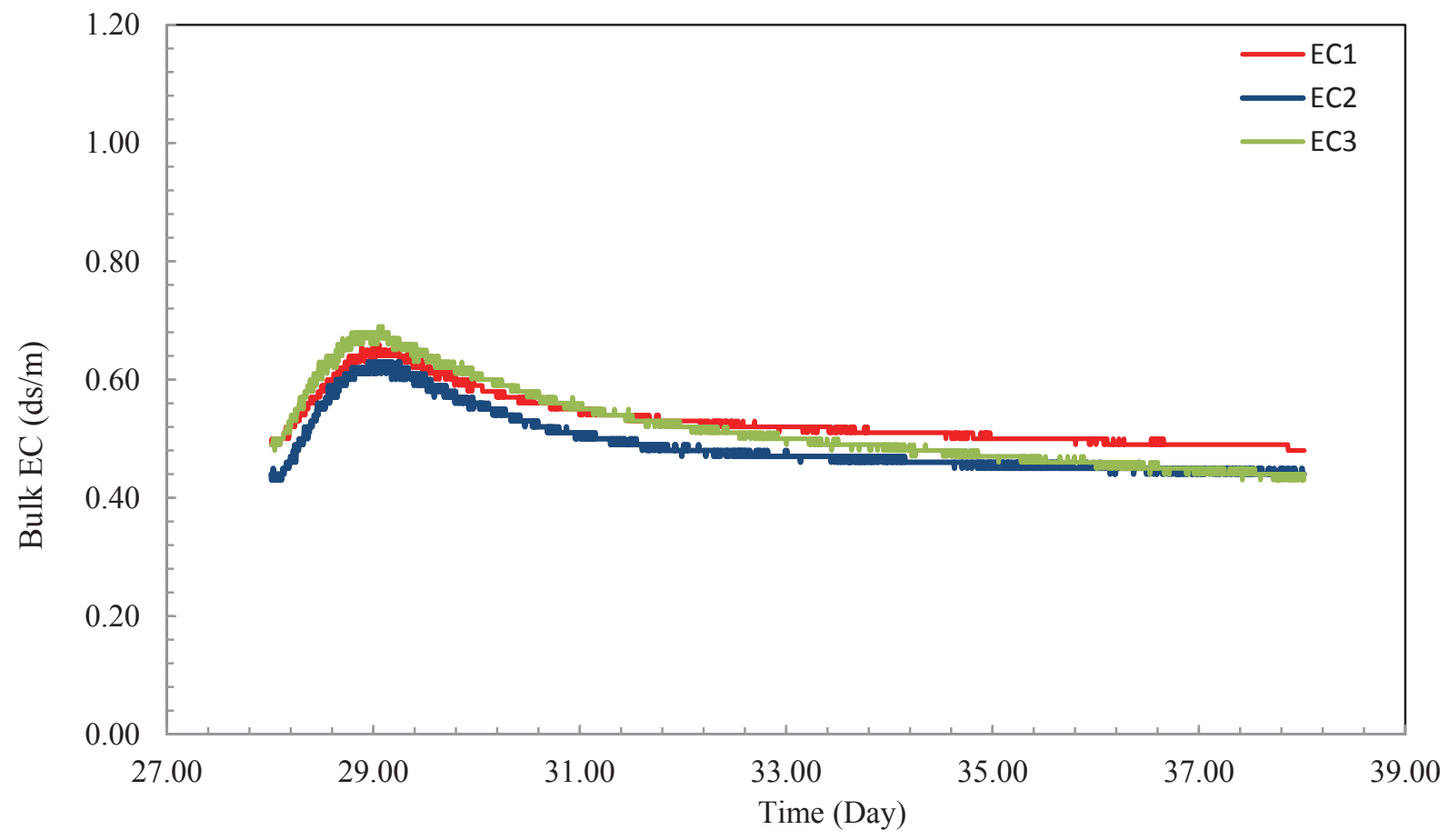

Figure AI8. Bulk Electrical Conductivity of first layer after fourth layer deposition 


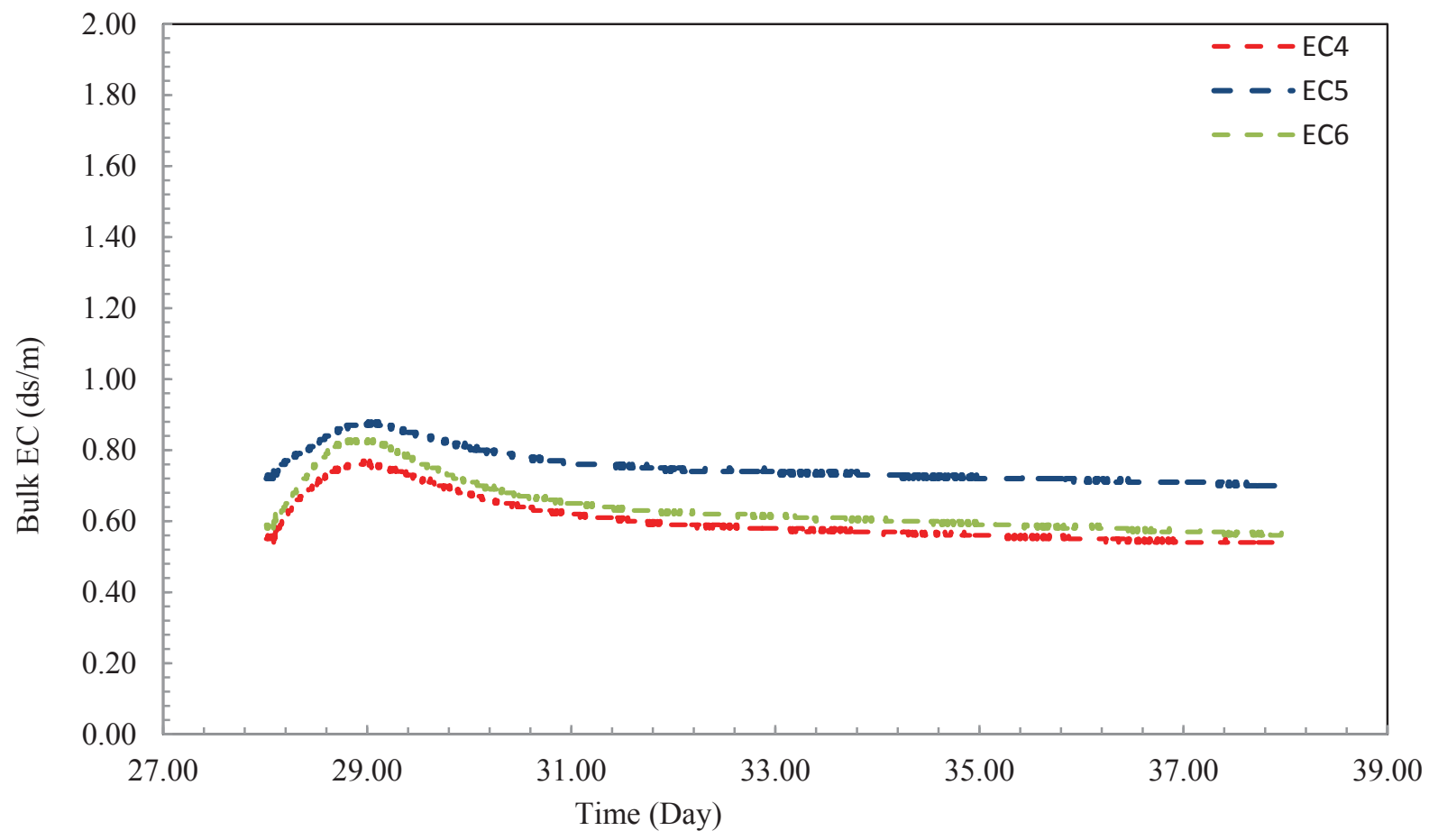

Figure AI9. Bulk Electrical Conductivity of second layer after fourth layer deposition

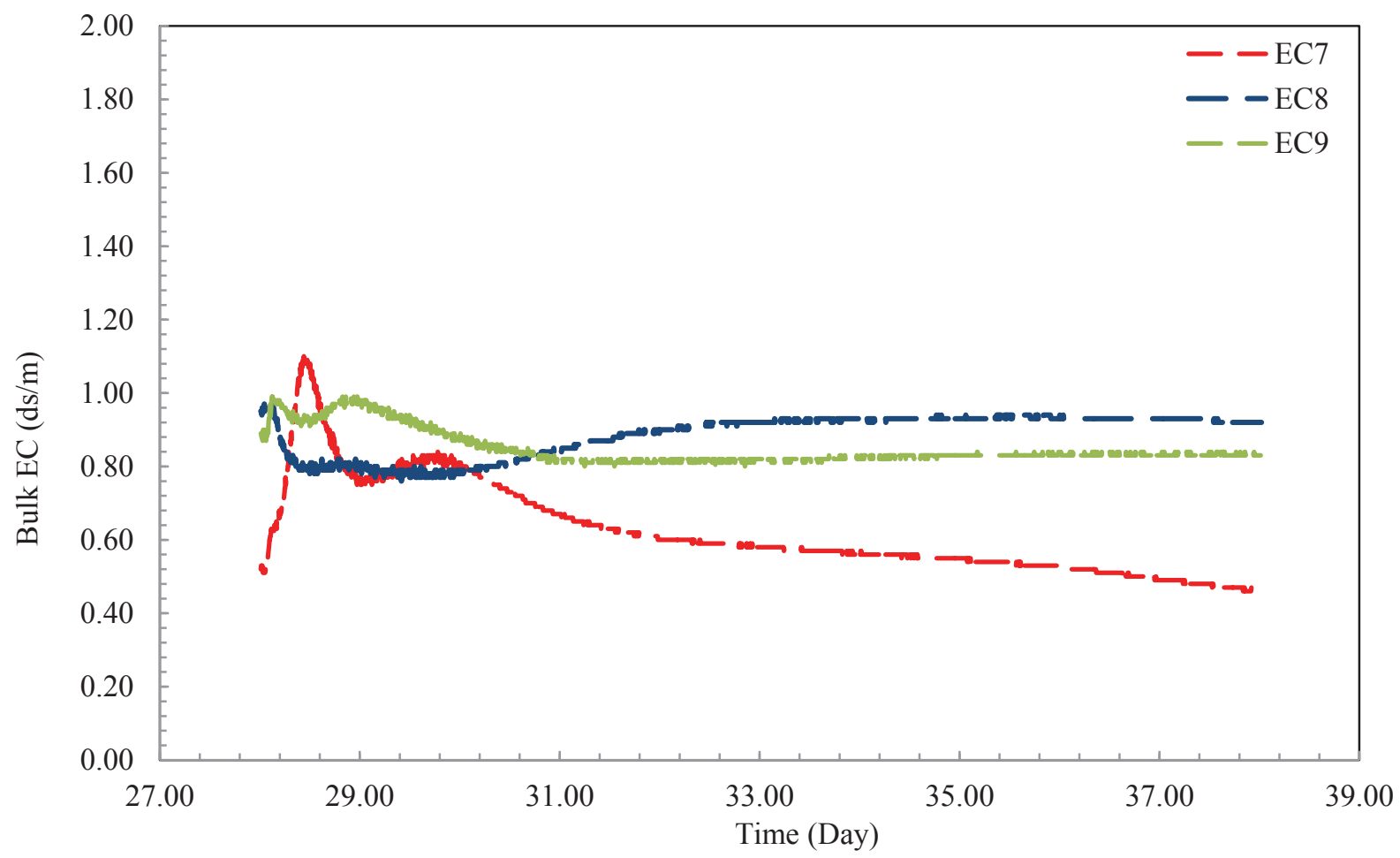

Figure AI10. Bulk Electrical Conductivity of Third Layer after Fourth Layer Deposition 


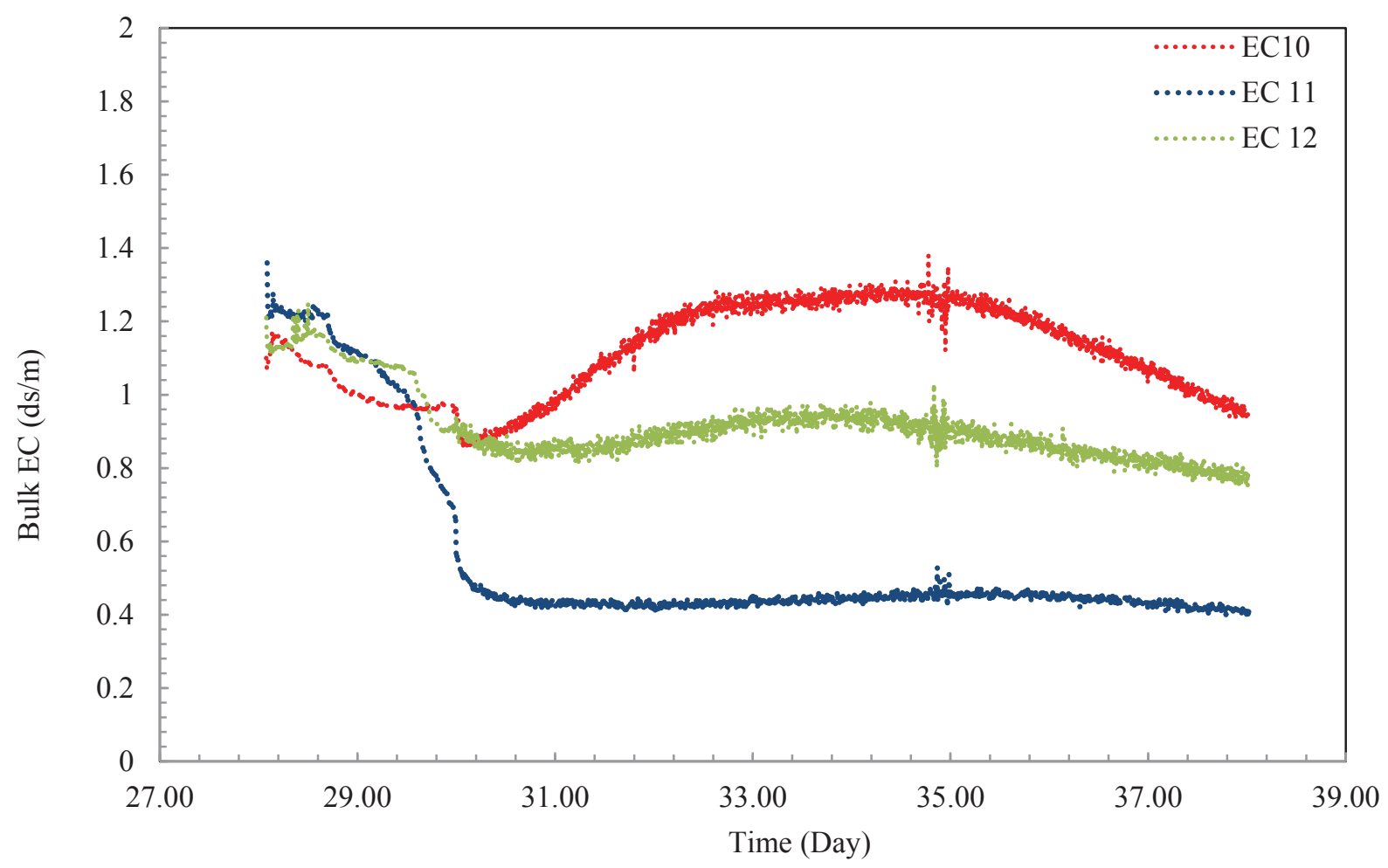

Figure AI11. Bulk Electrical Conductivity of Fourth Layer 


\section{APPENDIX II: 3D volume change stress history of desiccated-}

\section{rewetted samples}
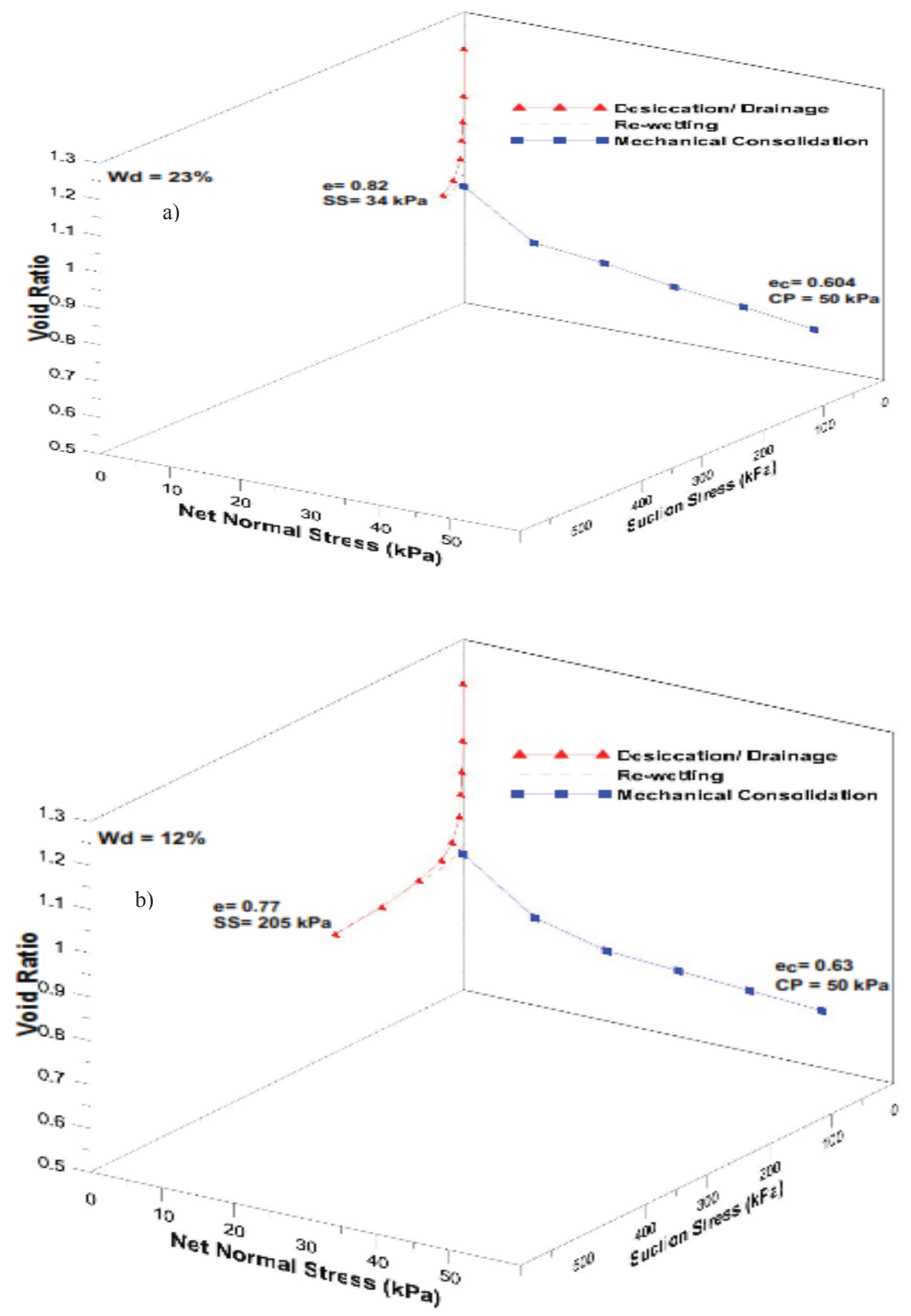

Figure AII.1 3D volume change stress history of samples desiccated to a) $W=23 \%, b) W=12 \%$, rewetted and consolidated to $50 \mathrm{kPa}$ 


\section{APPENDIX III: Generation of excess pore pressure during shearing for desiccated-rewetted and desiccated-consolidated-rewetted}

\section{samples}

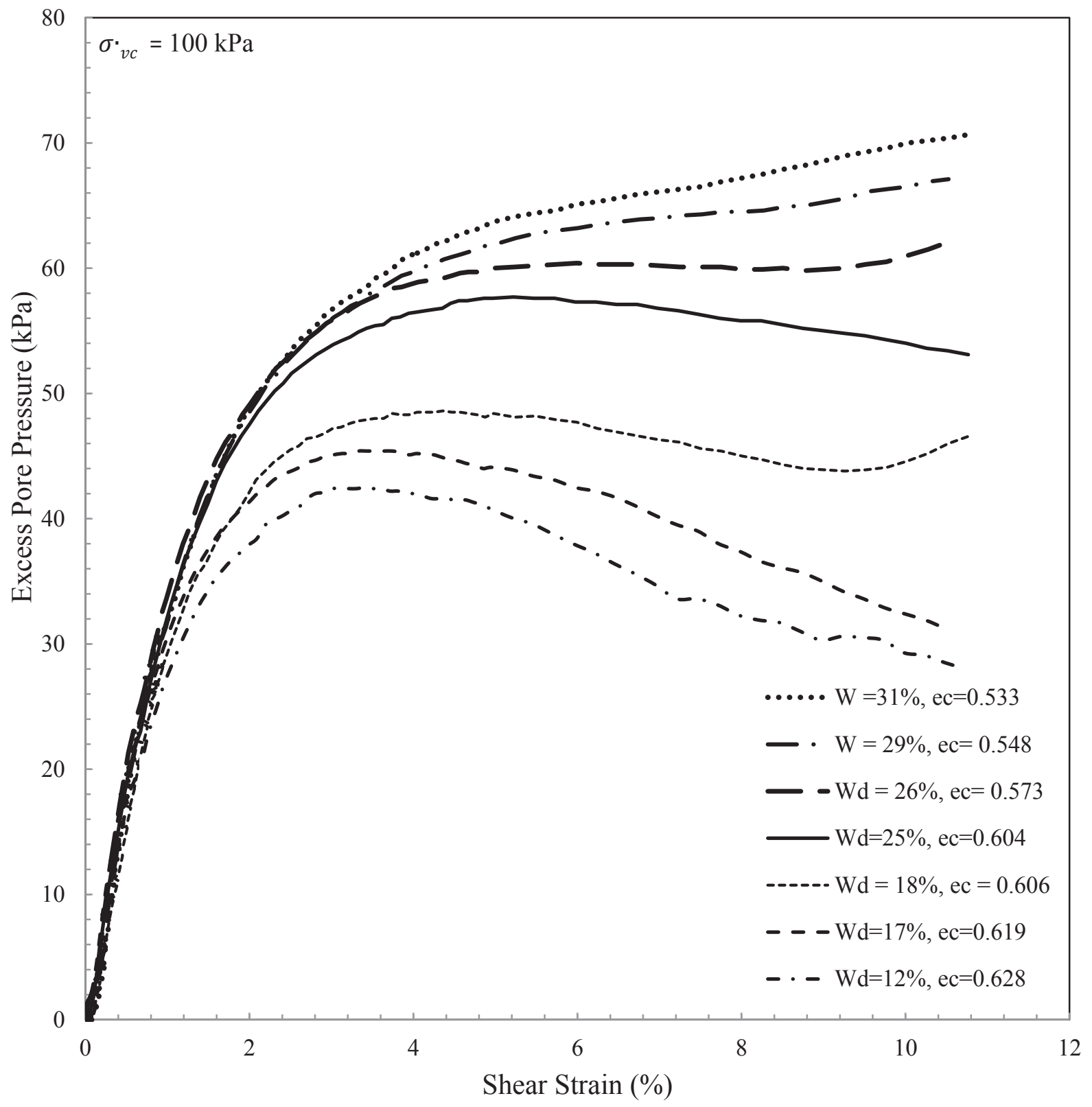

Figure AIII.1 Generation of excess pore pressure during shearing for desiccated and rewetted samples prepared by small scale method under $100 \mathrm{kPa}$ 


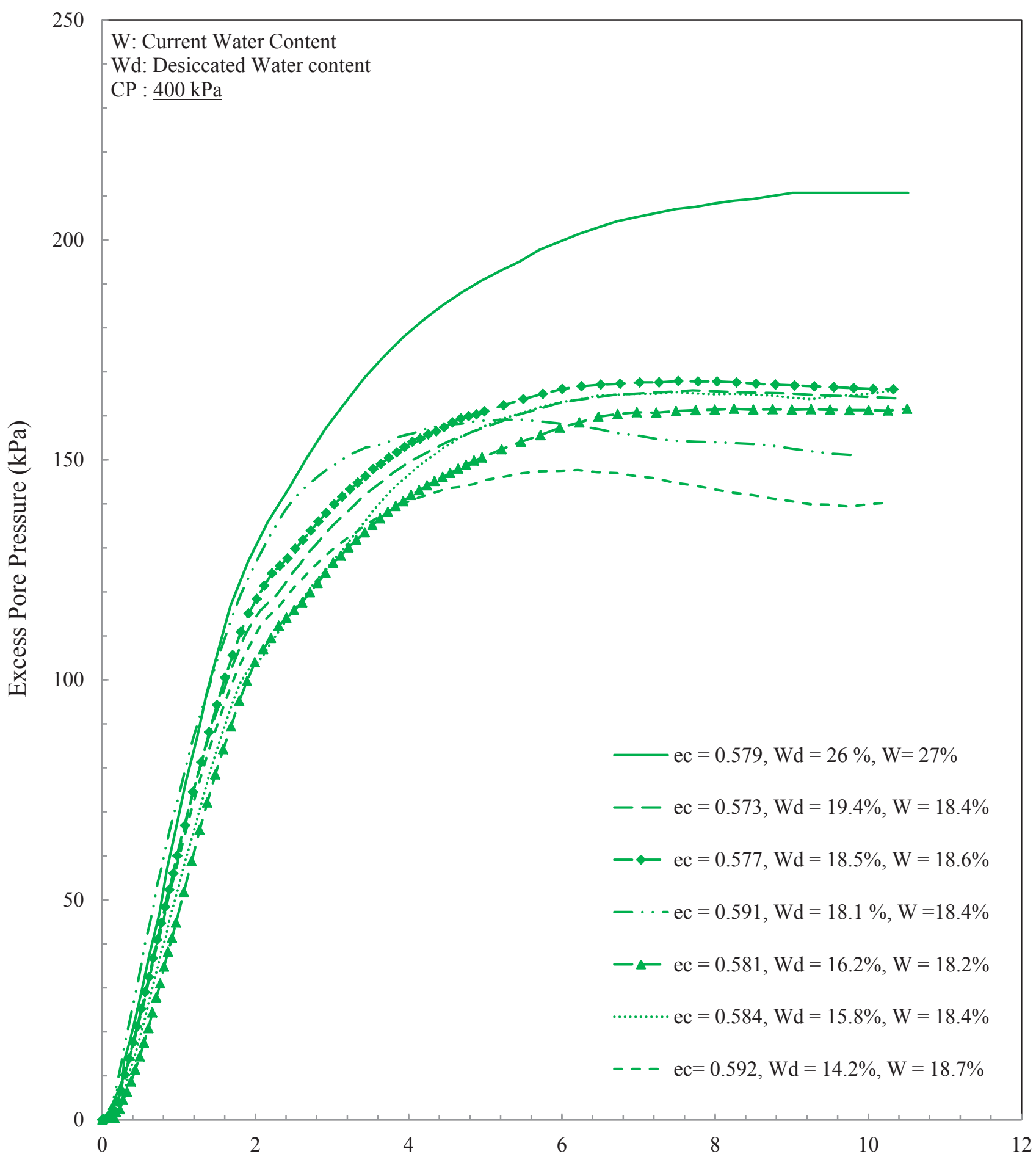

Effective Normal Stress (kPa)

Figure AIII.2 Excess pore water pressure generation of desiccated-consolidated-rewetted samples prepared under $400 \mathrm{kPa}$ 


\section{APPENDIX IV: Cyclic simple shear results}
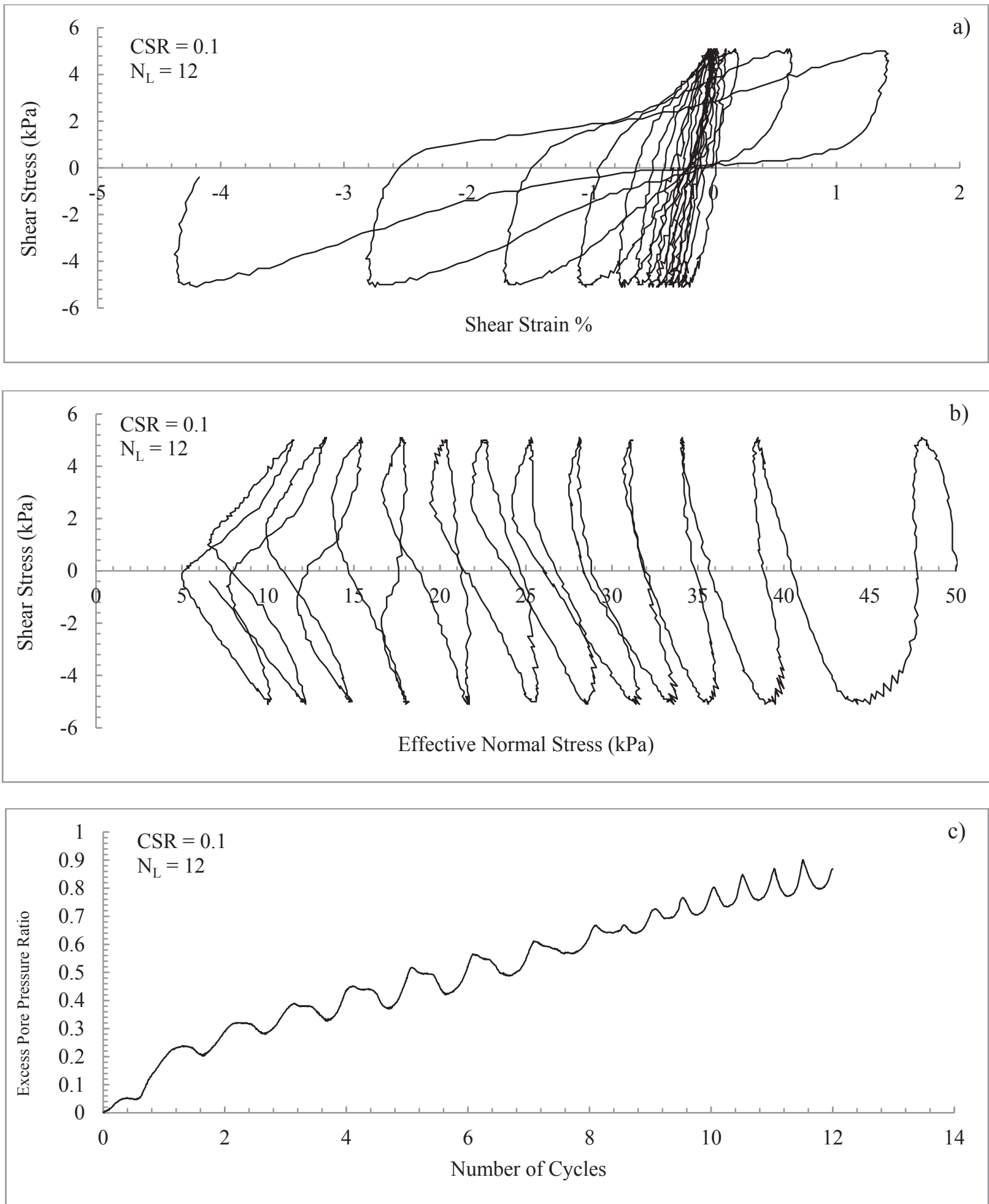

Figure AIV.1 Cyclic simple shear response of desiccated/rewetted sample $(\mathrm{Wd}=20 \%)$ under $\mathrm{CSR}=0.12$ 

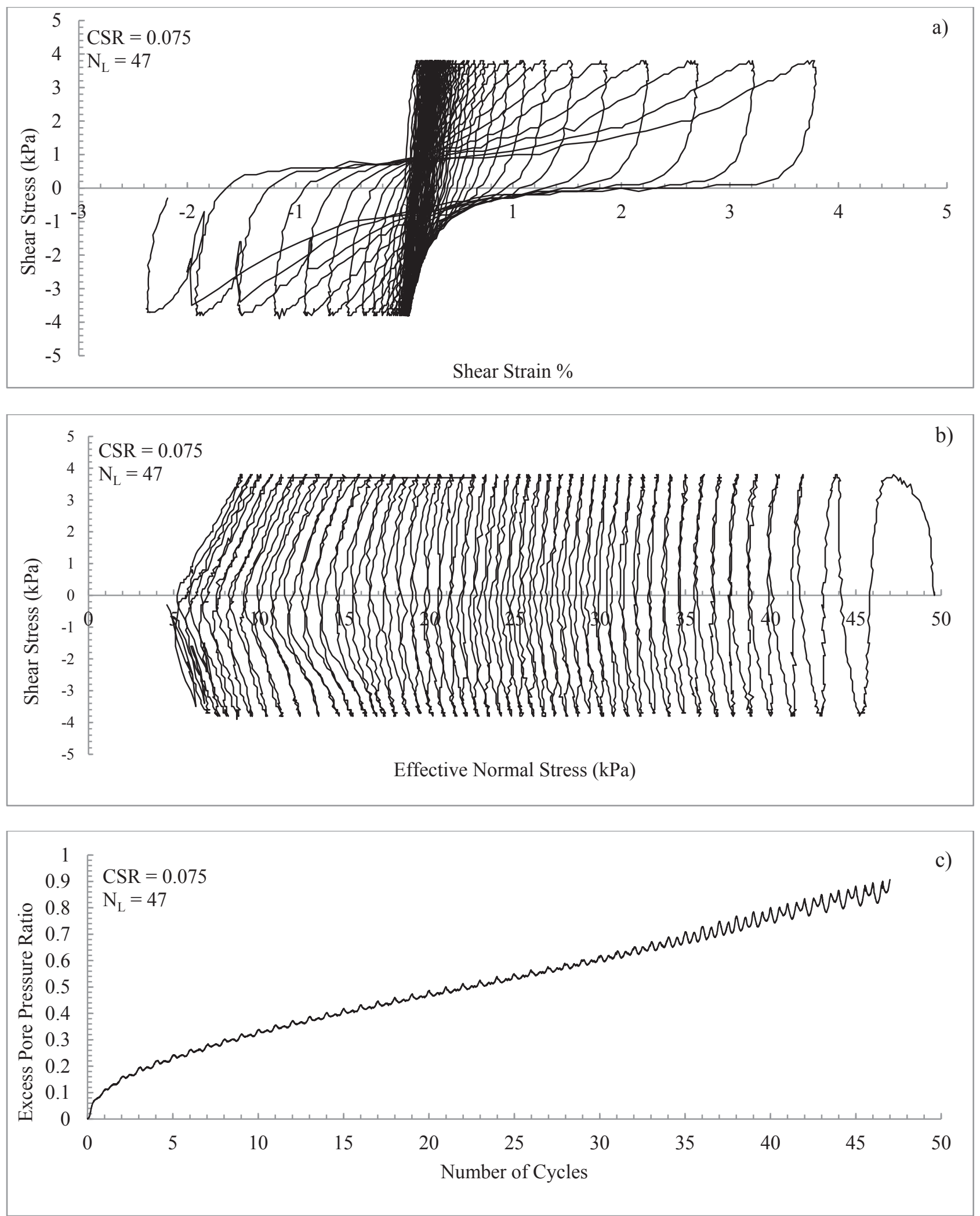

Figure AIV.2 Cyclic simple shear response of desiccated/rewetted samples $(\mathrm{Wd}=17 \%)$ under $\mathrm{CSR}=0.075$ 

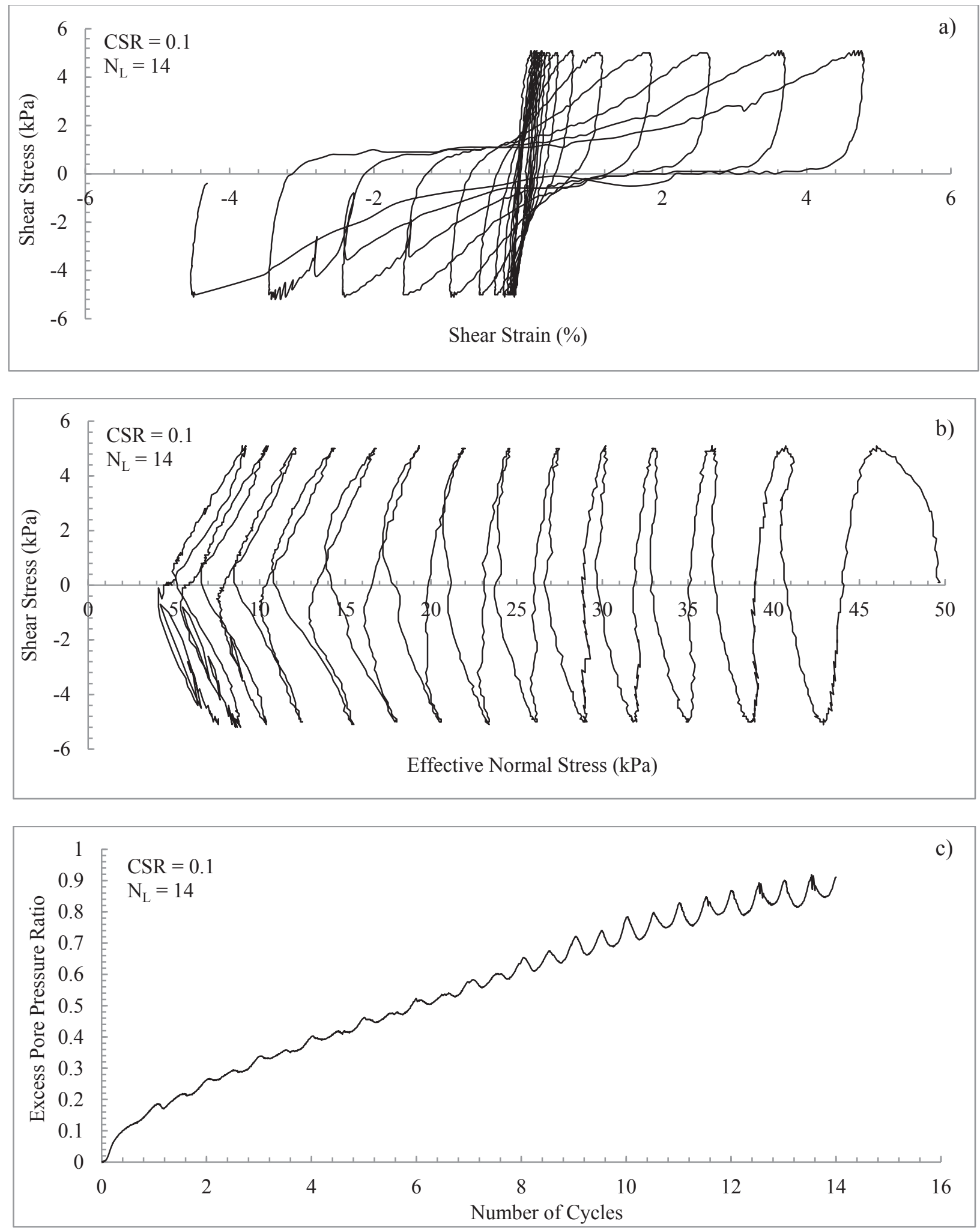

Figure AIV.3 Cyclic simple shear response of desiccated/rewetted sample $(\mathrm{Wd}=17 \%)$ under $\mathrm{CSR}=0.1$ 

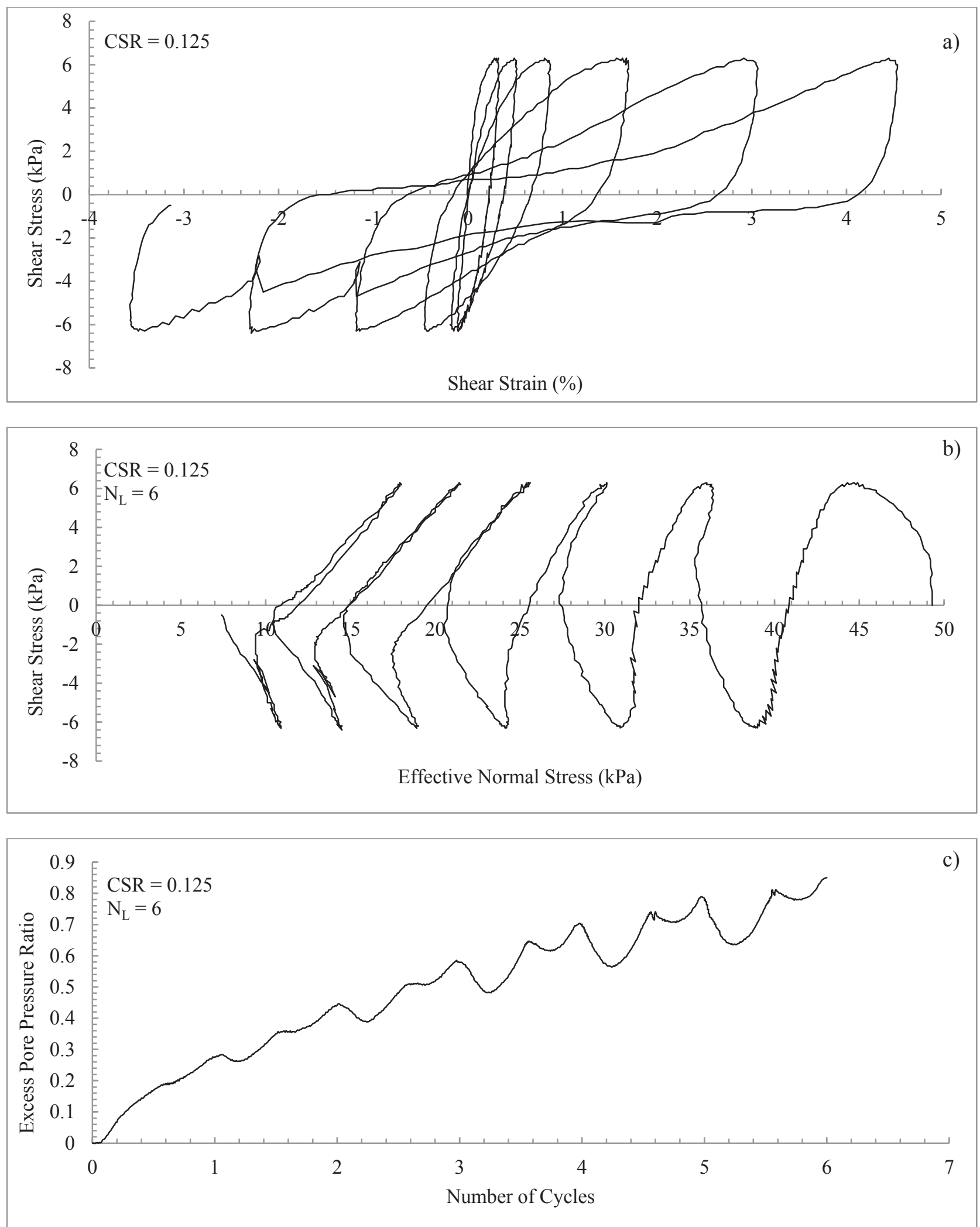

Figure AIV.4 Cyclic simple shear response of desiccated/rewetted samples $(\mathrm{Wd}=17 \%)$ under $\mathrm{CSR}=0.125$ 

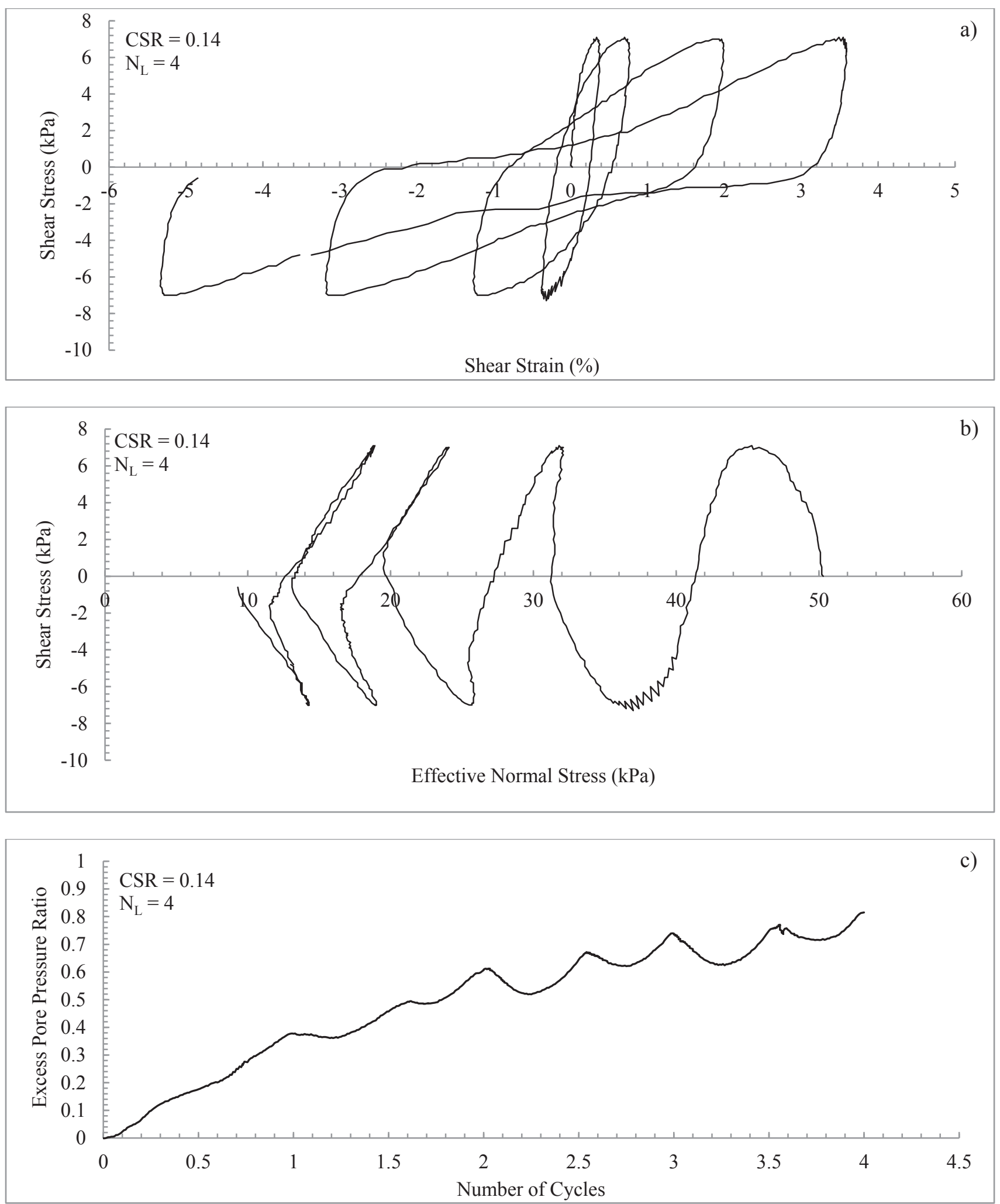

Figure AIV 5. Cyclic simple shear response of desiccated/rewetted sample $(\mathrm{Wd}=17 \%)$ under $\mathrm{CSR}=0.14$ 

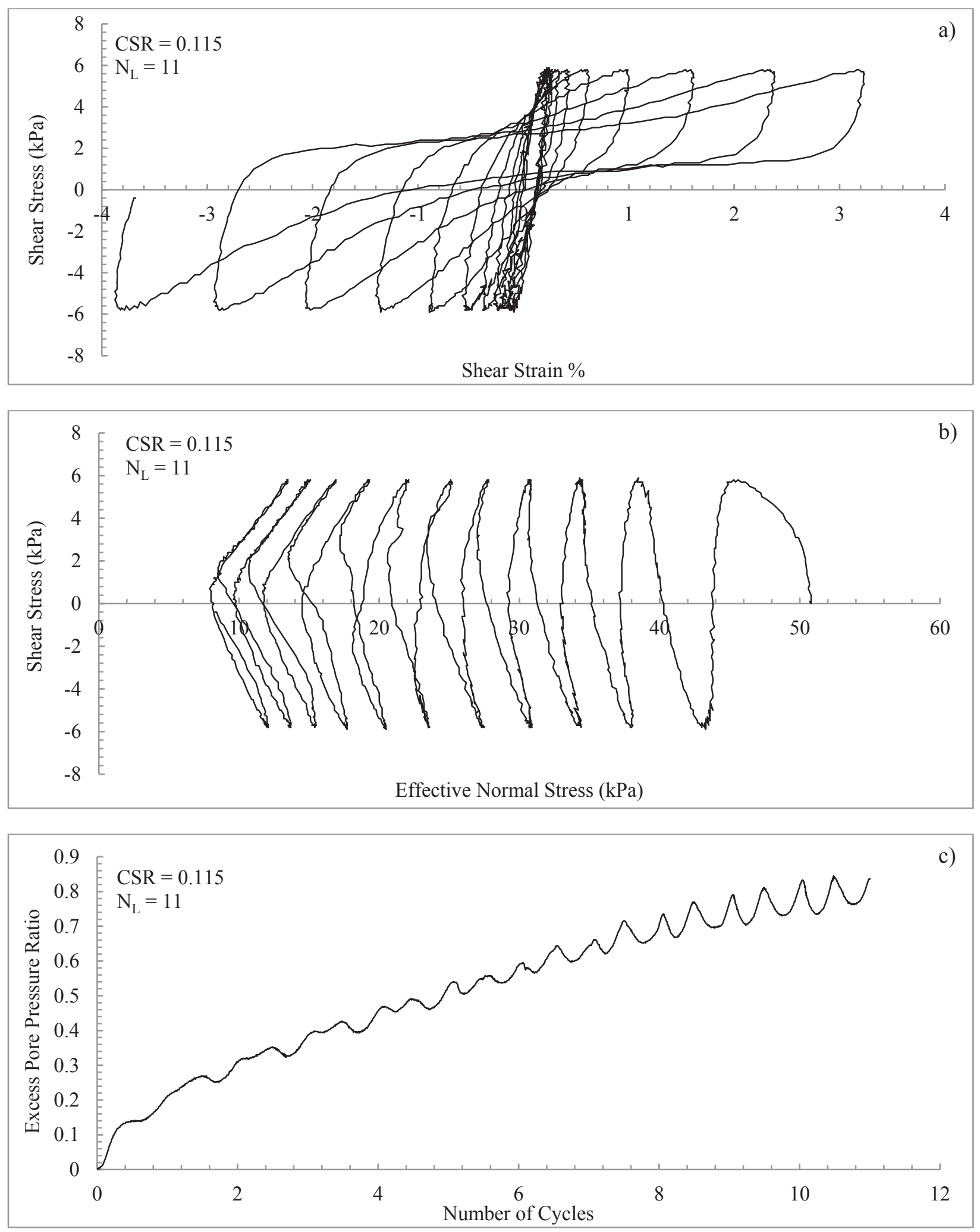

Figure A IV.6 Cyclic simple shear response of desiccated/rewetted sample $(\mathrm{Wd}=12 \%)$ at CSR $=0.115$ 

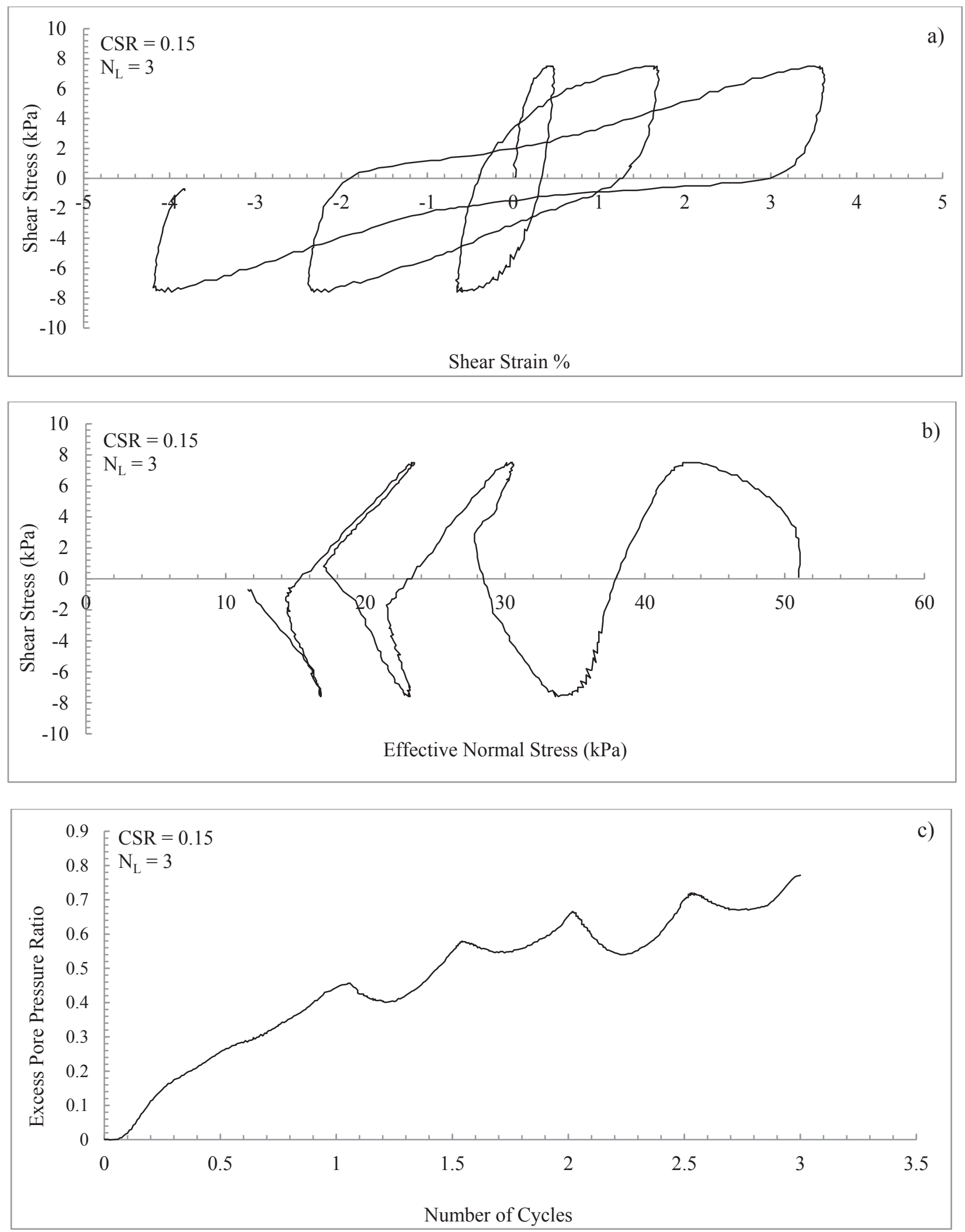

Figure AIV 7. Cyclic simple shear response of desiccated/rewetted sample $(\mathrm{Wd}=12 \%)$ under $\mathrm{CSR}=0.15$ 

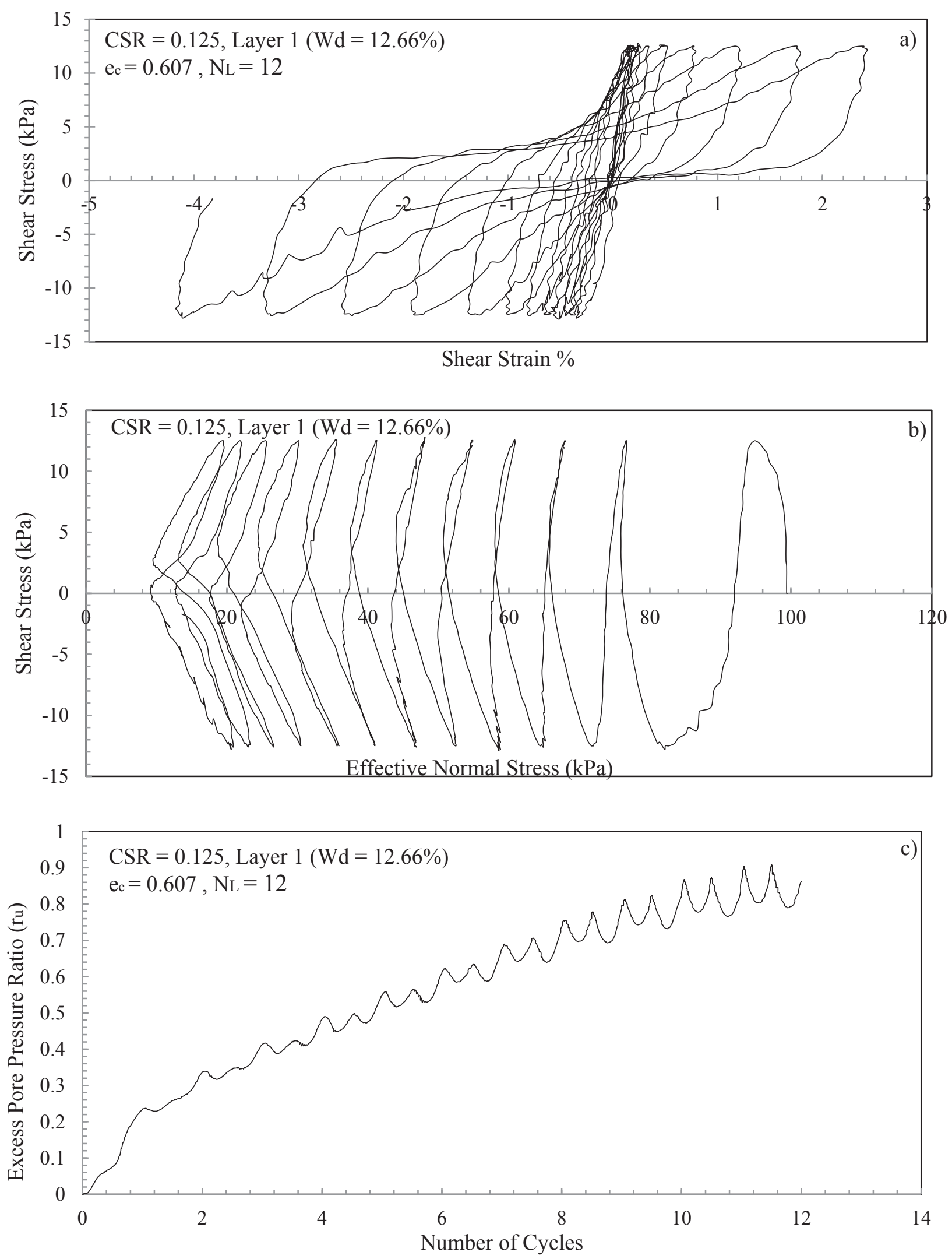

Figure AIV 8.Cyclic simple shear response of the sample obtained from Layer 1 at CSR $=0.125$ 

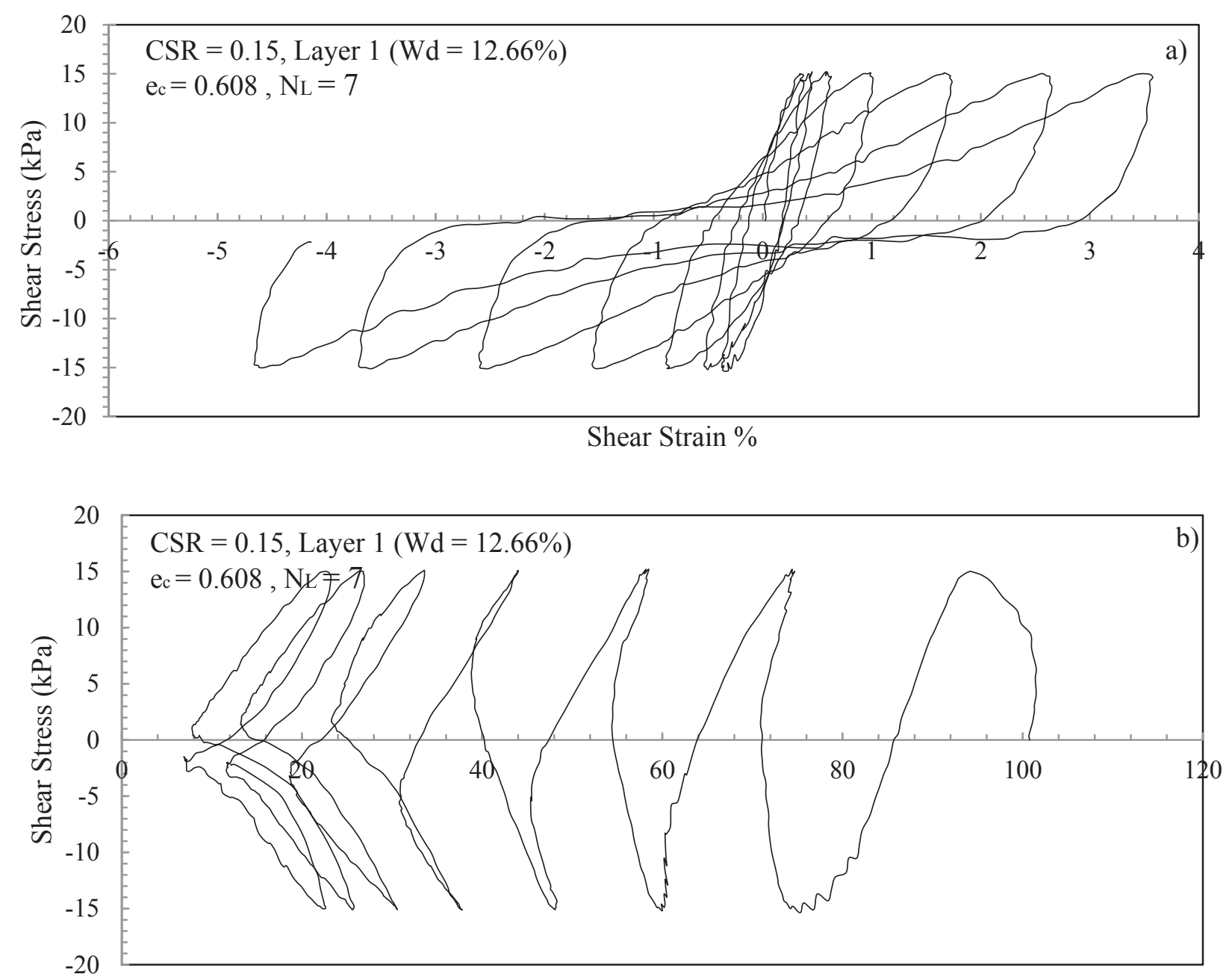

Effective Normal Stress $(\mathrm{kPa})$

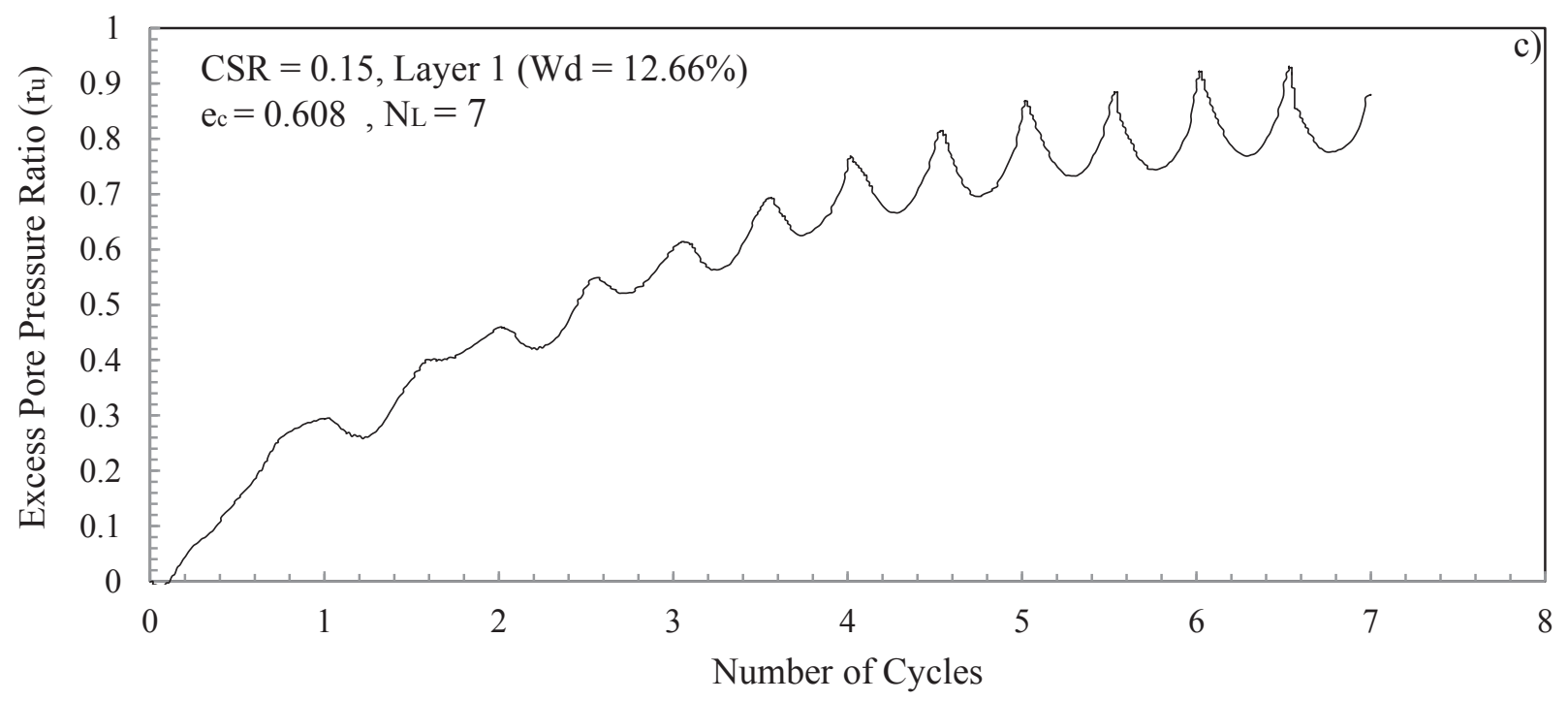

Figure AIV 9. Cyclic simple shear response of the sample obtained from Layer 1 at CSR $=0.15$ 

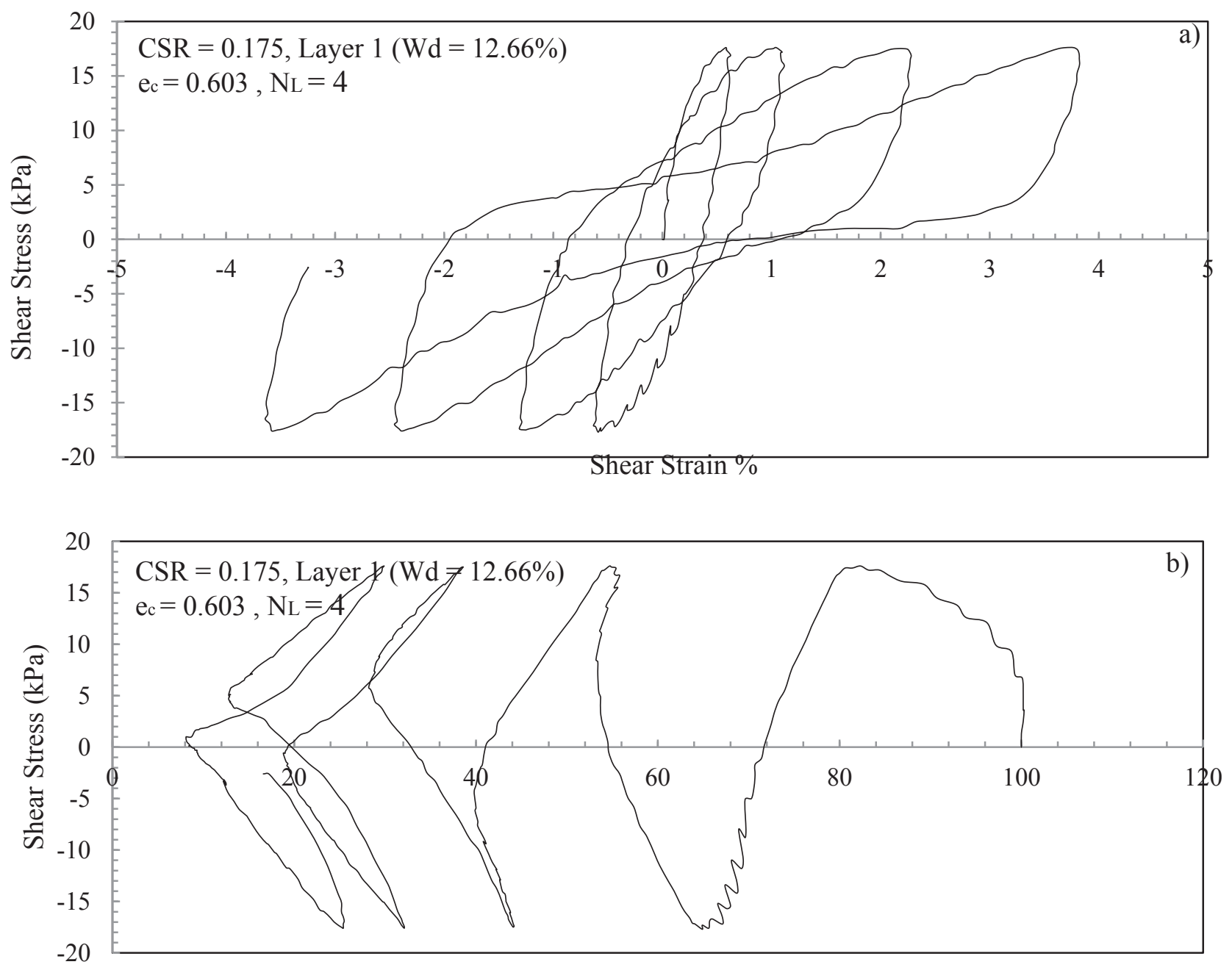

Effective Normal Stress $(\mathrm{kPa})$

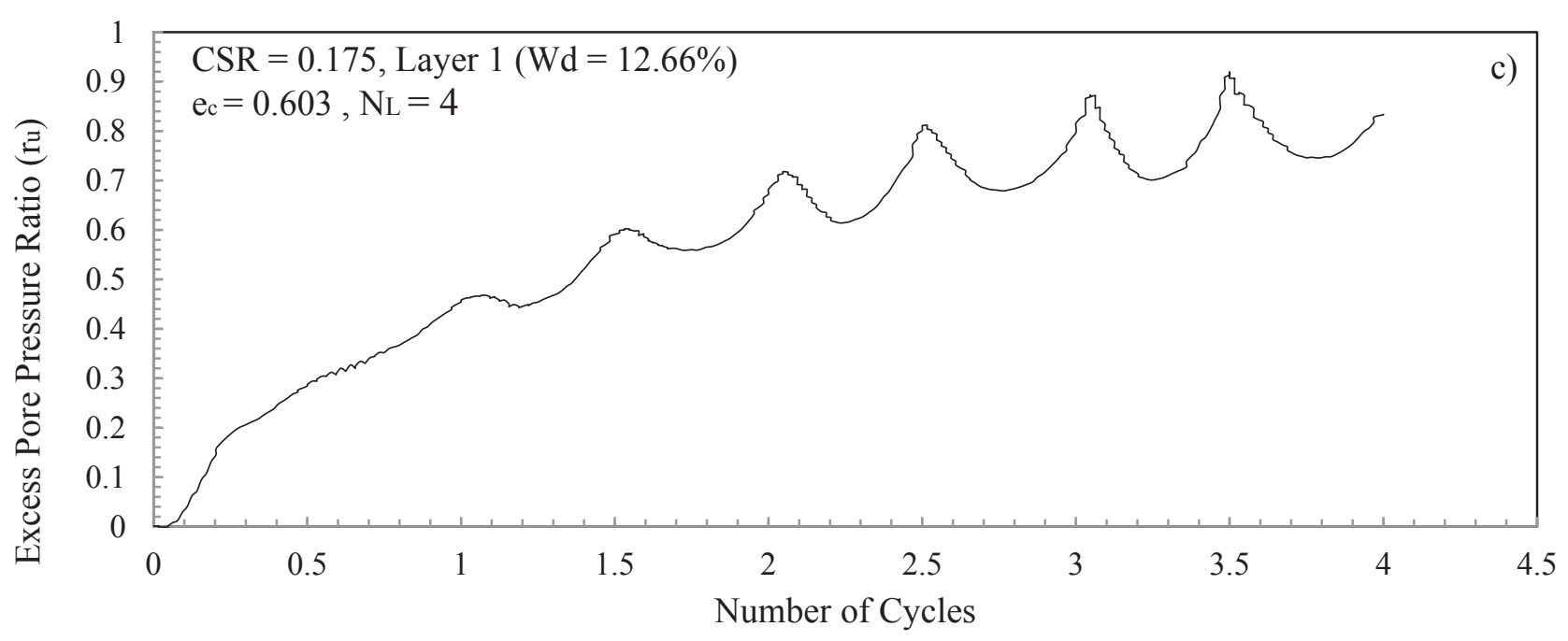

Figure AIV 10. Cyclic simple shear response of the sample obtained from Layer 1 at CSR $=$ 0.175 


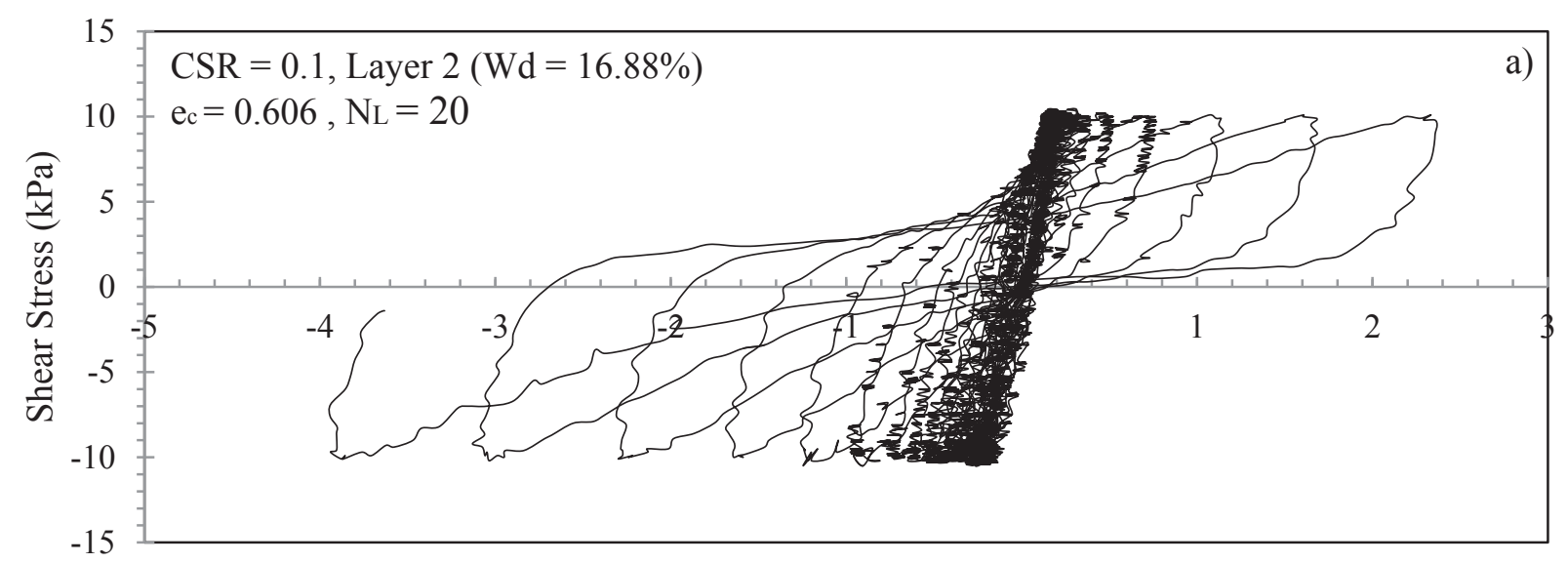

Shear Strain \%
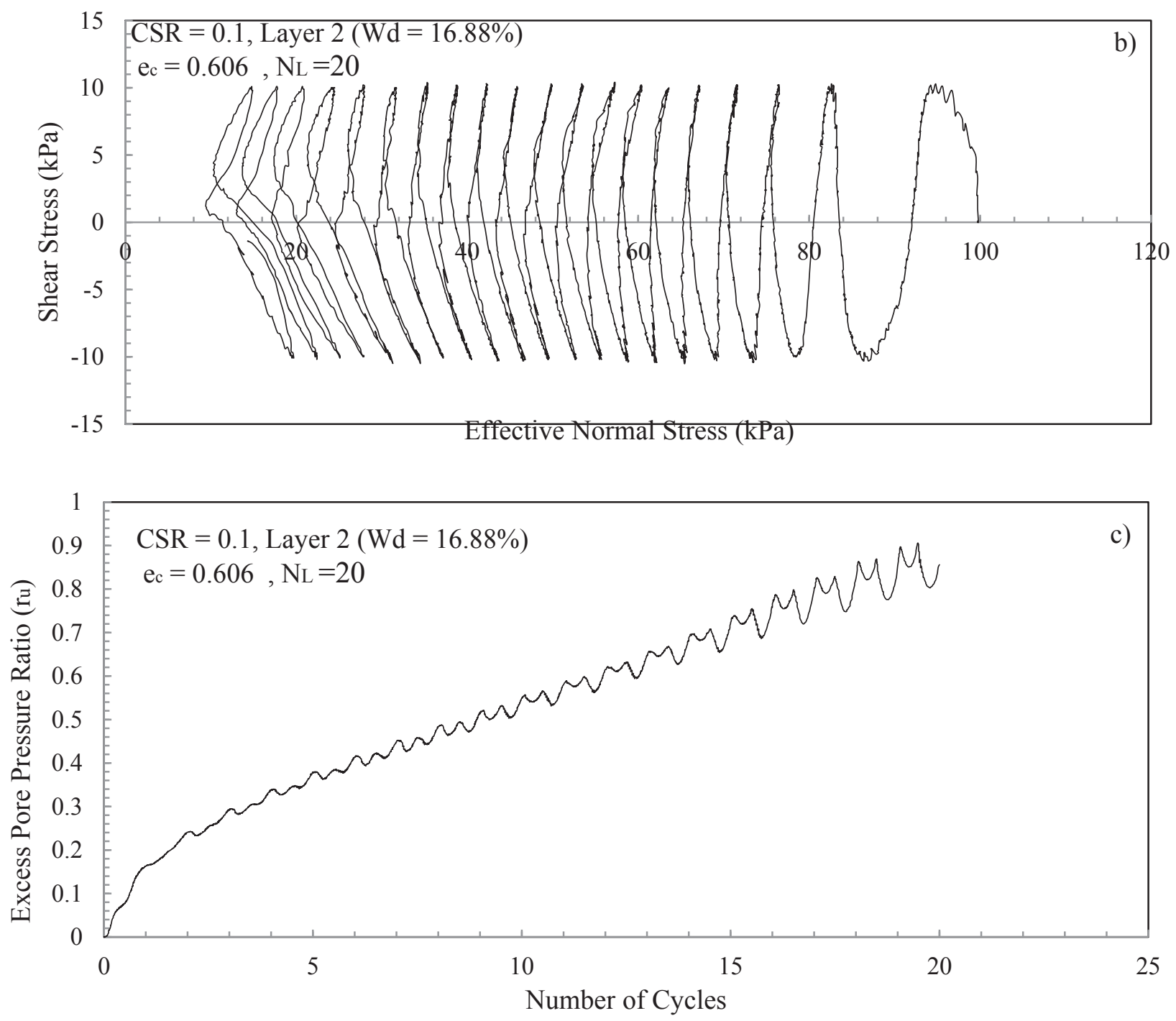

Figure AIV 11. Cyclic simple shear response of the sample obtained from Layer 2 at CSR $=0.1$ 

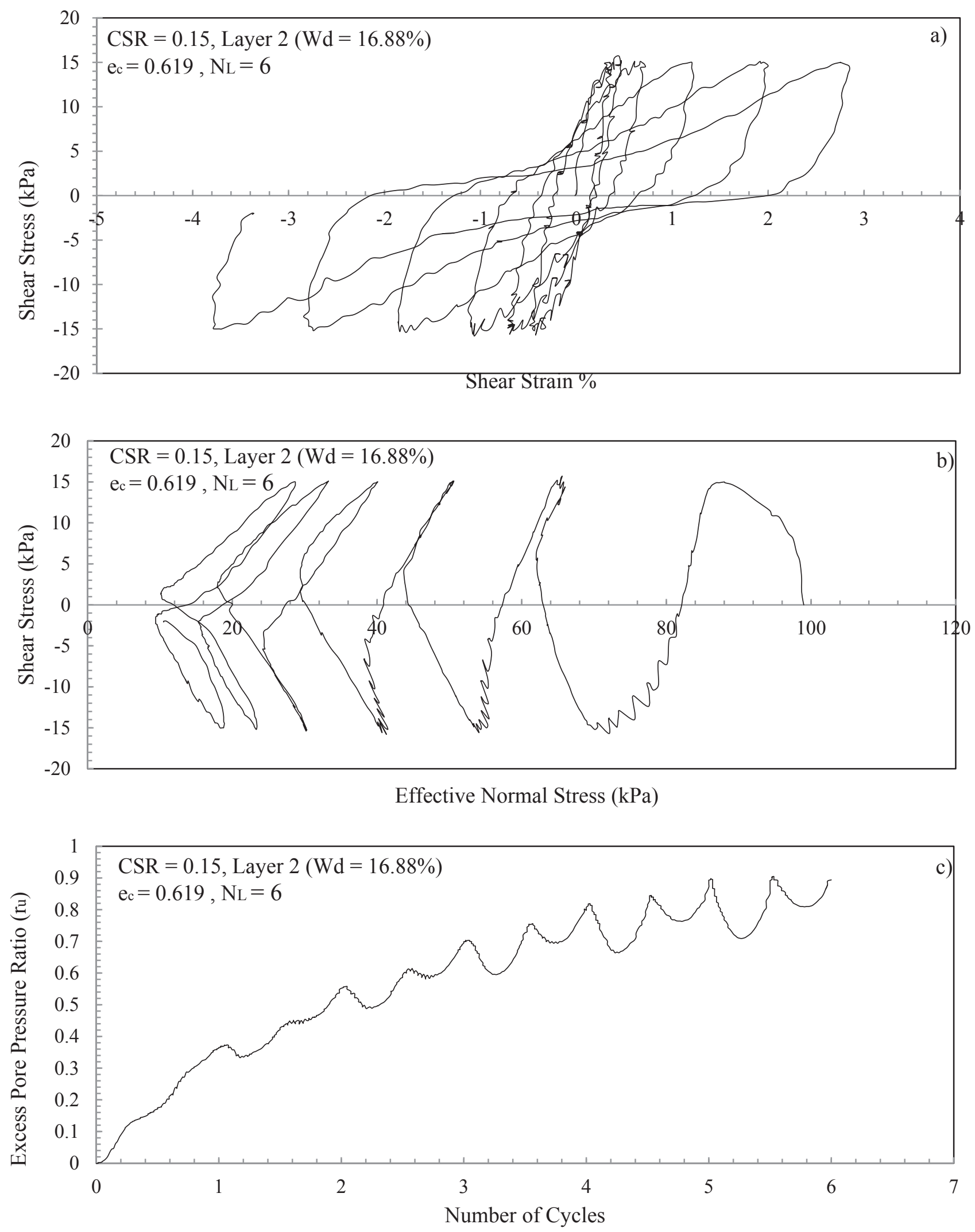

Figure AIV 12. Cyclic simple shear response of the sample obtained from Layer 2 at CSR $=0.15$ 

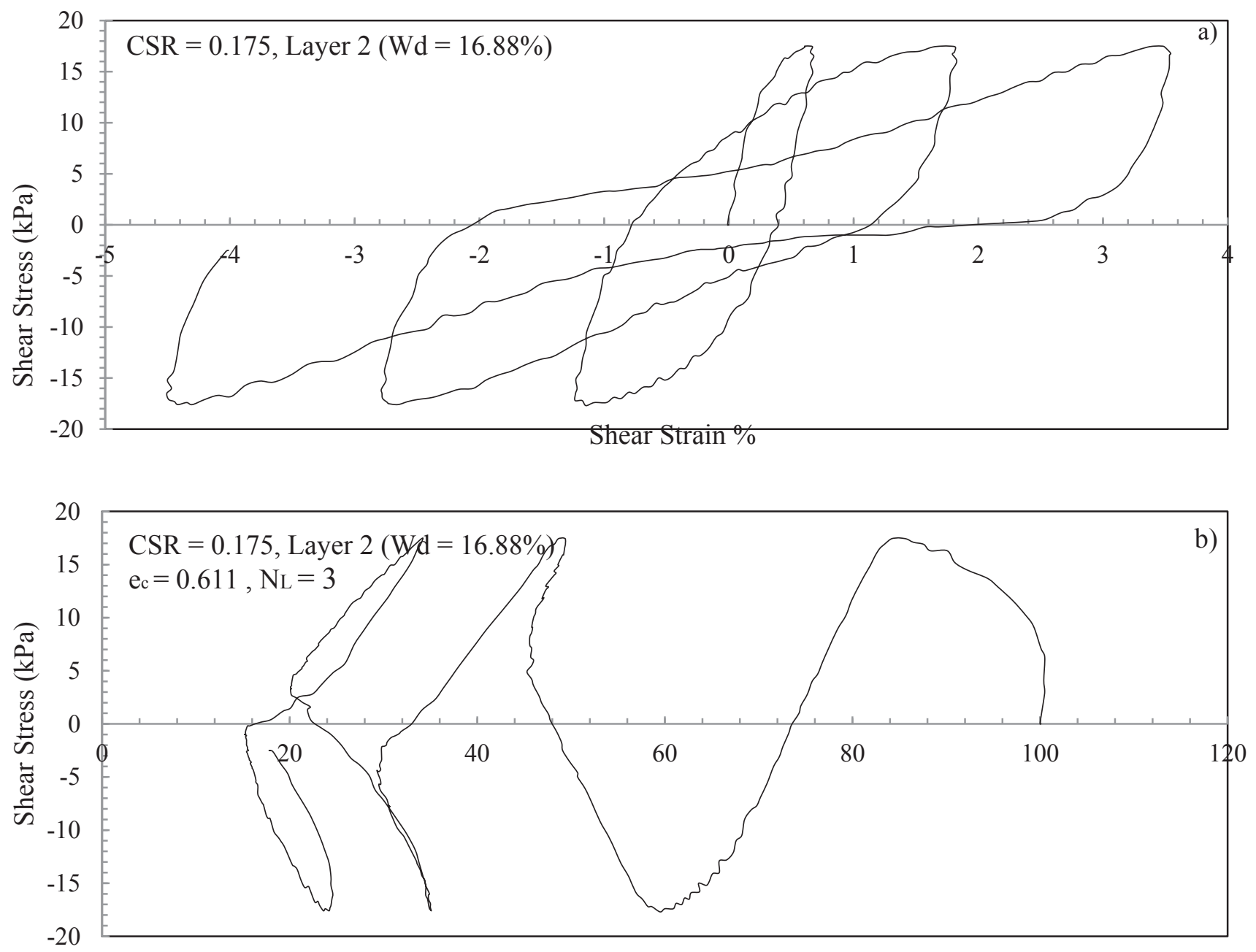

Effective Normal Stress $(\mathrm{kPa})$

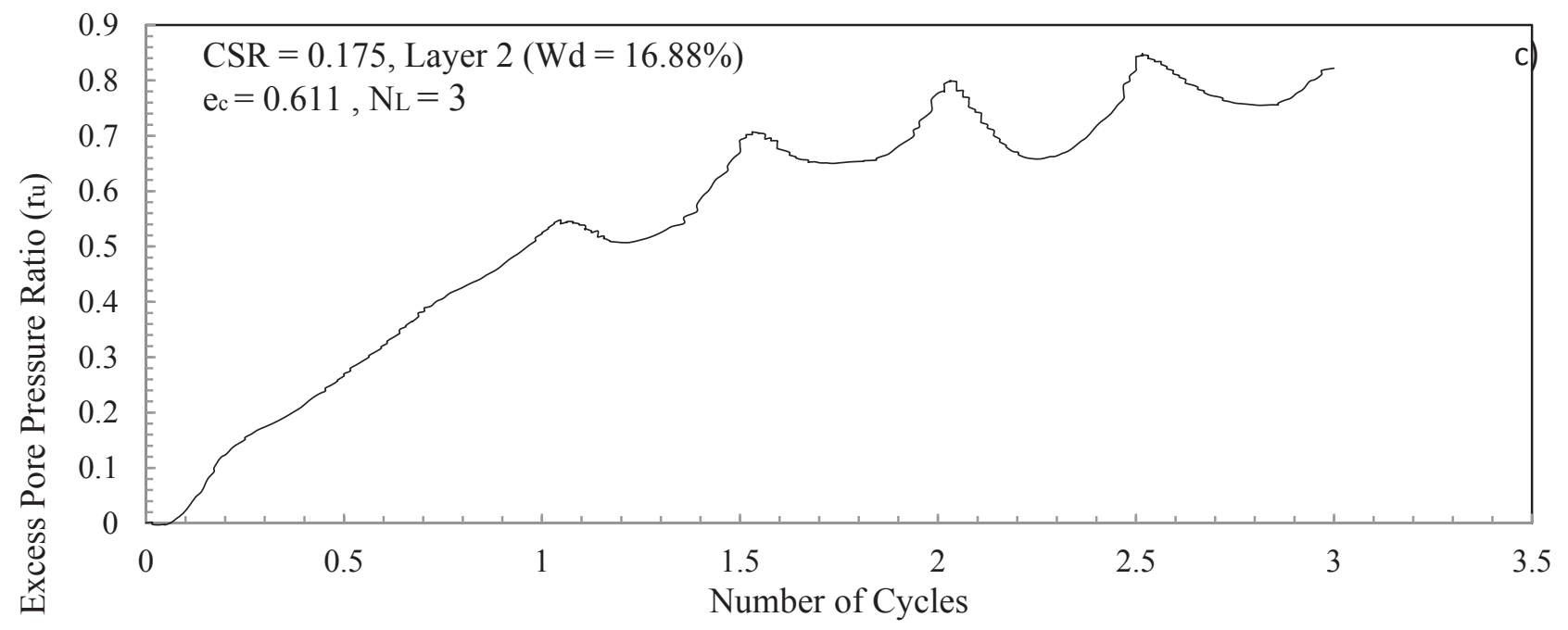

Figure AIV 13. Cyclic simple shear response of the sample obtained from Layer 2 at CSR $=$ 0.175 

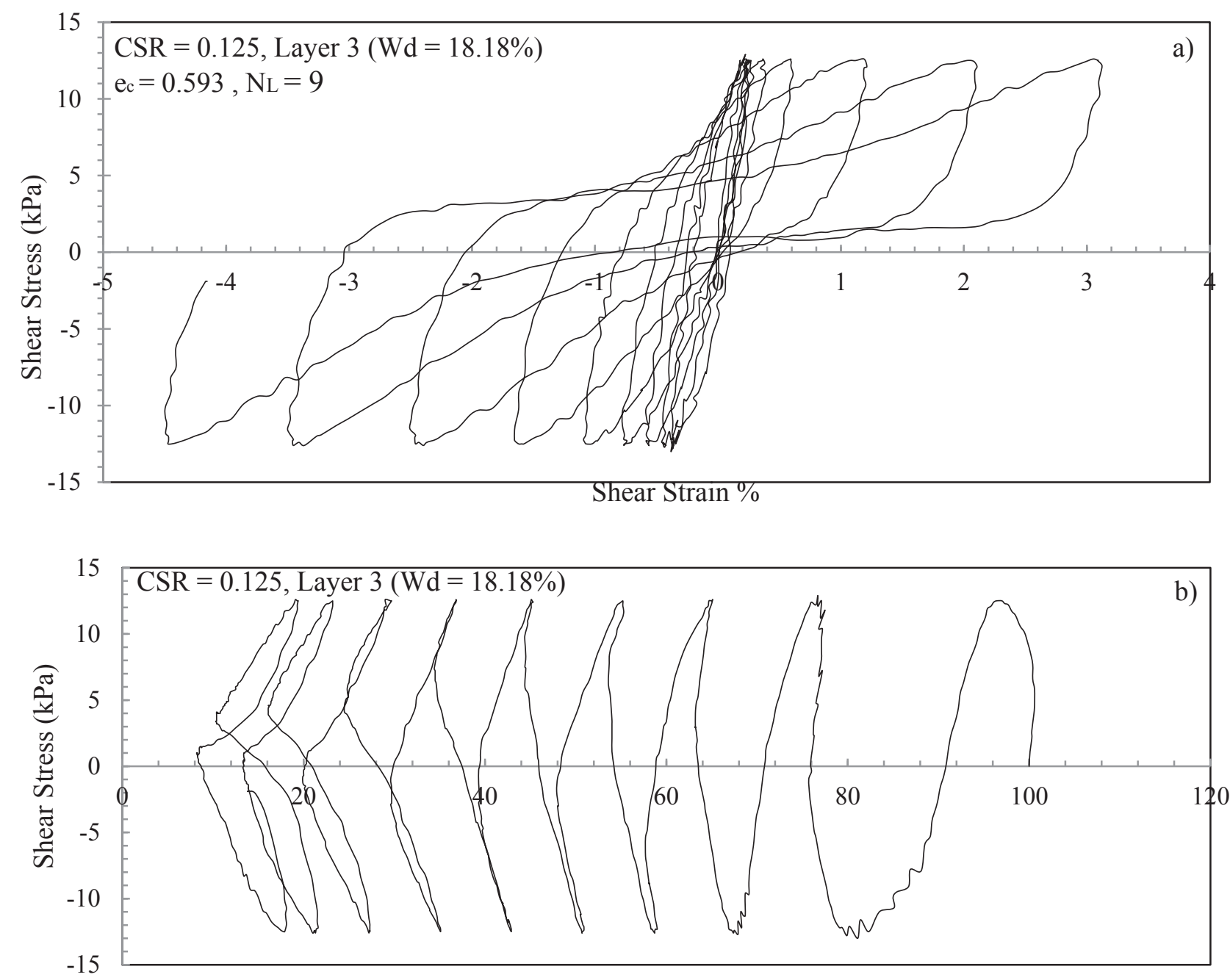

Effective Normal Stress $(\mathrm{kPa})$

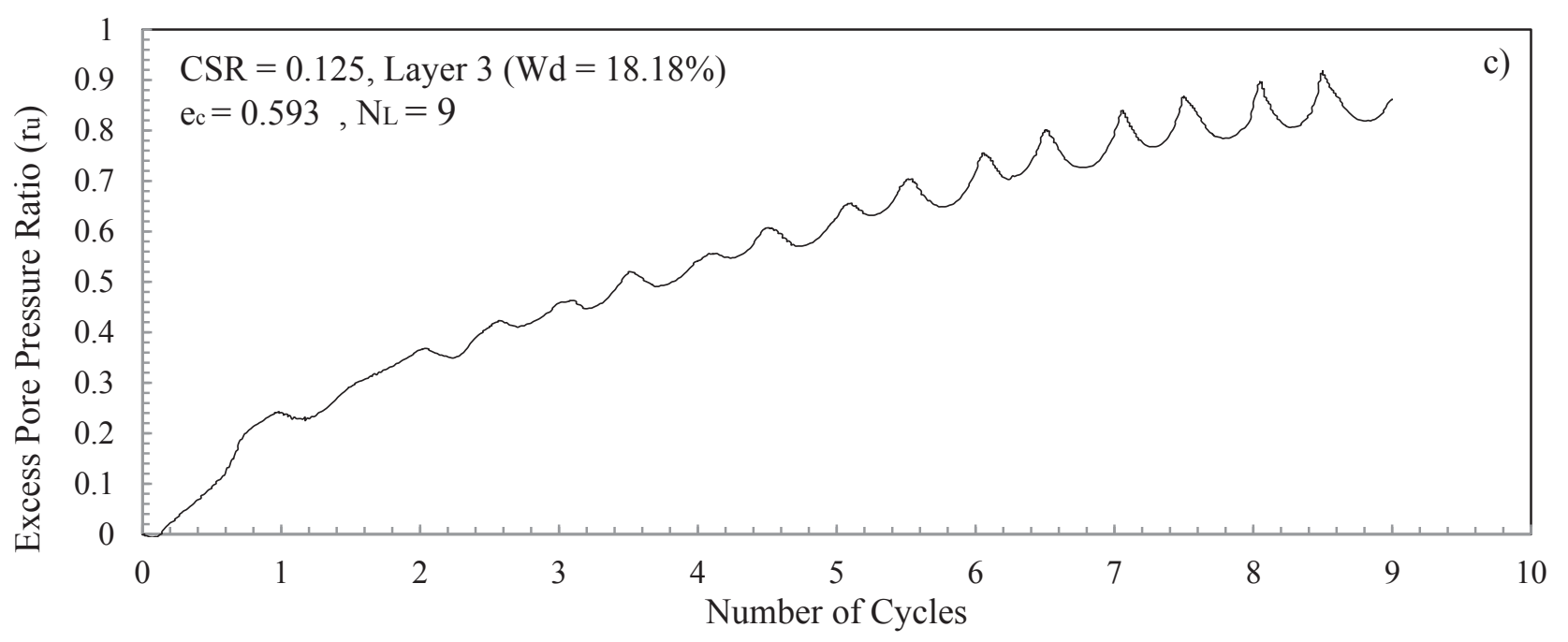

Figure AIV 14. Cyclic simple shear response of the sample obtained from Layer 3 at CSR $=$ 0.125 

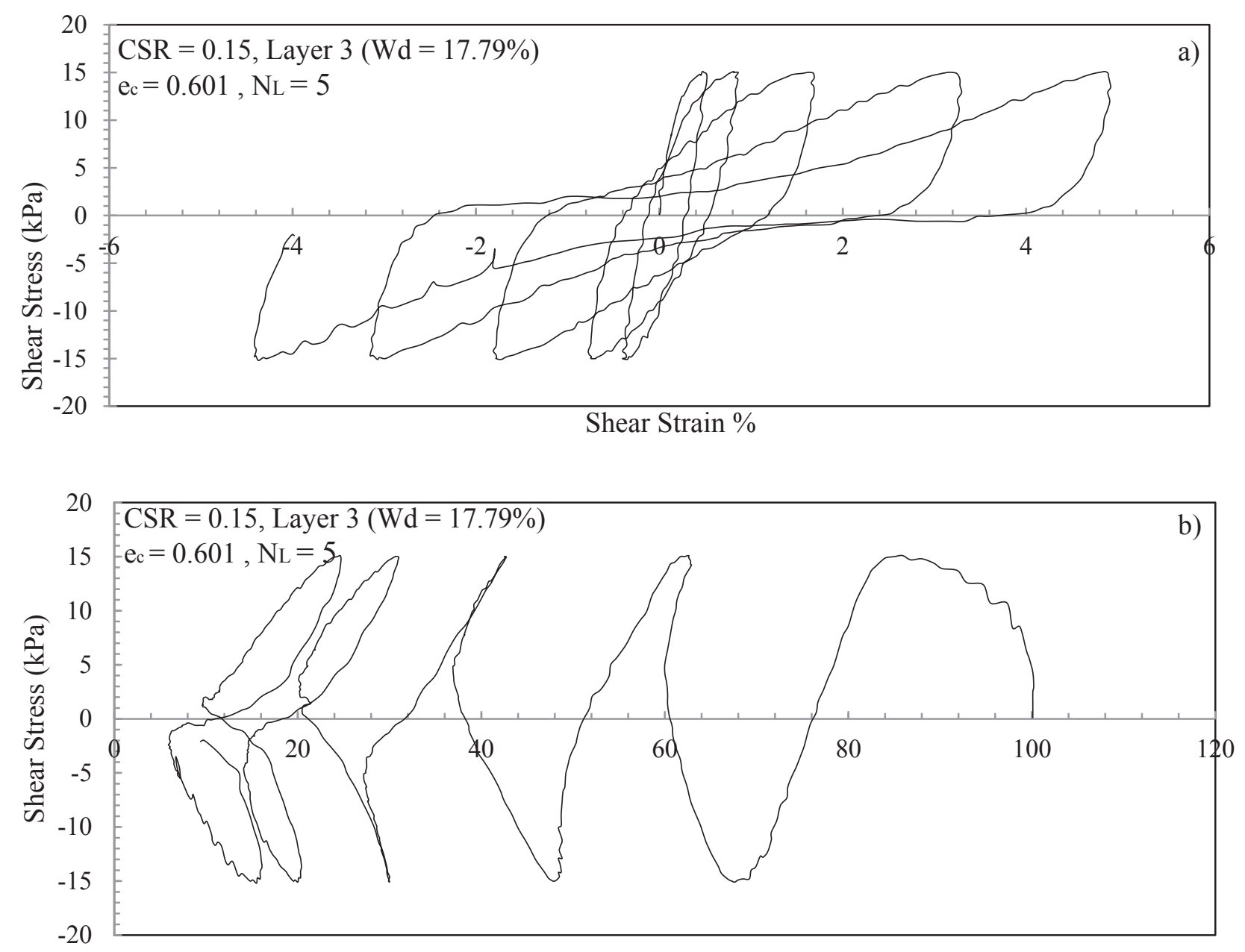

Effective Normal Stress ( $\mathrm{kPa})$

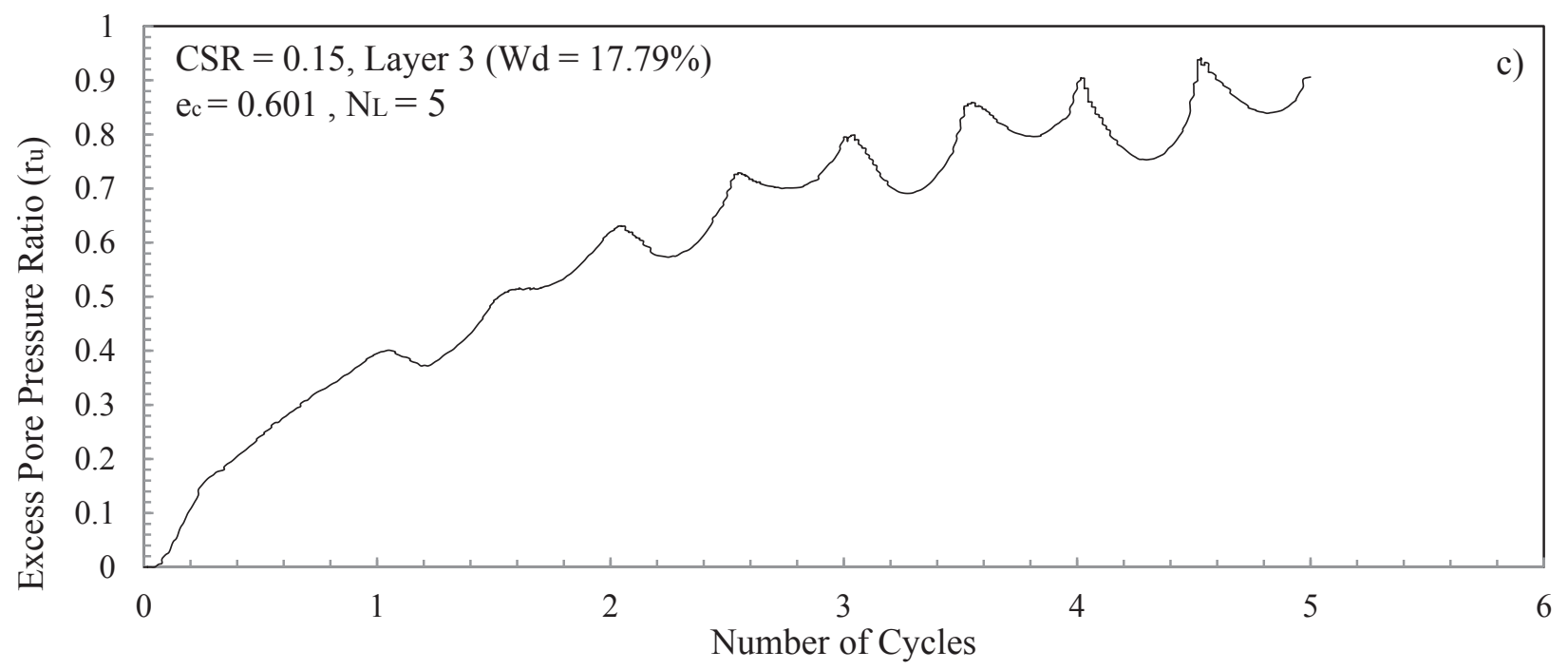

Figure AIV 15. Cyclic simple shear response of the sample obtained from Layer 3 at CSR $=0.15$ 

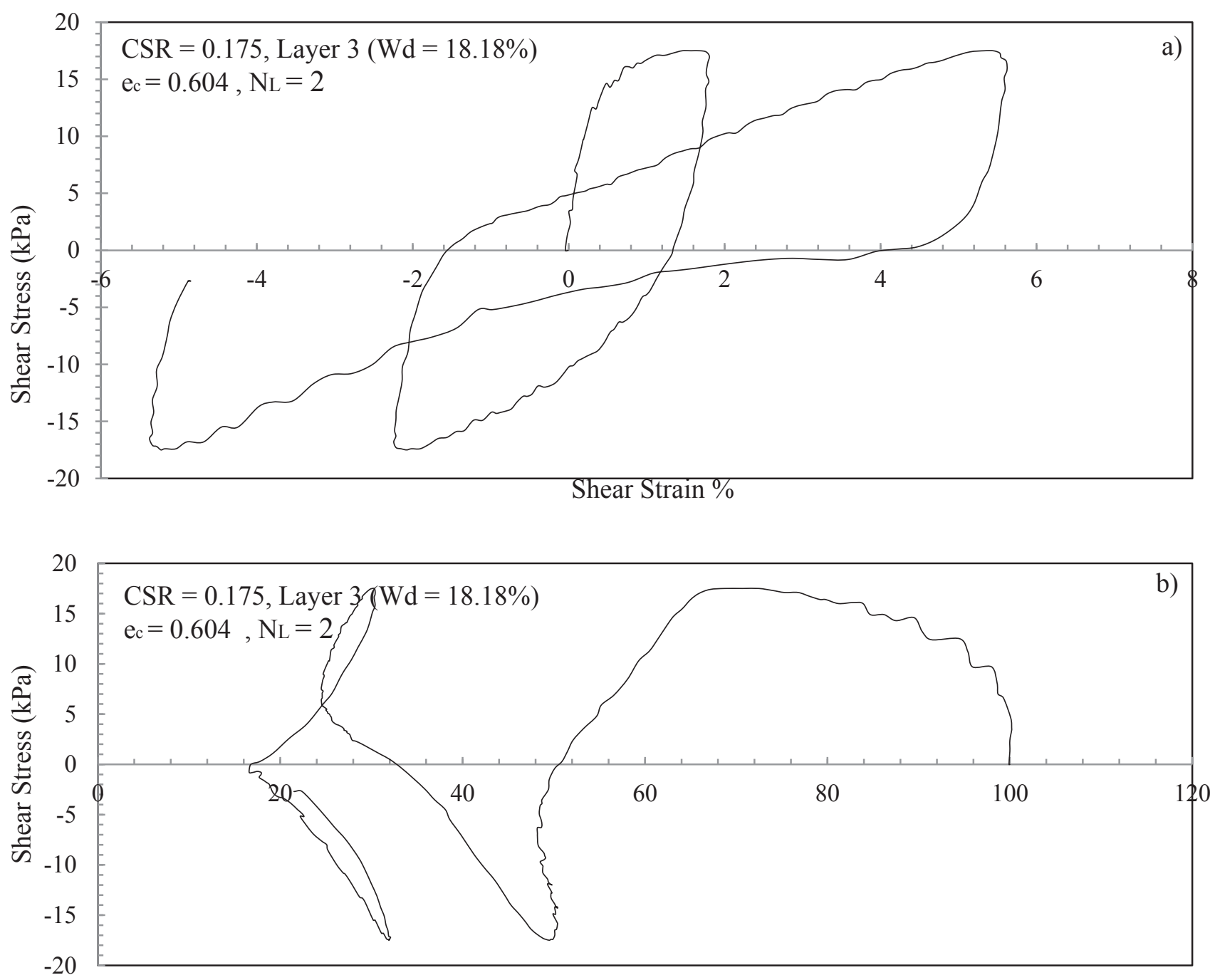

Effective Normal Stress $(\mathrm{kPa})$

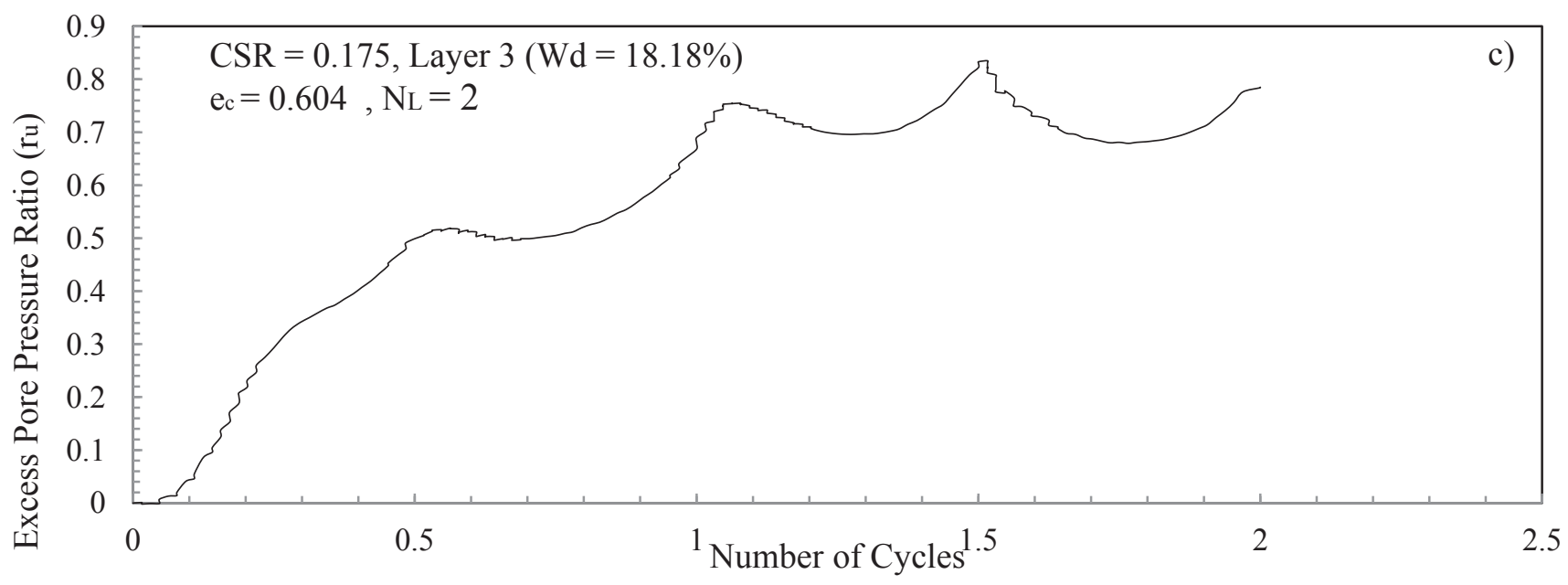

Figure AIV 16. Cyclic simple shear response of the sample obtained from Layer 3 at CSR $=$ 0.175 

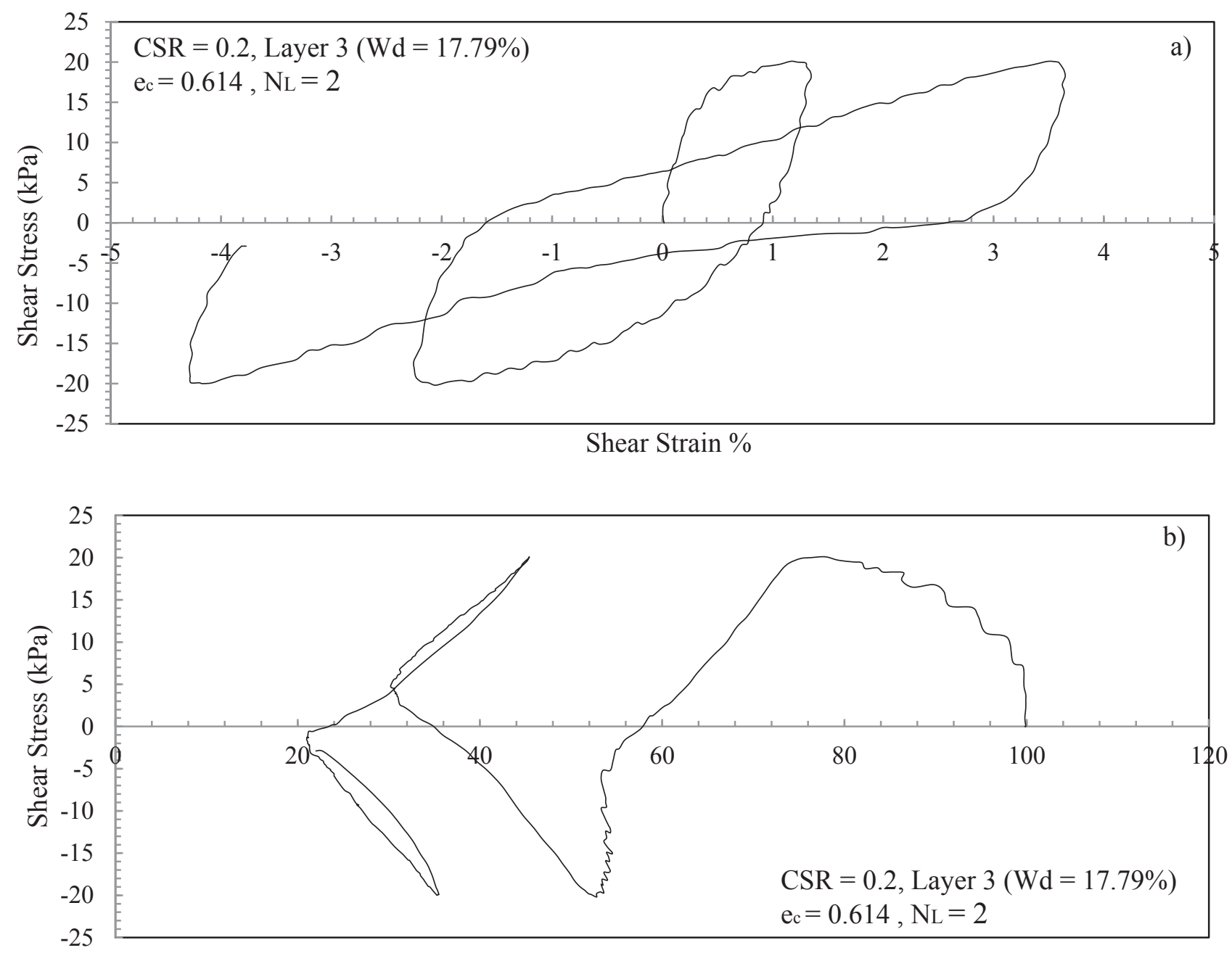

Effective Normal Stress $(\mathrm{kPa})$

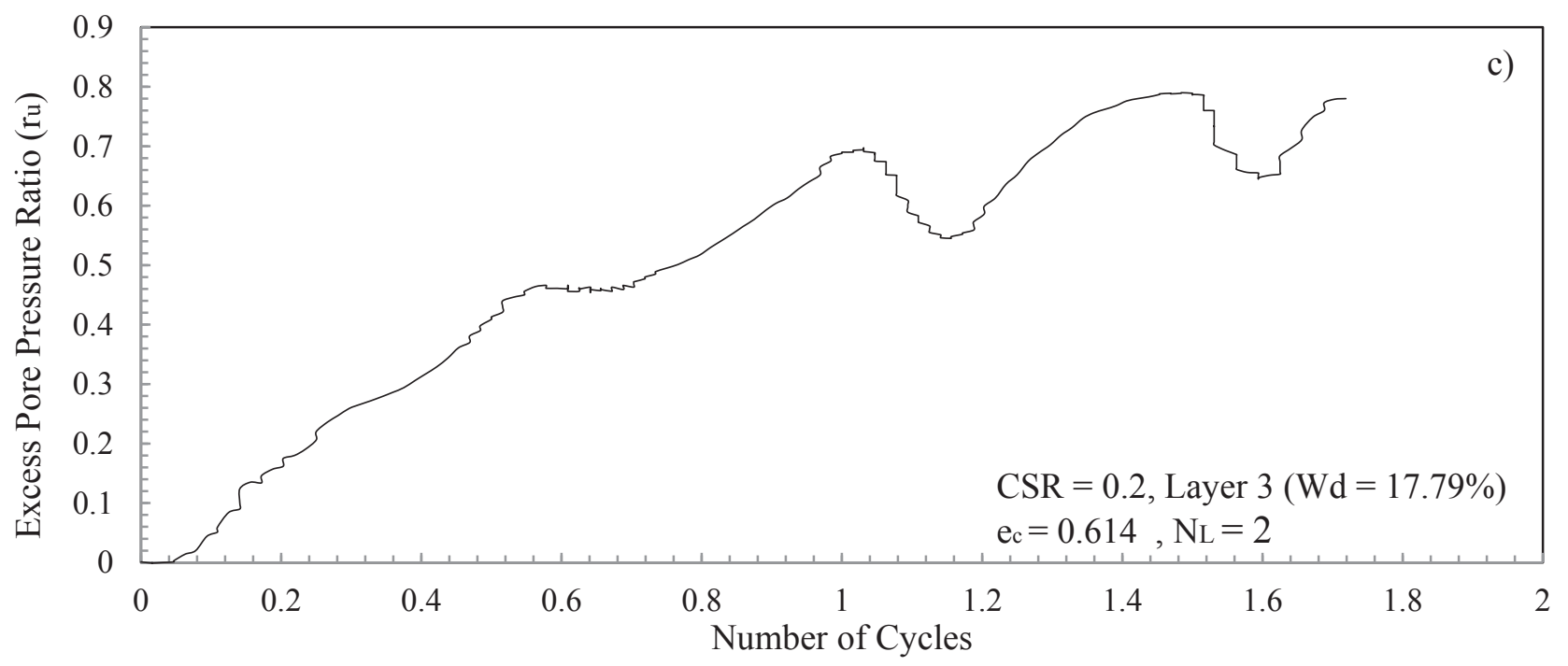

Figure AIV 17. Cyclic simple shear response of the sample obtained from Layer 3 at CSR $=0.2$ 


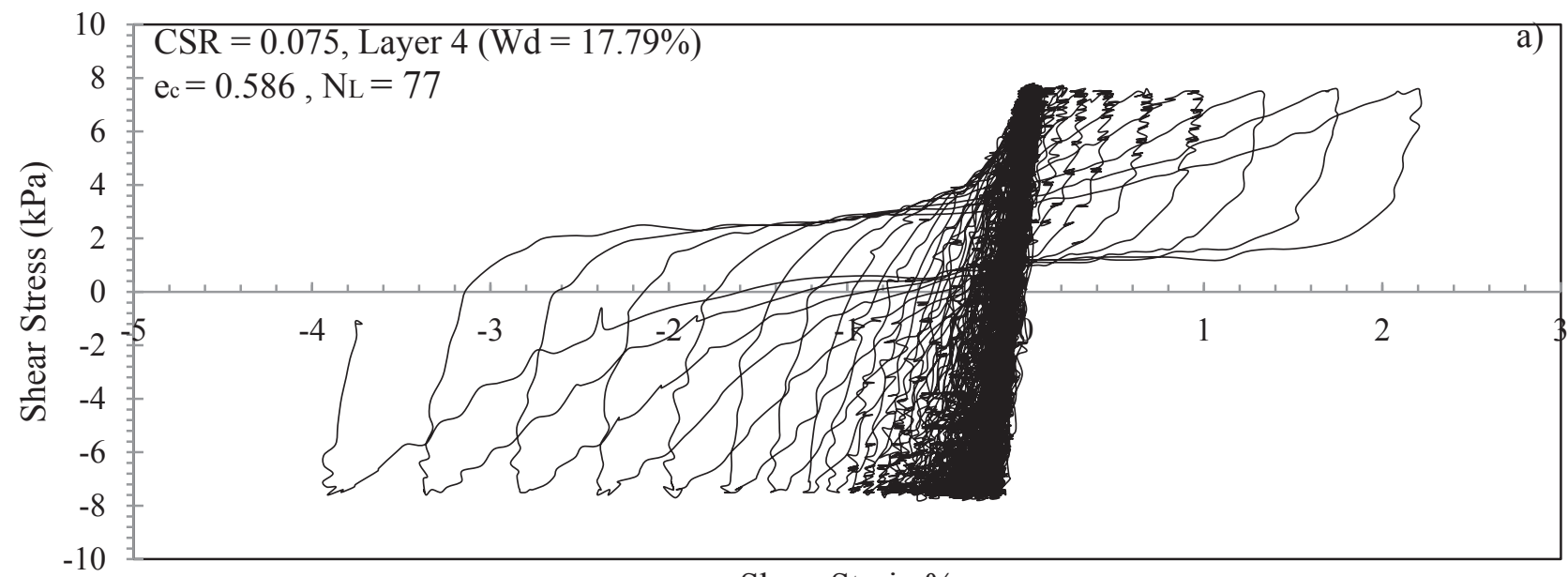

Shear Strain \%
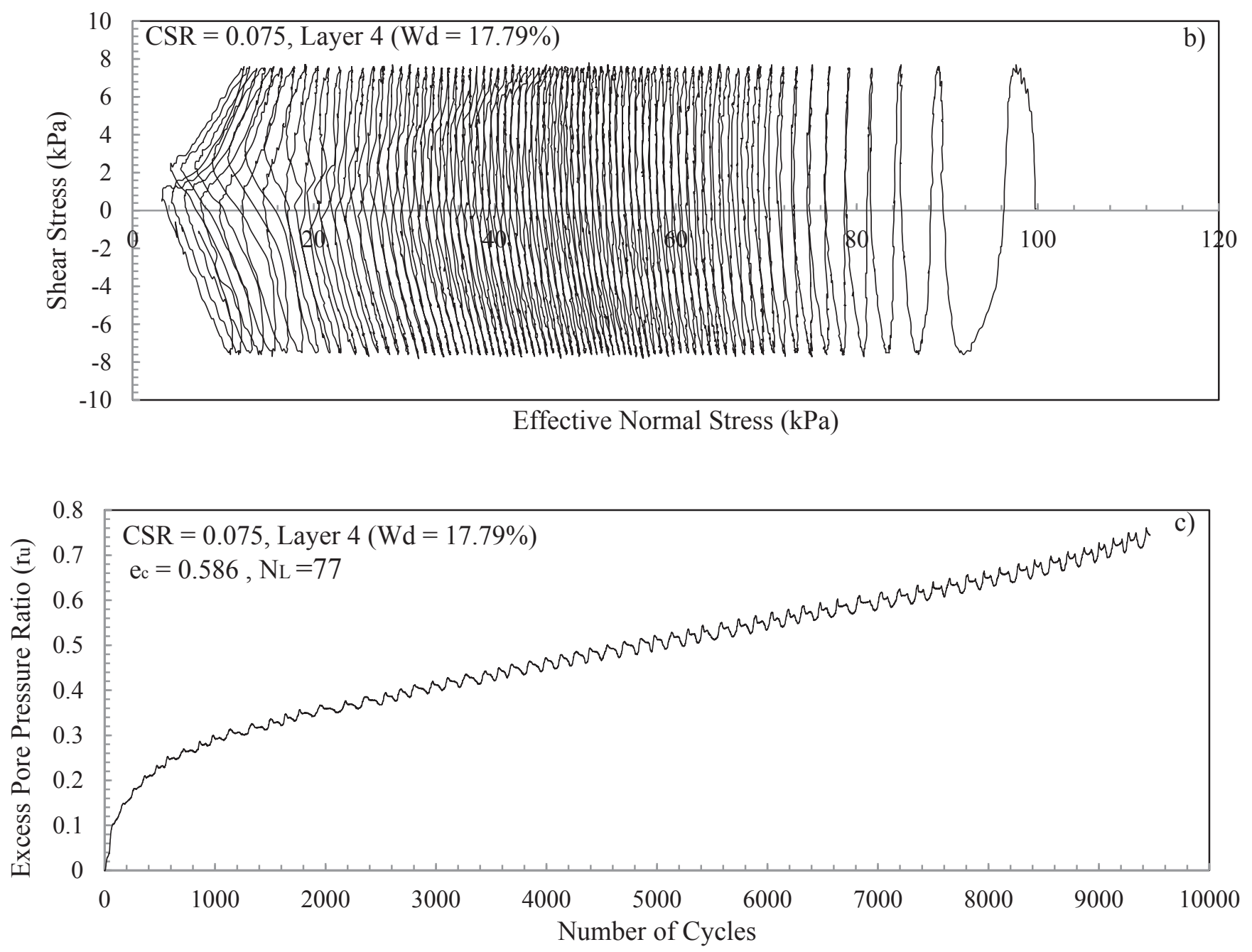

Figure AIV. 18 Cyclic simple shear response of the sample obtained from Layer 4 at CSR $=$ 0.075 

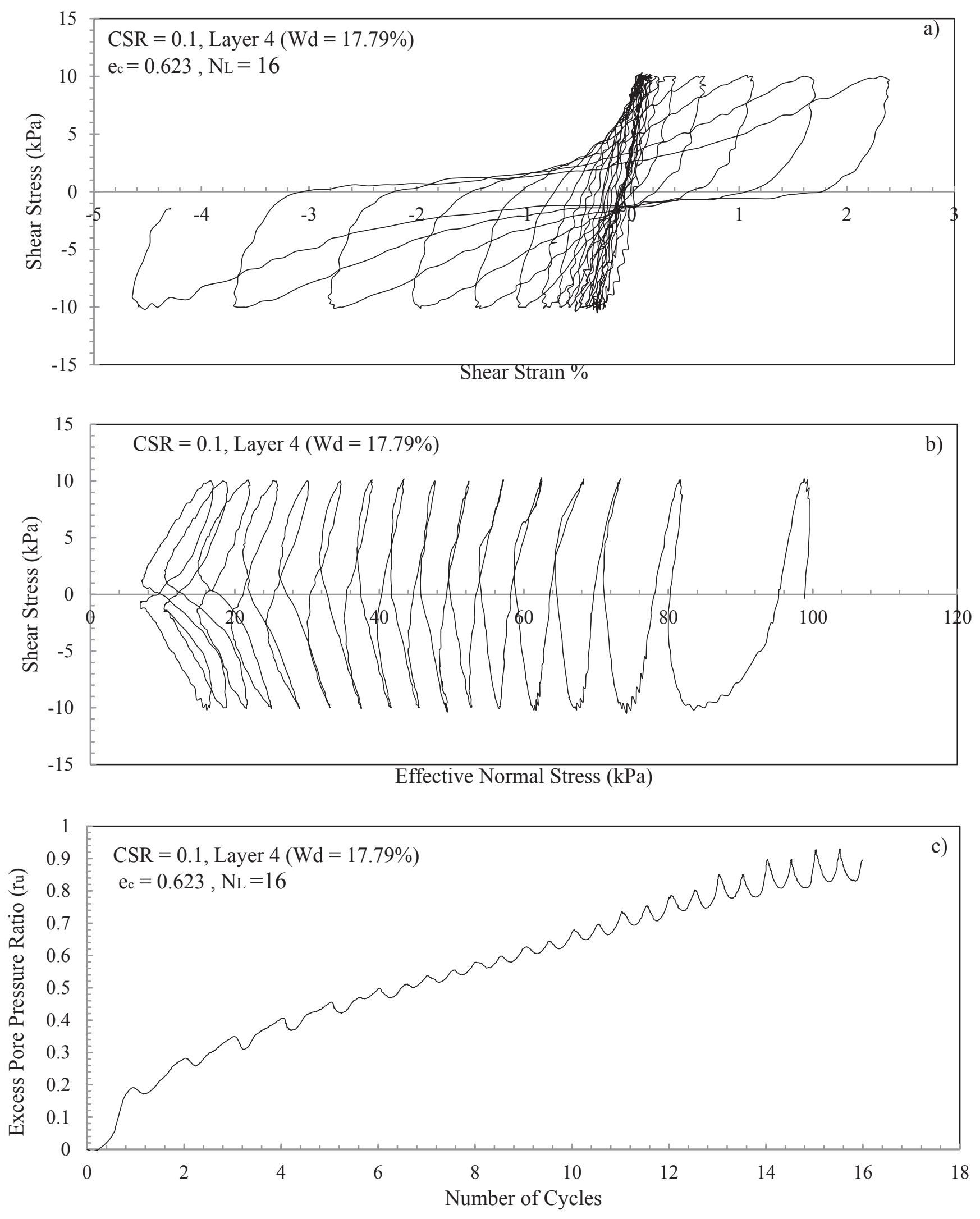

Figure AIV 19. Cyclic simple shear response of the sample obtained from Layer 4 at CSR $=0.1$ 

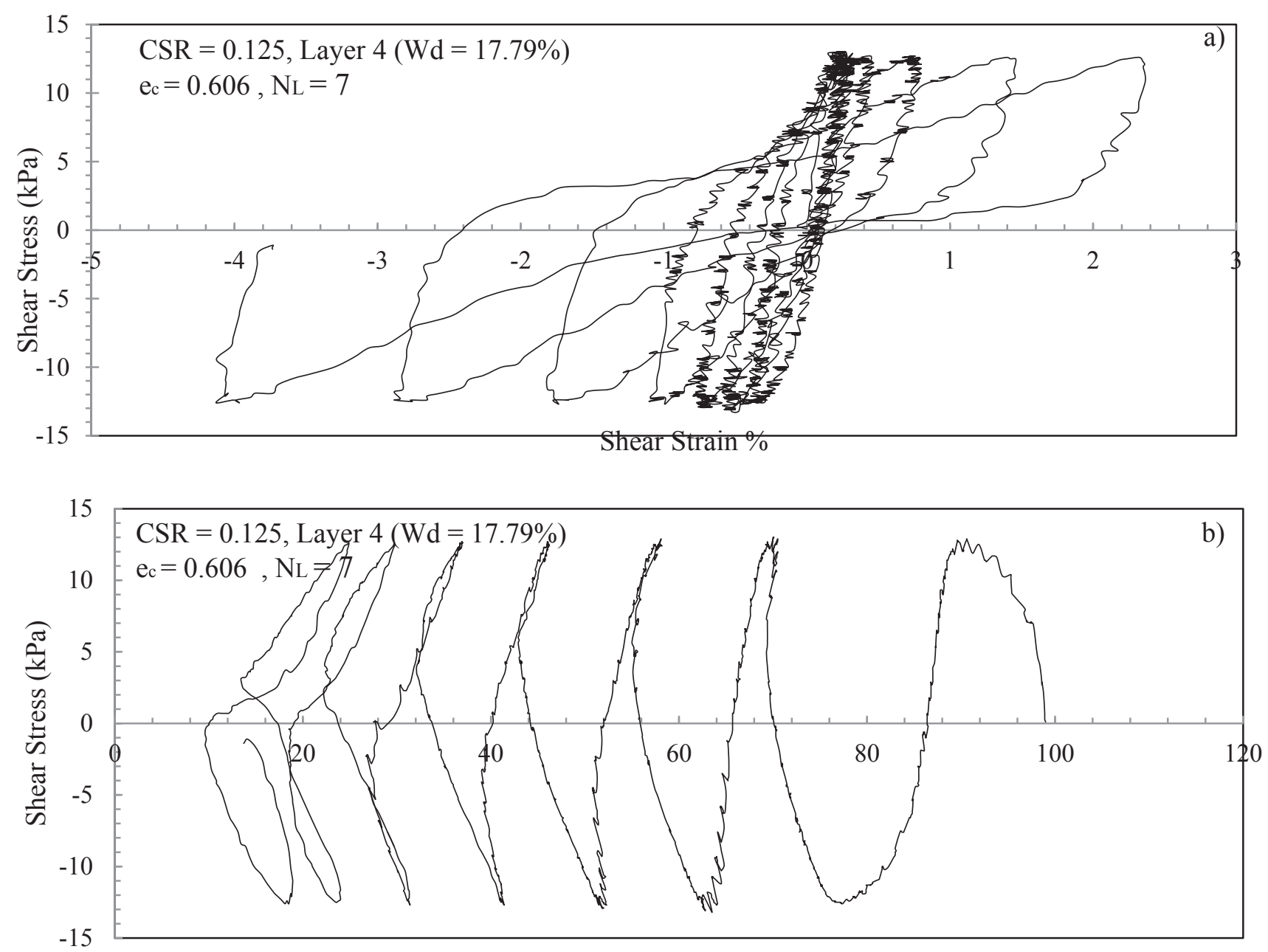

Effective Normal Stress $(\mathrm{kPa})$

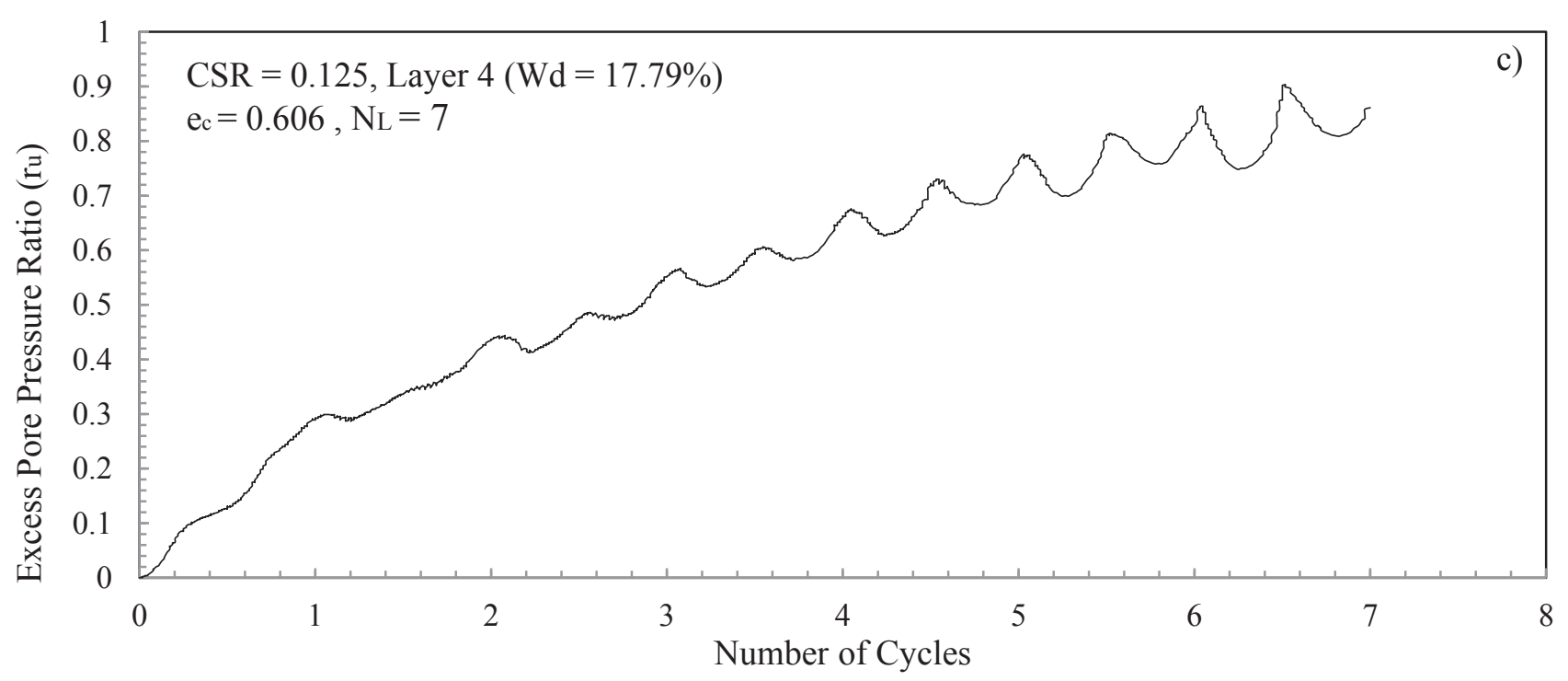

Figure AIV 20. Cyclic simple shear response of the sample obtained from Layer 4 at CSR $=$ 0.125 